Göttingen International Health Networt (GIHN) Uwe Groß and Kerstin Wydra (Eds.)

\title{
Maternal-Child Health
}

Interdisciplinary Aspects Within the Perspective of Global Health

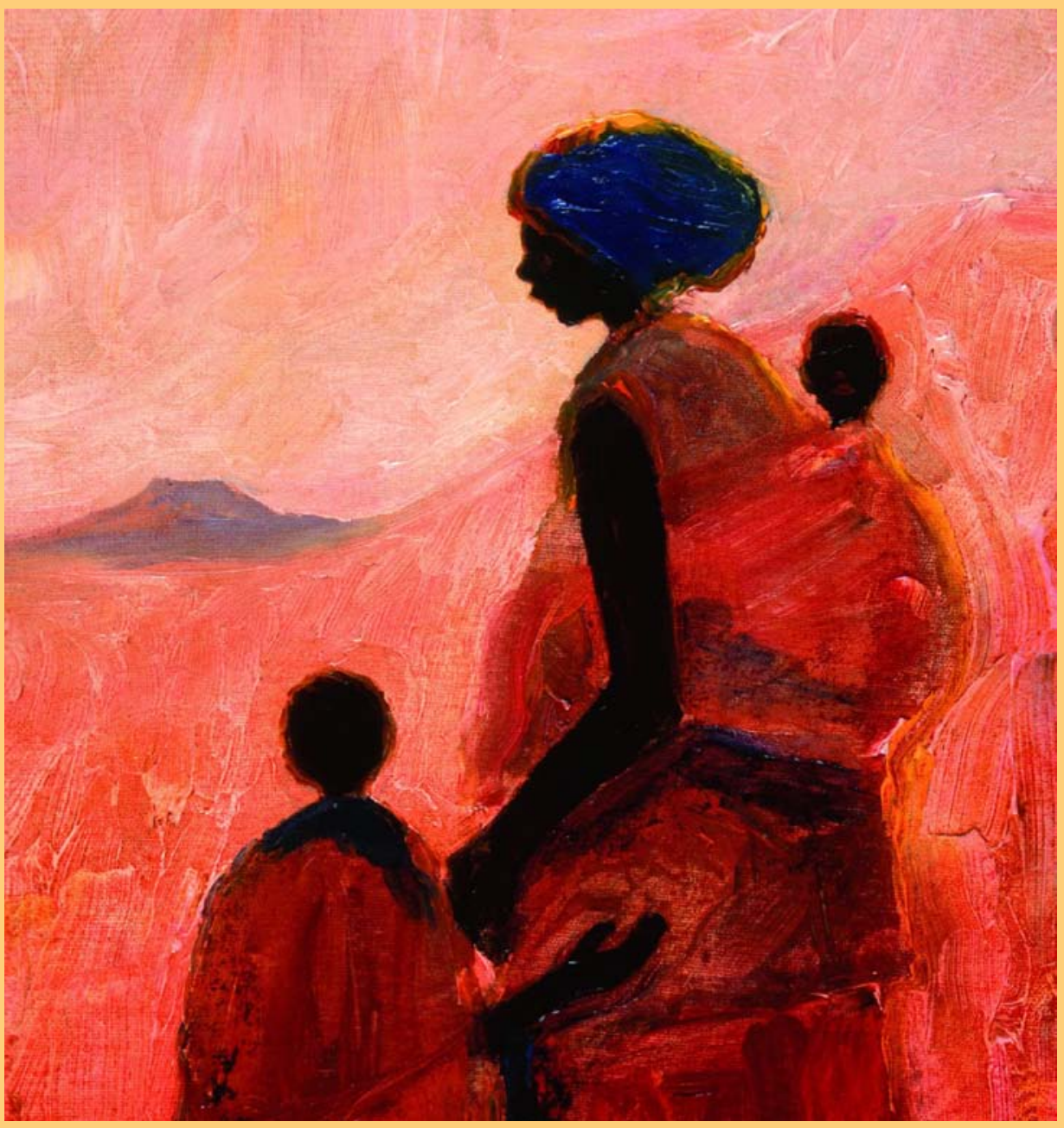

\section{Universitätsverlag Göttingen}



Göttingen International Health Network (GIHN)

Uwe Groß and Kerstin Wydra (Eds.)

Maternal-Child Health

This work is licensed under the Creative Commons License 3.0 "by-sa", allowing you to download, distribute and print the document.

(a)

SORIERIIGHISRESERVED 
erschienen im Universitätsverlag Göttingen 2013 
Göttingen International

Health Network (GIHN)

Uwe Groß and

Kerstin Wydra (Eds.)

\section{Maternal-Child Health}

Interdisciplinary Aspects Within the Perspective of Global Health

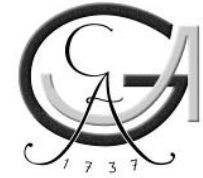

Universitätsverlag Göttingen 2013 


\section{Bibliographische Information der Deutschen Nationalbibliothek}

Die Deutsche Nationalbibliothek verzeichnet diese Publikation in der Deutschen Nationalbibliographie; detaillierte bibliographische Daten sind im Internet über $<$ http://dnb.ddb.de $>$ abrufbar.

Address of the Editor

Göttingen International Health Network

Prof. Dr. Uwe Groß

Institut für Medizinische Mikrobiologie

Universitätsmedizin Göttingen

Georg-August-Universität

Kreuzbergring 57

37075 Göttingen

e-mail: ugross@gwdg.de

www.gihn.net

This work is protected by German Intellectual Property Right Law.

It is also available as an Open Access version through the publisher's homepage and the Online Catalogue of the State and University Library of Goettingen

(http://www.sub.uni-goettingen.de). Users of the free online version are invited to read, download and distribute it. Users may also print a small number for educational or private use.

Setting and Layout: Josephine Meister

Cover: Franziska Lorenz

Cover Picture: Tony Hudson "Mother and Children"

(C) 2013 Universitätsverlag Göttingen

http:/ / univerlag.uni-goettingen.de

ISBN: 978-3-86395-084-2 


\section{Table of Content}

$\begin{array}{lr}\text { Editorial } & 5\end{array}$

$\begin{array}{lll}\text { I. Environment and Health } & 7\end{array}$

1. Maternal Mortality and Near Miss Events: the African Perspective 9

2. Nutrition, Sanitation and Reproductive Health in Maternal-Child Health: Challenges in Low Resource Settings

3. Water Sanitation Challenge: Prevalence of Diarrhoea in Children under Age 5 in Slums and Regular Localities in Enugu, South-East Nigeria

4. Improving Maternal and Child Health: The Role of Food Safety in the Developing World

5. Household Food Insecurity and Hunger: Implications for Maternal and Child Health in Nigeria

6. Children's Environmental Health Risks in Kenya 77

7. Metallic Iron for Clean Agriculture $\quad \mathbf{8 9}$

II. Socioeconomic Factors $\quad 99$

8. Maternal Health Care in Rural Areas of Tanzania: How do Beneficiaries Perceive the Quality of Services? 101

9. A Review of Selected Maternal and Child Health Indicators in Ghana 115

10. Health Financing and its Impact on Maternal and Child Health in Rwanda $\mathbf{1 3 7}$

11. Maternal and Child Health in Sub-Saharan Africa 145

12. Aspects of the Religious Context to Maternal-Child Health (Between Global and Local) 
13. Cultural Practices, Beliefs, Traditions and Norms in Atwima Nwabiagya District: A Blessing or a Curse for Maternal Health?

III. Infectious Diseases

14. Pregnancy-Related Infections

15. Malaria in Children and Adults - An Overview

16. Malaria in Pregnancy: The Parasite Infection Mechanism

17. Malaria and Blood Transfusion

18. The Role of Leptospirosis - Zoonotic Disease in Maternal-Child Health in Indonesia

19. Vertical Transmission of Hepatitis B Virus; the Role of Placental Barrier, Modes of Delivery, Viral Load \& Immunoprophylaxis of Neonates

20. Human Cytomegalovirus Infection in Pregnancy and in Newborns

21. Global aspects of Human Papillomavirus Infection in Feto-Maternal Health

22. Chlamydia Trachomatis Infection in Women

23. The Burden of Streptococcal Diseases in Maternal-Child Health in West Africa

24. Candidiasis in Pregnant Women

25. Salmonella Typhi Infection

26. Epidemiology and Characterization of Rotavirus Strains Causing Diarrhoea in Indonesia

27. Tuberculosis in Children

28. Diagnosis of Children Lung Tuberculosis based on Rapid Molecular Methods (MAS-PCR and Xpert MTB/RIF System)

29. The Role of the Tuberculosis Laboratory in Supporting the Clinical Examination of Various Specimens of Tuberculosis Patients in North Sumatera, Indonesia 
30. Buruli Ulcer: A Disease which Leads to Severe Disabilities and Mostly Affects Children Living in Wet Agro-Ecosystems of West and Central Africa.

31. Arboviral Infection during Pregnancy and Childhood: Implications for the Mother and Child

32. Social-Related and General Aspects of Ebola Virus in African Communities

33. Neglected Tropical Diseases (NTDs) in Women and Children

IV. Non-communicable Diseases

34. Significance of Iron Deficiency for Pregnancy and Childbirth in LowResource Countries

35. The Impact of Contraception on Maternal Mortality in Indonesia

36. Basic Sonography in Maternal Care in Resource-Poor Settings

37. Neonatal Health: The Key to Achieving Millennium Development Goal 4?

38. Ovarian Cancer in Pregnancy: What Should We Do?

39. Breast Cancer in Countries with Limited Resources 



\title{
Editorial
}

\author{
Uwe Gross and Kerstin Wydra
}

Improving maternal-child health as a challenging mission has been recognized by the United Nations already more than 10 years ago and resulted in the declaration of the Millennium Development Goals (MDG). Although much has been achieved already, several countries, especially those with low resources are far from track in reaching these goals, particularly when speaking of MDG 4 and 5.

Improving health conditions of pregnant women, young mothers, and their children requires multidisciplinary approaches. The Göttingen International Health Network (GIHN) is aiming to contribute to the MDG 4 and 5 by educational programmes including (human) capacity building, as well as by cooperating in scientific and clinical projects with partners from low- and middle-income countries. GIHN is formed by more than 30 scientists and clinicians, who come from 7 faculties of the Georg-August-University Göttingen (GAUG), including the University Medical Center Göttingen (UMG), and thus allowing interdisciplinary solutions from academia on questions related to Global Health.

Throughout the past years, several international summerschools focussing on interdisciplinary aspects of mother-child health, have been organized by GIHN in Ghana, Germany, Indonesia, and Tanzania. These summer schools enhanced our collaborations and broaden our understanding on maternal-child health and thus, lay the basis for collecting these findings and share them with a broad scientific community via a print AND open-access publication of this book. In this publication, more than 60 authors from 14 African, Asian and European countries reflect 
on the different dimension of maternal-child well-being within Global Health; its preconditions in our One World where the health of humans largely depends on the health of animals and plants, and thus being the result of adequate economic, ecological, social and cultural conditions. Therefore, to understand and interpret ONE Health requires a multifaceted view of scientists and clinicians, who should come from different disciplines. This book undertakes the challenge to reflect our view on how to tackle maternal-child issues and to improve their health conditions by bringing together experts from agricultural and environmental sciences, economical, social and theological sciences, biomedical and nutritional sciences, clinical human and veterinary medicine, as well as experts from epidemiology and the public health field. It is hoped that these interdisciplinary views of maternal-child health in a globalized world will offer ideas which finally will lead to new strategies on how to improve the health conditions of the most vulnerable populations.

This book consists of four sections (Environment and Health, Socioeconomic Factors, Infectious Diseases, and Non-Communicable Diseases) each including scientific reviews and original papers.

We thank the Federal Ministry for Economic Cooperation and Development (BMZ) and the German Academic Exchange Service (DAAD), particularly the DAAD Programme PAGEL for financially supporting this project including the realization of summer schools as well as the publication of this book. We would also like to express our appreciation to Gisela Bretzel and Helmut Eiffert for critically contributing to the review process of the manuscripts, Josephine Meister for her editing and fine-tuning the book chapters in endless hours, as well as Franziska Lorenz and Jutta Pabst as our publishers for their valuable advice on how to make such a book possible.

Göttingen and Erfurt, 1 September 2013

Uwe Groß and Kerstin Wydra 
I. Environment and Health 



\title{
1. Maternal Mortality and Near Miss Events: the African Perspective
}

\author{
Chibuike O. Chigbu, College of Medicine, University of Nigeria, \\ Enugu Campus, Nigeria.
}

\section{Maternal mortality}

Maternal mortality has remained a very important challenge to Africa and other resource-constrained regions of the world. Pregnancy and childbirth are special joyful and fulfilling life events for the African family. Maternal death is viewed as a very tragic and devastating event associated with enormous financial, social, emotional and psychological burden to the family.

The disparity in the health systems of developed and developing countries becomes obvious by comparing the statistics of maternal mortality in these two regions. Maternal mortality is rare in developed countries but sadly common in developing countries especially countries of sub-Saharan Africa. It is regarded by the United States Joint Commission on Accreditation of Health Care Organizations as a "sentinel event" as its application is a criterion to assess the quality of a healthcare system [1]. It is also a key indicator of population health, health system development, social and economic development. These functions are attributed to 
maternal mortality because it is almost entirely preventable due to adequate surveillance and intervention during pregnancy, childbirth and puerperium [2]. Surveillance and intervention mechanisms are well-integrated components of the health systems of developed countries. In sub-Saharan Africa, these mechanisms are very dysfunctional.

Maternal mortality became a global issue following the launch of the safe motherhood initiative in 1987 in Nairobi, Kenya. In 2000, world leaders met and reaffirmed their commitment to maternal mortality reduction with the Millennium Declaration of the United Nations General Assembly, the Millennium Development Goals (MDG). Reduction of maternal mortality was the focus of the $5^{\text {th }}$ Millennium Development Goal (MDG 5). While the safe motherhood initiative targeted a $50 \%$ reduction in maternal mortality by the year 2000, MDG 5 seeks to achieve a three-quarter reduction in global maternal mortality by the year 2015. To meet the target of MDG 5, a minimum annual decline rate of 5.5\% in maternal mortality ratio is required. Recent reports show an annual decline of $3.1 \%$ in maternal mortality ratio globally [3], and this falls short of the required minimum to achieve MDG 5. Africa is one of the poorest regions of the world and accounts for more than half of global maternal mortality [4]. Accruing evidence suggest that MDG 5 may not be achieved in Africa. Africa therefore deserves special attention in the global discourse on maternal mortality. This chapter will highlight the peculiarities of Africa and its special position in the global fight against maternal mortality and morbidity.

\subsection{Definitions and indices of maternal health measurement}

Maternal mortality: Maternal mortality is defined by the World Health Organization (WHO) as the death of a woman while pregnant or within 42 days of termination of pregnancy, irrespective of the duration and site of pregnancy, from any cause related to or aggravated by the pregnancy or its management, but not from accidental or incidental causes. Maternal mortality is further divided into direct and indirect maternal death.

Direct maternal death: Direct maternal death refers to maternal deaths resulting from obstetric complications of the pregnant state (pregnancy, delivery, postpartum), from interventions, omissions, incorrect treatments, or from a chain of events resulting from any of the above.

Indirect maternal death: These are maternal deaths resulting from previously existing diseases, or diseases that developed during pregnancy, and which are not due to direct obstetric causes but aggravated by physiological effects of pregnancy.

Pregnancy-related deaths: This refers to death of a woman during pregnancy, childbirth or the post-partum period, irrespective of the cause of death, including accidental and incidental causes. 
Late maternal death: This is defined as death of a woman from direct or indirect causes, more than 42 days but less than one year after termination of pregnancy.

\subsection{Measures of maternal mortality}

In determining the appropriate measures of maternal mortality, the main factors that determine a woman's lifetime risk of maternal death are taken into consideration. These factors are the risk of dying during a single pregnancy and the number of times a woman is exposed to such risks, i.e. the number of times she gets pregnant [5].

Maternal mortality ratio (MMR) reflects the risk of dying during a single pregnancy and is defined as the number of maternal deaths in a given time period divided by the number of live births during the same period. MMR = Number of maternal deaths / Number of live births.

Maternal mortality rate (MMRate) reflects both the risk of dying during a single pregnancy and the number of times a woman is exposed to such risks. It is defined as the number of maternal deaths divided by the number of women of reproductive age (age 15-49 years) in a population [5]. MMRate $=$ Number of maternal deaths / Number of women aged $15-49$ years.

\section{Global statistics on maternal mortality}

The global estimated total number of maternal deaths in 1990 was 543,000. This figure has declined to about 287,000 by 2010 [4]. The global drop in maternal mortality from the 1990 figure is $47 \%$. The global picture reflects huge disparities between developing and developed countries of the world. The maternal mortality ratio in developing countries is 240 per 100,000 births compared with 16 per 100,000 births in developed countries. A woman's life time risk of maternal death is 1 in 150 in developing countries compared to 1 in 3,800 in developed countries [4].

\section{African statistics on maternal mortality}

Almost all maternal deaths (99\%) occur in developing countries and more than half of these deaths occur in sub-Saharan Africa. Africa appears to be the least in the progress towards the attainment of the MDG 5. Most sub-Saharan African countries are not on track towards the achievement of MDG set targets due to poor commitments by governments of these nations to MDG 5 and poor translation of commitments where they exist to progressive action. Between 1990 and 2008, Africa recorded a $1.6 \%$ decline in maternal mortality ratio [6]. This is the least amongst developing regions of the world. Asia recorded $4.0 \%$ decline while Latin America and the Caribbean posted a 2.9\% decline. Sub-Saharan Africa is the greatest contributor to the poor performance of Africa in MDG 5, with an estimated annual decline of $1.7 \%$. North Africa's annual decline is $5.0 \%$. Despite the 
estimated decline in MMR in Africa over the period under consideration, Africa's contribution to total global maternal deaths is on the increase. Africa contributed $36 \%$ of global maternal deaths in 1990. In 2008, this figure has risen to $57 \%$ [6]. This increase is as a result of a regional shift in the burden of maternal mortality. A rising birth rate and slower decline in MMR, when compared to other regions of the world, are contributors to the regional shift. Between 1990 and 2008, the total number of births in sub-Saharan Africa increased by $1.5 \%$ per year, from 23 million to 32 million, while that of Asia decreased by $0.5 \%$ per year from 82 million to 74 million. Therefore, Asia's contribution to the total global maternal deaths fell from $58 \%$ in 1990 to $39 \%$ in 2008 [6].

In analyzing maternal mortality figures from Africa, one must take into consideration the fact that the figures might be under-estimates in some cases. This is due to difficulties associated with accurate and reliable collection of data in Africa, especially sub-Saharan Africa. Health care facilities are major sources of maternal mortality data from Africa. The under-utilization of health care facilities in Africa is well-known. Less than $50 \%$ of women in Africa receive skilled care during childbirth [7]. This translates to millions of births in non-formal health facilities. It is therefore possible that many cases of maternal deaths occurring in these facilities and attended to by unskilled birth attendants might not be captured in the maternal mortality data. Furthermore, formal data collection and collation mechanisms in many African countries are standardized in principle but not in practice. A lot more still needs to be done in this regard.

Table 1: Maternal mortality figures in Africa 2010.

Source: CIA World Factbook, January 2012.

\begin{tabular}{lrr}
\hline Country & $\begin{array}{c}\text { Annual } \\
\mathbf{1 0 0 , 0 0 0} \text { live births }\end{array}$ & death \\
\hline Chad & 1,100 & \\
Somalia & 1,000 & \\
Sierra Leone & 890 & \\
Central African Republic & 890 \\
Burundi & 800 & \\
Guinea-Bissau & 790 \\
Liberia & 770 \\
Sudan & 730 \\
Cameroon & 690 \\
Nigeria & 630 \\
Lesotho & 620 \\
Guinea & 610 \\
\hline
\end{tabular}




\begin{tabular}{|c|c|}
\hline Country & $\begin{array}{l}\text { Annual maternal death per } \\
100,000 \text { live births }\end{array}$ \\
\hline Niger & 590 \\
\hline Zimbabwe & 570 \\
\hline Congo, Republic of the & 560 \\
\hline Congo, Democratic Republic of the & 540 \\
\hline Mali & 540 \\
\hline Mauritania & 510 \\
\hline Mozambique & 490 \\
\hline Malawi & 460 \\
\hline Tanzania & 460 \\
\hline Angola & 450 \\
\hline Zambia & 440 \\
\hline Cote d'Ivoire & 400 \\
\hline Senegal & 370 \\
\hline Kenya & 360 \\
\hline Gambia, The & 360 \\
\hline Ghana & 350 \\
\hline Benin & 350 \\
\hline Ethiopia & 350 \\
\hline Rwanda & 340 \\
\hline Swaziland & 320 \\
\hline Uganda & 310 \\
\hline Burkina Faso & 300 \\
\hline Togo & 300 \\
\hline South Africa & 300 \\
\hline Comoros & 280 \\
\hline Equatorial Guinea & 240 \\
\hline Eritrea & 240 \\
\hline Madagascar & 240 \\
\hline Gabon & 230 \\
\hline Djibouti & 200 \\
\hline Namibia & 200 \\
\hline Botswana & 160 \\
\hline Morocco & 100 \\
\hline Algeria & 97 \\
\hline Cape Verde & 79 \\
\hline Sao Tome and Principe & 70 \\
\hline Egypt & 66 \\
\hline Mauritius & 60 \\
\hline Libya & 58 \\
\hline Tunisia & 56 \\
\hline
\end{tabular}




\section{Causes of maternal mortality}

There are various causes of maternal mortality. The relative importance and distribution of each identified cause of maternal mortality varies from region to region and from country to country. There are clear differences in the distribution of causes of maternal mortality between developed and developing countries [7]. A systematic analysis which was organized by the World Health Organization showed that causes classified under "other direct causes" ranked highest in maternal etiology of maternal mortality in developed countries. These "other direct causes" mainly refer to complications arising from interventions like those relating to caesarean section and anesthesia. These account for $21 \%$ of maternal mortality in developed countries, followed by hypertensive disorders and embolism [8].

In Africa, hemorrhage is the leading cause of maternal deaths, accounting for $33.9 \%$ of maternal deaths, followed by "other indirect causes" and sepsis. HIV/AIDS is also an important cause of maternal death in Africa and it contributes to $6.2 \%$ of maternal mortality [8]. It is important to note that in developed countries, Asia and Latin America, the contribution of HIV/AIDS to maternal mortality is $0 \%$. Although it is possible that some of the deaths resulting from $\mathrm{HIV} / \mathrm{AIDS}$ in the regions reporting $0 \%$ might have been misclassified; the impact of HIV/AIDS on maternal mortality in Africa should not be underestimated. In Africa, HIV/AIDS remains a huge problem. Stigmatization and under-reporting are key issues. Documented proportions of maternal death caused by HIV/AIDS

Table 2: Causes of maternal deaths in Africa and developed countries

\begin{tabular}{lcr}
\hline $\begin{array}{l}\text { Cause of maternal } \\
\text { death }\end{array}$ & $\begin{array}{l}\text { Africa } \\
\text { (Percentage) }\end{array}$ & $\begin{array}{c}\text { Developed countries } \\
\text { (Percentage) }\end{array}$ \\
\hline Hemorrhage & 33.9 & 13.4 \\
Other indirect causes & 16.7 & 14.4 \\
Sepsis & 9.7 & 2.1 \\
Hypertensive disorders & 9.1 & 16.1 \\
HIV/AIDS & 6.2 & 0 \\
Unclassified deaths & 5.4 & 4.8 \\
Other direct causes & 4.9 & 21.3 \\
Obstructed labor & 4.1 & 0 \\
Abortion & 3.9 & 8.2 \\
Anemia & 3.7 & 0 \\
Embolism & 2.0 & 14.9 \\
Ectopic Pregnancy & 0.5 & 4.9 \\
\hline
\end{tabular}

Adapted from: Khan KS, Wojdyla D, Say L, Gülmezoglu AM, Van Look PF. WHO analysis of causes of maternal death: a systematic review. Lancet 2006, 367(9516):1066-74. 
in Africa are probably underestimates. The major causes of maternal deaths in this continent are largely preventable.

\subsection{Non-medical causes of maternal mortality}

The medical causes of maternal mortality enumerated above may not tell the entire story about the etiology of maternal mortality in Africa. A comprehensive look will reveal a confluence of scenarios operating collectively and together with the medical causes to ensure that pregnant African women remain endangered species. These scenarios are operational at the individual level, family level, community level and national level. They are manifested in weak health systems, poor infrastructure, family and communal poverty, poor education and cultural barriers. The 3-D (3-delay) model captures most of these levels of neglect [9]. The first delay is at the individual/family and community level and this represents delay in seeking skilled maternity care. The second delay is the delay in reaching an appropriate health facility and the third delay is at the level of the health facility and represents delay in prompt institution of appropriate interventions.

Delay in seeking appropriate care: Why do many African women delay in seeking care? The low status of the African woman at the family and community level is the starting point on the road to maternal death in Africa. Illiteracy and poverty are implicated as major determinants of maternal mortality in Africa [10,11]. Illiteracy runs a vicious cycle with poverty. Many women in Africa are illiterate (cannot read nor write). Chad, Burkina Faso, Niger, South Sudan, Benin, Sierra Leone, Senegal Somalia, Gambia, Mozambique, Central African Republic, Somalia and Nigeria have female literacy rates ranging between $13 \%$ and $50.4 \%$ [12]. Poor education leads to poverty and poverty hinders the education of the girl child. Illiteracy, poverty and gender inequality work together to maintain the low status of the African woman. Poor education makes it difficult for many African women to recognize obstetric emergencies, and to recognize the importance of skilled maternity care, as well as access relevant information on maternal health. Poverty means that cost becomes an important consideration in seeking medical help during obstetric emergencies, and will lead to delays. Gender inequality guarantees that the African woman has no control over her health. In many African societies, decision to seek maternity care can only be made by the men [13]. Imagine a pregnant African woman (Mrs. A), living in a remote rural African village, who suddenly started bleeding and needs to wait for her husband to return from work to decide if and where she will receive medical care. She may not take the vital and life-saving decision to visit a health facility probably because she does not have the full autonomy to such decisions [13]. Even where the family or society allows her such autonomy, she may not have the money to pay for the services at the health facility. Most health facilities operate a 'pay at the point of service' policy. This policy entails that medical services are paid for before they are rendered. Where money is available within the family, it might have been earmarked for a vital purpose like payment of 
older children's school fees or payment of previous family loans. These will contribute to delay in Mrs. A seeking appropriate maternity care for her emergency obstetric condition. Such delays can sometimes end up in fatalities and maternal death.

Delay in reaching an appropriate health facility: sub-Saharan African nations have very poor road infrastructure and poor transport systems. In rural communities, functional health facilities, where available, may not be within reasonable distance. The word functional is important here because many primary health facilities are located for political reasons and are very ill-equipped and poorly staffed. Even when Mrs. A above has overcome the barriers to the first type of delay, she may still not get to the nearest health facility in time. It may take several hours for her to arrange transport to the health facility. When the transport becomes available, the bad road to the health facility may become a serious issue to be contend with.

Delay in receiving adequate care in a health facility: the majority of health facilities in Africa exist only in name. They are poorly staffed, electricity and water supplies are virtually non-existent, and medical supplies are unavailable. Even in some uncommon situations where everything is available, bureaucratic bottlenecks and attitude of the medical staff may become serious issues causing avoidable and fatal delays. If Mrs. A eventually gets to a health facility alive, she may not leave the facility alive due to the unavailability of appropriate staff to assist her, unavailability of appropriate medical supplies and blood bank services, poor coordination between the various cadres of health staff, extensive and tardy bureaucratic processes, electricity and/or water outage or unavailability. At the end, hemorrhage would be recorded as the cause of maternal death.

Mrs. A's counterpart in a developed country would have called emergency medical services immediately she noticed the bleeding. She would have been picked up by an air or vehicle ambulance within minutes and appropriate interventions instituted even while in the ambulance. She may not have to make any direct payments for the cost of her health care. Her survival and that of the baby is guaranteed.

\subsection{Maternal mortality as a human right issue}

Maternal mortality is no longer seen as only a human development issue. Maternal mortality is now considered a human rights issue. Maternal death is viewed through the prism of denial of rights of women to health, equality and nondiscrimination. It is estimated that about $74 \%$ of maternal deaths are preventable. Preventable maternal death therefore represents a violation of a woman's right to life. Many international conventions and treaties recognize the right to the highest attainable standard of health as a fundamental human right and maternal health is entrenched within the right to highest attainable health [14].

In Africa, maternal mortality within the context of human right violation bears particular mention. "There is no single cause of death and disability for men be- 
tween the ages of 15 and 44 that is close to the magnitude of maternal death and disability" [14]. Obvious inequalities in the exercise of the right to highest attainable health do exist between men and women in Africa, with the men enjoying these rights while the women are left at the mercy and dictates of the men. The patriarchal culture of male dominance and privilege existent in many African societies ensures the persistence of these inequalities at the family and community levels [13]. The low status and subordination of women make it difficult for women to be placed in decision-making positions in African politics and governance. Thus, correction of these inequalities through appropriate legislation and policy formulation at the national level has been difficult. It is no surprise then that the most marginalized women are the most vulnerable to maternal deaths in Africa. These include indigent women, and those from ethnic minorities, as well as those dwelling in rural communities [15]. These women are also those most exposed to 'women-unfriendly' patriarchal systems and policies.

Many African nations are signatories to a variety of international treaties that legally guaranty the right to highest attainable standard of health. This right is also recognized in the national constitutions of many African countries. In these nations, it is the responsibility of the state to ensure adequate provision of goods and services required to prevent maternal death. Emergency obstetric care is one amongst many of these services. Almost all sub-Saharan African countries are clearly in violation of these treaties. It is time for the world to call African governments to change in this regard.

\subsection{Cross-linking environment, agriculture and maternal mortality and morbidity}

Environmental issues like climate change, deforestation, water scarcity, water pollution (e.g. by crude oil exploration), decreasing biodiversity and soil erosion are taking heavy tolls on Africa leading to low agricultural outputs. Africa is one of the continents of the world that have continued to show the largest net losses of forests. The majority of African women live in rural and semi-rural settings. These women rely almost completely on agriculture for daily survival, which in turn relies on favorable environment. The day-to-day well-being of these women is closely tied to the natural environment. Consequently, adverse environmental changes lead to disproportionately high levels of poverty, hunger, poor nutrition and economic deprivation. Gender inequality existing in many African countries ensures that these adverse effects are tilted more to the disadvantage of women and the girlchild. Poor family income in most African societies translates to less education for the girl-child, prostitution and early marriage. Poor nutrition limits the development of the female pelvis and together with early marriage sets the stage for obstructed labor. Utilization of maternal health services decreases with decreasing education [13]. 


\subsection{Prevention of maternal mortality in Africa}

Since the launch of the safe motherhood initiative in 1987 in Nairobi, Kenya, reduction of maternal mortality in Africa and other developing regions has been an international priority. A wide variety of efforts have been made towards reducing maternal mortality in Africa. Some of these efforts have met with limited successes. The safe motherhood initiative, which goal is to reduce maternal mortality by $50 \%$ by the year 2000 did not achieve its target at destination time. At the current rate, the MDG 5 target may also not be achieved. The interventions needed to prevent maternal deaths are well known. The commitment of governments of African nations towards the reduction of maternal mortality is poor. Where adequate commitments exist, translating such commitments into results is the major problem. Provision of essential obstetric care will not be enough to reduce maternal mortality as provision can not always be translated into utilization. Provision of essential obstetric care must be coupled to effective utilization to achieve success. The quality of the health care systems as well as the general social infrastructure must be adequately addressed. Female education and empowerment (both economic and social empowerment) should be an essential part of any prevention strategy in Africa. This will be the most effective way to eliminate type 1 delay from the road to maternal death.

Provision of safe and timely physical access to health care will eliminate type 2 delays. This should be in the context of provision of adequate social infrastructure like good roads, functional transport systems, security, equitable distribution of health facilities, and functional and effective referral systems between health facilities.

Functional and effective maternal health care facilities that provide adequate emergency obstetric care services will be key to eliminating type 3 delays. Adequate number of well-trained, well-remunerated and motivated maternal health care professionals, availability of quality assurance mechanisms within the health care system, availability of essential medicines and supplies, and elimination of pay at the point of service policies should form the back bone of interventions to prevent type 3 delays.

The interventions at the different levels of delays enumerated above must be well integrated and coordinated in a holistic manner and high-level integrity ensured in the implementation process, in order to achieve meaningful successes in maternal mortality reduction in Africa.

\section{Maternal near miss: definition of indicators}

Maternal near miss (MNM): This refers to a woman who nearly died but survived a complication that occurred during pregnancy, childbirth or within 42 days of termination of pregnancy. 
Severe maternal outcome: This is a life-threatening condition including all maternal deaths and maternal near-miss cases.

Women with life-threatening conditions (WLTC): This refers to all women who either qualified as maternal near-miss cases or those who died. These are women with severe maternal outcome. It is the sum of maternal near-miss and maternal deaths. Severe maternal outcome ratio (SMOR): The number of women with life-threatening conditions per 1,000 live births. This indicator gives an estimate of the amount of care and resources that would be needed in an area or facility.

Maternal near miss ratio (MNMR): This is the number of maternal near-miss cases per 1,000 live births. Maternal near miss ration also gives an estimation of the amount of care and resources that would be needed in an area or facility.

Maternal near-miss mortality ratio: This refers to the ratio between maternal near miss cases and maternal deaths.

Classification of maternal near miss: The world health organisation has identified three distinct approaches to identifying maternal near miss. These are disease-specific criteria, intervention based or management specific criteria and organ-system dysfunction criteria. Each criterion has its merits and demerits. The organ system dysfunction based approach appears to be the most popular.

African Perspective: There is a growing interest in near miss events in recent times. For the developed countries, this interest stems from the rarity of maternal mortality. Maternal deaths are now rare events in developed countries and may not be sufficient to assess the quality of care rendered by the health system; hence the need for near miss events as an adjunct to maternal mortality. In developed countries, maternal near miss is seen as a credible alternative to maternal mortality in quality of care assessment.

The growing interest in maternal near miss in Africa is not due to rarity of maternal deaths. Maternal deaths in Africa remain high enough for quality assessment. However, maternal near miss is being considered as a useful outcome measure and complimentary to maternal mortality. Maternal deaths in Africa usually result from regular life-threatening complications. Therefore more robust conclusions on the road to maternal death in Africa can be drawn from maternal near miss audits [16, 17]. Reliable data on maternal near miss is scarce in Africa. Some countries are just beginning to appreciate the importance of reliable and accurate maternal near miss data in maternal health and MDG 5. In Nigeria, the process of creating a national data system on maternal near miss is progress. More African countries should be encouraged to create national data systems on maternal near miss. Such data are invaluable towards MDG 5. It could serve as reference points for measuring progress, policy making and advocacy [18]. 


\section{References}

1. The Joint Commission (2010). Sentinel alert, issue 44: preventing maternal deaths. January 26.

2. Hill K, Thomas K, AbouZahr C, Walker N, Say L, Inoue M et al. (2007). Estimates of maternal mortality worldwide between 1990 and 2005: an assessment of available data. Lancet; 370(9595):1311-9.

3. World Health Organization (2012). Global Health Observatory. Available at

www.who.int/gho/maternal_health/mortality/maternal_mortality_text/e n/index.html [Accessed November 20, 2012].

4. World Health Organization (2012). Maternal mortality. Fact sheet No 348.

5. World Health Organisation (2007). Maternal mortality in 2005. Estimates developed by WHO, UNICEF, UNFPA, and the World Bank.

6. Wilmoth JR, Mizoguchi N, Oestergaard MZ, Say L, Mathers CD, ZureickBrown S (2012). A new method for deriving global estimates of maternal mortality. Statistics, Politics and Policy. Vol.3: Iss. 2, Article 3.

7. World Health Organization (2012). Global Health Observatory. Available at www.who.int/gho/maternal_health/en/index.html [Accessed November 20, 2012].

8. Khan KS, Wojdyla D, Say L, Gülmezoglu AM, Van Look PF (2006). WHO analysis of causes of maternal death: a systematic review. Lancet, 367(9516):1066-74.

9. United Nations Fund for Population Activities (2003). Maternal mortality update 2002, a focus on emergency obstetric care. Available at www.unfpa.org/upload/lib_pub_file/201_filename_mmupdate-2002.pdf [Accessed November 20, 2012].

10. Karlsen S, Say L, Souza JP, Hogue CJ, Calles DL, Gülmezoglu AM, Raine $\mathrm{R}$ (2011). The relationship between maternal education and mortality among women giving birth in health care institutions: analysis of the cross sectional WHO Global Survey on Maternal and Perinatal Health. BMC Public Health; 11:606.

11. Buor D, Bream K (2004). An analysis of the determinants of maternal mortality in sub-Saharan Africa. J Womens Health (Larchmt); 13(8):92638.

12. Central Intelligence Agency. The World Factbook. Available at https://www.cia.gov/library/publications/the-worldfactbook/fields/2103.html [Accessed December 12, 2012]. 
13. Chigbu CO, Onah HE (2009). Ethical and social issues facing obstetricians in low-income countries. Clin Obs Gynecol 52(2): 237-249.

14. Yamin SA, Maine D (1999). Maternal mortality as human rights issue: measuring compliance with international treaty organizations. Human rights quarterly; 21(3).

15. Hunt P, De Mesquita JB (2012). Reducing maternal mortality: the contribution of the right to the highest attainable standard of health.

UNFPA. Available at www.unfpa.org/webdav/site/global/shared/documents/publications/red ucing_mm.pdf [Accessed November 25, 2012].

16. Cochet L, Pattinson RC, MacDonald AP (2003). Severe acute maternal morbidity and maternal death audit-a rapid diagnostic tool for evaluating maternal care. S Afr Med J, 93: 700-702.

17. Vandecruys HIB, Pattinson RC, MacDonald AP, Mantel GD (2002). Severe acute maternal morbidity and mortality in the Pretoria academic complex: changing patterns over 4 years. Eur J Obstet Gynecol Reprod Biol, 102: 6-10.

18. Oladapo OT, Olalekan OA, Fakeye O, Ekele BA, Fawole AO, Abasiattai A et al. (2009). National data system on near miss and maternal death: shifting from maternal risk to public health impact in Nigeria. Reprod Health, doi: 10.1186/1742-4755-6-8. 



\title{
2. Nutrition, Sanitation and Reproductive Health in Maternal-Child Health: Challenges in Low Resource Settings
}

\author{
Stellamaris Muthoka and Elizabeth Kamau-Mbuthia, Department of Human Nutri- \\ tion, Egerton University, Kenya \\ Wilkister Moturi, Department of Environmental Science, Egerton University, Kenya \\ Douglas K. Ngotho, Department of Reproductive Health, Egerton University, Kenya
}

\section{Introduction}

Maternal, new born and child health continue to be a major concern globally. This has given rise to many interventions towards alleviating maternal and child morbidity and mortality. The interventions also aim to address the Millennium Development Goals (MDGs) by 2015. WHO (2010) reported that although globally there has been some progress on maternal and child health over the past decade (19902008) - much still needs to be done to reach targets set by the UN and WHO on halving 1990's levels of child and maternal morbidity and mortality by 2015 . Although the global maternal mortality has decreased by $34 \%$ since 1990, it still remains high at 358,000 deaths in 2008. South Asia and Sub-Saharan Africa accounted for $87 \%$ of the burden of maternal deaths in 2008 , and in total ninety nine percent of the maternal deaths have been occurring in developing countries (WHO, 
2010). Poor maternal pre-pregnancy and post delivery nutrition status and health are major predictors of child morbidity and mortality, with low access to safe water and poor environmental sanitation making the situation worse.

WHO (2002) estimates that environmental risk factors account for $25 \%$ of the overall burden of disease, and $30 \%$ of that burden falls on children under-five, particularly in developing countries. Globally, some of the preventable risks include childhood and maternal underweight; unsafe water, sanitation and hygiene; high iron deficiency and overweight/obesity.

These health risks have been mainly subject to the developing world, however, there is a growing number of risks which have become global. Some 170 million children in poor countries are underweight, mainly from lack of food, while more than one billion adults worldwide - in middle income and high income countries alike are overweight or obese. About half a million people in North America and Western Europe die from overweight/obesity-related diseases every year (WHO, 2002).

Intervention is necessary now, otherwise $\mathrm{WHO}$ warns that inaction is likely to result to loss of up to 110 million underweight children by the year 2020 which is unacceptably high. The Joint Monitoring Programme for water supply and sanitation 2010 estimates 1.2 billion people used unsafe water from sources or systems with significant sanitary risks by 2010 and that the set goal is not achievable despite efforts made (WHO/UNICEF, 2010; Onda, 2012) .

Approximately 3.1 per cent of deaths (1.7 million) and 3.7 per cent of Disability Adjusted Life Years (DALYs $\left.{ }^{1}\right)$ (= 54.2 million) worldwide are attributable to unsafe water, sanitation and hygiene. Of this burden, about one-third occurred in Africa and one-third in south-east Asia. Overall, 99.8 per cent of deaths associated with these risk factors are in developing countries, and 90 per cent are deaths of children. Child malnutrition is a primary indicator of monitoring a population's nutritional status (WHO, 2011a). Various forms of infectious diarrhoea make up the main burden of disease associated with unsafe water, sanitation and hygiene (WHO, 2002).

There is a large unmet need for family planning, as the WHO estimates that 215 million women who wanted to avoid pregnancy could not access or are not using contraception (WHO, 2011b). Contraception use in developing countries has increased from $8 \%$ in the 1960s to $62 \%$ in 2007 (WHO, 2011c). Pregnancy and childbirth, however, are more risky for young adolescents, and in developing countries these complications are the leading cause of death of women between ages 15 and 19 years (WHO, 2011d).

An overall improvement in maternal and child health globally is anticipated; however, neither the world as a whole, nor the developing regions are expected to achieve the Millennium Development goals. This is largely due to the deteriorating

\footnotetext{
${ }^{1}$ The sum of years of potential life lost due to premature mortality and the years of productive life lost due to disability.
} 
situation in Africa where all sub-regions, except Northern Africa, are expected to fail to meet the goal.

\section{Nutrition, health and sanitation nexus}

Under-nutrition is mainly a consequence of inadequate diet and frequent infection, leading to deficiencies in calories, protein, vitamins and minerals. Underweight remains a pervasive problem in developing countries, where poverty is a strong basic cause, contributing to household food insecurity, poor childcare, maternal under-nutrition, unhealthy environments, and poor health care as implied in the conceptual framework on malnutrition ${ }^{2}$. Populations in poor resource setting are worse off compared to mid and high income groups that afford access to better quality health care and sanitation. A study among low-resource setting in Kenya (Muthoka et al., 2010) indicated worse nutrition status among urban slums compared to rural areas although household food security was the reverse. This can be explained by the unsanitary conditions in the urban slums. In the study, mean number of persons using a toilet facility was 32 compared to 9 in the rural areas (Muthoka et al., 2010). With a single sanitation facility serving so many people, there is a likelihood of "flying toilets" developing (exposed human excreta scattered in the open), especially within the slum. This means that yards, fields, and consequently, water sources are likely to be contaminated with faecal material that may contain pathogens. Flies will get access to exposed faeces, thus they are likely to contaminate other sources causing infection, for example food and water. Children playing in the yards and on the streets are likely to come into contact with the faeces. When faecal matter dries up, it is likely to be contained in dust that can be blown up and be inhaled.

Child-health has been improving as the global under-five mortality declined by $27 \%$ from 1990 (90 deaths per 1,000 live births) to 2008 (65 deaths per 1,000 live births). There were about 8.8 million under-five deaths in 2008 globally, with the WHO African Region accounting for half of all child deaths in the world (WHO, 2011e). The child mortality rates in low-income countries were almost 20 times larger than in high-income countries.

Child malnutrition which is measured by poor child growth is an important indicator for monitoring population nutritional status and health. In 2011, an estimated $17 \%$, or 99 million children under five years of age in developing countries were underweight (low weight-for-age according to the WHO child-growth standards). Global underweight is most common in the regions of South-Central Asia (30\%), followed by Western, Eastern, and Middle Africa (22\%, 19\% and 17\%, respectively) and South-Eastern Asia (17\%). The situation is better in other regions

${ }^{2}$ UNICEF, 2012 
of Eastern and Western Asia, Northern Africa and Latin America and the Caribbean, where less than $10 \%$ of children were underweight (de Onis et al., 2004).

Children in the poorest households are twice as likely to be underweight as those in the least poor households. Children living in rural areas are more likely to be underweight than those living in urban areas. Childhood malnutrition, including poor growth and micronutrient deficiencies, is an underlying cause of death in an estimated $35 \%$ of all deaths among children under five years of age.

\section{Maternal mortality}

Maternal mortality is a health indicator that shows very wide gaps between rich and poor, both between countries and within them. Every day in 2010, about 800 women died due to complications of pregnancy and child birth, including severe bleeding after childbirth, infections, hypertensive disorders, and unsafe abortions. Out of the 800, 440 deaths occurred in sub-Saharan Africa and 230 in Southern Asia, compared to five in high-income countries. The risk of a woman in a developing country dying from a pregnancy-related cause during her lifetime is about 25 times higher compared to a woman living in a developed country (WHO, 2011f). Poor maternal nutrition also implies post delivery complications. A study in Kenya among mothers in poor resource settings from both urban and rural area demonstrated delayed health seeking behaviour in rural environments which has a negative impact on health and nutrition status of mothers (Muthoka et al., 2010). Maternal health challenges commonly experienced include long distances to the nearest equipped health facility, inadequate skilled health personnel, high costs of transport, and inability to recognise risks early in order to seek timely care.

\section{Child underweight}

Child malnutrition is a primary indicator of monitoring a population's nutritional status, food security situation and as a whole the development process of a country. Although the proportion of underweight children under the age of five in developing countries has declined by $11 \%$ from 1990 to 2010, nutritional services are likewise inadequate (WHO, 2011c). About 103 million children under the age of five, an estimated $18 \%$, in developing countries are underweight according to the WHO child-growth standards (WHO, 2011c). Childhood malnutrition, which encompasses micronutrient deficiencies and poor growth, causes 35\% of all deaths among children under five years (WHO, 2011c). Child malnutrition, when not attended to in time is likely to increase the child mortality rates. WHO, (2002) reports under-nutrition as a contributing factor in more than half of all child deaths accounting for about 138 million DALYs in developing countries. Trends in the past decade (1990-2008) indicate global improvements in the reduction of under-five mortality rates by $27 \%$. The low-income countries yet lag behind the high-income countries as they are 20 times worse off (WHO, 2011b) and the Afri- 
can region still accounting for half of the child deaths in the world. Africa as a region still accounts for half of the child deaths in the world.

\section{Maternal and child health challenges}

\section{Reproductive bealth}

In some African countries, the maternal mortality has increased despite commitment by most states to the Millennium Development Goals of reducing it by $75 \%$ by 2015 (Lema et al., 2005). Women nutritional status and health is further compromised by closely spaced births thus impacting on their capacity as care-givers. Increase in unmet needs in reproductive health can be explained by socio-cultural attitude and poor access to health services. Post-delivery complications are not only a factor of maternal age but are common among undernourished mothers and those within low-resource settings (Muthoka et al., 2010). It implies that low income or poor resource base are contributing factors in limiting access to health services and are likely to impact negatively on maternal health care.

\section{Poor decision making and late health-seeking behaviour}

Education, low incomes, culture and inability to make independent decisions for some rural mothers influences their health-seeking behaviours. Late decision making compromises the management of reproductive related illnesses and infections and easily leads to maternal death, or long admission days at the health centres. These deprive other younger children and maternal care and can lead to the vicious cycle of malnutrition. Education during the visit of antenatal clinics is aimed at sensitising mothers of pregnancy-related risks and enhancing positive attitude on the need to seek treatment once infection or any abnormality is noted.

\section{Inadequate resources}

Low income is usually co-related to education level, with mothers who are less educated having less chances in good-income jobs that can support them and their families. Inadequate resources complex health especially when there is a cost related to treatment at the health facilities. Thus, mothers opt to other cheaper means of delivery or treatment which also increases the risks of infections and complications. Although most mothers make at least one antenatal visit during their pregnancy, delivery assisted with skilled health personnel still remains low among communities with low-resource settings and those with limited access to health services either due to distance to health facility and poor road infrastructure (Muthoka et al., 2010). 


\section{Interventions}

Appreciably many interventions have been made to improve the situation of mother and child health. Focussing on the first 1,000 days of pregnancy and early childhood (24 months), due to high impact nutrition interventions (HiNi) aims at preventing malnutrition and reducing child mortality. These include, improving the nutritional status of women of reproductive age through diet counselling at anteand postnatal clinics, iron and folate supplementation during pregnancy and nutrition and health education during clinic visits (MOHPS, 2012). Among the children under five, nutrition well-being has been achieved through improved infant and young child infant feeding practices (IYCF) among mothers and guardians. The adoption of IYCF guidelines in all health facilities has ensured constant nutrition messaging to mothers/guardians thus reducing confusion especially on length of exclusive breastfeeding and optimising complementary feeding.

Micronutrient deficiency still remains a challenge. This has been addressed by the provision of vitamin A and zinc supplementation and introduction of multiple Micro-Nutrient Powders (MNPs) for children under the age of five. The supplementations have also been supported by iodisation of all domestic salts to improve the health outcomes of the population. In Kenya with the legislation of salt iodization, $98 \%$ of all households consume iodised salt (MOHPS, 2012).

Improved access to quality curative care through free health care services in all public health facilities for all under-five children has encouraged many mothers to seek health care postnatally. Access to health services gives the mothers a chance to enjoy other hospital-based interventions such as integrated management of childhood illness. This includes immunization and deworming, coupled with nutrition, sanitation and health education.

Among the children who are ill, both Integrated Management of Acute Malnutrition (IMAM) and Integrated Management of Severe Acute Malnutrition (SAM) are available at health facilities to prevent progression to severe stage and deaths.

Diet-related intervention for school-going children and other children include improving nutrition in schools through food supplementation for those malnourished or in marginal ecological zones and school-feeding programmes in public and private institutions; improve nutrition knowledge on and control of dietrelated non-communicable diseases (NCDs) such as over-nutrition and obesity and value of exercise; attitudes and practices among the population.

Other cross-sector intervention include strengthening nutrition surveillance, monitoring and evaluation systems and strengthening coordination and partnership among key nutrition actors and mobilizing essential resources. However, more effort in scaling up nutrition and health are still required to meet the Millennium Development Goals. 


\section{Policy implication}

- Improving access to food at all times to especially households with low resource base is crucial to enhance adequate dietary intake which directly impacts on nutrition and health of both mother and child.

- Improve availability and accessibility to clean safe water.

- Poor maternal health outcomes, raise concerns of service delivery and access, quality of service in rural areas as a public health concern

- Attendance to ANC is not indicative of assisted skilled delivery at the health facilities. The low facility-assisted deliveries are a public health concern and indicative of poor access or combination with socio-cultural factors which may not be addressed at the ANC clinics.

- Empowerment of rural mothers to diversify basic household resources and improve income base as a way to mitigate short-medium term household food insecurity and poverty which may increase access to food and health services.

\section{References}

De Onis, M., Blössner, M., Borghi, E., Frongillo, E. A. \& Morris R. (2004). Estimates of global prevalence of childhood underweight in 1990 and 2015. JAMA, 291, 2600-6.

Lema, V.M.,Changole, J., Kanyighe, C.\& Malunga, E.V. (2005). Maternal mortality at the Queen Elizabeth Central Teaching Hospital, Blantyre, Malawi. East Afr Med J., 82, 3-9.

Ministry of Public Health and Sanitation (MOPHS). (2012). High impact nutrition intervention: Scaling up nutrition.

Muthoka, S., Mbuthia, E. K., Moturi, W. \& Ngotho, D.K. (2010). Enhancing sustainable community health and nutrition security and safe motherhood among rural and low income urban households. Unpublished.

Onda, K., LoBuglio, J. \& Bartram J. (2012). Global access to safe water: accounting for water quality and the resulting impact on MDG progress. Int J Environ Res Public Health, 9, 880-894.

World Health Organization (WHO). (2002). The World Health Report 2002 Reducing Risks, Promoting Healthy Life.

World Health Organization (WHO). (2010), UNICEF, UNFPA, World Bank. Trends in maternal mortality: 1990-2008. Available from: http://whqlibdoc.who.int/publications/2010/9789241500265_eng.pdf 2010 [Accessed April 15, 2011]. 
World Health Organization (WHO). (2011a). Global Health Observatory (GHO): Women fertility and family planning [Main Page]. Available from: http://www.who.int/gho/women_and_health/health_interventions/fertility_t ext/en/index.html 2011 [Accessed December 1, 2012].

World Health Organization (WHO). (2011b). Global Health Observatory (GHO): Underweight. Available from: http://www.who.int/gho/mdg/poverty_hunger/underweight_text/en/index. html 2011 [Accessed December 1, 2012].

World Health Organization (WHO). (2011c). Global Health Observatory (GHO): Maternal and reproductive health. Available from: http://www.who.int/gho/maternal_health/en/index.html [Accessed November 15, 2012].

World Health Organization (WHO). (2011d). Skilled attendants at birth. Available from:

http://www.who.int/gho/maternal_health/skilled_care/skilled_birth_attenda nce_text/en/index.html [Accessed December 1, 2012].

World Health Organization (WHO). (2011e) Global Health Observatory (GHO): Maternal and reproductive health. Available from: http://www.who.int/gho/maternal_health/en/index.html [Accessed December 2, 2012].

World Health Organization (WHO). (2011f).Global Health Observatory (GHO): Women fertility and family planning. Available from: http://www.who.int/gho/women_and_health/health_interventions/fertility_t ext/en/index.html 2011 [Accessed December 1, 2012].

World Health Organization (WHO)/ UNICEF.(2010).Progress on Sanitation and Drinking-water: 2010 Update. WHO/UNICEF Joint Monitoring Programme for Water Supply and Sanitation.

UNICEF. (2012). State of the World Children 2012: Children in the urban world. UNICEF, New York. pp 4-5. 


\section{Water Sanitation Challenge: Prevalence of Diarrhoea in Children under Age 5 in Slums and Regular Localities in Enugu, South-East Nigeria}

Pat Obiageli Ndu, Chica Onwasigwe, Silva M. Anika

Centre for Environmental Management and Control, University of Nigeria, Enugu Campus, Nigeria.

\section{Summary}

As the world inches close to the 2015 deadline on the Millennium Development Goals (MDG's), it is becoming more glaring that many nations would be off track and off target on the eight goals. The 2012 update on drinking water shows that even though the world has met the drinking water target, about 780 million people still lack safe potable water for drinking and other uses. The coverage in sub Saharan Africa is currently 61 percent and for Nigeria 42 percent as against the 75 percent stipulated target on the goal (WHO/UNICEF/JMP, 2012). There are also indications that globally, sanitation targets would not be met with coverage in subSaharan Africa, where 30 percent and 45 percent use either shared or unimproved facilities, respectively, and 25 percent practice open defecation. An estimated 109 
million (69\%) Nigerians lack access to improved sanitation facilities and 34 million people still practice open defecation (WHO/UNICEF/JMP, 2012).

These challenges in the water-sanitation sector have been shown to have linkages to diarrhoeal morbidity in children under the age of five. About 6.9 million children under the age of five were reported to have died by the end of 2011 (WHO, 2012). The deaths were linked to dehydration, chronic diarrhoea, neonatal diseases, pneumonia, malaria and HIV/AIDS. Diarrhoea, a water-borne disease, remains the third biggest killer of children in sub-Saharan Africa and kills more children in the region than HIV/AIDS and malaria combined. In Nigeria alone, about 85,921 children under the age of five, die each year from diarrhoeal diseases linked to inadequate sanitation, unsafe water and poor hygiene. The country has recorded very modest progress on under-five mortality rate which dropped from 199 deaths per 1,000 live births between 1993 and 1998, to 157 deaths per 1,000 live births between 2003-2008 (National Population Commission (NPC, 2009). The MDG target expectation is 36 per 1,000 live births by 2015 .

The current Joint Monitoring Program put together by WHO/UNICEF assesses progress based on proxy measures for both water and sanitation. The improved drinking water sources are those that by nature of their construction are protected from outside contamination particularly with fecal matter and improved sanitation those facilities that hygienically separate human excreta from human, animal and insect contact (WHO, 2002). The systematical testing of the microbial, physical and chemical quality of water at national and regional levels is considered expensive and logistically complicated. Consequently in the current set of indicators; the safety and reliability of drinking water supply sources and sanitation facilities were not addressed. This implies that complete information about drinking water safety is not available for global monitoring. There is a chance that some of these sources may not have been adequately maintained and would not provide safe water and a possibility that the number of people using safe water supplies may be overestimated.

There is now a push to include water quality in the monitoring program to accommodate testing for the presence of E. coli. Also a process to develop new water, sanitation and hygiene goals, targets and indicators beyond 2015 is now in progress (WHO/UNICEF/JMP, 2012). This would address the information gap created by the current regimen which has a limiting effect on the usability of data obtained with the proxy method for water quality.

To address this gap, the methodology adopted for this study though adapted from WHO/UNICEF (2006) core questions on drinking water and sanitation was combined with an investigation of the quality of the different water sources used at the household level within the study areas. This study took into account the importance of parameters like drinking water quality, water availability, per capita water consumption per person within households, water treatment at point of use, correct hand washing practices among others. The household survey was conducted in three regular localities and three slums in Enugu Metropolis of South-East 
Nigeria; Trans Ekulu, New Haven, Uwani, Agu-Owa, Ugbo-Okonkwo, and Ikirike.

Of the 610 questionnaires shared across localities, 578 were duly completed and returned $(94.75 \%$ return rate). The questionnaire captured information on demographic and socio-economic variables, water indicators covering water sources, access, availability, per capita consumption and water treatment practices; sanitation indicators covering facility types, adequacy of facility, hand-wash practices and waste management. The health indicators tracked diarrhoeal morbidity in children under five. Correlation and regression analysis were used to determine relationship and strength of association between use of unimproved water and unimproved toilet facilities and diarrhoea prevalence in children.

The quality of the water from the different sources was assessed at National Agency for Food and Drugs Administration and Control (NAFDAC) Water Laboratory in Agulu, Anambra State to determine whether they met the Nigerian Drinking Water Quality Standards (NDWQS) (Standard Organization of Nigeria (SON), 2007). All the water samples analysed did not meet the stipulated standards and branded unsatisfactory including pipe-borne and table water samples collected from the localities. The findings showed a moderate correlation of 0.537 for water and 0.608 for sanitation. There was a significant relationship between the use of unimproved water sources and prevalence of diarrhoea and $\mathrm{R}$ as the measure of the strength of relationship was 0.823 (very high). The correlation for relationship between prevalence of diarrhoea in children under the age of 5 and the use of unimproved sanitation facilities was 0.608 and the $R$ value 0.752 showed a high strength of relationship also.

These findings on diarrhoeal prevalence were both startling and in conflict with the picture painted by the diarrhoea records of the University of Nigeria Teaching Hospital and studies conducted by Nnodu et al. (2008) and Jibrum (1988) in the same area of South-East Nigeria. The dry season was captured as the season for diarrhoea in these studies and records. This survey was conducted in a six-months time frame. Two months in the period were dry season March to April; four months May to August were in the wet season. Four weeks in the dry season belt was used for the pilot survey leaving only one month of dry season for the main survey.

Of the six localities in the study, Trans Ekulu recorded a diarrhoea prevalence rate of 14.29 percent, Agu-Owa 24.10 percent, New-Haven 14.16 percent, UgboOkonkwo 33.80 percent, Uwani 26.64 percent, and Ikirike 43.40 percent. The very high prevalence rates in relation to the national average raises questions around possible confounding variables. The rates were higher in the slums where both water and sanitation infrastructure were either absent or not enough. These disparities within localities show that water and sanitation challenges require a multifaceted intervention program that would address the heterogeneity of the issues in the sector. Location-specific plans should be generated for each locality. 
In addition, NAFDAC may have to review monitoring and evaluation procedures of table water pushed into the market that carry their registration numbers and seemingly connoting safety. Government at the three tiers should allocate human and material resources to tackle issues in the sector and also begin to see slum eruptions as a 'cry for help'. There must be a conscious effort to mount programs and intervention schemes that would reduce prevalence of diarrhoeal disease in children under the age of five. The life of every child must count irrespective of the localities they find themselves in by accident of birth.

\section{Introduction}

Water is not scarce because fresh water constitutes $2.5 \%$ of the water on earth and would be abundant if it were evenly distributed (Shiklomanov, 1993). If all the fresh water on the planet were divided equally among the global population, there would be 5,000-6,000 $\mathrm{m}^{3}$ available for everyone per year. This global calculation gives an impression of abundance but in reality, nearly one billion people in the world do not have access to potable water to meet their daily needs (WHO/UNICEF/JMP, 2012). While the world's population tripled by the 20th century, the use of renewable water resources has grown six-fold. This population growth coupled with industrialization and urbanization concentrates large numbers of people in small areas resulting in increased demand for potable water.

One of the health problems linked to the water-sanitation challenge is diarrhoea loosely defined as; a symptom of gastrointestinal infection characterized by the passage of loose watery stools more frequently than normal. Severe cases may mean three or more watery stools in a span of 24 hours (WHO, 2004). Diarrhoea could be just watery as in cholera or passed with blood as in dysentery. It can be caused by a bacteria, viruses and parasitic organisms spread by contaminated water or food. It is not a common condition in the developed world where access to sanitation facilities and safe water is high and personal and domestic hygiene relatively good (WHO, 2000). Diarrhoea is the third leading cause of death in children after respiratory infections and HIV/AIDS.

Enugu Metropolis, South East Nigeria has witnessed tremendous population growth in over 100 years of its existence. The population has grown from 3,170 in 1921 (Coleman, 1971) to 722,664 in 2006 (NPC, 2006). This expansion could be linked to the loss of investments to the 'abandoned property saga' in various parts of the country by the Igbos of South East extraction following the Nigeria-Biafra civil war. Many rebuilt their post-war lives in their homeland and Enugu the capital of the then East Central State was a popular choice resulting in the birth of new localities; a trend not matched with expansion of water and sanitation facilities. Slums whose basic features are the absence of clean water, electricity, good road network, toilet facilities, and waste management plans (Wambui et al., 2007) erupted at city fringes. 


\section{Study area}

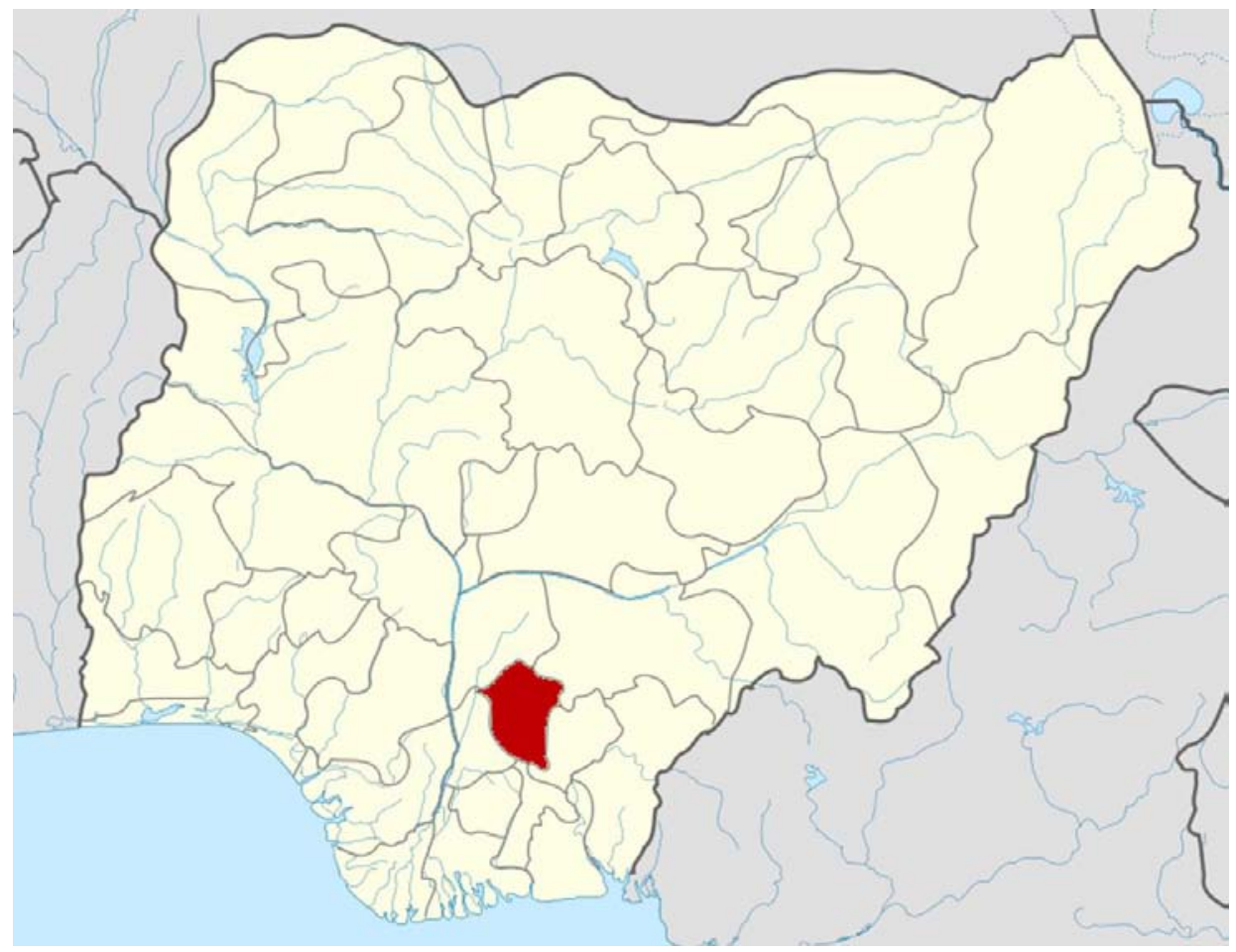

\section{Figure 1: Map of Nigeria showing Enugu State}

Source: Wikipedia, 2013

The founding of Enugu was influenced by the discovery of coal by an itinerant party of geologists between 1908 and 1909. Prior to this discovery, the virgin land had no historical, economic or political importance. Although the British authorities transformed Enugu into a beautiful city; their interest was purely economic. Enugu became a second-class township in 1917 under the name Enugu-Ngwo but Ngwo was dropped in 1928 to distinguish the town from the Enugu village in Ngwo town. The topography of the area influenced the decision of the natives to call the area enu-ugwu (Enugu) meaning "hill top" (Fig. 1).

Enugu Metropolis is in the tropical rain-forest zone with a derived savannah experiencing between 1,520 and 2,030 $\mathrm{mm}$ of rain annually (Egboka, 1985). The city has only the wet and dry seasons. The wet season lasts from May to October with the heaviest rainfall occurring between June and July and a break in August referred to as 'August Break'. The dry season lasts from November to April. The other weather condition is the Harmattan; a dusty trade wind originating from the Sahara desert blowing southwards lasting a few weeks from December to January. 
Enugu Metropolis became the headquarters of the Eastern Province in 1936, capital of Eastern Nigeria in 1951, and capital of East Central State in 1967 in the wake of the Nigerian civil war. In 1976, it was made the capital of the old Anambra State and finally the capital of the present Enugu State in 1991. The Capital Territory is made up of three local Governments Areas - Enugu East, Enugu North and Enugu South. Each local government area is further subdivided into localities.

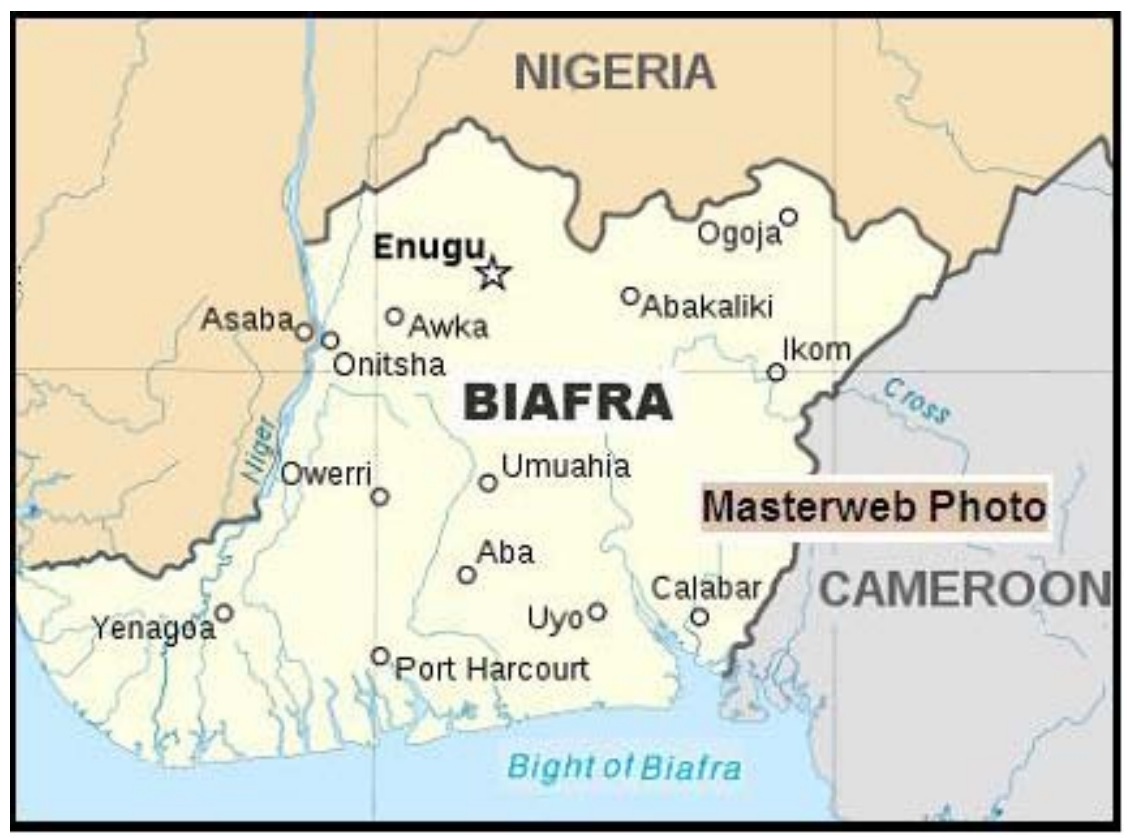

Figure 2: Map of Nigeria showing Biafra

Source: nigeriamasterweb.com, 2013

Beginning with the January 15, 1966 coup d'etat and the counter coup organised by Northern Nigeria officers, 185 Igbo officers and over thirty thousand Igbos and south-easterners were massacred in a spate of four months. The event got them terrified and they fled home to Eastern Nigeria for safety. The seeming nonchalance by the federal government left the people no option than to self-preserve because the people figured that a government that won't safeguard the lives of its citizens had no claim to their allegiance and the victims sought their safety in other ways - including secession (Achebe, 2012).

Crushing the Biafran dream took three years (Fig. 2). The investments accumulated over the years in the banks and real estate was suddenly labelled 'abandoned' and confiscated by the different military governors in the states. It did not matter how much money they left in the banks prior to the war, each individual was given twenty pounds at the end of the pogrom. In spite of the 'no victor, no vanquished' declaration; the average Igbo man felt defeated and lost faith in the project called 
Nigeria. Playing safe and survival meant settling in Enugu Metropolis in 1970. The water and sanitation facilities were not expanded adequately to accommodate the influx of the people into Enugu.

\section{Background of the problem}

In the 1960's, 70's up to early 80's, Nigerian leaders at the two tiers of government provided treated drinking water for the people. Suddenly, rapid population growth, corruption, poor planning, and insensitivity of the leaders changed all that. Potable water became scarce due to the spatial limits of water coverage. The current water supply service coverage in the country according to WHO/UNICEF/JMP report for 2012 is $58 \%$ (87 million people) translating to lack of potable water to about 70 million people. This report ranked Nigeria third behind China and India in the list of countries with the largest population without access to improved drinking water.

Enugu Municipal in that time frame also used to discharge the water needs of the residents into homes through pipes and faucets. Households were informed through radio and television announcements when Water Corporation needed to do turnaround maintenance of equipment in their facility. This gave residents ample time to store enough water to last through the period of system shut down. In time all that changed. Load shedding and rationing few days at a time started, cascading into weeks of taps remaining dry with no explanations from anyone. Households resorted to collecting water from not so good sources mainly from the shallow streams called 'mmiri ani' literarily meaning 'ground water' that dot the city landscape. Residents took baths, did laundry and washed their cars in these shallow streams. Water for drinking and cooking were procured for a fee.

With this development; it is now common place to be woken up by the chatter of women and children heading to shallow streams and ponds oftentimes contaminated to draw water at the crack of dawn. The blaring horns of water tankers and push-cart vendors searching for clients gives one an undesirable wake up call. One of the strategies adopted by the people to mitigate the water crisis is the drilling of wells from which ground water is harvested. As dependence on hand dug wells gained prominence as a water source, the dependence on shallow streams began to decline or so it seemed. Ground water mining without adequate planning, legal framework and governance has opened a new debate on the sustainability of the intensive use of ground water resources (UNDP, 2006). Also new in the sector is the dependence on table water packaged in sachets and bottles as sources of drinking water. The different brands in the market carry National Agency for Food and Drugs Administration and Control (NAFDAC) registration numbers, a subtle way of informing prospective consumers that the products have been endorsed for wholeness and safety. 
Of the 725,767 households in Enugu State, only about 14.4\% have pipe-borne water inside their homes; the rest $(85.6 \%)$ are compelled to use alternative sources (NPC, 2006). In the sanitation sector there are reports that 34 million Nigerians practice open defecation placing her fifth in the world behind India, Indonesia, Pakistan and Ethiopia for open defecation (Allwell, 2012). This 'flying toilet' habit is endangering the water bed through the hydro geo-pollution cycle and adding to the burden of environmental degradation. Currently, Enugu State Waste Management Authority (ESWAMA) dumps the solid wastes collected around the city in land fill sites at the city fringes. These mounds of refuse are covered with soil to form manure. This method of disposal constitutes a source of contamination for ground water from where domestic water supplies are drawn exposing the consumers to the different pathogenic organisms in the water some of which can cause diarrhoeal diseases.

Diarrhoeal prevalence in children is one of the conventional ways for accessing the quality of water used at the household level. Increasing the quantity of water used at household level allows for better hygiene practices no doubt but raising the quality of drinking water reduces the ingestion of pathogens (Billig et al., 1999). Health benefits from improved water supply occur through better water quality with reduced ingestion of pathogens (Esrey et al., 1991).

\section{Methodology}

In the course of carrying out this research, 610 questionnaires were distributed in three regular localities and three slums in Enugu Metropolis between March 2010 and June 2011. The determination of the sample size for the different localities was based on the Nigerian population census figures of 1991 projected to 2011. The survey instrument was allotted as follows: Trans Ekulu (79), New Haven (130), Uwani (220) all regular localities and Agu-Owa (40), Ugbo-Okonkwo (52), and Ikirike (88) the contiguous slums. A total of 578 of the 610 questionnaires were properly completed and returned. The questionnaire was an adaptation of the core questions on drinking water and sanitation for household surveys developed by WHO/UNICEF/JMP, (2006). The harmonized questions were derived from an in-depth study of several international survey instruments; www.wssinfo.org. The aim of the standardized prototype was to improve survey comparability over time and harmonize them for purposes of global monitoring.

Water samples were taken from streams, hand dug wells, tanker-truck water, water from push cart vendors, table water in sachets and bottles, pipe borne water and taken to NAFDAC laboratory within twenty-four hours of harvesting the water from the various sources for analysis to determine whether they met the National Drinking Water Quality Standards. Samples were collected in new one litre-capacity white transparent plastic bottles. The transparency of the bottles made physical observation of the content easy. The containers were washed and 
rinsed with methylated spirit and left to dry for 24 hours. Thereafter, containers were rinsed with the water meant for analysis and appropriately labelled. The Physical and Chemical Analysis were carried out with the Rapid Smart Spectrophotometer, which is a menu-driven instrument with over 80 pre-programmed tests and a wide wavelength range of $350-1,000 \mathrm{~nm}$. The instrument measures the transmittance and absorbance of the different elements in the water sample and automatically gives the concentration of the substance without having to go through tedious mathematical formulae and calculations. The water samples were subjected to these bacteriological examinations and analysed in line with NSDWQ (SON, 2007); enumeration of total aerobic mesophilic count, coliform test, E.coli test and Pseudomonas test.

Parametric tests and inferential statistics were used for analysis using a computer program; Statistical Package for Social Sciences (SPSS version 17). Both qualitative and quantitative responses were collected and analysed to address the relationship of the data to the research questions as basis for testing the hypotheses in the study.

\section{Results and discussion}

Access to potable water and sanitation remain basic needs that must be met to ensure that the health of household members especially children under five years is not undermined. In focusing our lens on the challenge of water and sanitation interventions and linkages to diarrhoea in children under five years, some of these parameters were assessed; water availability and access, quantity of water used by households daily, water quality, proportion of people not served by improved water, treatment of water before drinking, water sources, types of toilet facilities, access to improved toilet facilities, proper hand-washing practices, proportion of people not served by improved toilet facilities among others.

These sources of drinking water were elicited from the measurement instrument; pipe-borne water, protected well, rain water harvests, sachet (pure water), bottled water, tanker/truck vendor water, push cart vendor water and surface water. There was a heavy dependence on sachet water in all localities save for New Haven; Ugbo-Okonkwo (80\%), Agu-Owa (77\%), Trans Ekulu (60\%), Uwani $(59 \%)$ and Ikirike $(58 \%)$. Dependence on pipe-borne water as drinking water source was high in New Haven compared to other localities which explains why they had fewer households depending on sachet water. Trans Ekulu residents also depended heavily on tanker/truck vended water (44\%) and Ikirike residents on surface water $(70 \%)$ (Table 1$)$. 


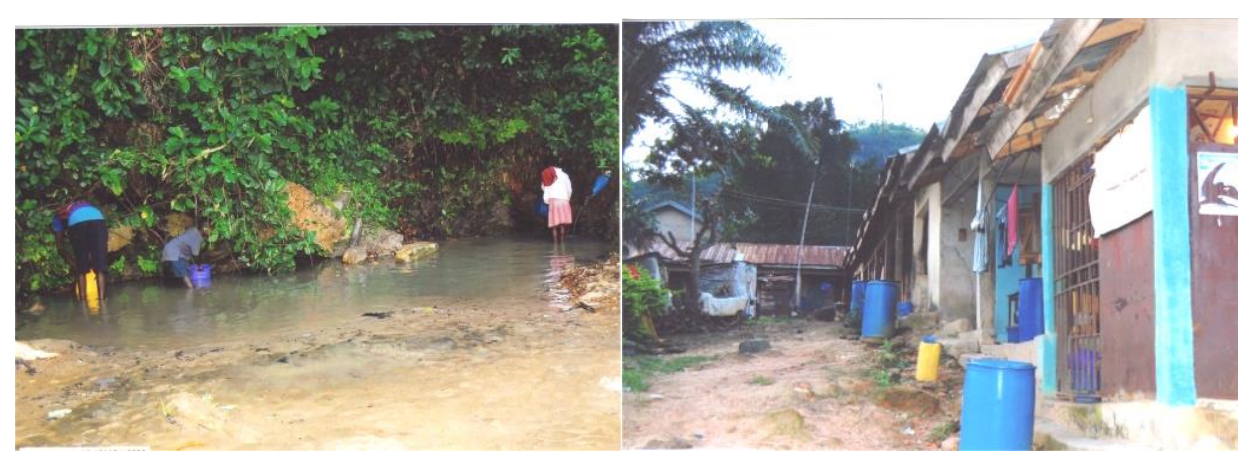

Figure 3: Ikirike stream/spring

Source: Researcher's fieldwork, 2012
Figure 4: Typical slum house type

Source: Researcher's fieldwork, 2012

Table 1: Sources of drinking water; number $(\mathrm{N})$ and percentage $(\%)$ of respondents

\begin{tabular}{|c|c|c|c|c|c|c|c|c|c|c|c|c|}
\hline \multirow[t]{2}{*}{ Water Source } & \multicolumn{2}{|c|}{$\begin{array}{l}\text { T/Ek } \\
\text { ulu }\end{array}$} & \multicolumn{2}{|c|}{$\begin{array}{l}\text { Agu- } \\
\text { Owa }\end{array}$} & \multicolumn{2}{|c|}{$\begin{array}{l}\text { New } \\
\text { Haven }\end{array}$} & \multicolumn{2}{|c|}{$\begin{array}{l}\text { U/Okon } \\
\text { kwo }\end{array}$} & \multicolumn{2}{|c|}{ Uwani } & \multicolumn{2}{|c|}{ Ikirike } \\
\hline & $\mathbf{N}$ & $\%$ & $\mathbf{N}$ & $\%$ & $\mathbf{N}$ & $\%$ & $\mathbf{N}$ & $\%$ & $\mathbf{N}$ & $\%$ & $\mathbf{N}$ & $\%$ \\
\hline $\begin{array}{l}\text { Tap } \\
\text { water inside }\end{array}$ & 10 & 13 & 2 & 5 & 80 & 66 & 7 & 16 & 32 & 15 & 0 & 0 \\
\hline Public tap & 6 & 8 & 8 & 21 & 12 & 10 & 24 & 55 & 39 & 19 & 0 & 0 \\
\hline $\begin{array}{l}\text { Protected } \\
\text { well }\end{array}$ & 10 & 13 & 4 & 10 & 16 & 13 & 10 & 23 & 31 & 15 & 5 & 6 \\
\hline Rain water & 16 & 21 & 18 & 46 & 11 & 9 & 29 & 66 & 51 & 24 & 27 & 31 \\
\hline $\begin{array}{l}\text { Sachet (pure) } \\
\text { water }\end{array}$ & 45 & 60 & 30 & 77 & 43 & 35 & 35 & 80 & 124 & 59 & 51 & 58 \\
\hline Bottled water & 17 & 23 & 0 & 0 & 15 & 12 & 0 & 0 & 12 & 6 & 0 & 0 \\
\hline Tanker water & 33 & 44 & 4 & 10 & 14 & 11 & 3 & 7 & 83 & 40 & 25 & 28 \\
\hline $\begin{array}{l}\text { Push cart } \\
\text { water }\end{array}$ & 8 & 11 & 8 & 21 & 0 & 0 & 5 & 11 & 2 & 0.9 & 14 & 16 \\
\hline Surface water & 5 & 7 & 16 & 41 & 0 & 0 & 5 & 11 & 2 & 0.9 & 62 & 70 \\
\hline
\end{tabular}

Source: Researcher's fieldwork (2010-2011)

The habit of proper water treatment at point-of-use varied across localities. The respondents from New Haven, who depend largely on pipe-borne water, do not think there is a need to apply further treatment with only $8 \%$ of the respondents captured as applying appropriate water treatment before use. In the category of respondents that did nothing to make water safe more than $50 \%$ of the respondents across localities admitted to doing nothing (Table 2). 
Table 2: Water treatment; number $(\mathrm{N})$ and percentage (\%) of respondents

\begin{tabular}{|l|l|l|l|l|l|l|l|l|l|l|l|l|}
\hline Methods & \multicolumn{2}{l}{ T/Ekul } \\
& \multicolumn{2}{l|l}{$\begin{array}{l}\text { Agu- } \\
\text { Owa }\end{array}$} & \multicolumn{2}{l|l}{$\begin{array}{l}\text { New } \\
\text { Haven }\end{array}$} & \multicolumn{2}{l|}{$\begin{array}{l}\text { U/Okonk } \\
\text { wo }\end{array}$} & \multicolumn{2}{l|}{ Uwani } & \multicolumn{2}{l|}{ Ikirike } \\
\hline & $\mathrm{N}$ & $\%$ & $\mathrm{~N}$ & $\%$ & $\mathrm{~N}$ & $\%$ & $\mathrm{~N}$ & $\%$ & $\mathrm{~N}$ & $\%$ & $\mathrm{~N}$ & $\%$ \\
\hline $\begin{array}{l}\text { Boil, } \\
\text { filter, add } \\
\text { chlorine }\end{array}$ & 34 & 45 & 9 & 23 & 10 & 8 & 11 & 25 & 84 & 40 & 11 & 13 \\
\hline $\begin{array}{l}\text { Add } \\
\text { alum, } \\
\text { strain, } \\
\text { settle }\end{array}$ & 3 & 4 & 1 & 3 & 33 & 27 & 4 & 9 & 20 & 10 & 4 & 4 \\
\hline Nothing & 38 & 51 & 29 & 74 & 79 & 65 & 29 & 66 & 106 & 50 & 73 & 83 \\
\hline TOTAL & 75 & 100 & 39 & $\begin{array}{l}10 \\
0\end{array}$ & $\begin{array}{l}12 \\
2\end{array}$ & 100 & 44 & 100 & 210 & 100 & 88 & 100 \\
\hline
\end{tabular}

Source: Researcher's fieldwork (2010-2011)

The proportion of respondents from the different localities who do not have access to improved sanitation facilities are as follows; Trans Ekulu 17 (23\%), AguOwa 30 (77\%), New Haven 38 (31\%), Ugbo-Okonkwo 34 (77\%), Uwani 115 (55\%) and Ikirike 84 (95\%). On the whole, 318 (55\%) of the 578 respondents captured in the study had no access to improved sanitation facilities.

Table 3: Types of toilet facilities and number (No) of respondents

\begin{tabular}{|l|l|l|l|l|l|l|l|l|l|l|l|l|}
\hline Toilet Type & \multicolumn{2}{|l|}{ T/Ekulu } & \multicolumn{2}{|l|}{ Agu-Owa } & $\begin{array}{l}\text { New } \\
\text { Haven }\end{array}$ & $\begin{array}{l}\text { U/Okon- } \\
\text { kwo }\end{array}$ & & \multicolumn{2}{l|}{ Uwani } & \multicolumn{3}{l|}{ Ikirike } \\
\hline $\begin{array}{l}\text { Water } \\
\text { Closet }\end{array}$ & 68 & 12 & 4 & 0 & 119 & 36 & 20 & 14 & 163 & 74 & 4 & 1 \\
\hline $\begin{array}{l}\text { Pit With } \\
\text { Cover }\end{array}$ & 7 & 5 & 10 & 5 & 3 & 2 & 14 & 10 & 18 & 12 & 6 & 5 \\
\hline $\begin{array}{l}\text { Pit No } \\
\text { Cover }\end{array}$ & 0 & 0 & 8 & 5 & 0 & 0 & 7 & 3 & 12 & 12 & 8 & 3 \\
\hline $\begin{array}{l}\text { Bucket } \\
\text { latrine }\end{array}$ & 0 & 0 & 0 & 0 & 0 & 0 & 0 & 0 & 17 & 15 & 11 & 9 \\
\hline $\begin{array}{l}\text { Bush, field } \\
\text { etc. }\end{array}$ & 0 & 0 & 17 & 0 & 0 & 0 & 3 & 0 & 0 & 0 & 59 & 0 \\
\hline TOTAL & 75 & 17 & 39 & 10 & 122 & 38 & 44 & 27 & 210 & 113 & 88 & 18 \\
\hline
\end{tabular}

Source: Researcher's fieldwork (2010-2011)

About 378 (93\%) of 407 respondents from regular localities had water closet/flush toilet facilities in their homes and $122(30 \%)$ shared their facilities making them unimproved. Of the 171 respondents from the slums, only 28 (16\%) had water closets, $79(46 \%)$ indulged in open defecation and $35(20 \%)$ used toilet facilities classified as unimproved like pit toilets without cover and bucket latrines (See Table 3). Generally the respondents from the regular localities used toilet types classified as improved. 


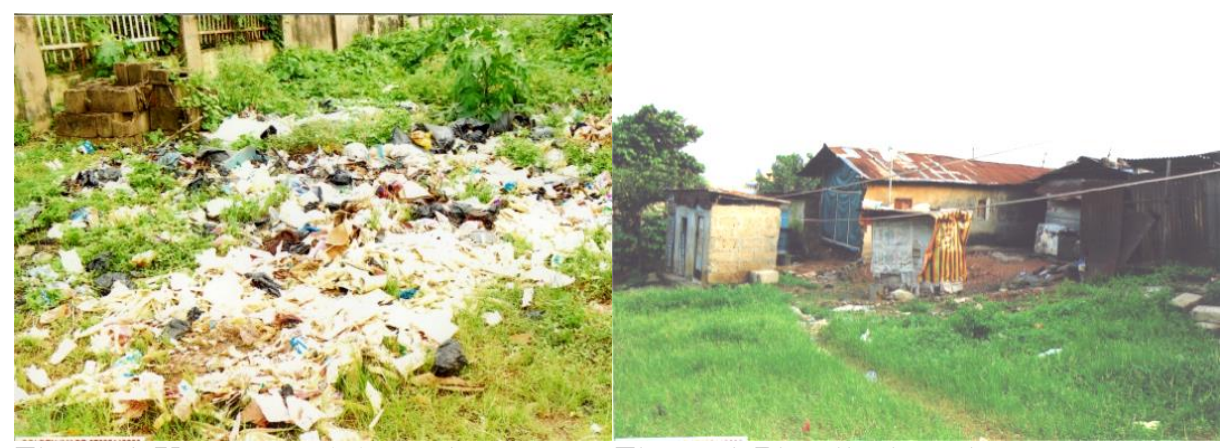

Figure 5: Unapproved dumpsite

Source: Researcher's fieldwork 2012
Figure 6: Pit toilet in a slum

Source: Researcher's fieldwork, 2011

Table 4: Water analysis of different water sources

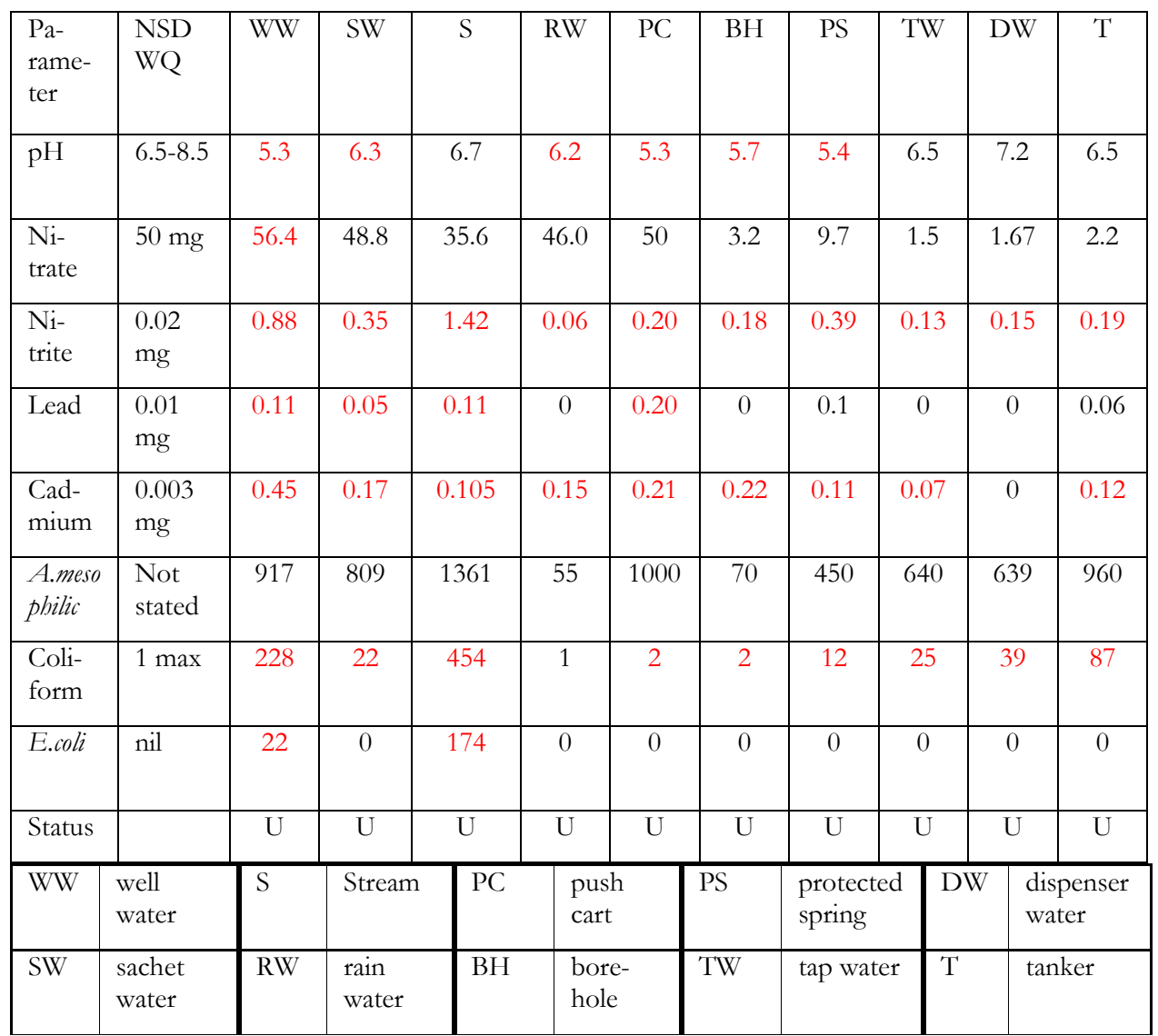

Source: Researcher's field work 2010-2011; U = Unsatisfactory.

The red highlights are values above normal. 
About 35 water samples were collected from different drinking water sources at various localities and sent to NAFDAC laboratory for analysis. The means of the different parameters were taken for wells, sachet water, bottled water, streams and pipe-borne water making 30 samples. Rain water harvest, tanker vendor water, protected spring, bore hole and pushcart vendor water were drawn randomly and sent in for analysis as well. The report in Table 4 shows that none of the samples met the Nigerian stipulated standards for drinking water. All except the rain water (RW) had coliform counts above tolerable levels. The well water (WW) and stream (S) showed presence of E. coli, an indicator of recent faecal contamination. There were high levels of aerobic mesophilic bacteria in all the samples. The maximum count was not stated in NSDWQ. Cadmium levels were high in all the samples except the dispenser bottle table water (DW). The nitrite content was high in all the samples and nitrate high in the well water (WW). The lead content of water from these sources were high; well (WW), sachet water (SW), stream (S), pushcart vended (PC), protected spring (PS) and tanker vended (T). The $\mathrm{pH}$ of water from some sources were lower than stipulated values; well (WW), sachet water (SW), rain water harvests $(\mathrm{RW})$, borehole $(\mathrm{BH})$, protected spring (PS) and push cart vended water $(\mathrm{PC})$.

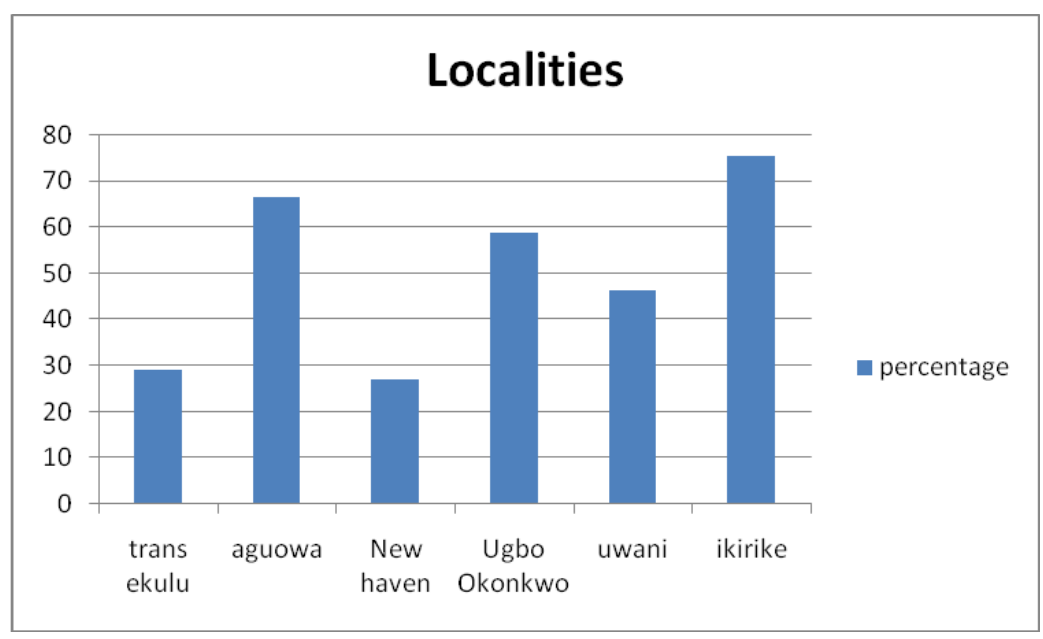

Figure 7: The prevalence of diarrhoea in children under five

Source: Researcher's fieldwork 2010-2011

Figure 7 above shows that prevalence of diarrhoea was higher in the slums compared to the regular localities contiguous to them. Among the slum localities, Ikirike had the highest prevalence of diarrhoea in children under five and UgboOkonkwo the lowest prevalence. Uwani had the highest prevalence followed by Trans Ekulu and New Haven for the regular localities. Only New Haven and its contiguous slum Ugbo-Okonkwo depended largely on pipe-borne water for their 
drinking water needs. It is therefore not surprising that they had the lowest diarrhoeal prevalence as shown in Table 1.

To capture a bird's eye view of the diarrhoeal prevalence in children under the age of five in the area, the researcher had to look at the hospital records of some hospitals in the metropolis including the University of Nigeria Teaching Hospital (UNTH) a foremost tertiary level care in the region. The data were lifted from the diarrhoea register and the researcher isolated the cases for children under the age of five. The record used in generating the chart on Figure 8 was kept for diarrhoea cases treated and managed by the unit. The prevalence rate could therefore not be calculated because the total number of children under age five that came for treatment was not trapped in the record. However, the diarrhoea cases were shown to be more ubiquitous in the wet season as against the dry.

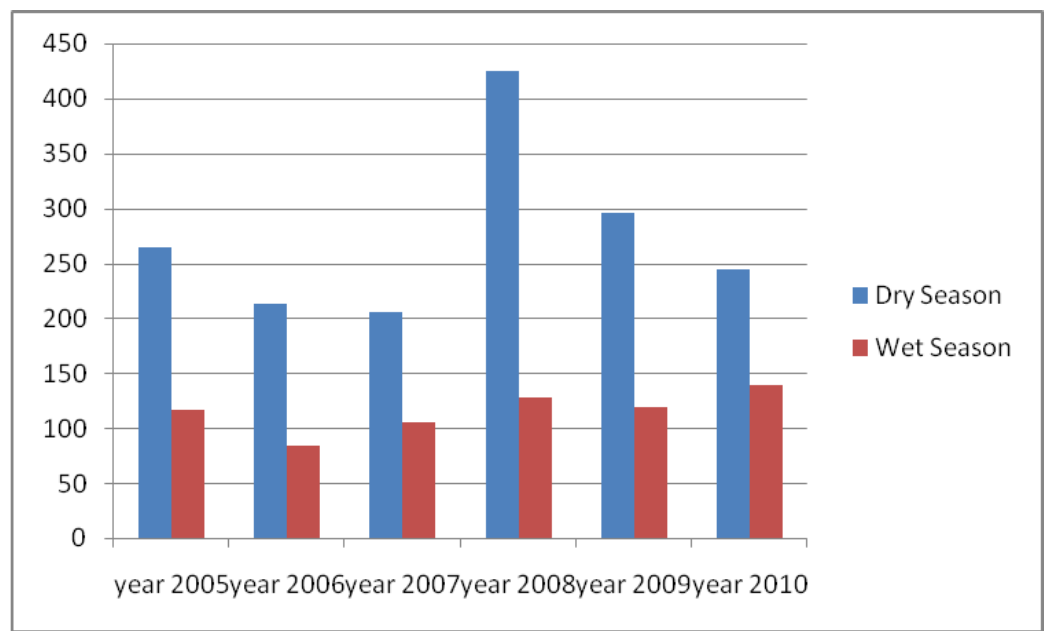

Figure 8: Diarrhoea cases at the Children's Emergency, UNTH 2005-2010

Source: Diarrhoea Register Children's Emergency UNTH, Enugu

The results from the statistical analysis for correlation between prevalence of diarrhoea in children under five and use of unimproved water and unimproved sanitation facilities showed a moderate correlation of 0.537 for water and 0.608 for sanitation (Table 5 and 6 ). The two week time frame was considered adequate to control for recall bias. 
Table 5: Correlations of diarrhoea prevalence and unimproved water

\begin{tabular}{|l|l|l|l|}
\hline & & $\begin{array}{l}\text { diarrhoea } \\
\text { prevalence }\end{array}$ & $\begin{array}{l}\text { unimproved } \\
\text { water }\end{array}$ \\
\hline diarrhoea prevalence & $\begin{array}{l}\text { Pearson Correlation } \\
\text { Sig. (2-tailed) } \\
\mathrm{N}\end{array}$ & 1 & $\begin{array}{l}.319 \\
.537 \\
6\end{array}$ \\
\hline unimproved water & Pearson Correlation & .319 & 1 \\
& Sig. (2-tailed) & .537 & 6 \\
\hline $\mathrm{N}$ & 6 & 6 \\
\hline
\end{tabular}

Source: Researcher's fieldwork 2010-2011

The researcher obtained information on the prevalence of diarrhoea among the children under age five in the households by asking mothers or other caregivers of the children whether they had diarrhoea within two weeks preceding the interview.

Table 6: Correlations of diarrhoea prevalence and unimproved sanitation

\begin{tabular}{|l|l|l|l|}
\hline & & $\begin{array}{l}\text { diarrhoea } \\
\text { prevalence }\end{array}$ & $\begin{array}{l}\text { unimproved } \\
\text { sanitation }\end{array}$ \\
\hline diarrhoea prevalence & $\begin{array}{l}\text { Pearson Correlation } \\
\text { Sig. (2-tailed) }\end{array}$ & 1 & .719 \\
& N & 6 & .608 \\
unimproved sanitation & Pearson Correlation & .419 & 6 \\
\hline & Sig. (2-tailed) & .408 & 1 \\
& $\mathrm{~N}$ & 6 & 6 \\
\hline
\end{tabular}

Source: Researcher's fieldwork 2010-2011

Table 7: Model summary ${ }^{\mathrm{b}, \mathrm{c}}$ for diarrhoeal prevalence and unimproved water

\begin{tabular}{|l|l|l|l|l|}
\hline Model & $\mathrm{R}$ & $\begin{array}{l}\mathrm{R} \\
\text { Square }\end{array}$ & Adjusted R Square & $\begin{array}{l}\text { Std. Error of } \\
\text { the Estimate }\end{array}$ \\
\hline 1 & $.823 \mathrm{a}$ & .677 & .596 & 55363515 \\
\hline
\end{tabular}

Source: Statistical analysis 2012

There is a significant relationship between the use of unimproved water resources and diarrhoeal prevalence (Table 7). The measure of the strength of relationship (R) was .823 and quite high. 
Table 8: Model Summary ${ }^{\mathrm{b}, \mathrm{c}}$ for Diarrhoeal Prevalence and Unimproved Sanitation

\begin{tabular}{|l|l|l|l|l|}
\hline Model & $\mathrm{R}$ & $\mathrm{R}$ Square & Adjusted R Square & $\begin{array}{l}\text { Std. Error of } \\
\text { the Estimate }\end{array}$ \\
\hline 1 & $.752 \mathrm{a}$ & .565 & .456 & 60083467 \\
\hline
\end{tabular}

Source: Statistical Analysis 2012

a) Predictors: (Constant), sample localities

b) Dependent Variable: diarrhoea

c) Weighted least squares regression weighted by unimproved water (Table 7) or sanitation (Table 8)

There is a significant relationship between the use of unimproved sanitation facilities and prevalence of diarrhoea (Table 8). The $\mathrm{R}$ value of 0.752 shows a high strength of relationship which is however slightly lower than that for unimproved water sources.

These findings in significance and strength of association is startling and in conflict with the picture painted by the diarrhoea records of the University of Nigeria Teaching Hospital. The dry season was captured as the season for diarrhoea and most of the data harnessed for this survey happened in the wet season. It is not disputable that unimproved water sources and unimproved sanitation facilities affect the prevalence of diarrhoea in children. That is consistent with the findings of Esrey (1991) and Fewtrell (2005) as well as the studies done by Nnodu et al. (2008) and Nnajide (1988) conducted in the South East Nigeria. The only difference between this study and that of Nnodu et al. (2008) and Nnajide (1988) is that their surveys were carried out in the dry season.

Of the six localities in the study, only New Haven enjoyed semblance of regular water supply from water board. The lower diarrhoeal prevalence found in the analysis was a reflection of this fact. However, the very high prevalence rates in relation to the national average raises questions around possible confounding variables. There are a number of indicators that are known to affect diarrhoeal prevalence in children under age 5; hygiene practices, access to water, per capita consumption of water per person in the household and literacy status of the mother.

The measurement instrument used in the survey captured the attitude of respondents to hygiene particularly correct hand-wash practices. The reports show that about $41 \%$ of the respondents and $58 \%$ of slum residents did not routinely wash hands properly (wash both hands with soap or ash and water) after activities that compromise the hygienic status of the hands. Data on the literacy status of the mothers revealed that 32\% had tertiary-level education, 35\% had high-school diplomas, 23\% had only elementary school-level education and 10\% had no formal education but acquired vocational skills from informal schools. Very few slum residents had tertiary-level education. Some studies have shown that the literacy 
level of a mother reduced diarrhoeal prevalence in children under 5 (Boadi and Kuitunen, 2005).

The damning report from the NAFDAC laboratory water analysis of the water sources should make it imperative that these unimproved water sources should be subjected to adequate treatment before consumption. However, many of the respondents did nothing to the water prior their consumption. Of the 578 respondents captured in the study; a total of $159(28 \%)$ respondents used appropriate water treatment for their drinking water, $65(11 \%)$ used alum, strained or allowed water to settle; an inappropriate treatment method and 354 (61\%) did nothing to the water prior to consumption. Many erroneously believed that a NAFDAC registration number on a product meant safe.

Water is a major nutrient for children and when from an unimproved source increases their risk of exposure to water-borne pathogens. Few health issues are as common and insidious as diarrhoea. Each year more than four billion episodes of diarrhoea account for about $20 \%$ mortality in children under five years globally (Fuentes et al., 2006). The average under-five child in the developing countries suffers an average of three episodes of diarrhoea per year, and unsafe drinkingwater along with inadequate supplies of water for personal hygiene and poor sanitation are the main contributors (Kosek et al., 2003). The findings from this study corroborate all these views on diarrhoeal disease prevalence and relationship with unimproved water and unimproved sanitation.

There are other variables that may have contributed to the high prevalence rates and the departure from the national average of $10 \%$. The microbial quality of the water from various sources showed high levels of coliform bacteria in all the samples except harvested rain water. There was E. coli in the well and stream water samples indicative of recent fecal contamination. The plot sizes within the metropolis make it hard for residents to maintain a safe lateral distance between hand dug wells and septic tanks or pit toilets. Without the right kind of distance in this regard, the ground water is compromised by movement of pathogens through the different aquifers. In addition to the coliforms is the mesophillic Aeromonas whose maximum permissible level was not stated. Some species in the genus are however known to be causative agents of gastroenteritis. Reports from Australia suggest that there may be a connection between cases of Aeromonas-associated diarrhoea and the number of Aeromonas in the drinking water (Burke, 1984).

Nigeria is endowed with abundant water resources to support sustainable provision of water supply and adequate sanitation but the enormous potential remain largely untapped due to uncoordinated haphazard implementation of policies and programs (National Planning Commission, 2012). During the product launch for Vision 20:20, the Enugu State Government initiated a community needs assessment of the 471 rural communities in the State and in the key development needs; $342(72.61 \%)$ communities requested for potable water and 304 (64.5\%) communities requested for healthcare facilities ranking 2 nd and 3 rd next to access roads that was the topmost; 385 (81.7\%) (Enugu State Vision 4: 2020, 2012). 
Studies on water are often extremely variable spatially. Locations of a few kilometers apart are found to be different in terms of water quality and availability. Such variations require monitoring at the local level or they would be lost in the averaging process (Douglas et al., 2006). In Enugu State, South-East Nigeria for instance, the main sources of water for households at the State level are rivers, springs and streams (NPC, 2006). A finer resolution and limited spatial radius shows that hand-dug wells were the most popular for Enugu East and Enugu South Local Government Areas while tanker-water vendor water source was more common in Enugu North Local Government Area (NPC, 2006). Global or country-level statistics also hide important regional or national or local heterogeneity in water resource availability and access, masking the true numbers of people suffering from water crisis. Many more of these location-specific studies are the direction to go such that all the pieces of the puzzle in the water-sanitation sector can be unmasked and tackled.

\section{Conclusion/recommendations}

A lot of research has been carried out globally that looked at the water-sanitationhealth tripod. The challenge has remained the spatial variability of issues in the sector. At the moment, the development at the various levels of governance has been the top-bottom approach with the federal government, ministers and state governors exclusively deciding where public funds should go. Obviously, this approach is not working. Regarding budget preparation, a new paradigm should be guided by needs assessment which is not only aiming the allocation of resources but substantially fosters the reduction of poverty through championing of peoplecentric development plans (Flides, 2009). A bottom-up view brings the priority needs to the fore because it presents a specific approach to the problems of different localities (Easterly, 2006). Only a thorough understanding of the situation can enable to create a uniquely effective solution.

It is time for Nigeria to set realizable and realistic targets, develop and domesticate achievable action plans and allocate financial and human resources needed to bring safe water and basic sanitation to the people in a sustainable manner. Such information could be used to measure progress towards meeting national and global goals. The Federal Government of Nigeria has continued to pay lip service to the problem considering the steady decline in budgetary allocation to water and sanitation, 112 billion Naira in 2010, 62 billion Naira in 2011 and 39 billion Naira in 2012 (Allwell, 2012). In the absence of improved water, millions of Nigerians are forced to consume what is available from rain, wells, ponds, boreholes and streams.

The problem is not that of water availability as such. Rather the issue is whether the water is within reasonable proximity, reliable, safe and sufficient to meet daily water needs of the different households. Water and sanitation challenges 
require a multifaceted intervention program that would address the current diarrhoeal prevalence rate among children under five years. This can only happen as policy makers and stake holders pay attention to the various factors within the localities contributing to the scourge. The heterogeneity of the issues demand that location-specific plans should be generated for each locality or zone as needed.

The data elicited from this survey shows marked differences from one location to another which means that the right solution may have to ride on the back of a needs assessment done for an assigned locality. The stakeholders must learn to see slum residents as real people compelled by circumstances to be in the places they squat in. The life of every child must count irrespective of the localities they find themselves in by accident of birth.

Policy makers should address some of their issues by allocating more resources to address the problems that surround them. It can start with as little as a low maintenance water and sanitation scheme that would make available potable water and improved sanitation facilities. This would ultimately reduce water-borne diseases and save the water bed from pollution powered by environmental degradation from open defecation and 'flying toilets'.

NAFDAC would need to review the current policy on monitoring and evaluation of table water in the market. It is not enough to pay scheduled visits like they do now. The integrity of such visits may be marred by emergency 'fix it' acts by the producers. In its stead they should pick samples from neighborhood shops and markets. They should also enforce the shelf life policy that demands that a best before date be stamped on all products. Defaulters should be prosecuted accordingly. It is scandalous that products carrying their insignia would not meet stipulated standards.

The United Nations recognize potable water and good sanitation as a human right following a report that the world lost 4,000 children daily to water-borne diseases. In their collective opinion, water and sanitation crisis constitute an infringement on a child's right to life (Mchangama, 2010). Children when born, should grow, develop and thrive in environments with clean air, clean water, safe food and minimal exposure to harm. A sustainable and integrated water-sanitation scheme is required to provide an enabling environment for that to happen (UNEP/WHO, 2010).

\section{References}

Achebe, C. (2012). There was a country: a personal history of Biafra. The Penguin Press, New York.

Allwell, O. (2012). 66 Million Nigerians lack potable water and 32 million defecate openly. www.punchnig.com/news/32-million:nigerians-defecate-openlyworldbankreport. 
Billig P., Bendahmane D., and Swindale A. (1999). Nutrition Technical Assistance Series: Water and sanitation measurement guide, United States Agency for International Development (USAID).

Boadi, K.,O. and Kuitunen, M. (2005). Childhood diarrhoeal morbidity in Accra metropolitan area, Ghana; socio-ecnomic, environmental and behavioural risk determinants, Journal of Health and Population in Developing Countries.

Burke, V. et al (1984). Isolation of Aeromonas species from an unchlorinated domestic water supply. Applied and Environmental Microbiology, 48: 367-370.

Coleman, James (1971). Nigeria; Background to nationalism, 1974. University of California Press.

Douglas, E.,M., Githui, F., W., Mtafya, A., R., Green, P., A., Glidden, S., J.,Vorosmarty, C., J., (2006). Characterizing water scarcity in Africa at different scales. Journal of Environment, 2006.

Easterly, W. (2006). The white man's Buren. New York; Penguin Books.

Egboka, B. C. E., (1985). Water resources problem in the Enugu area of Anambra State, Nigeria. Water Resources Environment Pollution Unit (WREPU), Department of Geology, Anambra University of Technology, awka.

Enugu State Vision 4; 2020, (2010). The First Enugu Vision 4 2020, Medium Term Implementation Plan, 2010-2013. An economic blue print for the transformation of the coal city. Published by 1415 Design.

Esrey, S., Potash, J., Roberts, C., and Shiff, C. (1991). Improved water supply and sanitation and ascariasis, diarrhoea, drancunculiasis, schistosomiasis and trachoma. Bulletin of the World Health Organization, 69 (5): 609-21.

Flides, J. (2009). The winds of change for Africa. BBC News: http://news.bbc.co.uk [Accessed, November 29, 2010]

Fewtrell, L. et al. (2005). Water, sanitation and hygiene interventions to reduce diarrhoea in less developed countries; a systematic review and meta-analysis. Lancet Infectious Diseases, 5 (1): 42-52.

Fuentes, R., Pfutze, T. and Seek, P. (2006) A logistic analysis of diarrhoea incidence and access to water and sanitation. Human Development Report, UNDP, 2006.

Kosek, M., Bern, C., Guerrant, R., L. (2003). The global burden of diarrhoeal disease as estimated from studies published between 1992 and 2000. Bulletin of World Health Organization, 81: 197-204.

Mchangama, J. (2010). Water: This right is wrong. The Guardian newspaper, Volume 28, No 11552, Guardian Press Limited, Lagos, Nigeria. 
National Planning Commission (2010). The First National Implementation Plan For Nigeria Vision 20:20:20 (2010-2013), Volume 2, Sectoral Plans and Programmes Economic Transformation Blue Print, Published by 1st October Publishing.

National Population Commission (NPC) Nigeria and I.C.F. Macro (2009). Nigeria Demographic and Health Survey 2008 Abuja Nigeria: National Population Commission and I.C.F. Macro.

National Population Commission, (2006). National and State Population and Housing Tables. Priority Tables. Volume 1, NPC, Abuja, 2009.

Nnajide, N. (1988). Study of diarrhoea disease in children aged 1-5 in some hospitals in Enugu (Abakpa, UNTH, and Symbol Hospital) between 19801985. Unpublished MBBS Dissertation, Department of Medicine and Surgery, University of Nigeria, Nsukka.

Nnodu, V., C., Okoye, C., O. and Onwuka, S., U. (2008). Sources and quality of domestic water in Aguluezechukwu in Aguata Local Government Area in Anambra State, Nigeria. Journal of Environemental Sciences, 12: 54-72.

Shiklomanov, I., A. (2000). Appraisal and assessment of world water resources Water International, 25: 11-32.

Standard Organization of Nigeria (SON), (2007). Nigeria Industrial Standards, NIS 554:2007, Nigeria standard for drinking water quality ICS 13060 20, Price Group D, Lagos and Abuja

United Nations Development Program (2006). Human Development Report; Beyond scarcity, power, poverty and global water crisis. UNDP, New York.

United Nations Environment Program (UNEP)/WHO (2010). Healthy environment for healthy children: key message for action. UNEP/WHO Publications, France.

United Nations-Water (2006). Coping with water scarcity: a strategic issue and priority for system-wide action, United Nations, New York.

Wambui, E., Murage, K. and Ngindu, A. (2007). Quality of water the slum dwellers use: the case of a Kenyan slum. Journal of Urban Health, 84: 829-838.

WHO/UNICEF/JMP (2012). Progress on drinking water and sanitation: 2012 update. UNICEF Division of Communication, New York.

WHO (2012). Water sanitation and health: water related diseases. Geneva Switzerland

WHO/UNICEF (2006). Core questions and drinking water and sanitation for household survey. Geneva, Switzerland. 
WHO/UNICEF (2004). Meeting the MDG's on drinking water and sanitation target: a mid-term assessment of progress. Geneva, Switzerland.

WHO (2002). Guidelines for drinking water quality, second edition. Geneva, Switzerland.

WHO, (2000). Global water supply and sanitation assessment. Geneva Switzerland. 


\section{Improving Maternal and Child Health: The Role of Food Safety in the Developing World}

Ken Okwae Fening, University of Ghana, Accra, Ghana

\section{Abstract}

Food safety is the absence of unacceptable levels of contaminants, adulterants, naturally occurring toxins or any other substance that may make food injurious to health on an acute or chronic basis. Food safety has become a growing public health concern in developed and developing countries. Globally, governments and stakeholders are intensifying efforts to improve and promote food safety. These hazards have received much attention: naturally occurring toxins, pesticide residues, parasites, adulterants, antibiotic drug residues, zoonotic diseases, microbial pathogens, persistent organic pollutants, heavy metals and genetically modified foods. The impact of these hazards seem to be more severe in the developing world due to illiteracy, lack of awareness, inadequate access to modern research 
laboratories to monitor food contaminants and residue levels, poor enforcement of food safety regulations and standards, and poor food handling practices. Many developing nations do not have the political will, capacity and resources to respond to the challenges in the sector. Food safety is sometimes not considered a priority by some of them. Perhaps looking at food safety as an instrument of public health and a tool for economic development may earn it the attention required. The promotion of food safety has been linked to improved nutrition and health outcomes. Consequently, policy makers and stakeholders should drive programs that would secure safe food for all, especially infants, young children and pregnant women. This would contribute to the realization of the targets spelt out in Millennium Development Goals (MDGs) 4 and 5, aimed at reducing child mortality and improving maternal health.

\section{Overview and important aspects of food safety}

Safe food is free from toxins, pesticides, chemical and physical contaminants, microbiological pathogens such as bacteria, parasites, and viruses (Cynthia, 2003). Food safety is an important public health issue as consumers are becoming more conscious of what they eat or drink (WHO, 2012). Food safety should be a shared responsibility between producers, industry, government, researchers and consumers (Altekruse et al., 1995; Acheson et al., 1998). Unsafe food is a major contributor to foodborne illnesses defined as diseases caused by agents that enter the body through the ingestion of food (WHO, 2012). Among the predisposing factors are improper holding temperatures, inadequate cooking, contaminated equipment, food from unsafe sources and poor hygiene (Cynthia, 2003; Fening and Edoh, 2009; WHO, 2012). Threats to food safety have always been there, but new challenges are coming up as a result of changing demographics and lifestyles, new food production practices and constant evolution of microorganisms (Acheson et al., 1998).

The targets for MDG4 and 5 are about reducing child mortality and improving maternal health (MDGs report 2011). Achieving the goal for child survival requires actions that would address the many challenges that expose children to the causes of diseases and death. In spite of the modest progress recorded for MDG 5, pregnancy remains a major health risk for women in the developing regions. Therefore, promoting safe food for improved maternal and child health, especially in the developing world would contribute to the realization of MDGs 4 and 5. 


\section{Food safety challenges in the developing and developed world}

Food safety issues and foodborne diseases are a growing public health problem in developed and developing countries (WHO 2012). Globally, policy makers and stakeholders are intensifying efforts to improve and promote food safety in response to an increasing number of food safety problems and rising consumer concerns. Food safety and foodborne illnesses are not well documented in the developing countries. However, there are studies linking food safety problems to the high prevalence of diarrhoeal diseases in developing countries (Fening and Edoh, 2009).

Unsafe food has also been proven to contain hazards and toxins. Prominent among these naturally occurring toxins are mycotoxins, marine biotoxins, cyanogenic glycosides and toxins occurring in poisonous mushrooms. Mycotoxins, such as aflatoxins, fumonisins and ochratoxin A, are found at detectable levels in many staple foods. In Nigeria, some food items used for weaning purposes like groundnuts, maize, and other oilseeds, are vulnerable to moulds, especially Aspergillus parasiticus and $A$. flavus that produce aflatoxins (Jimoh and Kolapo, 2008; Oluwafemi and Ibeh, 2011). The health implications of long-term exposure to these toxins are still poorly understood, but some studies have established positive associations between them and the incidence of oesophageal and liver cancer (Aly, 2002; Fandohan et al., 2003). Ankrah et al. (1994) studied the relationship between a diet containing aflatoxins and liver inflammation in Ghana. The major food sources that the subjects ate were cassava (as konkonte), maize (as kenkey), or a mixture of the two (as akple). The results indicated the participants were predisposed to inflammation of the liver rather than cancer of the liver. However, Bulatao-Jayme et al. (1982) found that the risk of developing primary liver cancer increases with the quantity of aflatoxin in the ingested food. Similarly, Yu et al. (1989) studied the relationship between primary liver cancer and aflatoxin intake in local foods in five villages in China and found a significant correlation between the disease and aflatoxin prevalence. Rensburg (1977) also reported a significant statistical relationship between the incidence of liver cancer and the intake of aflatoxin in Africa and Asia.

Food safety is also known to be affected by pesticides (insecticides, herbicides and fungicides) used by farmers in crop production. There is a limit to what the average consumer can ingest pesticides and remain safe (Patrick, 2012). Exposure to pesticides can occur directly from occupational, agricultural and household use, and indirectly through the diet (Cohen, 2007). High levels of pesticide residues are usually present in foods like vegetables, fruits and cereals. In developing countries, farmers still utilize hazardous pesticides indiscriminately in their farms. There are studies showing pesticide residues in milk, vegetable, fruits, meat and fish in Ghana (Fianko et al., 2011). High levels of pesticides, heavy metals, microorganisms and mycotoxins were also detected in street-vended food samples in Accra, Ghana from 1999-2000 (Pesticides News, 2011). Chlorpyrifos was detected in six out of 
eight samples of waakye (rice and beans) and one out of eight samples of fufu (cassava and plantain dough). Other studies confirmed that some vegetables such as lettuce, cabbage, tomatoes and onions being sold to consumers in a Ghanaian market contained detectable levels of chlorpyrifos, lindane, endosulfan, lambdacyhalothrin, and DDT residues (Ntow, 1998; Ntow et al., 2006).

There is now a conscious effort to replace insecticides like organochlorines (OCs) that persist for years in nature with products like pyrethroids, biopesticides and botanicals that are more nature-friendly (Fening et al., 2011). In the past few decades, different studies have attempted to establish a relationship between chronic pesticide exposure and adverse health effects. A recent systematic review that examined the literature on human health effects of currently used pesticides, excluding OCs found a high level of clinical and subclinical effects including significant positive associations with solid tumours, haematological cancers and genotoxic effects (Sanborn et al., 2004). In addition, pesticides were found to affect mental and emotional functioning, the nervous system (causing neurodegenerative disease) and the reproductive system (causing birth defects, infertility, foetal death, and intrauterine growth retardation) (Sanborn et al., 2004).

A recent review that examined how pesticides affected children revealed that in addition to causing reproductive effects, pesticide residues were linked to childhood cancer, neurological, neurobehavioral and endocrine disorders (Garry, 2004). Children appear to be very vulnerable to the effects of pesticides. The reason is not far-fetched. Children have less developed detoxification pathways. Newborn infants have low levels of the enzyme paraoxanase-1 responsible for detoxifying organophosphates (OP) in pesticides (Chen et al., 2003). Also children with a longer life span ahead enjoy a long latency period during which time they may stay asymptomatic (Sanborn et al., 2004). However, children may be at greater risk of pesticide exposure as they eat and drink more per kilogram of body weight than adults and their diets are often rich in foods which contain high levels of pesticides including breast milk (Cohen, 2007). Furthermore, OP pesticides are neurotoxic at high doses and chronic exposure of pregnant women could adversely affect children's cognitive development (Maryse et al., 2011).

Persistent organic pollutants (POPs) are another group of compounds that can accumulate in the environment and the human systems. Some popular examples are dioxins and polychlorinated biphenyls (PCBs). These compounds are unwanted by-products of some industrial processes and waste incineration. Exposure to POPs has been linked to a variety of adverse health effects in humans. In the same way, heavy metals such as lead and mercury, cause neurological damages in children. Exposure to cadmium can cause kidney damage, in the elderly. Both heavy metals and POPs may wind up in food chains through pollution of air, water and soil.

There are other food safety issues brought about by contact with microorganisms that result in foodborne diseases. One of the major foodborne diseases in the developing countries is salmonellosis caused by the Salmonella species. Some of 
the symptoms are fever, headache, nausea, vomiting, abdominal pain and diarrhoea. There are foods usually involved in outbreaks of salmonellosis such as eggs, poultry, raw milk and chocolate. Campylobacteriosis is yet another widespread infection. It is caused by some species of Campylobacter. Some of the noticeable symptoms of campylobacteriosis include severe abdominal pain, fever, nausea and diarrhoea. Some microorganisms like variants of Escherichia coli and Listeria are known to cause intestinal bleeding along with gastric disorders. Although their incidence is relatively low, their severe and sometimes fatal health consequences, particularly among infants, children and the elderly, places them among the most serious foodborne infections. A recent study in Ghana identified farmlands as the main source for lettuce microbial contamination (Amoah et al., 2007a). According to the study, aside from the irrigation water, contamination was also attributed to manure application and already contaminated soil. In another study, high counts of several pathogenic microbes including Salmonella and E. coli were also found in fruits and vegetables sold in Sango Ota, Nigeria (Eni et al., 2010).

The challenge does not end with what we have come to know as microorganisms. There is another group of organisms also factored into the big debate on food safety; genetically modified organisms (GMOs). These GMO's and their derivatives are prohibited in organic food production but there is currently insufficient evidence to reach any definitive conclusions regarding the safety of genetically modified foods for humans. However, some negative health effects have been observed in animal trials (Patrick, 2012). It is believed that genetically modified foods may contain allergens or toxins that are not found in conventional foods. Typical examples of such foods include crops modified to resist pests, foods with allergens removed, food whose essential nutrients are increased and foods with anti-microbial markers. The potential risks and benefits of genetically modified foods is an important aspect of assessment of foods derived from biotechnology that has not received the right level of attention (WHO, 2012). Even the addition of low levels of antibiotics in animal feed to increase growth rate in livestock production has been linked with drug resistance in animals and humans (WHO, 2012).

Diarrhoea, one of the fallouts arising from unsafe foods is a pandemic and has claimed the lives of many children, particularly of those under 5 years of age from developing countries (Kosek et al., 2003). Diarrhoeal diseases, especially cholera is in the front burner of public health domain because of the linkages to enormous economic losses that perpetuate poverty in developing countries especially Africa, South Asia and Latin America (Parashar et al., 2003; Fening and Edoh, 2009). In addition to the microorganisms cited so far, water, contaminated foods and drinks are also known to be channels of infection. Different foods, including rice, vegetables, fruits and various types of seafood have been implicated in outbreaks of cholera. Some of the cholera symptoms include abdominal pain, vomiting and profuse watery diarrhoea, which may lead to severe dehydration and possibly death (Fening and Edoh, 2009). 


\section{Promoting safe and nutritious food intake in the developing world}

The major problem-limiting activities needed for food safety promotion and prevention of foodborne illnesses is the lack of material resources required for driving programs that would generate the required good. Governments in the developing world face multi-faceted demands, but have very limited capacity to respond to them as they come. The question on everyone's mind continues to be; where should food safety be placed in the priority list of the community? One of the ways this can be done is by seeing food safety as a public health concern and tool for economic development. It therefore means that promoting safe food consumption would lead to improved nutrition and health outcomes. This should be entrenched in any strategy meant for poverty alleviation and hunger reduction.

If the developing nations desire to meet the scheduled targets for MDGs 4 and 5 , there must be a commitment by the different tiers of government, the organized private sector and donor agencies to drive schemes that would make that happen. There is now a need to put in place intervention programs that would guarantee the safety of foods consumed by infants, young children and pregnant women. Food safety should be driven along with food hygiene in awareness campaigns especially at the grass roots. Pregnant women can reduce their unborn child's exposure to pesticides by thoroughly washing fruits and vegetables before they are eaten. The findings of a study in selected cities in West Africa show that washing vegetables before consumption is an important component of a multiple barrier approach for health risk reduction (Amoah et al., 2007b). Also eating organic foods grown without synthetic pesticides can limit exposure (Patrick, 2012). In addition, the standards and policies entrenched at country levels must be enforced.

The World Health Organization (WHO) is increasingly getting involved in the activities of Food and Agricultural Organization (FAO)/WHO Codex Alimentarius Commission, whose standards, guidelines and recommendations are regarded as the international reference point for food safety requirements by the World Trade Organization. In line with this, the WHO is expanding its global network of participating institutions to monitor chemical contamination of the food supplies particularly in developing countries. WHO has initiated projects that would establish a knowledge base focusing on a broader evaluation of risks, benefits and other considerations related to the production and consumption of foods derived from biotechnology. Current and future research must therefore focus on developing environmentally friendly options for pests and diseases management in both field crops and stored produce. There is also the need to provide clean water for irrigation to minimize the risk of microbial contamination of crop produce.

There should be intensified efforts in monitoring and enforcing food standards and laws in the developing world by building the capacity of the enforcement agencies. There is the need to equip and expand laboratories and other facilities needed for assessment of these foods prior to consumption especially for pro- 
cessed products. All stakeholders must speak with one voice if meeting the targets of MDGs 4 and 5 would ever be a reality.

\section{References}

Acheson, D. W. K.. and Robin, K. L. (1998). Safe Eating. New York: Dell.

Altekruse, S. F., Street, D. A. et al. (1995). Consumer knowledge of foodborne microbial hazards and food-handling practices. Journal of Food Protection, 59, 287-294.

Aly, E. (2002) Distribution of aflatoxins in product and by-products during glucose production from contaminated corn. Nahrung/Food 46, 341-344.

Amoah,P., Drechsel,P., Henseler,M., Abaidoo, R.C.(2007a). Irrigated urban vegetable production in Ghana: microbiological contamination in farms and markets and associated consumer risk groups. Journal of Water Health, 5, 4566.

Amoah,P., Drechsel,P., Abaidoo,C., Klutse,A. (2007b). Effectiveness of common and improved sanitary washing methods in selected cities of West Africa for the reduction of coliform bacteria and helminth eggs on vegetables, Tropical Medicine and International Health, s2, 40-50.

Ankrah, N. A., Rikimaru, T., Ekuban, F. A. (1994). Observations on aflatoxins and the liver status of Ghanaian subjects. East African Medical Journal, 71, 739-74.

Bulatao-Jayme, J., Almero, E. M., Castro, M. C. A., Jardeleza, M. T. R., Salamat,L. A. (1982). A case-control dietary study of primary liver cancer risk from aflatoxin exposure. International Journal of Epidemiology, 11, 112-119.

Chen, J.K.M., Chan, W., Berkowitz, G., Wetmur, J. G. (2003). Increased influence of genetic variation on PON1 activity in neonates. Environmental Health Perspectives, 111, 1403-10.

Cohen, M. (2007). Environmental toxins and health. The health impact of pesticides. Australian Family Physician, 36 (12).

Cynthia. A. R. (2003). Food safety. Encyclopedia of Food and Culture http://www.encyclopedia.com/topic/Food_safety.aspx, [Accessed October 29, 2012].

Eni, A.O., Oluwawemitan, I. A., Solomon, O.U. (2010). Microbial quality of fruits and vegetables sold in Sango Ota, Nigeria. African Journal of Food Science, 4, 291- 296. 
Fandohan, P., Hell, K.., Marasas, W.F.O., Wingfield, M.J. (2003). Infection of maize by Fusarium species and contamination with fumonisin in Africa. African Journal of Biotechnology, 2, 570-579.

Fening, K.O., Owusu-Akyaw M., Mochiah, M.B., Amoabeng, B.W., Narveh, E., Ekyem, S.O. (2011). Sustainable management of insect pests of green cabbage, Brassica oleraceae var. capitata L. (Brassicaceae), using homemade extracts from garlic and hot pepper. Pages 567-570. In: Pest and disease management. Organic Crop Production Volume 1: Organic is Life, Knowledge for Tomorrow. Proceedings of the Third Scientific Conference of the International Society of Organic Agriculture Research (ISOFAR), held at the 17th IFOAM Organic World Congress, Namyangju, Korea, 28 September - 1 October 2011.

Fening, K.O. and Edoh, D. A. (2009). The impact of socio-economic status and sanitation levels on the prevalence of diarrhoeal diseases in the Akim Oda area of Ghana: The Internet Journal of Epidemiology, Volume 6, Number 2.

Fianko, J.R., Donkor, A., Lowor, S.T., Yeboah, P. O. (2011). Agrochemicals and the Ghanaian Environment, a Review. Journal of Environmental Protection, 2, 221-230.

Garry, V. F. (2004). Pesticides and children. Toxicology and Applied Pharmacology, 198, 152-63.

Jimoh, K.O. and Kolapo, A.L. (2008). Mycoflora and aflatoxin production in market samples of some selected Nigerian foodstuffs. Research Journal of Microbiology, 3, 169-74.

Kosek, M., Bern, C., Guerrant, R. L. (2003). The magnitude of the global burden of diarrhoeal disease from studies published 1992-2000. Bulletin of WHO, 81, 197-204.

Maryse, F. B., Jonathan, C., Kim, G. H., Katherine, K., Michelle, V., Norma, C., Celina, T., Caroline, J., Asa, B., Dana, B. B., Brenda, E. B. (2011). Prenatal Exposure to Organophosphate Pesticides and IQ in 7-Year-Old Children. Environmental Health Perspectives, 119, 1189-1195.

Millennium Development Goals (MDGs) report (2011). United Nations, New York, 72pp.

Ntow J.W, Gijzen, H.J., Kelderman, P., Drechsel, P, (2006): Farmer perceptions and pesticide use practices in vegetable production in Ghana. Pest Management Science, 62, 356-365.

Ntow, W. J. (1998). Pesticide misuse at Akumadan to be tackled," NARP Newsletter, Vol. 3, No. 3. 
Oluwafemi, F. and Ibeh, I.N. (2011). Microbial contamination of seven major weaning foods in Nigeria. Journal of Health Population and Nutrition, 29, 415-419.

Parashar, U.D., Bresee, J.S., Glass, R.I. (2003). The global burden of diarrhoeal disease in children. Bulletin of WHO, 81, 236.

Patrick, H. (2012). Organic farming food quality and human health. A review of the evidence. Soil Association, Bristol, UK, 88pp. ISBN 09052000802. www.soilassociation.org [Accessed November 12, 2012].

Pesticides News (2001). Food contamination in Ghana. No. 52, page 17, June 2001.

Rensburg, V. (1977). In Rodricks, R.J., Hesseltine, C.W. \& Mehlamann, M.A. (1977). Mycotoxins in human and animal health, pp 699. Path. tox. Fubl. Inc. Fark Forrest, IL.

Sanborn, M., Cole, D., Kerr, K., Vakil, C., Sanin, L.H., Bassil, K. (2004). Systematic review of pesticide on human health effects. Ontario College of Family Physicians.

World Health Organisation, WHO (2012). Food safety and foodborne illness. Geneva, Switzerland, Factsheet No. 237.

Yu, S.Z., Cheng, Z.Q., Liu, Y.K., Huang, Z.Y. and Chao, Y.F. (1989). The aflatoxins and contaminated water in the etiological study of primary liver cancer, in: S. Natori, K. Hashimoto and Y. Ueno (Eds.), Mycotoxins and Phycotoxins '88, Elsevier, Amsterdam, pp. 37-44. 



\section{Household Food Insecurity and Hunger: Implications for Maternal and Child Health in Nigeria}

Adeola Olajide and Adeyemo Temitayo, Department of Agricultural Economics, University of Ibadan, Oyo State, Nigeria

\section{Introduction}

Health infrastructure is one of several resource systems harnessed for the development of a country. Such an infrastructure and the services rendered through it should be equitably distributed to that the wellbeing of individuals will be enhanced. Where this is not the case, the socio-economic development of the society will be impaired with the long-term effect of jeopardizing the future of the nation. However, if the best of infrastructure exists in an environment where people have poor health and nutrition habits, the future of the nation will still be at risk. This is what escalates problems like maternal ill health and acute malnutrition in children. Maternal health may be defined as the state of a mother before, during and after pregnancy which affects the physical and neurological development of the child. It is a product of a lifestyle of good nutrition and healthy living. If a mother is unhealthy before and during the pregnancy, the probability that her child would be 
poorly formed is high; and the chances of survival for her and her child are minimized.

The status of maternal and child health in Nigeria is such that it has drawn attention at the national and international levels. According to Ogunjimi et al. (2012), several national programs have been instituted by the Federal Government to curb the level of malnutrition in children and high maternal morbidity. These include National Child Health Weeks in which millions of children are vaccinated; National Midwives Service Scheme - a capacity-building scheme for midwives and free medical treatment for women and children under the age of five years. Two notable international efforts include the USAID's Maternal and Child Health Integrated Program being administered by the John Hopkins University in 22 Local Government Areas in Kano, Katsina and Zamfara states (USAID, 2012 b); and the UNICEF's Save the Children Community Management of Acute Malnutrition in 383 sites across the northern states of Nigeria (Abubakar, 2012). Efforts at curbing these malnutrition issues built up, particularly in the northern areas of Nigeria, where signs of food problem were identified (USAID FEWSNET, 2012) and statistics show that millions of children suffer from severe and moderate malnutrition which cannot easily be treated through the primary health care system. Nigeria accounts for $10 \%$ of the world's maternal and under five mortality rates; 800 women die per 100,000 births; 54,000 women die each year due to pregnancy-related complications; $1,080,000$ to $1,620,000$ Nigerian women suffer annually from disabilities as a result of complications during pregnancy and childbirth (USAID, 2012 b); about 1 million children under the age of five years die every year and of these, 250,000 are babies less than a month old. Both maternal and neonatal mortality rates in Nigeria have wide geographical variation, the highest rates are seen in the North-East and Northwest zones of the country, the lowest rates noticed are in the South-West and South-East (The Nigerian Academy of Science, 2009).

Many factors have been linked to maternal and child ill health but one of the most prominent is food insecurity. This is a situation which arises when people cannot meet their basic calorie needs and have limited means to access the balance required from markets. It is often a product of economic, political, demographic, social, cultural and technical factors. Food insecurity is exacerbated when the knowledge of food utilization is poor and the supply of related resources such as drinking water, health care services and sanitation is inequitably distributed (Ajieroh, 2010). Food insecurity can lead to hunger and it manifests as malnutrition and chronic illness (UNICEF, 2012).

\section{The food security situation in Nigeria}

Food insecurity implies that there is no regular physical and economic access to enough safe and nutritious food for a healthy life. Being food insecure is not a static situation because several factors combined make people vulnerable to food 
insecurity. For example, increasing prices of agricultural products make poor families become more vulnerable (Obamiro, 2004). Also, price instabilty in food markets is driving changes in general food habits, where new forms of consumption are being embraced both in urban and rural centers and by people of different work background (Muhammad-Lawal and Omotesho, 2010). Social, economic and environmental conditions have an impact on food insecurity of individuals and families and determine the length of time these affected people are insecure and cope with strategies to improve the situation. As such, food insecurity is often said to be transitory or chronic (IFPRI, 2004).

Hunger is an extreme case of food insecurity where the people concerned experience prolonged deprivation of physical food irrespective of its quality and quantity. It is a chronic form of food insecurity accentuated by poor governance, poor health care, water and sanitary supply. Rural communities, most especially women and children are often the victims of hunger - people who are hardly heard or seen, and people who suffer from absolute lack of financial access, productive resources and markets (Akinyele, 2009). Such people are chronically vulnerable and suffer from malnutrition. This is reflected in a permanent state of ill health in a woman and chronic/acute malnutrition as well as stunting in children and thereby undermining the country's development capacity.

In Nigeria, food imports are still high. Rural areas in particular are vulnerable to food shortage, malnutrition, high food cost and poor quality of foods. While children in rural households are especially vulnerable to stunting and acute malnutrition, all age groups suffer from one form of food insecurity or the other. Since the majority of the population lives in rural areas, a picture of the food situation there would give a fair representation of the situation (Akinyele, 2009).

Food security tends to vary with the seasonal production calendar, especially in the rural areas. At the harvest period, with above average harvest and good market flows, minimal food insecurity is experienced. But from May to July in the south and July to October in the north, the lean or hunger season during which staple food stuffs are scarce and expensive occurs. At this period, homes without stocks are most vulnerable because they deplete the stock in exchange for using cash in order to meet other household needs and ensure enough food to eat. Apart from insufficient stocks, low market prices during the harvest period reduce the income and foster emergency situations where food needs are not met. The increasing rates of flooding and dry spells, particularly in the north, are climate-related factors that jeopardize the food security status of the people due to problems of delayed harvest and spoiled food. Increasing tribal and religious unrest from the middle belt to the north is hampering the flow of food stuff across the regions and thereby leading to high food prices (Damisa et al., 2011). Due to such market dependencies poor households are at a higher risk of hunger and malnutrition.

The trend of food prices in Nigeria shows fluctuations over the past 9 years, from 2003 to 2011 (Anidi and Olajide, 2012). Nationally, there has been an increase in prices of rice, yam, beef, chicken, egg, palm oil, fruits and vegetables. The 
high prices of food could be witnessed in all geopolitical zones of the country, however the highest prices occurred in the South-Eastern zone, where it increased six years in a row (Akinyele, 2009). In the South-Western zone, there was also a greater fluctuation of prices of foods in comparison to other zones in the country. The trend of increased food prices is less severe in rural areas, where most vegetables are still gathered and need not be purchased. Likewise, farm-gate prices of items commonly consumed in rural areas have not risen as sharply in the rural areas as in the urban areas. However, since there is an increasing preference of rural dwellers for long grain rice, food prices increased for those foods in both rural and urban areas (Anidi and Olajide, 2012; Akinyele, 2009). The increasing food prices and price instability foster the problem of food insecurity as there is a reduced access to adequate food needed by these households due to the reduction of purchasing power of the people in an inflation period where they are unable to correspond to the decrease of their income.

\subsection{Women's access to food in Nigeria}

The rural woman is known to be the back bone of farm and household activities. Apart from working on family plots or husband's plots, women cultivate their own plots for the purpose of having additional supplies for the household. They are involved in the processing and marketing of farm products. Having to live in an environment conditioned by norms, they often find themselves with unequal opportunities compared to men. This plays out in the form of low literacy level, low income generation capacity, poor access to productive resources, poor access to adequate and nutritious food etc. In the past, the concern for the low quality of life of rural women led to the implementation of programs such as Better Life for Rural Women (BLW) in 1987 and the Family Economic Advancement Program (FEAP); while the Country Women Association of Nigeria (COWAN) represents one of several private sector efforts in this direction (Odurukwe et al., 2006). Through them, a general awareness across the nation was created and some definite changes were made, particularly with respect to food processing technologies. However, the direct impact on food security situation through women empowerment is hardly empirically supported. The issue of being food secure or not is related first to the household status and secondly to intra-household relations.

\subsection{Rural household food security situation in Nigeria}

Most households in the rural areas are poor and cannot meet their calorie requirement on a regular and consistent basis. According to Obamiro (2004), the month of April is a critical period when rural household in South-West Nigeria are known to be most vulnerable to food insecurity. At such a period, farm operations begin in earnest, necessary inputs are obtained, and household composition is at its full size. However, food reserves are low during that period. The food situation gradu- 


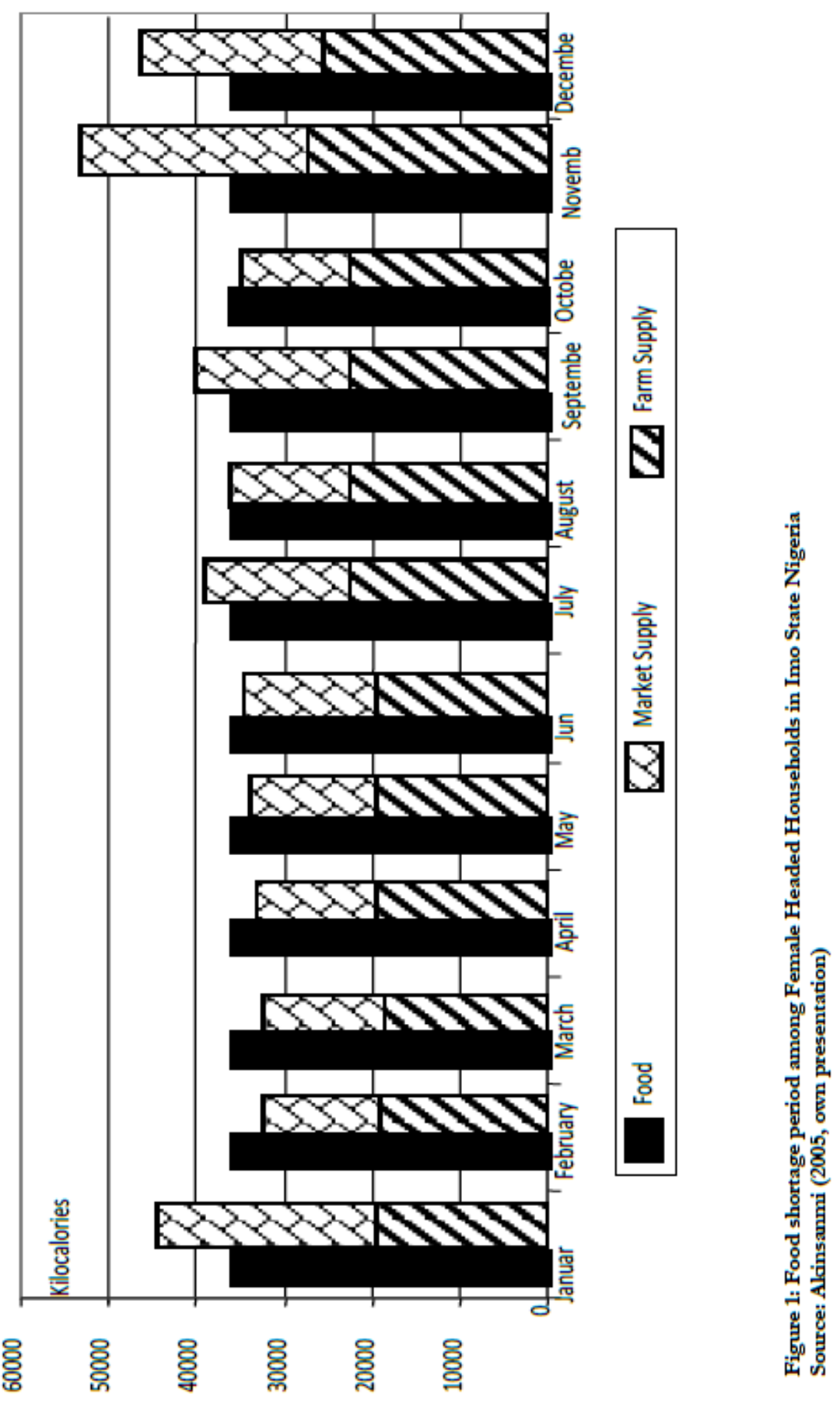




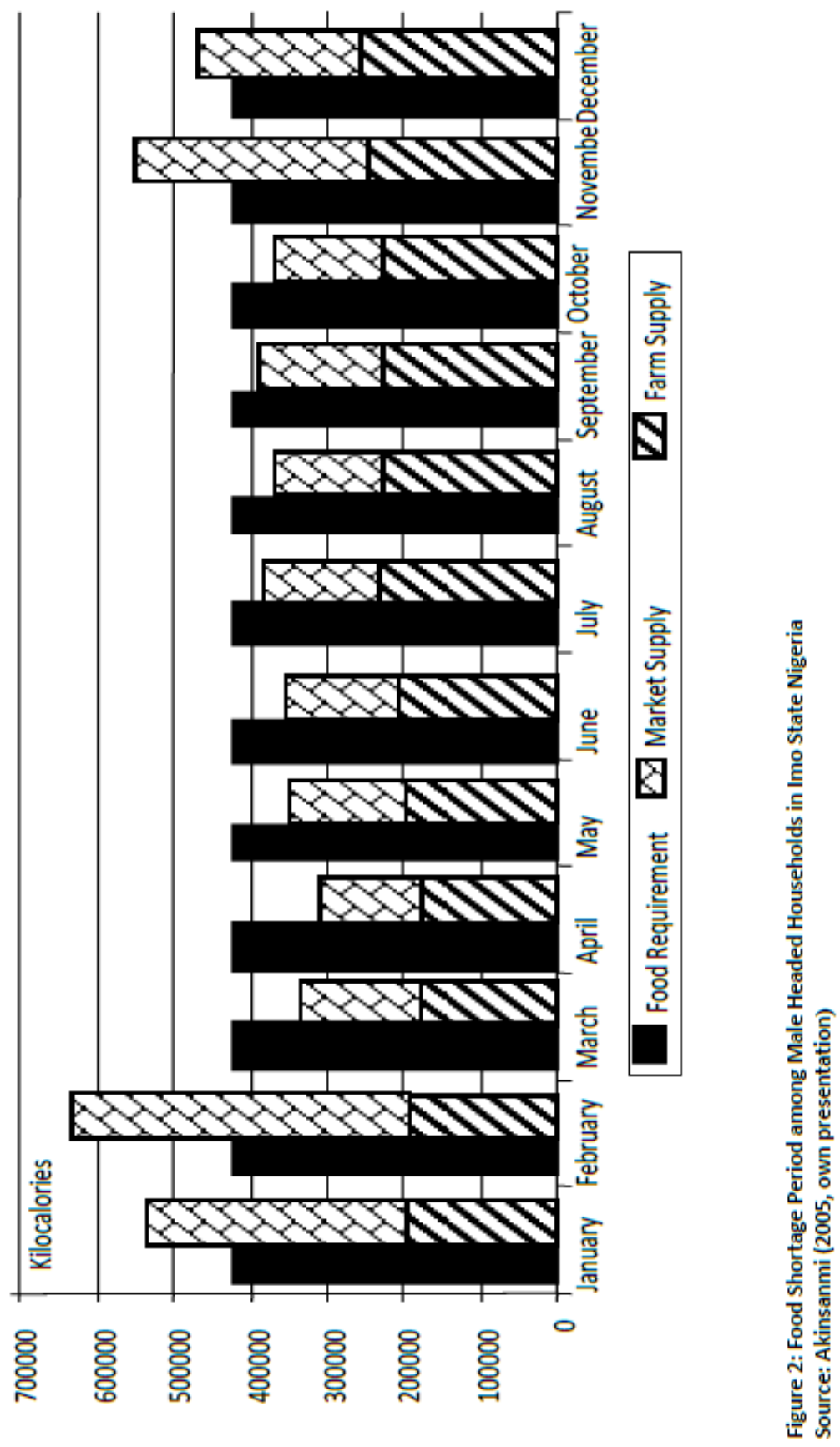


ally worsens as the season progresses. As the study in this region showed, about $40 \%$ of households could not meet their daily calorie requirements in the 'hungry' month. However, since it was measured for just a month, it could not be said that those people who appeared to have access to a sufficient amount of calories did not suffer from inadequate amount later on in the season. Neither could it be said that those who were calorie insufficient remained in this situation throughout the entire season as social networks where they can escape during such periods still exists. However the study emphasizes the vulnerable nature of rural households.

Akinsanmi (2005) studied the food security situation in South-East Nigeria and as it is shown below, farm families could not meet their calorie needs for a period of 5 months within one year even when they tried to combine the output from their own production with purchased food from the market. The quantities of calories consumed were also examined and the investigation showed that protein deficiency was highest during such periods. The food shortage of femaleheaded households was, as demonstrated in this study, equally to the poor situation of male-headed households (Figures 1 and 2). But women in the male-headed households could be relatively more vulnerable to hunger because of their tendency to sacrifice their food allocation for other household members, particularly their children.

The level of dietary diversity recognized in the poor households was quite broad but it was a case of substitution as the lack of money to purchase one item led to the consumption of another. For example, some households indicated that they consumed more fruits when they experienced hunger. Others ate fish or meat accompanied with "garri". In their view, quite a number associated being food secure with having a large quantity of carbohydrate food such as cassava and yams, it also meant being able to eat at least twice a day irrespective of the composition of the food.

In the Northern Guinea Savanna zone of Nigeria, Damisa et al. (2011) stated that farm families experienced hunger 2 to 3 months after the main harvest. The intensity of the hunger experienced is progressive; it becomes harsh in the month of January overlapping into February and in the months of March to June it becomes severe. The cycle as noted by the authors is a regular one; the output produced is not enough to meet home consumption but most of it has to be sold immediately, between October and December, in order to pay back credit incurred and meet other domestic obligations. With floods occurring in the study area, the hunger period was pronounced and led to severe malnutrition of farm families, children in particular. One of the coping strategies in the food shortage period was that at least one member of the household would go without food for a day; other coping strategies in the area included sale of livestock and household assets and borrowing of foodstuff. Women, who lived in such households, deprived themselves of food for their husbands and children.

Oluwatayo (2008) in his study of the food security situation in Ekiti state, SouthWest Nigeria, showed that more male-headed households were vulnerable to food 
insecurity compared with female-headed households due to the fact that resources were pooled together in the female-headed households. Also they had a higher likelihood of participating in cooperative societies which enhanced their capacity to meet their calorie needs. Muhammad-Lawal et al. (2009) developed an ultimate food composition plan for food-insecure households in the rural areas of Kwara state since the nature of food insecurity associated with the households was tied to poor knowledge of nutritious and low-cost food items. The dietary pattern of rural households surveyed did not show a calculated attempt at meeting household food security and nutritional requirement but rather focus on staples which are typically produced and are mainly carbohydrates.

In response to the pervasive situation, the Federal Government of Nigeria instituted a Special Program for Food Security across the nation with the aim of improving national and household food security and reducing rural poverty in an economically and environmentally sustainable way (FAO, 2010). The specific objectives were:

- Improve household food security and incomes through increases in productivity, diversification and sustainable use of natural resources

- Enhance food security of consumers through improved access to and availability of food and also increase income of producers through more efficient marketing

- Enhance farmers "and consumers" access to support services such as extension, credit, nutrition and health education

- Foster participation of the poorer section of the rural population in the development of the community

Several program activities were instituted through which the objectives of the program were to be achieved. The program successfully increased the production of priority crops such as cassava, yam, millet, sorghum etc; it also increased the supply of food crops in marginal areas. However, beyond increased food production and food supply there is the question of access and knowledge and use of food items and intra-household allocation of food.

\subsection{Intra-household allocation of food in Nigeria}

Aromolaran (2004) in his study in South-Western Nigeria found that while food consumption pattern were essentially the same between males and females, adults received a higher percentage of their calorie intake from high quality sources compared with their children while children below the age of 6 years received food energy intake from roots and tubers alone. It was also reported that children and male adolescents received calorie intake less than the daily minimum required. This is due to the fact that the absolute value of what male adolescents require is higher 
than what females require, however implying also that women's calorie needs are not met either. The study showed that calorie allocation was skewed towards household members who worked in the food sector. According to Akerele (2011), unequal distribution of food within a household is a factor that promotes undernourishment among household members in Nigeria. In the study examining the calorie distribution pattern and calorie adequacy of household members in SouthWestern Nigeria, households appeared to be nutritionally sufficient while some members were under-nourished. Those who were negatively affected were male pre-school children and female school-aged children while the most favored were adult male within the family. This implies that children growing up became progressively more malnourished than adult males who are able to keep their level of security.

The study showed that female adolescents were more food secure than the males but female adults were less food secured than the adult males. This scenario might be a reflection of the practice of maternal buffering in which mothers deliberately limit her own food portion size in order to ensure that children (particularly the pre-school children) get enough to eat in the face of food insufficiency at home (Maxwell, 1995 cited in Akerele, 2011). This might be a reason for relative higher calorie adequacy level of pre-school children than other younger members of the households (Akerele, 2011). In the region, larger food portions are given to the adult male (especially if he is the household head) as a sign of respect. Likewise, where the household engages in farming activities, the older household members are the main sources of farm labor. These often make them receive higher allocation to compensate them for the increased energy demand for agricultural activities. The studies indicate that equitable distribution of food calorie is more likely achieved in households where members engage in farming (Tables 1 and 2).

Table 1: Test of difference of means of calorie adequacy by age and gender groups

\begin{tabular}{lcccl}
\hline \multicolumn{1}{c}{ Age group } & \multicolumn{2}{c}{ Calorie adequacy ratio (\%) } & \multicolumn{2}{c}{$\boldsymbol{t}$-test } \\
\cline { 2 - 6 } & Male & Female & $\boldsymbol{t}$-computed & $\boldsymbol{P}>/ \boldsymbol{t}$ / \\
Less than 6 & 94.72 & 94.18 & 1.002 & 0.312 \\
(pre-school children) & & & & \\
6-10 & 86.93 & 82.56 & 1.101 & 0.342 \\
(school-age children) & & & & \\
11-18 (adolescent) & 87.71 & 102.14 & $2.263_{-}^{*}$ & 0.028 \\
19-59 (adult) & 133.24 & 90.97 & $4.667_{-}^{*}$ & 0.000 \\
\hline Entire households & \multicolumn{3}{c}{105.45} \\
Variable significant at 5\% $\alpha$-level
\end{tabular}

Source: Akerele, (2007) Intra-household food distribution patterns and calorie inadequacy in SouthWestern Nigeria. 
Table 2: Intra-household relative calorie allocation

\begin{tabular}{lcc}
\hline \multicolumn{3}{c}{ Relative calorie allocation in \% } \\
\hline \multicolumn{1}{c}{ Age group } & Male & Female \\
Less than 6 (preschool children & 0.898 & 0.894 \\
6-10 (school-age children) & 0.824 & 0.783 \\
11-18 (adolescent) & 0.832 & 0.969 \\
19-59 (adult) & 1.264 & 0.862 \\
60 and above (aged) & 0.978 & 0.972 \\
\hline
\end{tabular}

Source: Akerele, (2007) Intra-household food distribution patterns and calorie inadequacy in South-Western Nigeria

Odurukwe et al. (2006) reported that the adoption of women in agriculture technology packages actually led to all members of the family having animal protein sources such as chicken and fish included in their diets, however the sufficiency of the protein sources for the family was not reported. Aju and Popoola (2010) found that various vegetables were consumed regularly among households in South-Eeast Nigeria, particularly Imo state. However, no discrimination in the sharing of such meals was reported.

The forgoing paragraphs give an interesting picture of the complex nature of intra-household allocation of food. But it tells clearly that food allocation is influenced by the structural composition of the household, the economic viability of individuals and other sentiments related to the culture. This explains why adults, particularly males, seem to be favored at the expense of female adults and children. It also shows the knowledge gap in food composition which is why large quantities of food consumed in the household do not automatically translate to nutritional security and explains why children are fed from roots and tubers alone. The general trend supports the idea that women are less likely to be nutritionally secure but more empirical evidence is required.

\section{Malnutrition: implications for maternal and child health}

Malnutrition starts from conception and is linked to several socio-economic characteristics such as education, poverty, access to health services. With the evidence from research, it is clear that when a household is poor, then the likelihood that the mother of the household and children will be malnourished is high, especially where the household has a limited social network. Malnutrition in women is especially responsible for maternal and child morbidity and mortality. When a woman does not have access to adequate food, it goes without saying that the children will be malnourished. Such women when pregnant do not have adequate nutrition to pass to the unborn child and even at birth, they are not able to provide enough nutrition for the child. Malnutrition in both, mother and child is the underlying 
cause of morbidity and mortality of a large proportion of children under 5 years in Nigeria, accounting for more than $50 \%$ of deaths of children in this age bracket (UNICEF, 2012).

Using the national survey, and other studies, Ajieroh (2010) revealed that 38$42 \%$ of Nigerian children under 5 years are stunted and $25-29 \%$ are underweight and $9 \%$ are wasted. According to the International Food and Policy Research Institute (IFPRI) "malnutrition can be perpetuated across generations in a cycle or, worse, a spiral of poverty and despair". The processes through which it functions can be described as follows: "[A girl] born to a mother that is undernourished will likely be born stunted in height and low in weight, even at full term. If the infant survives, her growth will be more likely to falter. Her ability to learn will be, to a large extent, irreversibly damaged as will her ability to develop other skills that pay off in the labor market, in the home, and in the community. She will be more susceptible to infectious diseases and to non-communicable diseases in later life. Throughout her life, her options and her power to make choices about those options will be as stunted as her growth will be. Society will be worse off in a social and economic sense. During her childbearing years, she will bear low birth-weight babies of her own. And so the cycle of intergenerational poverty and ill-health continues." (IFPRI 2004).

While there are many factors contributing to maternal and child health in Nigeria, one of the key issues is the nutritional status of the mother and the child. Food security includes availability, access and utilization of food as such women's nutrition is a combination of these dimensions. Inadequate nutritional education leads to poor utilization of the accessed food for the woman and her household.

\section{Conclusion}

In Nigeria, hunger and malnutrition have been shown to be prevalent especially among vulnerable groups, women and children and rural dwellers. It is shown that even with different policies and projects on maternal and child health, adequate nutrition is the key to ensure the success of achieving the goals of such programs. Malnutrition has led to death, stunting and wasting of a large percentage of children in Nigerian households. It is seen that there is inequality in nutritional status, maternal and child health between and among regions in Nigeria. Household food insecurity and hunger are found to be a significant factor in child and maternal health in Nigeria.

The fight against child and maternal ill health in Nigeria must be a fight against food and nutrition insecurity. Though direct empirical evidence do not abound, trends in existing research reports and development interventions suggest a strong link between food and nutritional insecurity and child and maternal mortality. A holistic approach to policy and strategy formulation which includes, production, access to resources, income, employment, health, education, food quality and safety, water and sanitation supply, good governance and political stability is required. Reducing under nutrition would make a significant contribution to reducing pov- 
erty and ending the vicious circle that connects the two. Also, increasing educational level of the mother increases the opportunities for economic empowerment as well as decision making within the households. This translates to increasing access to food as well as better decision making in the health and nutrition need of both herself and her child. Improved educational status also ensures proper utilization of food in the sense that food is presented both in the right quantity and proportion (Ajieroh, 2010).

While, Fayeye and Daramola (2007) opine that research should aim at increasing nutrient content of food, such that there is an increase in available nutrient for the staple foods consumed by the majority of households in Nigeria; the authors are of the opinion that intra-household dynamics which cause inequality in food allocation and consumption must be addressed from research and development perspective.

\section{References}

Abubakar, AA. (2012). North: saving malnourished children, Peoples Daily, 27th October, 2012, www.peoplesdaily-online.com [Accessed: October 27, 2012].

Ajieroh, V. (2010). A quantitative analysis of determinants of child and maternal nutrition, Nigeria IFPRI, National Strategy Support Program, Brief, No. 11.

Aju, PC. and Popoola, L. (2010). The dietary role of traditional vegetables in the rural communities of Imo state, Nigeria, Journal of Sustainable Development in Africa, 12:2010.

Akerele, (2007). Intra-household food distribution patterns and calorie inadequacy in South-Western Nigeria, International Journal of Consumer Studies, 35:545551.

Akinsanmi, OA. (2005). Gender relations and food security in Imo state, South East, Nigeria, Farming And Rural Systems Economics (Eds) W. Doppler Volume 68, pp 254 Margraf Verlag Weikersheim.

Akinyele, IO. (2009). Ensuring food and nutrition security in Nigeria, International Food Policy Research Institute, 2009 Appleton.

Anidi, O. and Olajide, OA. (2012). Variation in prices of selected food items in rural and urban markets in Oyo state, Paper Presented at the Annual Conference of Nigerian Association of Agricultural Economists "Agriculture in The National Transformation Agenda: The Policy Mix" 25th -27th September, 2012. Obafemi Awolowo University, Ile-Ife.

Aromolaran, BA. (2004). Intra-household redistribution of income and calorie consumption,South-Western Nigeria Economic: Growth Center Yale University Center, Discussion Paper No. 890. 
Damisa, MA., Saleh, MK.and Aliyu, RS (2011). Rural household perception and response strategies to seasonal food shortages in the Northern Guinea Savanna of Nigeria, Journal of Sustainable Development in Africa, Volume 13, No. 6, 2011.

Fayeye, TR. and Daramola, JO. (2007). Strategies for food security and health improvement in Sub Sahara Africa, World Journal of Agricultural Sciences, 3:808-814.

Food and Agricultural Organization (2010). Supporting Programs for Food Security, www.fao.org [Accessed October 27, 2012].

IFPRI (2004). Africa's food and nutrition security situation: where are we and how did we get here?, 2020 Discussion Paper 37.

Maxwell, DG. (1995). Measuring food insecurity: the frequency and severity of coping strategies. IFPRI, Food Consumption and Nutrition Division

Discussion Paper No. 8, pp 31, International Food Policy Research Institute, NW, Washington, DC, USA.

Muhammad-Lawal, A. and Omotesho OA. (2010). Optimal food plan for rural households' food security in Kwara State, Nigeria: The goal programming approach, Journal of Agricultural Biotechnology and Sustainable Development, 2:007-014.

The Nigerian Academy of Science (2009). Reducing child mortality, Nigeria Olumide, Y.M and Odubanjo O.M (Eds) West African Book Publishers Lagos.

Obamiro, OE. (2004). Rural food security and living standard in humid zones of Nigeria, Farming And Rural Systems Economics (Eds) W. Doppler, Volume 68, pp 254 Margraf Verlag Weikersheim.

Odurukwe, SN., Matthews-Njoku, EC. and Ejiogu-Okereke, N. (2006). Impacts of the women-in-agriculture (WIA) extension program on women's lives; implications for subsistence agricultural production of women in Imo State, Nigeria, Livestock Research for Rural Development, Volume 18, Article 18.

Ogunjimi, LO., Ibe, RT. and Ikorok, MM. (2012). Curbing maternal and child mortality: The Nigerian experience, International Journal of Nursing and Midwifery, 4:33-39.

Oluwatayo, IB. (2008). Towards assuring households-food security in rural Nigeria: have cooperatives got any place?, Nigerian Journal of Rural Sociology, 7:57-69.

UNICEF (2012). Maternal and Child Health, Online Resource [Accessed November 28, 2012].

USAID (2012 a). Famine Early Warning Systems Network, October 2011 to March 2012. 
USAID (2012 b) Maternal and Child Health Integrated Program http://nigeria.usaid.gov/programs/health-population-andnutrition/projects/maternal-and-child-health-intergrated-program-mchi [Accessed November 28, 2012] 


\title{
6. Children's Environmental Health Risks in Kenya
}

\author{
Wilkister Nyaora Moturi, Environmental Science Department, \\ Egerton University, Kenya
}

\section{Abstract}

The major causes of morbidity and mortality in children in Kenya are due to diseases that are preventable through immunization, proper environmental management or observing basic hygiene. A case study was conducted in 2005-2006 in 350 rural households in upper River Njoro watershed to determine selected environmental health risks to children aged 1-4 years in the home environment. These risks included food handling practices, handwashing at critical times and faecal disposal practices. Data was collected using information from key informants, questionnaires, continuous and spot observations. Results indicate that about $41 \%$ of study households did not have toilets and faecal disposal for children was suboptimal, implying poor faecal disposal methods. This could explain the reason why in $69.4 \%$ of households, faecal matter was spotted in the compound at least once during the visits made to the homesteads. This predisposes the children, who play around the compound, to faecal-oral infections. Food safety is threatened because $68.4 \%$ of caretakers were observed not to wash their hands before handling food. Of those children who were capable of feeding themselves, $32.5 \%$ did not wash their hands before eating. In $21.4 \%$ of households, leftover food was stored in a 
manner likely to allow food contamination. Despite the numerous programmes put in place to deal with issues of children's environmental health, many children especially those under the age of five are severely exposed to environmental health risks in the home environment. More efforts should be directed towards enhancing child environmental health within the home environment.

Key words: Diarrhoea, food safety, home, mortality, sanitation.

\section{General information on Kenya}

Kenya is located within the eastern side of the continent of Africa and forms an important geopolitical, economic and infrastructural part of East Africa. It lies astride the equator and is situated between longitudes $34^{\circ} \mathrm{E}$ to $42^{\circ} \mathrm{E}$ and latitudes $5.5^{\circ} \mathrm{N}$ to $5^{\circ} \mathrm{S}$. Kenya has an area of about $582646 \mathrm{~km}^{2}$. In 2008 , the population was 38 million, of which about $70 \%$ lived in rural areas.

The population distribution is uneven from an average of 230 persons per $\mathrm{km}^{2}$ in high population areas to an average of 3 persons per $\mathrm{km}^{2}$ in arid areas. Over $50 \%$ of the population is younger than 15 years. The country's population is characterized by high death rates, low life expectancy, increasing fertility rates (from 4.7 children per woman in 1998 to 5.0 in 2006), high infant mortality and low life expectancy (57 Years). There are 42 ethnic groups in Kenya, each with its own culture and traditions, some of which are influenced by the extent of economic development in their native geographical locations (Republic of Kenya, 2009).

Some $52 \%$ of Kenya's population lives below the poverty line as compared to $23 \%$ internationally. In addition, $36 \%$ of Kenya's population suffer from malnutrition (stunting) and are clearly vulnerable to environmentally related diseases. The total disease burden caused by environmental disease in Kenya is 24\% (WHO, 2004). In the 2006 WHO report on behaviour and environmental health indices, it was estimated that access to improved water sources in 2002 was $67 \%$ (urban $89 \%$, rural $46 \%)$ and deteriorated to $61 \%$ in $2004(83 \%$ urban, $46 \%$ rural) whereas access to improved sanitation in 2000 was $49 \%$ (urban 56\%, rural 43\%) and declined to $43 \%$ in 2004 (46\% urban and $41 \%$ rural).

High-risk infectious diseases can be categorized into three groups, namely: food or waterborne diseases; vector-borne diseases and water-contact diseases. They include bacterial and protozoan diarrhoeal diseases, hepatitis A, typhoid fever, malaria and schistosomiasis. In most rural public health facilities, diarrhoea is ranked number three of the leading causes of outpatient attendance (Ministry of Public Health and Sanitation, 2010). In Kenya, about $80 \%$ of hospital attendance is due to preventable diseases and $50 \%$ of these diseases are water, sanitation and hygiene related (Ministry of Health, 2007). 


\section{Overview of children's environmental health}

Children are our future, numbering over 2.3 billion worldwide (aged 0-19) and representing boundless potential. Child survival and development hinge on basic needs to support life; among these, a safe, healthy and clean environment is fundamental (WHO, 2004). Interest in child environmental health has mounted in recent years, spurred by concern that this vulnerable group - especially children under five years of age - are disproportionately exposed to and affected by health risks from environmental hazards. The World Health Organization has defined environmental health as "all the physical, chemical, and biological factors external to a person, and all the related factors impacting behaviours. It encompasses the assessment and control of those environmental factors that can potentially affect health. It is targeted towards preventing disease and creating health-supportive environments" (WHO, 2008).

Over $40 \%$ of the global burden of disease attributed to environmental factors falls on children below five years of age, who account for only about $10 \%$ of the world's population (WHO, 2009). Children under age five are in the dynamic stage of growth. Their immune, respiratory, and digestive systems are still developing. The impact of an unhealthy environment is felt among them because they are always close to the ground, where many contaminants settle (WHO, 2003). Environmental risk factors often act in concert, and their effects are exacerbated by adverse social and economic conditions, particularly conflict, poverty and malnutrition. Six factors within environmental health issues have been identified as priorities, as they cause the bulk of environment-related deaths and diseases among children. These are: household water security, hygiene and sanitation, air pollution (including indoor air pollution and environmental tobacco smoke), vector-borne diseases, chemical hazards (for example lead, mercury, arsenic and the unsafe use of pesticides), and unintentional injuries.

Children are often exposed to many of these environmental health risk factors at a time, often simultaneously in the places, or settings, where they live, learn, play and sometimes work. Health risks in these settings are complex and often interconnected. Using an integrated approach to tackle the multiple risks to children's environmental health in the places where children spend their time provides a focus for local action across a diverse array of environmental risk factors. The home, school and neighbourhood settings are the places that children spend most of their time. These settings must promote good health and be free from risks to children's health and safety, yet they are often the places where children are most at risk (Healthy Environments for Children Alliance, HECA, 2005).

In Kenya, key environmental health problems that had been identified by key stakeholders include inadequate access to safe water supply and sanitation, overcrowding especially in informal settlements, disease causing vectors and air pollution, particularly indoor. Malaria, acute respiratory infections, diarrhoea, measles, malnutrition, skin infections and intestinal worms are documented to contribute to 
over $70 \%$ of the deaths in children under five. These are diseases that are preventable through immunization, proper environmental management or observing basic hygiene (Ministry of Health, 2004).

\section{Environmental health risks in the home environment: case study of Upper River Njoro watershed, Nakuru County}

A longitudinal study was conducted from 2005 to 2006 in 350 rural households in upper River Njoro watershed, Nakuru County to determine selected environmental health risks to children aged 1-4 years in the home environment. Nakuru County is located $160 \mathrm{~km}$ North West of Nairobi which is the capital city of Kenya. These risks included food storage practices, faecal disposal and handwashing practices. Households in the sampling frame included those with at least one child aged 1-4 years. One such child in each household was selected as the index child. Where there was more than one such child, the youngest was selected. Questionnaire, continuous and spot observations were used to collect data. Information was also obtained from key informants who coupled as research assistants.

\subsection{Household demographic characteristics}

Average household size for the sampled households was 6 (range 2 -15). Average number of children per household was 4 (range 1-13). Average number of children per household aged 1-4 was 2 (range 1-4). Most (91.7\%) households were male headed. About $7 \%$ of primary males and $6.6 \%$ of primary females did not have any formal education at all. $56 \%$ of fathers and $59 \%$ of mothers had attained about $7-8$ years of formal schooling (primary). Tertiary education had only been undertaken by few $(2.4 \%)$ of the primary adults of the households.

According to Lopez et al. (2006), rural children, children of uneducated mothers and children in the poorest households continue to have higher mortality risks than better-off children - as they did 10 years previously - even though overall mortality levels of children have declined. Analysis of Demographic Health Surveys in Niger, Burkina Faso, and Mali by Fayehun (2010) indicate that the level of parental education is closely related to the household environments and that more than $90 \%$ of children whose mothers have tertiary education live in households with low or non-health hazards. The higher the parent's educational level, the lower is the likelihood of a health-hazardous household environment for children under age five $(p<0.001)$.

\subsection{Food storage practices}

About $60 \%$ of the households either covered their leftover food or stored it in an enclosure where flies were excluded. These included covering and storing in a cup- 
board, on table, floor or a rope hanging from the roof such as that shown in Figure 1. Leftover food was left uncovered on the floor by $21 \%$ of the households, where flies, dust and roaming animals could have access to it. The reason given was that since children are left on their own when parents go to tend to their farms, they could help themselves to the food whenever they felt hungry. The remaining households constituting 19\% stored their food uncovered either on hanging ropes, tables or open shelves.

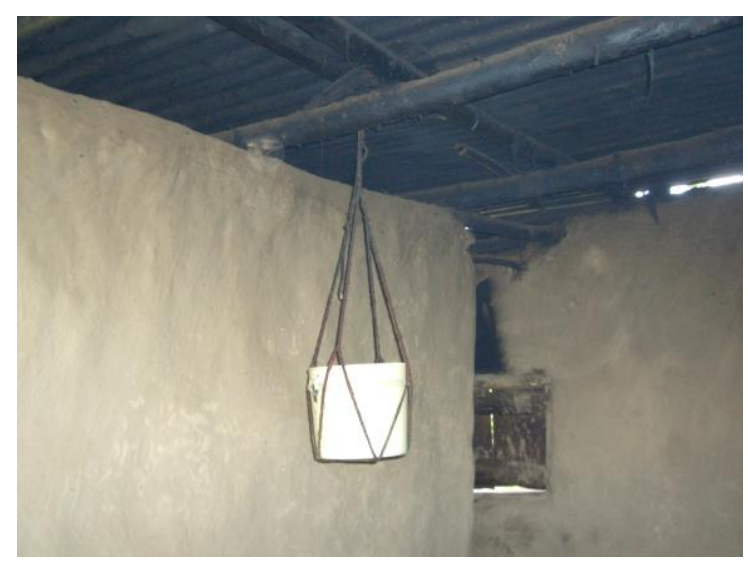

Figure 1: Leftover food stored on hanging rope

The issue of food safety is closely related to water, sanitation and hygiene, particularly with regard to reducing the incidence of diarrhoeal diseases among children. The global incidence of foodborne diseases is difficult to assess accurately. According to the Ministry of Health (Kenya) report presented at the Bamako Conference in 2002, there are inadequate mechanisms for the prevention and control of foodborne diseases and less than $1 \%$ of cases are reported $(\mathrm{MoH}, 2004)$. In developing countries, up to $70 \%$ of diarrhoeal diseases may be caused by foodborne pathogens (HECA, 2005). This imposes a heavy social and economic burden on communities and their health systems. A number of effective interventions exist to address food safety at different points along the food chain and all of these reduce the risks to children's health downstream. However, potentially the most effective interventions are those that are closest to children and in settings where they spend most of their time - within the home environment.

\subsection{Handwashing practices}

Thirty-six per cent of the index children in the study households did not wash their hands before eating. This was observed mostly when the children were left under the care of their older siblings or caretakers (other than the mother) or when the children were so hungry and crying for food, such that cleaning of hands was over- 
looked as unimportant. $35 \%$ cleaned their hands on their own and only $29 \%$ had their hands cleaned either by their mother, caretaker or older sibling. For those who washed their hands before eating, the whole hand was washed among $41 \%$ of the children; palms only among $55 \%$ and fingers only among $4 \%$.

The mother or caretaker was observed if she washed her hands at two critical times: after purging the child (and/or after handling the child's faecal matter) and before handling food (other than before eating). The results are shown in Table 1. A very small percentage used the recommended method of clean water and soap.

Table 1: Washing of hands by mother/caretaker in study households

\begin{tabular}{|l|l|l|l|l|}
\hline & \multicolumn{2}{|l|}{ After purging child } & \multicolumn{2}{l|}{ Before serving food } \\
\hline & Frequency & Percent & Frequency & Percent \\
\hline Not washed & 128 & 51 & 240 & 69 \\
\hline Washed with used water & 49 & 19 & 33 & 9 \\
\hline Washed with clean water & 45 & 18 & 71 & 20 \\
\hline $\begin{array}{l}\text { Washed with clean water and } \\
\text { soap }\end{array}$ & 28 & 11 & 5 & 1 \\
\hline Used water and soap & 3 & 1 & 1 & 0.3 \\
\hline Total & 253 & 100 & 350 & 100 \\
\hline
\end{tabular}

Hands serve as vectors, transmitting pathogens to foodstuffs and drinks and to the mouth of susceptible hosts. Children are an important reservoir of diarrhoeal pathogens and the caretaker who purges the child after defecation is often a major preparer of food for the household. According to Cairncross et al. (2003), most cases of endemic diarrhoea in children are not waterborne but transmitted from person to person on hands, food and other fomites because of poor hygiene practices. Rosen and Vincent (2001) state that almost all endemic diarrhoeal diseases that take a heavy toll on health in sub-Saharan Africa are transmitted through the faecal-oral pathway and are therefore very often water-washed. It has been estimated that up to $70 \%$ of diarrhoeal diseases are faecal-oral (HECA, 2005). Reviews of various studies by Huttly et al. (1997) and Curtis and Cairncross (2003) indicate a reduction in severe enteric infections by between $35-66 \%$ when handwashing is observed. Lack of handwashing is also responsible for some respiratory infections which are responsible for two thirds of child deaths (WHO, 2002). 


\subsection{Faecal disposal practices}

A large number of study households (41\%) possessed no latrines. This was far below the national average of $83 \%$ and the average for rural households, which was given as 46.7\% (Ministry of Health, 2004). The remaining $59 \%$ had mainly simple pit latrines with a wooden floor that normally consists of a log with a hole drilled in the middle. Only one household had the improved pit latrine. Table 2 shows the various defecation and faecal disposal practices for children aged between one and four years. About $80 \%$ of faecal disposal encouraged environmental contamination and only $20 \%$ could be classified as safe.

Table 2: Defecation and faecal disposal for children aged 1-4 years in the study households

\begin{tabular}{|l|c|c|}
\hline Defecation and disposal site & Frequency & Percent \\
\hline Yard then latrine & 47 & 13 \\
\hline Latrine floor & 6 & 2 \\
\hline In yard then covered with soil & 7 & 2 \\
\hline $\begin{array}{l}\text { Bush/anywhere, } \\
\text { then swept and thrown out of compound }\end{array}$ & 269 & 77 \\
\hline Wearing loincloth & 21 & 6 \\
\hline Total & 350 & 100 \\
\hline
\end{tabular}

When a child defecated in loincloths, it was washed immediately in only $8 \%$ of the studied households. All the other practices regarding handling of soiled loincloths could be classified as unsafe. These practices included keeping the soiled cloths in open containers without water, polythene bags and sacks; wiping and reusing; leaving them lying around in the yard or house, roof or fence or the child moving around in the soiled loincloth.

Of at least five visits made to each household for both continuous and spot observation, no human faeces were spotted in the yard in 31\% of the homesteads. For the remaining 69\%, human faeces were spotted at least once in the yard during the visits. For animal faeces, they were spotted once or more times in the yards in $98 \%$ of all the study households.

At least 20 viral, bacterial and protozoan enteric pathogens, including Salmonella spp., Shigella spp, Vibrio cholerae and rotavirus multiply in the human gut, exit in the excreta and transit through the environment, causing diarrhoea in new hosts (Curtis and Cairncross, 2003). That is why most of the children's diseases revolve around poor sanitation (UNICEF, 1998; Rutsein, 2000; WHO, 2009) and especially so in rural areas (GoK and UNICEF, 2003). Child mortality results show that access to an improved sanitation facility is associated with a 5.5 to $12 \%$ point lower probability of infant and child death as compared to no sanitation facility (Demombynes and Trommlerová, 2012). 


\section{Children's environmental health initiatives in Kenya}

Kenya signed several conventions and declarations with a commitment to health and environmental safety that were all basis for the agenda of the Libreville meeting. These are: Stockholm Declaration on the Human Environment (1972); AlmaAta Declaration on Primary Health Care (1978); Bamako Convention on Hazardous Wastes (1991); Declaration of the UN Conference on Environment and Development Rio de Janeiro (1992); Millennium Declaration and the subsequent Millennium Development Goals (2000); Johannesburg Plan of Implementation adopted by the World Summit on Sustainable Development (2002); Health Strategy of the African Union (2006); Algiers Declaration on Desertification (2006); Algiers Declaration on Health Research in Africa (2008); Ouagadougou Declaration on Primary Health Care and Health Systems (2008); The Ethekwini Declaration on Hygiene and Sanitation (2008) and Bali Declaration on Waste Management for Human Health and Livelihood (2008).

Milestones related to the above include the development of national frameworks such as the National Environment Plan (1994) which addresses actions required to reduce risks to human health and the environment; Session Paper No. 6 on environment and development (1996) which provides a roadmap on policy, legal and administration actions to address sustainable development issues; Poverty Reduction Strategy (2001) which underscores the need for proper environmental management within the major urban centers as the nuclei for industrial development; National Poverty Eradication Plan which emphasizes special circumstances for poor urban women and other natural resource constraints as a foundation on which poverty eradication and environmental planning must be anchored; Environment, Sanitation and Hygiene Policy (2007) which addresses the state of environment and hygiene in Kenya and provides guidance on areas for improvement; Vision 2030 whose social pillar seeks to build a just and cohesive society with social equity in a clean and secure environment; National Initiatives on Health and Environment Linkages including EMCA (1999) where NEMA is the enforcement arm; Occupational Safety and Health Act - OSHA (2007) and the National School Health Policy and Guidelines whose goal is to enhance the quality of health in school communities by creating a healthy and child-friendly environment for teaching and learning.

Other than the government, there are other organizations which also play an important role in child-environmental health. These include UN organizations such as UNICEF, NGOs like Christian Children's Fund and World Vision as well as community-based organizations in various parts of the country. These work either independently or in conjunction with government departments.

Despite having well-defined national health policies and a reform agenda whose overriding strategies are focused on improving healthcare delivery services and systems through efficient and effective health management systems and reform, there has not been a breakthrough in improving the situation of having 
healthy settings for children within the home environment. Whereas the schoolgoing child has been catered for, the most vulnerable children are those who are not at school age yet. They have been left out because little control can be assured for private homes. According to analyses undertaken between 2003 and 2008 in Kenya, child mortality was seen to have declined. The major driver for this was seen to be increased availability of insecticide-treated nets (ITNs) and extensive anti-malarial campaigns (Demombynes and Trommlerová, 2012). If similar efforts to improve hygiene, sanitation and food safety are put in place, more positive results are bound to be felt.

\section{Recommendation to improve child environmental health in Kenya}

- Greater efforts need to be put in place to ensure provision of basic services like water for all. Availability of safe sources of drinking water will significantly reduce child mortality and therefore investments in this sector will be rewarding.

- Access to sanitation facilities like constructing toilets entail a private cost but do have significant social benefits. The government should work closely with both the private sector and civil society to ensure that households have universal access to sanitation facilities as this will to a great extent reduce the number of infant deaths.

- The proposed housing policy should make it mandatory for each housing unit to have a sanitation facility such that all households have access to sanitation facilities.

- Government policy should be focused towards promoting the use of lowpolluting fuels and in particular discouraging the use of firewood and charcoal. Through the use of economic instruments, incentives should be created for the promotion of cleaner fuel sources. This would also create employment opportunities that lead into increased earnings and reduced poverty. In addition, this will reduce the incidence of respiratory-tract infections in children.

- The government in conjunction with other partners can (i) set priorities for children's environmental health and determine information needs, (ii) identify lead and participating agencies and (iii) implement data collection and indicator reporting and link this to policies and programmes to improve children's environmental health. 


\section{References}

Cairncross S., O’Neill D., McCoy A. and Sethi D. 2003. Health, environment and the burden of disease; a guidance note. Department for International Development. London, UK.

Curtis V. and Cairncross. 2003. Effect of washing hands with soap on diarrhoea risk in the community: a systematic review The Lancet Infectious Diseases 3: 275-81.

Demombynes G. and Trommlerová S.F. 2012. What has driven the decline of infant mortality in Kenya? The World Bank, Africa Region. Poverty Reduction and Economic Management Unit. Policy Research Working Paper 6057. http://econ.worldbank.org. [Accessed August 26, 2013].

Fayehun O.A. 2010. Household environmental health hazards and child survival in Sub-Saharan Africa. Demographic and health research. ICF Macro.

USAID. Calverton, Maryland, USA

Government of Kenya (GOK). 1999. National Poverty Eradication Plan 19992015. Department of Development Coordination, Nairobi, Kenya.

GOK/UNICEF. 2003. Country Programme of Cooperation 1999-2003, Situation Analysis and Strategy by Sub-Project, 2003.

Healthy Environments for Children Alliance (HECA). 2005. Improving children's environmental health in settings. Experiences and lessons for policies and action. Presented in Entebbe, Uganda in Inter-regional Consultation Meeting on 29 Nov.-2 Dec. 2005.

Huttly S.R., Morris S.S. and Pisani V. 1997. Prevention of diarrhoea in young children in developing countries. Bulletin of World Health Organization 75: 163-174.

Lopez A.D., Mathers C.D, Ezzati M, Jamison D.T, Murray J.L.C. 2006. Global burden of disease and risk factors. World Bank and Oxford University Press. New York, USA.

Ministry of Finance and Planning (2001). Poverty Reduction Strategy Paper for the Period 2001-2004. Ministry of Finance and Planning, Government of Kenya.

Ministry of Health Kenya. 2004. National Profile: The status of children's environmental health. Government of Kenya, Nairobi.

Ministry of Health (2007). National environmental sanitation and hygiene policy. Government printer, Nairobi. Ministry of Public Health and Sanitation. Division of Sanitation and Hygiene profile. www.publichealth.go.ke 
Republic of Kenya. 2009. Report on Kenya country situation analysis and needs. Assessment for the preparation of national plans of joint action for implementation of the Libreville Declaration on Health and Environment in Africa. Ministry of Public Health and Sanitation and Ministry of Environment and Mineral Resources. Kenya Sana Report.

Republic of Kenya (2010) Ministry of Public Health \& Sanitation. Government printer, Nairobi, Kenya.

Rosen S. and Vincent J.R. 2001. Household water resources and rural productivity in Sub-Saharan Africa: A review of the evidence. African Economic Policy Discussion Paper Number 69. USAID, Washington DC.

Rutstein, S.O. 2000. Factors associated with trends in infant and child mortality in developing countries during the 1990s. Bulletin of the World Health Organization 78: 1256-1270.

UNICEF. 1998. A situation analysis for children and women in Kenya. Nairobi, Kenya.

World Health Organization. 2002. World Health Report 2002. Reducing risks, promoting healthy life. World Health Organization, Geneva.

World Health Organization (WHO). 2003. The physical school environment: an essential element of a health-promoting school. The World Health Organization's Information Series on School Health.

WHO, 2004. The World Health Report. Changing History. World Health Organization, Geneva.

WHO, 2006. Working Together for Health. http://www.who.int/whr/2006/en/ [Accessed June 20, 2012]

WHO. 2008. "Environmental Health.” WHO, Geneva. http://www.who.int/topics/environmental_health/en/[Accessed August 26, 2013].

World Health Organization (WHO). 2009. World Health Statistics 2009. Geneva: The World Health Organization. 



\title{
7. Metallic Iron for Clean Agriculture
}

\author{
Chicgona Noubactep, Department of Applied Geology, Georg-August-University \\ Goettingen, Germany
}

\section{Abstract}

Contaminated surface water used for irrigation has been identified as an important source of pathogens for waterborne diseases. The problem is crucial in peri-urban areas of the developing world where poor families rely on mixed wastewaters for the irrigation of crops that are partly consumed raw. As a consequence, cholera, typhoid fever and paratyphoid are almost endemic in cities like Dakar, Douala or Kampala. There is an urgent need for an affordable and appropriate technology for the treatment of irrigation water. The amendment of sand filters with metallic iron $(\mathrm{Fe} 0)$ has been identified as a universal technology for decentralised water treatment. The present communication introduces a concept for the design of $\mathrm{Fe} 0 /$ sand filters to decontaminate irrigation water. The suitability of $\mathrm{Fe} 0$ /sand filters for irrigation water arises from the fact that chemical, microbial and physical contamination is collectively addressed. The operating mode of Fe0 filters is explained and some research needs are discussed. It is hoped that more researchers will be interested in co-developing this promising health-keeping technology. 


\section{Introduction}

Filtration is the oldest way to improve the quality of water. Basically, filtration is a sieving or a size-exclusion process. Filtration is generally used as a step in the production of water for general domestic purposes. Filtration is also commonly used to further purify safe drinking water to meet the requirements of some industrial applications (Shannon et al., 2008; Sutherland, 2012). Water filtration as presented herein is the treatment of unconventional sources of water (wastewater and sludge) so as to increase the quantity of fresh water.

Unconventional sources of interest include the collection and recycling of: (i) grey water from residences, (ii) industrial and municipal wastewaters, and (iii) irrigation wastewater (drainage water). The variability of the source of water to be treated suggests that a wide array of inorganic (e.g. arsenic, cadmium, chromium, chloride, cobalt, fluoride, lead, mercury, nitrate, phosphate, selenium, sulfate, uranium, zinc), organic (e.g. biphenyls, detergents, fertilizers, greases, hydrocarbons, pharmaceuticals, pesticides, phenols, plasticizers, oils), and microbial (e.g. algae, amoebas, bacteria, fungi, planktons, viruses) pollutants may be expected (Shannon et al., 2008; Carr et al., 2010; Ali, 2012; Allred, 2012; Ingram et al., 2012; Ntangmo Tsafack et al., 2012). The question arises, how to treat such a mixture in an affordable manner?

The state-of-the-art knowledge on the treatment of multi-pollutant systems can be summarized by the following statement by Doula (2009): "there is no one piece of treatment equipment that manages all contaminants. All treatment methods have limitations. The treatment system that is best for any particular situation (rural or municipal water system, household, industry, well, underground, irrigation, etc) depends on the contaminant(s) present, concentration(s), size of equipment and maintenance and operational requirements of the system." This statement clearly shows that designing a system for the treatment of unconventional water sources is a real challenge to the scientific community.

While the statement of Doula (2009) is funded on the evidence that adsorptive affinity of micro-pollutants for available solid surfaces determine their removal extents (Nödler et al., 2010), it has been demonstrated recently (Noubactep, 2010, 2011) that contaminant removal in $\mathrm{Fe} 0$ filters is primarily a non-specific adsorption size-exclusion process. Accordingly, even species with no specific or poor affinity to $\mathrm{Fe} 0$ and its corrosion products (iron oxides) could be quantitatively removed, if the system is properly designed: a thicker $\mathrm{Fe} 0 /$ sand layer or appropriate pre-treatment before the $\mathrm{Fe} 0$ / sand layer.

The objective of this communication is to present a concept for the treatment of unconventional waters used for irrigation using $\mathrm{Fe} 0 /$ sand filters. An inventory of contaminant likely to be present in unconventional waters will first be given, followed by the state-of-the-art knowledge on the processes occurring in $\mathrm{Fe} 0 /$ sand filters. Some hints for the design of $\mathrm{Fe} 0 /$ sand filters will be given at the end. 


\section{Urban wastewaters}

The present section focuses on wastewater which is often the only source of irrigation water for urban and peri-urban farmers in the developing world. The issue of using irrigation wastewater (drainage water) for domestic purposes (Ensink and van der Hoek, 2009; van der Hoek et al., 1999 and 2001) is not explicitly included. However, the presented technology could be useful for: (i) the recycling of drainage water for safe irrigation, and (ii) the treatment of drainage water for safe drinking water (Noubactep et al., 2012). In general, the use of wastewater in agriculture can be a health hazard for farmers and consumers (Ensink and van der Hoek, 2009; Ntangmo Tsafack et al., 2012).

\section{Domestic wastewater}

Domestic effluent consists mainly of blackwater (faeces, urine and associated sludge) and greywater (kitchen and bathing wastewater). Domestic wastewater is particularly well suited to irrigated agriculture. It contains significant quantities of plant beneficial ions (nutrients) such as nitrogen, phosphorus and potassium. Domestic wastewater can be regarded as a 'free' source of nutrients. The crop yields can be enhanced without the addition of chemical fertilizers. However, domestic effluent is just a fraction of urban wastewater. Moreover, domestic wastewater may contain hazardous chemicals like heavy metals from personal care products.

\section{Institutional wastewaters}

Institutional wastewaters are effluents from commercial establishments and institutions, including hospitals and educational facilities. This water also contains human excreta and essentially more hazardous chemicals than domestic wastewater. The degree of contamination depends partly on the size and the prestige of the city. For example a university city like Abidjan, Accra, Gaborone, Lagos, Lusaka, Stellenbosch or Yaoundé necessarily releases various pollutants into the wastewater depending on which chemicals are used in teaching and research.

\section{Industrial/ commercial wastewaters}

Industrial wastewater is the effluent from manufacturing and commercial activities such as printing, food and beverage processing and production. Industrial wastewater may include various amounts of potentially plant-toxic ions (e.g. heavy metals). Therefore, crop productivity can be jeopardized if suitable management of water is not employed (Carr et al., 2010).

\section{Storm water / urban runoff}

Urban areas are characterized by impervious surfaces created by buildings and pavements. These "cemented" surfaces cause rainwater to flow quickly over the landscape. The rapid flow is necessarily coupled with increased transport or species 
which are not significantly soluble in water (e.g. hydrocarbons, grease, vehicle byproducts).

\section{Summary}

Urban wastewater certainly contains human excreta, microbial contamination and probably hazardous chemicals from industry, households, and other sources. Polluted urban wastewater constitutes a serious health hazard to local populations (farmers and consumers of agricultural products). Local residents should be protected by constructing a sewer system to evacuate the wastewater from the neighbourhood. Whether sewerage is available or not, such hazards should be reduced or controlled by treating the wastewater before it is used for irrigation.

\section{$4 \mathrm{Fe} 0 /$ sand filters}

\subsection{State-of-the-art knowledge}

Metallic iron $(\mathrm{Fe} 0)$ has been demonstrated for almost two decades to remove aqueous biological and chemical contaminants. In recent years, scientific efforts have optimized the design of Fe0 filters for contaminant removal (Noubactep and Schöner, 2010; Ingram et al., 2012; Noubactep et al., 2012 and 2013). It has been demonstrated in particular that a pure $\mathrm{Fe} 0$ filter $(100 \% \mathrm{Fe} 0)$ is not sustainable. Sustainable $\mathrm{Fe} 0$-based filters are those containing less than $50 \% \mathrm{Fe} 0$ (volumetric proportion). Preliminary experimental results demonstrate that the optimal Fe0 volumetric proportion is even lower than 40\% (Miyajima, 2012; Miyajima and Noubactep, 2013).

The technology has been developed to treat high-volume of low-contaminated groundwater which is slowly flowing through a reactive barrier. Successful treatment of irrigation water with $\mathrm{Fe} 0 /$ sand filters requires that the two basic subsurface conditions are mimicked: darkness and a low level of dissolved oxygen $\left(\mathrm{O}_{2}\right)$. A third relevant condition is a low temperature, however it is difficult to achieve. The first condition is very easy to achieve as choosing the filter container will be sufficient. As concerning the second condition, many systems are known as $\mathrm{O}_{2}$ scavengers, the most appropriate being a slow sand filter.

\subsection{Mechanisms of contaminant removal in $\mathrm{Fe} 0$ filters}

Contaminants are fundamentally adsorbed onto and/or co-precipitated within the mass of transforming corrosion products at the surface of $\mathrm{Fe} 0$ (or in its vicinity). This experimental observation makes $\mathrm{Fe} 0$ a universal medium for water treatment (Noubactep et al., 2009; Noubactep and Schöner, 2010). Upon proper design, a $\mathrm{Fe} 0$ filter will remove all contaminants by adsorption, adsorptive size exclusion and 
co-precipitation. This characteristic challenges the common assumption, that due to differences in chemical and physical properties (e.g. adsorptivity, solubility, degradability), multiple contaminant mixtures may not efficiently be remediated by a single material (e.g. Doula, 2009). Although all contaminants are quantitatively removed in $\mathrm{Fe} 0 / \mathrm{H}_{2} \mathrm{O}$ systems, contaminants with higher adsorptive affinity to iron and thus, corrosion products will be more efficiently removed. In other words, contaminants with lower affinity to iron oxides (e.g. cationic species) should be removed before the $\mathrm{Fe} 0$ bed. Fortunately such contaminants (e.g. methylene blue) are strongly adsorbed onto sand (Mitchell et al., 1955). Sand and Fe0 are sufficient for water treatment as a rule (Fig. 1). Depending on their availability, other minerals and biomaterials could be advantageously tested and used.

It is essential to recall that a Fe0-amended sand filter is a self-filtration system in which efficient "filter aids" (iron corrosion products) are progressively generated. The filter aids improve the process of size exclusion by filling the porous network and worsening the hydraulic conductivity (permeability loss). According to this, designing a $\mathrm{Fe} 0 /$ sand filter means basically to find out the balance between increased efficiency for contaminant removal (more $\mathrm{Fe} 0$ ) and increased permeability (less $\mathrm{Fe} 0$ or more sand). As discussed above, the $\mathrm{Fe} 0 /$ sand filter should be placed after a sand filter which consumes dissolved $\mathrm{O}_{2}$. Under anoxic conditions, permeability loss is minimal (Caré et al., 2013).

\section{3 $\mathrm{Fe} 0$ filters for irrigation water}

Designing Fe0-based filters for irrigation water could be regarded as an easy task since the fundamental operating mode of $\mathrm{Fe} 0$ filtration has been recently achieved (Caré et al., 2013). At any location, the proper filter design will depend on: (i) the water quality, (ii) available materials (e.g. gravel, pumice, sand, wood) and (iii) the suitability of available materials to mitigate contamination alone and in admixture with $\mathrm{Fe} 0$. If these characteristics are known, then designing $\mathrm{Fe} 0$-based filters is a monitored routine operation. The number of: (i) slow sand filters, (ii) $\mathrm{Fe} 0$ /sand filters, (iii) the amount and the proportion of $\mathrm{Fe} 0$ in each filter and (iv) the number of filters containing other materials (e.g. pumice, zeolite) should be optimised in pilot studies. Figure 1 gives an illustration of a treatment plan built with coarse sand, fine sand and granular iron (Fe0). The number of each filter type and the use of other materials can be flexibly adjusted to meet the treatment goal.

\subsection{Requirements for efficient $\mathrm{Fe} 0$ filters}

A filtration plan designed according to Fig. 1 may require an appropriate $\mathrm{Fe} 0$ material to be efficient. However, if slow sand filters alone can efficiently treat the available raw water, no $\mathrm{Fe} 0$ use is necessary. This statement may be surprising. However, it should be considered that cities like Berlin are still largely using slow sand filters as stand-alone technology for safe drinking water provision (Urfer and 
Fortier, 2012). This statement highlights the crucial importance of modern water analytic laboratories in the developing world. However, before laboratories are built and equipped, people must be protected from avoidable diseases. Therefore, designing systems that are able to address multi-element contamination can be regarded as a bridging solution: cue $\mathrm{Fe} 0$ filters.

The Fe0-containing unit is the heart of the treatment system. The selected $\mathrm{Fe} 0$ material should be able to considerably ameliorate the quality of water for at least six months. Therefore, making suitable $\mathrm{Fe} 0$ materials quantitatively available is the first task to win the battle against avoidable diseases. Possible sources of iron include: construction steel, steel wood, material from old tyres and indigenous iron.

Indigenous iron is related to metal iron that results from African knowledge before the colonial era (Chirikure, 2007; Onipede, 2010). For example, Onipede (2010) reported on specialized iron mining villages in Oyo (Nigeria) recorded in 1904 to have "100 to 120 people engaged in iron mining, smelting and smiting". The development of the African 'iron industry' was stultified with the advent colonialism. The brilliant African blacksmith for example had to abandon his ancestral mission of manufacturing tools for daily life. He was forced to earn his life as a farmer of export products like cocoa, coffee or rubber as subsistence and communal system was replaced by a capitalist economy. Nevertheless, the iron making skills are still available in the African society and could be revived once the market is identified. The advent of $\mathrm{Fe} 0$ filters is regarded as such an opportunity. For the first time the African research community is given a real opportunity to solve the long lasting water crisis with their own expertise. The gains in terms of "devise saving" are immense and should be the motivation for individuals and research groups.

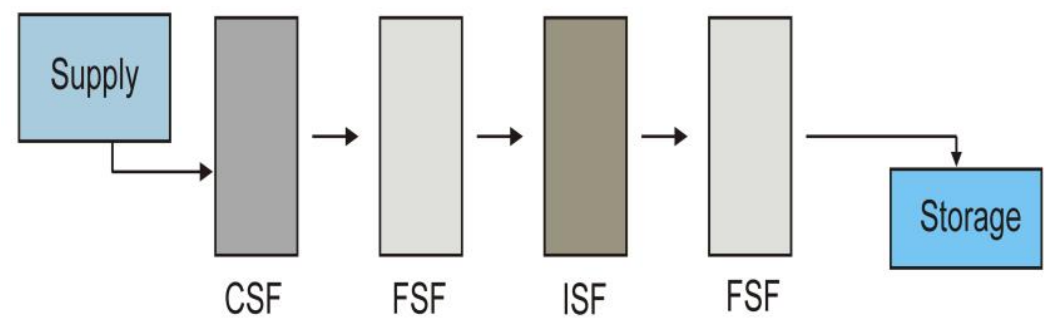

Figure 1: Illustration of a water treatment option using metallic iron (Fe0) beds. CSF $=$ coarse sand filter, FSF $=$ fine sand filter, $I S F=$ iron sand filter.

\section{Concluding remarks}

Using $\mathrm{Fe} 0$ filters to treat irrigation water is an appropriate, affordable and efficient mitigation step in decontamination of irrigation water for small growers of leafy greens. Because $\mathrm{Fe} 0$ was proven a universal technology for water treatment, using 
$\mathrm{Fe} 0$ filters is regarded as a powerful tool to diversify sources of irrigation water to be used when irrigating leafy green commodities. In particular, both wastewater and drainage water (recycled irrigation water) could be efficiently treated upon proper filter design.

It is hoped that the research community in the developing world will use the opportunity to co-develop the $\mathrm{Fe} 0$ filtration technology to win the battle against water-related diseases. As it was well formulated by Kofi Annan: "We shall not finally defeat the infectious diseases that plague the developing world until we have also won the battle for basic health care, sanitation and safe drinking water." Fe0 filtration fortunately addresses both sanitation and safe drinking water.

The use of $\mathrm{Fe} 0$ filtration for safe drinking water could have the positive 'side effect' that traces of iron remain in the treated water. If this is the case, then water treated by $\mathrm{Fe} 0$ filters could be regarded as "iron-fortified drinking water" which is known to prevent mother and child from iron deficiency and iron anemia. Dutrade-Oliveira et al. (2011) reported that the worldwide prevalence of iron deficiency is $47 \%$ in children under five, $30 \%$ in non-pregnant women of childbearing age, and $42 \%$ in pregnant women. It appears that a coordinated multidisciplinary research is needed to exploit the great potential of $\mathrm{Fe} 0$-ammended filtration systems for a more healthy society.

\section{Acknowledgments}

Thoughtful comments provided by Dr. Mohammad A. Rahman (University of Göttingen) on the draft manuscript are gratefully acknowledged. Dr. Rahman is also thanked for fruitful collaboration on the design of iron/sand filters and the design of Figure 1. The manuscript was improved by the insightful comments of anonymous reviewers.

\section{References}

Ali, I. (2012). New generation adsorbents for water treatment. Chemical Reviews 112, 5073-5091.

Allred, B.J. (2012). Laboratory evaluation of zero valent iron and sulfur-modified iron for agricultural drainage water treatment. Ground Water Monitoring \& Remediation 32, 81-95.

Caré S., Crane R., Calabro P.S., Ghauch A., Temgoua E., Noubactep C. (2013). Modelling the permeability loss of metallic iron water filtration systems. Clean - Soil, Air, Water, doi: 10.1002/clen.201200167.

Carr, G., Nortcliff, S. \& Potter, R.B. (2010). Water reuse for irrigated agriculture in Jordan: challenges of soil sustainability and the role of management strategies. Philosophical Transactions Royal Society A 368, 5315-5321. 
Chirikure S. (2007). Metals in society: Iron production and its position in Iron Age communities of southern Africa. J. Social Arch. 7, 72-100.

Doula, M.K. (2009). Simultaneous removal of $\mathrm{Cu}, \mathrm{Mn}$ and $\mathrm{Zn}$ from drinking water with the use of clinoptilolite and its Fe-modified form. Water Research 43, 3659-3672.

Dutra-de-Oliveira J.E., Marchini J.S., Lamounier J., Almeida C.A.N. (2011). Ironfortified drinking water studies for the prevention of children's anemia in developing countries. Anemia, Volume 2011, Article ID 815194, 5 pages.

Ensink J.H.J., van der Hoek W. (2009). Implementation of the WHO guidelines for the safe use of wastewater in Pakistan: balancing risks and benefits. Journal of Water Health 7, 464-468.

Ingram, D.T., Callahan, M.T., Ferguson, S., Hoover, D.G., Shelton, D.R., Millner, P.D., Camp, M.J., Patel, J.R., Kniel, K.E. \& Sharma M. (2012). Use of zerovalent iron biosand filters to reduce E. coli O157:H12 in irrigation water applied to spinach plants in a field setting. Journal of Applied Microbiology $112,551-560$.

Mitchell G., Poole P., Segrove H.D. (1955). Adsorption of methylene blue by highsilica sands. Nature 176, 1025-1026.

Miyajima, K. (2012): Optimizing the design of metallic iron filters for water treatment. Freiberg Online Geoscience 32, 60 pp.

Miyajima, K., Noubactep, C. (2013). Impact of Fe0 amendment on methylene blue discoloration by sand columns. Chemical Engineering Journal 217, 310-319.

Nödler, K., Licha, T., Bester, K. \& Sauter, M. (2010). Development of a multiresidue analytical method, based on liquid chromatography-tandem mass spectrometry, for the simultaneous determination of 46 micro-contaminants in aqueous samples. Journal of Chromatography A 1217, 6511-6521.

Noubactep, C., Schöner, A., Woafo, P. (2009). Metallic iron filters for universal access to safe drinking water. Clean: Soil, Air, Water 37, 930-937.

Noubactep, C. (2010). The suitability of metallic iron for environmental remediation. Environmental Progress \& Sustainable Energy 29, 286-291.

Noubactep C., Schöner A. (2010). Metallic iron: dawn of a new era of drinking water treatment research? Fresenius Environmental Bulletin 19, 1661-1668.

Noubactep, C. (2011). Aqueous contaminant removal by metallic iron: Is the paradigm shifting? Water SA 37, 419-426.

Noubactep C., Temgoua E., Rahman M.A. (2012). Designing iron-amended biosand filters for decentralized safe drinking water provision. Clean: Soil, Air, Water 40 (8), 798-807. 
Noubactep, C. (2013). Metallic iron for water treatment: A critical review. Clean Soil, Air, Water, doi: 10.1002/clen.201200502.

Ntangmo Tsafack, H., Temgoua, E. \& Njine T. (2012). Physico-chemical and bacteriological quality of the vegetable watering water in the Dschang Town, Cameroon. Journal of Environmental Protection 3, 949-955.

Onipede K.J. (2010). Technology development in Nigeria: The Nigerian machine tools industry experience. Journal of Economics 1, 85-90.

Shannon M.A., Bohn P.W., Elimelech M., Georgiadis J.G., Marinas B.J., Mayes A.M. (2008). Science and technology for water purification in the coming decades. Nature 452, 301-310.

Sutherland, K. (2012). Drinking and pure water: Filtration improvements progress global clean water provision. Filtration \& Separation 49, 12-14.

Urfer D., Fortier S.P. (2012). Traitement décentralisé de l'eau potable - kls®filter. ARPEA 252, (avril 2012) 6-13.

van der Hoek W., Konradsen F. \& Jehangir W.A. (1999). Domestic use of irrigation water: health hazard or opportunity? International Journal of Water Resources Development 15, 107-119. 

II. Socioeconomic Factors 



\title{
8. Maternal Health Care in Rural Areas of Tanzania: How do Beneficiaries Perceive the Quality of Services?
}

\author{
Andrew Makoi, SIKIKA Company Ltd (a health advocacy NGO), \\ Dar es Salaam, Tanzania
}

John M. Msuya, Sokoine University of Agriculture, Department of Food Science and Technology, Dar es Salaam, Tanzania

\section{Abstract}

A cross-sectional descriptive study was conducted in Morogoro Rural District in eastern part of Tanzania to determine the perception and satisfaction of pregnant women and mothers, regarding the quality of maternal health care services they get in government-owned public health facilities. The study involved 216 pregnant women and 67 mothers selected using convenience sampling in 3 health centres. Exit interviews and interview schedules were used to collect data. Five-point Likert scale was used to measure the level of satisfaction, while perceived quality of care was measured by using a rating scale of 1-5. SPSS-computer program was used to analyze the data. Results were presented in frequencies and percentages while rela- 
tionship between perceived quality and level of satisfaction was tested using Chisquare statistics.

Five inquiry statements were used to test the perception of mothers and pregnant women about the services they receive in the sampled public health facilities. The statements included the questioning about maternal health worker's promptness in providing services, their willingness to assist, capability to respond to the client's requests, capacity to provide information on the timing of the service, and their willingness to speak in a respectful language to the client. The study findings showed that the majority of pregnant women $(77.3 \%)$ and mothers $(67.2 \%)$ perceived the quality of maternal care to be acceptable. The perception of quality of maternal care was associated with levels of satisfaction $\left(\chi^{2}=56.7\right.$, p-value $<$ $0.001)$, perceived waiting time $\left(\chi^{2}=31.1, \mathrm{p}\right.$-value $\left.<0.001\right)$, perceived maternal health workers' behaviour $(\chi 2=27.5$, $\mathrm{p}$-value $<0.001)$, and responsiveness of the workers $\left(\chi^{2}=37.6\right.$, p-value $\left.<0.001\right)$.

\section{Introduction}

\subsection{Quality of health care}

The concept of quality of health care services is multidimensional; it does not apply to all situations equally (De Geyndt, 1995). Good quality of health care can be defined as the one that meets technical standards as well as the needs and expectations of users and community (Ministry of Health of Tanzania, 2000). The most widely used conceptual framework for assessing quality is the one introduced by Donabedian (1980), who used the elements of structure, process and outcome. The structural aspect of quality health care includes factors such as the infrastructure of buildings, availability of equipment, drugs supply, number of staff and their qualification and other organizational activities (Gilson, 1994).

Even though the judgment of the patients (clients) may differ from those of professionals and how they would judge it to be best for them, the evaluation of quality health care through client's perception is increasingly being used now, mainly to reflect on quality of health care in relation to structure, processes and outcome (Baltussen et al., 2001). The framework of maternal health by Hulton et al. (2007) defines quality of care as something which includes multiple elements such as clinical and technical aspects as well as experience in receiving services. The latter encompasses women's perspectives and interactions with providers. Perception of quality of maternal care is influenced by the expectations of women before they receive the various services, and whether these expectations are met when receiving health care services (Smith and Englebrecht, 2001). 


\subsection{Client's satisfaction versus perception}

A simple and practical definition of satisfaction would be the degree to which desired goals, needs, desires or expectations have been achieved. Satisfaction is achieved when the patient/client perceives the quality of care and services as something positive and satisfying that meets expectations. Patient or client satisfaction can be described as an attitude - a person's general orientation towards a total experience of health care. Satisfaction is an example of a perception, but it is by no means the only example. Satisfaction does not imply superior service, only the perception of an adequate or acceptable service and thus, satisfaction is a relative concept in a way that one person is maybe satisfied whereas the other one is not although they received the same service.

Even though studies of client satisfaction which often focus on patientprovider interactions, have lately increased in numbers, particularly in industrialized nations (Clemes et al., 2001), considerable difficulties have been outlined regarding the conceptualization and measurement of satisfaction. In addition, there is the opinion that respondents have been seen to report unrealistically high levels of satisfaction which are likely to derive from preferences related to courtesy or gratitude (Williams and Schutt-Aine, 2000; Langer et al., 2002; Nigenda et al., 2003).

Satisfaction ratings, being both a measure of care and a reflection of the respondent (Sitzia and Wood, 1997) and thus, do not often reflect objective reality. To overcome this challenge, some organizations emphasize the measurement of client perception instead. For example, the Joint Commission on Accreditation of Healthcare Organizations has replaced the term 'satisfaction' with 'perception of service' (JCAHO, 1999). The patients' perception of quality of care is critical to understand the relationship between quality of care and utilization of health services and is now considered an outcome of healthcare delivery (Turkson, 2009). As an important predictor of satisfaction, perceived quality of care has evolved as a concept over several decades of research and professional practice (Banzart and Koenig, 2009). The current study intended to determine the perceived quality of maternal health care services among mothers and pregnant women attending health facilities in Morogoro Rural District in Tanzania.

\section{Methodology}

\subsection{Health situation in the study area}

Morogoro Region is located on the eastern side of Tanzania mainland, where it lies between latitudes $5058^{\prime \prime}$ and 10000 ' south of the equator and between longitudes 35025 ' and 380 30" east of Greenwich and it is bordered by seven regions. In the north are Tanga and Manyara and in the eastern side the Coast Region and Lindi Regions. On the western side there are Dodoma and Iringa Regions while Ruvuma 
is located in the southern part of the region (Ministry of Planning, Economy and Empowerment Report, 2007).

The current study was conducted in Morogoro Rural District. Administratively, Morogoro Rural District is one of the six districts that constitute the Morogoro Region. The other districts include Ulanga, Mvomero, Kilosa, Kilombero and Morogoro Municipality. The region is located at the eeast coast of Tanzania. The district has an area of 11,731 square $\mathrm{km}$ with an estimated population of 288,986.

Available statistics from the Tanzania Demographic and Health Survey (NBS, $2010)^{1}$ for the whole of Morogoro Region indicate that approximately $58 \%$ of deliveries were administered in health facilities, a rate which is far below those reported in the neighbouring regions of Iringa (80.4\%), Ruvuma $(85.9 \%)$ and Pwani (73.1\%). The same survey also indicated that only $46.7 \%$ of sexually active married women in Morogoro Region were using any method of contraceptive. The contraceptive methods were grouped into two categories of modern and traditional methods. Modern methods include female sterilization, male sterilization, the pill, intrauterine device (IUD), injectables, implants, male condom, female condom, diaphragm, foam, jelly, and lactational amenorrhoea (LAM). Traditional methods included periodic abstinence or rhythm, withdrawal, and folk methods. In this respect, the modern methods accounted for 39.9\% while the traditional methods were $6.8 \%$. The neighbouring regions also showed a similar situation.

Similarly, other maternal care indicators for the Morogoro Region are not encouraging either. The percentage of women aged 15-49 years with their last baby born during the past five years and who received a vitamin A dose in the first two months after delivery (postpartum) was only $28.9 \%$. However although the percentage was not very high it was slightly above the neighbouring regions (NBS, 2010). In contrary, the extent of protection against neonatal tetanus is generally very high throughout the country, including Morogoro Region where the coverage reached 90.7\% during the Tanzania Demographic and Health Survey of 2010, which is the most recent so far.

A good picture of the health situation in the study district (Morogoro Rural District) can perhaps best be captured by considering the number of available health facilities in the area. The Tanzanian health system distinguishes three main categories of facilities that provide health services depending on the type and extent of services. The categories include hospitals (which are further classified as national, regional and district referral hospitals, as well as normal hospitals), health centres, and dispensaries. As of 2006, there were a total of 53 dispensaries in the district, of which 42 were government owned while the remaining were privately owned (Ministry of Planning, Economy and Empowerment Report, 2007). By the end of year 2010, the number of dispensaries had increased to 93. On the other hand, the district has 3 health centres namely Tawa, Ngerengere and Duthumi. The three are well placed within the district to serve as referrals for a number of sur-

\footnotetext{
${ }^{1}$ It is difficult to obtain district-specific statistics
} 
rounding dispensaries, in which each one caters for not less than 30 dispensaries. At the time of this study, the Morogoro Rural District did not have any designated hospital. Considering the size of manpower, the district had by the end of 2005 one fully qualified medical doctor, 71 clinicians, 51 nurses, 10 health officers and 108 medical attendants. The doctor to population ratio in 2006 was estimated to be 1 per 278,824 . The study aimed to determine the perceived quality of maternal health care by the women who are served by the 3 named health centres, including the surrounding dispensaries.

\subsection{Study design, data collection and analysis}

Descriptive cross-sectional study design was employed to collect information between July and August 2011. The study included two target population groups, namely pregnant women who were attending the ANC services and mothers who had given birth in all the 3 named health centres in the district and their surrounding 'catchment' dispensaries, as it has been described above. Information on the respondents' perception and satisfaction or dissatisfaction with the health care services were solicited using an interview schedule. Some of the statements in the interview were adapted from the perceived service quality items of the SERVQUAL survey (quality of service questionnaire) developed by Parasuraman et al. (1988) where a Likert's scale was used to measure the variables. Dimensions such as satisfaction, responsiveness and empathy/understanding were adopted from the named questionnaire, and other independent variables were substituted and/or added. Specifically, the statements inquired on maternal health workers' promptness in providing services, willingness to assist, responding to client's requests, providing information on when services would be given, and acceptability of the language they use to their clients. SPSS statistical software was used for data analysis whereby frequency, percentage and mean were calculated to describe the sample statistics while Chi-square testing was used to determine the relationship between independent and dependent categorical variables.

For convenience of analyses, particularly the Chi-square testing of whether a relationship existed between a dependent and an independent variable, it was necessary to shorten some of the categorical variables from the five-point ordinal scale scoring into a 2-point nominal scale such as acceptable and unacceptable, or satisfied and dissatisfied.

For example, the dependent variable of perceived quality of care was re-coded as "acceptable" from very good and good category while "not acceptable" was adopted for the fair, poor and very poor categories. Similarly, the perceived waiting time was scaled down to "long" (unacceptable) and "adequate" (acceptable). 


\subsection{Ethical considerations}

The research proposal for the study was submitted for ethical clearance to the Muhimbili University of Health and Allied Sciences (MUHAS) Research and Publications Committee. Permission to implement the study was sought from the relevant district authorities. A verbal consent was obtained from each respondent before an interview was conducted. Confidentiality was observed throughout the study, whereby participants were not identified by names but rather by assigned code numbers.

\section{Results}

\subsection{Perceptions and satisfaction towards quality of care}

The results indicated that more than three quarters of pregnant women, $(77.3 \%)$ and approximately two thirds of mothers, $(67.2 \%)$, perceived the quality of maternal care to be acceptable.

Results in Fig. 1 show that about one quarter (27\%) of the pregnant women were very satisfied with the services received, and almost two thirds (61\%) were satisfied. The remaining proportions were either unsure or dissatisfied. Results also show that $18 \%$ of mothers who gave birth in the health facilities were very satisfied with the delivery services while $61 \%$ were satisfied. The remaining were either unsure $(13 \%)$ or dissatisfied $(7.5 \%)$.

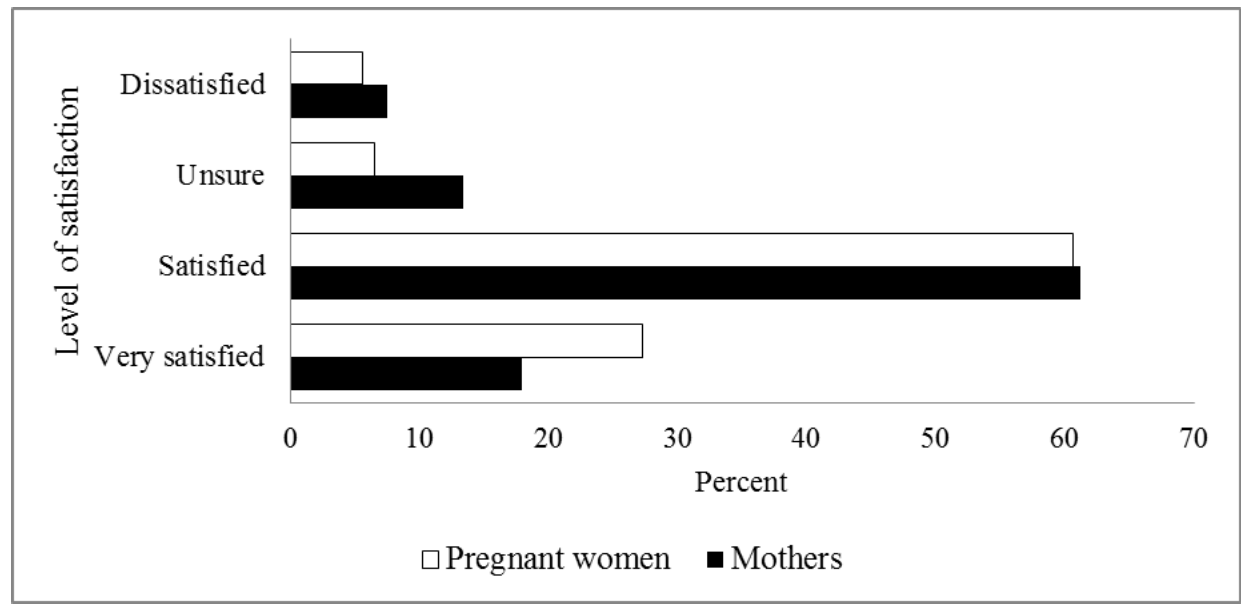

Figure 1: Level of satisfaction with ANC and delivery services among pregnant women and mothers delivering in health facilities in Morogoro Rural District 
Chi-square testing for relationship between level of satisfaction with Antenatal Care (ANC) services and perceived quality of maternal care indicated a strong relationship $\left(\chi^{2}=56.7\right.$, p-value $\left.<0.001\right)$. Eighty five percent of the interviewed pregnant women who were satisfied with the level of ANC perceived the quality of maternal care received to be acceptable, while $81 \%$ of those who were dissatisfied perceived the quality of care to be non-acceptable.

\subsection{Satisfaction with waiting time}

The minimum waiting time for pregnant women waiting to enter the consultation room recorded was 6 minutes while the maximum waiting time was 7 hours, where the mean was 2.1 (S.D = 1.6) hours. On the other hand, $87 \%$ of mothers waited for less than an hour to enter delivery room while $12 \%$ waited between 1 and 3 hours, leaving a small proportion (1.5\%) that waited for more than 3 hours. The minimum waiting time for mothers to enter the delivery room was 2 minutes and the maximum was 3.5 hours with a mean of $40(\mathrm{SD}=23)$ minutes. Results (Fig. 2 ), indicate that $18 \%$ and $51 \%$ of pregnant women respectively reported to be very satisfied and satisfied with the waiting time to enter the consultation room while the remaining one quarter $(24 \%)$ were dissatisfied with the waiting time. Similar findings were reported by mothers, whereby $25 \%$ and $52 \%$ respectively were very satisfied and satisfied with the waiting time before entering the delivery room.

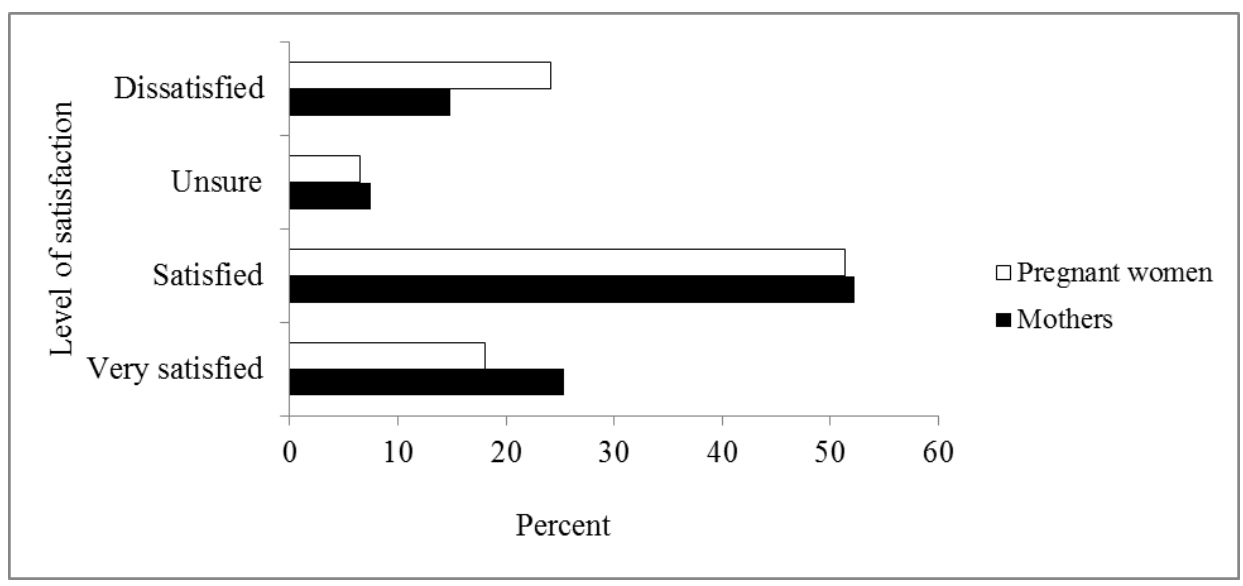

Figure 2: Level of satisfaction with waiting time among pregnant women and mothers delivering in health facilities in Morogoro Rural District

Chi-square testing for relationship between time spent waiting by pregnant women before entering the consultation room and the perceived quality of care showed a strong relationship $\left(\chi^{2}=31.1, \mathrm{p}\right.$-value $\left.<0.001\right)$. More than three quarters $(78 \%)$ of pregnant women perceived that the time spent waiting before entering the consul- 
tation room was adequate and thus theywere satisfied with waiting time. However, about two thirds $(68 \%)$ perceived the waiting time to be too long and thus, dissatisfying

\subsection{Perception on behaviour and responsiveness of maternal health workers}

Results of the five inquiry statements that were meant to show the perception of mothers and pregnant women are summarized in Table 1. More than two thirds of the respondents agreed (total of strongly agree and agree) with all the four statement. Test for relationship between perceived responsiveness of women towards providers regarding their specific needs and the perceived quality of maternal care showed a strong relationship $(\chi 2=37.6, \mathrm{p}$-value $<0.001)$. More than $80 \%$ of pregnant women who positively perceived maternal health workers to respond to their specific needs perceived the quality of maternal care to be acceptable, while $61 \%$ of those who perceived the maternal health workers negatively also perceived the quality of maternal care to be non-acceptable.

\section{Discussion}

\subsection{Perceived quality of care and level of satisfaction}

The finding of the study showed that a majority of pregnant women and mothers perceived the quality of maternal health care to be acceptable. This has also been reported in a study by Stein et al. (1996), whereby respondents perceived high level of quality of care in $\mathrm{MCH}$ services. In comparison, pregnant women appear to have a slightly higher percent of acceptability $(77.3 \%)$ than the mothers $(67.2 \%)$. The difference could be that ANC processes or services are much better organized or planned than delivery services. Similar findings were reported by a study done in a rural district in Ghana (Turkson, 2009).

\subsection{Waiting time}

Waiting time in this aspect was taken to be the time duration a woman spends waiting before receiving maternal health services, specifically when pregnant women and mothers were waiting to enter the consultation and delivery rooms to obtain various services respectively. More than three quarters of the pregnant women and mothers perceived the waiting time to be adequate which implied high levels of satisfaction. Similar findings have been reported in other parts of Tanzania (Msamange et al., 1996; Andaleb et al., 2001). 
Table 1: Perception of women and mothers on health care services and responsiveness of maternal health workers towards their needs

\begin{tabular}{|c|c|c|c|c|c|}
\hline \multirow[t]{3}{*}{ Inquiry statement } & \multirow{3}{*}{$\begin{array}{c}\text { Level of } \\
\text { agreement }\end{array}$} & \multicolumn{2}{|c|}{ Pregnant women } & \multirow{2}{*}{$\begin{array}{l}\text { Mothers } \\
(\mathrm{n}=67) \\
\end{array}$} & \\
\hline & & \multicolumn{2}{|c|}{$(n=216)$} & & \\
\hline & & No. & Percent & No. & Percent \\
\hline \multirow{5}{*}{$\begin{array}{l}\text { Health workers in the facility } \\
\text { give prompt services }\end{array}$} & Strongly agree & 30 & 13.9 & 7 & 10.4 \\
\hline & Agree & 132 & 61.1 & 37 & 55.2 \\
\hline & $\begin{array}{l}\text { Neither agree or } \\
\text { disagree }\end{array}$ & 27 & 12.5 & 15 & 22.4 \\
\hline & Disagree & 27 & 12.5 & 8 & 12 \\
\hline & Strongly disagree & 0 & 0 & 0 & 0 \\
\hline \multirow{5}{*}{$\begin{array}{l}\text { Health workers are always } \\
\text { willing to help }\end{array}$} & Strongly agree & 64 & 29.6 & 14 & 20.9 \\
\hline & Agree & 126 & 58.3 & 37 & 55.2 \\
\hline & $\begin{array}{l}\text { Neither agree or } \\
\text { disagree }\end{array}$ & 24 & 11.1 & 15 & 22.4 \\
\hline & Disagree & 2 & 0.9 & 1 & 1.5 \\
\hline & Strongly disagree & 0 & 0 & 0 & 0 \\
\hline \multirow{5}{*}{$\begin{array}{l}\text { Health workers respond to } \\
\text { requests } \\
\text { when you need it }\end{array}$} & Strongly agree & 41 & 19 & 16 & 23.9 \\
\hline & \begin{tabular}{|l|} 
Agree \\
\end{tabular} & 137 & 63.4 & 29 & 43.3 \\
\hline & $\begin{array}{l}\text { Neither agree or } \\
\text { disagree }\end{array}$ & 22 & 10.2 & 14 & 20.9 \\
\hline & Disagree & 16 & 7.4 & 8 & 11.9 \\
\hline & Strongly disagree & 0 & 0 & 0 & 0 \\
\hline \multirow{5}{*}{$\begin{array}{c}\text { Health workers tell them } \\
\text { exactly when services will be } \\
\text { performed }\end{array}$} & Strongly agree & 51 & 26.6 & 13 & 19.4 \\
\hline & Agree & 137 & 63.4 & 47 & 70.1 \\
\hline & $\begin{array}{l}\text { Neither agree or } \\
\text { disagree }\end{array}$ & 14 & 6.5 & 6 & 9 \\
\hline & Disagree & 14 & 6.5 & 1 & 1.5 \\
\hline & Strongly disagree & 0 & 0 & 0 & 0 \\
\hline \multirow{5}{*}{$\begin{array}{l}\text { Health workers use language } \\
\text { that shows respect to clients }\end{array}$} & Strongly agree & 55 & 25.5 & 5 & 7.5 \\
\hline & Agree & 149 & 69 & 56 & 83.6 \\
\hline & $\begin{array}{l}\text { Neither agree or } \\
\text { disagree }\end{array}$ & 9 & 4.2 & 5 & 7.5 \\
\hline & Disagree & 2 & 0.9 & 1 & 1.5 \\
\hline & Strongly disagree & 1 & 0.5 & 0 & 0 \\
\hline
\end{tabular}


About $40 \%$ of pregnant women waited for less than 1 hour while the remaining waited for more than an hour. This was different for the case of mothers where the majority $(86.6 \%)$ waited for less than one hour. Similar findings have also been reported in Ghana (Turkson et al., 2009). The difference could be attributed to the understanding of the health care providers that women who are giving birth need immediate attention so as to reduce risks to the woman and the child. Such concerns in developing countries have also been reported by others (Singh et al., 1999; Newman et al., 1998).

\subsection{Perception on the behaviour and responsiveness of health providers}

The relationship between the client's level of satisfaction with the behaviour of maternal workers and perceived quality of maternal care has been shown in the current study. Similar observations have been reported in other developing countries (Aldana et al., 2001; Baltussen et al., 2001). It appears that behaviour of maternal health workers plays a vital role in influencing women's level of satisfaction, whereby politeness and showing respect for the clients are critical issues. Regarding the behaviour of the workers, the question of their responsiveness is interesting as the majority of mothers and pregnant women in the study agreed that maternal health workers were responsive enough towards their needs. Some of the statements given by the respondents include: "maternal health workers are always willing to help and provide the necessary support" and "workers tell us exactly when services will be provided". A study in India observed a similar situation (Krishna et al., 2006) where women were very positive about their health providers and reported that medical workers provided all the information on exact services they were interested in or needed.

Although there is no standard procedure, protocol or process that dictates the way providers should respond to women's needs, and since this is more deeply rooted in the behaviour of providers due to long-term mental conditioning guided by ethical principles, measures should be put in place to constantly monitor and evaluate perceptions of quality of care according to women, taking into consideration component of behaviour of health workers so as to maintain the sustainability of women's satisfaction. Also, prompt attention has been shown to be a key dimension in surveys of community satisfaction with health services (Cohen et al., 1996; Sitzia and Wood, 1997). However, prompt attention on its own is not a function of health improvement, but it is a dimension of a patient's satisfaction (Cohen et al., 1996). The study findings showed that almost $80 \%$ of pregnant women and mothers agreed that maternal health workers were providing them prompt individual attention. In general it appears that understanding women's health care and health needs is a basis for patient-centred care and for effective health services. 


\section{Conclusion}

It appears that despite of the very often claimed poor quality of health services in poor countries like Tanzania - and especially in the rural areas, the majority of the beneficiaries (in most cases women) are still satisfied with the services they get. The current study observed that pregnant women and mothers perceived the quality of maternal healthcare to be acceptable. However, it should be remembered that satisfaction does not necessarily reflect true quality. It may be true that because of the limited access to health services in the rural areas, the respondents did not have much choice to compare with, and therefore they were narrowly constrained in their judgement. It would be interesting to undertake a comparison study in an urban setting where respondents have more choices ranging from governmentowned public facilities (with minimal requirement for paying) to the privately run commercial facilities where purchasing power tends to dictate the terms of services to a greater part. It can also be recommended to conduct a qualitative study to have an in depth understanding regarding women's perceptions and satisfaction regarding specific types of services received in the different health facilities.

\section{References}

Aldana JM, Piechulek H, al-Sabir A (2001). Client satisfaction and quality of healthcare in rural Bangladesh. Bull World Health Organ, 79:512-517.

Andaleb SS (2001). Service quality perception and patient satisfaction: A study of hospitals in developing countries. Social Sciences and Medicine, 40:487-490.

Baltussen RMPM, Ye Y, Haddad S, Sauerborn RS (2001). Perceived quality of care of primary health services in Burkina Faso. Health Policy and Planning, 17:4248.

Banzart ES, Koenig (2009). Women's satisfaction with delivery care in Nairobi's informal settlements. Int J Qual Health Care, 21:79-86.

Clemes MD, Ozanne LK, Laurensen WL (2001). Patients' perceptions of service quality dimensions. An empirical examination of health care in New Zealand. Health Mark Q, 19:3-22.

Cohen G, Forbes J, Garraway M (1996). Can different patient satisfaction survey methods yield consistent results? Comparison of three surveys. Br Med J, 313: $841-4$.

De Geyndt W (1995). Managing the quality of care in developing countries. World Bank Technical papers No; 258, Washington DC. 
Donabedian A (1980). Exploration in quality assessment and monitoring. The definition of quality and approaches to its assessment. Health administration press.

Epstein KR, Laine C, Farber NJ, Nelson EC, Davidoff F (1996). Patients' perceptions of office medical practice. Judging quality through the patients' eyes. Am J Med Qual, 11:73-80.

Gilson L, Alilio M, Heggenhougen H (1994). Community satisfaction with primary health care services. An evaluation undertaken in the Morogoro region of Tanzania. Social Science and Medicine, 39:767-80.

Hulton LA, Matthews Z, Stones RW (2007). Applying a framework for assessing the quality of maternal health services in urban India. Soc Sci Med, 64:208395.

Joint Commission on Accreditation of Healthcare Organizations (JCAHO), (1999) Hospital Accreditation Standards. Oakbrook Terrace, IL, USA: Joint Commission on Accreditation of Healthcare Organizations.

Krishna DP, David P, Karen BR (2006). Towards patient-centered health services in India - a scale to measure patient perceptions of quality. Int J Qual Health Care, 18:414-421.

Langer A, Nigenda G, Romero M, Rojas G, Kuchaisit C (2002). Conceptual bases and methodology for the evaluation of women's and providers' perception of the quality of antenatal care in the WHO Antenatal Care Randomised Controlled Trial. Paediatr Perinat Epidemiol.12 Suppl, 2:98-115.

Measurement of patient satisfaction guidelines (2003). Health strategy implementation project. Irish Society for Quality and Safety in Health Care.

Ministry of Health of Tanzania (2000). Quality assurance training guidelines for health workers.

Ministry of Planning, Economy and Empowerment Report (2007). Morogoro Region Profile Report.

Msamange GI, Urrassa DP, Mujinja PGM (1996). Equity of access to public, private not for profit and private for profit health facilities in two regions of Tanzania. A research report submitted to UNICEF, New York and IDRC, Ottawa Canada.

National Bureau of Statistics (NBS) (2010). Tanzania Demographic and Health Survey (TDHS) 2010. Dar es Salaam: NBS and ORC Macro.

Newman RD, Gloyd S, Nyangezi JM, Machabo F, Muiser J (1998). Satisfaction with outpatient healthcare services in Manica Provinca, Mozambique. Health Policy Plan, 13:174-180. 
Nigenda G, Langer A, Kuchaisit C, Romero M, Rojas G, Al Osimy M, Villar J, Garcia (2003). Women's opinions on antenatal care in developing countries. Results of a study in Cuba, Thailand, Saudi Arabia and Argentina. BMC Public Health, 3:17.

Parasuraman A, Zeithaml VA, Berry LL, Servqual (1988). A multiple-item scale for measuring consumer perceptions of service quality. J Retail, 4:12-37.

Singh H, Haqq ED, Mustapha N (1999). Patient's perception and satisfaction with health care professionals at primary care facilities in Trinidad and Tobago. Bull World Health Organ, 77:356-360.

Sitzia J, Wood N (1997): Patient satisfaction: A review of issues and concepts. Social Science \& Medicine, 45:1829-43.

Smith M, Englelbrecht B (2001). Developing tools to assess client satisfaction at district hospital. http://www.hst.org.za/uploads/files/clienttool

Stein K (1996). Service quality among women receiving $\mathrm{MCH}$ and family planning services. African Journal of Fertility, Sexual and Reproductive Health, 1:14652.

Tanzania Demographic and Health Survey (TDHS) 2004-2005. National Bureau of Statistics Dar es Salaam, Tanzania (2005).

Turkson PK (2009). Perceived quality of health care delivery in a rural district in Ghana. Ghana Med J, 43:65-70.

WHO (2010). Trends in Maternal Mortality: 1990 to 2008. Estimates developed by WHO, UNICEF, UNFPA and The World Bank.

Williams T, Schutt-Aine J (2000). Measuring family planning service quality through client satisfaction exit interviews. International Family Planning Perspectives, 26:63-71. 



\title{
9. A Review of Selected Maternal and Child Health Indicators in Ghana
}

\author{
Edward Nketiah-Amponsah, Department of Economics, \\ University of Ghana, Legon, Accra, Ghana \\ Gloria Afful-Mensah, Department of Banking and Finance, \\ University of Professional Studies, Accra, Ghana
}

\section{Abstract}

This chapter reviews selected maternal and child health indicators in Ghana drawing heavily on the five Demographic and Health Surveys (1988, 1993, 1998, 2003 and 2008) conducted in Ghana since independence. The period under review witnessed, among others, a reduction in child and maternal mortalities, increased antenatal care utilization, contraceptive usage, facility delivery, postnatal care, immunization coverage, use of insecticide treated bednets among both pregnant women and children aged under-five. It is also apparent that the government of Ghana through the Ministry of Health and the Ghana Health Service has implemented a number of health policies including free-maternal healthcare and the national health insurance scheme which have far reaching consequences on the health of 
women and children. The review further indicates that improvement in maternalchild health outcomes over the period is somehow explained by socio-economic attributes particularly educational attainment of the mother and urban residence.

\section{Introduction}

Over the past two decades, maternal and child health has become a major issue in most developing countries, especially in the Sub-Saharan African region because of the high under-five and maternal mortality rates coupled with high incidence of malaria. This is because ensuring good maternal and child health is very essential to a country's growth and development process. Literature abounds on the positive association between good health in general and maternal health in particular and its role in poverty alleviation and human development (Thomas and Strauss, 1997; Ronsmans and Campbell, 2006; Odwee et al., 2006). The health of women is more at risk in periods related to childbirth. Aside the risk of dying during pregnancy and childbirth, some women may suffer from temporal or even permanent disabilities or illnesses. In order to improve maternal health globally, the Safe Motherhood Initiative (SMI) was launched in 1987 as a way of increasing the awareness of the scope and dimensions of maternal mortality and to stimulate commitment among governments, donors, United Nation agencies and other relevant stakeholders in order to help address this public health challenge. This initiative was put in place mainly to reduce the burden of maternal morbidity and mortality in developing countries. Shortly, the Inter-Agency Group made up of the World Bank, World Health Organisation, UNFPA, UNICEF and UNDP was established to help make the goals of the SMI achievable. Since then, there has been series of programmes and initiatives aimed at addressing maternal health problems particularly in developing economies. Maternal health was broadly defined in an International Conference on Population and Development (ICPD) which was held in Egypt to include "education on safe motherhood, prenatal care (that is focused and effective), maternal nutrition programmes, adequate delivery assistance that avoids excessive recourse to caesarean sections and provides for obstetric emergencies; referral services for pregnancy, childbirth and abortion complications; postnatal care and family planning" (The Safe Motherhood Initiative, 1987 - 2005).

Although Ghana's current maternal mortality ratio (MMR) of 350 per 100,000 women is behind schedule in Ghana's quest to achieve its Millennium Development Goal (MDG) target of 158 per 100,000 women by 2015, modest progress has been achieved over the past two decades (WHO, 2011). In fact, between 2005 and 2011, Ghana's MMR reduced from 560 per 100,000 women to 350 per 100,000, representing an approximate $37 \%$ improvement over the period. Protecting the health of women during reproduction is very crucial in order for society to benefit from their future contributions to society and ensuring the health and productivity of future generations. In addition, given that children are the future leaders, dan- 
gers to their health are likely to lead to serious negative consequences for any economy. Maternal mortality is also identified as very crucial to the health and survival of children such that if the health of mothers is compromised, there will be serious negative consequences for their families, communities and perhaps the entire process of economic and social development. Given the direct association of maternal health to child health, maternal health is regarded as a means to ensure childhood survival rather than an end in itself (AbouZahr, 2003). This is why the United Nations (UN) has set as one of eight MDGs, the reduction of the maternal mortality ratio (MMR) by two-thirds in the developing world by the year 2015 (Ajakaiye and Mwabu, 2010). In Ghana, available evidence shows that pregnancyrelated complication is among the leading causes of death among women of reproductive age. There is a general perception within the country that rural women, the poor (vulnerable) and the uneducated/less educated are the most affected as compared to their urban and educated counterparts. Investing in maternal health saves individual women's lives and safeguards their wellbeing (The Safe Motherhood Initiative, 1987-2005).

A more recent health policy with far reaching consequences for maternal and child health is the implementation of the National Health Insurance Scheme (NHIS) in 2005. The government had in 2003 introduced a policy for delivery fees for intrapartum care in public, private and mission facilities in the four mostdeprived regions of the country in an effort to improve maternal health and survival (Ministry of Health, 2004). This policy was extended in the following year to cover the remaining six regions. Subsequent to the 2003 policy, the government of Ghana announced a free maternal care regime for pregnant women in July 2008 with initial funding from the British government to quicken the attainment of MDGs 4 and 5. Under this programme, expectant mothers are to receive the full package of antenatal, prenatal and postnatal care (NHIS, 2010). However, due to misuse of the scheme, NHIA revised the implementation guidelines in 2010 to encourage pregnant women to register with the NHIS before accessing healthcare. At the end of 2010, a cumulative total of 1,394,445 pregnant women representing $7.7 \%$ of the total number registered had subscribed to the scheme (NHIS, 2010).

Another intervention worth-mentioning is the government's establishment of community-based health planning services (CHPS) as the first point for primary health care especially in deprived and rural communities. From a low of 39 in 2002, functional CHPS zones increased to 1,034 in 2010 (NHIA Report, 2010) to help scale up access to health in the remotest and most deprived parts of the country (ISSER, 2012). The progress of Ghana in health care delivery can be partly seen in its elimination of guinea worm since May 2010. A potential threat to the Ghana Health Service in executing its healthcare programmes is however the fiscal deficit being experienced by the sector in recent times as the year 2011 recorded a deficit of GHC 132.98 , representing $22 \%$ of the revenue generated by the sector in same year. 


\section{$3 \quad$ Fertility profile of the country}

The management of population growth to support sustainable development is a major objective of most governments. Ghana's population since independence has seen a continuous increase even though the rate of increase in recent times has recorded some marginal declines as compared to periods between 1960s and 1990s. This is evident in the figures provided by the Population and Housing Census of the Ghana Statistical Service (GSS, 2010). Despite this increment, various governments since independence have recognised the role of the country's population in growth and development and have therefore made efforts at achieving rapid social and economic development by incorporating the population-related factors into the country's general development agenda. Ghana became the first country in Sub-Saharan Africa to adopt a population policy mainly to reduce population growth to a rate of 2.0 percent per annum by the year 2000. In addition, the Ghana National Family Planning Programme (GNFPP) was instituted to give family planning services to couples who had interest in limiting or spacing their births through their various outlets. However, in 1984, Ghana's population according to the census office was estimated to be 12.3 million, representing an increase of about 44 percent compared to the figure recorded in 1970 (GDHS Report, 1988). In a related development, pharmacies and chemical sellers in the country were permitted to sell some contraceptives (condoms, vaginal foaming tablets, oral contraceptives) through 3,600 retail outlets (Ampofo, 1988: cited in 1988 GDHS Report, pg. 3). The purpose of this initiative was to manage the existing high fertility rates in the country which obviously had a direct association with the increasing population trends. Though Ghana's total fertility rate is one of the highest in Africa, data from the Demographic and Health Surveys indicate a consistent decline in the rates (see Table 1).

Although the total fertility rate (TFR) consistently declined in all the five rounds of the survey, there exists a striking difference in fertility levels by the background characteristics considered in the study. High fertility in the country continues to be a phenomenon in the rural area with women having almost two children more than their urban counterparts. Specifically, there was a consistent rise in the rural-urban fertility differential between 1988 and 2003. However, the TFR for rural women was 1.8 child higher (4.90) than for urban women (3.10) in the five years preceding the 2008 GDHS. An interesting revelation (though not surprising) is the differences in TFR across the various levels of education. Women with no education on average have four children more than their counterparts with at least secondary education. Again, while the difference in the number of children with respect to women with no formal education, primary and middle or Junior Secondary School (JSS) is not very great, it appears that having secondary or higher education has a significant inverse relationship with the total number of children a woman will have at the end of her reproductive age. 
Table 1: Fertility rates by background characteristics

\begin{tabular}{lccccc}
\hline Characteristic & 1988 & 1993 & 1998 & 2003 & 2008 \\
\hline \hline Place of residence & 5.05 & 3.99 & 2.96 & 3.10 & 3.10 \\
Urban & 6.64 & 6.36 & 5.41 & 5.60 & 4.90 \\
Rural & & & & & \\
Woman's level & & & & & \\
of education & 6.77 & 6.67 & 5.83 & 6.00 & 6.00 \\
None & 6.09 & 6.10 & 4.94 & 5.30 & 4.90 \\
Primary & 5.87 & 4.71 & 3.78 & 3.50 & 3.50 \\
Middle/JSS & 3.55 & 2.90 & 2.80 & 2.50 & 2.10 \\
Secondary/Higher & & & & & \\
Total fertility rate & 6.43 & 5.35 & 4.55 & 4.40 & 4.00 \\
(15 - 49 years) & & & & & \\
\hline \hline
\end{tabular}

The data based on the 2008 Ghana Demographic and health Survey (GDHS) therefore suggests that on average, Ghanaian women will have four children at the end of their reproductive age in 2008 indicating a decline from 6.4 in 1988. The obvious conclusion that can be drawn from the TFR trend is that our society still places "high prestige" on large family size, albeit less true for better educated women. One probable reason why better educated women place a lower premium on large family size is that with education, they are able to break away from the traditional notion that having more children constitute potential future stream of wealth. Besides, given that women with at least secondary education had spent part of their reproductive years in school, they are more likely to postpone child bearing which may eventually lead to having relatively small family sizes. Generally, while this shows an improvement, the TFR as of 2008 shows that the nation was unable to achieve the target of a 2 percent population growth rate at the year 2000 .

\subsection{Fertility regulation (family planning) in Ghana}

The main contributor to population growth in Ghana is not necessarily migration but rather the high fertility rate as evidenced by Ghana's net migration rate of -0.58 migrants $/ 1,000$ population, suggesting that more people leave Ghana than enter ${ }^{1}$. The act of spacing births or limiting the number of births a couple can have in itself is not a recent development in the country given that there were some already existing traditional methods. However, the realisation of the harsh consequence of rapid population growth on the nation's growth and development necessitated the

${ }^{1}$ http://www.indexmundi.com/ghana/net_migration_rate.html [Accessed Januray 17, 2013]. 
controlling of population growth. In this regard, the Christian Council of Ghana was the first to set up a Family Advice Centre in 1961 purposely to give advice to married couples on family planning and responsible parenthood. Six years later, the Planned Parenthood Association of Ghana (PPAG) was also set up with branches throughout the country. As part of its objectives, the PPAG was to educate the general public on issues concerning the benefits of family planning and offer modern family planning services to them. In 1969, the government of Ghana got involved in this campaign by coming up with a national population policy mainly to encourage people to plan and control their family sizes. Hence, a secretariat was established and charged with the responsibility to manage family planning logistics, clinics, public education and field activities (1988 GDHS Report). Since then some other organisations and individuals have joined in educating and providing modern family planning services to the general public at less or no cost to them. Table 2 shows some trends in the knowledge and use of such methods based on GDHS data.

Table 2: Knowledge of fertility regulation methods (in percentages)

\begin{tabular}{|c|c|c|c|c|c|}
\hline $\begin{array}{l}\text { All Women } \\
\text { Variable }\end{array}$ & 1988 & 1993 & 1998 & 2003 & 2008 \\
\hline \multicolumn{6}{|c|}{ Knowledge of method } \\
\hline any method & 76.2 & 91.1 & 92.9 & 97.7 & 97.8 \\
\hline any modern method & 73.8 & 90.6 & 92.5 & 97.5 & 97.7 \\
\hline \multicolumn{6}{|l|}{ Knowledge of source } \\
\hline any method & 69.8 & 72.4 & 78.4 & na & na \\
\hline any modern method & 66.4 & 71.9 & 78.0 & na & na \\
\hline \multicolumn{6}{|c|}{ Currently married women } \\
\hline \multicolumn{6}{|c|}{ Knowledge of method } \\
\hline any method & 79.4 & 91.3 & 93.6 & 98.0 & 97.9 \\
\hline any modern method & 76.5 & 90.7 & 93.1 & 97.8 & 97.8 \\
\hline \multicolumn{6}{|l|}{ Knowledge of source } \\
\hline any method & 73.4 & 74.0 & 81.1 & na & na \\
\hline any modern method & 69.6 & 73.5 & 80.6 & na & na \\
\hline
\end{tabular}

Source: GDHS Reports from 1988 to 2008; na: not applicable 


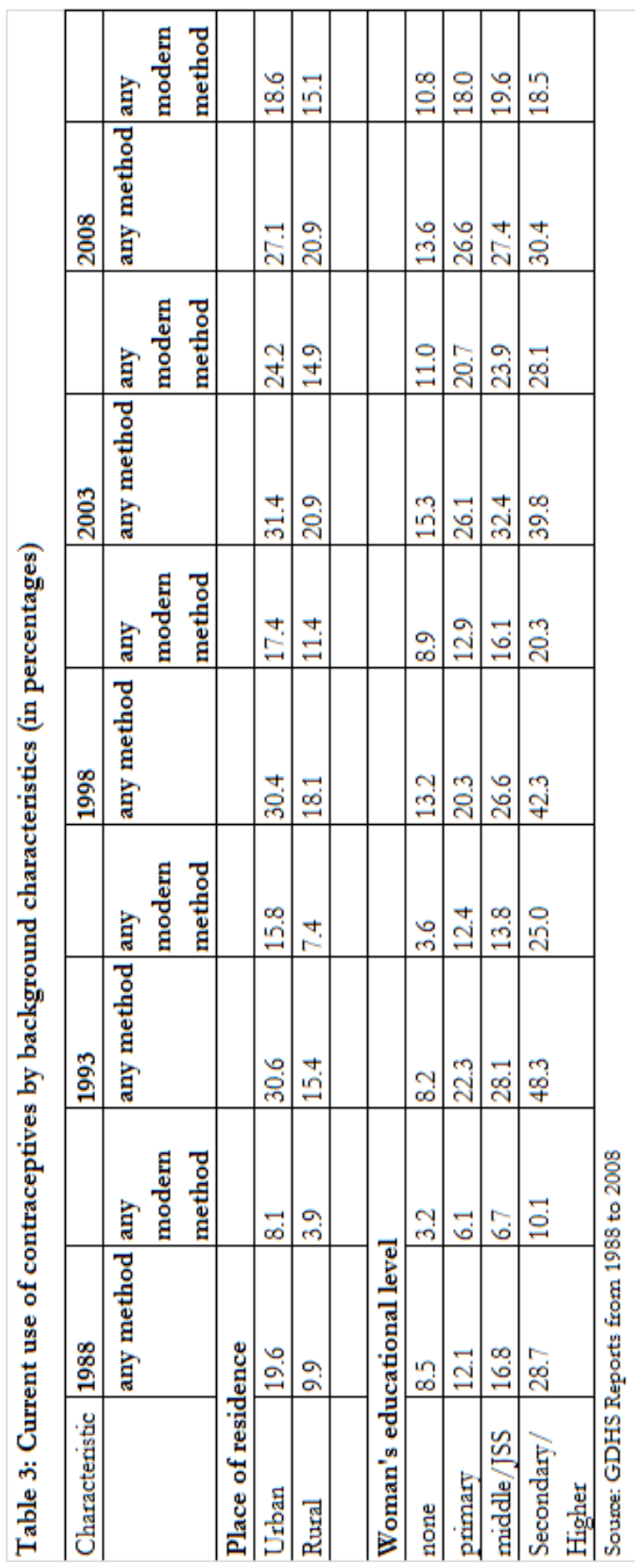


The knowledge of family planning methods currently is almost universal in the country as recorded in the last GDHS survey. For instance, in the 2008 survey, as many as $97.7 \%$ and $97.8 \%$ of women aged $15-49$ indicated that they had knowledge of any method and modern methods of contraceptives, respectively. However, the major challenge is whether or not people are really using the services given that the population growth rate between 1984 and 2000 was 2.7 (instead of the target of 2 percent). In addition, the latest census indicates a growth rate of 2.5 between the period 2000 and 2010 (GSS, 2012).

Despite the high knowledge of family planning methods in the country, the rates of use are not very encouraging. There are also differences with regard to the use of contraception by background characteristics (Table 3). Here, even though there have been some marginal improvements, on average, only 1 out of every 5 women in the rural areas uses any family planning method. Even though the rates for urban women were slightly higher than their rural counterparts, they are still low. Also, while there was a consistent increase in the percentage of usage of any family planning method (except for 2008 where there was no change) in the rural areas, that of the urban areas was alternating. Although not surprising, the use of family planning services was revealed to be positively related to a woman's level of education. Thus, the higher the educational level of a woman, the higher the possibility of using any family planning method. Generally, women with at least secondary education are almost three times more likely to use any method compared to their counterparts with no formal education.

Table 4 presents the three most important reasons given for the non-use of contraceptives in the country based on all the five rounds of the Ghana Demographic and Health Surveys. Reasons are captured based on two major age groups (i.e. women who are less than 30 years and those at least 30 years). From Table 4, there appears to be a particular trend with regard to reasons given for the non-use of contraception. Younger women were less likely to use any family planning method mainly because of their perceived side-effects associated with the methods. Thus, except for 1988 and 1993 where "lack of knowledge" was ranked the top reason, the period 2003 and 2008 recorded "side-effects" as the top ranked reason for non-usage of contraceptives. On the other hand, the reasons for non-use of contraception by older women were mainly "desire to have children" and the "side-effects".

\section{Selected maternal and child health indicators}

Over the years, various governments have come up with policies aimed at achieving improved health for women and children. This is evident in some policies like the abolishing of the "cash-and-carry" system, the introduction of free maternal health care services, „the health insurance scheme, etc. One main strategy by the government in improving the accessibility of health care is the Primary Health Care 
Table 4: Top three ranked reasons for non-use of any family planning method

\begin{tabular}{|c|c|c|}
\hline Reason & $\begin{array}{l}<30 \\
\mathrm{yrs}\end{array}$ & $\begin{array}{l}30+ \\
\text { yrs } \\
\end{array}$ \\
\hline \multicolumn{3}{|l|}{1988} \\
\hline lack of knowledge & 29 & 18.3 \\
\hline infrequent sex & 12.7 & \\
\hline menopausal/subfecund & & 18 \\
\hline other & 12.2 & 13.8 \\
\hline \multicolumn{3}{|l|}{1993} \\
\hline lack of knowledge & 15.8 & \\
\hline want children & 45.9 & 25.9 \\
\hline menopausal/hysterectomy & & 16.8 \\
\hline side effects & 7.1 & \\
\hline difficult to get pregnant & & 16.3 \\
\hline \multicolumn{3}{|l|}{1998} \\
\hline want children & 21.2 & 18.1 \\
\hline side effects & 23.7 & 15.7 \\
\hline menopausal/hysterectomy & & 16.6 \\
\hline respondents opposed & 16.2 & \\
\hline \multicolumn{3}{|l|}{2003} \\
\hline method related (side effects) & 47.5 & 33.8 \\
\hline fertility-related (wants more children, menopausal) & 20.3 & 47.7 \\
\hline respondents opposed & 16.9 & 10.7 \\
\hline \multicolumn{3}{|l|}{2008} \\
\hline method related (side effects) & 48.8 & 38.5 \\
\hline fertility-related (mainly infrequent sex, menopausal) & 11.1 & 37.4 \\
\hline opposition to use (mainly respondents opposed) & 30.8 & 19.2 \\
\hline
\end{tabular}

Source: GDHS Reports from 1988 to 2008

programme (PHC). The main purpose of the programme was to decentralise health services to the districts by training more people to provide health care services. Also, recognising the role of traditional birth attendants particularly in rural areas, the programme was designed to train and improve their services. The PHC programme, however, focused on maternal and child health care, family planning, nutrition, control of diarrhoeal diseases and expansion of the programme on immunisation and malaria control (GDHS Report 1988).

\subsection{Antenatal care in Ghana}

Complications of pregnancy and childbirth are the major cause of death for women of reproductive age in Ghana (Nyarko et al., 2006). Antenatal care (ANC) visits 
promote and maintain the health of pregnant women by identifying and treating problems during pregnancy. During ANC visits, screening for complications and advice on a range of issues including birth preparedness, place of delivery and referral of mothers are made. In Ghana, midwives and other mid-level providers make up the largest proportion of health professionals providing antenatal care, except for the Greater Accra region, where ANC provided by midwives and other mid-level providers is almost equal to that provided by doctors (GDHS Report, 2008). Doctors are generally the most expensive providers of maternal health care services (MHC) particularly for ANC services based on the prices they charge for a visit followed by midwives, nurses and medical assistants. However, the traditional providers are the cheapest means of providing such maternal health care services (Overbosch et al., 2004). Although, the free maternal health services via the national health insurance scheme has removed the financial burden associated with MHC, these services still remain expensive for women who are not registered with the scheme and/or patronize health facilities which are not accredited by the scheme. Currently, there is a new strategy for delivering ANC service and this is known as focused antenatal care service (FANC). The FANC strategy encourages a minimum of four (4) antenatal care visits. That is, before the 16th week, at the 26th week, 32nd week and the 36th week of pregnancy (Nyarko et al., 2006). This initiative has the objective of promoting the health and survival of mothers and their babies by doing the following:

- Targeting assessments of pregnant women to ensure a normal childbearing cycle and newborn period, and to facilitate the early detection of complications, chronic conditions, and other potential problems that could affect the pregnancy and

- Individualising the care to help maintain the normal progress of pregnancies, including preventive measures, supportive care, health messages and counselling of women and families for effective self care, birth preparedness and planning for complications (Nyarko et. al., 2006).

Unlike the previous antenatal care system which categorised the ANC clients into risk groups based on their previous obstetric history, medical conditions, age, parity, weight and laboratory investigations, the focused ANC system considers all pregnant women to be at risk and consequently do away with the risk assessment criteria (Nyarko et al., 2006). Generally, the country has seen a remarkable improvement in ANC coverage over the past two decades. Statistics from the 2008 GDHS indicates that the ANC coverage was approximately 95 percent. Despite the improvements, some women fail to either make all the recommended four visits or go for $\mathrm{ANC}$ check up late. Table 5 presents the number and timing of such visits among Ghanaian women. 
Table 5: Number and timing of ANC visits in percentages

\begin{tabular}{lrrrr}
\hline Characteristic & 1993 & 1998 & 2003 & 2008 \\
\hline \hline Number of ANC visits & & & & \\
0 & 12.6 & 10.5 & 6.3 & 3.5 \\
1 & 4.8 & 5.2 & 4.0 & 2.8 \\
2 - 3 & 22.0 & 20.2 & 16.7 & 13.3 \\
at least 4 & 58.9 & 62.3 & 69.4 & 78.2 \\
Don't know/missing & 1.7 & 1.9 & 3.7 & 2.3 \\
& & & & \\
Months pregnant at time of first visit & & & & \\
no ANC & 12.6 & 10.5 & 6.3 & 3.5 \\
$<4$ months & 33.1 & 38.9 & 46.4 & 55.0 \\
4 - 5 months & 35.4 & 35.8 & 34.7 & 31.9 \\
6 - 7 months & 15.0 & 12.4 & 10.7 & 8.0 \\
$8+$ & 2.4 & 1.7 & 1.2 & 1.1 \\
& & & & \\
\hline \hline
\end{tabular}

Source: GDHS Reports from 1988 to 2008

Over the past two decades, the country has moved from a situation where approximately 13 per 100 expectant mothers (12.6\%) to just about 4 per $100(3.5 \%)$ did not attend antenatal care. It is quite encouraging to see a consistent increase in the percentage of women making the recommended number of antenatal care visits of 4 or more since 1993 (see Table 5). What is worrying is the timing of the first visit given that a considerable percentage of women wait until their second or third trimester before making their first ANC visit. Early ANC check up is necessary in order to detect any potential problem so that women are given the appropriate medication before the problems worsen.

\subsection{Delivery care in Ghana}

Access to obstetric services from qualified health professionals is essential in preventing maternal deaths given that labour and delivery are the shortest, but a very critical period of the pregnancy-childbirth process. Despite the importance of ANC visits, it does not guarantee a safe delivery since any delivery is subject to complications. This therefore requires the presence of a skilled attendant in order to ensure a safe delivery. During an International Conference on Population and Development that was held in Cairo in the year 1994, one of the targets that was set to be achieved by the year 2015 was that deliveries assisted by skilled birth attendants should reach 90 percent globally and 60 percent in countries with high maternal death rates including Ghana (Odoi-Agyarko, 2003). The utilisation of 
health care facilities in Ghana is still low with assistance during labour and delivery proving insufficient. Surprisingly, in some regions in the north, unsupervised delivery is the norm (Heyen-Perschon, 2005). Table 6 provides the choice of place of delivery by selected background characteristics.

Generally, Table 6 suggests that older women (35 years and above) are more likely to deliver at home compared to their younger counterparts. On the other hand, women who are less than 20 years are the ones who mostly deliver in health facilities (public or private facilities). Although not surprising, women expecting their first child are almost twice as likely to deliver in health facilities compared to their counterparts with six or more children. One probable reason is that going through their first pregnancy may scare them which may induce them to seek qualified assistance during delivery. This possible explanation is evident in the fact that women expecting children of birth order at least six usually do not deliver in health facilities possibly because of the experience they have gained in their previous deliveries. Again, the results also indicate that unsupervised delivery (delivery at home or delivery without the presence of a health professional) is more of a rural phenomenon given that urban women are more than twice as likely to deliver in health facilities. Education has a direct relationship with delivering in a health facility.

Except for women with middle/JSS level education which recorded a marginal decline in the percentage of facility-based delivery in 1998, all other levels of education recorded consistent improvements with regard to supervised/institutional delivery between 1993 and 2008. Of particular interest is the significant difference in women with no education and their counterparts with at least secondary education. Thus, women with at least secondary education are generally more than three times more likely to deliver in a health facility compared to their counterparts with no formal education. Finally, the surveys reveal that attending at least the minimum required number of ANC visits improves the chances of the woman delivering in a health facility. For instance in 2008, the percentage of women delivering at a facility who did not go for any ANC check-up or made at most three visits and their counterparts who made at least four visits was 54.7 and $33.3 \%$, respectively. In all, deliveries attended by trained health workers have increased over the past three decades. The WHO reports that $59 \%$ of births in Ghana were supervised or attended by skilled personnel in 2011 (WHO, 2011), a 16 percentage points improvement compared to 1998 . 


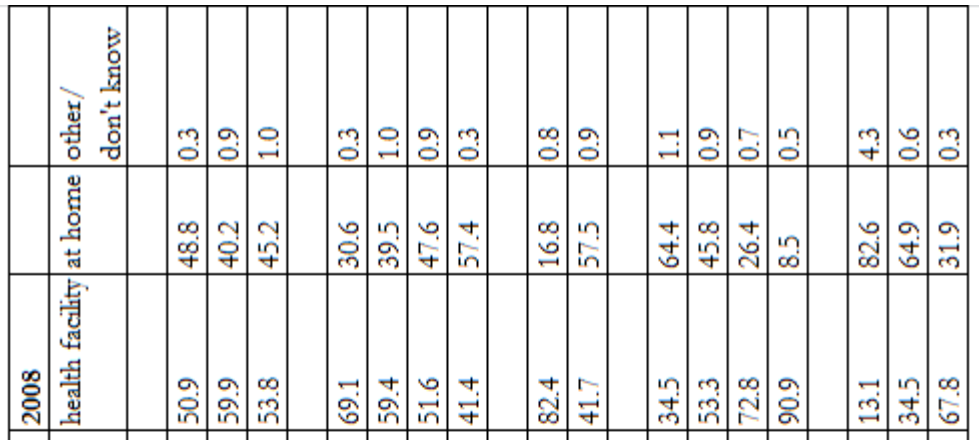

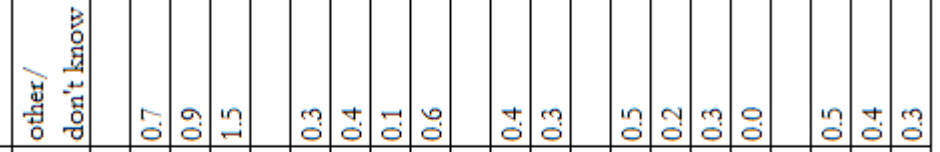

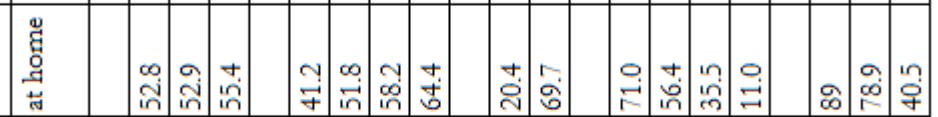

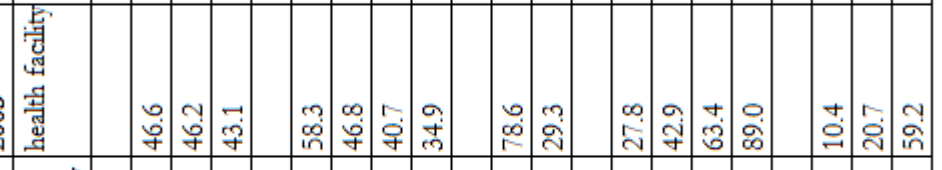

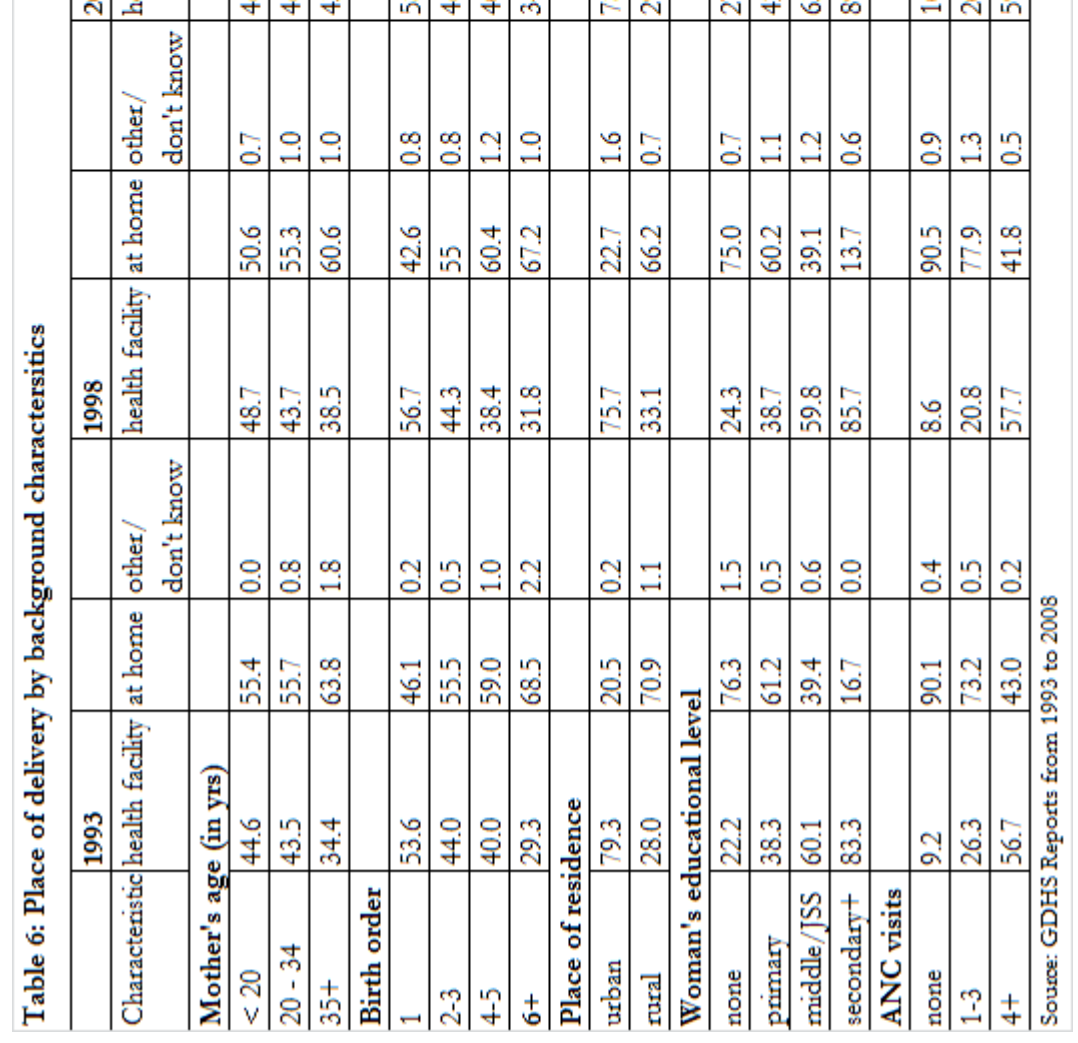




\subsection{Postnatal care in Ghana}

The postnatal period starts immediately after delivery and through the first six weeks of life. This period is very critical for mothers and their newborns in terms of survival. The period is very important for treatment of complications arising from delivery, especially for non-institutional deliveries. According to the 2008 GDHS report, approximately 45 percent of all maternal deaths occur within one day of delivery and about 65 percent of maternal deaths occur within the first week. This means that the most critical condition for Ghanaian women in their reproductive age is the postnatal period. Despite the extent of deaths that occur during this period, early postnatal care (PNC) is the ideal time to help improve the health and survival of both the mother and the newborn child by encouraging preventive behaviours and practices, and to increase the likelihood that potentially life-threatening complications in both mothers and newborns are detected, referred and treated as early as possible (Sines et al., 2007). In Ghana, it is recommended that mothers go for their postnatal check-up within the first three days of delivery and subsequent check-ups depending on their health status (GDHS Report, 2008). Unlike the ANC visits, postnatal check up rates are very low among Ghanaian women as shown in Table 7.

Table 7: Percentage of women who did not receive any postnatal check up by background characteristics

\begin{tabular}{lccc}
\hline Characteristic & $\mathbf{1 9 9 8}$ & $\mathbf{2 0 0 3}$ & $\mathbf{2 0 0 8}$ \\
\hline \hline Woman's age (in years) & & & \\
$<20$ & 44.3 & 47.9 & 28.5 \\
$20-34$ & 48.7 & 52.0 & 21.0 \\
at least 35 & 54.4 & 58.8 & 27.4 \\
& & & \\
Place of Residence & & & \\
urban & 42.3 & 39.4 & 11.8 \\
rural & 50.2 & 55.5 & 30.5 \\
& & & \\
Woman's educational level & & & 36.5 \\
none & 54.4 & 60.5 & 26.9 \\
primary & 47.3 & 48.1 & 13.7 \\
middle/JSS & 41.5 & 44.0 & 3.7 \\
secondary/higher & $(36.8)$ & $*$ & \\
& & & \\
\hline \hline
\end{tabular}

Source: GDHS Reports 1998 to 2008

* means the figure was less than 25 unweighted cases and was therefore suppressed

Even though the data in Table 7 shows that more rural women do not go for PNC check-up, there is still a significant number of women in the urban areas who also 
do not go for postnatal check ups. In the rural areas, the percentage of women who did not go for such checks increased marginally from $50.2 \%$ to $55.5 \%$ between the period 1998 and 2003. However, the 2008 survey recorded a significant fall of about 25 percentage points. A possible reason for the significant decline in the postnatal check up in the rural areas is that most women (especially those who look physically fit after delivery) may see the time spent in receiving the postnatal care as an opportunity cost in terms of the incomes they could have earned if they had worked given that such women are mostly engaged in labour-intensive agricultural activities either directly or indirectly. A worrying issue is the effect of education on the possibility of going for PNC check up. Here, although there is a direct relationship, the differences were not very significant especially for 1998 and 2003.

Table 7 shows that there is still a high percentage of women who do not go for PNC check up regardless of their background characteristics. It is imperative that innovative educational campaigns are used to highlight the benefits of PNC services in order to scale up its utilization.

\subsection{Childhood immunisation}

Immunising children ensures that they are protected against the childhood killer diseases, hence being one of the priority areas of the primary health care programmes. Currently, the government of Ghana has adopted the World Health Organisation (WHO) and UNICEF guidelines for vaccinating children. Therefore, for a child to be considered fully vaccinated, he or she must have BCG at birth or soon after, three doses of DPT and polio (first dose for both when the child is 6 weeks old, second dose when 10 weeks old and the last dose when 14 weeks old). In addition, it is recommended that the vaccine for measles is taken at 9 months old. This means that, by 12 months, a child should have received all vaccinations. Figure 1 presents the trend in the vaccination for all five rounds of the survey.

Generally, there is an improvement in the vaccination coverage in the country as Figure 1 reveals a consistent increase in terms of the percentage of children receiving all the vaccines by age 1 . According to the 2008 GDHS, only 1 percent of children received no vaccination at all. Table 8 however, gives the immunisation coverage by some selected background characteristics. Data beyond 2008 lend further credence to the forward march in immunization coverage in Ghana. The percentage of fully immunized children increased from $87.4 \%$ in 2010 to $88.1 \%$ in 2011, albeit lower than the targeted figure of $89 \%$ (ISSER, 2012). Again the table emphasises the importance of maternal education and place of residence in explaining immunization coverage. Low educational attainment and place of residence partly explain inequities in maternal and child health outcomes (Zere et al., 2012). 


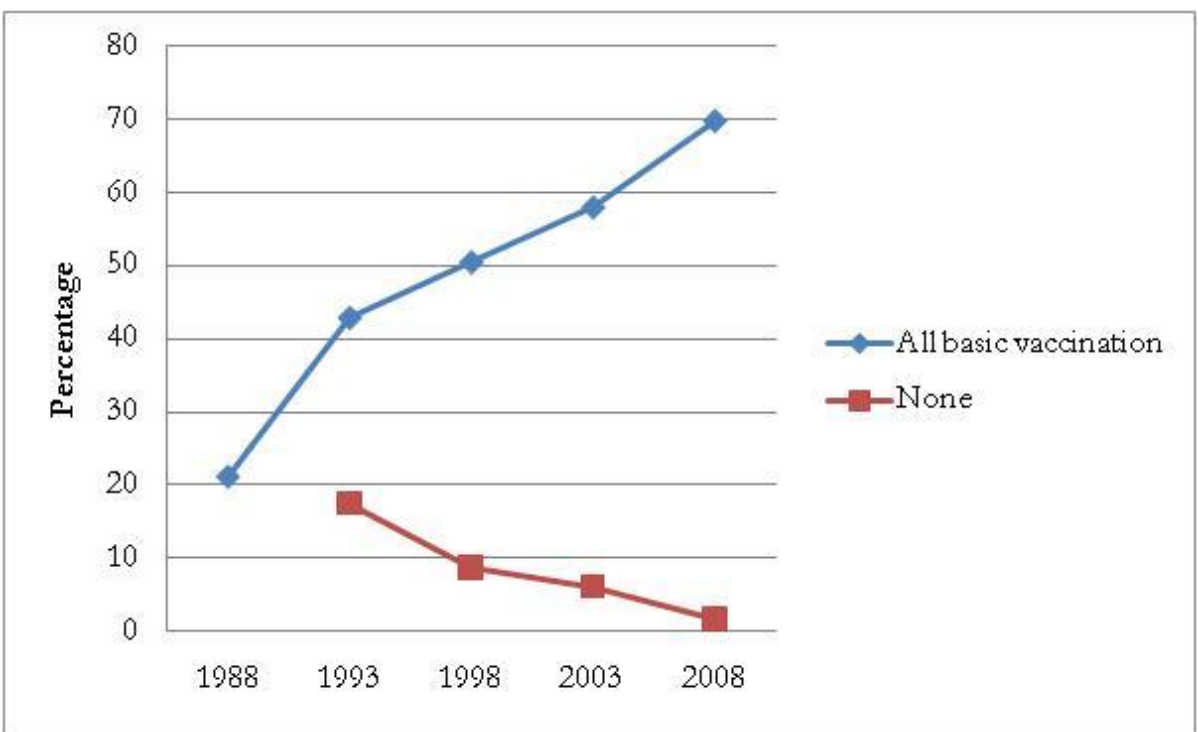

Figure 1: Vaccination by 12 months of age

Source: GDHS Reports from 1988 to 2008

\subsection{Early childhood mortality rates}

Early childhood mortality as used in this study is made up of neonatal mortality ${ }^{2}$, postneonatal mortality ${ }^{3}$, infant mortality ${ }^{4}$, childhood mortality ${ }^{5}$ and under-five mortality ${ }^{6}$. Causes of under-five mortality in Ghana encompass early neonatal conditions, malaria, pneumonia, diarrhoea with HIV and measles contributing less than 10\%. Generally, Ghana has recorded declines in its mortality rates since 1988. Figure 2 shows the trends in these mortality rates and Table 9 further gives the differences in the mortality rates based on some selected background characteristics. The rapid decline in mortality over the past two decades is closely associated with increased immunization coverage, especially against measles. It can be inferred from the table that following the introduction of the EPI programme in 1978, immunization coverage (children fully immunized) has increased from about $20 \%$ in 1988 to a little over $70 \%$ by 2008 . Other factors that have contributed to the decline in under-five mortality include eradication of small pox, the sustained reduction in poverty, increased provision of portable water, promotion of oral rehydration therapy and improvement in medical care with the NHIS playing a major role over the past 7 years.

\footnotetext{
2 Probability of dying within the first month of life.

3 Difference between infant and neonatal mortality.

${ }^{4}$ Probability of dying between birth and exactly age one.

5 Probability of dying between exactly age one and exactly age five.

${ }^{6}$ Probability of dying between birth and exactly age five.
} 


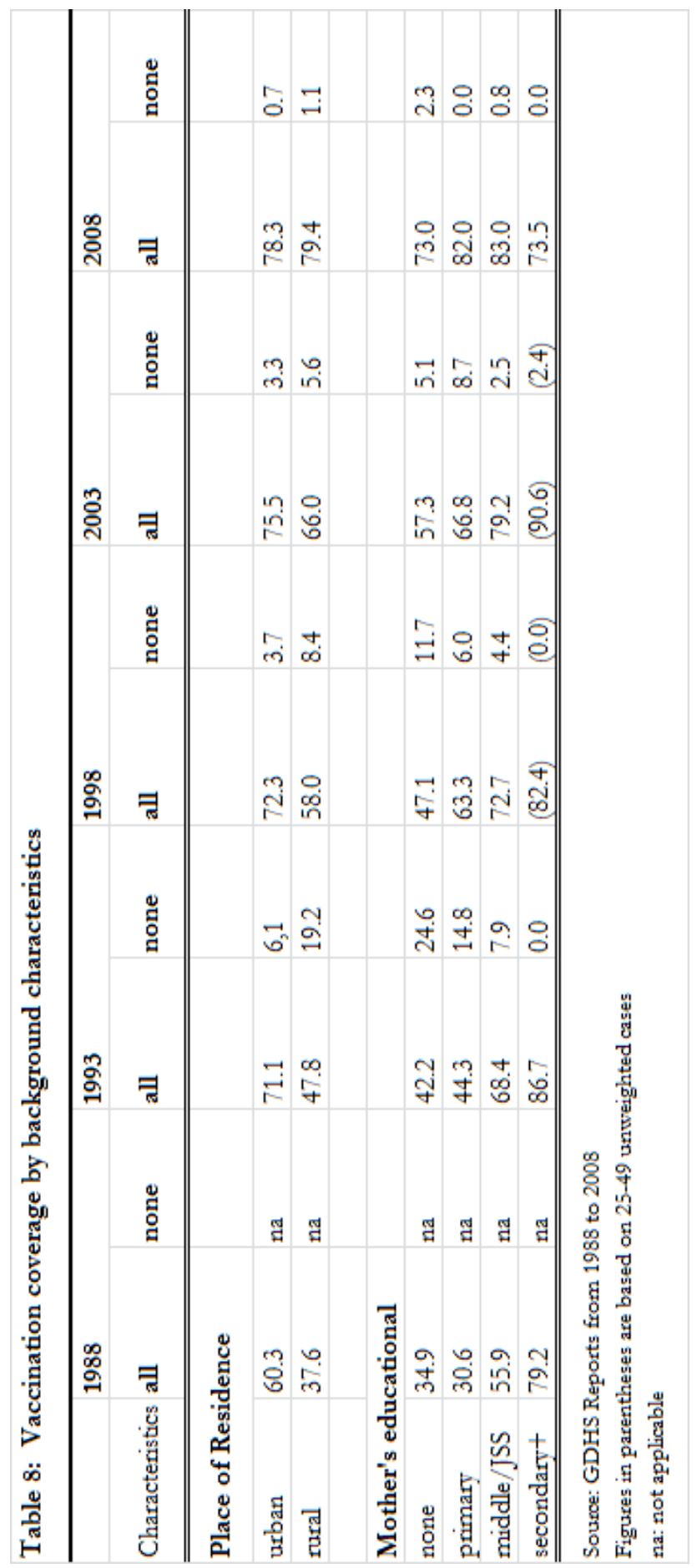




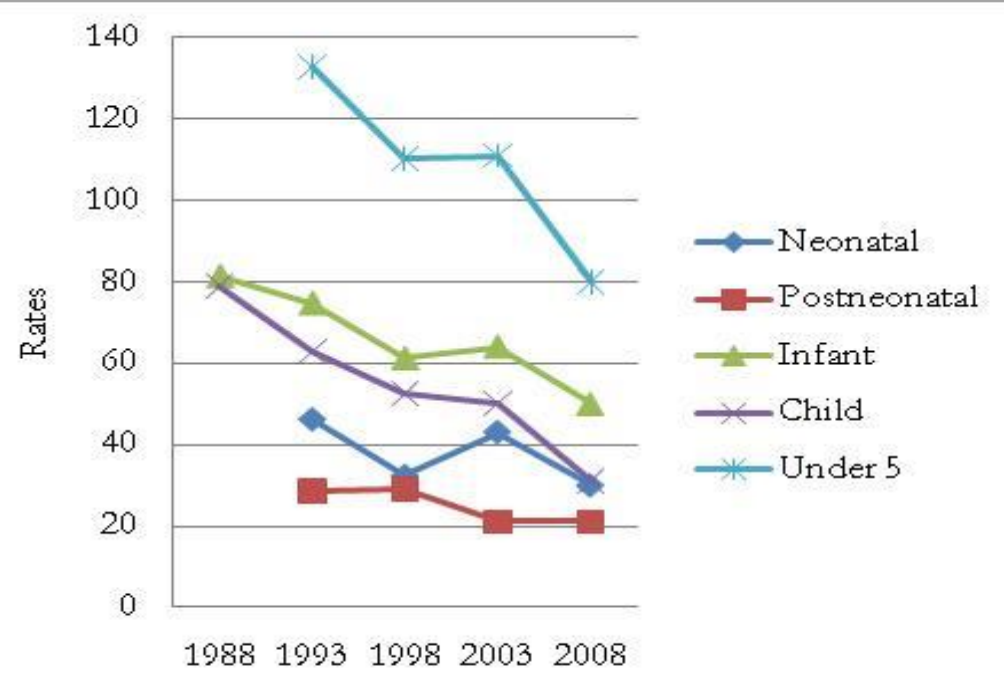

Figure 2: Early childhood mortality rates (per 1,000 live births)

Source: GDHS Reports from 1988 to 2008

Evidence from Figure 2 suggests a consistent decline (except for the period between 1998 and 2003) in almost all the early childhood mortality rates. Specifically, the neonatal, infant and under-five mortalities rose marginally in these periods. Again, the mortality rates appear to be very high in the rural areas and improve with educational attainment of the mother although in 2008, women with primary education rather recorded a lower postneonatal mortality (20.0) compared with their counterparts with middle or junior secondary level of education (23.0). With regards to the age groups, generally, women who are less than 20 years old consistently recorded high mortality rates throughout the five rounds of the survey. A perusal of all the DHS on Ghana shows significant variations by urban-rural residence, region, wealth quintiles and educational status of mothers (GSS, 2008).

\section{Conclusion}

This paper reviews the performance of selected maternal and child health indicators in Ghana drawing heavily from the five Demographic and Health Surveys (1988, 1993, 1998, 2003 and 2008). The review points out that steady progress has been made in improving maternal and child health outcomes over the past two decades. However, the progress is not enough to enable Ghana to achieve the 


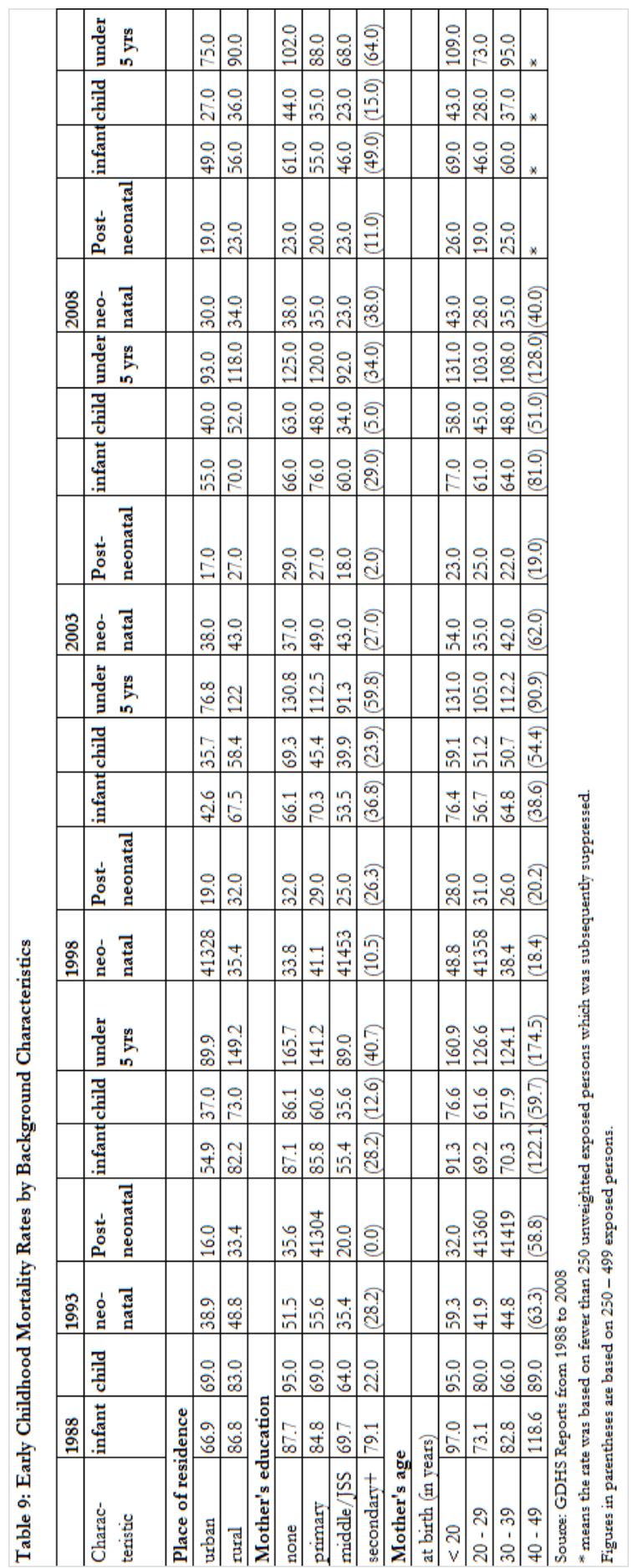


maternal and child-health related MDGs. Hence, sustaining the current progress via increased investment in health and health care coupled with massive health education will boost the uptake of maternal and child health services. With Ghana's economic growth averaging approximately $8 \%$ over the past five years and in particular the record growth of $14.4 \%$ in 2011 which was propelled by oil production and exports, it is envisaged that more resources will be channelled into the health sector in order to achieve improved population health and especially the health of women and children.

\section{References}

AbouZahr, C. (2003). Safe motherhood: a brief history of the global movement 1947 - 2002. British Medical Bulletin, 67:13 - 25.

Ajakaiye, O. and Mwabu, G. (2010). Reproductive health, economic growth and poverty reduction in Africa, Framework of Analysis. University of Nairobi Press.

Ampofo, D. A. (1988). Maternal-child health and family planning in Ghana: Population Impact Project, University of Ghana.

Ghana Health Service (2011). 2010 Annual Report. Ghana Health Service, Accra

Ghana Statistical Service (GSS) and Institute for Resource Development/Macro Systems Inc. (IRD), (1989). Ghana Demographic and Health Survey 1988. Columbia, Maryland USA: GSS and IRD.

Ghana Statistical Service (GSS) and Macro International Inc. (MI), (1994). Ghana Demographic and Health Survey 1993. Calverton, Maryland: GSS and MI.

Ghana Statistical Service (GSS) and Macro International Inc. (MI) (1999). Ghana Demographic and Health Survey 1998. Calverton, Maryland: GSS and MI.

Ghana Statistical Service (GSS), Noguchi Memorial Institute for Medical Research (NMIMR), and ORC Macro. (2004). Ghana Demographic and Health Survey 2003. Calverton, Maryland: GSS, NMIMR, and MI.

Ghana Statistical Service (GSS), Ghana Health Service (GHS), and ICF Macro (2009). Ghana Demographic and Health Survey 2008. Accra, Ghana: GSS, GHS, and ICF Macro.

Ghana Statistical Service (2012). Population and Housing Census. Ghana Statistical Service Accra

Heyen-Perschon, J. (2005). Report on current situation in the health sector of Ghana and possible roles for appropriate transport technology and transport 
related communication interventions. European Section of the Institute for Transportation and Development Policy.

Institute of Statistical, Social and Economic Research (2009). The state of the Ghanaian economy in 2009. ISSER, University of Ghana, Legon

Institute of Statistical, Social and Economic Research (2010). The state of the Ghanaian economy in 2009. ISSER, University of Ghana, Legon

Institute of Statistical, Social and Economic Research (2011). The state of the Ghanaian economy in 2009. ISSER, University of Ghana, Legon

Nyarko, P., Birungi, H., Armar-Klemesu, M., Arhinful, D., Degansu, S., OdoiAgyarko, H., and Brew, G. (2006). Acceptability and feasibility of introducing the WHO focused antenatal care package in Ghana. http://www.Popcouncil.org/pdfs/frontiers/FR_FinalReport/ghana_who_anc .pdf. [Accessed October 10, 2012].

Odoi-Agyarko, H. (2003). Profile of reproductive health situation in Ghana. http://www.who.int/countries/gha/publications/Reproducative_Health_Prof ile.pdf. [Accessed October 17, 2012].

Odwee, J., Okurut, F. and Adebua, A., 2006, The determinants of health care in Uganda: the case study f Lira District, Northern Uganda. Research Paper No. 155. Africa Economic Research Consortium, Nairobi.

Overbosch, G. B., Nsowah-Nuamah, N. N. N., van den Boom. G.J.M., Damnyag L. (2004). Determinants of antenatal care use in Ghana" Journal of African Economies, 13: 277301.

Sines, E., Syed, U., Wall, S. and Worley, H. (2007). Postnatal care: a critical opportunity to save mothers and newborns. Population Reference Bureau. http://www.prb.org/pdf07/SNL_PNCBriefFinal.pdf. [Accessed October 10, 2012].

Thomas, D. and Strauss, J., 1997, Health and wages: evidence on men and women in urban Brazil, Journal of Econometrics, 77:159-85.

WHO (2011). World Health Report. World Health Organization, Geneva.

Zere, E., Kirigia, J.M., Duale, S. and Akazili, J (2012). Inequities in maternal and child health outcomes and interventions in Ghana. BMC Public Health, 12:252. 



\title{
10. Health Financing and its Impact on Maternal and Child Health in Rwanda
}

\author{
Edmond Nkurikiyimana, Administration and Finance, \\ Kabutare District Hospital, Huye District, Rwanda
}

\section{Abstract}

Towards achieving the goal of universal coverage of health services in Rwanda, the health system financing should play a vital role in making the service geographically and financially available. Health-service utilization and consumption depend on their availability on peripheral level where all service seekers could achieve them easily. Financial resources play a key role within the health-care sector as they are needed for health infrastructure development, training of health personnel and also as incentives for all health-care sector partners.

In Rwanda as in other African countries, health care performances depend on a variety of factors, not only the financial ones. They are related to the history and culture as well as to the economic situation such as poverty and employment.

This chapter elaborates the situation of maternal health in Rwanda and how the health-sector financing has an impact on maternal health indicators. It states 
also how the Rwandans have overcome cultural and other barriers to maternal and child health in order to achieve the Millennium Development Goals 4 and 5.

\section{Concept of health financing}

The term health financing represents the provision of financial resources for the health-care sector. The purpose of health financing is to make funding available, as well as to set the right financial incentives for providers, to ensure that all individuals have access to effective public health and personal health care ${ }^{1}$. This means reducing or eliminating the possibility that an individual will be unable to pay for such care, or will be impoverished as a result of trying to do so.

The stability and sustainability of health financing is considered as the main achievement of populations' health goals. And the well arranged health-financing system helps governments to mobilize adequate financial resources, to allocate them equitably, and make an efficient, effective and equitably use ${ }^{2}$.

Health-care financing differs from one country to another because of the differences in macroeconomic and fiscal policy, the burden of diseases, the population and health-care market growth, health-care financial and geographical accessibility. And the function of financing differs from national health-care provision to self payment to health-care seekers. They are categorized in:

- National health service system

- Social health insurance system

- Community-based health insurance

- Direct purchase.

\section{Health financing in Africa and in Rwanda}

Most low-income countries are being severely challenged to provide essential services to their populations and to provide financial protection. Without substantial increases in external assistance, meeting the Millennium Development Goals (MDGs) is highly unlikely.

Most regions will not reach the Millennium Development Goals for health because of slow progress in the 1990s. In Africa, the decline of child mortality of about $0.5 \%$ a year since 1990 needs to be accelerated to a decline of $8 \%$ a year in order to reach the target of halving childhood mortality by 2015 . Neither increased health spending nor growth alone will do the job. Reaching the

\footnotetext{
${ }^{1}$ Innovative Medicine South Africa (2000). Understanding health care financing, NHA background and report, P 5 .

2 World Health Organization (2005). Strategy on healthcare financing for countries of western pacific and south East Asia, WHO library, P 7.
} 
goals requires growth and a multisectoral effort. For example, Rwanda would need a twentyfold increase in public spending on health to achieve the Millennium Development Goals basis on public expenditure alone ${ }^{3}$.

In most countries, internal resources are not sufficient enough to provide comprehensive health-care services to the population. The user fee method of health financing was used in most low-income countries to substitute the inability of governments to allocate adequate funds to their health-care system. To support the user fee method, external financial assistance towards the health system is necessary as a basis for achieving the Millennium Development Goals in health care. Due to the incapacity of health-care seekers who suffer from extreme poverty, the risk pooling was thought in the way to improve the financial accessibility and towards an effective health insurance.

\section{Financing of maternal and child health in Africa and in Rwanda}

The Millennium Development Goals on maternal and child health are lagging far behind target. Although Africa has just $12 \%$ of the global population, it accounts for half of all maternal deaths and half the deaths of children under five 4 .

In Rwanda, the attendance in prenatal clinics is higher than $95 \%$; all of them receive educational and clinical necessaries to give normal birth like iron pills, maternal education, and tetanus vaccination. But when it comes to the time of delivery, only $28 \%$ give birth at health facilities ${ }^{5}$.

The role of traditional birth attendants is crucial for deliveries in Rwanda. This is due to the poor services in health facilities as there is a lack of adequate health material and skilled personnel, especially in remote areas. Another aspect is that culturally, Rwanda's women believe more in their old mum than in the young graduates when it comes to the safety in delivery. The long distance between health facilities and villages also become an obstacle which leads women rather to deliver in their home than in health facilities. Most of our health facilities are far from the community (more than $5 \mathrm{~km}$ ) and the result is that a mother in labor can not walk such distances anymore.

To attain the 4th and 5th Millennium Development Goals, African countries have to adopt strategies on maternal and child health financing. The scarcity of health personnel and health infrastructure are big challenges which our African governments are facing to deliver a proper service that satisfies our customers. Though global maternal deaths are in decline and women's health has lately become a global priority, our goal of reducing maternal mortality by $75 \%$ in 2015 is

\footnotetext{
${ }^{3}$ Gottret P., Schieber G. (2006). Health financing revisited, the World Bank, P 15-18.

${ }^{4}$ Mills S. (2009). Reducing maternal mortality, strengthening the World Bank response, The World Bank, June 2009.

${ }^{5}$ Ministry of Health Rwanda (2006). Maternal, neonatal and child health assessment. March 2006.
} 
still a long way off. Women and children are still dying needlessly. Another risk associated with maternal child death is malaria where in most African countries the deaths associated to malaria are more than $60 \%$ in pregnant women and under five years old children.

The lack of adequate financial resources is a key promoter of all these problems of maternal child health. The low-income countries still have the problem to receive sufficient resources to finance their health sector. Even if according to the Abuja declaration, some governments are trying to allocate $15 \%$ of their budget to the health sector, the needs are more than the resources available. For example Rwanda, where health expenditures are around $16 \%$ of total expenditures, most of the health facilities are still in a situation where the health worker can not offer a competitive quality care to the population due to the lack of key personnel such as physicians, nurses and midwives. The use of available resources is also subjected to non-optimal use and the lack of cost-effective analysis for interventions.

\section{Socio-cultural aspects of maternal and child health in Rwanda}

Maternal health in most African countries is strongly associated with cultural and social aspects of the family. In Rwanda, the reproductive health decisions are mostly made by the husband and a strong pressure is exert to woman to have many children, especially boys in the way to ensure family protection. The lack of prenuptial consultation leads to non-empowering women who can not decide on their future reproductive health. The result of this can be viewed in the increasing number of unwanted pregnancies. Non-empowering women are expected to be more frequently found if educated only in primary school compared to young graduates in university. Even if the government is empowering women, they are still reluctant to change especially when it come to rural poor women who are limited to access to information and therefore to new ideas ${ }^{6}$.

\section{Eradication of cultural barriers in the area of maternal and child health}

The strategy to address maternal and child health issues in Rwanda must go first by eradicating cultural barriers especially the pronatalism concepts such as greeting and congratulating newly married couples with statements like "be fruitful, may you have many sons and daughters", "children are your wealth", "congratulations and give birth to another baby" to the mothers. Another strategy should be the consideration of factors that hold back the use of family planning strategies such as

\footnotetext{
${ }^{6}$ Ministry of Health Rwanda (2003). National reproductive health policy, Kigali, P 15-25.
} 
poverty and accessibility-related health care issues, sociocultural and religious influences, insufficient information, counseling and side effects on using family planning methods, difficulty in using natural method of family planning. This should be associated with eradicating the informal relationship with multiple partners and empowering women in decision making in the household 7 .

\section{Financing maternal and child health in Rwanda}

The scarcity of resources is a major constraint to ensure that all mothers receive the interventions they need in a timely fashion. The demand is affected by financial barriers to care-seeking which interacts with geographical and cultural barriers, and combined with inadequate quality of care within the formal health sector, serve to discourage service use. In addition, substantial inequity exists in maternal mortality rates and coverage of maternal healthcare both within and between low-income regions.

The provision of effective maternal health services requires money for staff, drugs, medical supplies, and food. These costs will differ between health-care facilities and home delivery. Seeking care at a health facility has additional financial implications for the household in terms of "travel costs and patients" and their companion's time, which are subject to seasonal variation.

Faced with these constraints, giving incentives to health-care providers in maternal health aspect and subsidizing these services become a priority of Rwandan government. Besides the implementation of a cost-sharing system in 2007, a performance indicator was established on which health care providers should benefit from "Performance Based Financing (PBF) Incentives".

To seek antenatal care in Rwanda was made free of charge in all public health facilities. And, in addition a PBF scheme was established to insure incentives to health-care providers. Major areas of maternal health concerns that are targeted by PBF interventions include: poor quality of delivery care; low rates of delivery with a skilled attendant or in a health facility; inadequate utilization of antenatal care; high caesarian section rates; low coverage of pregnant women receiving three doses of interpersonal therapy (i.e. counseling and support sessions during pregnancy); low coverage of pregnant women receiving iron supplements at antenatal care; and management of obstetric and neonatal complications.

\footnotetext{
${ }^{7}$ Intrahealth international (2008). Family planning in Rwanda, how a taboo topic become the priority number one, www.intrahealth.org [accessed December 14, 2012]
} 


\section{The effects of health financing on maternal and child health}

\subsection{Effects of expenditure on maternal and child health}

Providing enough resources for health in Rwanda has a good effect on maternal child health in all levels. Increasing health expenditures have made health services accessible financially and geographically. Especially the PBF scheme provides incentives to health workers to deliver quality maternal services as they are remunerated on quality and quantity indicators. The increase in health spending in Rwanda has impacted the high performance in maternal and child health especially in the poorer quintile of the population ${ }^{8}$. The considerable decrease in maternal death and in under five years mortality rates was due to the increase in birth attended by skilled health workers and giving birth at health facilities. The increase in necessary health equipment and material also has reduced the risk of death during delivery.

\subsection{Effects associated to the access of antenatal care and health facility deliveries}

Due to the increase of financing of health in Rwanda, the delivery at health facilities and antenatal care attendance have increased considerably. As the cost of attending the services have become lower in financial terms, the percentage of antenatal care and delivering at health facilities has increased. The statistics show that in 2005 at least $94 \%$ of the pregnant women visited antenatal care services once during the pregnancy. Among them, 24\% delivered in health facilities. Indeed in $2010,98 \%$ of pregnant women visited the antenatal care service three times during the pregnancy and among them $69 \%$ delivered in health facilities 9.

\subsection{Effects on the coverage of maternal health services}

Financial and geographical accessibility of health services plays a key role in the improvement of maternal and child health services. In 2006, the maternal and child health services were delivered in approximately 420 public health facilities where more than $60 \%$ of the targeted population has to walk more than $5 \mathrm{~km}$ to reach the service delivery point. The number of providers was also very low especially in rural areas. The increase in health spending in maternal and child health accounts for a considerable improvement in maternal and child health indicators such as the decrease in under 5 mortality from 127/1,000 lives to 50/1,000 lives in 2012. We

\footnotetext{
${ }^{8}$ Kinoti S. (2011). Effect of performance based financing on maternal health in developing countries. USAID- trac project.

${ }_{9}^{9}$ Abbot P., Rwirarira J. (2012). Against odds: achieving of health MDGs in Rwanda, IPAR- Rwanda.
} 
can also note the increase in the percentage of deliveries with skilled attendant from $31 \%$ in 2000 to $70 \%$ in $2010^{10}$.

\subsection{Effects on services utilization}

The less difficult access to health services has led to higher maternal and child health services utilization rates. The statistics of Democgraphic and Health Survey (DHS) 2011 shows that the use of contraceptives among women at reproductive age is over $45 \%$ in modern method and over $52 \%$ in all methods. And as said above, the increase in deliveries assisted by a skilled attendant shows us that if the services are accessible they are used by beneficiaries.

The free services of antenatal care and vaccination have shown their preference by the Rwandan community where recent statistics show that $98 \%$ of women during pregnancy pass at least once a health facility by seeking antenatal care. And $99.1 \%$ of all newborns are immunized.

These statistics show that if there is a higher financial influx on the health system available and if other arrangements are made to make the health services more accessible, it positively affects the service utilization and thus, the life of the population.

\subsection{Enhancing equity through targeting population}

With these important aspects of financing maternal and child health, another issue derives from the fact that these services are consumed by the poorer and the rich, by educated and non-educated mothers, people living in cities and those from rural remote areas, male and females.

In Rwanda, these characteristics were taken into consideration when planning maternal and child health services and the results are the following: starting by the first point after birth, the coverage of vaccination is still high in all categories, $99.2 \%$ of newborn males and $98.5 \%$ of females, $99.7 \%$ of newborns in the cities against $99.1 \%$ of newborns in rural area. When looking into the subject of mother's education, $98.5 \%$ of the newborns of non-educated mothers are immunized, $99.2 \%$ of newborns of mothers with primary education and $99.5 \%$ of newborns of mothers with secondary and higher education are immunized. These statistics confirm the equity in immunization services ${ }^{11}$. These statistics are similar to the statistics on the treatment of childhood illness and the equitable distribution of child care among the population in Rwanda.

Taking into account the contraceptive utilization in reproductive health, the statistics show nearly the same use in cities and rural areas. In cities, the coverage (use) of contraceptives is $53.1 \%$ and in rural areas $51.4 \%$. A small difference can be

\footnotetext{
${ }^{10} \mathrm{Idem}$

${ }^{11}$ National Institute of Statistics of Rwanda (2011). Demographic and Health Survey 2010.
} 
observed regarding the level of education of the users of contraceptives. Where the portion of none educated is $43.3 \%, 52.6 \%$ of those with primary education and $60.3 \%$ of those with secondary and higher education are using them. When looking on the aspect of gender, males often do not use contraceptive methods as they think that the family planning is the issue of females ${ }^{12}$. The statistics show that the use of contraceptives in Rwanda is still low in all population categories. Even if these services have been made free of charge their use by males is still a challenge to achieve within the goals of family planning.

When looking for those aspects related to seeking antenatal care, the same observation can be made in regards to vaccination where $98.3 \%$ of women living in cities seek these services and $98.0 \%$ of those living in rural areas. And taking in consideration the level of education, $96.4 \%$ of non-educated women seek antenatal care compared to $98.4 \%$ of educated women with primary level education and $98.7 \%$ with secondary and higher education ${ }^{13}$.

And when looking on delivery assisted by a skilled birth attendant, $82.7 \%$ of women living in cities give birth in health facilities, however only $67.2 \%$ of those living in rural areas are accompanied by a skilled health-care worker. And taking into account the level of education, respectively 56.7 of non-educated women, $69.9 \%$ of women educated at primary level and $87.3 \%$ of those with secondary and higher education are assisted by a professional birth attendant ${ }^{14}$. These statistics confirm the diversity of seeking maternal health services among the urban and rural area and the role of education in seeking health services in general.

\subsection{Effects on maternal and child health services and outcomes}

Rwanda has achieved a wide range of targets within the Millennium Development Goals on improving maternal and child health. And even if it is not easy to link these performances to financial inputs; we cannot forget that the financial resources are necessary for any realization. During the decade of 2000-2010, many developments have been observed in maternal and child health. The considerable decrease in infant mortality from 125/1,000 lives in 1994 to 25/1,000 lives in 2010 and the one in maternal mortality from 130/1,000 in 1990 to 48/1,000 in 2010 are the main indicators of progress towards the achievement of MDGs and the improvement of better maternal child health in Rwanda ${ }^{15}$.

\footnotetext{
${ }^{12}$ National Institute of Statistics of Rwanda (2011). Demographic and Health Survey 2010.

${ }^{13}$ National Institute of Statistics of Rwanda (2011). Demographic and Health Survey 2010.

${ }^{14}$ National Institute of Statistics of Rwanda (2011). Demographic and Health Survey 2010.

${ }^{15}$ Abbot P., Rwirarira J. (2012). Against odds: achieving of health MDGs in Rwanda, IPAR- Rwanda
} 


\section{Maternal and Child Health in Sub-Saharan Africa}

Jan Paulick, Faculty of Economic Sciences, Georg-August-University Goettingen, Germany

Sebastian Vollmer, Faculty of Economic Sciences, Georg-August-University Goettingen and Harvard School of Public Health

\section{Introduction}

This chapter gives an overview of the socio-economic literature on maternal and child health in Sub-Saharan Africa. First, we describe the levels and trends of maternal and child health in Sub-Saharan Africa and compare them to other parts of the world. In the second section, we discuss macroeconomic effects on health and the role of health systems. In the third section, we describe the relationship between health, health behavior and their socio-economic determinants at the microeconomic level. In the last section, we conclude.

The United Nations Millennium Development Goals target maternal and child health with a reduction of maternal mortality by three quarters and a reduction of child mortality by two thirds between 1990 and 2015 (UN, 2010). Availability of good data is a necessity in order to evaluate progress towards these goals and to 
conduct research on its determinants. Bryce et al. (2008) find that none of the 68 priority countries have appropriate health systems to gather data on all relevant indicators of maternal and child health. Thus, analyses have to rely on estimates. In many parts of Sub-Saharan Africa progress was insufficient, especially compared to other parts of the world; the relative share of worldwide child deaths has been increasing in the region. It is generally argued that scaling-up proven health interventions and more financial resources are needed to achieve the targets set by the Millennium Development Goals (Bryce et al., 2008).

Not all measures of child health receive the same attention. For example, still births are often not counted and have not been included as a priority in the Millennium Development Goals. They are often associated with stigma, aggravating women's pain, even though they could be averted in many cases. Frøen et al. (2011) document that research and policy programs give greater attention to infant and child mortality. One reason is that still births are more difficult to elicit, as local definitions of still births and miscarriages often differ (Haws et al., 2010).

Over the past four decades, child mortality has been declining worldwide, from 16 million in 1970 to 11.9 million in 1990 and 7.7 million in 2010, according to estimates from Rajaratnam et al. (2010). The authors find that rates of decline were smallest in Sub-Saharan Africa, although the situation improved slightly in more recent years.

Figure 1 shows under-five mortality rates for different world regions, as defined by UNICEF, for the years from 1990 to 2010. Sub-Saharan Africa is divided into Eastern and Southern Africa and West and Central Africa (each shown separately).

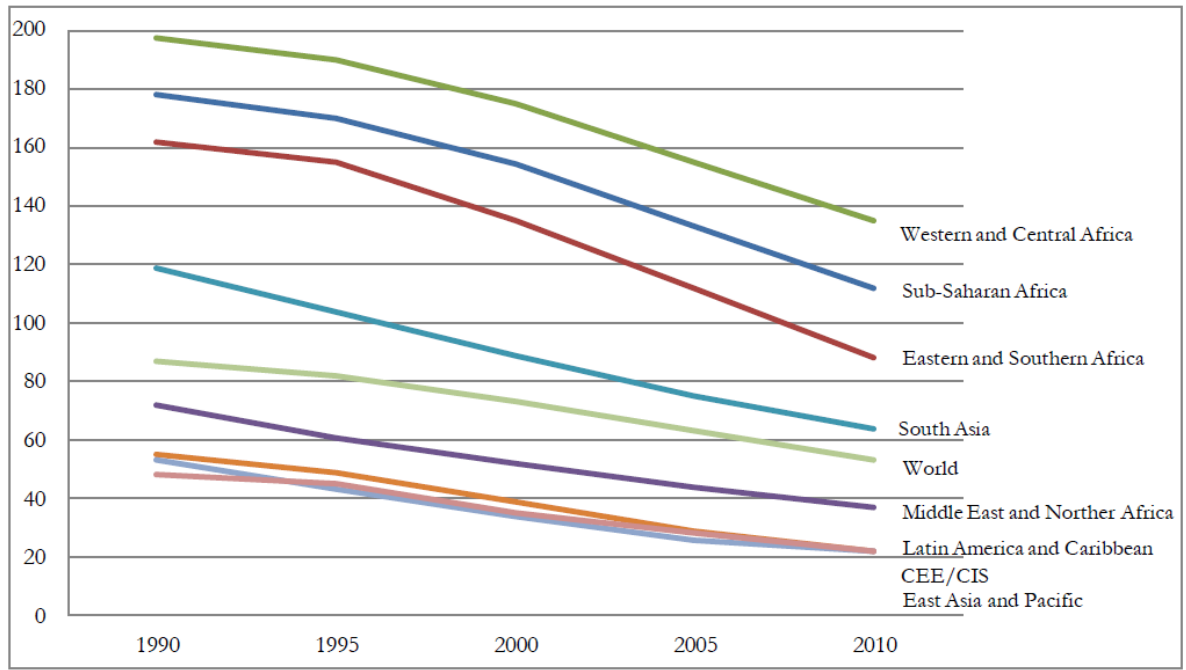

Figure 1: Under-five mortality rate (per 1,000 live births)

Source: WHO (2012), own presentation. 
Child mortality has been declining since 1990 in all world regions, but regions differ substantially in their absolute levels. The lowest levels of under-five mortality, with around 20 deaths per 1,000 live births are found in Central Eastern Europe and the Commonwealth of Independent States (CEE/CIS), Latin American and East Asian countries. Child mortality in Northern African and Middle Eastern countries is almost twice as high with 36 deaths occurring per 1,000 live births. South Asia has a rate of around 60 deaths per 1,000 live births in 2010, which declined from 120 deaths in 1990. The highest child mortality rates are found in SubSaharan African countries. In Western and Central African countries child mortality is more than six times higher than in CEE/CIS countries. In the Eastern and Southern Africa, child mortality is somewhat lower, but still at 88 deaths per 1,000 live births in 2010.

Progress towards the Millennium Development Goals for child mortality in 2015 is labeled insufficient for Sub-Saharan Africa and South Asia. Estimates predict that most countries in Sub-Saharan Africa will miss the target of the Millennium Development Goals for 2015 (Burguet and Soto, 2011).

According to estimates of Liu et al. (2012), the majority of under-five deaths $(64 \%)$ in 2010 occurred due to infectious diseases. Neonatal mortality accounts for $40 \%$ of these deaths with the leading causes being preterm (14\%) and intrapartumrelated complications (9\%). Pneumonia (14\%) diarrhoea (10\%) and malaria $(7 \%)$ account for the majority of deaths among older children. The authors attribute reductions in child mortality from 2000 to 2010 mainly to infectious diseases rather than non-communicable diseases. Figure 2 displays causes of under-five mortality for Sub-Saharan Africa in 2010. Pneumonia and malaria are the leading causes of death. Neonatal deaths, prematurity and birth asphyxia account for $21 \%$ of all deaths. The five most important causes of death account for $64 \%$ of all deaths. Awareness of this mortality pattern can be useful for designing targeted policy interventions at the regional or country level.

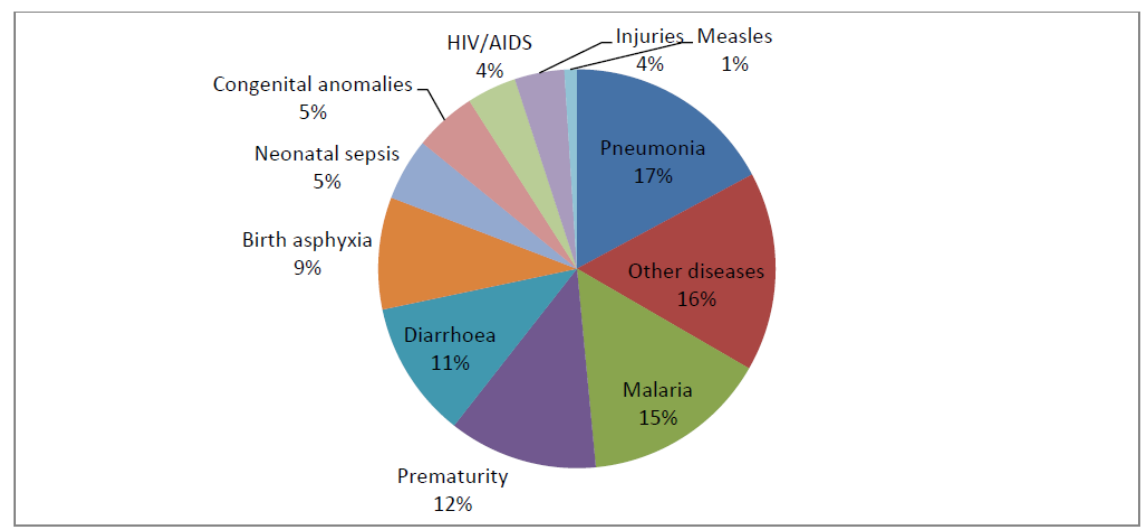

Figure 2: Under-five mortality in Africa by cause (2010)

Source: (IGME, 2012), own presentation. 
A strong downward trend is also visible for maternal mortality (deaths during pregnancy and up to six weeks after delivery). The maternal mortality ratio, i.e. maternal deaths per 100,000 live births, fell from 422 in 1980 to 322 in 1990 and 251 in 2008. However, the share of global maternal deaths in Sub-Saharan Africa increased from $23 \%$ in 1980 to $52 \%$ in 2008, as improvements in maternal mortality in Sub-Saharan Africa did not keep up with population growth and improvements in other regions (Hogan et al., 2010). Figure 3 shows the maternal mortality ratio for different world regions. Sub-Saharan Africa exhibits the highest maternal mortality ratio. Maternal mortality has been declining in all world regions, but regional differences still remain high.

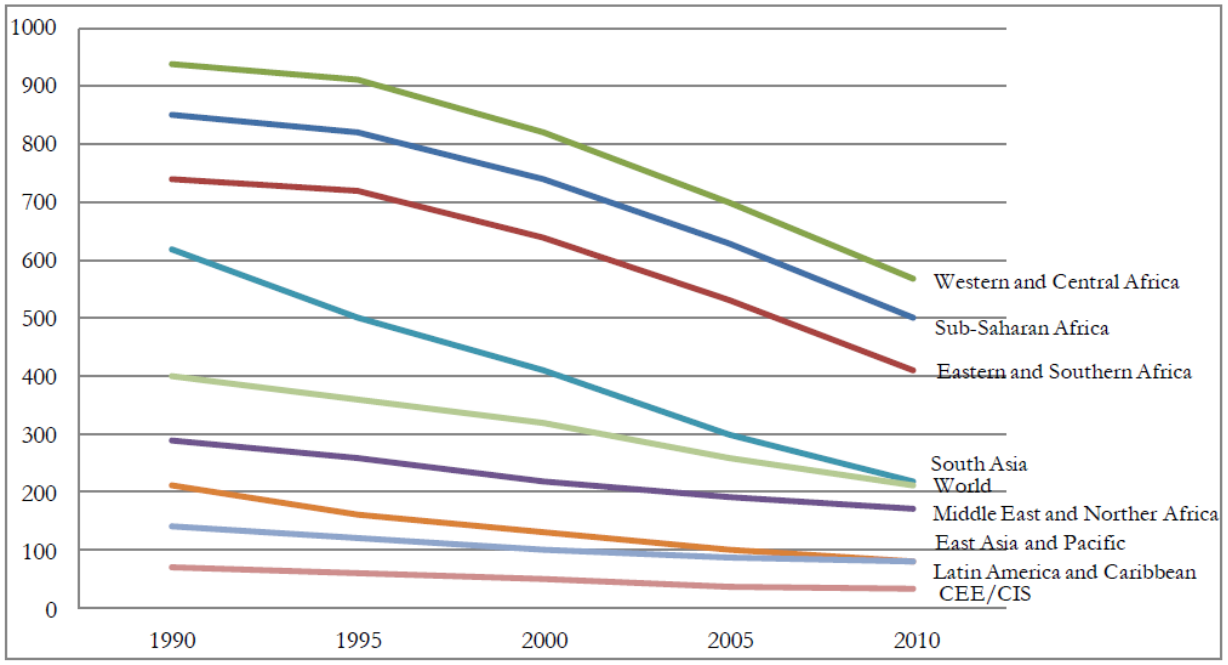

Figure 3: Maternal mortality ratio (maternal deaths per 100,000 live births)

Source: WHO (2012), own presentation.

There are not only high differences between world regions, but differences within Sub-Saharan African countries are also remarkably high. Table 1 summarizes some key health-system and economic indicators for African countries. GDP per capita differs widely with a range of only $\$ 199$ in the Democratic Republic of Congo to more than $\$ 20,000$ in Equatorial Guinea. Expenditures on health range from 3 to $13 \%$ of GDP, reflecting differences in priorities, effectiveness and also absolute levels of GDP. Births attended by skilled professionals are nearly universal in countries like Mauritius and Algeria, but only happen in one out of ten cases in Ethiopia. Cross-country variations can be explained by differences in health care quality, priority setting, country-specific conditions and historic trends. Measures of health care coverage, such as physicians, hospital beds, midwives and nurses per 10,000 people differ widely across countries. Richer countries in terms of GDP per capita tend to have greater health care coverage and tend to do better in terms of health outcomes. However, this pattern is not universal. 


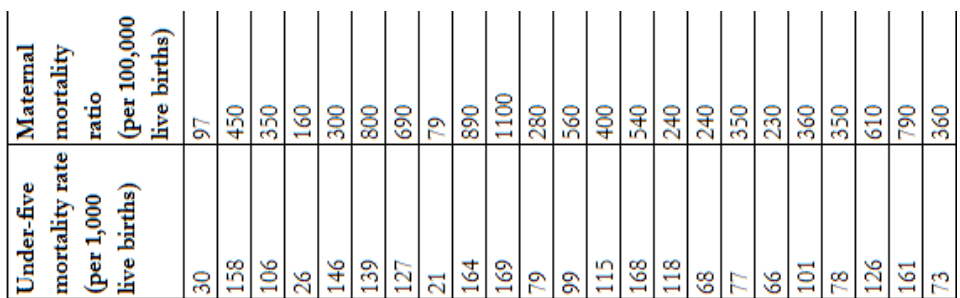

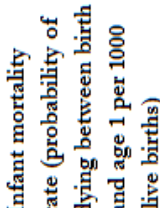

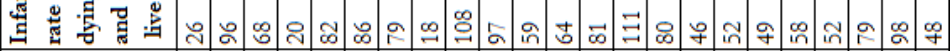

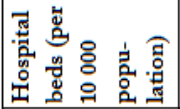

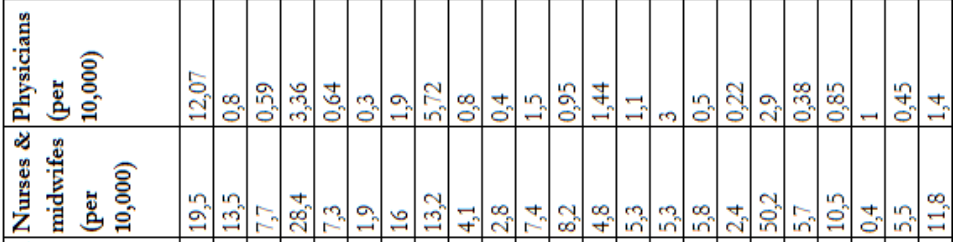

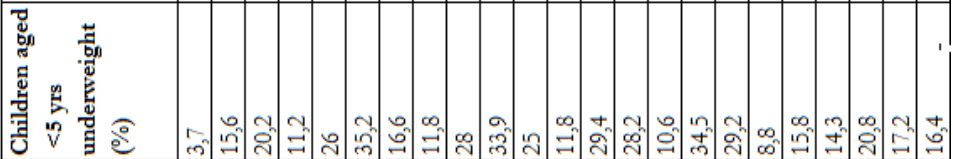

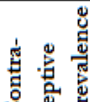

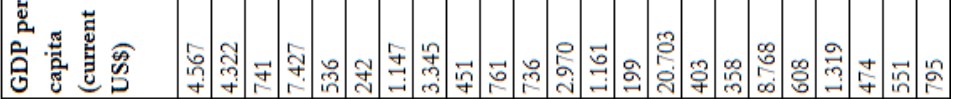

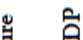
毒专若

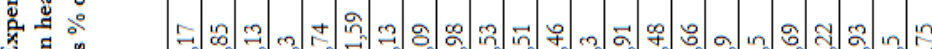

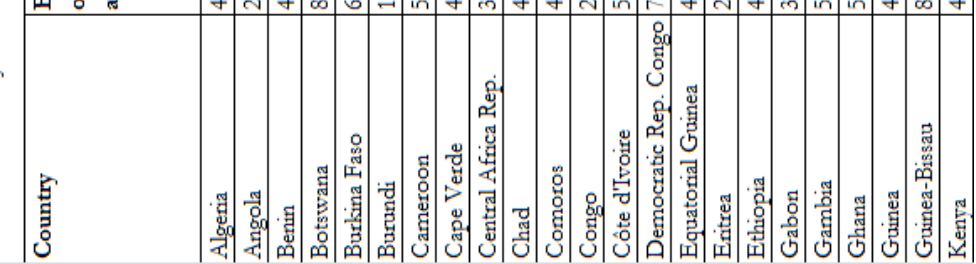



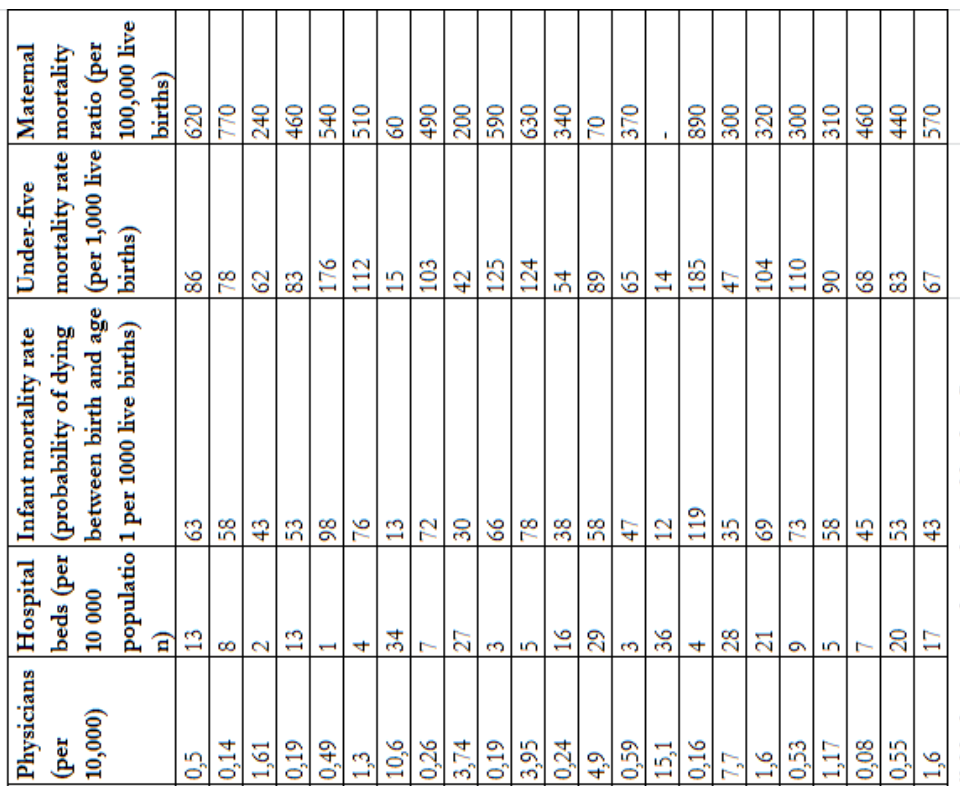

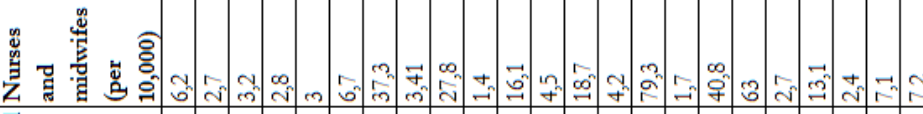

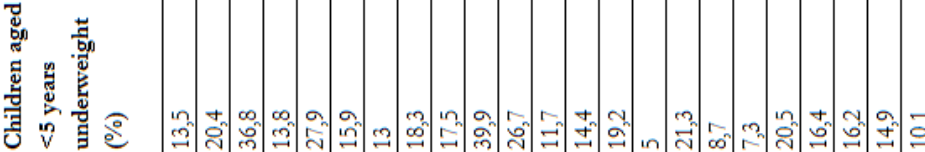

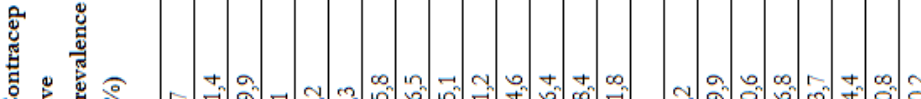
ठ.

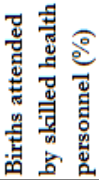

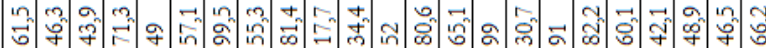
范茑

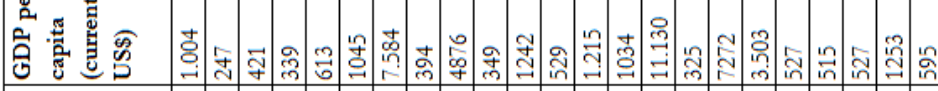

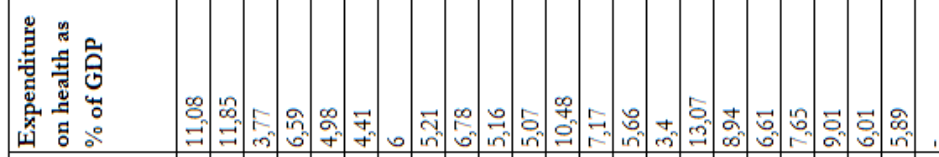


Health is clearly linked to economic prosperity. A causal link is hard to establish, but it seems unlikely that Sub-Saharan Africa faces highest poverty rates and worst health conditions in the world simply by coincidence. In the remainder of the paper we will examine the links between economic indicators and health in greater detail.

\section{Macroeconomic dynamics}

\subsection{Economic development and health}

The wealth and health of nations are correlated (and so are health and income). Infant and child mortality are lower and life expectancy is higher in developed countries. A straightforward explanation for a positive correlation of income and health would be that health improves with higher income due to better health resources, but it may also be the case that good health increases income due to higher productivity, leaving the question of causality wide open. Furthermore, both health and income might be simultaneously affected by other factors (Pritchett and Summers, 1996).

There is also a debate about the magnitude of the effect of income on health. Alsan et al. (2008) argue that income is less important in improving health outcomes than other factors such as technological progress. Increases in GDP do not necessarily improve child health status. Distribution of income matters, as poorer individual's health might not benefit from economic growth (Harttgen et al., 2012). Ickowitz (2012) finds that income cannot explain low child mortality in Ghana relative to its neighbors. She finds that geography and health policy are significant determinants of child mortality, although these variables cannot fully capture the differences between countries either. Alderman et al. (2005) argue that income growth is not enough to reach the Millennium Development Goals in Tanzania.

The question of how health is affected by times of economic turmoil is closely related to the fundamental question about the link between health and economic development. Friedman and Shady (2012) estimate the impact of income fluctuations on infant mortality in 30 countries in Sub-Saharan Africa during the financial crisis of $2008 / 2009$. Their results suggest that 28,000 to 50,000 children died as a consequence of the economic downturn. Girls were more heavily affected than boys with a $1 \%$ reduction in GDP causing excess child mortality of 0.33 per 1,000 live births for boys and 0.61 per 1,000 live births for girls. These results are even more striking as boys are generally more prone to infant mortality. The result only holds for heavy negative economic shocks. During mild recessions boys and girls are affected equally. Cornia et al. (2011) estimate 15,000 excess child deaths occurred as a consequence of the economic crisis in 2008/2009.

Cogneau and Jedwab (2010) study the effects of falling cocoa prices on child well-being by comparing children of cocoa producers with children of farmers in 
other sectors over the period from 1988 to 1993 . They find that school enrollment, child labor, height and morbidity worsened after negative cocoa price shocks. Children of cocoa producers were 3-4\% more ill after cocoa price shocks than the reference group.

Fay et al. (2005) emphasize that GDP per capita is not the only determinant of child health. They identify access to infrastructure as important explanatory variable for the variance between richer and poorer households in child health. They argue that infrastructure is particularly important for child malnutrition and to a lesser degree for child mortality. Moreover, the authors identify female literacy, inequality, urbanization and access to basic health services as important determinants of child health. Ravallion (2007) shows that the effect of infrastructure on child health is not robust to alternative model specifications. Instead, his findings underline the importance of basic health care provision.

Wang (2003) finds that water and sanitation in developing countries are negatively associated with child mortality, but insignificant for infant mortality. For both outcomes, access to electricity is significant. Harttgen et al. (2013) find that a $10 \%$ increase in GDP per capita is associated with $1.5-1.7 \%$ lower odds of being stunted, 2.8-3.0\% lower odds of being underweight, and 3.5-4.0\% lower odds of being wasted for children in sub-Saharan African countries. The education level of the household head and mother, socio-economic status and the mothers' nutritional status are more important determinants of child nutrition. In general, household characteristics appear to play a more important role than aggregate macrolevel variables.

Klasen (2008) finds that standard explanatory variables fail to explain high levels of child undernutrition in South Asia and high levels of child mortality in SubSaharan Africa. He argues that unreliable data, measurement error and concepts of measurement can explain a substantial part of the regional variation in these variables. In particular, the measure of caloric input is rather noisy and should be replaced by a measure of caloric deficiency based on expenditure surveys. Also zscores, which are used to measure weight-for-height standard deviations from a reference population, bear some problems. Using U.S. children as reference standard neglects genetic differences that some argue matter in child development. New efforts have been made in developing a broader reference standard.

Patterns of child mortality within countries differ between rural and urban areas, with child mortality typically being lower in urban areas. Bocquier et al. (2011) argue that empirical research on the determinants of child mortality may be biased if migration between rural and urban areas is not taken into account. They find that child mortality is still higher in rural areas even after adjusting for migration flows. Differences between rural and urban areas tend to disappear after controlling for education, birth intervals, wealth and infrastructure, implying that these factors drive differences between rural and urban regions. In addition, Silal et al. (2012) identify differences in access to health services of households in rural and urban areas of South Africa. 
Related to the literature on wealth and health outcomes is the question of whether development aid improves population health. In theory, aid flows in-crease available resources that can be spent on private or government health projects, essentially working through the same channels as increases in GDP per capita. However, the empirical literature on aid and health is rather inconclusive. Burguet and Soto (2011) for example find no effect of aggregate aid flows on child mortality. The authors suggest distinguishing between aid flows to different areas of the health sector, namely basic health, population policies and reproductive health and food aid and food security programs. Based on the cumulative effects within the three different areas, they project that in the absence of aid, child mortality would be around 2-4\% higher by 2015 .

\subsection{Health systems and public spending}

Whether or not economic wealth translates into better health depends on the effectiveness of the health system in place. The financing of a health system has consequences for equity, efficiency and utilization. Financing of the health system can be direct via out-of-pocket user charges or indirect via pooling through taxes and insurance. Taxes and health insurance typically include some redistributive component from the rich and healthy to the sick and poor while user fees put a higher burden on sick people (Ensor and Ronoh, 2005).

In maternal health care, financing plays an especially critical role. While basic services are more or less predictable, complications during pregnancy or delivery need urgent attention and cannot be anticipated. Direct user charges therefore could have disastrous consequences for poorer households. Households might try to delay treatment as long as possible or avoid it completely. Also incentives for preventive treatment are reduced. The empirical literature confirms that user charges reduce utilization. In contrast, the introduction of social insurance schemes generally leads to increased utilization of antenatal care and delivery at health facilities (Ensor and Ronoh, 2005). Smith and Sulzbach (2008) find that membership in community-based health insurance schemes significantly increases utilization if maternal care is covered and high user fees apply for non-members.

However, user charges also have advantages. Over-utilization does not occur and payments are limited to actual usage, which can be considered fair. However, the need for exempting poor households is generally recognized in the literature. In developing countries user charges for delivery procedures make up a substantial amount of per-capita income. Even in the absence of user charges informal payments and transport costs are a financial burden. For providers, the structure of reimbursements matters. Payments based on performed procedures have been found to increase cesarean sections beyond reasonable rates. At the same time budget allocations can lead to inadequate service provision due to a lack of incentives (Ensor and Ronoh, 2005). The optimal design of a health system also needs to take preferences in society into account. Ternent et al. (2010) evaluate willing- 
ness to pay for improvements in maternal health in Burkina Faso using a hypothetical bidding game. They find men are willing to pay more in absolute terms, but women are willing to pay a higher percentage of their income $(11 \%$ versus $4 \%$ for men). Prior maternal complication in the household had a positive effect on willingness to pay.

Filmer and Pritchett (1999) find that public spending, both related and unrelated to health care, does have little impact on infant and child mortality. They find other factors such as income, income distribution, female education and religion are better able to explain differences between countries. Public spending is thus not per se health improving. The authors hypothesize that their finding is due to the fact that effectiveness of spending differs among countries and health systems.

In a case study of health systems in four countries (including Uganda and South Africa), Parkhurst et al. (2005) find that skilled assistance at birth, the mix of private and public service providers and reforms in the health system are the most important determinants of maternal health. While skilled attendance is an important factor, it does not necessarily reflect timeliness or quality of treatment. The role of the private sector is diverse and either supplements or substitutes public health care. Private care ranges from modern (and also more expensive) services to traditional care. Private health care can be detrimental to the quality in the public sector, for example through higher wages that make the public sector less attractive for high-skilled health professionals.

Qualified staff is critical for the provision of health services. Fako et al. (2004) highlight the importance of training for midwives in reducing maternal mortality. Gerein et al. (2006) point to a shortage of qualified staff for maternal care in SubSaharan Africa. The problem is most severe in rural areas. They identify outmigration of qualified staff and high HIV/AIDS prevalence as main causes of shortages. Additionally, qualified staff seeks employment in the private sector and at international organizations. Staff shortage does not only reduce availability of services and prolong waiting times, it also increases the workload of the remaining staff and thus deteriorates their work performance and morale.

There is a rich literature on specific components of health systems and smallscale health interventions. The external validity of these studies has to be evaluated on a case-by-case basis, nevertheless, one might be able to learn from specific health systems and health interventions in improving other health systems.

For example, Ahmed et al. (2012) estimate that contraceptive use averted roughly 270 thousand maternal deaths in 2008. Meeting the unmet need for contraceptives could lower maternal deaths by another hundred thousand worldwide. $33 \%$ of all maternal deaths in Sub-Saharan Africa could be averted this way. Unmet need for contraceptives affects maternal mortality through unwanted pregnancies, unsafe abortions and high frequency of pregnancies (Ahmed et al., 2012).

Kasirye (2010) finds positive effects of vaccinations on children's nutritional status in East African countries. However, the introduction of national programs, cash transfers and extension of secondary schooling are difficult to accomplish due 
to resource constraints. Evaluating the success of interventions in Millennium Development Villages, Pronyk et al. (2012) find a reduction of under-five mortality by $22 \%$ after three years. They speculate that the reduction is the result of interventions in immunization, distribution of bed nets, investments in infrastructure, agriculture, nutrition and access to health care.

\subsection{Conflict and health}

Conflict is a particularly important topic in Sub-Saharan Africa, because almost three out of four countries in the region were affected by conflict over the last three decades (Verwimp et al., 2010). In 2011, there were 14 state-based armed conflicts in African countries (Themnér and Wallensteen, 2012).

Armed conflict reduces a nation's productive resources and wealth. Direct effects on health include casualties, injuries and malnourishment. Exposure to conflict also has indirect effects on health outcomes, such as economic stress, healthrelated stress, forced migration and detrimental impact on the functioning of public health systems (Minoiu and Shemyakina, 2012; Agadjanian and Prata, 2003). Even after the end of conflict, recovery to pre-conflict levels might be slow (Chandrasekhar et al., 2011).

Child health is of particular concern, because improvements in quality of life at older ages cannot fully compensate for deprivations in early childhood. Children affected by conflict tend to do worse at school and earn lower wages in adulthood (Verwimp et al. 2010).

Minoiu and Shemyakina (2012) use a differences-in-differences approach to investigate the effects of armed conflict on regions in Côte d'Ivoire that were tormented by war. Children who were born during conflict in exposed areas had a half standard deviation lower z-score for height-for-age than children in the control group. This finding underlines the importance of conditions in early childhood for long term development.

Similarly, Agadjanian and Prata (2003) find that children in regions that were severely affected by civil war in Angola were more likely to be malnourished and less likely to have received immunizations. The control group includes children that were conceived after the conflict ended temporarily in 1994.

In a later study, Avogo and Agadjanian (2010) investigate the impacts of forced migration in Angola's capital with a dataset from 2004, two years after peace was restored. The authors distinguish between groups that migrated because of immediate effects of war and groups that migrated for other reasons. The authors find that children are at higher risk in terms of survival when parents migrate for reasons related to war. In the long term no differences were found in health seeking behavior, illness and immunization compared to the non-migrant group.

Verwimp et al. (2010) observe lower height-for-age scores among children, who live in areas exposed to civil war in Burundi. The scores were lower the longer violence affected the area. The authors identify displacement and loss of crops as 
the main channels through which conflict deteriorated child health. Children exposed to these hardships were more likely to be malnourished and more prone to infectious diseases. In Rwanda, crop-failure and civil conflict emerged simultaneously. Here, the authors find a negative impact of conflict on child health; cropfailure however, only had a significant impact on girls.

\section{Microeconomic dynamics}

\subsection{Health behavior and utilization}

Health behavior and utilization are important determinants of individual health outcomes. Preventive care and treatment-seeking behavior are of particular importance. Treatment-seeking behavior, i.e. the demand for health services, is affected by supply and perceived quality of care. Onah et al. (2006) identify characteristics of places where women choose to deliver in a case study of Enugu, Nigeria. Among others, presence of specialists, availability of equipment, promptness of treatment, and affordability were frequently reported by women, who delivered three months prior to the survey. Kruk et al. (2010) find that positive communitylevel perception of medical staff's skills, quality of care and negative views of traditional birth attendants increase the utilization of health facilities. Gage (2007) shows that distance is an important barrier to utilization of maternal health services at health facilities.

Individuals' decisions to utilize health facilities rely on a variety of determinants. Gyimah et al. (2006) discover higher utilization among Christians compared to Muslims and traditional women, controlling for socio-economic characteristics. Brazier et al. (2009) evaluate an intervention in Burkina Faso, which promoted maternal care at primary health facilities and improved access and quality of care. In addition to an increase in utilization of about $30 \%$, they also find that differences in utilization stemming from wealth differences have declined.

McTavish et al. (2010) study the determinants of maternal health care utilization at the individual and national level. Within countries maternal care utilization is determined by income, education, age, rural or urban residence and marital status of women. In countries with low female literacy rates poor households are less likely to use maternal health services than in countries with high female literacy rates. Magadi et al. (2007) find lower utilization of maternal care services for teenage mothers. Ahmed and Mosley (2002) document a correlation between the use of maternal health services and contraceptives in six developing countries. One explanation could be that experience with one health activity reduces perceived barriers for the other. For intervention strategies this suggests simultaneous efforts could improve results.

Behavioral patterns influence the effectiveness of health programs and interventions. Yarnoff (2011) studies the effect of vitamin A supplementation on child 
mortality with an explicit focus on behavioral responses. Better educated mothers more often complemented vitamin A supplementation with investments in malaria prevention. Information may also change treatment-seeking behavior. Agee (2010) studies the role of information on child nutrition in Nigeria. The percentage of mothers reporting problems with information on health care in a community is negatively correlated with child nutrition. Information problems also reduce the positive effects of wealth on child nutrition.

The effectiveness of information depends on which members of a household are targeted and what information is provided. Information is better processed by better educated individuals (Dupas, 2011). Aubel et al. (2004) identify grandmothers as an influential group within communities of Western Senegal. Targeting grandmothers with nutritional education proves more effective for improving nutritional behavior of younger women than targeting women of reproductive age directly. The authors argue that health campaigns should adapt to local cultural context.

\subsection{The role of individual socio-economic characteristics}

Education can promote healthy behavior, while wealthier households can afford better food, sanitation, and better health care. Higher education generally transfers into higher income and wealth. It is thus difficult to disentangle the effects and to assess relative importance (Heaton et al., 2005). Health expenditures make up a relatively large share of household expenditures in low-income countries and are arguably an aggravating factor in causing poverty. Only little resources are spent on preventive care, resulting from financial constraints but also from low supply (Dupas, 2011).

Fuchs et al. (2010) try to disentangle the effects of income and education on child health. The authors argue that education is more substantial in reducing infant mortality in developing countries than wealth. However, it is questionable whether the data really support such a strong claim. Similarly, Heaton et al. (2005) find education and socio-economic status to have positive effects on child health outcomes with about equally strong effects across developing countries. Gakidou et al. (2010) argue that half of the 8.2-million-reduction in child deaths from 1970 to 2009 can be attributed to increased educational attainment.

Desai and Alva (1998) examine the effect of maternal education on child mortality, nutrition and immunization status in 22 countries. After controlling for infrastructure, place of residence, marital status and husband's education, maternal education is significant for child immunization status in half of the countries but only in a few countries for the other health outcomes. The authors conclude that maternal education is more important for health-seeking behavior than for actual health outcomes.

Burchi (2010) links nutritional knowledge and education of mothers in Mozambique. Primary education improves child nutrition, but higher education 
does not make an additional difference. However, the effect of higher education is partly picked up by household wealth, which has a profound positive influence on child health in the study. Knowledge on nutrition also increases children's heightfor-age.

Parents' health status is also a strong predictor of child health. This observation might be partly due to the fact that healthier parents also tend to be wealthier and better educated. However, there are also reasons to believe that parent's health has direct effects on child health, as child care depends on healthy parents. Orphans whose parent(s) died of HIV/AIDS attend school less frequently (Mather, 2011). Kidman et al. (2010) find that children suffer more often from illness and injury if their parents are HIV-positive.

\section{Conclusions}

We have discussed the socio-economic literature on maternal and child health in Sub-Saharan Africa. Indicators of maternal and child health have been improving over time, but progress has been rather slow, even in countries with high growth rates. It is questionable whether Sub-Saharan African countries will reach the Millennium Development Goals on improving maternal and child health. Financial constraints in low-income countries make it difficult to formulate clear policy recommendations.

There is evidence that reductions in child mortality have benefited wealthier households more than poorer households. Improvements have also been less pronounced in rural areas where poorer households are more concentrated (Wang, 2003). Even with average mortality rates declining over the last decades, households at extreme ends of the income and wealth distribution deserve attention.

The literature suggests that health outcomes depend on a breadth of factors that are interlinked in a complex way. Despite the breadth and richness of the literature, we have a rather limited understanding of the relative importance of various causal effects of socio-economic characteristcis on maternal and child health. It will be a challenge for future research to add further evidence to this literature and to translate this evidence into policy action. 


\section{References}

Agadjanian, V. and Prata, N. (2003). Civil war and child health: regional and ethnic dimensions of child immunization and malnutrition in Angola. Social Science \& Medicine, 56: 2515-2527.

Agee, M. (2010). Reducing child malnutrition in Nigeria: Combined effects of income growth and provision of information about mothers' access to health care services. Social Science \& Medicine, 71: 1973-1980.

Ahmed, S. and Mosley, W. (2002). Simultaneity in the use of maternal-child health care and contraceptives: evidence from developing countries. Demography, 39: 75-93.

Ahmed, S., Li, Q., Liu, L., Tsui, A. (2012). Maternal deaths averted by contraceptive use: an analysis of 172 countries. Lancet, 380: 111-125.

Alderman, H., Hoogeveen, H., Rossi, M. (2005). Reducing child malnutrition in Tanzania - combined effects of income growth and program interventions. World Bank Policy Research Working Paper 3567.

Allegri, M., Ridde, V., Louis, V., Sarker, M., Tiendrebéogo, J., Yé, M., et al. (2011). Determinants of utilisation of maternal care services after the reduction of user fees: A case study from rural Burkina Faso. Health Policy, 99: 210-218.

Alsan, M., Bloom, D. E., Canning, D., Jamison, D. (2008). The consequences of population health for economic performance. In S. Bennett, L. Gilson, \& A. Mills, Health, economic development and household poverty (pp. 21-39). New York: Routledge International Studies in Health Economics.

Aubel, J., Touré, I., Diagne, M. (2004). Senegalese grandmothers promote improved maternal and child nutrition practices: the guardians of tradition are not averse to change. Social Science \& Medicine, 59: 945-959.

Avogo, W. and Agadjanian, V. (2010). Forced migration and child health and mortality in Angola. Social Science \& Medicine, 70: 53-60.

Bocquier, P., Madise, N., Zulu, E. (2011). Is there an urban advantage in child survival in Sub-Saharan Africa? Evidence from 18 countries in the 1990s. Demography, 48: 531-558.

Brazier, E., Andrzejewski, C., Perkins, M., Themmen, E., Knight, R., Bassane, B. (2009). Improving poor women's access to maternity care: Findings from a primary care intervention in Burkina Faso. Social Science \& Medicine, 69: 682690.

Bryce, J., Daelmans, B., Dwivedi, A., Fauveau, V., Lawn, J., Mason, E., et al. (2008). Countdown to 2015 for maternal, newborn, and child survival: the 2008 report on tracking coverage of interventions. Lancet, 371: 1247-1258. 
Burchi, F. (2010). Child nutrition in Mozambique in 2003: The role of mother's schooling and nutrition knowledge. Economics \& Human Biology, 3: 331-345.

Burguet, R. and Soto, M. (2011). Seeds of hope: Assessing the effect of development aid on the reduction of child mortality. Barcelona GSE Working Paper Series, Working Paper No. 591.

Chandrasekhar, S., Gebreselassie, T., Jayaraman, A. (2011). Maternal health care seeking behavior in a post-conflict HIPC: The Case of Rwanda. Population Research and Policy Review, 30: 25-41.

Cogneau, D. and Jedwab, R. (2010). Commodity price shocks and child outcomesThe 1990 cocoa crisis in Côte d'Ivoire. CEPREMAP Working Papers 1018.

Cornia, G., Rosignoli, S. and Tiberti, L. (2011). The impact of the food and financial crisis on child mortality: The case of Sub-Saharan Africa. Innocenti Working Paper 2011-01.

Desai, S. and Alva, S. (1998). Maternal education and child health: Is there a strong causal relationship? Demography, 35: 71-81.

Dupas, P. (2011). Health behavior in developing countries. Annual Review of Economics, 3: 425-449.

Ensor, T. and Ronoh, J. (2005). Effective financing of maternal health services: A review of the literature. Health Policy, 75: 49-58.

Fako, T., Forcheh, N., Ncube, E. (2004). Prospects of safe motherhood in Botswana: midwifery training and nurses' ability to complete the Botswana obstetric record. Social Science \& Medicine, 58: 1109-1120.

Fay, M., Leipziger, D., Wodon, Q., Yepes, T. (2005). Achieving child-healthrelated Millennium Development Goals: The role of infrastructure. World Development, 33: 1267-1284.

Filmer, D. and Pritchett, L. (1999). The impact of public spending on health: does money matter? Social Science \& Medicine, 49: 1309-1323.

Fink, G., Günther, I., Hill, K. (2011). The effect of water and sanitation on child health: evidence from the demographic and health surveys 1986-2007. International Journal of Epidemiology, 40: 1196-1204.

Friedman, J. and Shady, N. (2012). How many infants likely died in Africa as a result of the 2008-2009 global financial crisis? Health Economics, forthcoming.

Frøen, J. F., Cacciatore, J., McClure, E. M., Kuti, O., Jokhio, A. H., Islam, M., et al. (2011). Stillbirths: why they matter. Lancet, 377: 1353-1366. 
Fuchs, R., Pamuk, E., Lutz, W. (2010). Education or wealth: which matters more for reducing child mortality in developing countries? Vienna Yearbook of Population Research, 8: 175-199.

Gage, A. (2007). Barriers to the utilization of maternal health care in rural Mali. Social Science \& Medicine, 65: 1666-1682.

Gakidou, E., Cowling, K., Lozano, R., Murray, C. (2010). Increased educational attainment and its effect on child mortality in 175 countries between 1970 and 2009. Lancet, 376: 959-974.

Gerein, N., Green, A., Pearson, S. (2006). The implications of shortages of health professionals for maternal health in Sub-Saharan Africa. Reproductive Health Matters, 14: 40-50.

Gyimah, S., Takyi, B., Addai, I. (2006). Challenges to the reproductive-health needs of African women: On religion and maternal health utilization in Ghana. Science \& Medicine, 62: 2930-2944.

Harttgen, K., Klasen, S., Vollmer, S. (2013). Economic growth and child undernutrition in sub-Saharan Africa. Population and Development Review, 39: 397-412.

Haws, R., Mashasi, I., Mrisho, M., Armstrong-Schellenberg, J. (2010). “These are not good things for other people to know": How rural Tanzanian women's experiences of pregnancy loss and early neonatal death may impact survey data quality. Social Science \& Medicine, 71: 1764-1772.

Heaton, T., Forste, R., Hoffmann, J., Flake, D. (2005). Cross-national variation in family influences on child health. Social Science \& Medicine, 60: 97-108.

Hogan, M., Foreman, K., Naghavi, M., Ahn, S., Wang, M., Makela, S., et al. (2010). Maternal mortality for 181 countries, 1980-2008. Lancet, 375: 1609-1623.

Ickowitz, A. (2012). Wealthiest is not always healthiest: what explains differences in child mortality in West Africa? Journal of African Economies, 21: 192-227.

IGME. (2012). Levels and trends in child mortality: Report 2012, UNICEF, New York, 2012. New York, Geneva and Washington, DC: The UN Interagency Group for Child Mortality Estimation.

Kasirye, I. (2010). What are the successful strategies for reducing malnutrition among young children in East Africa? Human Development Research Paper $2010 / 15$.

Kidman, R., Hanley, J., Subramanian, S., Foster, G., Heymann, J. (2010). AIDS in the family and community: The impact on child health in Malawi. Social Science \& Medicine, 71: 966-974. 
Klasen, S. (2008). Poverty, undernutrition, and child mortality: Some inter-regional puzzles and their implications for research and policy. Journal of Economic Inequality, 6: 89-115.

Kruk, M., Rockers, P., Mbaruku, G., Paczkowski, M., Galea, S. (2010). Community and health system factors associated with facility delivery in rural Tanzania: A multilevel analysis. Health Policy, 97: 209-216.

Liu, L., Johnson, H., Cousens, S., Perin, J., Scott, S., Lawn, J., et al. (2012). Global, regional, and national causes of child mortality: an updated systematic analysis for 2010 with time trends. Lancet, 379: 2151-2161.

Magadi, M., Agwanda, A., Obare, F. (2007). A comparative analysis of the use of maternal health services between teenagers and older mothers in sub-Saharan Africa: evidence from Demographic and Health Surveys (DHS). Social Science \& Medicine, 64: 1311-1325.

Mather, D. (2011). Poverty, AIDS, orphanhood, gender and child schooling in sub-Saharan Africa: A review of the evidence. MSU International Development Working Paper 116. .

Mc'Tavish, S., Moore, S., Harper, S., Lynch, J. (2010). National female literacy, individual socio-economic status, and maternal health care use in sub-Saharan Africa. Social Science \& Medicine, 71: 1958-1963.

Minoiu, C. and Shemyakina, O. (2012). Child health and conflict in Côte d'Ivoire. American Economic Review, 102: 294-299.

Onah, H., Ikeako, L., Iloabachie, G. (2006). Factors associated with the use of maternity services in Enugu, southeastern Nigeria. Social Science \& Medicine, 63: $1870-1878$.

Parkhurst, J., Penn-Kekana, L., Blaauw, D., Balabanova, D., Danishevski, K., Rahman, S., et al. (2005). Health systems factors influencing maternal health services: a four-country comparison. Health Policy, 73: 127-138.

Pritchett, L. and Summers, L. (1996). Wealthier is healthier. Journal of Human Resources, 31: 841-868.

Pronyk, P., Muniz, M., Nemser, B., Somers, M.-A., McClellan, L., Palm, C., et al. (2012). The effect of an integrated multisector model for achieving the Millennium Development Goals and improving child survival in rural subSaharan Africa: a non-randomised controlled assessment. Lancet, 379: 21792188.

Rajaratnam, J., Marcus, J., Flaxman, A., Wang, ,. H., Levin-Rector, A., Dwyer, L., et al. (2010). Neonatal, postneonatal, childhood, and under-5 mortality. Lancet, 375: 1988-2008. 
Ravallion, M. (2007). Achieving child-health-related Millennium Development Goals: The role of infrastructure - A comment. World Development, 35: 920 928.

Silal, S., Penn-Kekana, L., Harris, B., Birch, S., McIntyre, D. (2012). Exploring inequalities in access to and use of maternal health services in South Africa. BMC Health Services Research, 12: 120-131.

Smith, K. and Sulzbach, S. (2008). Community-based health insurance and access to maternal health. Social Science \& Medicine, 66: 2460-2473.

Ternent, L., McNamee, P., Newlands, D., Belemsaga, D., Gbangou, A., Cross, S. (2010). Willingness to pay for maternal health outcomes: Are women willing to pay more than men? Applied Health Economics and Health Policy, 8: 99-109.

Themnér, L. and Wallensteen, P. (2012). Armed conflict, 1946-2011. Journal of Peace Research, 49 (4).

UN (2010). Millennium Development Goals. United Nations, Department of Public Information, http://www.un.org/millenniumgoals/ [Accessed November 13, 2012].

UNSD (2012). United Nations Statistics Division National Accounts Main Aggregates Database. United Nations Statistics Division, http://unstats.un.org/unsd/snaama/dnllist.asp [Accessed October 23, 2012].

Verwimp, P., Bundervoet, T., Akresh, R. (2010). The impact of violent conflict on child health: What are the channels? MICROCON Policy Briefing 6.

Wang, L. (2003). Determinants of child mortality in LDCs: Empirical findings from demographic and health surveys. Health Policy, 65: 277-299.

WHO (2012). Global Health Observatory - World Health Statistics. World Health Organization, http://apps.who.int/gho/data/\# [Accessed October 20, 2012].

Yarnoff, B. (2011). Household allocation decisions and child health: can behavioral responses to vitamin A supplementation programs explain heterogeneous effects? Journal of Population Economics, 24: 657-680. 



\section{Aspects of the Religious Context to Maternal-Child Health (Between Global and Local)}

Martin Tamcke, Faculty of Theology, Georg-August-University Goettingen, Germany

\section{Introduction}

"One cannot use electric light and radio, making use of medical and clinical processes in cases of illness or injury, and at the same time believe in the spirits and magical world of the New Testament." 1 This sentence does not come from a critic of the Christian religion, but rather from a leading 20th century German theologian whose concern it was to free the religion by offering a realistic picture of both Christianity and of the world in which it is engaged. It is not surprising that Bultmann's theology was heavily opposed by fundamentalists. Nevertheless, it quickly became accepted due to its being adopted by the educational system in Germany and its immediate implementation in classrooms. Bultmann's approach became the most important exegetical theology of the 20th century. In this sense, it was always important for the religious leaders to advocate for health as a human right. Thus, religious institutions—-such as religious or church hospitals, church development

\footnotetext{
${ }^{1}$ Rudolf Bultmann, Neues Testament und Mythologie. Das Problem der Entmythologisierung der neutestamentlichen Verkündigung, München 3rd Edition 1988, P. 16.
} 
services, social movements in Hinduism and Islam-are always involved in the fight against disease and often play a leading role. Around the same time as Bultmann, Albert Schweitzer developed his concept of "reverence for life". ${ }^{2}$ There has yet to be produced a detailed concept regarding the ethical basis for reverence for life in the case of maternal death. Neither has there been any conclusive sociological research about the influence of religion on the behavior of mothers and family members (and the entire community) in the case of pregnancy ${ }^{3}$. Even today there are such initiatives as the fight against maternal mortality in the context of church development services which distinguish itself as the Catholic Women's Movement. With their commitment to developmental policy in the fight against maternal mortality they hold public demonstrations in favor of increased spending on the relevant ministries 4 . The reality is, however, quite different. Rudolf Bultmann had to fight against belief in miracles, a belief which can cause fundamentalists to refuse necessary medical procedures, yet today the religious environment in other countries of the world have not adopted this approach and are not in line with modern religion. The multitude of problems which can arise from the realm of religion, when striving to safeguard health, is just as formidable as the incalculable number of life-courses. That the following tentative and preliminary observations are collected from outside of Europe does not imply that the issue would not be of highest importance in both Europe and America-specifically in the European context would reflection upon the impact of the loss of religion be valuable. Nevertheless, my purpose here is to focus on the non-European context. Since such a brief overview has a different function than the specific studies for each local area, I necessarily remain general and broad in my approach.

\section{Traditional healers and circumcision}

Traditional healers and midwives were often criminalized in Africa specifically because their religiously motivated healing methods were not able to be brought in line with the current, immediate, and plausible requirements of medicine ${ }^{5}$. This has had disastrous consequences. Still today only $42 \%$ of pregnant women in Uganda

\footnotetext{
${ }^{2}$ Martin Tamcke, Maternal mortality: A consequence of a lack of reverence for life?, in: Martin Kappas, Uwe Groß, Dermot Kelleher, Global Health, A Challenge for Interdisciplinary Research, Göttingen University Press 2012, P. 109-116.

${ }^{3}$ Eunice Kamara, Reproductive and sexual health problems of adolescent girls in Kenya - a challenge to the Church, Reproductive Health Matters, 7 (14), 1999, P. 130-133.

${ }^{4}$ KFB: Flashmob gegen Müttersterblichkeit, http://religion.orf.at/stories/2558578/ [Accessed Dezember 1, 2012] and Muttertag-Mutternacht: Aktionstag für Mütter in Entwicklungsländern, http://religionv1.orf.at/projekt03/news/1205/ne120511_mutternacht.html [Accessed January 3, 2013].

${ }^{5}$ Uganda-Ostafrika, Aktuelles aus Uganda und den Nachbarländern: Schubkarre zur Entbindung, http://ugandaostafrika.ug.funpic.de/allgemein/mit-der-schubkarre-zur-Entbindung/[Accessed January 3, 2013].
} 
deliver their babies under the care of a midwife or at a hospital. Until the 1990s an intense battle was waged against traditional health services challenging their credibility —of primary concern was their experiential-knowledge of the ancestors and of ancestral religion. Finally, the WHO and government officials modified their stance in order to cooperate with the traditional healers who had, until that point, been criminalized. The traditional healers were incorporated into programs, however, it remains inconclusive whether or not they were even literate since the teaching approach left no room for student participation. The health authorities were working from training concepts intended for schools. They should have used role playing and practical exercises with a minor theoretical component and been aware of traditional healing powers, especially with regards to birth complications. Also, the timely delivery of the expecting mother should be guaranteed. TERRE DES FEMMES offered the female circumcisers small loans for the purpose of establishing a new existence in Burkina Faso if they would leave the profession. The result was that four-hundred circumcisers left the profession and consequently joined the campaign against female genital mutilation.

\section{The example of female circumcision}

Admittedly, there are often sanctioned practices, which are strictly religious, that also increase maternal mortality. The African Union has increased its commitment in the fight against maternal mortality with the General Assembly in Kampala (Uganda). It begins with the Maputo Protocol, which serves to safeguard the rights of women, a special project to accelerate the reduction of maternal mortality in Africa. In the Islamic context-and sometimes also in the traditional Christian context - female circumcision is represented as one of the most difficult problems. "Female genital mutilation is one of the major reasons for complications during childbirth. The risk of dying during childbirth increases greatly for both the mother and the infant if the mother has been genitally mutilated. Yet, statements on maternal mortality often fail to acknowledge the problem of FGM." ${ }^{6}$ This is the opinion that Austrian exile Waris Dirie came to in her fight for the rights of women in Africa and it is now gaining momentum by the measures which followed the Ma

\footnotetext{
${ }^{6} \mathrm{FGM}=$ Female Genital Mutilation. FGM major factor in high maternal mortality rates in Africa/FGM, http://warisdirie.wordpress.com/2010/08/02/fgm-major-factor-in-high-maternalmortality-rates-in-Africa [Accessed January 3, 2013]. For circumcision in Coptic Egypt, please see the literature cited in footnotes 10 and 11. For Ethiopian rejection of female circumcision see the UNICEF article: http://www.unicef.de/projekte/aethiopien/aethiopien-beschneid/[Accessed January 3,2013$]$.
} 
puto Protocol and the General Assembly of the African Union.7 "It is very important not to ignore this aspect when we talk about the problem of the high maternal mortality rate in Africa. Women who have been circumcised have a much higher risk of serious complications during childbirth than those who have not been. Strict laws against FGM and an increased awareness of the risks involved are important aspects in the fight against maternal mortality in Africa and should be included in any program which promotes women's health and family planning." 8 This call, however, is faced with the fact that in a country like Somalia, for example, $95 \%$ of young women are circumcised. ${ }^{9}$ Maternal mortality $(1,200$ per 100,000 live births) and infant mortality (before first birthday 108 per 1,000 and before fifth birthday 180 per 1,000) are extremely high. The battle of the old regime under Siad Barre against this practice proved to be as unsuccessful as the battle of the transitional government since that time. For this reason a strong division arose between the people who lived with these religious practices and the religious leaders who perceived the situation. On 26 October 2005, Islamic clerics in Mogadishu published a fatwa directed against the practice of female circumcision. The widespread practice in Africa was condemned in this fatwa as "un-Islamic". ${ }^{10}$ Also in the Coptic Orthodox Church of Egypt, female circumcision was never a religious requirement but was, however, an integral part of popular piety practiced by the poorer peasant families. The continuation of this practice is founded on the belief that it serves to protect the chastity of women. In the opinion of the Coptic Egyptian socialist Marie Assad, female circumcision has as much to do with religious beliefs as does a tonsillectomy and has no influence on the chastity of a woman. ${ }^{11}$ Devout women in the Coptic Orthodox Church have consistently denounced female circumcision as mutilation and they insist that their view is well within the teachings of the Church. ${ }^{12}$ However, female circumcision is just the tip of the "iceberg" con-

\footnotetext{
7 Waris Dirie (*1965 in Somalia, lived in Austria), winner of the "Deutsche Afrika Preis" from the Deutsche Afrika-Stiftung 1999, the "Erzbischof-Oscar-Romero-Preis" 2004 and the "Martin-BuberPlakette" 2008, she has also been the Chevalier de la Légion d'Honneur since 2007. She gained worldwide attention with her publications which focus on the maladministration of the position of women in traditional African-Islamic societies, and served as a UN special ambassador against female circumcision from 1997 to 2003. Waris Dirie, Wüstenblume, München 1998; Waris Dirie, Nomadentochter, München 2002; Waris Dirie, Schmerzenskinder, Berlin 2006; Waris Dirire, Briefe an meine Mutter, Berlin 2007; Waris Dirie, Schwarze Frau, weißes Land, München 2010.

${ }^{8}$ FGM major factor in high maternal mortality rates in Africa/FGM, http://warisdirie.wordpress.com/2010/08/02/fgm-major-factor-in-high-maternal-mortality-rates-inAfrica [Accessed January 4, 2013].

${ }^{9}$ Somalia, http://de.wikipedia.org/wiki/Somalia [Accessed January 4, 2013] Ioan M. Lewis, Understanding Somalia and Somaliland: Culture, History and Society, 2008; Markus Virgil Höhne und Virginia Luling, Milk and peace, drought and war: Somali culture, society and politics, London 2010. ${ }^{10}$ DPA-Report in the Medical Journal, 2nd November 2005.

${ }_{11}$ Marie Assad, Female circumcision in Egypt, Cairo 1980; Cairo Family Planning Association, Facts about female circumcision, Cairo 1990.

12 Jeanette Spenlen, Koptisch-orthodoxe Frauen im modernen Ägypten, in: Albert Gerhards und Heinzgerd Brakmann, Die koptische Kirche, Einführung in das ägyptische Christentum, Stuttgart 1994, P. 119-131, and especially P. $129 f$.
} 
cerning woman's rights in general. The patriarchal social structure is founded on religion. In every Christian marriage ceremony the Biblical passage Ephesians 5:226:30 is read which legitimizes the subordination of the woman to the man. ${ }^{13}$ Even more open-minded religious communities continue this practice despite the outcry to put an end to it and its being banned in most countries. It's not surprising that in the unrest in the Arab world, the so-called Arab Spring, particularly contemplative religious communities are loudly voicing their opinion that female circumcision should again be legalized where it had previously been forbidden. In Egypt for example, Professor Mohamed Kandil of the Unitversity of Menofiya campaigned in favor of this. ${ }^{14}$ Female circumcision is a custom in twenty-eight African countries, in Iraq, Yemen, Egypt, ${ }^{15}$ and even in Indonesia there are those who practice it emphatically as in the region of Java. ${ }^{16}$ Religious upheavals within Islam against female circumcision often result without lasting effect. The Islamic women are not always as successful as those within the Coptic Church since the Coptic women have had some moderate success in anchoring their fight within Church teaching against female circumcision. ${ }^{17}$ Historically, the practice is not rooted religiously; rather, it lingers as a part of popular religion. It makes the situation all the more difficult that the religious rules are anchored in the popular religion, especially in the core of a particular religion.

\section{Lack of rights due to a religiously sanctioned picture of the family}

The battle for the so called marriage under Sharia law is fought by pious Muslims who advocate for the legalization of child-marriage in places where it is banned by the state. The marriage age in Niger is ten years old, which speaks scorn against every prevention of maternal mortality, yet it is justified by Islamic jurists in most cases. ${ }^{18}$ Conservative Catholic theologians in Kenya fought against the deregulation

\footnotetext{
${ }^{13}$ Spenlen, J. 123.

${ }^{14}$ Birgit Gärtner, UN erlässt Resolution gegen weibliche Genitalverstümmelung, http://www.heise.de/tp/artikel/38/38279/1.html [Accessed January 4, 2013].

15 Ibid.

${ }^{16}$ Ibid.

${ }^{17}$ Spenlen, J. 123 (on Egypt); Birgit Gärtner (on Indonesia).

$18 \mathrm{https} / /$ diesiebtesichel.wordpress.com/tag/muttersterblichkeit/.The practice has also been introduced in Europe, see. Ryan Kisiel, The British child brides: Muslim mosque leaders agree to marry girl of 12, http:// europenews.dk/en/node/58322 (Daily Mail 10. September 2012) [Accessed January 4, 2013].
} 
of the abortion law. ${ }^{19}$ Abortion is punishable by up to seven years in prison. Those who assist in abortions are also punished. This restrictive abortion law will again be renewed when the new constitution comes into force in 2019. Paragraph 26 of Kenya's new constitution states, in agreement with the church, that life begins at conception. However, there is an exception clause which allows for an abortion, validated by a medical certificate, in cases where the mother's life is in danger. This caused church leaders and political figures to initiate protests against this law. The legitimate and illegitimate force of the churches was simultaneously exercised in a country where a widow is still often simply passed on to the deceased's brother, yet it remains a taboo. Maternal mortality as a result of unequal gender relations remains largely ignored politically and sanctioned religiously. Religion is not the sole cause of maintaining the traditional patterns of behavior, but it becomes an obstacle to action in the struggle against maternal mortality where it regards its own task as that of preserving the traditional social order and the traditional family morality. "Between religion and state public policy, between patriarchal tradition and societal norms, there is not much room for protecting the interests of women." 20 Such obstacles, which are virtually the cause of increased maternal mortality, are also present in Latin America. This can be seen in Nicaragua where similar laws have been created. ${ }^{21}$ Resistance to traditional social norms concerning family and the

${ }_{19}$ AG Bildung, Martina Backes 2011, Factsheet Muttersterblichkeit

http://www.frauen-rechte-gesundheit.org/corpus/Muettersterblichkeit_Rechte_Kenia.pdf [Accessed January 4, 2013], and Kenya National Commission on Human Rights (2009): Women, Reproduction and the State in Kenya. Briefing Paper:

http://www.knchr.org/dmdocuments/briefing\%20paper\%20women \%20reproduction $\% 20$ and $\% 20$ st ate $\% 20$ in $\%$ 20kenya.pdf. [Accessed January 4, 2013], and A Shadow Report to the 5th and 6th combined report of the Government of the Republic of Kenya, on the international Convention on the Elimination of all Forms of Discrimination against Women (CEDAW), Presented by FIDA Kenya to the 39th session of the United Nations Committee on the Elimination of all Forms of Discrimination against Women, 23 July - 10 August 2007, NEW YORK,

U.S.A. http://www.iwraw-ap.org/resources/pdf/Kenya\%20SR\%20final.pdf [ Accessed January 4, 2013], and Centre for Reproductive Rights (2010): In Harm's Way. The impact of Kenya's restrictive abortion law.

http://reproductiverights.org/sites/crr.civicactions.net/files/documents/ES_inharmsway_FINAL.p df [Accessed January 4, 2013], and Failure to deliver. Violations of Women's Human Rights in Kenyan Health Facilities. Centre for Reproductive Rights and FIDA 2007,

http://reproductiverights.org/sites/crr.civicactions.net/files/documents/pub_bo_failuretodeliver.pd f [Accessed January 4, 2013].

20 Afya Yangu. It's my Right!, https://www.iz3w.org/buch-cd-dvd/dvds/afya [Accessed January 4, 2013]. This report draws from a movie in which women in Kenya talk about their rights to health and sexual self-determination.

${ }^{21}$ Heidemarie Wieczorek-Zeul, Frauen bringen den Wandel - Gender, Entwicklung und Milleniumsziele, in: Frauen bringen den Wandel, Bilanz und Perspektiven einer geschlechtergerechten Entwicklungszusammenarbeit, Dokumentation der Veranstaltungen am 20. And 21. April 2007 in Bonn and Hamburg 2007, P. 6-8, especially P. 7. The volume also contains fundamental contributions to gender issues and the Church Development Service of Brot für die Welt (Jens Koy, Geschlechtergerechtigkeit und Afrika, Die Genderarbeit des Evangelischen Entwicklungsdienstes, P. 15-16, and Reinhard Koppe, Frauenförderung und Gender-Mainstreaming, Das Genderengagement von Brot für die Welt in Afrika, P. 19-21, table of contents statement is inaccurate); also: http://www.venro.org/fileadmin/Publikationen/gender/VENRO_Gender_final.pdf 
"mother image" forces women into social ostracism. Women's rights activist Esther Mwaura describes the situation: "For a woman to talk about abortion here is to make a pact with the devil. Any discussion about abortion condemns both the women and the culprits-but concerning the reproductive rights of women, no one wants to know anything." 22

\section{Outlook}

An applied strategy which is only dedicated to medical intervention is doomed to fail in countries like Somalia or Afghanistan among others. Many factors of the social construction of motherhood are legitimized by religion or even based on religion. A sustainable strategy that will potentially change the social structure must involve both men and women. Expectations towards the motherly role, knowledge (appropriately including experiential knowledge), and daily practices (for example, when young mothers have to restrict themselves from food so as not to bring about resentment from their in-laws who are in charge of the house in which they have lived since marriage) often have clearly religious undertones. In India, both Aryuveda and Unami doctors are active in the field of maternal mortality. The family's emotional preparation, the society, and the religious community indicate the context in which a young woman must face her motherhood as she is completely at their mercy. An aspect that obviously needs to be treated is whether religious factors become barriers which contribute to the strengthening of mothers by medical and other types of care. To date, however, the theological-ethical aspect has not been comprehensively evaluated, nor has the sociological component been sufficiently determined. In societies where religion has a self-evident position, the European interviewer is presented with no small challenge since his/her list of questions stems from circumstances that do not apply to these types of societies. Lest the struggle against maternal mortality be undermined from the outset by predetermined cultural and religious structures, a broadening of strategies for religious-dialogue is immediately necessary. This need can be seen in the return of African theologians towards traditional African religions, the return of Muslim scholars to the traditional code of ethics of Islam, and the specific moral values of Hindu religious leaders. ${ }^{23}$ Even the memorandum of the Evangelian Church of Germany (EKD) is limited to medical and financial aspects in the fight against maternal mortality. ${ }^{24} \mathrm{~A}$ statement from the World Council of Churches says: "The situation in which Mary found herself is like the misery which, after two thousand

\footnotetext{
22 Ibid.

${ }^{23}$ See, Volker Küster, Einführung in die Interkulturelle Theologie, Göttingen, 2011, P. 145-147.

${ }^{24}$ Aus Gottes Frieden leben - für gerechten Frieden sorgen, Eine Denkschrift des Rates Evangelischer Kirchen in Deutschland, 2. Auflage Gütersloh 2007, P. 81f
} 
years, millions of mothers are still enduring." ${ }^{25}$ However, the idea and impulse for action certainly includes multiple levels such as, "in the home, in the parish, in the community, and in the church (across denominations)," but it is generally limited to the realm of "in the home": "Take the necessary information, the health services, the safe room, the support and the resources for leading a healthy life to the mothers, the young women and girls at their homes? What steps can you take to make new mothers less vulnerable?"26 Are these general questions attributable to the context of the author, Manoj Kurian, who is a doctor from Malaysia working as a Program Officer for Health and Healing at the World Council of Churches? In any case critical, tangible results must emerge that first allow for the realization that their own actions obligate rather non-conducive social behavioral patterns. Otherwise, such common sharpening-of-conscience remain with the believers on a level that is not capable of changing society's acceptance of misconduct.

25 Manoj Kurian, Gesundheit der Mütter, http://www.oikoumene.org/fileadmin/files/wccmain/documents/p4/health/2011/Health_month_week2_GER.pdf [Accessed February 21, 2013]. 26 Ibid. 


\title{
13. Cultural Practices, Beliefs, Traditions and Norms in Atwima Nwabiagya District: A Blessing or a Curse for Maternal Health?
}

\author{
Beatrice Asenso Barnieh, Ghana Education Service, Ghana
}

\section{Abstract}

Many policies and programmes have been implemented and intensified in Ghana to ensure that the country is on track in achieving the Millennium Development Goal 5 (MDG 5). Acceleration on the progress of the achievement of this goal has received a renewed attention by the government through the introduction of free medical care for women during partum and postpartum period, as well as through the expansion of health facilities and training of health personnel in the country. Yet, maternal mortality still remains a deforming scar on the progress towards the achievement of the MDG 5 in the country. Ghana has been ranked 41st on the world maternal mortality rate index. Reports indicate that the current maternal mortality rate in Ghana is $385 / 100,000$ live births as compared to the required rate of 185/100,000 live births needed to achieve the MDG 5. Factors that impede healthcare delivery, access and utilization are often addressed using medical explanatory models. Policies and programmes to address the maternal mortality are focused on the provider side. Approaches to address the acceptance and uptake of 
the programmes and policies are neglected. However, research has proven that health is influenced by biological, social, cultural and psychological elements which could also have a negative impact. It is therefore important to study and understand the underlying factors that impede the uptake of services in Ghana. This study was conducted in Atwima Nwabiagya District in Ghana to identify the cultural practices, traditions, norms and beliefs in the district that have implications on maternal health. Qualitative research methods (document review, focus group discussions and in-depth interviews with key informants) were used to explore the cultural practices, traditions, beliefs and norms in this district. Snowball method was used to identify the key informants. The findings from this study were coded into themes and interpreted to assure the clarification of cultural practices towards health. The results indicated that some cultural practices, traditions, beliefs and norms in Atwima Nwabiagya District delay the access to healthcare, have a negative impact on health-seeking behaviour, as well as health utilization, compliance, acceptance and the uptake of health programmes, particularly by pregnant and nursing mothers and thus, have a great impact on their health.

\section{Introduction}

The deadline for the Millennium Development Goals (MDGs) is fast approaching. With less than two years left to meet the deadline, the target for MDG 5 has not been reached yet. Close to 342,900 women die of pregnancy and childbirth complications every year. The estimated maternal deaths in 2010 were 287,000 worldwide. Giving rise to a maternal mortality rate (MMR) of 210 maternal deaths per 100,000 live births in 180 countries. The tragedy is that approximately $99 \%$ (287,000 maternal deaths in 2010) out of the total amount of deaths occurred in the developing countries. Sub-Saharan Africa recorded the highest maternal death rate $(162,000)$ followed by South Asia region $(83,000)$.

The MMR of 2010 shows that Ghana is making progress towards the attainment of MDG 5 (WHO, 2010) however, there could be more room for improvement. Though Ghana's MMR declined from 540/100,000 live births in 2000, to $451 / 100,000$ live births in 2007 to $350 / 100,000$ lives birth in 2008, the reduction is predicted to be at a rate of $3.3 \%$ annually compared to the $5.5 \%$ annual rate required to achieve the MDG 5 target of 185/100,000 live births by 2015 (WHO, World Bank, UNFPA, 2008). Ghana will therefore unlikely attain its aim for MDG 5 by 2015. The Alliance for Reproductive Health Right (ARHR) stated that this is intolerable and agrees with the World Health Organisation (WHO) that the implementation of simple interventions by all stakeholders could contribute to the reduction of these "worthless deaths" (Okine, 2012). 


\subsection{The global maternal health policies}

Maternal health can be defined as the health of a mother during partum and postpartum periods. Globally, the maternal health policy journey began in 1948 during the foundation of the World Health Organization (WHO). The WHO recognized maternal and child health $(\mathrm{MCH})$ as one of the priority areas. In 1978, when the primary healthcare policy was greatly promoted by the $\mathrm{WHO}$ and $\mathrm{MCH}$ has been included into the strategies and first line health services were expanded as well as antenatal and delivery care, and the training of $\mathrm{MCH}$ workers focusing on the survival of the child.

However, this was not enough because the maternal component in the $\mathrm{MCH}$ services and traning received little attention. Professor Alan Rosenfield in 1985 raised awareness towards maternal mortality and he questioned the neglected $\mathrm{M}$ component in MCH, saying, "Where is the M in the MCH?" (Rosenfield, 1985). With reference to this, Safe Motherhood Initiative was introduced in 1987 with a greater emphasis on community-based care and risk rediction - focusing on the training of traditional birth attendants, obstetric care at first referral level as well as emergency obstetric care as an integral part of $\mathrm{MCH}$. The journey continued with the International Conference on Population and Development in Cairo in 1994. The world leaders would have come together and made an informed choice about antenatal care; referral- and skilled delivery care; obstetric care that avoids excessive use of Caesarian sections; abortion care and the enhancement of effective ongoing programs. Then in 1997, there was a paradigm shift in antenatal care. According to Maine (1997) the focus was now on "improving the accessibility, quality, and utilization of emergency obstetric care for women who developed complications, rather than on having contact with all pregnant women."

Later, there was an increasing realization that every pregnancy faces risks and so Safe Motherhood Initiative was expanded in 1999 to include advanced safe motherhood through human rights; women empowerment to ensure choices, economic and social investment; delay in early marriage and first birth; improved access to skilled attendance at delivery; improved access to quality reproductive health services; prevention of unwanted pregnancy; and addressing unsafe abortion, progress measurement and partnership (Safe Motherhood Initiative, 1999). In 2000 , the maternal health once again received global attention through its embedment in the MDG declaration. The WHO also became more committed in making pregnancy safer by providing technical and policy support, by strengthening government capacity to advocate, promote and coordinate research and dissemination as well as monitoring and evaluation. $\mathrm{MCH}$ was entirely integrated when WHO took all necessary steps to institutionalize the integrated management of pregnancy and childbirth. In addition, safe motherhood received more attention through human rights approach in 2001. 


\subsection{The implementation of maternal health policy in Ghana}

Ghana is one of the many developing countries swift to endorse international conventions aimed to improving reproductive and maternal health. Ghana ratified the International Conference on Population and Development (ICPD); the Maputo Plan of Action, the Abuja Target of allocating at least 15\% of the national budgets to health and the Campaign to Accelerate Reduction of Maternal Mortality in Africa (CARMMA) and many others. The adoption of these policies by the government led to the expansion of health facilities and training of more health personnel in Ghana (Okine, 2012).

Despite all these policies, maternal mortality still remained a problem in the country. Fewer mothers had access to healthcare and so in 1996, the government of Ghana introduced an exemption health policy for mothers. The policy covered $28 \%$ of the delivery costs of women who delivered by caesarean section and $26 \%$ of the costs of delivery of women who had a normal birth. The exemption policy was confronted with funding challenges, and so in 2004 the policy was abolished. Consequently, the number of pregnant women who had access to skilled attendants dropped from 54\% to 34\% between 2005 and 2007 (NPC, 2011; Witter et al., 2007; SHFSG, 2001; IMMPACT, 2008).

In 2008, as part of the National Health Insurance Policy, the government instituted a free antenatal and postnatal care for all mothers so as to curb the maternal and child mortality menace. To date, reducing maternal and child mortality remains unattained in Ghana as described by Okine (2012). Unmet need for family planning remains as high as $35 \%$, skilled delivery as low as $45.6 \%$, acute shortage of midwives persists while health staff remain inequitably distributed with $43 \%$ of doctors in the Greater Accra Region and 4\% for the three Northern Regions (Okine, 2012). This suggests that when health service planning is focused on the provider side, the question as to whether the services will be an utilised need to be addressed. It is evident that most often factors that limit acceptance and uptake of health services are neglected (UNFPA, 2011). Health system strengthening alone may not lead to a positive outcome.

It is therefore imperative to study and understand the underlying factors that impede the uptake of health services in Ghana. Senah explained that all the attempts to reduce maternal mortality in Ghana have led to the implementation of policies and programmes which derived their interpretation from medical explanatory models. Meanwhile, many studies have shown that factors, other than medical, contribute immensely to maternal and child mortality in Ghana. Seneh cited that socio-economic and cultural practices are critical underlying factors affecting maternal health (Senah, 2003). For example, the cost of health facility may deny the poor of the access to health care (Bour, 2003). Also, cultural practices, traditions, beliefs and norms in pregnancy and child birth may oppose modern antenatal care and may lead to negative impact on acceptance of the service (Boudier, 1984). He maintained that factors that promote health and have a negative impact on it could 
be explained as a combination of biological, social, cultural and psychological elements which are inseparable and therefore, must be invariably addressed together.

\subsection{Cultural practices, traditions, norms and beliefs}

The meaning of cultural practices, traditions, norms and beliefs used in the context of this study was adapted from Tylor's definition of the "culture" (1871). According to Tylor, culture is a complex whole which includes beliefs, norms, traditions, customs, morals, laws, knowledge, arts and any other capabilities and habits acquired by a man as a member of a society. And hence, the four words: culture practices, traditions, norms and beliefs can be used interchangeably in this study. Tylor described culture as a way a group of people live in one geographical area and a host of inherited and established guidelines that help people to make informed choices. According to him, culture influences how a society will feel and judge the world, and behave in relation to other people, supernatural forces and the natural environment. He indicated that culture offers the opportunity to transfer guidelines to the next generation through the use of symbols, language, art and rituals. He viewed culture as the lens by which a society perceives and understands the world and learns how to live within it. From Talyor's perspective, culture is a very powerful tool for survival (Tylor, 1871).

Cultural practices, norms and beliefs influence healthcare enormously in Ghana. Some diseases are regarded as not for hospitals resulting in different healthseeking behaviour among the community. The decision to visit a healthcare centre and even the utilization of healthcare information are all culturally constructed. Notably, women in Ghana are made to observe some dietary regulations, practices and taboos during and after pregnancy in order to ensure safe delivery and good child health (Arhin, 2001). Some of these cultural practices are beneficial to $\mathrm{MCH}$, whilst others have negative effects on the health of the mother and the child.

Cultural practices, traditions, norms and beliefs may have an indirect effect on women health. For example, they may actively restrict pregnant women from seeking antenatal care in modern health facility (Browner et al., 1990; Rawlins, 1991; Sindiga, 1995). They delay access to health, exacerbate non-compliance of health information, guidelines and protocols, affect utilization, uptake and acceptance of health programmes and projects, affect nutritional status of women and children resulting in malnutrition. With the exception of female genital mutilation and early child marriage which has a direct health outcome and have attracted international attention, some other cultural practices, traditions, norms and beliefs which have indirect effect on maternal health have received little attention in Ghana. This paper exposes cultural practices, traditions, norms and beliefs in Atwima Nwabiagya District in Ghana which may have an impact on maternal health. 


\section{Methodology}

\subsection{Ethical consideration}

Permission was obtained from the district health director and the medical superintendent in charge of the district hospital before the beginning of the study. Verbal consent was also obtained from the traditional leaders, family heads and the participants of this study.

\subsection{Document review}

The data collection began in July 2010 and ended in November 2012. The researcher used qualitative research method to understand the cultural practices, traditions beliefs and norms in Atwima Nwabiagya District. Health reports, district profiles, organizational charts, manuals, medical records, textbooks, diaries, autobiographies and archived data on cultural practices, norms and beliefs in the district were reviewed with the aim to gain better understanding of the cultural practices, traditions, norms and beliefs in this district.

\subsection{Focus group discussion}

Four communities (from the north, south, east and west) were randomly selected from every subdistrict in Atwima Nwabiagya to reflect the district range of geographical location. The total numbers of the subdistricts are five (Abuakwa, Nkawie, Berekese, Akropong and Asuofuo) giving rise to 20 communities involved in this study, out of a total of 126 communities in the district. The participants were selected from different households after it has been discussed with traditional leaders. They were selected from different ethnic groups to ensure that the results could be generalized. This was done by the help of key informants who identified the target groups from the different ethnic groups.

In each of the 20 communities, ten (3 Akans, 1 Ewe, 1 Ga-Adangbe, 1 MoleDagbane, 1 Guan, 1 Frafra, 1 Dagomba and 1 Akwamu) participants from educated first-time pregnant women, uneducated first-time pregnant women, educated women with past record of child birth and uneducated women with past record of child birth who were willingly to participate in the study were conveniently and purposively recruited from different households to form a focus group. This focus group was necessaary to ensure that the views of all participants, the educated, uneducated, first-time pregnant women and women with past record of child birth were collected. Completely, 40 participants were recruited from each community. To sum up, 800 individuals participated in the focus groups discussion in the district.

Four focus groups with 10 members (the recommended maximum focus group size) were formed in every community. The focus group discussions were 
held in the communities to explore the women's ideas, knowledge on cultural practices, traditions, beliefs and norms in the communities. Open-ended interview guidelines were used with the flexibility of incorporating emerging issues linked to the research for further probing and investigations. All the interviews were conducted in Asante-Twi language because this is the language which is widely spoken and understood by all the different ethnic groups in the district. Every interview lasted for about an hour at each occasion.

\subsection{In-depth interview}

In order to triangulate the results, twenty in-depth interviews with key informants were carried out. Snowball sampling technique was adopted to identify key informants in this area to be involved in the in-depth interview. As key informants pregnant women, mothers, fathers, traditional leaders with in-depth knowledge about cultural issues, beliefs and norms, birth attendants, as well as health officers in the district who were all very much familiar with the health situation and healthseeking behaviour were recruited for this study.

\subsection{Data analysis}

The approach to the data analysis was both inductive and deductive. The content of the discussions and interviews with the participants has been analysed. Interview text, field diaries, and recorded tapes were used to generate transcript and codes (similar ideas in the data were matched and grouped). This involved identification of major themes and sub themes, recurring languages and meanings. Contrasting ideas were not discarded but included in the analysis. The ideas from the discussions and the interviews were written on cards and displayed on a table. Important themes were identified and posted on a flip chart. Logical connection within themes were identified and supported with comments made by the participants. The transcripts were repeatedly reviewed carefully and systematically to ensure there was a link between the research questions and the emerging issues.

\section{Findings}

The age of the participants was between 18-70 years. Fifty per cent of the participants were literates who had completed 12-16 years of education and the remaining $50 \%$ were illiterates. Eighty per cent were gainfully employed or were either trading or farming whilst $20 \%$ were housewives, jobless, retired or were not working due their old age. 


\subsection{Nutrition and diet}

\section{Consumption of non-food items}

The study revealed that some pregnant women in the district consume clay, which is known as "hyire" in Akan language to ensure that the baby will obtain a smooth skin. "The only thing I prefer eating now is 'hyire'. I like it because I do not vomit when I eat it. Ever since I was born, pregnant women in this village have been eating it without any side effect. My grandmother told me, it will give my baby a smooth skin when I deliver" (pregnant woman, 30 years).

"The pregnancy itself comes with the food that one must eat. For me, no one had ever prescribed any food item for me during pregnancy. My system tends to reject certain food items automatically whenever I am pregnant. I tend to vomit when I eat any food my baby does not like" (A biology tutor, 35 years old mother).

\section{Dietary taboos}

Whilst the health personnel think the expectant and lactating mothers are free to eat any food provided it is well balanced, the responses from the women indicated that traditionally, women who are pregnant as well as lactating mothers are forbidden to eat certain foods to ensure healthy and sound delivery. According to them, the food taboos had been in existence for many generations. They also mentioned that pregnant women are restricted to eat salty foods, any fish or meat which contains salt and some other foods containing proteins. As an example, pregnant women are not supposed to eat salted fish which they referred to it as ('Koobi', 'momoni), meat, eggs, beans and snails. Pregnant women stated that they are not allowed to consume oily and fatty foods. In addition, carbohydrates such as banana and ripe plantain are also not allowed for consumption during pregnancy. It has been acknowledged that pregnant women are not allowed to enjoy vegetables such as okra, garden eggs, tomatoes, green cocoyam leaves, and pepper. And also fruits including pineapple, mango, guava, coconut are excluded from their diets during pregnancy.

\section{Reasons for the pregnancy-related food taboos.}

When the participants were asked about the reasons for observing the food taboos, they explained that the reasons for not eating meat, chocolate, eggs are that they intensify complication during child birth (maternal death, rupture of the uterus) due to delivery of strong babies associated with consumption of such foods. Another reason is that the baby will grow to become a thief if the mother consumes eggs during pregnancy. They specified that eating eggs whilst pregnant may result in giving birth to babies who are asthmatic and they added that the consumption of beef will dry out breast milk. They further affirmed that ripe plantain and banana are major causes of preterm contractions and miscarriages, as well as forced 
labour and delay in removing the umbilical cord. It was also stated that "drooling and slime babies" are a result of mothers who consumed okra and snail during pregnancy.

The restriction of salted fish consumption was associated with swollen feet in mothers and babies with low IQ. Beans and green cocoyam leaves were believed to cause stomach upset and also prolonged delivery as in the case of banana and ripe plantain. Similarly, jaundice in both mothers and babies were linked to consumption of oils and fatty foods during the time of pregnancy. They mentioned that the consumption of animal coat delays the removal of umbilical cord and miscarriage. They added that pineapple and coconut consumption also bring about miscarriage and natural abortion. Whitish blind eyes in infants were also attributed to the consumption of coconut. Mangoes and Guava were associated with appendicitis.

When the participants were asked to speak about the origin of the pregnancyrelated food taboos, they mentioned that the taboos have been there since they were born. They believed that the taboos are passed on from generation to generation. How the taboos were instituted was not known to them. Perhaps, when one person consumes certain food items and then experiences complications which may have been the result of meat that was not well cooked or taking the example of vegetables which were not well washed, it was affirmed that people tend to generalize these experiences and restrict every pregnant woman from consuming these foods.

\subsection{Care during pregnancy}

According to the participants, pregnancy is not a disease and hence, women must carry on with their duties in the same way than before their pregnancy. Indeed, it was observed that pregnant women in the study area carried basketful of farm products to find their way back to the farm and mostly with their babies at their back whilst the husband held the cutlasses and walked along with them. Walking behind a pregnant woman was also believed to initiate miscarriage. They indicated that women must also avoid leaning or sitting on a cement floor during pregnancy to avoid complications. Women who are pregnant are advised to fill their buckets to the brim when fetching water to prevent the baby from suffering from myopia, an eye defect. They mentioned that swollen legs in pregnant women indicate twins in the mother's womb.

Generally, the participants explained that it is culturally immodest to show early signs of pregnancy until it is visible. This, they said is to prevent evil eyes from haunting the unborn baby. They also indicated that expectant mothers are admonished to delay the attending of antennal care at the early stage of pregnancy to avoid giving birth to babies who are small. Some others stated that they avoided the antenatal care because they wanted to keep their privacy. They said that they feel uncomfortable when they are examined by male practitioners. They stressed that it is culturally unacceptable for women to expose their nakedness to any other 
man apart from their own husbands. Some explained that they are scared of the medical procedures. Some women stated that they were unable to hide their pregnancy in the early stages. Others refused antenatal care because they felt fit during pregnancy. The majority of these women visited the health facility when they felt that some complications may occur during their pregnancy. They indicated that it would be safer if trained health personnel handle such complications.

The women explained that they are advised to keep their bodies clean, especially the private parts during pregnancy, and thus, it is very common that they insert ginger and pepper into their anus and vagina, introduce enema prepared from herbs, or sit above hot water to ensure good health of the mother and the baby. The results indicated that women should be alert during pregnancy to avoid accidents which may be harmful to the health of the unborn child. Similarly, they stated that women are asked to avoid contact with all forms of chemicals such as hair relaxers during pregnancy. It is their belief that having sex when pregnant will ensure that the baby resembles with the father. Likewise, standing behind a pregnant woman may cause the baby to resemble with the person.

\subsection{Hospital delivery}

Among the women who attended antenatal care, only a few women delivered at the health facility. They mentioned that an hospital delivery restrict them from performing some important rituals soon after birth. They cited the bad attitudes of health personnel such as shouting on them and their discouragement for them although they deliver at the hospital. Another point which was mentioned referred to the women's fear that their babies may being swapped at the hospital. It was also common among pregnant women that they believed that it is also a taboo to cross some rivers and streams during certain times of pregnancy. So although they want to deliver at a specific hospital it is not possible because the refusal of these particular beliefs may result in death.

"When I got pregnant for the first time, I went to about three different health facilities for check-ups and screening. They all confirmed that I had three babies in my womb. When it was time for me to deliver, I went to the hospital. I was unconscious but I saw most of the things the nurse did. She pulled my three babies one after the other, but I did not see where exactly she placed them. After I was conscious, I asked for my babies and she handed only one baby to me. I challenged her but all my efforts were fruitless. After five years, I saw a small boy in the market and as soon as I set eyes on him, I felt he was one of my two stolen babies. I followed him to his residence and told his guardian that the boy was my son. She reported me to the police station and I was arrested. Unfortunately, I had no witness and so I was charged guilty. Since then I had vowed never to deliver at a health facility nor encourage anybody to deliver there" (A woman, 50 years old).

"I delivered my first baby at a public hospital. I entered in to labour and when I was screaming; the nurses shouted and rebuked me. When it was time for me to deliver, the nurses sacked all my relatives out of the delivering room. The tap was not flowing at the health facility. The nurses asked me to go and fetch some water from the downstairs. It was 
very difficult for me because I was in a severe pain. I delivered on the staircase when I had fetched the water and was climbing back to my ward. I saw my baby and it was a girl. One of the nurses came and picked it and sent it to the ward. When I got back and checked on my baby, I saw that my baby has been swapped. The nurse rather placed a baby boy in the court besides me. I complained but nobody paid attention to me" (A woman, 30 years).

\section{Treatment of postpartum wounds and bygiene after delivery}

Some women use enema prepared from some herbs to facilitate delivery. According to them, the herbalists do not disclose the names of the herbs to them which they think; it is a way to preserve their business. Others disclosed that they use enema from time to time during postpartum period. Some mentioned that they are given some herbal concoctions to drink it in order to avoid the uterus from rupturing. They believed that the abdomen must be tied after delivering to ensure that the uterus returns to the normal position. After birth, they insert grounded pepper, ginger, 'esorowesa' (black pepper), and ashes into their vagina to enable the internal wounds to be healed. They also drink medicinal herbs to help get rid of all clotted blood from the uterus. Some of the participants mentioned that wrist band made from rags containing carcass from wall gecko and other concoctions is put on the baby's wrist to protect the baby from getting convulsion.

"The herbalist pierced the wrist of my child and inserted some concoction to prevent the baby from being attacked by the evil spirit and subsequent convulsion. He gave us some herbal medicine to be used as enema for the child to avoid any future sickness. As soon as the enema was introduced in to my baby's anus, he started passing out stool stained with mucus and blood. My baby became weak. We called the herbalist and he told us he would be there. Three days passed and he had not showed up. We were scared and thus, we were searching for him. He was nowhere to be found. He showed up a week after and assured us that our baby will be fine, when all the mucus has come out of him. Indeed, when our baby stopped passing out the mucus he became very strong than ever before" (In-depth interview with a man, 40 years).

They further confirmed that they are admonished to sit on a hot water bucket. The modern mothers recounted that they place hot water bottle on their belly to ensure that all the internal wounds due to delivery are healed. Nson, bonto and nkyine' (ash, tobacco leaves, and salt respectively) are combined for treating the stump of the umbilical cord to ensure quicker removal and also to prevent nasty smell of the area. They explained that when visting a mother and in the case the room is smelling, then it is probably a sign that the cord stump is not being well treated. The pregnant women also acknowledged that they eat soups prepared from herbs. According to them, soup prepared from palm fruit, 'abenduro', 'kwaonsusua' (turkey berry) is considered appropriate for enhancing the production of breast milk. A mother without enough breast milk is supposed to smear the breast with salt solution to improve the production of the breast milk.

These women also talked about the fact that the current sanitary pads are not able to manage the vaginal discharge and excessive blood flow and hence they still 
stick to the old traditional way of managing it with old clothes. The baby is kept indoors and covered entirely to wade off evil spirit from harming it. The mother is supposed to stay indoors for about three months before resuming duty. They are supposed to keep their bodies clean and put on nice clothes when they come outof-doors. When the baby is born and there is mucus running down the nose, the mother must suck it with the mouth to demonstrate the love she has for the baby.

\section{Cutting of the umbilical cord, circumcision and placental disposal}

Many of the women also mentioned that their baby's umbilical cord has been cut at the hospital. Those who delivered at home also mentioned that sharp razor blade was used to cut the umbilical cord either by themselves or by the traditional birth attendants. Male infants are circumcised and the ears of the females are pierced either by a hospital staff or a birth attendant depending whether the delivery occurred at home or at the hospital. Nobody mentioned that the female babies' genital organs were mutilated.

"I delivered by myself because nobody was around when I went into labour; I cut the umbilical cord with a sharp blade. I removed a thread from my plaited hair and tied the cord with it. There was no complication at all" (An old woman, 60 years).

Some mothers who delivered sometime back in a health facility disclosed that their placenta was given to them, so they could take it from the health facility to home in order to bury it. Others mentioned that the placenta was buried at the health facility.

"My placenta was not given to me to be sent home. I insisted that the placenta should be given to me to be buried at home because my mum told me that "where a baby's placenta is buried is where he or she comes from" (A woman, 35 years).

"I am not from this village; I am from Ewe land. When I was delivering my first born, I was asked to sit in the sand, covered myself till I delivered. Soon after delivering, the woman cut the umbilical cord with a razor blade and covered my baby's navel with a cloth and tied it with a thread" (A woman, 33 years).

\section{Compliance of the hospital guidelines}

The majority of women revealed that they delivered at a health facility. However, they disclosed that they did not comply with all the health facility guidelines after delivery. They admitted that they often apply the hospital guidelines alongside the traditional methods. But due to the fear of being rebuked by the hospital staff, particularly the doctors and nurses, they give them assurance of their compliance of the hospital guidelines. They declared that they prefer the traditional way of postpartum care to the hospital-based care. In their opinion, the traditional methods are effective, quicker and safer. Even the educated mothers attested to this fact. These suggest that there is a cultural barrier to the use of health information by lactating mothers. 
"When my wife was pregnant, she was advised to see a traditional herbalist for some medicine, to enable her to deliver a very strong baby. Initially, I stopped her because I am a modern man. Later, I allowed her to go for the medicine because I was told; my child will suffer from 'asram' if my wife does not go for the herbal medicine. I gave it a thought and realized the herbalist was a male and so I did not entertain so much fear. Truly, the herbs were effective. My wife delivered a very strong baby" (In-depth interview with mathematics tutor, 45 years).

"I delivered at the hospital. My baby's umbilical cord was cut at the health facility. I was advised by the nurse to apply spirit at the umbilical cord stump to facilitate the removal of the umbilical cord. I did not comply because my friend told me that will cause the chord stump to develop a bad odour. I was given some concoction prepared from ash and some leaves by an old woman in my residential area to apply to the area. The woman is known to be very experienced when it comes to child's care. I was told to apply the medicine to the chord stump every one hour. That was what I used to facilitate the removal of the umbilical cord. Three days after, I reported at the hospital, when I was asked by the nurse of my compliance of the hospital guidelines, I assured her I was complying because I was afraid she will rebuke me. Later, I was gripped with fear when the rest of the umbilical cord had not removed. Fortunately, it fell off before my next visit to the health facility"(ICT Tutor, 35 years).

\section{Conclusion}

Intensification of programmes, projects, policies and massive expansion of maternal health facilities as well as training of health personnel alone does not lead to the required maternal health outcome in Atwima Nwabiagya District of Ghana. Maternal mortality is still high because women are losing their lives in the name of cultural practices, traditions, beliefs and norms in the district. The results from the study indicated that cultural practices, traditions, beliefs and norms are deeply rooted in the society to the extent that access to healthcare is delayed. Those who utilize the health services do not comply with most of the hospital guideline. Many women complement traditional practices with modern medical care and fail to comply with hospital guidelines even if they deliver at a health facility. The decision to deliver at a healthcare centre and the utilization of health information are all culturally constructed. However, some pregnancy-related diseases do not have anything to do with hospital care at all. Many pregnant women and mothers are adviced to stick to dietary taboos which most often pose risks to their health and the health of the baby.

In order to create required maternal health outcomes, the root causes of cultural practices, beliefs and norms must be addressed to ensure the uptake and acceptance of health projects, programmes and policies by all members of the society. All stakeholders must be made to understand the implications of some of the cultural practices, traditions, beliefs and norms that are harmful to maternal and child health and thus, must be encouraged to continue with more useful ones that promote good health and development. Though some few cultural practices, tradi- 
tions, beliefs and norms such as male circumcision have been found to have some health benefits (e.g. prevention of HIV/AIDS infection), it can be concluded that many of them are detrimental to maternal health in Atwima Nwabiagya District and thus, some people characterize them as curses.

\section{Recommendation}

Health education programmes in the district must be expanded to include cultural issues so as to create community awareness of the health implications of some of the practices and beliefs. Pregnant and lactating mothers and all the other stakeholders must be taught about the negative impact of food taboos and the importance of a healthy diet during partum and postpartum period. Additionally, women should follow hospital guidelines more strictly. Health practitioners training curricula must be revised to embrace health-related cultural practices in the country to ensure that health practitioners will understand the critical issues in the communities in order to adopt and implement positive practices and develop strategies to discourage the harmful ones.

Also the government must train health practitioners from the communities and ensure that knowledge will be transferred to the communities. People in this setting tend to understand and appreciate the advices of people who have already experienced the same conditions before. Further, more women must be trained as health practitioners and be assigned to handle antenatal and postnatal cases in the district. Health practitioners must be provided with interpersonal training and communication skills development to improve interpersonal relation with the patients. Health education outreach programmes must disseminate health information in a dialect which is understood by all communities in the region.

The "barefoot doctor approach" must be adopted to ensure that health personnel are in constant contact with the communities. All stakeholders capable of influencing women in healthcare-seeking decision making must be brought on board to tackle the problem of the health-related cultural practices and beliefs. They must also be involved in the health education processes. The efficacy and effectiveness of some of the traditional medicines and methods need to be studied, since many of the women who participated in the study, not excluding the educated women, attested that they were effective. The government must incorporate health education and cultural issues in the general school curricula starting from the basic school to the university. 


\section{References}

Arhin, J.Y.K. (1996). The management of pregnancy in a rural community: A case study of Anyanam. Unpublished MPHIL Thesis. Department of Sociology, University of Ghana.

Atim, C. (Ed) (2001). A survey of health financing schemes in Ghana. PHR plus.

Browner, C.H. \& Sargent C F (1990). Anthropology and studies of human reproduction. In (T.M. Johnson and C.E. Sargent, eds.) Medical Anthropology: Contemporary Theory and Method, pp. 215-229. Praeger Publishers, New York.

Bour D (2003) Analysing the primacy of distance in the utilisation of health services in the Ahafo-Ano South District, Ghana. International Journal of Health Planning and management, 18:293-311.

Bourdieu P (1984) Distinction : A social critique of the judgement taste, MA: Harvard University Press, Cambridge.

Darko, M.O. (1992). Taboos on women: A case study of Akuapim traditional area. Unpublished B.A. dissertation. Department of Sociology, University of Ghana.

District Profile (2009). Atwima Nwabiagya District, Ashanti.

Hogan, C. M., Foreman, J. K., Naghavi, Ahn, Y. S ., Wang, M., Makela, M. S., Lopaz, A. D., Lozano, R \& Murray, J. L. Y. (2010). Maternal mortality for 181 countries, 1980-2008: A systematic analysis of progress towards millennium development goal 5. The Lancet, 375: 1609-163.

Initiative for Maternal Mortality Programme Assessment (2008). Extending coverage of maternal healthcare through the National Health Insurance Authority in Ghana: Lessons from the IMMPACT evaluation of the delivery care policy.

International Conference on Population and Development (1994). Cairo Egypt. Summary of the Programme of Action.

Maine, D.A., Rosenfield, A., Wallace, A.M., Kimball, B., Kwast, E., Papierniek \& White, S. (2001).Presentation of maternal deaths in developing countries: Program options and practical considerations. Paper presented at the International Safe Motherhood Conference, Nairobi.

Maine D (1993) Safe Motherhood Programmes: Options and issues. Centre for Population and Family Health and School of Public Health, Faculty of Medicine, Columbia University New York. 
National Population Council (2011). Ghana population stabilization report. Ohio State University (2009). Nutritional needs of pregnancy and breastfeeding. Family and consumer facts sheet. HYG-5573-09.

Okine, V. (2012). Moving from promises to action to improve maternal health. Ghana web, health news.

Rosenfield, A \& Maine, D. (1985). Maternal mortality: A neglected tragedy: where is the $\mathrm{M}$ in MCH? Lancet, 2 (8446): 83-85.

Safe Motherhood Initiative (1989). Critical issues. Reproductive health matters

Sargent, C. and J. Rawlins 1991. Factors influencing prenatal care among lowincome Jamaican women. Human Organization, 50: 179-187.

Senah, K.A. (2003). Maternal mortality in Ghana: The other side. Research Review NS 19.1; 47-55-(1993). The socio-cultural context of infant/Child morbidity and mortality in Kessena Nankana District, unpublished report for IDRC/ Navorango Health Research Center.

Tylor, E.B. (1871). Primitive Culture: Researches into the development of mythology and philosophy, religion, art, and customs. Volume 1 (Google eBook), reissued by Cambridge University Press (2010).

UNICEF, WHO, New Economic Partnership for African Development Bank of Southern Africa, Micro nutrient initiative \& global alliance for improved nutrition.(2004).Vitamins and minerals deficiency. A partnership to end hidden hunger in Sub-Saharan Africa. New York, USA.

United Nations Children's Emergency Fund \& World Health Organization (2004). Antenatal care in developing countries: promises, achievements and opportunities: an analysis of trends and differentials.

United Nations Population Fund. (2011). The global programme to enhance reproductive health commodity security. Annual Report.

Witter, S., Arhinful, D.K., Kusi, A., Zakariah-Akoto, S. (2007). The experience of Ghana in implementing a user fee exemption policy to provide free delivery care. Reprod. Health Matters, 15: 61-71.

WHO, UNICEF, UNDPA\& World Bank (1990-2010). Trends in maternal mortality. 


\section{Infectious Diseases}





\section{Pregnancy-Related Infections}

Uwe Groß, Institute of Medical Microbiology, German Consulting Laboratory for

Toxoplasma, University Medical Center Goettingen, Georg-August-University, Germany

\section{Introduction}

A general dilemma of pregnancy-related infections is the fact that most of them are either clinically asymptomatic or uncharacteristic in the pregnant mother, but pose a high or even fatal risk for the foetus or neonate.

Based on the time and route of transmission to the foetus, pregnancy-related infections are classified as either prenatally (via the placenta), perinatally (during delivery) or postnatally (after birth) acquired (Figure 1). Although several different bacteria, viruses and parasites can cause these infections, it has generally been accepted to use the abbreviation TORCH (infections caused by Toxoplasma gondii, rubella virus, cytomegalovirus (CMV), and herpes simplex virus; HSV) for some of the most serious infections that may cause embryo- or foetopathy following diaplacental infection. In addition to these, varicella-zoster virus (VZV), parvovirus B19, human immunodeficiency virus (HIV), and the bacterial species Treponema pallidum and Listeria monosytogenes can cause prenatal infections of the foetus or embryo. In most cases, clinical symptoms of prenatal infections are present in utero, 
at birth or within the first days of life; however, some infections (e.g. toxoplasmosis) may manifest at much later age.

Perinatal infections are especially caused by the bacterial species Listeria monozytogenes, Streptococcus agalactiae (group B streptococci; GBS), Escherichia coli, Neisseria gonorrhoeae, and Chlamydia trachomatis. In addition, hepatitis B and $\mathrm{C}$ virus (HBV, HCV), herpes simplex virus 2 (HSV-2), human papillomavirus (HPV), enteroviruses, and CMV may lead to infection of the newborn during the perinatal period (Figure 1).

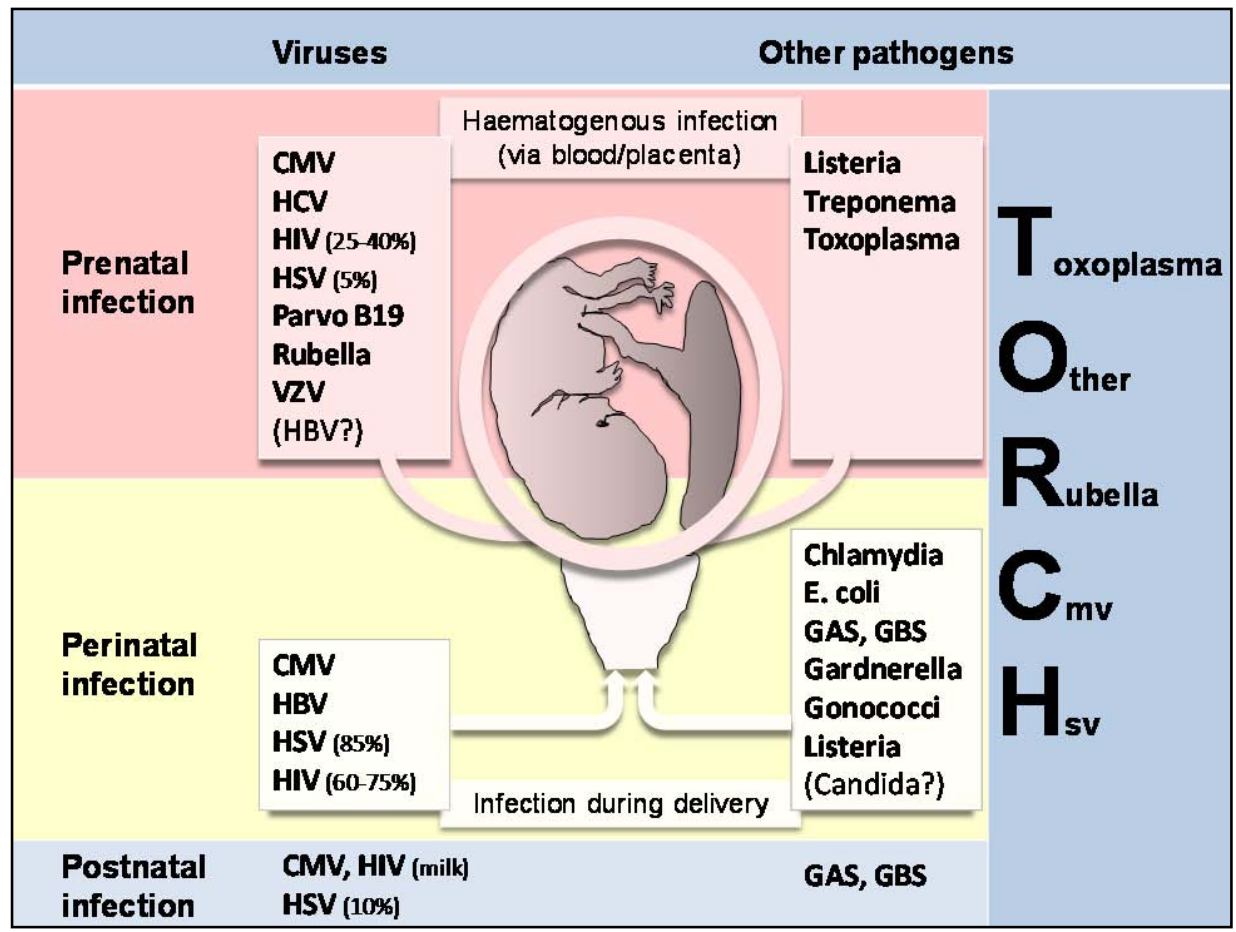

Figure 1: Overview of pregnancy-related infections.

In addition to the above mentioned pathogens, several other infectious agents have to be considered to be a risk for the foetus and newborn infant (Table 1).

Prenatally acquired infections are further classified into those causing embryopathy and those causing foetopathy:

Embryopathy: prenatal infection acquired during the first trimester leading to intrauterine disorders of the embryo during the development of organs

Foetopathy: prenatal infection acquired after the third month of pregnancy leading to intrauterine disorders of the foetus after the development of organs has been completed. 
Some of the most important pregnancy-related infections, such as those caused for instance by CMV, HBV, C. trachomatis or GBS, will also be presented in more detail in other

Table 1: Clinical symptoms, diagnosis and therapy of pregnancy-related infections

\begin{tabular}{|c|c|c|c|c|}
\hline Pathogen & $\begin{array}{l}\text { Risk during } \\
\text { pregnancy }\end{array}$ & $\begin{array}{l}\text { Symptoms of the } \\
\text { infected child }\end{array}$ & Diagnosis & Therapy \\
\hline CMV & $\begin{array}{l}\text { entire preg- } \\
\text { nancy, post- } \\
\text { natal (milk) }\end{array}$ & $\begin{array}{l}\text { in most cases no } \\
\text { symptoms, but } \\
\text { severe disease } \\
\text { possible (hepatitis, } \\
\text { CNS) }\end{array}$ & $\begin{array}{l}\text { mother and } \\
\text { child: } \\
\text { serology } \\
\text { (IgM), } \\
\text { PCR from } \\
\text { amniotic } \\
\text { fluid }\end{array}$ & $\begin{array}{l}\text { in pregnancy: hy- } \\
\text { perimmunoglobulin } \\
\text { newborn: ganciclovir, } \\
\text { valganciclovir }\end{array}$ \\
\hline $\mathrm{HBV}$ & perinatal & $\begin{array}{l}\text { chronic infection, } \\
\text { eventually late } \\
\text { manifestations }\end{array}$ & $\begin{array}{l}\text { HBsAG / } \\
\text { PCR from } \\
\text { blood }\end{array}$ & $\begin{array}{l}\text { mother: evt. lamivu- } \\
\text { din or tenofovir, } \\
\text { child: active/passive } \\
\text { immunization }\end{array}$ \\
\hline HIV & $\begin{array}{l}\text { perinatal: } \\
(60-75 \%) \\
\text { prenatal: } \\
(25-40 \%) \\
\text { postnatal: } \\
(10-15 \%)\end{array}$ & $\begin{array}{l}\text { mostly no symp- } \\
\text { toms at birth }\end{array}$ & $\begin{array}{l}\text { mother and } \\
\text { child: PCR } \\
\text { from blood, } \\
\text { serology } \\
(\mathrm{AG} / \mathrm{AB})\end{array}$ & $\begin{array}{l}\text { mother: e.g. combivir } \\
\text { (= Zidovudin }+ \\
\text { Lamivudin) } \\
\text { + lopinavir } \\
\text { child: zidovudin }\end{array}$ \\
\hline $\mathrm{HSV}$ & $\begin{array}{l}\text { perinatal } \\
(85 \%), \text { post- } \\
\text { natal }(10 \%), \\
\text { prenatal }(5 \%)\end{array}$ & $\begin{array}{l}\text { neonatal herpes } \\
\text { encephalitis }\end{array}$ & $\begin{array}{l}\text { PCR from } \\
\text { vesicles }\end{array}$ & acyclovir \\
\hline $\begin{array}{l}\text { Parvovirus } \\
\text { B19 }\end{array}$ & $\begin{array}{l}\text { entire preg- } \\
\text { nancy }\end{array}$ & $\begin{array}{l}\text { hydrops foetalis in } \\
\text { utero, miscarriage } \\
\text { or healthy new- } \\
\text { born }\end{array}$ & $\begin{array}{l}\text { mother and } \\
\text { child: se- } \\
\text { rology, } \\
\text { (PCR from } \\
\text { blood) }\end{array}$ & $\begin{array}{l}\text { none, } \\
\text { eventually intrauterine } \\
\text { blood exchange }\end{array}$ \\
\hline Rubella & $\begin{array}{l}\text { entire preg- } \\
\text { nancy, high } \\
\text { risk in } 1-17 \\
\text { wop }\end{array}$ & $\begin{array}{l}\text { organ malfor- } \\
\text { mation: heart, eye, } \\
\text { ear, brain } \\
\text { (= Gregg syn- } \\
\text { drome) }\end{array}$ & $\begin{array}{l}\text { mother: } \\
\text { IgM } \\
\text { child: PCR } \\
\text { from urine; } \\
\text { serology } \\
\text { (IgM) }\end{array}$ & $\begin{array}{l}\text { none, } \\
\text { (abortion during first } \\
12 \text { wop?) }\end{array}$ \\
\hline
\end{tabular}




\begin{tabular}{|c|c|c|c|c|}
\hline Pathogen & $\begin{array}{l}\text { Risk during } \\
\text { pregnancy }\end{array}$ & $\begin{array}{l}\text { Symptoms of the } \\
\text { infected child }\end{array}$ & Diagnosis & Therapy \\
\hline VZV & $\begin{array}{l}\text { 1-2 trimester } \\
\text { \& perinatal }\end{array}$ & $\begin{array}{l}\text { limb malfor- } \\
\text { mations, skin } \\
\text { scarifications, } \\
\text { neonatal chicken } \\
\text { pox }\end{array}$ & $\begin{array}{l}\text { mother \& } \\
\text { child: PCR } \\
\text { from vesi- } \\
\text { cles; child: } \\
\text { AB persist }\end{array}$ & $\begin{array}{l}\text { acyclovir, } \\
\text { hyperimmunoglobulin }\end{array}$ \\
\hline $\begin{array}{l}\text { Chlamydia } \\
\text { trachomatis }\end{array}$ & perinatal & $\begin{array}{l}\text { inclusion conjunc- } \\
\text { tivitis; late-onset } \\
\text { pneumonia }\end{array}$ & AG, PCR & macrolides \\
\hline GBS & $\begin{array}{l}\text { perinatal, } \\
\text { especially } \\
\text { preterm deliv- } \\
\text { eries }\end{array}$ & $\begin{array}{l}\text { early onset: sepsis } \\
\text { late onset: menin- } \\
\text { gitis }\end{array}$ & $\begin{array}{l}\text { mother: } \\
\text { culture } \\
\text { from vagi- } \\
\text { na, } \\
\text { child: cul- } \\
\text { tures from } \\
\text { blood, CSF } \\
\text { etc. }\end{array}$ & penicillin or ampicillin \\
\hline $\begin{array}{l}\text { Listeria } \\
\text { monocytogenes }\end{array}$ & $\begin{array}{l}\text { entire preg- } \\
\text { nancy \& peri- } \\
\text { natal }\end{array}$ & $\begin{array}{l}\text { granulomatosis } \\
\text { infantiseptica, } \\
\text { sepsis, meningitis, } \\
\text { hepatitis }\end{array}$ & $\begin{array}{l}\text { mother and } \\
\text { child: cul- } \\
\text { ture from } \\
\text { vagina, } \\
\text { amniotic } \\
\text { fluid etc. }\end{array}$ & amoxicillin/ampicillin \\
\hline $\begin{array}{l}\text { Neisseria } \\
\text { gonorrboae }\end{array}$ & perinatal & $\begin{array}{l}\text { neonatal ophthal- } \\
\text { mitis }\end{array}$ & $\begin{array}{l}\text { culture } \\
\text { from eye }\end{array}$ & $\begin{array}{l}\text { mother: penicillin/ } \\
\text { cephalosporin }\end{array}$ \\
\hline $\begin{array}{l}\text { Treponema } \\
\text { pallidum }\end{array}$ & $\begin{array}{l}\text { high risk: } \\
\text { syphilis II \& } \\
>18 \text { wop }\end{array}$ & $\begin{array}{l}\text { rash, bloody rhini- } \\
\text { tis; } \\
\geq 2 \text {. year of life: } \\
\text { Hutchinson trias }\end{array}$ & $\begin{array}{l}\text { child: IgM } \\
\text { positivity } \\
\text { until } 3 \\
\text { month of } \\
\text { life }\end{array}$ & penicillin $G$ \\
\hline $\begin{array}{l}\text { Toxoplasma } \\
\text { gondii }\end{array}$ & $\begin{array}{l}\text { entire preg- } \\
\text { nancy } \\
\text { high risk of } \\
\text { transmission: } \\
\geq 2 \text { trimester }\end{array}$ & $\begin{array}{l}\text { retinochoroiditis, } \\
\text { intracerebral cal- } \\
\text { cifications, hydro- } \\
\text { cephalus }\end{array}$ & $\begin{array}{l}\text { serology } \\
(\operatorname{lgM}, \lg \mathrm{A}), \\
\text { PCR from } \\
\text { amniotic } \\
\text { fluid }\end{array}$ & $\begin{array}{l}<16 \text { wop: spiramycin, } \\
\geq 16 \text { wop: sulfadiazine } \\
+ \text { pyrimethamine }+ \\
\text { folinic acid }\end{array}$ \\
\hline
\end{tabular}

Abbreviation: wop $=$ week of pregnancy, $A B=$ antibody, $A G=$ antigen, $C S F=$ Cerebrospinal fluid

chapters of this book. It should generally be advised to screen women late in pregnancy ( $>32^{\text {nd }}$ week of pregnancy) for the presence of $\mathrm{HBs}$ antigenaemia; children 
of HBs-antigen-positive women should then be vaccinated within the first hours of life to prevent them from developing chronic hepatitis.

\section{Infections with the human immunodeficiency virus (HIV)}

Since its discovery in 1981, HIV has become a pandemic that not only killed thousands of people but also changed daily life. Although incidence rates have decreased during the last years, worldwide and at the moment, roughly 34 million persons live with HIV. With more than 22 million, the vast majority of infected persons live in sub-Saharan Africa followed by some 4 million affected persons in south and south-east Asia (1). However, seroprevalence rates between countries can vary extremely. For instance, a study performed in 2003 did not reveal a single HIV-seropositive case among 2,450 pregnant women in Bali, Indonesia (2). Therefore and based on the high prevalence rates in sub-Saharan Africa, it is especially there that treatment during pregnancy has resulted in a so to speak dramatic decline of peripartal infections of the newborns. However, still more than 1.5 million persons die of the acquired immunodeficiency syndrome (AIDS) each year, and the total death toll has reached more than 30 million persons since 1981 (1).

Our current knowledge indicates that HIV originated from several primate-tohuman species barrier jumping events. HIV is an enveloped RNA virus belonging to the retrovirus family and exists in two major lineages; HIV-1 and HIV-2. Whereas HIV-1 is worldwide distributed, HIV-2 is predominantly found in West Africa.

Both types of viruses are primarily transmitted through sexual contact or via blood. Several lines of evidence show that female sex workers were the main drivers for the spread of HIV through heterosexual contacts in Africa, whereas men who have sex with men (MSM) continue to be the major risk group in the northern hemisphere, especially USA and Europe. At risk for HIV infection are also injection drug users (IDU), especially those living in countries of southern Europe, the former Soviet Union and countries in south and south-east Asia (3). In regards to pregnancy-related infections, it should be noted that HIV-2 is less frequently transmitted from mothers to their children (4). However, breastfeeding poses an additional risk of transmission to the child, which can even increase when performed for prolonged times as it is usual in most countries of sub-Saharan Africa (5). When summing up prenatal, perinatal and postnatal transmission, the overall transmission risk from untreated mothers to their children is calculated to be 30 45\% (6). Approximately 2.5 million children worldwide are currently living with $\mathrm{HIV} / \mathrm{AIDS}$, the vast majority of them in sub-Saharan Africa with an incidence rate of 330,000 new infections each year (7).

If not treated, infection with HIV generally causes subsequent immunodeficiency whereby several opportunistic infections evolve which finally could be lethal. Although the member states of the United Nations are aiming in the Millennium De- 
velopment Goals (MDG) "to have halted by 2015 and begun to reverse the spread of HIV/AIDS" and "to achieve, by 2015, universal access to treatment for HIV/AIDS for all those who need it" (8), the reality is rather disillusioning: whereas the availability of highly active antiretroviral therapy (HAART) in most countries of the northern hemisphere has turned AIDS into a chronic rather than lifethreatening disease, still a major proportion of low-income and middle-income countries face a lack of appropriate diagnosis and treatment of HIV/AIDS for all those who need it (7).

This is a tragedy, particularly because of the fact that prenatal or perinatal infection and thus, the risk for a child of a HIV-positive mother to get the disease can be reduced from $45 \%$ to less than $5 \%$ if treated. Even more dramatic is the fact that approximately $50 \%$ of $\mathrm{HIV}$-infected children die by the age of 2 years if left untreated (9). It is therefore an urgent need to identify and treat those pregnant women who need antiretroviral therapy (ART) for their own health.

The diagnosis of HIV infection is based on the detection of specific antibodies, HIV antigen (e.g. p24) or HIV RNA by using PCR from the blood. Although screening tests that simultaneously detect HIV antigen and antibodies are available even in low- and middle-income countries, the overall coverage rate still depends on the accessibility to antenatal care. Own data from a rural hospital in Ghana indicates a HIV testing coverage of nearly $90 \%$ if the pregnant women have access to antenatal care (Völker et al., unpublished data). This is in sharp contrast to the reported $26 \%$ of HIV testing coverage of pregnant women in low- and middleincome countries from 2009 (10). Likewise, financial costs and logistics have also prevented satisfying coverage rates of HIV diagnosis in infants who are at risk (7). In many low- and middle-income countries an important additional limitation is the availability of equipment for determining $\mathrm{CD}^{+} \mathrm{T}$ cell counts. Therefore, and in contrast to high-income countries, the treatment of HIV-infected pregnant women does not consider viral load or $\mathrm{CD}^{+} \mathrm{T}$ cell counts.

In most cases, treatment for maternal health should start immediately when HIV-infected pregnant women have either symptoms that are indicative for AIDS (WHO clinical stage 3 or 4 ) or if $\mathrm{CD}^{+} \mathrm{T}$ cell counts are $<350 / \mu \mathrm{l}(10)$. In asymptomatic pregnant women with $\mathrm{CD}^{+} \mathrm{T}$ cell counts $>350 / \mu \mathrm{l}$ or those with rather untypical symptoms (WHO clinical stage 1 or 2 ) and $\mathrm{CD}^{+} \mathrm{T}$ cell counts $>350 / \mu \mathrm{l}$, ART prophylaxis is not considered for their own health but for preventing mother-to-child transmission of HIV. In most African countries, initiation of therapy for these groups of HIV-infected pregnant women starts from the 14th week of pregnancy and should also be continued during delivery and breastfeeding. The current prophylactic scheme in most African countries consists of either zidovudine monotherapy or HAART (zidovudine + lamivudine (3TC) + either lopinavir/ritonavir, abacavir or efavirenz, respectively). The current British Guidelines recommend elective caesarian section for those women who are under zidovudin monotherapy. In addition, if viral loads can be determined, elective caesarian section is also justified at $>50$ viral copies $/ \mathrm{ml}$ at $36^{\text {th }}$ week of pregnancy (reviewed in 
12). The HAART scheme for asymptomatic HIV-infected pregnant women with CD4+ T cell counts of $>350 / \mu \mathrm{l}$ is similar in high-income countries such as Germany where the initiation of therapy also depends on the viral load and therapy usually starts at the $24^{\text {th }}$ or $28^{\text {th }}$ week of pregnancy (13). Together with caesarian section in the $36^{\text {th }} / 37^{\text {th }}$ week of pregnancy, this strategy has reduced the motherto-child transmission rate of HIV to $1-2 \%$.

Nevirapine or/and zidovudine for 4-6 weeks is the current prevention measure for the child. Infected children who were exposed to maternal or infant prevention/treatment schemes should receive a combination of 2 nucleoside reverse transcriptase inhibitors (NRTIs) plus a protease inhibitor; those who were not exposed to maternal or infant prevention/treatment schemes, should receive a combination of 2 NRTIs plus 1 non-nucleoside reverse transcriptase inhibitor (NNRTI) (reviewed in 7).

\section{Neonatal herpes simplex}

Herpes simplex virus (HSV) infection in the adult typically occurs with multiple vesicular lesions either at the orofacial area (HSV-1) or in the genital tract (HSV-2; HSV-1 with increasing frequency). Prenatal transmission is a rather rare event. It can occur (i) when primary infection during pregnancy leads to viraemia or (ii) when primary infection of the genital area ascends and reaches the foetus. With 30$80 \%$ infected pregnant women, HSV-2 is more prevalent in sub-Saharan Africa than in high-income or in Asian countries (10-30\%) (reviewed in 14).

Most problematic, however, is perinatal infection that may occur when primary genital infection with HSV-2 (less common HSV-1) is present at the time of delivery. It is not unusual that genital herpes remains undiagnosed because clinical symptoms may be only mild or even uncharacteristic. Since the newborn is not protected by maternal antibodies at this stage, HSV can easily disseminate into different organs resulting in either (i) superficial localized neonatal herpes (affecting only skin, eyes and mouth without involvement of internal organs), (ii) disseminated neonatal herpes (affecting also several internal organs, especially the liver), or (iii) central nervous system (CNS) neonatal herpes. The overall death rate without therapy is $65 \%$, but even treatment cannot prevent most infants with CNS neonatal herpes to develop abnormally. If the pregnant mother has recurrent HSV2 infection at the time of delivery, the infant is usually protected from viraemia by diaplacentally transferred maternal $\operatorname{IgG}$ antibodies. However, even in this case the virus is still able to disseminate within the axons finally resulting in life-threatening encephalitis of the infant. Therefore, caesarian section or prenatal acyclovir treatment is necessary if the mother has active genital herpes (primary infection as well as recurrent episodes) at the time of delivery. Although being relatively rare, postnatal HSV infection occurs especially when infants born by non-infected mothers have contact with family members or health care workers who have active orofa- 
cial HSV-1 infection. Irrespective of the mode of transmission, acyclovir is the treatment of choice for infected infants suffering from neonatal herpes simplex.

Since up to $40 \%$ of the infected neonates have no characteristic vesicular skin lesions (15), diagnosis and prevention of active maternal infection is most important. In the majority of cases, diagnosis in the adult patient relies on the identification of typical vesicular skin lesions. If symptoms are uncertain, laboratory diagnosis of primary or reactivated infection is achieved by direct detection of viral DNA using PCR from skin lesions. Although the detection of specific IgM antibodies in the serum of the patients is indicative for active infection, only true seroconversion confirms primary infection. However, most important is prevention of the acquisition of HSV infection, especially in late pregnancy. In this case, serological screening is helpful in order to identify HSV-seronegative women, who are advised to avoid unprotected sex or oral contact with actively HSV-infected partners in late pregnancy.

\section{Infections with varicella zoster virus (VZV)}

Viraemia as a prerequisite for prenatal transmission of VZV from the pregnant woman to the foetus occurs only when following a primary infection but not in cases of endogenous reactivation of a former infection. Since the vast majority of women become already infected before conception, less than $5 \%$ of all pregnancies are at risk for acquiring primary infection. Since viraemia of the mother begins before she develops typical skin lesions, diagnosis usually comes too late to prevent diaplacental VZV transmission. Transmission in early pregnancy may result in embryopathy; this so-called congenital varicella syndrome is characterized by lesions of the skin, eyes, CNS and skeleton (malformation of the limbs).

Even more dangerous is the situation when the mother acquires infection in the perinatal period. In this case, the newborn develops severe neonatal chicken pox between the $1^{\text {st }}$ and $10^{\text {th }}$ day of life that might be accompanied by pneumonia and has a mortality rate of $30 \%$.

The clinical presentation is usually guiding the diagnosis; detection of viral DNA using PCR from skin lesions might be useful to confirm the diagnosis in the mother and the infant, especially in atypical cases. Persistence of specific antibodies over the first months of life is another diagnostic indication for an infection of the infant.

Following exposure and if available, seronegative pregnant women should receive passive immunization with VZV-specific immunoglobulins (VZIG) as soon as possible (within $48 \mathrm{hrs).} \mathrm{If} \mathrm{VZIG} \mathrm{is} \mathrm{not} \mathrm{available,} \mathrm{oral} \mathrm{acyclovir} \mathrm{should} \mathrm{be} \mathrm{given}$ as post-exposure prophylaxis (16). The same immune prophylaxis should be applied to newborns of mothers who have acquired chicken pox during the period of 7 days before delivery until 2 days after birth. 


\section{Rubella embryopathy}

Most people worldwide acquire rubella virus infection in childhood. Nevertheless, before the introduction of vaccination, rubella virus had been a threat for prenatal infections. Nowadays approximately $90 \%$ of women in high-income countries are seropositive before becoming pregnant. A much smaller proportion of pregnant women is protected from primary infection in countries of sub-Saharan Africa or Southeast Asia, e.g. approximately $53-65 \%$ in Nigeria and Sudan $(17,18)$ or $70-90 \%$ in China, Vietnam, Indonesia or the Philippines (19). In 2008 it has been estimated that worldwide more than 110,000 children are born with congenital rubella syndrome annually making this infectious disease a leading cause of preventable congenital defects (20).

Diaplacental transmission is common when primary infection takes place during the $1^{\text {st }}$ trimester of pregnancy and then often results in abortion of the embryo. An additional $30-50 \%$ of those infected children develop rubella embryopathy with an overall mortality rate of $20 \%$. Typical for infections at this stage of embryonic development are organ malformations which are clinically overt before the $5^{\text {th }}$ months of life and which are termed GREGG syndrome. It affects the eyes (retinopathy, cataract, microphthalmus), ears (sensorineural hearing loss or deafness), heart (pulmonary stenosis, defect of the ventricular septum, Ductus Botalli remains open etc.), and the CNS (microcephaly). In addition, symptoms such as low birth weight or hepatosplenomegaly could occur as well. The late-onset rubella syndrome manifests between the $4^{\text {th }}$ and $6^{\text {th }}$ months of life and is characterized by zero growth with exanthema and pneumonia. Laboratory diagnosis of prenatal infection of the child is rather based on a positive PCR result obtained from amniotic fluid and foetal blood or on the detection of specific IgM antibodies in the newborn or infant.

Viraemia occurs before the typical rash is present in the infected patient, posing a substantial risk for undetected prenatal transmission during pregnancy. The strategy to combat this problem is to screen women before they become pregnant. Non-immune women should then be actively vaccinated before becoming pregnant with a life-attenuated vaccine that usually combines measles, mumps, and rubella (MMR). If a non-immune pregnant woman is exposed to rubella virus, and if available, she might get protected by receiving specific hyperimmunoglobulins.

In most high-income countries, MMR vaccination early in childhood is generally recommended for the entire population. Since measles and rubella still pose high risks, especially for populations living in sub-Saharan Africa and SoutheastAsia, the WHO together with other stakeholders has set-up a program to achieve measles and rubella elimination in at least five WHO regions by the year 2020 (20). 


\section{Congenital syphilis}

The sexually transmitted disease syphilis (syn. lues) is caused by the bacterium Treponema pallidium ssp. pallidum. In the adolescent or adult patient, the disease manifests in stage 1 locally most often in the genital tract as an ulcus with regional lymphadenitis. After some months, it disseminates haematogenously causing systemic infection. The stage 2 of the disease is characterized by an exanthema and enanthema and it is posing a major risk for diaplacental transmission to the foetus. Finally, after some years, the disease might proceed to stage 3 which is characterized mainly by gummata, neurosyphilis, and psychosis.

As mentioned above, congenital syphilis might occur when the pregnant woman experiences stage 2 of the disease in late pregnancy, especially within the last trimester. Approximately 50\% of the cases result in miscarriage or either in earlyor late-onset congenital syphilis. The early-onset form has a poor prognosis and already manifests at the time of birth, causing newborns suffering from exanthema, enanthema, hepatosplenomegaly and bleeding of the lungs. The late form of the disease manifests between the $5^{\text {th }}$ and $25^{\text {th }}$ year of life and is characterized by typical symptoms of congenital syphilis: deformation of the front teeth and the limbs, peculiar form of the nose, keratitis, sensorineural hearing loss, and rhagades.

Laboratory diagnosis of syphilis is mostly based on serological tests. For this, the rapid plasma test (RPR test) is in use in most low- and middle-income countries. This test is measuring antibodies directed against lipoid-containing antigens from mitochondria which are exposed during tissue damage caused by T. pallidium. Penicillin is a treatment of choice for both the pregnant woman and the newborn. The efficacy of treatment can be monitored by the RPR test which should significantly decrease or even become negative upon successful treatment.

\section{Neonatal sepsis with group-B streptococci (GBS)}

It is estimated that sepsis is responsible for $10 \%$ of maternal deaths and $26 \%$ of neonatal deaths (21). Irrespective of the economic situation of a country, one of the most prevalent bacterial causes of neonatal sepsis are group-B streptococci (GBS). As an example, approximately 10-15\% of all pregnant women in Germany or $13-22 \%$ of those living in countries of sub-Saharan Africa are asymptomatic carriers of these bacteria which are also termed Streptococcus agalactiae (21).

If no prophylactic antibiotics are given during the time of delivery the child may get infected and develops early-onset sepsis within the first 5-8 days of life. Severe signs of sepsis are often accompanied by meningitis and pneumonia. Mortality is high and may reach more than 50\%. Late-onset sepsis is resulting from either perinatal or postnatal infection and is less common $(<2$ cases $/ 1000$ live births in high-income countries). It usually develops starting from the $2^{\text {nd }}$ week of life and is characterized by meningitis with a relatively good prognosis. 
Carriers among pregnant women can principally be identified by culturing GBS from vaginal/anal swabs. Positive women should then receive intrapartum chemoprophylaxis with either penicillin or ampicillin. In resource-poor settings, an alternative approach based on common risk factors for neonatal sepsis (e.g. prematurity, premature rupture of membranes, maternal fever) might be more feasible for the decision-making process of whether to start chemoprophylaxis or not. (21). Infection of the newborn can also be confirmed by culture from respective specimen (e.g., blood, cerebrospinal fluid or urine). Either penicillin or cephalosporins, respectively, are the antibiotics of choice for treating infected newborns.

\section{Listeriosis}

Listeria monocytogenes is found in the environment and may infect food animals as well as humans. Especially the consumption of raw milk and milk products, such as cheese, poses a major risk for temporary colonization or infection of humans who - if immunocompetent - usually do not experience clinical symptoms. If $L$. monocytogenes invades the epithelial layer of the intestine, bacteriaemia with subsequent diaplacental transmission to the embryo or foetus is possible. Since the bacteria are shed through the faeces, inadequate body hygiene might favor contamination of the female genital tract, eventually resulting in perinatal infection of the child as well. So far, only very few data is available from resource-poor countries on listeriosis acquired during pregnancy. Depending on the route of transmission, one has to differentiate between the following types of listeriosis of the child.

Early-onset neonatal listeriosis: This type of infection results from prenatal transmission to the embryo or foetus. Infection of the embryo in early pregnancy most often leads to septic miscarriage whereas foetal infection in late pregnancy results in septic preterm birth of a severely affected child who suffers from hepatosplenomegaly, pneumonia, and systemic multiple granulomas in the lungs, CNS and skin.

Late-onset neonatal listeriosis: The development of meningitis within the $2^{\text {nd }}$ to $5^{\text {th }}$ week of life is a typical clinical manifestation of perinatal infection of the newborn.

Since listeriosis acquired during pregnancy is often accompanied by either no or only a few uncharacteristic symptoms in the pregnant women, diagnosis is only possible by cultivating $L$. monocytogenes from swabs taken from the vagina or from the rectum. In contrast, symptoms of the newborn are quite typical and should lead to diagnosis by bacterial cultivation from blood culture, cerebrospinal fluid, skin swabs or stool samples, respectively.

Avoidance of the consumption of raw milk or milk products would be most efficient for preventing exposure to listeria. In this regards, it should be noted that L. monocytogenes are even able to replicate at refrigerator temperatures of $4-7^{\circ} \mathrm{C}$. Infection of the mother during pregnancy and of the newborn should be treated for 
at least two weeks with ampicillin. It should be noted that cephalosporins are not effective against L. monocytogenes.

\section{Congenital toxoplasmosis}

The protozoan parasite Toxoplasma gondii is shed with the feces of its definite hosts which are felides (cats, lions, tigers) and can persist in soil for several months if not years. Nearly all mammals and many other animals can also become infected and harbor the parasite as persistent cyst stages, especially within their CNS and muscle tissue. Infections of humans therefore result either from contact with cat feces, contaminated soil or from ingesting raw cyst-containing meat products.

It is estimated that one quarter to one third of the world's human population is infected with T. gondii, however the seroprevalence rates differ significantly between countries. In almost $95 \%$ of all cases, infection of the immunocompetent adolescent or adult patient proceeds without clinical symptoms; approximately 5\% may develop cervical lymphadenitis. Following primary infection, the parasite persists lifelong, especially in the brain without causing symptoms in the immunocompetent individual. However, immunodeficiency such as AIDS might later induce reactivation of latent $T$. gondii infection, resulting in life-threatening toxoplasmic encephalitis.

If an infection had already occurred before pregnancy, the child is usually protected from getting infected. However, primary infection during pregnancy may allow diaplacental transmission in approximately $50 \%$ of all cases resulting in congenital toxoplasmosis of the child. Although an infection in early pregnancy has a low risk of diaplacental transmission, it can result in miscarriage of the child or in serious embryopathy. Prenatal infection in the $2^{\text {nd }}$ trimester mostly leads to foetal hydrocephalus, intracerebral calcifications or retinochoroiditis, respectively. Infections in late pregnancy have a high risk of diaplacental transmission with the consequence that most of the infected children remain clinically asymptomatic at birth. However, a large proportion of more than $50 \%$ of them may develop retinochoroiditis or seizures later in life if they are not treated within the first months after birth.

Diagnosis of primary infection during pregnancy is mainly based on serological detection of specific IgM or IgA antibodies. Since screening during pregnancy is not frequently performed in most countries irrespective of the given economic situation, true seroconversions are hardly identified and thus, making it difficult to suppose the exact time of infection in regards to the beginning of pregnancy. In addition to conspicuous ultrasound results performed in pregnancy (e.g. dilatation of ventricles) and indicative clinical symptoms at birth, congenital toxoplasmosis of the child is diagnosed by the detection of specific IgM or IgA antibodies and by the persistence or rise of IgG antibody titers over the first 6-12 months of life. 
Where available, pregnant women with primary infection should be treated with spiramycin until the $16^{\text {th }}$ week of pregnancy and thereafter with the combination of pyrimethamine plus sulfadiazine plus folinic acid (22). Infected newborns should also receive the combination therapy for their first 3-12 months of life.

\section{Conclusion}

Clinicians worldwide face a similar dilemma when dealing with pregnancy-related infections. Most of these infections do not cause typical symptoms in the pregnant women, however they have life-threatening impact on the health of the child. Therefore, the most reliable mean for ensuring timely diagnosis would be overall implementation of screening programs for pregnant women. However, the economic situation in nearly all countries worldwide has made a priorization necessary for only some of the pregnancy-related diseases. As a consequence, screening programs for congenital syphilis exist even in most low- and middle-income countries. In addition, at least in high-prevalence countries and irrespective of the economic situation, screening for malaria, group-B streptococci, HIV and HBs antigenaemia should be a matter of implicitness.

\section{References}

1. UNAIDS. http://www.unaids.org [Accessed February 15, 2013].

2. Surya IG, Kornia K, Suwardewa TG et al. 2005. Serological markers of hepatitis B, C, and E viruses and human immunodeficiency virus type-1 infections in pregnant women in Bali, Indonesia. J Med Virol 75: 499-503.

3. De Cock KM, Jaffe HW, Curran JW. 2012. The evolving epidemiology of HIV/AIDS. AIDS 26: 1205-1213.

4. Adjorlolo G, De Cock KM, Ekpini E, et al., 1994. Prospective comparison of mother-to-child transmission of HIV-1 and HIV-2 in Abidjan, Ivory Coast. JAMA 272: 462-466.

5. Dunn DT, Newell ML, Ades AE, Peckham CS. 1992. Risk of human immunodeficiency virus type 1 transmission through breast-feeding. Lancet 340: 585-588.

6. De Cock FM, Fowler MG, Mercier E et al. 2000. Prevention of mother-tochild HIV transmission in resource-poor countries. Translating research into policy and practice. JAMA 283: 1175-1182.

7. Kenny J, Mulenga V, Hoskins S, Scholten F, Gibb DM. 2012. The needs for HIV treatment and care of children, adolescents, pregnant women and older 
people in low-income and middle-income countries. AIDS 26 (Suppl 2): S105S116.

8. United Nations Millennium Development Goal 6- Combat HIV/AIDS, malaria and other diseases: http://www.un.org/millenniumgoals/aids.shtml [Accessed February 15, 2013].

9. Newell ML, Coovadia H, Cortina-Borja M, Rollins N, Gaillard P, Dabis F. 2004. Mortality of infected and uninfected infants born to HIV-infected mothers in Africa: a pooled analysis. Lancet 364: 1236-1243.

10. WHO Progress Report 2009 (with UNAIDS and UNICEF). Towards universal access. Scaling up priority HIV/AIDS interventions in the health sector: http://www.who.int/hiv/pub/tuapr_2009_en.pdf [Accessed February 15, 2013].

11. WHO HIV/AIDS Programme 2010. Antiretroviral therapy for HIV infection in adults and adolescents. Recommendations for a public health approach: http://www.who.int/hiv/pub/arv/adult2010/en/index.html [Accessed February 15, 2013].

12. Senise J, Bonafé S, Castelo A. 2012. The management of HIV-infected pregnant women. Curr Opin Obstet Gynecol 24: 395-401.

13. AWMF-Online. Deutsch-Österreichische Empfehlungen zur HIV-Therapie in der Schwangerschaft und bei HIV-exponierten Neugeborenen, DAIG Deutsche AIDS-Gesellschaft e.V.: http://www.awmf.org/leitlinien/detail/ll/055-002.html [Accessed February 15, 2013].

14. Anzivino E, Fioriti D, Mischitelli M et al. 2009. Herpes simplex virus infection in pregnancy and in neonate: status of art epidemiology, diagnosis, therapy and prevention. Virol J 2009 Apr 6;6:40. doi: 10.1186/1743-422X-6-40.

15. Jacobs RF. 1998. Neonatal herpes simplex virus infections. Semin Perinatol 22: 64-71.

16. Daley AJ, Thorpe S, Garland SM. 2008. Varicella and the pregnant woman: Prevention and management. Aust N Z J Obstet Gynaecol. 48: 26-33.

17. Hamdan HZ, Abdelbagi IE, Nasser NM, Adam I. 2011. Seroprevalence of cytomegaovirus and rubella among pregnant women in western Sudan. Virol J 2011 May 11;8:217. doi: 10.1186/1743-422X-8-217.

18. Onakewhor JU, Chiwuzie J. 2011. Seroprevalence survey of rubella infection in pregnancy at the University of benin Teaching Hospital, Benin City, Nigeria. Niger J Clin Pract 14: 140-145. 
19. Tseng HF, Chang CK, Tan HF, Yang SE, Chang HW. 2006. Seroepidemiology study of rubella antibodies among pregnant women from seven Asian countries: evaluation of the rubella vaccination program in Taiwan. Vaccine 24: 5772-5777.

20. WHO 2012. Global measles and rubella strategic plan 2012-2020: http://www.who.int/immunization/newsroom/Measles_Rubella_StrategicPla n_2012_2020.pdf [Accessed February 15, 2013].

21. Seale A, Mwaniki M, Newton CRJ, Berkley JA. 2009. Maternal and early onset neonatal bacterial sepsis: burden and strategies for prevention in sub-Saharan Africa. Lancet Infect Dis 9: 428-438.

22. Hotop A, Hlobil H, Groß U. 2012. Efficacy of rapid treatment initiation following primary Toxoplasma gondii infection during pregnancy. Clin Infect Dis 54: 1545-1552. 



\section{Malaria in Children and Adults - An Overview}

Raihan, Faculty of Medicine, Syiah Kuala University, Banda Aceh, and

Zainoel Abidin Hospital, Department of Child Health, Jln. Tgk. M. Daud Beureueh, Banda Aceh, Indonesia

\section{Introduction}

Malaria remains a major health problem in tropical areas of the world. Almost half of the world's population lives in countries where the disease is endemic. Children are the worst affected, especially children aged 6 months to 5 years. Most of the 13 million who die each year from malaria are children, mainly in Africa, which is hyperendemic for malaria. In parts of the world where malaria is endemic, it may cause as many as $10 \%$ of all deaths in children.

The symptoms of malaria are nonspecific and parasitological diagnosis uncommon, making precise calculation of disease burden difficult and causing both overtreatment with antimalarial drugs and undertreatment of nonmalarial causes of fever. In older children, malaria has a similar course as in adults. However, in children below the age of 5 years, particularly infants, the disease tends to be atypical and more severe. This chapter includes a summary of epidemiology, etiology, pathogenesis, clinical manifestation, diagnosis, treatment, and prevention of malaria in children. 


\section{Epidemiology}

According to the World Malaria Report 2011, there were about 216 million cases of malaria and an estimated 655.000 deaths in 2010. Malaria mortality rates have fallen by more than $25 \%$ globally since 2000 , and by $33 \%$ in the WHO African Region. Most deaths occur among children living in Africa where a child dies every minute from malaria (WHO, 2010).

People of all races are affected by malaria, with some exceptions. People of West African origin who do not have the Duffy blood group are not susceptible to P.vivax malaria (Miller et al., 1976). Children with heterozygous sickle cell trait have lower parasite rates and less fatal infections as compared to normal children (Glickman et al., 2007). Thalassemias may also confer some protection, may be due to higher levels of foetal haemoglobin (HbF) (Williams et al., 1996). Glucose 6phosphate dehydrogenase (G6PD) deficiency has been found to have a protective effect against malaria in some studies. It has been observed that congenital malaria and malarial parasitemia in newborns are very rare, in spite of significant maternal parasitemia and sequestration of the parasites in the placenta. Passive immunity due to maternal antibodies, retarded growth of the parasites in erythrocytes containing $\mathrm{HbF}$ and resistance for parasite growth in old red cells with $\mathrm{HbF}$ may be the causes (Mehta, 2012).

In the first two months of life, children may not contract malaria or the manifestations may be mild with lowgrade parasitemia, due to the passive immunity offered by the maternal antibodies. Children of all ages living in nonmalarious areas are equally susceptible to malaria. In endemic areas children younger than 5 years old may have repeated and often serious attacks of malaria. Partial immunity develops in the survivors. Thus older children and adults often have asymptomatic parasitemia (Kakkilaya, 2006).

In endemic and hyperendemic areas, the parasite rate increases with age from 0 to $10 \%$ during the first three months of life to 80 to $90 \%$ by one year of age, and persists at a high level during early childhood. The mortality rate is highest during the first two years of life. By school age, a considerable degree of immunity would have developed and asymptomatic parasitemia can be as high as $75 \%$ in primary school children. On the contrary, in areas of low endemicity, where the immunity is low, severe infection occurs in all age groups including adults. The morbidity and mortality due to malaria in children tends to be very high in these areas. Malnutrition does not increase susceptibility to severe P.falciparum malaria. In fact, it has been observed that wellnourished children are more likely to develop severe disease than those with malnutrition (Kakkilaya, 2006).

The intensity of transmission depends on factors related to the parasite, the vector, the human host, and the environment. Transmission is more intense in places where the mosquito's lifespan is longer and where it prefers to bite humans rather than animals. It also depends on climatic conditions that may affect the number and survival of mosquitoes, such as rainfall patterns, temperature and 
humidity. Malaria can also occur when people with low immunity move into areas with intense malaria transmission. Human immunity is another important factor, especially among adults in areas of moderate or intense transmission conditions. Partial immunity is developed over years of exposure, and although it never provides a complete protection, it does reduce the risk of suffering severe disease. For this reason, most malaria deaths in Af-rica occur in young children, whereas in areas with less transmission and low immunity, all age groups are at risk (WHO, 2010).

\section{Etiology}

Malaria is caused by Plasmodium species, which are protozoal blood parasites. The following 4 species can infect humans: P.vivax, P.falciparum, P.malariae, and P.ovale, each with different patterns of endemicity, virulence, incubation period, and morphology (Stauffer and Fischer, 2003). Plasmodium falciparum and P.vivax cause the vast majority of infections worldwide, but the majority of severe disease and death is related to infection with P.falciparum (Crawley et al., 2010). In recent years, some human cases of malaria have also occurred caused by P.knowlesi - a species that causes malaria among monkeys in certain forested areas of South-East Asia (WHO, 2010).

\section{Pathogenesis}

Malaria is transmitted exclusively through the bites of infected female Anopheles mosquitoes to the human host. Malaria can also be transmitted through blood transfusion. Among people living in malarious areas, semi-immunity to malaria allows donors to have parasitemia without any fever or other clinical manifestations. Organ transplantation is another malarial transmission route. Transplacental malaria can be significant in populations who are semi-immune to malaria (Crawley et al., 2010).

The bite of an infected mosquito introduces asexual forms of the parasite, called sporozoites, into the bloodstream. Sporozoites enter the hepatocytes and form schizonts, which are also asexual forms. Schizonts undergo a process of maturation and multiplication known as preerythrocytic or hepatic schizogony. In $P$. vivax and P.ovale infection, some sporozoites convert to dormant forms, called hypnozoites, which can cause disease after months or years. Preerythrocytic schizogony takes 6-16 days and results in the host cell bursting and releasing thousands of merozoites into the blood. Merozoites enter the erythrocytes and initiate another asexual reproductive cycle, known as erythrocytic schizogony. The parasite successively passes through the stages of trophozoite and schizont, ultimately giving rise to several merozoites. Upon maturation of these merozoites, the erythrocyte ruptures, releasing the merozoites and multiple antigenic and pyrogenic substances 
into the bloodstream. These merozoites again infect new erythrocytes. After a few cycles of this erythrocytic schizogony, some merozoites differentiate into the sexual forms: the male and female gametocytes. A mosquito that takes a blood meal from a patient with gametocytemia acquires these sexual forms and plays host to the sexual stage of the plasmodial life cycle. Rupture of a large number of erythrocytes at the same time releases a large amount of pyrogens, causing the paroxysms of malarial fever. The periodicity of malarial fever depends on the time required for the erythrocytic cycle and is definite for each species. P.malariae needs 72 hours for each cycle, leading to the name malaria quartana. Plasmodium vivax and P.ovale each take 48 hours for one cycle and cause fever on alternate days (malaria tertiana). However, this periodicity requires all the parasites to be developing and releasing simultaneously; if this synchronization is absent, periodicity is not observed. This may happen in P.falciparum infections (Mehta, 2012).

Malaria disease appears secondary to the parasite's interaction with its host including invasion into erythrocytes, attachment to microvasculature, glucose metabolism, and host response. Multiple genetic host factors protect from severe disease, and in addition there may be variation in the virulence of parasite strains. Severe morbidity and mortality in general are caused by P.falciparum infection in young children or non-immune adults. Newborns of immune mothers appear to be protected from severe disease until approximately 6 months of age. After this time period, young children are at risk for severe disease, particularly severe anemia and cerebral malaria, which are related to age and transmission intensity. Overlap of these two clinical syndromes is common (Chandy and Idro, 2003; Crawley et al., 2010).

Plasmodium falciparum has particular biologic properties that are postulated to confer its deadly potential. First, this parasite, unlike the other species, can invade a wider range of ages of red blood cells. This results in a higher burden of parasites as compared to other species, and, in general, parasitemias $>2 \%$ are common in P.falciparum infections. Second, P.falciparum late stage trophozoites and schizonts can adhere to microvasculature and sequester out of the circulation. This may provide the parasite a possibility to avoid clearance by the spleen. Sequestration likely plays a role in patients who proceed to develop cerebral malaria and, in addition, may play a role in other clinical manifestations. Finally, it has been postulated that P.falciparum schizonts release a malaria toxin at the time of schizont rupture; this results in cytokine responses, which contribute to the clinical illness (WHO, 2000; Moroushek and Aguilar, 2002; Mackintosh and Beeson, 2004).

Loss of erythrocytes due to parasitization is an obvious contributor to anemia. In fact, there are multiple mechanisms that result in malaria-induced anemia, including extravascular hemolysis or erythrophagocytosis of both parasitized and nonparasitized erythrocytes, bonemarrow suppression, and dyserythropoiesis. Patients from endemic areas often have concomitant processes that contribute to anemia, such as malnutrition, iron or folate deficiency, hemoglobinopathies, sickle- 
cell disease, human immunodeficiency virus (HIV) infection, or helminthic infections (Berkley et al., 2009; Crawley et al., 2010).

The pathophysiology of coma in cerebral malaria occurs through distinct and various mechanisms. The striking feature of this syndrome is that the vast majority of patients regain consciousness within 2 to 3 days, with no neurologic sequelae. Children often have high intracranial pressure presumably followed after an increase in the cerebral blood volume. Seizures, hyperthermia, anemia, and sequestration of parasitized and unparasitized erythrocytes are thought to contribute to this phenomenon. The sequestration of infected erythrocytes via parasite proteins expressed on their surface with cerebral microvascular endothelial ligands is thought to be a critical component of coma. Sequestered parasites likely compromise the local blood flow of the microcirculatory system. Seizures are frequent, prolonged, and repeated and are believed to contribute to the comatose state. Together, production of tumor necrosis factor (TNF), hypoglycemia, the release of nitric oxide and free radicals, seizures, and microvasculature obstruction contribute to the encephalopathy (Idro et al., 2005).

\section{Clinical manifestation}

\subsection{General presentation}

Patients with $P$. falciparum malaria present with high fever that may be accompanied by chills, rigors, sweats, and headache. Other common findings include generalized weakness, backache, myalgias, vomiting, and pallor. In children, these symptoms resemble and are frequently mistaken for a viral syndrome or acute gastroenteritis. Children with partical immunity against P.falciparum frequently present with signs such as hepatosplenomegaly, anemia, and jaundice. It is not unusual for these patients to have very minimal symptoms, such as anorexia or decreased activity, or even being asymptomatic. Of children with positive test results, one third were asymptomatic, and splenomegaly was the only manifestation of disease in one-third (Maroushek and Aguilar, 2002). In addition to specific tests for malaria, several other nonspecific laboratory abnormalities are common in patients with this disease, such as elevated C-reactive protein, procalcitonin and liver enzyme levels, thrombocytopenia, and neutropenia. Although it is unusual in the travelling population, the partially immune patient may have additional laboratory abnormalities on presentation, such as anemia, hypoalbuminemia, and hematuria. Hyponatremia and hypoglycemia are of note, because they are associated with more-severe morbidity and occur more common in children than in adults (English et al., 1996).

Although P.falciparum generally manifests days to weeks after initial exposure, P.vivax and P.ovale, which have the hepatic hypnozoite stage, may present much later. Patients with P.vivax infection commonly present with paroxysmal fevers, chills, headaches, and myalgias. In chronically infected children, anemia and hyper- 
splenism are common. Splenic rupture is a serious complication in children with hypersplenism. Plasmodium malariae and P.ovale generally cause fever but not a toxic appearance. In some individuals, however P.malariae may co-exist as a commensallike organism, causing infection but no clinical disease (Stauffer and Fischer, 2003).

Malaria frequently occurs in patients who have a history of recent or ongoing use of a malaria chemoprophylactic agent. This may be attributed to several factors, such as drug resistance, noncompliance with treatment, or inadequate or inappropriate administration especially in children, because of the difficulties in administering bitter medications. It may be very difficult to diagnose malaria in these children, because they may have minimal symptoms and the malaria blood smears are frequently false negative. Malaria should be considered and the diagnosis be pursued under these circumstances (Stauffer and Fischer, 2003).

\subsection{Plasmodium falciparum malaria with complications}

Complications of P.falciparum infection appear as the result of cytokine release and of the parasite's unique ability to cause parasitized erythrocytes to adhere to vascular endothelium and cause erythrocyte sequestration, altered blood flow, and ischemia. The patient's immune status also greatly affects the manifestations of malaria. In populations originating from areas of constant, high-intensity malaria transmission, most deaths occur in younger children, as a result of severe anemia. In the same populations, infected adults and older children may have minimal symptoms or may be asymptomatic. Conversely, in areas where malaria is less prevalent, partial immunity may not develop or it may develop at an older age. In these cases, the major cause of deaths is cerebral malaria (WHO, 2000).

In a patient with P.falciparum parasitaemia and no other obvious cause of symptoms, the presence of one or more of the following clinical or laboratory features determines the diagnosis of severe malaria: impaired consciousness or unrousable coma, prostration (i.e. generalized weakness so that the patient is unable to walk or sit up without assistance), failure to feed, multiple convulsions (more than two episodes in $24 \mathrm{~h}$ ), deep breathing, respiratory distress (acidotic breathing), circulatory collapse or shock, systolic blood pressure $<50 \mathrm{~mm} \mathrm{Hg}$, clinical jaundice plus evidence of other vital organ dysfunction, haemoglobinuria, abnormal spontaneous bleeding, and pulmonary oedema (radiological). While laboratory findings are hypoglycaemia (blood glucose $<2.2 \mathrm{mmol} / \mathrm{L}$ or $<40 \mathrm{mg} / \mathrm{dL}$ ), metabolic acidosis (plasma bicarbonate $<15 \mathrm{mmol} / \mathrm{L}$ ), severe normocytic anaemia $(\mathrm{Hb}<5 \mathrm{~g} / \mathrm{dL}$, packed cell volume $<15 \%)$, haemoglobinuria, hyperparasitaemia $(>2 \% / 100$ $000 / \mu \mathrm{L}$ in low intensity transmission areas or $>5 \%$ or $250000 / \mu \mathrm{L}$ in areas of high stable malaria transmission intensity), hyperlactataemia (lactate $>5 \mathrm{mmol} / \mathrm{L}$ ), and renal impairment (serum creatinine $>265 \mu \mathrm{mol} / \mathrm{L})(\mathrm{WHO}, 2000)$.

Cerebral malaria is defined by $\mathrm{WHO}$ as unrousable coma in a patient with P.falciparum parasitaemia not attributable to other causes of encephalopathy. Although the term implies a distinct disease entity, the clinical syndrome is highly 
variable, with most cases falling into one of three main categories: coma with marked physiological derangement (severe anaemia, metabolic acidosis, respiratory distress, shock); coma with protracted or multiple seizures, where unconsciousness might be caused by a long $(>1 \mathrm{~h})$ postictal state or by subclinical or subtle seizure activity, characterised by conjugate eye deviation, nystagmus, salivation, and hypoventilation; or a pure neurological syndrome of coma and abnormal motor posturing, which might be complicated by high intracranial pressure and recurrent seizures (Idro et al., 2005). Although most children with cerebral malaria regain consciousness within $48 \mathrm{~h}$ and seem to make a full neurological recovery, approximately $20 \%$ die and 10\% have persistent neurological sequelae. Headache, confusion, and irritability may precede cerebral malaria, but a nonimmune child's condition can precipitously decline from normal sensorium to coma within hours. Seizures are common, and children as opposed to adults, frequently have increased intracranial pressure (Chandy and Idro, 2003). Other typical findings include decorticate or decerebrate posturing, nystagmus, dysconjugate gaze, papilledema, retinal hemorrhages, and altered respiration. The findings of examination of the cerebrospinal fluid (CSF) are generally unremarkable (white blood cell (WBC) count of $<20$ cells $/ \mu \mathrm{L}$, slightly elevated protein level, and normal glucose level) (WHO, 2000; Mackintosh et al., 2004).

Hypoglycemia, an important complication of severe malaria in children, results from parasite-induced suppression of gluconeogenesis in the liver and induction of insulin secretion from the pancreas. The excess secretion of insulin is intensified by the initiation of quinine treatment and can result in devastating neurologic sequelae. Respiratory distress is another common complication in children, but unlike in adults, it is rarely primarily the result of pulmonary edema or respiratory distress syndrome and instead usually is a consequence of severe acidosis. Black water fever (severe hemolysis, hemoglobinuria, and renal failure) and algid malaria (vascular collapse, shock, and hypothermia) are rare presentations in children (Stauffer and Fischer, 2003).

There are no clinical features that reliably distinguish severe malaria from other severe infections in children. The most frequently reported clinical and laboratory features of severe P.falcifarum malaria in children, i.e. impaired consciousness (prostration or coma), seizures, respiratory distress, severe anaemia, hypoglycaemia, metabolic acidosis, and hyperlactataemia, are also recognized features of severe sepsis (Marsh et al., 1995; WHO, 2000). Since severe malaria is a multisystem and multi-organ disease, children frequently present with more than one of the classic clinical phenotypes: cerebral malaria, respiratory distress, severe malarial anaemia, hypoglycaemia. Respiratory distress (deep breathing, Kussmauls respiration) is a clinical sign of metabolic acidosis that can be misinterpreted as cardiac failure and circulatory overload, especially if associated with severe tachycardia (Crawley et al., 2010) 
Tabel 1. Severe malaria: differences between adults and children

\begin{tabular}{|c|c|c|}
\hline Clinical manifestation & adults & children \\
\hline $\begin{array}{l}\text { Duration of illness prior } \\
\text { to complications }\end{array}$ & 5-7 days & 1-2 days \\
\hline Convulsions & Common & $\begin{array}{l}\text { Very common; can be due to } \\
\text { severe infection, hypoglyce- } \\
\text { mia, febrile seizures, severe } \\
\text { anemia etc. }\end{array}$ \\
\hline $\begin{array}{l}\text { Abnormal brain stem reflexes (ocu- } \\
\text { lovestibular, oculocervical) }\end{array}$ & Rare & More common \\
\hline C.S.F. pressure & Usually normal & Variable, often raised \\
\hline Resolution of coma & 2-4 days & 1-2 days \\
\hline Neurological sequelae & $<5 \%$ & $>10 \%$ \\
\hline Cough & Uncommon & Common \\
\hline Anemia & Common & $\begin{array}{l}\text { More common and more } \\
\text { severe; may be the presenting } \\
\text { feature }\end{array}$ \\
\hline Jaundice & Common & Uncommon \\
\hline $\begin{array}{l}\text { Pre-treatment } \\
\text { hypoglycemia }\end{array}$ & Uncommon & Common \\
\hline Pulmonary oedema & Common & Rare \\
\hline Renal failure & Common & Rare \\
\hline Bleeding/clotting disturbances & Up to $10 \%$ & Rare \\
\hline
\end{tabular}




\section{Diagnosis}

Malaria is diagnosed using a combination of clinical observations, case history and diagnostic tests, principally microscopic examination of blood. Microscopy performed by an experienced operator is very sensitive, rapid, and inexpensive, and remains as the gold standard. Thick smears are more sensitive for detecting the presence of parasites, and thin smears can provide more details for species determination. Rapid diagnostic 'dipstick' tests, which facilitate the detection of malaria antigens in a finger-prick of blood in a few minutes are easy to perform and do not require trained personnel or a special equipment. Treatment solely on the basis of symptoms should only be considered when a parasitological diagnosis is not possible (Ohrt et al., 2002; Coleman et al., 2002)

\section{Treatment}

\subsection{Treatment of uncomplicated malaria}

Treatment of uncomplicated P.falciparum malaria has three main objectives. The first is to cure the infection, since this prevents progression to severe disease and the additional morbidity associated with treatment failure. The second is to prevent the development of antimalarial drug resistance, and the third is to reduce transmission (Crawley et al., 2010). WHO recommends artemisinin-based combination therapy (ACT) as firstline treatment for $P$. falciparum and chloroquine-resistant $P$. vivax infection (WHO, 2010).

Artemisinin and its derivatives achieve the highest parasite killing rates and target asexual and sexual stages of the parasite in the blood with two important therapeutic consequences: prevention of clinical deterioration and interruption of transmission. Combination of an artemisinin derivative with a long-acting antimalarial drug reduces treatment duration from 7 days to 3 days. The main ACTs are artesunate combined with either lumefantrine or amodiaquine or mefloquine or sulfadoxine-pyrimethamine (WHO, 2010):

1) artemether plus lumefantrine

- 6 dose regimen over a 3-day period.

- Dose: 5-14 kg: 1 tablet; 15-24 kg: 2 tablets; 25-34 kg: 3 tablets; and >34 kg: 4 tablets, given twice a day for $3 \mathrm{da}$

2) artesunate plus amodiaquine

- Fixed-dose formulation with tablets containing 25/67.5 mg, 50/135 mg or $100 / 270 \mathrm{mg}$ of artesunate and amodiaquine. Blister packs of separate scored tablets containing $50 \mathrm{mg}$ of artesunate and $153 \mathrm{mg}$ base of amodiaquine are also available. 
- Dose: $4 \mathrm{mg} / \mathrm{kg} \mathrm{BW/day}$ artesunate and $10 \mathrm{mg} / \mathrm{kg} \mathrm{BW} /$ day amodiaquine once a day for 3 days.

3) artesunate plus mefloquine

- available as blister packs with separate scored tablets containing $50 \mathrm{mg}$ of artesunate and $250 \mathrm{mg}$ base of mefloquine.

- Dose: $4 \mathrm{mg} / \mathrm{kg} \mathrm{BW/day} \mathrm{artesunate} \mathrm{given} \mathrm{once} \mathrm{a} \mathrm{day} \mathrm{for} 3$ days and $25 \mathrm{mg} / \mathrm{kg}$ $\mathrm{BW}$ of mefloquine either split over 2 days as $15 \mathrm{mg} / \mathrm{kg} \mathrm{BW}$ and $10 \mathrm{mg} / \mathrm{kg}$ $\mathrm{BW}$ or over 3 days as $8.3 \mathrm{mg} / \mathrm{kg} \mathrm{BW} /$ day once a day for 3 days.

4) artesunate plus sulfadoxine-pyrimethamine

- Available as separate scored tablets containing $50 \mathrm{mg}$ artesunate and tablets containing $500 \mathrm{mg}$ of sulfadoxine and $25 \mathrm{mg}$ of pyrimrthamine.

- Dose: $4 \mathrm{mg} / \mathrm{kg} \mathrm{BW} /$ day artesunate given once a day for 3 days and a single administration of $25 / 1.25 \mathrm{mg} / \mathrm{kg}$ BW sulfadoxine-pyrimethamine on day 1 .

5) dihydroartemisinin plus piperaquine.

- available as fixed-dose combination with tablets containing $40 \mathrm{mg}$ of dihydroartemisinin and 16-26 mg/kg BB/ dose piperaquine.

The aim of treatment for P.vivax and P.ovale malaria is to eliminate the blood and liver stages of infection (radical cure), thereby preventing a relapse and recrudescence. Chloroquine is recommended in countries where parasites are sensitive, with the addition of primaquine for 14 days to achieve radical cure. Primaquine should not be given to children known to have severe G6PD deficiency. Chloroquine is the recommended treatment for infections caused by P. malariae, P. ovale, and P. knowlesi. Children with HIV infection who develop malaria should receive prompt, effective antimalarial treatment according to WHO guidelines (WHO, 2010). Children receiving zidovudine or efavirenz should, if possible, avoid ACT regimens that contain amodiaquine (Berkley et al., 2009).

\subsection{Treatment of severe malaria}

Untreated P.falciparum malaria in a nonimmune individual can progress within hours to life-threatening illness. The primary objective of antimalarial treatment in severe malaria is to prevent death. In treating cerebral malaria, prevention of neurological deficit is also an important objective. Management comprises four main areas: clinical assessment of the patient, specific antimalarial treatment, adjunctive and supportive care (Crawley et al., 2010).

Therapeutic concentrations of an effective antimalarial drug need to be achieved as soon as possible. It is essential that effective parenteral antimalarial treatment in full doses is given promptly in severe malaria. Two classes of medicine are available: the cinchona alkaloids (quinine and quinidine) and the artemis- 
inin derivatives (artesunate, artemether and artemotil). Initial treatment of severe P. falciparum malaria: artesunate $2.4 \mathrm{mg} / \mathrm{kg} \mathrm{BW}$ IV or IM given on admission, then at $12 \mathrm{~h}$ and $24 \mathrm{~h}$, then once a day is the recommended treatment. If parenteral artesunate is not available: artemether $3.2 \mathrm{mg} / \mathrm{kg} \mathrm{BW}$ IM given on admission, then $1.6 \mathrm{mg} / \mathrm{kg} \mathrm{BW}$ per day; or quinine $20 \mathrm{mg}$ salt $/ \mathrm{kb} \mathrm{BW}$ on admission (IV infusion or divided IM injection), then $10 \mathrm{mg} / \mathrm{kg} \mathrm{BW}$ every $8 \mathrm{~h}$; infusion rate should not exceed $5 \mathrm{mg}$ salt $/ \mathrm{kg}$ BW per hour. Following initial parenteral treatment, once the patient can tolerate oral therapy, it is essential to continue and complete treatment with an effective oral antimalarian using a full course of an effective ACT (artesunate plus amodiaquine or artemether plus lumefantrine or dyhydroartemisinin plus piperaquine). The current recommendation (experts' opinion) is to give parenteral antimalarials in the treatment of severe malaria for a minimum of $24 \mathrm{~h}$, once started (irrespective of the patient's ability to tolerate oral medicatin earlier) or until the patient is about to tolerate oral medication, before giving the oral follow-up treatment (WHO, 2010)

Prostration with respiratory distress, lactic acidosis, delayed capillary refill, and severe tachycardia are indistinguishable from severe sepsis (Rivers and Abrens, 2008). Children with severe malaria should, when possible, have blood cultures taken at the time of admission, with lumbar puncture done on all children with impaired consciousness. Empirical antibiotic treatment should be given when the clinical condition prevents or delays lumbar puncture. The best choice of antibiotic in this case would be a quinolone or third generation cephalosporin (Bronzan and Taylor, 2007).

\subsection{Prevention of malaria with antimalarial drugs}

Children visiting malaria endemic countries are nonimmune and at increased risk of contracting malaria. Children should be protected from vectors between dusk and dawn (Crawley et al., 2010). Intermittent preventive treatment (IPT) is the administration of a full therapeutic dose of an antimalarial drug (or a combination of drugs) at specified timepoints, whether or not parasites are present (Greenwood, 2004). The most studied drug for IPT is sulfadoxine-pyrimethamine. IPT with sulfadoxine-pyrimethamine should not be given to HIV-infected infants who are receiving routine prophylaxis with cotrimoxazole or other sulfa containing drugs (Aponte et al., 2009). WHO has recommended IPT for infants (IPTi) with sulfadoxine-pyrimethamine in areas of Africa with moderate to high malaria transmission and low resistance to this drug combination (WHO, 2009).

In children aged 2 months to 10 years, a full treatment course of sulfadoxinepyrimethamine or amodiaquine (with or without artesunate), given at 1-2 monthly intervals to asymptomatic children during the transmission season, can reduce rates of clinical malaria by between $67.5 \%$ and $85 \%$ or more compared with placebo over a 3-4 month period of follow up (Cisse et al., 2006; Dicko et al., 2008). Piperaquine has shown promising results when given once a month to chil- 
dren, but data for its pharmacokinetics are still insufficient and its use as monotherapy should be avoided. In schoolchildren (aged 5-18 years) a treatment course of sulfadoxine-pyrimethamine and amodiaquine at 4 monthly intervals over 1 year reduced the prevalence of anaemia and improved school performance (Clarke et al., 2008).

\section{References}

Aponte, J.J., Schellenberg, D., Egan, A., Breckenridge, A., Carneiro, I., Critchley, J., Danguah, I., Dodoo, A., Kobbe, R., Lell, B., May, J., Premji, Z., Sanz, S., Sevene, E., Soulaymani-Bechleikh, R., Winstanley, P., Adjei, S., Anemana, S., Chandramohan, D., Issifou, S., Mockenhaupt, F., Owusu-Agyei, S., Greenwood, B., Grobusch, M.P., Kremsner, P.G., Macete, E., Mshinda, H., Newman, R.D., Slutsker, L., Tanner, M., Alonso, P. \& Menendez, C. (2009). Efficacy and safety of intermittent preventive treatment with sulfadoxinepyrimethamine for malaria in African infants: a pooled analysis of six randomised, placebo-controlled trials. Lancet, 374, 1532-1542.

Berkley, J.A., Bejon, P. \& Mwangi, T. (2009). HIV infection, malnutrition, and invasive bacterial infection among children with severe malaria. Clinical Infectious Disease, 49, 336-343.

Bronzan, R.N., Taylor, TE., Mwenechanya, J., Tembo, M., Kayira, K., Bwanaisa, L., Njobvu, A., Kondowe, W., Chalira, C., Walsh, A.l., Phiri, A., Wilson, L.K., Molyneux, E. \& Graham, S.M. (2007). Bacteremia in Malawian children with severe malaria: prevalence, etiology, HIV coinfection, and outcome. The Journal of Infectious Disease, 195, 895-904.

Chandy, J.C. \& Idro, RI. (2003). Parasitic diseases: cerebral malaria in children. Infections in Medicine, 20, 53-58

Cisse, B., Cairns, M., Faye, E., Ndiaye, O., Faye, B., Cames, C., Cheng, Y., Ndiaye, M., Colle Lo, A., Simondon, K., Trape, J.F., Faye, O., Ndiaye, J.L., Gaye, O., Greewood, B. \& Milligan, P. (2009). Randomized trial of piperaquine with sulfadoxine-pyrimethamine or dihydroartemisinine for malaria intermittent preventive treatment in children. PloS One, 4, e7164.

Cisse, B., Sokhna, C., Boulanger, D., Millet, D., Ba, E.H., Richardson, K., Hallett, R., Sutherland, C., Simondon, K., Simondon, F., Alexander, N., Gaye, O., Targett, G., Lines, J., Greenwood, B. \& Trape, J.F. (2006). Seasonal intermittent preventive treatment with artesunate and sulfadoxinepyrimethamine for prevention of malaria in Senegale children: randomised, placebo-controlled, double blind trial. Lancet, 367, 659-667. 
Clarke, S.E., Jukes, M.C.H., Njagi, J.K., Khasakhala, L., Cundill, B., Crudder, C., Estambale, B.B. \& Brooker, S. (2008). Effect of intermittent preventive treatment of malaria on health and education in schoolchildren: a clusterrandomised, double-blind, placebo-controlled trial. Lancet, 372, 127-138.

Coleman, R.S., Maneechai, N., Rachaphaew, N., Kumpitak, C., Miller, R.S., Soyseng, V., Thimasam, K. \& Sattabongkot, J. (2002). Comparison of field and expert laboratory microscopy for active surveillance for asymptomatic Plasmodium falciparum and Plasmodium vivax in western Thailand. American Journal of Tropical Medicine and Hygiene, 67, 141-144.

Crawley, J., Chu, C., Mtove, G. \& Nosten, F. (2010). Malaria in children. Lancet, 24, 1468-1481.

Dicko, A., Sagara, I., Sissoko, M.S., Guindo, O., Diallo, A.I., Kone, M., Toure, OB., Sacko, M. \& Doumbo, O. (2008). Impact of intermittent preventive treatment with sulfadoxine-pyrimethamine targeting the transmission season on the incidence of clinical malaria in children in Mali. Malaria Journal. 7, 123.

English, M.C., Waruiru, C., Lightowler, C., Murphy, S.A., Kirigha, G. \& Marsh, K. (1996). Hyponatremia and dehydration in severe malaria. Archives of Disease in Childhood, 74, 201-205.

Glikman, D., Nguyen-Dinh, P., Roberts, J.M., Montgomery C.P., Daum, R.S. \& Marcinak, J.F. (2007). Clinical malaria and sickle cell disease among multiple family members in Chicago, Illinois. Pediatrics, 120, e745.

Greenwood, B. (2004). The use of antimalarias to prevent malaria in the population of malaria-endemic areas. American Journal of Tropical Medicine and Hygiene, 71, 1-7.

Idro, R., Otieno, G., White, S., Kahindi, A., Fegan, G., Ogutu, B., Mithwani, S., Maitland, K., Neville, B.G. \& Newton, C.R. (2005). Decorticate, decerebrate, and opisthotonic posturing and seizures in Kenyan children with cerebral malaria. Malaria Journal, 4, 57.

Kakkilaya, B.S. (2006). Malaria in children. http://www.malariasite.com/malaria/Evolution.htm (accessed Sept 10, 2012).

Mackintosh, C.L. \& Beeson, J.G. (2004). Clinical features and pathogenesis of severe malaria.Trends in Parasitology, 20, 597-603.

Maroushek, S.R. \& Aguilar, E. (2002). Asymptomatic malaria in Liberian children. Pediatric Research, 51, 193A

Mehta, P.N. (2012). Pediatric malaria. http://emedicine.medscape.com/article/998942-overview (accessed October $23,2012)$. 
Miller, L.H., Mason, S.J., Clyde, D.F. \& McGinniss, M.H. (1976). The resistance factor to Plasmodium vivax in blacks. The Duffy-blood-group genotype, FyFy. The New England Journal of Medicine, 295, 302.

Ohrt, C., Purnomo, Sutamihardja, M.A., Tang, D. \& Kain, K.C., (2002). Impact of microscopy error on estimates of protective efficacy in malaria prevention trials. The Journal of Infectious Disease, 186, 540-546

Rivers, B.P. \& Abrens, T. (2008). Improving outcomes for severe sepsis and septic shock tools for early identification of at risk patients and treatment protocol implementation. Critical Care Clinics, 24 (suppl 3), s1-42.

Stauffer, W. \& Fischer, P.R. (2003). Diagnosis and treatment of malaria in children. Clinical Infectious Disease, 37, 1340-1348.

WHO. (2000). Severe P. falciparum malaria. Transsactions of the Royal Society of Tropical Medicine and Hygiene, 94(suppl 1), S1-90.

WHO. (2009). Report of the technical consultation on intermittent preventive treatment in infants (IPTi).

http:/ /www.who.int/malaria/publications/atoz/tegconsultiptiapr2009report/ en/index.html (accessed Oct 16, 2012)

WHO.(2010). Guidelines for the treatment of malaria, 2nd edn. http://whqlibdoc.who.int/publications/2010/9789241547925_eng. pdf (accessed October 16, 2012).

Williams, T.N., Maitland, K., Bennett, S., Ganczakowski, M., Peto, T.E., Newbold, C.I., Bowden, D.K., Weatherall, D.J. \& Clegg, J.B. (1996). High incidence of malaria in alphathalassaemic children. Nature, 383, 522. 


\section{Malaria in Pregnancy: The Parasite Infection Mechanism}

Ahmad Ghiffari, Emerging and Neglected Tropical Disease Unit, (BiK-F), Frankfurt am Main, Germany

Rostika Flora, Sriwijaya University Parasitology Department, Palembang, Indonesia

Rintis Noviyanti, Eijkman Institute for Molecular Biology, Jakarta, Indonesia

Plasmodium vivax (P.vivax) malaria is the most widely distributed species of human malaria, threatening nearly 3 billion people in 95 countries ranging from temperate to tropical in the Americas, Africa, Asia. In Indonesia, it is estimated that nearly 129,6 million people lived at risk of P.vivax transmission in 2010 (Elyazar et al., 2012). The number of patients with P.vivax malaria annually reaches $72-80$ million cases per year, and the highest rate is in Asia (Luxemburger et al., 2001). By contrast, in Africa the number of people with P.vivax malaria is low, due to low prevalence of Duffy antigen which serves as receptor for the invasion of merozoites of P. vivax into red blood cells (Mendis et al., 2001). Malaria in pregnancy can be caused by all human-pathogenic species of Plasmodium, but P.vivax and P.falciparum are the most common parasites that cause infection in pregnant wom- 
en. Plasmodium falciparum as the predominant parasite has the most severe impact on morbidity and mortality of mother and foetus (Mc Gregor, 1984).

In order to invade host erythrocytes, P.vivax requires a cell receptor, for example the Duffy antigen. Unlike P.falciparum that can invade red blood cells of all ages, P.vivax only invades reticulocytes. Plasmodium vivax also has a longer incubation period (12 days to several months) with the erythrocyte cycle of 42-48 hours and the production of 12-24 merozoites per schizont (Collins et al., 2004). The ability of P.falciparum to adhere tot he endothelium of blood vessels causes the occurrence of severe malaria in P. falciparum-infected patients (Rogerson et al., 2004). In contrast, P.vivax-infected erythrocytes change their shape cannot attach to the endothelium of blood vessels and thus, rarely progresses to severe malaria (Suwanarusk et al., 2004).

Pregnant women in malaria-endemic areas are highly susceptible, especially in primigravidae. The main factors are related to low cellular immunity and the placenta which unfortunately favors the parasite to multiply (Espinosa et al., 2004). Malaria has an impact on the mother and foetus causing maternal anemia and low birth weight (LBW).

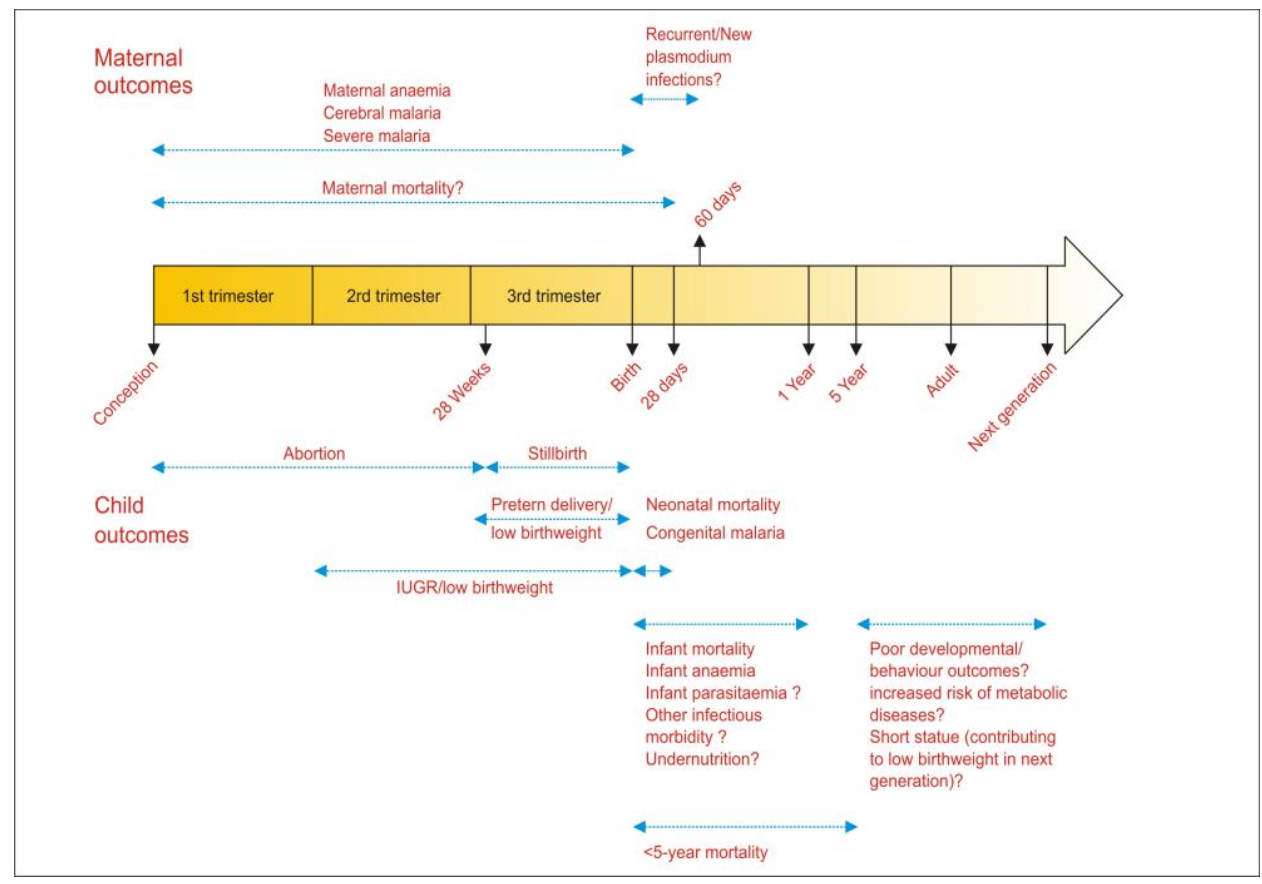

Figure 1: Effect of malaria in pregnancy on maternal, newborn, infant, and child health (Desai M, et all., 2007), own presentation. 
LBW is related with specific changes in the placenta where parasites mature and accumulate in high density of the placental intervillous space. Sequestration in the placenta is mediated by the receptor chondroitin sulphate A (CSA) and hyaluronic acid (HA), expressed by the syncytiotrophoblast placental intervillous space limit (Matejevic, 2001). To enable the attachment between parasitized erythrocytes with receptors in the placenta, the erythrocytes express variant surface antigens (VSA). The dominant VSA on the surface is P.falciparum Erythrocyte Membrane Protein 1 (PfEMP1) encoded by the var multigene family (Newbold et al., 1992, Smith et al., 1995; Noviyanti et al., 2007). Adhesion mediated by VSA results in changes of inflammatory cytokines in the placenta, such as an increase of TNF- $\alpha$, interleukin (IL) 2 and interferon (IFN)- $\lambda$ (Fried et al., 1998), which finally is associated with low birth weight and anemia.

The existence of TH1 cytokines as a response to the parasites results in excessive adverse effects. Despite this, IL-10 is important to regulate the inflammatory cytokine effect, foetal trophoblasts and maternal leukocytes. Expressing IL10 in high concentrations to protect the foetus from the inflammatory reaction will suppress anti-inflammatory responses against parasites and thus, the parasite persists in the placenta. This situation affects the incidence of severe anemia and preterm birth (Suguitan et al., 2003).

Unlike P.falciparum, P.vivax is not able to cause parasite sequestration in the placenta and placental pathologic changes. In the placenta, hemozoin can only be found slightly which is produced from hemoglobin catabolism within Plasmodiuminfected erythrocytes. Its presence is an indication of placental infection and it is associated with decreased foetal weight (Rogerson et al., 2003). Histological pictures of the active form of placental infection show black/gray colour, dense sinusoid with infected erythrocytes. Syncytial knots can occur with fibrinoid necrosis and thickening of the basal membrane and damage of trophoblasts (Suparman, 2005). 


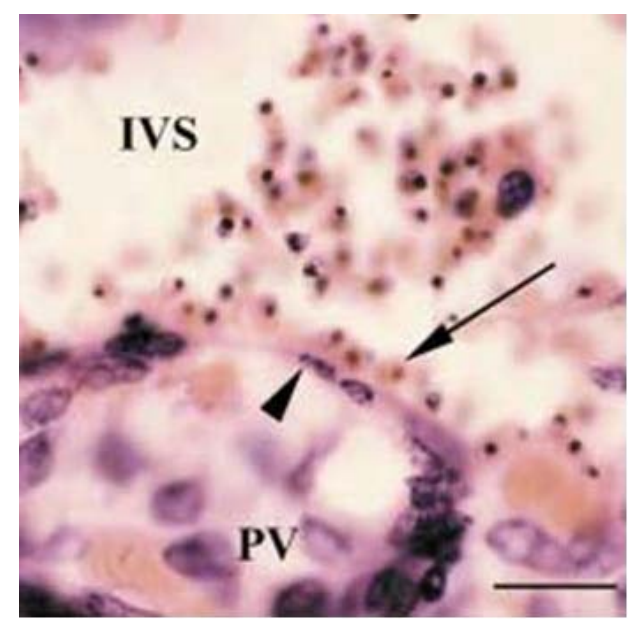

Figure 2: Histopathology of the infected placental tissue.

Some Plasmodium falciparum- infected erythrocytes (arrow) in the intervillous space (IVS) appear to be directly adherent to the surface of the syncytiotrophoblast cell layer (arrowhead) of a placental villus (PV). Scale bar $=\sim 20 \mu \mathrm{m}$. (Beeson et al. 2001). 


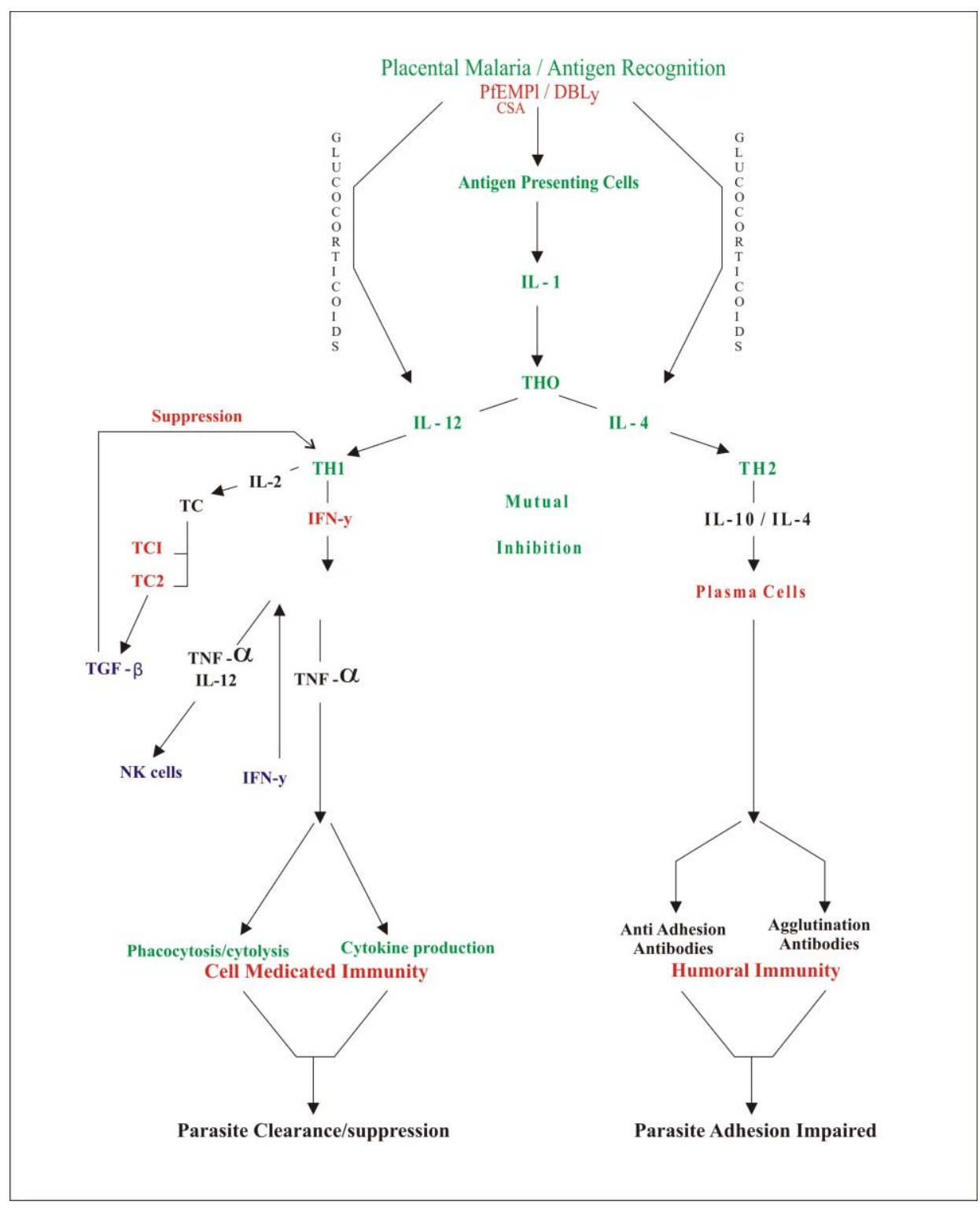

Figure 3: Placenta-related mechanisms of malaria parasite clearance, suppression and adhesion (Brabin BJ, et al., 2004), own presentation.

Abbreviation: PfEMP, P.falciparum erythrocyte membrane protein; DBL $\gamma$, Duffy binding like domain- $\gamma$; CSA, chondroitin sulphate A; NK, natural killer cells; IFN $\gamma$, interferon $\gamma$; TGF $\beta$, transforming growth factor $\beta$; TC, cytotoxic T cell; THO, T helper (precursor). 
The placenta has the function as a protective barrier against various pathogens present in the mother's blood, but the accumulation of erythrocytes infected with P.falciparum in the placental intervillous space can affect the mother and the foetus. In conclusion, malaria infection in pregnancy can cause severe anemia and low birth weight and increases the risk of death (Khong et al., 2006).

\section{References}

Beeson JG, Reeder JC, Rogerson SJ and Brown GV., (2001). Parasite adhesion and immune evasion in placental malaria. TRENDS in Parasitology, 17(7):331-337.

Brabin BJ., Romagosa C, Abdelgalil S, Menendez C, Verhoeff FH, McGready R, Fletcher KA, Owens A, d'Alessandro U, Nosten F, Fischer PR and Ordi J., (2004). The sick placenta— the role of malaria. Placenta, 25:359-378.

Collins EW, Sullivan JS, Nace D, Williams T, Williams A, and Barnwell JW., (2008). Observations on the sporozoite transmission of Plasmodium vivax to monkeys. Journal of Parasitology, 94(1):287-288.

Desai M., Kuile FO, Nosten F, McGready R, Asamoa K, Brabin B, Newman RD., (2007). Epidemiology and burden of malaria in pregnancy, Lancet Infect Dis 7:93-104.

Elyazar IRF, Gething PW, Patil AP, Rogayah H, Sariwati E, Palupi NW, Tarmizi SN, Kusriastuti R, Baird JK, Hay SI., (2012). Plasmodium vivax malaria endemicity in Indonesia in 2010. PLoS ONE, 7(5): e37325.

Espinosa F E M, Claudio TD, Wilson DA., (2004). Malaria during pregnancy in reference center from Brazilian Amazon: Unexpected increase of the frequency of Plasmodium falciparum Infections. Mem Inst Oswaldo Cruz, 99 (1):19-21.

Fried M and Duffy PE., (1998). Maternal malaria and parasite adhesion. J. Mol. Med,76:162-171.

Khong T. Y., (1991). Acute atherosis in pregnancies complicated by hypertension, small for gestational age infants, and diabetes mellitus, Archives of Pathology and Laboratory Medicine,115(7):722-725.

Luxemburger C, Rose McG, Am Kham, Linda M., (2001). Effects of malaria during pregnancy on infant mortality in an area of low malaria transmission. Am.J. Epidemiol,154(5):459-465.

Matejevic, D., (2001). Localization of hyaluronan with a hyaluronan-specific hyaluronic acid binding protein in the placenta in pre-eclampsia.

Gynecol.Obstet. Invest., 52:257-259. 
Mc. Gregor, I.A., (1984). Epidemiology, malaria and pregnancy. Am. J. Trop. Med. Hyg, 33:517-525.

Mendis K, Sina BJ, Marchesini P, Carter R., (2001). The neglected burden of Plasmodium vivax malaria. Am J Trop Med Hyg. 64(1-2):97-106.

Newbold C.I., Craig A.G., Kyes S. et al., (1992). PfEMP1, polymorphism and pathogenesis. Annals of Tropical Medicine and Parasitology, 91:551-57.

Noviyanti R, Trianty L, Poespoprodjo JR, Harsha Dadlani, Nursamsy N, Trimarsanto H, et al., (2007). Molecular mechanisms of Plasmodium falciparum infection in pregnancy-associated malaria in Timika, Papua, Indonesia. Personal communication.

Rogerson SJ, Pollina E, Getachew A, Tadesse E, Lema VM, Molyneux ME., (2003). Placental monocyte infiltrates in response to Plasmodium falciparum infection and their association with adverse pregnancy outcomes. Am J Trop Med Hyg, 68:115-119.

Smith JD, Chitnis CE, Craig AG, Robert DJ, DEH Taylor, DS Peterson et al., (1995). Switches in expression of Plasmodium falciparum var gene correlate with changes in antigenic and chytoadherent phenotypes of infected erythrocytes. Cell, 82:101-110.

Suguitan A.L, D. Channe G, Genevieve F, Lucy T, Ainong Z, Rosine D et al., (2004). Lack of an association between antibodies to Plasmodium falciparum malaria-associated glycosylphosphatidylinositols and placental changes in Cameroonian women with preterm and full-term deliveries. Inf. And Imm, 09: 5267-5273.

Suparman E., (2005). Malaria in pregnancy. Mirror World Medicine, 146:19-28.

Suwanarusk, R., Cooke, B.M., Dondorp, A.M., Silamut, K., Sattabongkot, J., White, N.J., Udomsangpetch, R., (2004). The deformability of red blood cells parasitized by Plasmodium falciparum and P. vivax. J. Infect. Dis, 189:190194. 



\title{
17. Malaria and Blood Transfusion
}

\author{
Nelly Al Audhah, Medical Faculty, \\ Lambung Mangkurat University, Indonesia
}

\section{Introduction}

Malaria remains a worldwide problem because of its morbidity and mortality, however due to the adoption of the Millennium Development Goals (MDGs) infectious diseases control became a global commitment The World Health Organization states that new cases of malaria in 2010 led to 216,000-655,000 deaths. In the United States, about 1,500 cases of malaria with 5 deaths were reported. Those cases were mostly tourists who had visited endemic countries or migrants who were diagnosed and treated in the United States $(1,2,3)$.

Transfusion-transmitted malaria is one of the incidents of infections transmitted by blood transfusion from the donor to the recipient. It has serious consequences for the patient as in many countries diagnosis is rarely considered in the recipients of a blood transfusion $(4,5)$. 


\section{Screening for blood transfusion}

When malaria screening with antigen detection methods has been carried out on more than 6,000 blood donors in India, $19.4 \%$ were tested positive with an enzyme-linked immunosorbent assay (ELISA) and $12.4 \%$ with an indirect fluorescent assay (IFA) (6). Research performed in 2012 by Hayatie et al (2012) showed a prevalence rates in endemic areas in Kalimantan, Indonesia: using a rapid diagnostic test (RTD) showed that $74.3 \%$ of blood donors were tested positive for Plasmo$\operatorname{dium}(7)$. These results indicate that the majority of blood which is used for transfusions in these areas contains antigens of either Plasmodium falciparum or P.vivax, respectively and pose a risk for the recipient (Fig. 1). Therefore, the donor should be examined for the occurrence of parasitaemia.

Picture 1. Diagnosis of malaria from thin blood smear and RDT.

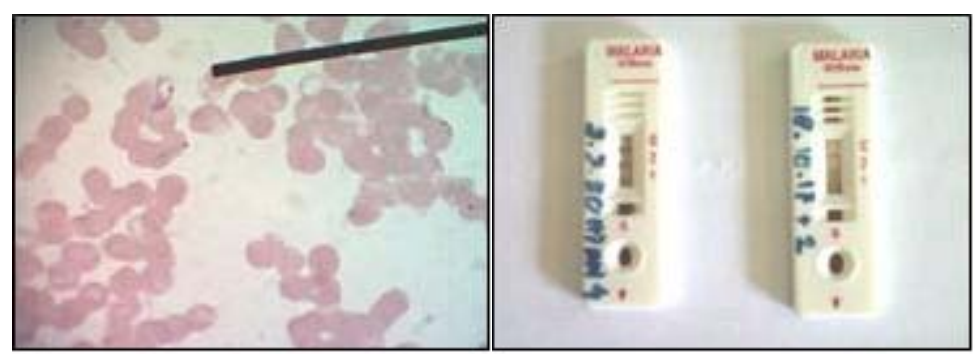

Old data from a study performed in former USSR indicate that with more than $80 \%$ of all Plasmodium-positive cases, P. malariae is most dominant in malaria transmitted by blood transfusion or haemotherapy (15).

Table 1. Reported malaria cases and deaths in Indonesia as given in the WHO World Malaria Report (WMR) 2012

\begin{tabular}{|l|l|l|l||}
\hline \multirow{2}{*}{ Year } & \multicolumn{2}{|l|}{ Malaria cases } & Malaria death \\
\cline { 2 - 4 } & $\begin{array}{l}\text { Presumed and } \\
\text { confirmed cases }\end{array}$ & $\begin{array}{l}\text { Confirmed with } \\
\text { microscopy }\end{array}$ & No data \\
\hline 2001 & $2,776,477$ & 267,592 & No data \\
\hline 2003 & $2,554,223$ & 223,074 & No data \\
\hline 2005 & $1,445,831$ & 437,323 & 494 \\
\hline 2006 & $1,320,581$ & 347,597 & No data \\
\hline 2007 & $1,140,423$ & 333,792 & \\
\hline
\end{tabular}




\begin{tabular}{|c|c|c|c|}
\hline \multirow[b]{2}{*}{ Year } & \multicolumn{2}{|l|}{ Malaria cases } & \multirow[b]{2}{*}{ Malaria death } \\
\hline & $\begin{array}{l}\text { Presumed and } \\
\text { confirmed cases }\end{array}$ & $\begin{array}{l}\text { Confirmed with } \\
\text { microscopy }\end{array}$ & \\
\hline 2008 & 746,120 & 266,277 & 669 \\
\hline 2009 & 544,470 & 199,577 & 900 \\
\hline 2010 & $1,849,062$ & 229,819 & 432 \\
\hline 2011 & $1,322,451$ & No data & 388 \\
\hline
\end{tabular}

The primary approach should be the screening of donors, referring to their health history and identifying any possible malaria risk associated with travel or residency. An important aspect which should also be considered is the geographical location. The question is whether or not a potential donor has visited or lived in a malariaendemic area or not. Within this context both the length of the time the donor stayed in a malarious area and the length of time since he has visited a malarious area are very important factors and need to be taken into account when trying to identify any potential risk for transfusion-transmitted malaria. Malaria that is transmitted by transfusion from the donor to the recipient manifests with a shorter incubation period of 2-4 days since the inoculum contains already the erythrocytic parasite stages (trophozoites and merozoites) and therefore a pre-erthrocytic liver cycle of the parasite is not necessary. Typical symptoms are fever, malaise, headache and vomiting. In the case of P.falciparum infection, especially in non-immune patients, the infection can progress rapidly and may end with the death of the patient. In fact, diagnosis of transfusion malaria requires a keen sense of clinical suspicion. In case of any blood transfusion, and if the recipient develops some of these symptoms, he should definitely be tested for malaria. If P.vivax is transmitted by transfusion, a relapse does not occur since the pre-erythrocytic liver cycle has not been passed by the parasite $(8,9,10,11)$.

After infection, individuals can remain infective for weeks to months, or even years, in the case of P.malariae infection. Therefore, those who have suffered from malaria cannot donate blood for at least 3 years after becoming asymptomatic, and proven carrier P.malariae should not donate blood. 


\section{References}

1. Abanyie FA, Arguin PM, Gutman J. State of malaria diagnostic testing at clinical laboratories in the United States, 2010: a nationwide survey. Malaria Journal 2011; 10: 340.

2. Gool TV, Marlies EVW, Rob K, et al. A simple and fast method to exclude high Plasmodium falciparum parasitaemia in travelers with imported malaria. Malaria Journal 2011; 10: 300.

3. Wurts N, Khadijetou ML, Herve B. Vivax malaria in Mauritania include infection of a Duffy-negative individual. Malaria Journal 2011; 10: 336.

4. Candolfi E. Transfusion-transmitted malaria, preventive measures, Transfus Clin Biol. 2005; 12: 107-113.

5. Kitchen AD and Chiodini PL. Malaria and blood transfusion. Vox Sanguinis 2006; 90: 77-84.

6. Choudhury N, Jolly JG, Ganguly NK, Mahajan RC, and Dubey ML. Plasmodial antigen detection by monoclonal antibody as a screening procedure for blood donors in transfusion medicine. I Indian Med Assoc. 1991; 89: 334-336.

7. Hayati L, Istiana, Al Audhah N. Screening malaria at blood transfusion in red cross unit, banjar district, south Kalimantan province. Unpublished results.

8. Milne LM, Kyi MS, Chiodini PL, Warhurst DC. Accuracy of routine laboratory diagnosis of malaria in the United Kingdom. J Clin Pathol 1994; 47: 740-742

9. Baird JK, Purnomo, Jones TR: Diagnosis of malaria in the field by fluorescence microscopy of QBC capillary tubes. Trans $\mathrm{R}$ Soc Trop Med Hyg 1992; 86: 3-5.

10. Bosch I, Bracho C, Perez HA: Diagnosis of malaria by acridine orange fluorescent microscopy in an endemic area of Venezuela. Mem Inst Oswaldo Cruz 1996; 91: 83-86.

11. Seed C, Cheng A, Keller A: Comparison of the efficacy of two malarial antibody enzyme immunoassays for targeted blood donor screening. Transfusion 2004; 44: 99A [Abstract].

12. McNamara DT, Thomson JM, Kasehagen LJ, Zimmerman PA: Development of a multiplex PCR-ligase detection reaction assay for diagnosis of infection by the four parasite species causing malaria in humans. J Clin Microbiol 2004; 42: 2403-2410. 
13. Rubio JM, Benito A, Berzosa PJ, Roche J, Puente S, Subirats M, LopezVelez R, Garcia L, Alvar J: Usefulness of seminested multiplex PCR in surveillance of imported malaria in Spain. J Clin Microbiol 1999; 37: 32603264.

14. Benito A, Rubio JM: Usefulness of seminested polymerase chain reaction for screening blood donors at risk for malaria in Spain. Emerg Infect Dis 2001; 7: 1068 [Letter].

15. Duhanna NN and Zukova TA. Transmission of malaria by blood transfusion: an epidemiological study in the USSR. Bull World Health Organ. 1965; 33: 853-856. 



\title{
18. The Role of Leptospirosis - Zoonotic Disease in Maternal-Child Health in Indonesia
}

\author{
Bambang Pontjo Priosoeryanto, Department of Veterinary Clinic, Reproduction and \\ Pathology, Faculty of Veterinary Medicine, Bogor Agricultural University (IPB), Indo- \\ nesia
}

Risa Tiuria, Department of Animal Diseases and Veterinary Public Health, Faculty of Veterinary Medicine, Bogor Agricultural University (IPB), Indonesia

\section{Introduction}

Leptospirosis is an acute febrile illness that occurs in humans and animals. The disease is caused by the Leptospira spp. The causative organisms are shed in the urine of affected animals and humans into the environment. Leptospirosis is presumed to be the most widespread zoonosis in the world (Hickey and Deemeks, 2003). In the tropical and sub-tropical areas, the seroprevalence of antibodies to leptospira may vary from 20 to $30 \%$ among subjects tested serologically (Gasem, 2005). Humans get infected by direct contact with the infected animals or indirect by contaminated water with urine of infected animals. The organism is invading the body through skin wounds or mucous membranes (gastrointestinal and respiratory tract) and conjunctiva (Faine, 1982). Rats, pet animals (dog and cat) and live- 
stock (cattle and pig) were reported serologically positive for leptospira in Indonesia.

The true incidence is not precisely known due to the fact that the disease is often overlooked or misdiagnosed and therefore under-reported in most endemic areas. Leptospirosis may be under-diagnosed because the diagnosis is difficult to confirm, it may be confused with other diseases, and the disease may be mild and not be investigated in the laboratory. On the other hand, laboratory facilities to perform standard diagnostic tests for leptospirosis are rarely available in endemic areas (Gasem, 2005).

The clinical signs of leptospirosis vary from fever, icterus, haemoglobinuria and abortion in pregnant animals. The severity of this disease depends on the serovar of Leptospira, the species of infected animal and the geographical area (Ebrahimi et al., 2004). During the last decade, leptospirosis outbreaks have been reported in many countries such as Indonesia, India and Malaysia. In Indonesia, in 2007, not less than 20 provinces were positive for the disease (Jakarta; Banten; West, Central and East Java; Yogyakarta; Lampung; South, West, and North Sumatera; Bengkulu; Riau; West and North Sumatera; Bali; West Nusa Tenggara; North and South Sulawesi; West and East Kalimantan) but in 2012, 33 provinces were reported positive.

Transmission occured when animals contract the disease by eating and drinking Leptospira-contaminated water, or by direct contact of broken skin or mucous membranes with mud, vegetation or aborted foetuses of infected or carrier animals. Recovered animals and animals with unapparent (subclinical) leptospirosis frequently excrete billions of leptospiras in their urine for several months or years.

\section{Animal leptospirosis in Indonesia}

Animal leptospirosis in Indonesia has been serologically detected in several species such as cattle, pig, sheep, goat, buffalo, dog, cat and rats. Partoatmojo (1964) reported and stated that since 1936 several serovars have been isolated from domestic and wild animals. In 1938 L. pomona and L. javanica were isolated from domestic cat in Java island by Esseveld and Colier, while Abdullah in 1961 isolated L. autumnalis, L. canicola, L. sarmini, L. schuffneri, L. benyamin, L. asam, L. javanica, L. grippotyphosa and $L$. bovis from rats (Rattus rattus regni sody) in Bogor and surrounding area. In Ambarawa, L. bataviae, L. icterobaemorrhagiae, L. javanica, L pyrogenes and L. semaranga were also isolated. Van Peenen et al. (1971) isolated L. bataviae and $L$. australis from rats (Rattus bartelini and Rattus fulvescens) from the Cibodas area of Bogor district.

Orr and Darodjat (1978) on their study in West, Central, East Java and Bali provinces reported cases of leptospirosis in cattle and pigs with more than 6 serovars of Leptospira. Sumariyanto (1981) also detected 3 serovars of Leptopsira 
from rats in Bali. In Medan of North Sumatera, Gani and Setyowati (1990) reported a case of leptospirosis in rats from pig farming.

Kusmiyati et al. (2005) from their serological investigation using Microscopic Agglutination Test (MAT) of leptospirosis, found that $77,78 \%$ of clinically asymptomatic cats were serologically positive for Leptospira, while $40.48 \%$ of dog sera, $24.65 \%$ of cattle sera, $4.21 \%$ of pig sera and $29.46 \%$ of rat sera were positive. In $2004,33.33 \%$ of dog sera, $17.38 \%$ of cattle sera and $48.00 \%$ of rat sera were serologically positive for Leptospira. Syamsudin et al. (1985) studied the reproduction disorders of Bali cattle in Sumbawa, and found that $29.9 \%$ of them were seropositive to more than 5 serovars of Leptospira.

The average number of leptospirosis which was found in the study of Kusmiyati et al. (2005) is shown in Figure 1. From this figure we can see that these animals could act as a reservoir in the environment and also as a source of infection to other animals as well as to humans.

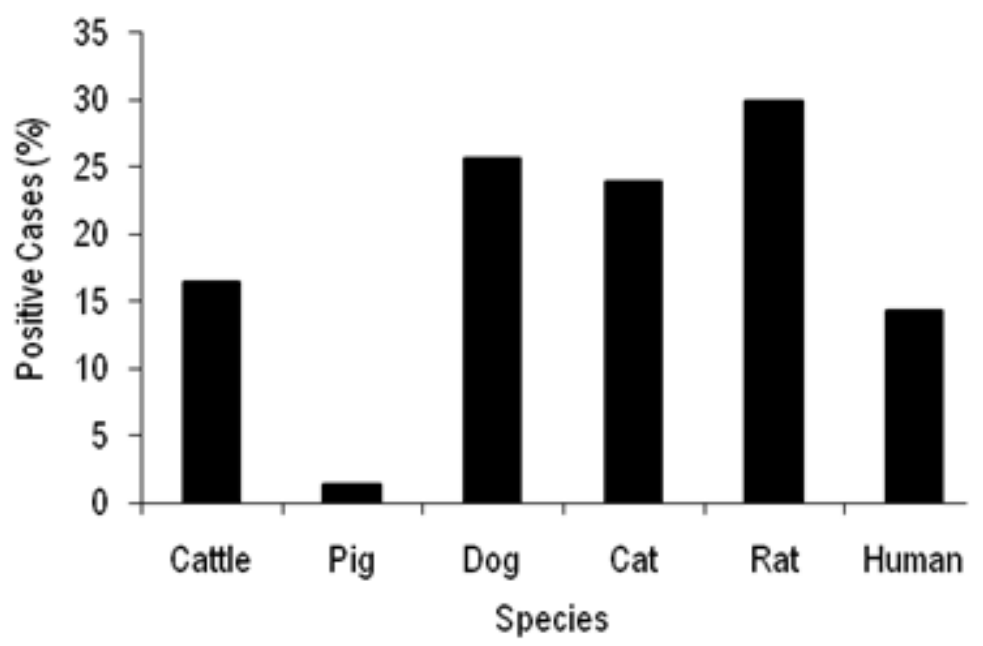

Figure 1: The average number of animals and humans in Indonesia with an immune response against Leptospira, collected and adapted from several reports (Kusmiyati et al., 2005; Ministry of Health; Ministry of Agriculture of the Republic of Indonesia).

The clinical signs of leptospirosis in animals vary. In the pregnant ruminant and pig, abortion and stillbirth are often found. In dairy cattle, fever and decreasing of milk production are common in infected animals; while reproduction disorders are found in pigs. In dogs, clinical signs of canine leptospirosis depend on the age and immunity of the host, environmental factors affecting the bacteria, and the virulence of the infecting serovar. Young animals are more severely affected than adults. The signs are fever, vomiting and jaundice while sub-clinical manifestation 
is also commonly found. The severity of the infection depends on the serovars of the agents. Leptospira interrogans serovar pomona in cattle causes fever, depression, acute anemia, haemorrhages and red water; while serovar hardjo in pregnant or lactating cows causes fever, decreasing milk production and abortion.

In dogs, four types of Leptospira infection have been found: 1) peracute infection, this type can be associated with massive leptospiremia and death which may occur rapidly with a few premonitory signs. 2) subacute infection, characterized by fever, anorexia, vomiting, dehydration, increased thirst; reluctance to move and paraspinal hyperesthesia in dogs that may result from muscular, meningeal, or renal inflammation; mucous membranes appear injected, petechial and ecchymotic hemorrhages are widespread, and progressive deterioration in renal function is manifested by oliguria or anuria. 3) acute infection, characterized by pyrexia (103$104^{\circ} \mathrm{F}$ ), shivering, and generalized muscle tenderness are the first clinical signs in acute leptospirosis; vomiting, rapid dehydration, and peripheral vascular collapse subsequently also occur; coagulation defects and vascular injury are characterized by hematemesis, hematochezia, melena, epistaxis, and widespread petechiae; icterus, intrahepatic cholestasis; dogs with chronic active hepatitis or chronic hepatic fibrosis as a sequela to leptospirosis, may eventually demonstrate overt signs of liver failure, including chronic inappetance, weight loss, ascites, icterus, or hepatoencephalopathy. 4) chronic/subclinical infection is the major leptospiral infection in dogs; serologic and microbiologic evaluation for leptospirosis should be performed in dogs with fever of unknown origin, unexplained renal or hepatic disease and anterior uveitis

The extent of damage to internal organs in post mortem observation in dogs is variable depending on the virulence of the organism and host susceptibility. Multifocal hepatic necrosis, multifocal haemorrhages of the lung and kidney (Figure 2), kidney enlargement, multifocal interstitial nephritis and renal tubular necrosis, edema and disseminated intravascular coagulation (DIC), extensive hepatic fibrosis and hepatic failure may result from this process (Monaham et al., 2009; Chandrasekaran et al., 2011; Ross et al., 2011). Other body systems, (e.g. CNS) are also damaged during the acute phase of leptospirosis.

\section{Human leptospirosis in Indonesia}

Clinically, leptospirosis in humans was already detected in Indonesia in 1892 by Van der Scheer, but the first isolation has only been sucessfully made in 1992 by Vervoort - a Dutch scientist during the Dutch colonization era (Partoatmodjo, 1964). The serovar hardjo was isolated by Wolf in 1938 from a rubber farmer in Deli of North Sumatera. In the early 1970s, human leptospirosis was reported by Fresh in South Sumatera and Bangka Island as well as in several hospitals in Jakarta. The outbreak of human leptospirosis at the transmigration area of Kuala Cina- 
ku in the Riau Province of Sumatera was reported by Simanjuntak et al (1986), pointing out that leptospirosis in humans was mostly transmitted by rats.

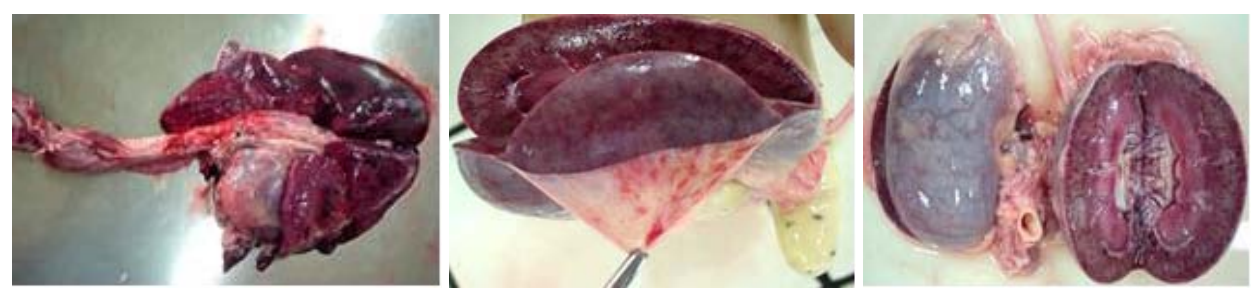

Figure 2. Gross lesions of leptospirosis in a doberman dog. Severe haemorrhages in the lung (left) and kidney (middle and right).

Leptospirosis is usually found in the rice farmer as well as in plantation, mining, slaughter house and military personnel. In some cases, swimmers were also infected by this organism. The fatality rate is high and could reach $2.5-16.45 \%$ (average $7.1 \%$ ). In people who are older than 50 years, the fatality rate can reach $56 \%$, in patients with hepatic leptospirosis and icterus the fatality rate could be higher.

The risk factors associated with human leptospirosis vary and they are mostly related to the exposure of humans to infected animals. The origin of infection in humans is usually a direct or indirect contact through urine or other body fluids of infected animals such as wild or feral animals, domestic pets or livestock. The organism can enter the body through skin wounds, intact skin or mucous membrane (gastrointestinal and respiratory tract) and conjunctiva. Leptospiral infection in human may be acquired via occupational or recreational activities. In wet tropical areas, where many rodents or farm animals live, human leptospiral infection is not limited to an occupation but more often to environmental contamination. Environmental factors that are associated with the transmission of leptospirosis are high population of rats, presence of dogs around the home, bad flow in gutters of the housing, and stagnant water. Outbreaks of leptospirosis are associated with water and animals in the context of swimming, drinking contaminated water or living in flood-prone areas etc.

In February - April 2002 (Widarso et al. 2003), an outbreak of human leptospirosis has occured in Jakarta-Indonesia after a long and severe flooding and 138 affected patients. 61 patients $(44.22 \%$ ) were positive by the MAT method; 103 people 21 people $(20 \%)$ died. In the same period 142 specimen of rodents serum $47 \%$ were positive to 4 serovar of Leptospira (pyrogenes, bataviae, icterohaemorrhagiae and canicola). In June 2002, in Bekasi near Jakarta, out of 117 random sample, 11 patients $(9.4 \%)$ were positive for Leptopsira and in the same month, 35 out of 43 rodent ser $(81.4 \%)$ were positive to 7 serovar of Leptospira (pyrogenes, bataviae, icterohaemorrhagiae, hardjo, pomona, javanica and gryppotyphosa). In March 2004 in the city of Makassar in South Sulawesi 7 patients were positive to Leptopsira of whom 5 people died. 
Between January-Juny 2007 human leptospirosis cases in several areas of Indonesia were reported by the Ministry of Health as follows: Jakarta with 248 cases; Banten with 34 cases; South Sulawesi with 1 case; Central Java with 3 cases; West Java with 9 cases and East Java with 1 case. In 2011 the cases increased especially in Jogyakarta when an outbreak of leptospirosis has occurred as seen in Figure 3; and also in one hospital in West Java up to January 2012 (unpublished data) 23 cases were recorded. In 2012, cases still occurred in Jogyakarta though the number went down in March 2012

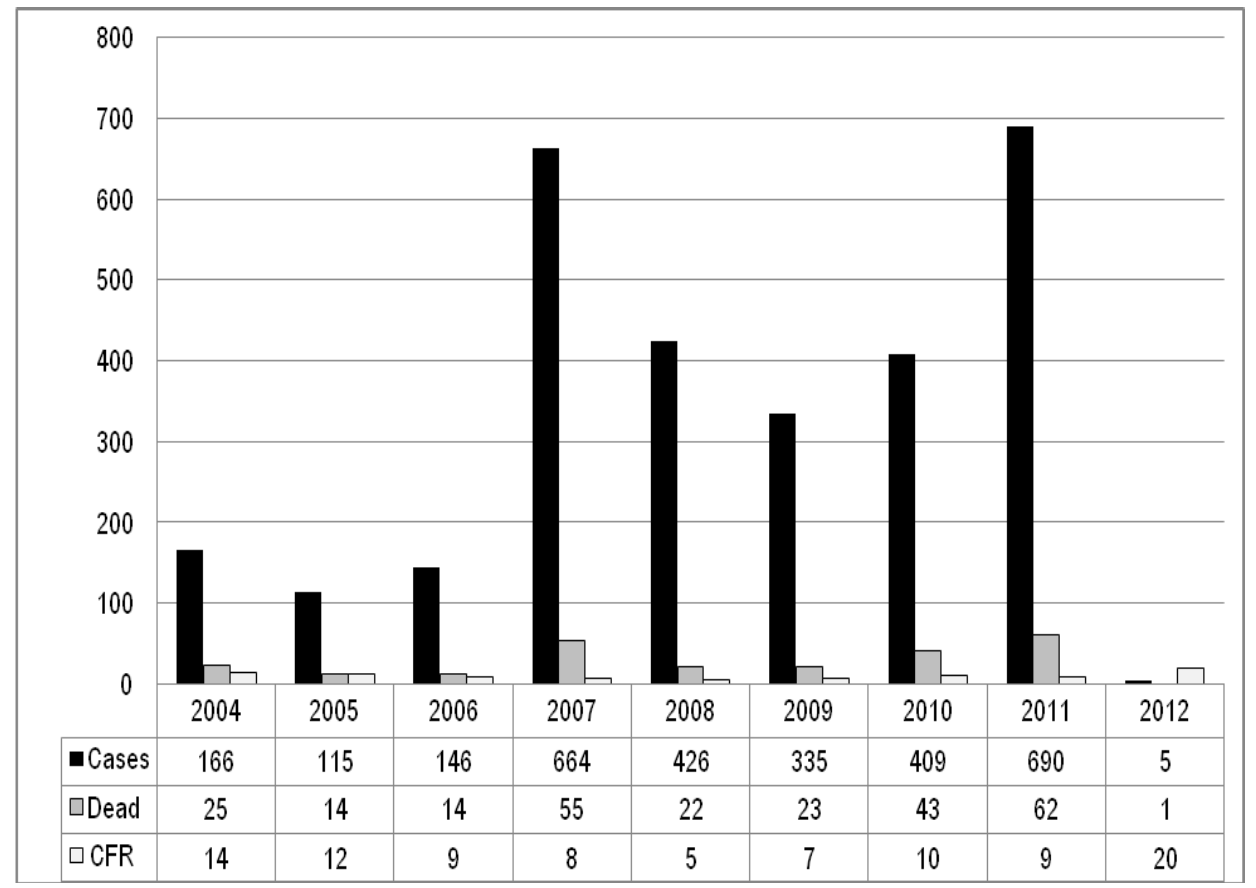

Figure 3. Yearly cases of human leptospirosis in Indonesia from 2004 - March 2012 (Aditama, National Coordination Meeting on Zoonoses, Ministry of Health, 2011). Year 2012 data only from Jogyakarta (Anonymous, 2012)

\section{Leptospirosis in mothers and children}

In human mothers, leptospirosis is extremely dangerous in all trimesters of pregnancy for both the mother and her foetus - there is a risk and also a potential issue for breast-feeding mothers. The foetus can be infected across the placenta due to the bacteria that could pass the barrier. This "transplacental" infection means that the foetus can become ill, independent of the mother - who may have such mild 
symptoms that she does not notice any illness herself. The foetus can be critically ill without the mother being aware of any symptoms.

Infection through transplacental transmission is very common where the mother is infected, and the outcome varies depending on the timing. The infected foetus will develop the same clinical symptoms as an adult - so it can suffer from jaundice, renal damage, vasculitis and haemorrhages especially in the first and second trimester where the foetal immunity response is absent. If the foetus is infected in the third trimester it can often present an immune response, and if it survives it will usually continue to develop and be born without abnormality. If the infection takes hold early the foetus will usually die.

Several studies from Vietnam, and Cambodia on the seroprevalence and the clinical signs of the disease including mild disease in children have been done (Thai et al., 2006 and 2008; Laras et al., 2002; Van Peneen et al., 1998; Wagenaar et al., 2004). Laras et al. (2002) reported that in one study from Cambodia, only 6 out of 202 (i.e., 3\%) cases of acute hemorrhagic fever in the study population were caused by leptospiral infection. On the other hand, a study at Binh Thuan province of Vietnam by Thai et al. (2006) on primary school children found that though frequently exposure to leptospira (as demonstrated by the seroprevalence of $11 \%$ at the age of 7 years), the incidence of reported symptomatic leptospirosis is usually low in these children.

In India, based on the report of Tullu and Karande (2009), the disease has been endemic in the Indian states of Kerala, Tamil Nadu, and the Andamans. Also, with improvement in diagnostic facilities over the past few years, leptospirosis is being reported from almost all the cities and states of India, including Delhi, Mumbai, Uttar Pradesh, Orissa, and Gujarat. Thirty out of 93 (32\%) urban slum children from Mumbai admitted for suspected leptospirosis.

In one case in a hospital in Central Java-Indonesia (unpublished data) the pregnant mother with her baby died during delivery and both were positive to leptospirosis. The figure of maternal-child leptospirosis infections in Indonesia seems to be bigger than it is, since Indonesia has only two seasons, the dry and the wet (rainy) season and it mostly occurs during the flooding in the rainy season when the contact of people/animals with rat urine-contaminated water is most frequent. The same figures seem to occur in developing countries that lack infrastructure, medical aid and where poor environmental conditions exist. In developed countries young children are less susceptible to infection than adults as the general population has no innate immunity to the infection and there is no vaccination program. The issues, when dealing with young patients, are those of communication and medication. Young children are often exposed to leptospira because of their playing and sports activities - swimming in rivers, lakes, fishing, or throughout floodings - and when touching infected animals. 


\section{$5 \quad$ Preventive measures}

Leptospirosis in Indonesia and in other developing countries is still a health and medical problem, both in veterinary and human medicine. The spreading and outbreak of leptospirosis is most likely caused through contaminated water (e.g. urine of infected animal), especially after flooding. For preventing and control of leptospirosis in animals and humans, good collaboration and integration activities of veterinarian and medical doctors in all medical aspects are absolutely needed and improved education and socialization of the high-risk population is a must.

Leptospirosis infections might be prevented by avoiding or minimizing potential risk factors. Measures that might be useful to prevent leptospiral infections are : 1) drainage of contaminated waters, 2) improving quality of living environment including education, 3) water management (flood-prone area, farming etc.), 4) immunizations of domestic animals, 5) use protective clothing by workers, 6) prophylactic antibiotics for high risk activities, 7) prompt antiseptic treatment of wounds, 8) and most important is rodent control, and 9) the implementation of early warning systems, especially in endemic areas and during the rainy season.

\section{References}

Aditama T Y. 2011. National Coordination Meeting on Zoonoses, Ministry of Health, 2011.

Anonimous. 2012. Penderita Leptospirosis di Jateng Meningkat, Warga Diminta Waspada. Republika News paper - Wed, Jan 4th, 2012.

Ebraahimi A, Z Nasr and GA Kojouri. 2004. Seroinvestigation of bovine leptospirosis in Shahrekord distict, central Iran. Iranian J. Vet. Res. University of Shiraz. 5(2) Ser. (10). 1383:110-113.

Chandrasekaran D, S Prathaban, P Dhanabalan, C Balachandran, BM Manohar and KS Venkataraman. 2011. Pathological changes in canine leptospirosis. Tamilnadu J. Veterinary \& Animal Sciences. 7(3): 180-183.

Faine S. 1982. Guidelines for the control of leptospirosis. WHO. Geneva. 171 p.

Gani MS and Setyomati. 1990. Isolasi Leptospira sp. pada tikus rumah dari perusahaan peternakan babi di Medan. Bulletin Veteriner BPPH Wil I 1:14-16.

Gasem MH, JFP Wagenaar, MGA Goris, MS Adi, BB Isbandrio, RA Hartskeerl, JM Rolain, D Raoult, and ECM van Gorp. 2009. Murine typus and leptospirosis as causes of acute undifferentiated fever, Indonesia Dispatch, 15(6).

Gasem MH. 2005. Management of human leptospirosis. Prosiding Lokakarya Nasional Penyakit Zoonosis. Pusat Penelitian dan Pengembangan Peternakan. Bogor 15 September 2005. 
Hickey PW and D Dememeks. 2003. Leptospirosis. Emedicine. pp.1-9.

Kusmiyati, SM Noorand Supar. 2005. Leptospirosis pada hewan dan manusia di Indonesia. Wartazoa 15(4): 213 - 220.

Laras K, Van CB, Bounlu K, Tien NT, Olson JG, Thongchanh ST, et al. 2002. The importance of leptospirosis in Southeast Asia. Am J Trop Med Hyg; 67:278-86.

Masniari P and I Komala. 2006. Mewaspadai leptospirosis di Indonesia sebagai penyakit zoonosis. Lokakarya Nasional Penyakit Zoonosis.

Monahan AM, JJ. Callanan and JE Nally. 2009. Host-pathogen interactions in kidney during chronic leptospirosis. Vet Pathol. 46: 792-799.

Orr HS and M Darodjat. 1978. Leptospirosis: Kerja pendahuluan di LPPH. Bulletin Lembaga Penelitian Penyakit Hewan 16:35-45.

Orr HS, M Darodjat, J Achdijati and M Soeroso. 1980. Kejadian leptospirosis dan brucellosis pada ternak di Indonesia. Prosiding Seminar Penyakit Reproduksi dan Unggas LPPH. Bogor 13 -15 Maret 1980. 31-58.

Partoatmodjo S. 1964. Penyelidikan mengenai leptospirosis: Penyakit zoonotik yang berarti bagi ekonomi dan kesehatan masyarakat di Indonesia. Thesis. Institut Pertanian Bogor. Bogor.

Partodihardjo S, M Noordin, SM Darodjat, Sugijanto and S Djojosedarmo. 1979. Survei serologik terhadap brucellosis dan leptospirosis pada ternak potong di Jawa Tenga, Jawa Timur dan Bali. Media Vet. 1:30-34.

Ross L, R Jakowski, C Bolin and M Kiupel. 2011. Retrospective immunohistochemical detection of leptospirosis in dogs with renal pathology. Intern. J. Appl. Res. Vet. Med. 9(4): 324-33.1

Sumariyanto JA. 1981. Pengisolasian Leptospira serogroup javanica dari ginjal tikus (Rattus rattus) asal Pulau Bali. Bulletin Lembaga Penelitian Penyakit Hewan, 21:29-32.

Syamsudin A, M Darodjat, A Sarosa and Gerhat. 1985. Inventarisasi penyakit reproduksi pada sapi Bali di Sumbawa. Penyakit Hewan. Balitvet, 17(30): 2831.

Simanjuntak G, C Koesharjono and S Harjoutomo. 1986. Leptospirosis di daerah transmigrasi Kuala Cinaku Propinsi Riau tahun 1981. Penyakit Hewan. Balitvet, 18(31):6-13.

Thai KT, Binh TQ, Giao PT, Phuong HL, Hung LQ, Van Nam N, et al. 2006. Seroepidemiology of leptospirosis in southern Vietnamese children. Trop Med Int Health,11:738-45. 
Thai KT, Nga TT, Phuong HL, Giao PT, Hung le Q, Binh TQ, et al. 2008. Seroepidemiology and serological follow-up of anti-leptospiral $\operatorname{IgG}$ in children in Southern Vietnam. Acta Trop,106:128-31.

Tullu MS and Karande S. 2009. Leptospirosis in children: A review for family physicians. Indian J Med Sci, 63:368-78.

Van CT, Thuy NT, San NH, Hien TT, Baranton G, Perolat P. 1998. Human leptospirosis in the Mekong delta, Vietnam. Trans R Soc Trop Med Hyg, 92:625-8.

Van Peneen PFD, RH Light and JS Sarosa. 1971. Isolation of Leptospira in wild rodent. South East Asian J. Trop. Med, Pub. Health, 2:496-502.

Wagenaar JF, Falke TH, Nam NV, Binh TQ, Smits HL, Cobelens FG, et al. 2004. Rapid serological assays for leptospirosis are of limited value in southern Vietnam. Ann Trop Med Parasitol; 98:843-50.

Widarso, MH Gasem, W Purba, T Suharto and S Ganefa. 2003. Pedoman diagnosa dan penatalaksanaan kasus penanggulangan leptospirosis di Indonesia. Sub Direktorat Zoonosis, Direktorat Jenderal Pemberantasan Penyakit Menular dan Penyehatan Lingkungan, Departemen Kesehatan RI. 


\section{Vertical Transmission of Hepatitis B Virus; the Role of Placental Barrier, Modes of Delivery, Viral Load \& Immunoprophylaxis of Neonates}

Maisuri T. Chalid, Department of Obstetrics and Gynecology, Medical Faculty, Hasanuddin University, Makassar, Indonesia

David H. Muljono, Chairman of the National Committee on Viral Hepatitis and Head of Department of Hepatitis and Emerging Disease of Eijkman Institute of Molecular Biology, Jakarta, Indonesia

\section{Abstract}

Hepatitis B virus (HBV) is a major public health problem that affects a significant proportion of the world's population. Approximately 2 billion people worldwide have been infected with the Hepatitis B virus. Globally 50 million new cases are diagnosed annually. Worldwide, 240 million people are chronically infected, comprising 5-10\% adults and up to $90 \%$ of infants (World Health Organization, 2012). About $75 \%$ of them reside in the Asia-Pacific. Africa has the second largest number with 50 million chronic carriers, whereas in Europe and North America the prevalence is less than $1 \%$. In endemic areas, where up to $20 \%$ of women at childbearing age may have $\mathrm{HBV}$, vertical transmission remains the most frequent route of infection. Estimated $40-50 \%$ of chronic $\mathrm{HBV}$ infection originates from perinatal transmission. Factors that may contribute to vertical transmission are 
viral load, modes of delivery, and placental barrier. Although HBV transmission can be reduced by universal infant vaccination programs, it continues to occur in high-prevalence countries. The high prevalence of Hepatitis B among pregnant women that leads to a high risk of vertical transmission, not only has an impact on maternal and child health, but also makes Hepatitis B a serious threat to the quality of life of future generations. Studies of vertical transmission of Hepatitis B may help to establish the prevalence and risk factors and provide data on recommendations for inter-ventions to break the transmission cycle.

Keywords: hepatitis B virus, vertical transmission, perinatal pregnancy

\section{Introduction}

Hepatitis B viral infection is one of the most important public health problems all over the world. Approximately 2 billion people worldwide have been infected with the hepatitis B virus (HBV) and approximately 240 million people have chronic infection of Hepatitis B which may develop into acute, fulminant, cirrhosis and liver carcinoma (World Health Organization, 2012).

In high prevalence areas, the transmission of hepatitis $\mathrm{B}$ virus from mothers to neonates is the main route of transmission of hepatitis B. In the absence of immunoprophylaxis $90 \%$ of infants who get infected during pregnancy, delivery or breastfeeding, become chronic carriers of HBV (Chang, 2007). Even if the provision of active and passive immunization soon after birth will reduce the rate of vertical transmission of hepatitis B virus, immunoprophylaxis is not $100 \%$ protective and up to $2 \%$ of infants can still become infected, where the maternal viral load is high. Meanwhile, according to WHO data of 1985, an estimated $73-100 \%$ of infants were born to mothers with positive $\mathrm{HBeAg}$ and $\mathrm{HBsAg}$. This makes the vertical transmission of hepatitis B a global health problem that needs serious attention, particularly in moderate to high endemic areas like Indonesia with low in public awareness on HBV.

The following illustration will explain the role of viral load, mode of delivery, the placental barrier and immunoprophylaxis of neonates in the mechanism of vertical transmission. A case report on how this mechanism occurs will also be discussed in this paper.

\section{Epidemiology}

Global distribution of hepatitis B is divided into 3 categories: mildly endemic areas such as Northern Europe, North America and Australia, where the HBV carrier status is less than $0.1 \%$. Moderately endemic areas such as eastern Europe, the Mediterranean, South America and West Asia where the carrier status is about 5\%, 
and severely endemic areas such as China, Southeast Asia, the Pacific, and Africa where up to $20 \%$ are estimated to be chronic carriers.

Transmission of hepatitis B virus can be vertical or horizontal. In Africa, which is one of the hyperendemic HBV regions, it was found that infection with hepatitis $B$ virus (HBV) is transmitted horizontally. The main source of infection is horizontal family members who have been infected with HBV infection through gloves and needles which are not sterile (Chang, 2007; Wishnuwardhani, 1997).

There are approximately 50 million chronic carriers of HBV in Africa with a $25 \%$ risk of mortality. In sub-Saharan Africa, the carriers rate ranges from $9-20 \%$. Many studies have outlined that a horizontal transmission in childhood is predominantly in Africa rather than perinatal transmission (Kiire CF, 1996). ). Studies in Ghana, West Africa, have shown that $16 \%$ of 1368 pregnant women who had been screened were chronic carriers of HBV (Candotti et al., 2007).

Chronic hepatitis B infection is an important health issue in Eastern Asia. In a serological survey in China in 2006, HBsAg carrier status was around 7.18\% (Liang et al., 2009). In Taiwan, the HBsAg carrier status was found to range from 10 to $20 \%$, whiles in Taipei it was about 5\% among infants increasing to $10 \%$ by the second birthday (Sun et al., 2012).

The rate of transmission was found to increase with age and infection rates as high as $50 \%$ were noted at the age of 15 years (Chang, 2007).

The incidence of hepatitis B in pregnant women varies widely, averaging around $10 \%$. Rohtagi et al. reported a rate of $25 \%$, Gupta et al. reported a figure of around $39 \%$ and Stevans et al. reported 17\%. The rate of transmission of HBV from the mother to the baby ranges from $0.12 \%$ to $60 \%$ (Sharma et al. 1996).

Research in Southeast Asia showed that the HBsAg prevalence in the population ranges between 4-13\% (Wishnuwardhani, 1997). An estimated 40-50\% of them are chronic carriers and were proven having obtained the infection during birth (Chen CJ et al., 2000; Merican I et al., 2000).

In Indonesia, hepatitis B is moderately-to-highly endemic, with low public awareness. Data on hepatitis B prevalence in pregnant women are limited in number as well as study coverage. Research in West Java (1985) stated 4.7\% of HBV carriers and $1.9 \%$ in Bali (2005) with women from low socioeconomic and education levels being more prone to this infection than others (Putu Surya IG et al., 2005; Raniers J et al., 1987).

Previous data published by Soemohardjo et al. (1984) and Bandasho (1985) remarked that the rate of pregnant women with $\mathrm{HBsAg}$ carrier status were around $3.4 \%$ and $4.5 \%$ (Raniers et al., 1987).

North America, southern Europe and the Oceania region are areas with low prevalence (approximately $0.1 \%$ of the population), and HBV obtained in many teens and adults (Chang, 2007). 


\section{Virology}

Hepatitis B virus belongs to the family of Hepadnaviruses. The virus particle (virion) consists of a capsule of lipids on the outside and an icosahedral nucleocapsid core composed of proteins. The diameter of the particle size is $42 \mathrm{nM}$. The core nucleocapsid genome consists of DNA, and is sometimes called "Dane particle" (Drew, 2001).

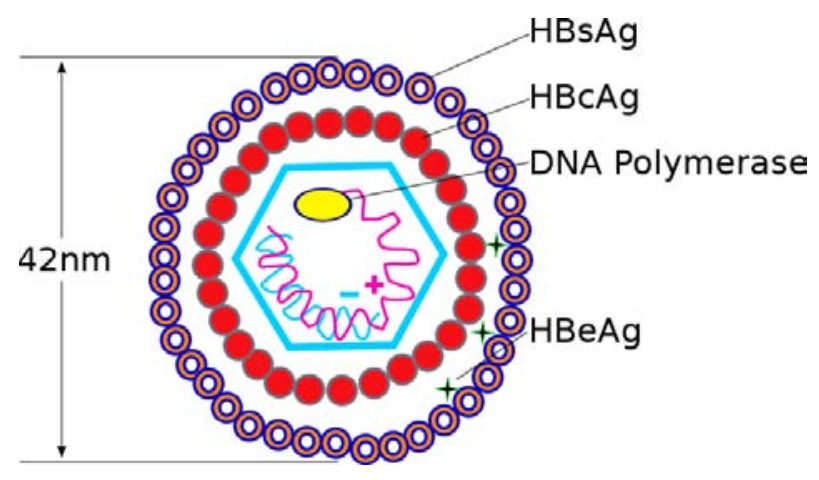

Figure 1. Hepatitis B Virus Structure

The viral genome consists of double-stranded DNA with 3200 nucleotides. Another core component of $\mathrm{HBV}$ is the core antigen $(\mathrm{HBcAg})$ and $\mathrm{HBeAg}$ which is a glycoprotein with a light molecular weight (Drew, 2001).

The viral envelope containing HBsAg, consists of one major protein and two other proteins. HBsAg aggregates are often found in large quantities in the serum during infection. They can have a spherical or filamentous shape with an average diameter of $22 \mathrm{~nm}$ and can contain parts of the nucleocapsid. When HBV DNA in the serum then active infection is proven (Drew, 2001).

In the liver of infected patients, $\mathrm{HBcAg}, \mathrm{HBeAg}$, and $\mathrm{HBV}$ DNA are found in the nucleus of infected hepatocytes, whereas HBsAg is found in the cytoplasm. Hepatitis B virus replication involves a reverse transcription step and this is something that is unique among DNA viruses. During viral replication, viral RNA transcripts positively incorporate into the mature core particle at the end of the cycle of replication (Zuckerman, 2010; Drew, 2001).

\section{Transmission mechanisms}

Women of childbearing age in high prevalence area constitute a reservoir for HBV perinatal transmission which is associated with a very high rate of chronicity (Lok AS, 2002). The vertical transmission is influenced by several factors such as maternal levels of HBV-DNA, HBeAg, variations in gene $\mathrm{S}$ of hepatitis B virus (HBV), 
duration of labor, and neonatal immune deficiency (Raniers et al.; Shen et al., 2008; Singh et al., 2011; Sun et al., 2012). Gestational age during hepatitis B infection is an important factor affecting infant outcome (Chang, 2007).

\subsection{The role of the placental barrier}

Intrauterine infection is rare, less than $5 \%$ occurring in infants born to mothers with positive $\mathrm{HBsAg}$ and $\mathrm{HBeAg}$. Research in Taiwan revealed that, over a period of 10 years only $2.4 \%$ of 665 infants born to mothers who were both HbsAg and $\mathrm{HBeAg}$ positive, only $2.4 \%$ were $\mathrm{HBsAg}$ positive at birth. Rupture of blood vessels in the mother and the placental barrier breakdown is the main route leading to perinatal (vertical) hepatitis B infection (Chang, 2007). Intrauterine infection with HBV is caused by a damage of placental barrier (Bai et al., 2007; Jonas, 2009; Papaevangelou et al., 1974; Xu et al., 2002).

The materno-foetal transmission rate varies from 0.6 to $20 \%$. This variation is depending occurs upon many factors such as the time of onset of hepatitis in mother. There is a higher incidence of transmission $(76 \%)$ in the third trimester or early post-partum, compared to the transmission $(10 \%)$ in the first or second trimester (Sharma et al., 1996).

It has been reported that the rate of transmission of $\mathrm{HBV}$ in mothers with positive $\mathrm{HBeAg}$ and about $85 \%$ of infants will become chronic carriers (Sharma et al., 1996).

$\mathrm{Xu}$ et al. (2002) examined 101 placental tissues from pregnant women at term by in-situ hybridization immunohistochemistry and obtained the following results: HBsAg was found in 34 placentas (33.7\%), HBeAg in 38 placentas $(37,6 \%)$, $\mathrm{HBcAg}$ in 21 placentas (20.8\%) and HBV-DNA in 45 placentas (44.6\%). Intrauterine Infection was found in eight of the 101 women.

Intrauterine $\mathrm{HBV}$ infection correlated with the presence of the virus in villous capillary endothelial cells lining the foetal parts than other placental linings (Xu et al., 2002).

\subsection{Mode of delivery}

Prolonged labor has an influence on the risk of vertical transmission from mother to her foetus, which revealed a linear correlation between the incidence of HBsAg in the cord and the length of first stage of labor of which the correlation can be observed if the first stage lasts $\geq 9$ hours. Research by Jonas (2009) on 447 infants born to $\mathrm{HBs} A g$-positive mothers revealed that $24.9 \%(96 / 385)$ of vaginally born babies had HBV infection compared to about $10 \%$ delivered by caesarean section. However, in other studies no significant association between the mode of delivery and transmission of HBV was found, and no difference between vaginal delivery and caesarean section was observed (Bai et al., 2007; Sharma et al., 1996). 


\subsection{Viral load}

The risk for HBV transmission through the placenta depends on $\mathrm{HBeAg}$ positivity, titer of HBsAg and HBV-DNA levels. In one study, the mother's level of HBV DNA $>108$ copies $/ \mathrm{ml}$ was associated with increased risk of intrauterine infection (Xu et al., 2002). Wiseman et al. reported that perinatal HBV transmission occurs only when the levels of HBV DNA exceeds 108 copies/ml (or about 1.7 x 107 IU $/ \mathrm{ml}$ ) and if the mother is $\mathrm{HBeAg}$ positive.

Determination of HBV-DNA plays an important role in the management and administration of $\mathrm{HBV}$ vaccination; however this is 10 times more expensive than that of HbsAg. Sun Kui-xia et al. conducted a study on the correlation between $\mathrm{HBsAg}$ and HBV-DNA. It was found that in pregnant HBeAg-positive pregnant women there was a correlation between the levels of HBsAg and HBV-DNA regardless of age and genotype.

HBsAg examination can be used as predictors of HBV-DNA levels: it has been shown that HBsAg levels of more than $4.1 \log I U / \mathrm{ml}$ are a predictive value for HBV-DNA levels $\geq 7.0 \log \mathrm{IU} / \mathrm{ml}$ in $\mathrm{HBeAg}$ positive women. This would be less expensive than HBV-DNA examination. By observing the levels of $\mathrm{HBs} A g$ in HBeAg mothers the provision of HBV or HBV immunoglobulin (HBIG) can be planned which also lowers the cost of universal vaccination at birth.

\subsection{Immunoprophylaxis}

Universal vaccination in newborns with active and passive immunoprophylaxis has dramatically reduced the prevalence of hepatitis B. In contrast, without immunoprophylaxis up to $90 \%$ of infants born to $\mathrm{HBeAg}$ - positive mothers become chronically infected. However, and even in cases of appropriate prophylaxis with HBV immunoglobulin (HBIG) and HBV vaccination, a significant risk $(>10 \%)$ of vertical transmission remains, particularly in mothers with high viral load (serum HBV DNA >10 6-7 IU/ml) and positive HBeAg status (Del et al., 1997).

Sun Kui-Xia et al. reported that out of 975 infants born to HBsAg-positive mothers, 25 infants $(2.6 \%)$ were identified to have suffered perinatal transmission despite the HBV vaccine and HBIG given at birth, one month and six months, respectively. In total $96 \%(24 / 25)$ of the newborns' mothers had HBV-DNA levels $\geq 107 \log \mathrm{IU} / \mathrm{ml}$ and were $\mathrm{HBeAg}$ positive. Mothers with high HBV-DNA levels have a high risk of transmitting HBV to their babies.

Some previous studies addressed that increasing doses of hepatitis B vaccine or HBIG can reduce the rate of transmission (Sun et al., 2012).

\subsection{Treatment}

When women in the childbearing age require antiviral therapy, the issue of pregnancy must be discussed before starting treatment. In pregnant women with 
chronic HBV infection who need antiviral therapy, the liver disease stage of the mother and potential benefit of treatment must be weighed against the risk to the foetus. IFN-based therapy is contraindicated because of its antiproliferative effect; the only choice is a nucleoside (NRTI) or nucleotide analogue (NtRTI) (Liaw YF et al., 2012; European Association for the Study of the Liver, 2012). However, pregnancy is usually a contraindication for all available drugs. Therapy with NRTI during pregnancy may be considered if the benefit seems to be higher than the risk. Tenovofir and Telbivudine are classified as category B drugs (no risk in animal studies, but unknown in humans), whereas Lamivudine, Adevofir, and Entecavir are classified as category $\mathrm{C}$ drugs (teratogenic in animals, but unknown in humans) by the US FDA (Petersen, 2011). Studies have shown that antiviral therapy administered in late pregnancy may further reduce the risk of perinatal HBV infection from highly viremic mothers, as compared with passive-active immunization alone. However, the extent of benefit, the threshold of serum HBV DNA level for initiating therapy, the optimal time to start therapy, the appropriate choice of an antiviral agent, and the optimal duration of therapy need to be further studied before they are applied in the algorithm of hepatitis B in pregnant women.

\subsection{Case illustration}

The illustrative case below is a small part of our research that describes the transmission mechanisms of HBV from the mother to the foetus. The occurrence of vertical transmission is defined by HBV DNA found in umbilical cord blood and foetal part of the placenta by PCR (Table 1, Figure 1-3).

Table 1: Mechanisms of vertical transmission through intrauterine

\begin{tabular}{|c|c|c|c|c|c|c|c|c|}
\hline \multirow[t]{2}{*}{ Sample } & \multirow[t]{2}{*}{$\mathrm{HBsAg}$} & \multirow[t]{2}{*}{$\mathrm{HBeAg}$} & \multicolumn{5}{|c|}{ HBV DNA } & \multirow[b]{2}{*}{ 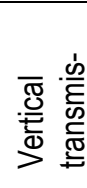 } \\
\hline & & & $\begin{array}{l}\text { maternal } \\
\text { blood }\end{array}$ & $\begin{array}{l}\text { cord } \\
\text { blood }\end{array}$ & $\begin{array}{l}\text { amniotic } \\
\text { fluid }\end{array}$ & $\begin{array}{l}\text { pars } \\
\text { maternal } \\
\text { placenta }\end{array}$ & $\begin{array}{l}\text { pars } \\
\text { foetalis } \\
\text { placenta }\end{array}$ & \\
\hline 1 & + & NA & + & NA & $\mathrm{NA}$ & NA & NA & NA \\
\hline 2 & + & + & + & + & NA & $t$ & + & + \\
\hline 3 & + & - & + & - & NA & NA & NA & - \\
\hline 4 & + & - & - & - & - & + & - & - \\
\hline 5 & + & + & + & NA & NA & + & + & + \\
\hline
\end{tabular}

NA: not available 


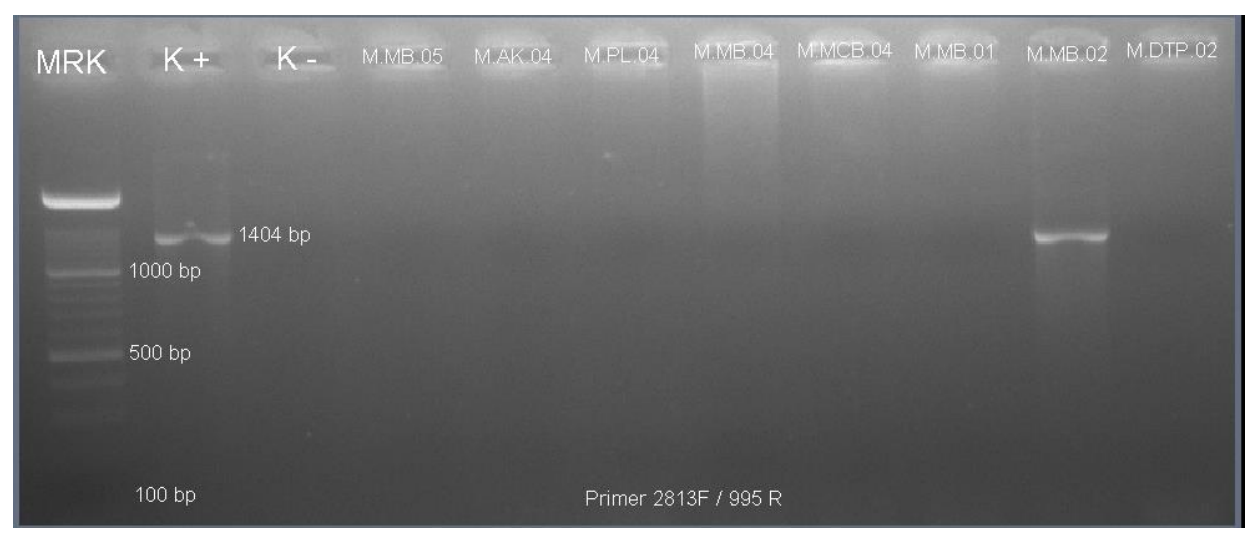

Fig. 2: HBV DNA examination by PCR in maternal blood, cordblood, amniotic fluid, and placental tissue

$\mathrm{M} . \mathrm{MB}=$ Maternal Blood, M.DTP $=$ Cord blood, $\mathrm{M} \cdot \mathrm{CB}=$ Cord Blood, M.AK $=$ Amniotic fluid, M.PL = whole Placenta, M.JP = Placental tissue pars maternal, M.JTP=Placental tissue pars foetalis

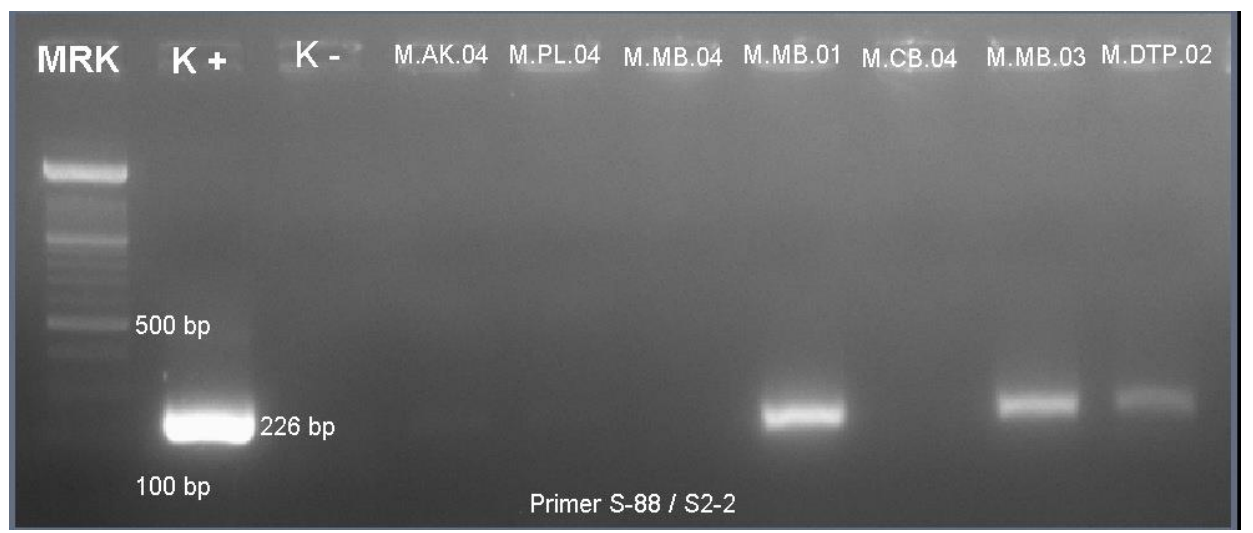

Fig. 3: HBV DNA examination by PCR in maternal blood, cordblood, amniotic fluid, placental tissue

M.MB $=$ Maternal Blood, M.DTP $=$ Cord blood, $\mathrm{M} . \mathrm{CB}=$ Cord Blood, M.AK $=$ Amniotic fluid, M.PL = whole Placenta, M.JP = Placental tissue pars maternal, M.JTP=Placental tissue pars foetalis 


\section{MRK K + M.JP.04 M.JP.06 M.JP.05 M.05.JTP}

Primer $\mathbf{8 8} 8$ dan $\mathrm{S} 22$

500 bp

249 bp

$100 \mathrm{bp}$

Fig. 4: HBV DNA examination by PCR in maternal blood, cordblood, amniotic fluid, placental tissue pars maternal and foetal

M.MB $=$ Maternal Blood, M.DTP $=$ Cord blood, $\mathrm{M} . \mathrm{CB}=$ Cord Blood, M.AK = Amniotic fluid, M.PL = whole Placenta, M.JP = Placental tissue pars maternal, M.JTP=Placental tissue pars foetalis

\section{Summary}

- HBV infection is diagnosed by seromarkers such as: HbsAg, HbeAg and HBV-DNA.

- HBeAg and HBV-DNA are the important factors affecting the vertical transmission of Hepatitis B.

- Vertical transmission occurs if HBV-DNA levels are $>108$ copy/ml ( or approximately $1.7 \times 107 \mathrm{IU} / \mathrm{ml}$ ) and if $\mathrm{HBeAg}$ is positive. 
- HbsAg level $\geq 4,1 \log \mathrm{IU} / \mathrm{ml}$ is a predictive value for HBV-DNA level $\geq 7.0$ $\log \mathrm{IU} / \mathrm{ml}$ in $\mathrm{HBeAg}$ positive women.

- HBsAg may be used as a main seromarker in determining vertical transmission to substitute the role of HBV-DNA due to the cost.

- For mothers having HBV-DNA $\geq 107 \log \mathrm{IU} / \mathrm{ml}$ and HBeAg positive, the rate of vertical transmission will remain despite the vaccination and HBIG at birth and theadministration of the second Hepatitis B vaccination at the age of 1 month and 6 months.

- Antiviral therapy in late pregnancy may reduce the risk of perinatal HBV infection from highly viremic mothers, however this still needs to be further investigated.

\section{References}

Bai H, Zhang L, Ma L, Dou XG, Feng GH, Zhao GZ. Relationship of hepatitis B virus infection of placental barrier and hepatitis $\mathrm{B}$ virus intra-uterine transmission mechanism. Worlds Journal of Gastroenterology 2007;26:362530.

Candotti D, Danso K, Allain JP. Materno-fetal transmission of hepatitis B Virus genotype E in Ghana, West Africa. Journal of General Virology 2007;88:268695.

Chang MH. Hepatitis B virus Infection. Science Direct 2007;12:160-7.

Chen CJ, LY W, Yu MW. Towards control of hepatitis B in the Asia-Pacific region. J Gastroenterol Hepatol 2000;15:E3-E6.

Del CR, Grosheide PM, Mazel JA, Heijtink, Hop WC. Ten year neonatal hepatitis B vaccination program, The Netherlands, 1982-1992: protective efficacy and long-term immunogenicity. Vaccine1997;15:1624-30.

Drew LW. Hepatitis. In: Wilson, R W, Sande M, editors. Current diagnosis \& treatment in infectious diseases. New York: Lipincot; 2001.

European Association for the Study of the Liver. EASL Clinical Practice Guidelines: Management of chronic hepatitis B virus infection. Journal of Hepatology 2012;57:167-85.

Kiire CF. The epidemiology and prophylaxis of hepatitis B in sub-Saharan Africa: a view from tropical and subtropical Africa. Gut 1996;38(suppl 2):s5-s12.

Liang XF, Bi SL, Yang WZ, Wang LD, Cui G. Epidemiological sero-survey of Hepatitis B in China - declining HBV prevalence due to Hepatitis B vaccination. Vaccine 2009;27:6550-7. 
Liaw YF, Kao JH, Piratvisuth T, Chan HLY, Chien RN, Liu JC, et al. Asian-Pacific consensus statement on the management of chronic hepatitis B: a 2012 update. Hepatology International 2012:DOI 10.1007/s12072-012-9365-4.

Lok AS. Chronic hepatitis B. N Engl J Med 2002;346:1682-3.

Jonas MM. Hepatitis B and pregnancy: an underestimated issue. Liver International 2009;29:133-9.

Merican I, Guan R, Amarapuka D, Alexander MJ, Chutaputti A, Chien RN, et al. Chronic hepatitis B virus infection in Asian countries. J Gastroenterol Hepatol2000;15:1356-61.

Papaevangelou G, Kremastinou T, Prevedourakis C, Kaskarelis D. Hepatitis B antigen and antibody in maternal blood, cord blood, and amniotic fluid. British Medical Journal1974;49:936.

Petersen J. HBV treatment and pregnancy. Journal of Hepatology 2011;55:1171-3.

Putu Surya IG, Kornia K, Agung Suwardewa TG, Mulyanto, Tsuda F, Mishiro S.

Serological markers of hepatitis B, C and $\mathrm{E}$ viruses and human immunodeficiency virus type-1 infection in pregnant women in Bali, Indonesia. Journal of Medical Virology 2005;75:499-503.

Raniers J, Vranckx R, Ngantung W, Sugita E, Meheus A. Prevalence and determinant of hepatitis B virus markers in pregnant women in West Java, Indonesia. J Trop Med Hyg 1987;5:249-53.

Sharma R, Malik A, Rattan, A, Iraqi A, Maheswari V, Dhawan R. Hepatitis B virus infection in pregnant women and its transmission to infants. Journal of Tropical Pediatrics1996;42.

Shen T, Yan X-M, Zou Y-L, Gao J-M, Dong H. Virulogic charateristics of hepatitis $\mathrm{B}$ virus in patients infected via maternal-fetal transmission. World Journal of Gastroenterology 2008;37:5674-82.

Singh AE, Plitt SS, Osiowy C, Surynicz K, Kouadjo E, Preiksaitis J, Lee B. Factors associated with vaccine failure and vertical transmission of hepatitis $B$ among a cohort of Canadian mothers and infants. Journal of Viral Hepatitis 2011;18:468-73.

Sun K-X, Li J, Zhu F-C, Liu J-X, Li R-C, Zhai XJ , Li Y-P, et al. A predictive value of quantitative $\mathrm{HBs} \mathrm{Ag}$ for serum HBV DNA level among HBeAg-positive pregnant women. Elsevier 2012;30:5335-40.

Wishnuwardhani SD. Penyakit Menular. In: Saifuddin AB, Rachimhadhi T, editors. Ilmu Kebidanan. Jakarta: Yayasan Bina Pustaka Sarwono Prawirohardjo; 1997. p. $551-78$. 
World Health Organization. Hepatitis B fact sheet No. 204. 2012 [cited 2012

December ]; Available from:

http:/www.who.int/mediacentre/factsheets/fs204/en/.

Xu D-Z, Yan Y-P, Choi BCK, Xu JQ, Men K, Zhang JX, Liu ZH, Wang F-S. Risk factors and mechanism of transplacental transmission of hepatitis $B$ virus : A case control study. Journal of Medical Virology 2002;67:20-6.

Zuckerman AJ. Perinatal transmission of hepatitis B. Journal of Hepatology 2010;52:282-4. 


\section{Human Cytomegalovirus Infection in Pregnancy and in Newborns}

Gerhard Jahn, Institute of Medical Virology and Epidemiology of Viral Diseases, Universitätsklinikum Tübingen, Germany

\section{Introduction}

Human cytomegalovirus (HCMV), the prototype of the beta-herpesviruses, has a worldwide distribution. In healthy adults, clinical symptoms are rare and not specific, but the virus is associated with opportunistic disease and many variable symptoms can be observed in immunosuppressed patients. Mother-child-infection of HCMV is worldwide the leading cause of birth defects and developmental disabilities such as hearing loss, opticus atrophy or CNS damage. Congenital HCMV infection occurs in about $0,6 \%$ of all newborns worldwide. The highest risk for adverse outcome in the newborn results from primary infection during the first half of pregnancy. The seroconversion rate during pregnancy differs depending on sexual exposure, but in industrialised countries it is about $2 \%$. In many countries in Africa and Asia there is a high seroprevalence of HCMV antibodies in young women. Numbers of $70 \%$ up to $100 \%$ are not unusual. The transmission rate of HCMV from mother to child in these high seroprevalence regions is low at about $1 \%$. This is in clear contrast to the transmission rate of about $40 \%$ during primary 
infection in regions of low seroprevalence. The risk for bad outcome in newborns from preconceptional seropositive women is very low. If seropositive women however acquire a secondary infection during the first half of pregnancy, there is a risk for adverse outcome too. The risk to acquire secondary infection depends on lifestyle such as the number of sexual partners or close contact to children less than three years during pregnancy. Breastfeeding of HCMV- positive mothers is an additional major route of HCMV transmission accounting for about $35 \%$ of infected newborns from seropositive mothers.

\section{Cell tropism and transmission routes}

HCMV is a promiscuous virus concerning cell types infected in various organs (Sinzger et al., 2008; Bissinger et al., 2002). Major target cells for the virus are epithelial cells, endothelial cells, fibroblasts, monocytes/macrophages, smooth muscle cells and in the liver in addition hepatocytes or in the placenta in addition trophoblasts. The cell tropism of HCMV and the importance for the major transmission routes within an infected body or between individuals is illustrated in Figure 1. The importance of cell tropism and organs productively infected by HCMV is demonstrated in Figure 2.

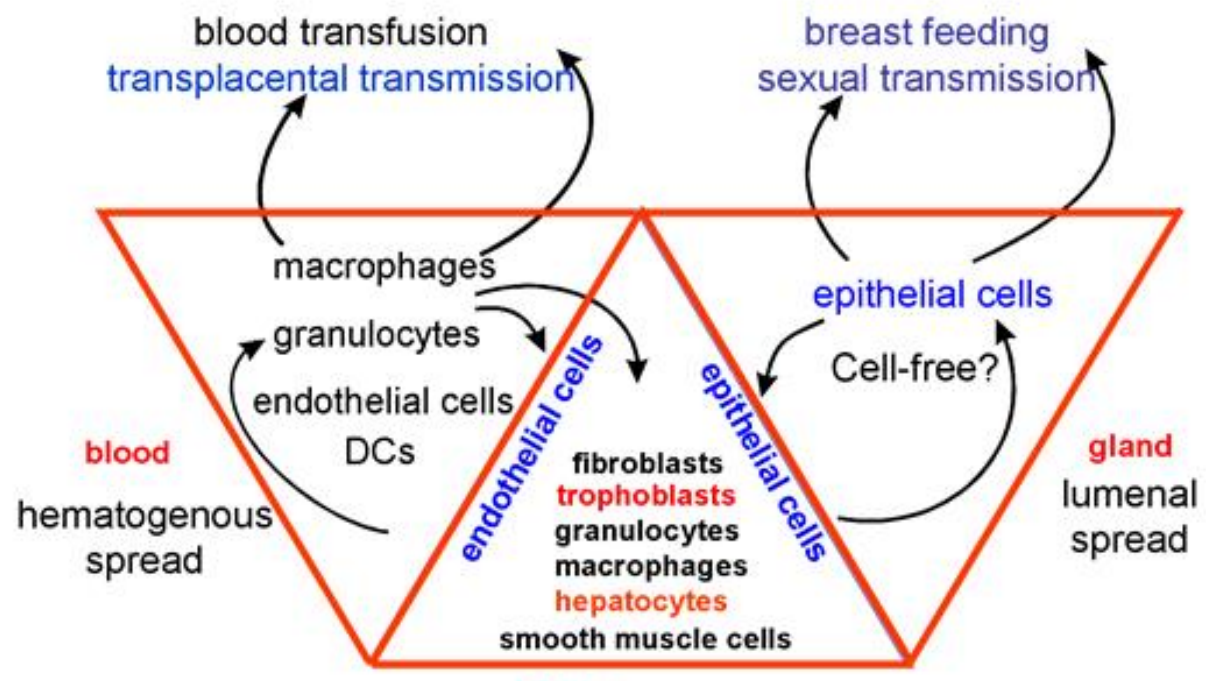

tissue spread

Fig. 1: HCMV cell tropism and major transmission routes 
In this Figure 2, the end organ disease of HCMV infection in immunosuppressed patients is mentioned. Remarkably, in healthy individuals hepatitis is the most frequent organ infection. The most important disease in pregnancy is illustrated by the congenital HCMV syndrome (congenital inclusion disease, CID). Another important point concerning transmission of HCMV is that in blood the infectivity is strictly cell associated in contrast to breast milk where the virus is found independent of cells.

\section{Transmission by}

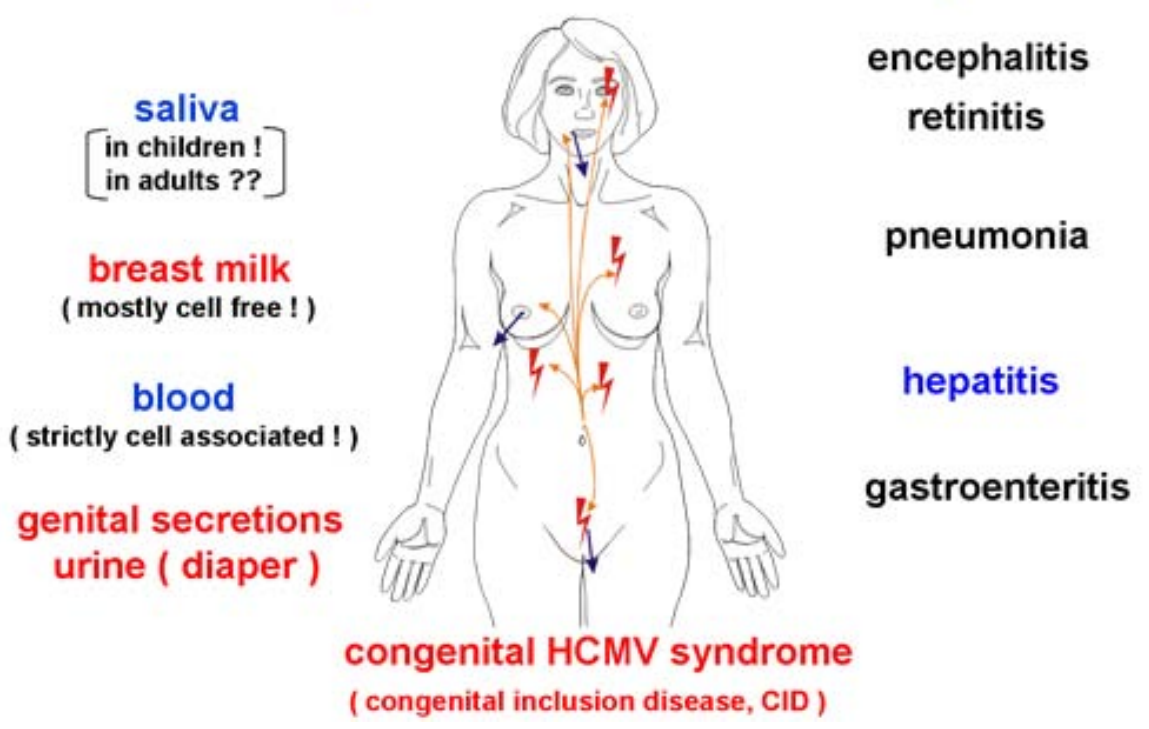

\section{End organ disease}

encephalitis

retinitis

pneumonia

hepatitis

gastroenteritis

Fig. 2: HCMV infection and epidemiology: production, transmission, organ manifestation

The transmission route of HCMV depends on lifestyle and the seroprevalence in the population. In Table 1, the major transmission routes of HCMV are listed. Worldwide the major transmission routes are breastfeeding, sexual intercourse and close personal contact to children less than three years.

Horizontal transmission of HCMV is well known by direct and indirect exposure to secretions of an infected individual shedding the virus.

Congenital infection is very important concerning adverse outcome in the newborn with postnatal sequelae, mainly sensory neural hearing loss. Blood-borne infection by HCMV can be avoided by IgG testing or filtered blood, since the virus /infectivity is exclusively found in blood cells. 
Tab. 1: Transmission routes of HCMV

\section{Breast-feeding \\ 2. Sexual intercourse}

$\uparrow$ ?

3. Close personal contact to children $<3$ years

4. Direct and indirect exposure to secretions ( saliva, urine, toys, inseminations )

5. Congenital infection (vertical infection)

6. Blood products ( transfusions )

\section{Organ transplantation}

No evidence for zoonotic origin (animals)

In patients with solid organ transplantation or stem cell transplantation HCMV infection and organ disease are well known and managed by diagnostic screening procedures and by pre-emptive therapy or prophylactic treatment.

\section{Epidemiology}

The incidence of congenital HCMV infection varies in different countries and the range is described between $0,2 \%$ and $1 \%$ (Table 2 ).

Most data were published from countries with low antibody prevalence in young women $(30 \%-70 \%)$. Some data are available from countries of high HCMV antibody prevalence $(70 \%-100 \%)$. There is a need for more good studies in countries with high prevalence.

Tab. 2: Incidence of congenital HCMV infection in different countries (newborns shedding HCMV in urine $<3$ weeks)

\begin{tabular}{|c|c|c|c|c|}
\hline USA & UK & Canada & Sweden & Germany \\
\hline $1 \%$ & $0,3 \%$ & $0,42 \%$ & $0,5 \%$ & $0,2 \%$ (Survey) $!$ \\
\hline
\end{tabular}

The epidemiology of congenital HCMV infection is well studied in the United States and in Europe (Cannon et al., 2010; Enders et al., 2011; Revello et al., 2011). In the US, there are about 4 million live births, $1 \%$ of newborns are congenitally 
infected (40,000). From these about 7\% are symptomatic at birth and $93 \%$ are asymptomatic at birth. In $10 \%$ of the symptomatic once there is a fatal disease described and $90 \%$ survive with sequelae. It is important to point out that in the group of those who are asymptomatic at birth about $15 \%$ of the survivors develop sequelae (hearing loss). Taken together all the data from the US studies, there is a fatal outcome or sequelae of all live births per year of about $8,000(0.2 \%)$. The estimation from the US data for China, a country with about 20 million live births per year, would bring up to 40,000 fatal outcomes or sequelae. This is most likely not realistic, because China is a high prevalence country and the risk of primary infection is very low. The risk for secondary infection during pregnancy is however unknown. Future studies will shed more light on the situation of countries like China. The estimation of the US data for Germany, a country with 700,000 live births per year would bring up a total of about 1,400 fatal outcomes or sequelae of all live births per year. This is again most likely not realistic. We observe 200 cases per year in our preliminary data on mother-child-infection during pregnancy resulting in about 200 cases per year.

To understand the risk of HCMV transmission in pregnancy in relationship to the seroprevalence Figure 3 illustrates the situation for HCMV seropositive mothers and for HCMV seronegative mothers with seroconversion during pregnancy.

In seropositive women there is a low risk of reinfection during pregnancy resulting in symptomatic congenital infection (Fig. 3).

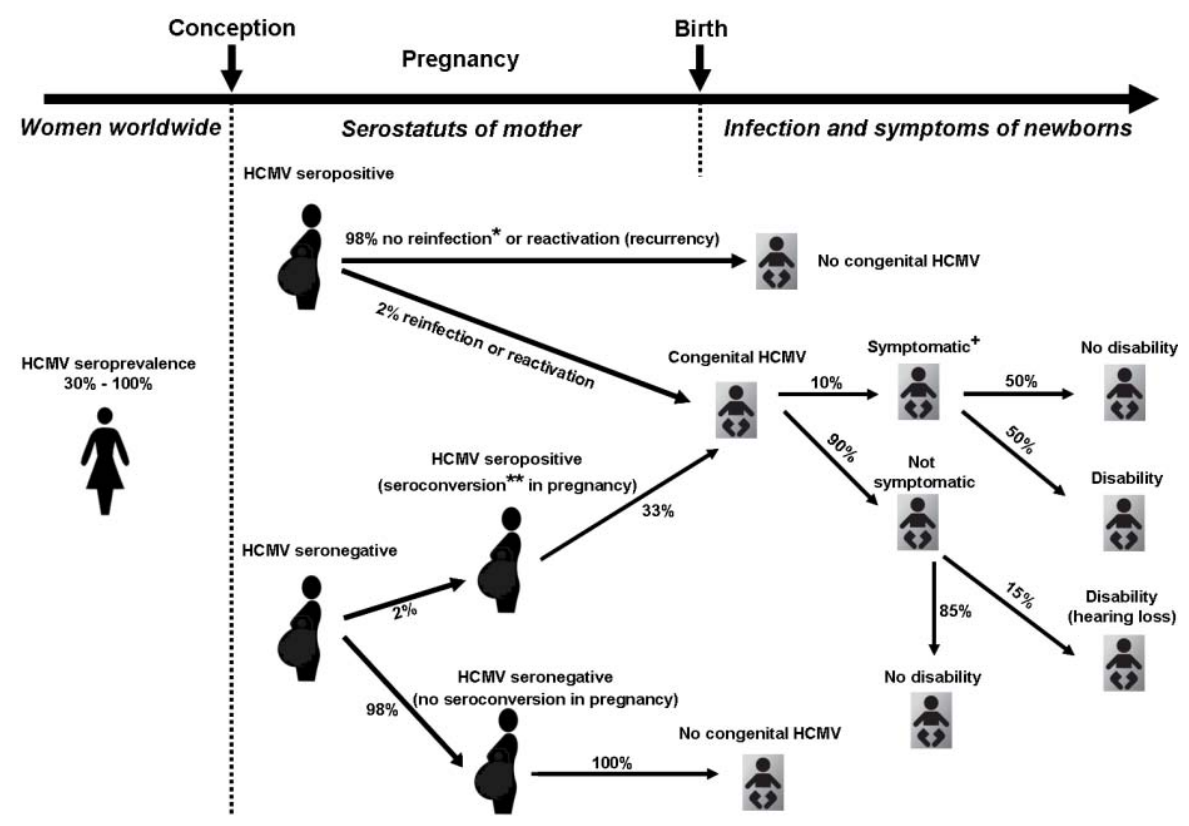

Fig.3: Risk of HCMV transmission in pregnancy in relationship to seroprevalence 
Severe congenital HCMV disease and intrauterine foetal death have been reported with secondary infection. The major risk of symptomatic congenital HCMV infection results from primary infection within the first half of pregnancy (lower part of Figure 3). The risk of seroconversion during pregnancy in seronegative women is described with $2 \%$. This is highly dependant on the lifestyle in a community, notably the numbers of sexual partners during pregnancy. The severity of congenital disease is related inversely to gestational age and is much greater than infection that occurs in the first trimester. The transmission rate is opposite. During the first trimester the transmission rate is less than $30 \%$ and during the third trimester it is about $70 \%$.

\section{Clinical outcome}

The clinical outcome in newborns with symptomatic HCMV infection after primary infection of the pregnant woman is listed in Table 3.

Tab. 3: Clinical outcome in newborns with symptomatic congenital HCMV infection (primary infection)

\begin{tabular}{|l|c|}
\hline Clinical symptomes & frequency (\%) \\
\hline Petechiae & 76 \\
\hline Icterus ( jaundice) & 67 \\
\hline Hepatosplenomegaly & 60 \\
\hline Microcephaly & 53 \\
\hline Intrauterine growth retardation (IUGR) & 50 \\
\hline Retinitis / optic atrophy & 20 \\
\hline Purpura & 13 \\
\hline Cramps & 7 \\
\hline Laboratory findings & \\
\hline ASAT-increase ( $>80 \mathrm{U} / \mathrm{L})$ & 83 \\
\hline Thrombozytopenia (<100.000/nm 3 ) & 77 \\
\hline Liquor protein increase (>120 mg/dL) & 46 \\
\hline
\end{tabular}

Hampreche K, Jahn G, Bundesposundheitsblatt - Gesundheitsschutz 20

Late onset complications: hearing loss in about $60 \%$ (primary infection )

( late onset of hearing loss in asymptomatic children at birth : 15 \%!) 
The relative percentage of each single symptom can vary and notably it is mentioned that the late onset complications like hearing loss occur at up to about $60 \%$. In addition, the late onset of hearing loss in asymptomatic newborns at birth is about $15 \%$. These data deliver evidence for postnatal antiviral treatment to avoid hearing loss in some infected newborns. Since hearing loss occurs postnatally in about $70 \%$ there should be a benefit of antiviral treatment. An open question is the duration of treatment: in a published study in which six weeks of ganciclovir treatment were selected, some benefit was observed. The more recent studies compare six months treatment versus six weeks treatment and the results are not published yet.

In clear contrast to the adverse outcome of congenitally infected newborns is the situation of newborns infected via breastmilk. The transmission of HCMV via breastmilk is well known documented since about 40 years and no symptoms were described in healthy newborns infected by breastmilk. The situation is a bit different in preterm infants infected by breastfeeding (Hamprecht et al., 2001). These very small preterm infants do have symptoms in about 50\% (Table 4).

Tab. 4: HCMV transmission to preterm infants by breastfeeding: clinical outcome

\begin{tabular}{|c|c|}
\hline $\begin{array}{l}\text { sepsis-like symptoms } \\
\text { (no bacteriaemia but: apnoea, bradycardia, } \\
\text { distended bowels and gray pallor) }\end{array}$ & $4 / 33(12 \%)$ \\
\hline hepatitis/hepatopathy & $5 / 33(15 \%)$ \\
\hline myoclonia & $2 / 33(6 \%)$ \\
\hline neutropenia $(<1500 / \mu \mathrm{l})$ & $14 / 33(42 \%)$ \\
\hline thrombocytopenia (< $150000 / \mu \mathrm{l})$ & $4 / 33(12 \%)$ \\
\hline asymptomatic HCMV infection & $17 / 33(52 \%)$ \\
\hline \multicolumn{2}{|c|}{$\begin{array}{l}\text { Hamprecht K, Maschmann J, Vochem M, Dietz K, Speer CP, Jahn G. Epidemiology of transmissior } \\
\text { of cytomegalovirus from mother to preterm infant by breastfeeding. ( 2001) Lancet. 371: } 513-51 \\
\text { Maschmann J, Hamprecht K, Dietz K, Jahn G, Speer CP. Cytomegalovirus infection of extremely } \\
\text { low-birth weight infants via breast milk. ( 2001) Clin Infect Dis. 33: 1998-2003 }\end{array}$} \\
\hline
\end{tabular}

The majority of symptoms are definitely transient and it is an open question if there is a late onset of permanent symptoms. In our study group of preterm infants coordination / navigation problems were observed in the breastmilk-infected group. Recently, a group in the Netherlands published cranial ultrasound findings 
(lenticulostriate vasculopathy) in preterm infants postnatally infected by HCMV (Nijman et al., 2012

\section{Diagnostics}

HCMV infection occurs with a low rate of symptomatic cases (fever, mild hepatitis), hence there is a need for specific virological tests. ELISAs are commercially available since more than 30 years for the detection of HCMV- specific IgG and IgM antibodies. However, the IgM kits available frequently yield discordant results, limiting their diagnostic value. Isolation of HCMV in cell culture is described in the mid 50ties. Molecular diagnostics for the detection of HCMV were developed in the 80ties and one example of a congenitally infected child is demonstrated in Figure 4. In this particular case, the mother was negative for HCMV by cell culture (urine and cervical swaps), negative by spot hybridization (urine) and even the placenta tissue was just slightly positive in the Southern blot hybridization. In addition, the mother was HCMV IgG positive and IgM negative. The child was IgG and IgM positive, cell culture was positive overnight and all investigated organs were overwhelmingly infected by HCMV (Jahn et al., 1988). Urine of the newborn was also positive as assayed by spot hybridization (Figure 4).
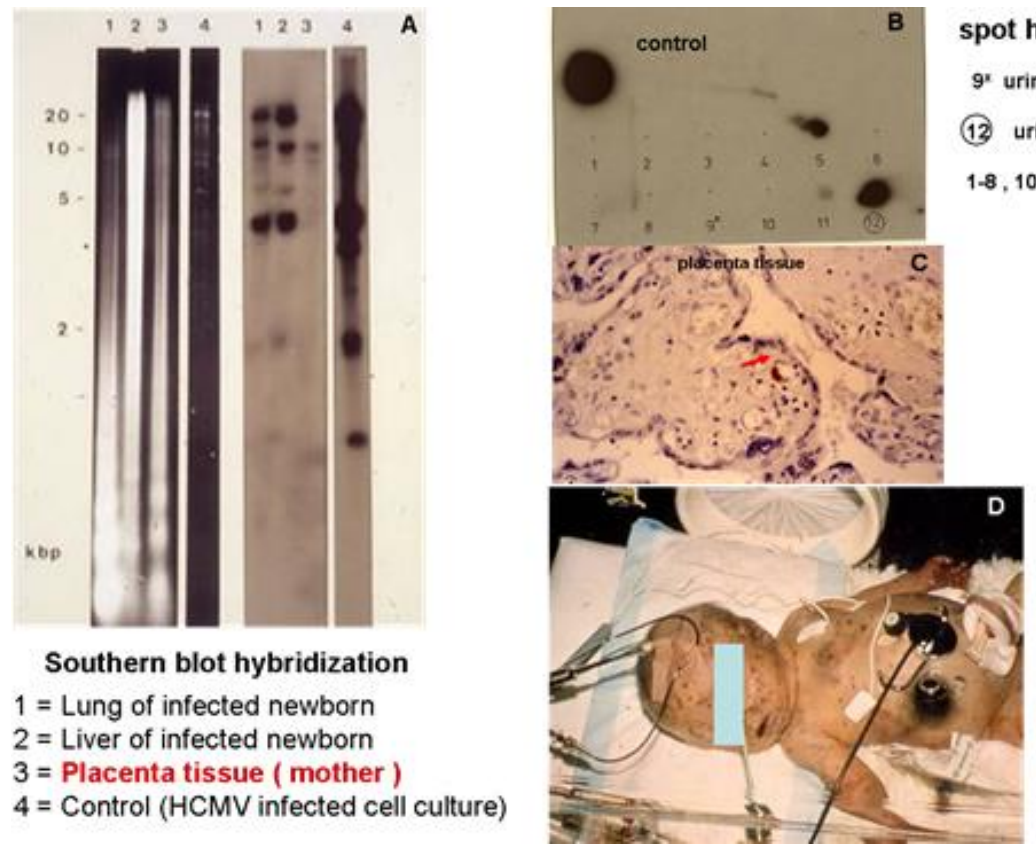

Fig. 4: Molecular diagnostics for HCMV infections in the 80ties (southern blot- and spot- hybridization) 
A detailed algorithm for diagnostic procedures in pregnancy is illustrated in Figure 5. All mentioned diagnostics are helpful to diagnose HCMV infection in pregnancy. The risk constellation in primary infection is pointed out in the centre of the Figure 5. For a more detailed diagnostic interpretation, the immunoblot with recombinant antigens of HCMV such as IE, pp150, p65, gB1 and gB2 is very helpful. In these assays, a combined interpretation of gB-reactivity, $\operatorname{IgG}$ avidity and IgM reactivity is possible to diagnose a high risk constellation: $\mathrm{gB}$ negative, $\operatorname{IgM}$ strongly positive and low IgG avidity.

The anti-HCMV IgG avidity testing (ELISA or immunoblot) seems to be the most reliable concept for the identification of a primary infection. Low avidity IgG indicates acute or primary HCMV infection and high avidity IgG indicates no current or no recent primary infection within the last three months. The measurement of $\operatorname{IgG}$ avidity is recommendated when IgM is present.

The tests are technically easy to perform, but the absence of standardization and the variability of the results makes the interpretation difficult especially in nonspecialized laboratories. In suspected cases, samples should be tested in a specialized / reference laboratory for IgG avidity and PCR should be performed from blood and / or amniotic fluid. The direct detection of HCMV in amniotic fluid by quantitative PCR is of special value to diagnose intra-uterine infection. PCR from dried blood spots or from saliva can be helpful to analyse congenital infection

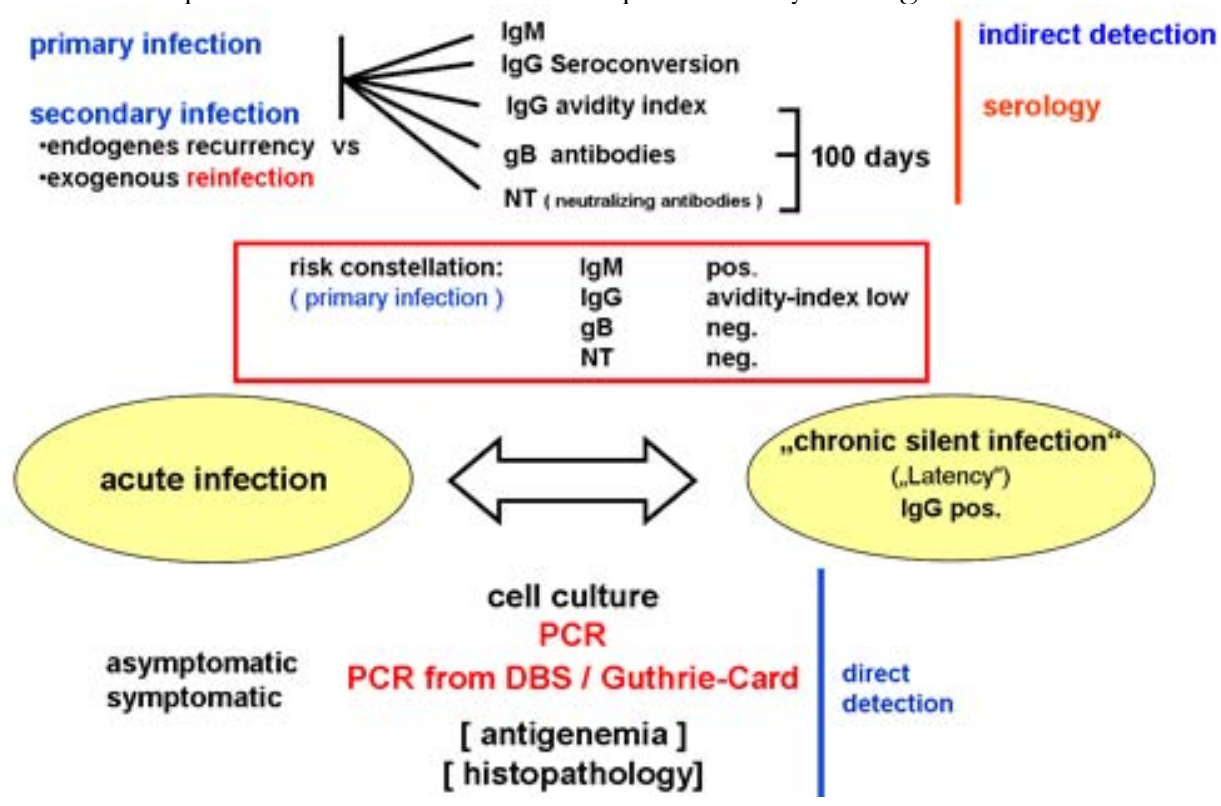

Fig.5: Diagnostic of HCMV infection in pregnancy in an experienced laboratory 
(particularly retrospectively) or to screen newborns (Boppana et al., 2010). Ultrasound identifies only around $25 \%$ of infected babies.

IgG avidity testing, specific glycoprotein B reactivity or neutralizing antibodiespatients after having received solid organ transplantation and one example of this delayed seroconversion of anti-gB is illustrated in Figure 6. In solid organ trans tested with fibroblasts are of value to diagnose primary infection, even retrospectively in a time period of about 100 days. This time period was originally defined in plantation, the antigenemia assay is of value. This quantitative assay is less helpful in patients with stem cell transplantation (lack of cells) and is also less helpful in pregnancy, because the time period for detection of positive cells in the cytospin preparation is too short (Fig. 6).

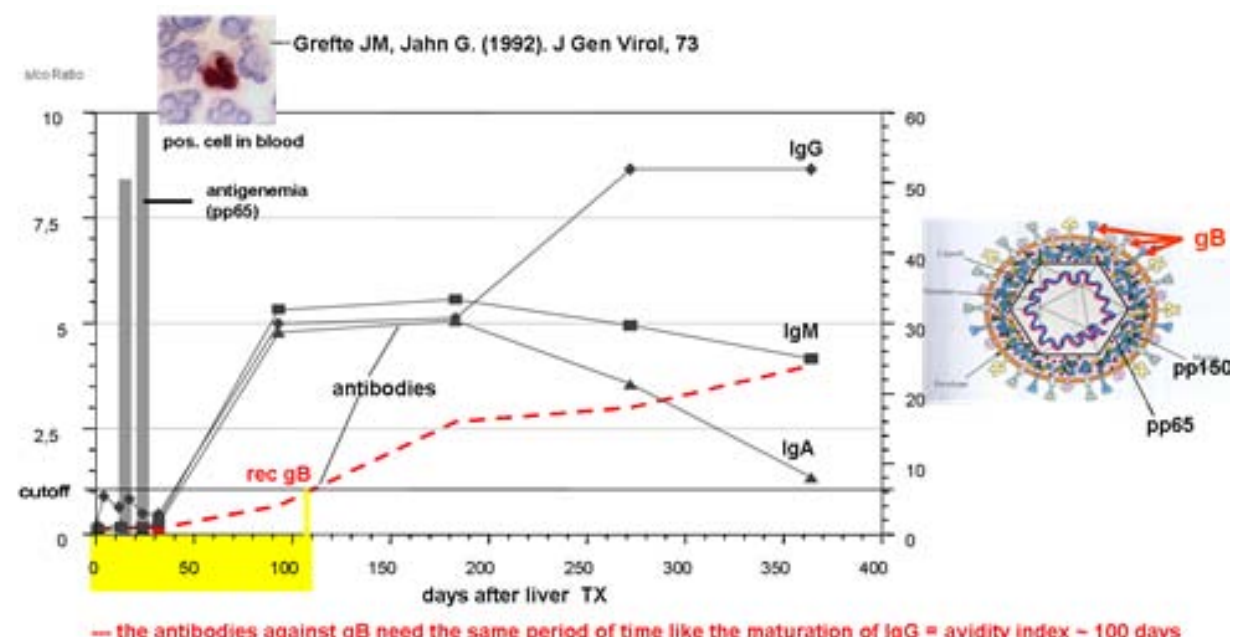

The delayed seroconversion of anti gB ( 100 days ) allows discrimination of primary infection vs secondary infection

(same time period was found for the detection of neutralizing antibodies as well as high avidity IgG-index)

Fig. 6: Antigenemia and antibody detection in primary infection of HCMV (worked out in solid organ transplant recipients as a model for pregnancy)

Figure 5 demonstrates many possibilities of HCMV testing and this concept is for an experienced laboratory and it is also expensive. Therefore, a more simple and much cheaper diagnostic recommendation for HCMV infection in pregnancy is illustrated in Figure 7. 


\section{Antiviral treatment and vaccines in development}

Antiviral treatment with ganciclovir in newborns is often given, however there are no clear guidelines or recommendations for it. There is a tendency to treat the newborns for more than six weeks as originally described. We observed for the first time in four individual cases antiviral resistance under ganciclovir treatment. After treatment of newborns a relapse of HCMV is frequently observed.

Despite promising observations with the application of antiviral compounds or the use of HCMV hyperimmune globulin (HIG) in pregnant women, results have to be interpretated with caution (Nigro et al., 2011). There is a need for placebocontrolled studies demonstrating the benefit of this treatment. Up to now there are no guidelines for antiviral / HIG treatment during pregnancy.

- HCMV IgG testing and / or storage of blood as early as possible in pregnancy

- Storage of blood for at least one year in any case !

- In case of HCMV IgG pos. women : information about the IgG status

(exclusion of recent infection in rare cases)

- In case of HCMV IgG neg. women : detailed information about HCMV infection (transmission routes, preventive procedures) re-testing depending on risk

- Symptoms during pregnancy : testing for seroconversion, IgM and IgG avidity

- Suspected recent HCMV infection : retesting of the stored serum in parallel with a recent serum, further tests

Symptoms for HCMV are never highly specific, but during pregnancy

when symptoms like fever, hepatitis, rush, lymphadenopathy come up, HCMV infection should be "ruled out"

$\rightarrow$ Contact specialists (reference laboratories) !

Fig. 7: Diagnostic recommendation for HCMV infection in pregnancy in an unexperienced laboratory

Studies with HCMV vaccines were done since more than 30 years with limited success. The recombinant $\mathrm{gB}$ vaccine is in trials since about 20 years and brings up a protection of about $50 \%$. Based on our knowledge today that $\mathrm{gB}$ is relevant for the entry in fibroblasts but not in epithelial or endothelial cells these results are not surprising. A more promising candidate for a non-living HCMV vaccine is definitely the $\mathrm{gH}$ pentameric complex, because this complex is relevant for entry into the major target cells of HCMV like epithelial cells and endothelial cells. A number of live HCMV vaccines, non-living HCMV vaccines under development are listed in Table 5 and Table 6. 
Tab. 5: Live HCMV vaccines in development

\begin{tabular}{|l|}
\hline Attenuated strain (Towne) \\
\hline Recombinants with wild virus (Towne-Toledo) \\
\hline Replication-defective virus \\
\hline Alphavirus replicon \\
\hline Vectored: poxvirus, adenovirus, LCMV \\
\hline
\end{tabular}

Tab. 6: Non-living HCMV vaccines in development

\begin{tabular}{|l|}
\hline Recombinant gB-glycoprotein with adjuvant \\
\hline DNA plasmids \\
\hline Peptides \\
\hline Dense bodies \\
\hline Virus-like-particles \\
\hline
\end{tabular}

The potential mechanistic correlates of protection against HCMV infection are listed in Table 7. These include epitopes for neutralization as well as epitopes for T-cells. It might be that in the near future there will be two vaccine candidates for HCMV: one for seronegative girls to generate neutralizing antibodies and one for seropositive immunosuppressed patients at risk with targets generating specific $\mathrm{T}$ cells.

Tab. 7: Potential mechanistic correlates of protection for HCMV

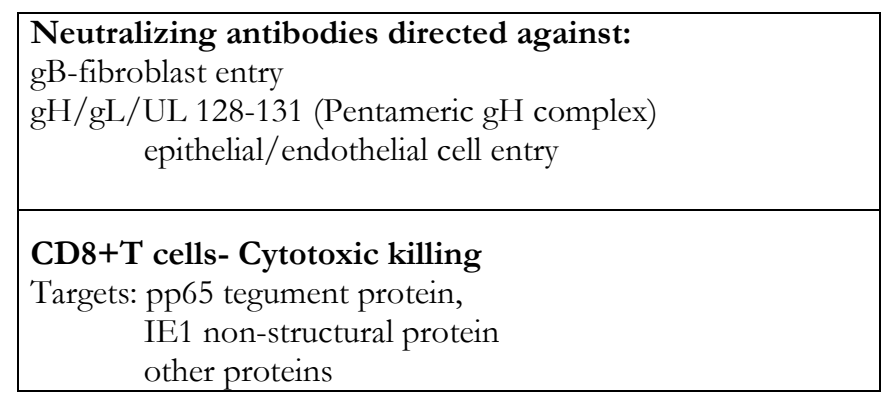




\section{Conclusion}

Congenital HCMV infection is the most important infection causing congenital neurological handicaps. The majority of symptomatic cases are the result of maternal primary infection during the first half of pregnancy. The problem is to diagnose the intra-uterine infection at the right time. Diagnostic procedures have improved and helped to diagnose primary infection. A congenitally acquired infection should be confirmed at birth. The management of foetal infection is complex and should be performed by a multidisciplinary team. Treatment options for foetal HCMV infection are limited. There are some promising results with antiviral drugs (ganciclovir) and hyperimmunglobulin.

It is very likely that the oral valganciclovir will replace the intravenous ganciclovir as the preferred treatment for newborns with symptomatic HCMV infection.

It is also very likely that new vaccine concepts with the gH pentameric complex will replace the $\mathrm{gB}$ vaccine as the future vaccine against HCMV infection. It seems however very likely that this might last for more than one decade.

Hygienic intervention for high-risk women, as defined by having close contact with children less than three years old, is appropriate. The important hygienic behaviours to prevent HCMV infection during pregnancy are: washing hands after changing babies' dirty diaper, not sharing the same drinking glass, spoon and fork with a young child, not kissing a young child on the mouth.

A prerequisite for this recommendation is the knowledge of HCMV. Therefore, an initial blood test for HCMV IgG-antibodies should be offered to all pregnant women or young women planning pregnancy. Regardless of the HCMV diagnostic strategy used, education of pregnant women about HCMV and the transmission routes as well as the risk of the infection for the newborn is necessary for prevention, particularly for seronegative women with contact to children younger than 3 years. Women have the right to know about HCMV, that they do have the chance to avoid the infection during pregnancy.

\section{References}

Bissinger AL, Sinzger C, Kaiserling E, Jahn G. (2002) Human Cytomegalovirus as a direct pathogen - correlation of mulitorgan involvement and cell distribution with clinical and pathological findings in a case of congenital inclusion disease. J Med Virol. 67. 200-206

Boppana SB, Ross SA, Novak z, Shimamura M, Tolan RW, Palmer AL, Ahmed A, Michaels MG, Sanchez PJ, Bernstein DL, Britt WJ, Fowler KB. (2010) Dried blood spot real-time polymerase chain reaction assays to screen newborns for congenital cytomegalovirus infection. JAMA. 303: 1375-1382. 
Cannon MJ, Scott Schmid D, Hyde TB. (2010). Review of cytomegalovirus seroprevalence and demographic characteristics associated with infection. Rev Med Virol. 20:202-213.

Enders G, Daiminger A, Bäder U, Exler S, Enders M. (2011). Intrauterine transmission and clinical outcome of 248 pregnancies with primary cytomegalovirus infection in relation to gestational age. J Clin Virol. 52: 244246.

Hamprecht K, Maschmann J, Vochem M, Dietz K, Speer CP, Jahn G. (2001) Epidemiology of transmission of cytomegalovirus from mother to preterm infant by breastfeeding. The Lancet. 357: 513-518.

Jahn G, Pohl W, Plachter B, von Hintzenstern. (1988) Kongenitale

Cytomegalovirus-Infektion mit letalem Ausgang. Dtsch med Wschr. 113: 424427.

Nigro G, Adler SP. (2011). Cytomegalovirus infections during pregnancy. Current Opinion in Obstetrics and Gynecology. 23: 123-128.

Nijman J, de Vries LS, Koopman-Esseboom C, Uiterwaal CSPM, van Loon AM, Verboon-Maciolek M. (2012) Postnatally acquired cytomegalovirus infection in preterm infants: a prospective study on risk factors and cranial ultrasound findings. Arch Dis Child Fetal Neonatal Ed. 97: 2959-2963.

Revello MG, Fabbri E, Furione M, Zavattoni M, Lilleri D, Tassis B, Quarenghi A, Cena C, Arossa A, Montanari L, Rognoni V, Spinillo A, Gerna G. (2011) Role of prenatal diagnosis and counseling in the management of 735 pregnancies complicated by primary human cytomegalovirus infection: a 20 -year experience. J Clin Virol. 50: 303-7

Sinzger C, Digel M, Jahn G. (2008) Cytomegalovirus Cell Tropism. in: Shenk T, Stinski MF. Human Cytomegalovirus. Berlin, Springer. Current Topics in Microbiol and Immunology, 325: 63-83. 


\section{Global aspects of Human Papillomavirus Infection in Feto-Maternal Health}

Fabienne D. Schwab, Department of Obstetrics and Gynaecology, University Hospital Basel, Switzerland

Andreas R. Günthert, Department of Obstetrics and Gynaecology, Luzerner Kantonsspital, Lucerne, Switzerland

\section{History, carcinogenesis and epidemiology}

Genital warts were considered as the result of sexual promiscuity and as potentially infectious among ancient Greek and Romans, and cervical cancer was already described as a frequent disease of married women, widows and prostitutes but uncommon in virgins and nuns in Italy in the 18th century (1). The link between plurality of human papillomavirus (HPV) types and the aetiology of genital warts and development of cancer, as well as virus-associated morphologic changes of cells like koilocytosis in cervical smears, became evident in the middle of the 1970s, $(2,3)$. Today it is known that more than $99 \%$ of cervical cancers, about $50-60 \%$ of vulvar and $60-90 \%$ of vaginal cancers develop over years by integration of high risk virus DNA, in particular HPV 16 and 18, into host cell DNA and selective 
transcription of E6 and E7 genes followed by immortalization and transformation of viral genome harbouring cells (4-6). HPV is the most common newly diagnosed sexually transmitted infection worldwide; the estimated lifetime risk in women for genital HPV infection is $50-80 \%$. Cervical cancer still represents the second most frequent cancer in women and, although vulvar cancer is rare, the incidence of HPV-associated vulvar intraepithelial neoplasia (VIN) increased over 300\% in developed countries during the last decades $(7,8)$. Screening and treatment for clinical manifestations of HPV infections like genital warts and cytology abnormalities is based on removing the infected cells and watching for recurrence, which consumes significant health care resources and costs, and also causes psychosocial morbidity (7). More than $40 \mathrm{HPV}$ types specifically infect the anogenital tract, these types can further be separated based on their oncogenic potential, and available HPV tests are able to identify 14 of these designated high-risk HPV types (9; Table 1).

Table 1: HPV classification

\begin{tabular}{|l|l|}
\hline High-risk & HPV types \\
\hline carcinogenic & $\begin{array}{l}16,18,31,33,35,39,45,51,52,56,58, \\
59\end{array}$ \\
\hline possibly carcinogenic & 68 \\
\hline probably carcinogenic & $26,53,66,67,70,73,82$ \\
\hline $\begin{array}{l}\text { tested for in available detection } \\
\text { systems }\end{array}$ & $\begin{array}{l}16,18,31,33,35,9,45,51,52,56,58,59, \\
66,68\end{array}$ \\
\hline Low-risk & $6,11,40,42,43,44,54,61,72,81,89$ \\
\hline
\end{tabular}

Source: Erickson BK et al. (9)

Except for a low number of e.g. iatrogenic infections, only sexually active women will acquire HPV infections, and the rate of acquisition correlates with the number of sexual partners (10). Age is the strongest predictor of prevalence, although race, geographic region and other risk factors like HIV infection together with immune suppression, female graft recipients, co-infection with Chlamydia trachomatis or Herpes simplex virus 2, smoking and promiscuity have also a significant impact on HPV prevalence. The 2011 National Health and Nutrition Examination Survey (NHANES) in the US tested 4,000 females for cervico-vaginal HPV infection showing an overall prevalence of $42.5 \%$, which was lowest among females 14-19 years of age (32.9\%) and highest among women $20-24$ years of age $(53.8 \%)$ (11). In comparison, a cohort study in Greece showed a prevalence of $42 \%$ in sexually active adolescent girls, and only $6 \%$ of this cohort had been vaccinated against 
HPV (12). In a cohort study in Beijing/China the prevalence of HPV infection was highest among women aged 30-34 years (35\%), and although HPV16 represented with $12.8 \%$ the most common genotype, it was significantly less frequent in comparison to previous European and US studies (13). In a cohort study in Indonesia, a country in which $90 \%$ of the population are Muslims, the overall HPV prevalence was only $11.4 \%$, the most prevalent subtype described was HPV52, and HPV16 and HPV18 were equally common in the general population. Although different age-specific patterns were seen in different regions of Indonesia, HPV positivity was most associated with the number of marriages (14). However, also in Islamic countries like the Extended Middle East and North Africa (EMENA) region the observed change in social and sexual behaviour contributes to a reported HPV prevalence of up to $25 \%$ in these countries (15). The reports on HPV prevalence in older women are controversial, some studies report on a second peak among postmenopausal women (16). Of course, women can acquire new HPV infections later in life which might also be associated with the number of new sexual partners (17), but a more recent study showed that incident HPV infection in older women was attributable to cumulative lifetime exposure in the past, and not current sexual behaviour, suggesting viral latency and reactivation (18). An Australian study was performed in indigenous women with a geographic cluster of vulvar cancer to investigate the type-specific and multiple infection prevalence of oncogenic HPV between sites. The prevalence of vulvar HPV was 39\%, which was significantly higher than the cervical HPV prevalence (26\%), and HPV16 was the most common genotype in both sites. However, there was a marked decline in cervical HPV16 prevalence with age, whereas following an initial decline, the prevalence of vulvar HPV16 remained constant in subsequent age groups (19). Thus, vulvar HPV infection might be a source for cervical reinfection after years.

A meta-analysis of 55 published studies worldwide including 8,308 patients with low-grade squamous intraepithelial lesion (LSIL) and HPV testing showed marked regional differences (20). HPV16 was the most common genotype (overall $26.3 \%)$ in all regions. The $2^{\text {nd }}$ most common type in Europe was HPV31, which was less frequent in Northern America and rare in Southern/Central America, Africa and Asia. In contrast, the $2^{\text {nd }}$ most common type in Northern America was HPV51, which was comparably rare on all other continents. HPV18 and HPV58 were both the predominant types in Asia and Africa. The number of included patients from Asia and Africa was low in that analysis, and these continents show of course very different cultural aspects, so it is unlikely that the results do represent HPV genotype distribution in females of the whole continents. Squamous cell carcinoma (SCC)/LSIL prevalence ratios indicated that HPV16 was 2-fold and HPV18 was 1.5-fold more common in SCC than in HPV-positive LSIL, thus appearing more likely to progress than other high-risk genotypes. A comparison of patients with cervical cancer in Suriname, Indonesia and the Netherlands demonstrated that HPV18 intratypic variants are differentially associated with adenocarcinoma (ADC) and SCC of the cervix, and the Asian Amerindian HPV18 variant 
was observed in $56 \%$ of ADCs compared to $15 \%$ of SCCs (21). HPV18 infection and intracervical transformation of cells might not be detected in conventional cervical smear. However, these results demonstrate that in addition to cervical smear, HPV testing is important to understand the different pathways of regional cancer development and to define patients at high risk. The overall estimated 1year clearance rate of incident HPV infections in women ranges from $40 \%$ to $70 \%$, and is almost $100 \%$ in young women after $2-5$ years. Among women who do not clear their infection, studies report variable rates of progression to cervical intraepithelial neoplasia ranging from $8 \%$ to $28 \%$. Additionally, an estimated $3-5 \%$ will eventually develop cervical cancer without any intervention after years, which therefore reflects the percentage of HPV-infected women without primary or secondary prevention $(9,22)$.

\section{Secondary prevention by screening}

Because of the high prevalence and clearance rates of HPV infection, particularly in young women, primary HPV testing is generally not yet recommended as an upfront screening strategy in developed countries, but HPV testing plays an important role in various screening algorithms. Cytological screening has been very successful in countries where adequate resources exist to ensure high quality and good coverage of the population at risk, and mortality reductions in excess of $50 \%$ have been achieved in many developed countries (22). In Central and South America, coverage may be high in places, but the quality of the cytology programs and access to treatment are poor, and rates of cervical cancer remain some of the highest documented in the world. Despite the low costs of consumables, high quality cytology screening is very expensive in absolute terms (23). In a pooled analysis of HPV testing in comparison to Pap smear in developed countries as primary screening strategy, HPV testing was substantially more sensitive than cytology in detecting high-grade lesions ( $96 \%$ vs. $53 \%$ ), but was less specific $(90 \%$ vs. $96 \%$ (22). Moreover, in populations vaccinated against high-risk HPV the predictive value of Pap smear screening will be reduced, because there will be fewer highgrade lesions among women with cytological abnormalities. In an attempt to make high-risk HPV testing more efficient, less invasive and less costly, self-collection of samples has been introduced. This screening method also has the advantage of reaching patients in remote locations or settings with limited resources, and although its sensitivity is clearly lower compared to clinician/nurse-taken samples, it is superior to cytology for detecting cervical intraepithelial neoplasia (CIN) grade $2 / 3$, which represent the precursors of cervical cancer $(22,24,25)$. HPV testing as upfront screening test should not be offered to women younger than 25 years because of the high clearance rate in this population. Cytology and visual inspection by colposcopy could be reduced to about $15 \%$ of all HPV-screened women and might be provided at higher quality and lower costs within an organised pro- 
gram. Visual inspection with acetic acid (VIA) means the examination with the naked eye, using a bright light, one minute after application of 3-5\% dilute acetic acid, and detection of well-defined aceto-white areas in the cervical surface indicates a positive test. This method is easy to perform, inexpensive and can be carried out by trained nurses, midwifes and paramedical health workers. Although its sensitivity is lower compared to HPV testing and Pap smear cytology, its effectiveness in reducing cervical cancer mortality was proven in India and it provides several advantages in low-resource countries (22). The major advantage is its costeffectiveness by the "screen and treat" approach, which means that detected lesions can be subsequently treated, for example with cryotherapy. VIA remains a successful first-step alternative in low-resource regions and might be combined with HPV testing as second-step "screen and treat" method as algorithm of organised programs in developing countries. This approach could be further combined with a mother-daughter approach in which daughters are vaccinated and mothers screened at the same visit, with treatment of the HPV-positive mothers scheduled to coincide with the next vaccine injection in the daughters $(22,26)$.

Taken together, obviously not only one screening algorithm will meet the criteria required for all populations around the world. Screening strategies have to be adapted to regional distribution of HPV genotypes and risk populations, the limits of acceptable expenditures and available health and financial resources. In addition, women tested for HPV require information and guidance both from their health care providers and through open sources, to avoid social and psychological consequences. The impact on quality of life, as well as the health and economic burden of HPV-related diseases and treatments worldwide are substantial (e.g. more than 4 billion dollars health care costs annually in the US), and require intense efforts to improve secondary prevention strategies and introduction of national immunization programs (27-30).

\section{Transmission and feto-maternal health aspects}

To date there is evidence that HPV can be transmitted not only sexually but also non-sexually. The modes of transmission include: sexual contact, non-sexual contact and maternal contact either directly or indirectly. HPV can be transmitted sexually such as in penetrating sexual intercourse as well as in intensive petting or intimate genital contact. HPV infection happens when microtrauma i.e. small lesions in the epithelium allows the HPV to penetrate and infect the basal layer of the epithelium. The frequent use of condoms can reduce the risk of infection of HPV, but 100\% protection cannot be provided as HPV transmission often occurs at the penile shaft, where the condom does not cover the skin. Winer et al. showed in their study, where they examined 82 women who reported their first intercourse with a male partner either during the study period or within two weeks before enrolment, that a regular use of condoms reduced the risk of transmission up to 
three times (31). They could show with this study that newly sexually active women with a consistent condom use by their partners can reduce the risk of cervical and vulvar-vaginal HPV infection. Also Bleeker et al. showed in a study, where women with CIN and their male sexual partners were randomized for condom use, that regular use of condoms promotes regression of CIN lesions and clearance of HPV in woman with persistent HPV infections, which have an increased risk of progressive CIN lesions (32).

Penile circumcision may reduce HPV prevalence and may reduce male-tofemale HPV transmission as several studies could show (33). Hernandez et al. showed that uncircumcised men have an increased risk of HPV infection, including with oncogenic HPV, specifically localized to the glans/corona, possibly because of its proximity to the foreskin, which may be particularly vulnerable to infection (34). Further Hernandez et al. examined in another study if there was a difference in acquisition and clearance of HPV in circumcised men compared to uncircumcised men (35). They examined a cohort of 357 adult males followed at 2months intervals for an average of 431 days. They demonstrated that there were no differences in HPV acquisition by circumcision status. Clearance of HPV infection, although including oncogenic types, was slower in the glans/coronal sulcus of the penis of uncircumcised compared to circumcised men. Circumcision may protect against $\mathrm{HPV}$-associated disease by enhancing the resolution of infection.

Although sexual contact is the primary mode of transmission other modes of transmission are discussed here. During pregnancy HPV can be transmitted prenatally: HPV DNA was detected in amniotic fluid, foetal membranes, cord blood and placental trophoblastic cells (36-39). Even children born with caesarean section showed HPV infection. HPV DNA detection did not differ by mode of delivery (37). Perinatal transmission can occur when there is contact of the foetus with infected cervical and vaginal cells of the mother during delivery. Vertical transmission of HPV to neonates in women without clinical evidence ranges from 1-18\% compared to women who had detectable HPV infection with transmission rates ranging from $5-72 \%$ (38). There is still controversial general opinion if the infants are really infected or only contaminated by HPV. Some studies indicate that HPV was only detectable for 2-4 days after delivery implicating a passive contamination and some studies showed detectable HPV in infants 6 weeks or even up to 3 years after delivery (37). Infants can also acquire HPV from breast-feeding, from siblings via kissing or from people via digital contact or during bathing or diapering (37) but they can also acquire HPV from sexual abuse. These different results raise the question of the optimal point of HPV vaccinations in children. It also still needs further studies to know the possible role of prophylactic HPV vaccination during family planning prior to pregnancy in order to prevent vertical transmission. The prevalence of HPV infection along with its subsequent clearance in adolescents is extremely high but the incidence of cervical cancer is negligible at 0.1 per 100,000 (40). Low-risk HPV clears faster than high-risk HPV. Genital warts (or condylomata acuminata, venereal warts, anal warts and anogenital warts) are a highly con- 
tagious sexually transmitted disease caused by some sub-types of HPV, especially 6 and $11(90 \%)$. Although condylomata acuminata are benign warts, they can cause symptoms such as burning, itching and bleeding. Above these symptoms condylomata acuminata also can cause false shame and irritation. There are several methods to remove condylomata acuminata, but HPV persistence and relapse is quite high. Especially high-risk HPV favours the transition zone of the cervix, infects the basal layer of the epithelium and causes CIN at different grades. Conisation is a surgical procedure to remove HPV-affected tissue in order to prevent further progression to cervical cancer. Several studies showed that conisation has a huge impact of preterm delivery, increased perinatal mortality and early rupture of membranes (41-43). Conisation was shown to be associated with a 2.8 -fold increased risk of perinatal death, most likely because of a 4.9-fold increase in extreme preterm delivery (42). The exact mechanism of conisation causing preterm delivery is not well known but the cervix is thought to be an important physical and immunological barrier to ascending infection. Treatment with topical imiquimod is less invasive and offers a feasible and efficacious treatment for patients with CIN2/3, but the recurrence rate is higher than after conisation (43). Vitamin B supplementation, such as riboflavin, thiamin, vitamin B12 and folate, might also be an option to increase the clearance rate (44). HPV infection is a serious psychological and physical burden for the patient, because of regular testing and colposcopy as well as incertitude regarding the further progression or regression. Women who receive abnormal Pap results report fear of future reproductive impairment, cervical cancer, and associated procedures. Of concern to women with HPV infection is its association with invasive cervical cancer, a cancer estimated to account for $12 \%$ of all cancers among women worldwide (45). The anxiety reported by these women also includes concern about disclosing information to partners, families, and friends due to stigma, and concerns about transmission and sexual relationships. Altered self-image, as represented by feeling contaminated, shame, guilt, selfblame, decreased self-esteem, and negative body image, is another theme frequently reported by these women (46).

\section{Vaccination}

At the moment there are two different vaccines commercially available: Gardasil ${ }^{\circledR}$ (Sanofi Pasteur MSD) that protects against HPV 6, 11, 16 and 18 and Cervarix ${ }^{\circledR}$ (GlaxoSmithKline Ltd.) that protects against HPV 16 and 18. The HPV vaccine inoculates against the strains of HPV 16 and 18 that cause $70-80 \%$ of cases of cervical cancers. HPV 6 and 11 cause $90 \%$ of genital warts. Clinical trials found that the effectiveness of these vaccines was greater than $98 \%$ and $93 \%$, respectively, against HPV 16 and $18(47,48)$. Since the vaccination does not cover all HPV types, regular screening is still required. HPV vaccination followed by regular screening is estimated to reduce lifetime risk of cervical cancer by $94 \%$ (49). HPV 
vaccines are given as three shots to protect against HPV infection and HPVrelated disease. It is recommended for girls before sexual contact and in some countries for boys at age 11 or 12 years to 26 years. A recent published study reported the decrease of high-grade cervical abnormalities by 38\% in girls younger than 18 years after introduction of the vaccination program in Australia, using the quadrivalent HPV vaccine for all women aged 12-26 years. This decrease was progressive and significantly different to the linear trend in incidence before introduction of the vaccination. No similar temporal decline was recorded for low-grade cytological abnormalities or in older age groups (50). However, a demonstrable reduction of the burden of cervical cancer by vaccination, which is the main goal of HPV vaccines, will take decades. Another point is the vaccine acceptability and the awareness towards HPV vaccination. Several studies reflect the positive attitude of mothers and girls towards vaccination, but even in countries with availability of vaccines, the uptake is still low among low-income populations and specific racial/ethnic minorities. To address limited vaccine uptake it may be useful to establish national or regional guidelines as well as culturally relevant interventions at the individual and community levels. Especially cultural aspects and barriers like existing beliefs and attitudes towards sexually transmitted disease and cervical cancer should be implemented in informational HPV vaccination campaigns (51-55).

\section{Summary}

HPV infection is the most common sexually transmitted infection and has major physical and psychological, as well as economic impact causing fear, anxiety and stigma and immense health costs. It shows global differences in prevalence, and management of prevention strategies and infection are manifold. Although most HPV infections clear spontaneously at 2-5 years, the impact of HPV associated morbidity, mortality and health costs is extremely high. The vertical transmission rate between mother and infant via contact with vaginal and cervical mucosa during delivery, transplacental transmission, transmission via amniotic fluid, and horizontal transmission during infancy is about $20-30 \%$. However, almost $100 \%$ of neonatal infections disappear spontaneously during the first year of life. Primary targets are primary and secondary prevention strategies, but the different global HPV genotype prevalence and the socio-economic differences do not allow a simple algorithm for all populations and is a challenge for each country or region, to introduce risk and resource-adapted organised screening and vaccination programs. These programs should also address the availability of information and health education about HPV infection to avoid confusion. 


\section{References}

1. Zur Hausen H. Papilllomaviruses in the causation of human cancers - a brief historical account. Virology 2009;384:260-265.

2. Zur Hausen H. Condylomata acuminata and human genital cancer. Cancer Res 1976;36:794.

3. Meisels A, Fortin R. Condylomatous lesions of the cervix and the vagina. I. Cytologic patterns. Acta Cytol 1976;20:505-509.

4. Schwarz E. Freese UK, Gissmann L, Mayer W, Roggenbruck B, zur Hausen H. Structure and transcription of human papillomavirus type 18 and 16 sequences in cervical carcinoma cells. Nature 1985;314:111-114.

5. Ikenberg H, Gissmann L, Gross G, Grussendorf-Conen EI, zur Hausen H. Human papillomavirus type 16 related DNA in genital Bowen's disease and in Bowenoid papulosis. Int J Cancer 1983;32:563-564.

6. Madsen BS, Jensen HL, van den Brule AJ, Wohlfahrt J, Frisch M. Risk factors for invasive squamous cell carcinoma of the vulva and vagina population based case-control study in Denmark. Int J Cancer 2008;20:505-509.

7. Ault KA. Epidemiology and natural history of human papillomavirus infections in the female genital tract. Inf Dis Obstet Gynecol 2006;2006 Suppl:40470.

8. van Seters M, van Beurden M, de Craen AJ. Is the assumed natural history of vulvar intraepithelial neoplasia III based on enough evidence? A systematic review of 3322 published patients. Gynecol Oncol 2005;97:645651.

9. Erickson BK, Alvarez RD, Huh WK. Human papillomavirus: what every provider should know. Am J Obstet Gynecol 2012;DOI 10.1016/j.ajog.2012.09.007.

10. Kjaer SK, Chackerian B, van den Brule AJ, et al. Cancer Epidemiol Biomarkers Prev 2001;10:101-106.

11. Hariri S, Unger ER, Sternberg M et al. Prevalence of genital human papillomavirus among females in the United States, the National Health And Nutrition Examination Survey, 2003-2006. J Infect Dis 2011;204:566573.

12. Michala L, Argyri E, Tsimplaki E et al. Human papilloma virus infection in sexually active adolescent girls. Gynecol Oncol 2012;126:207-210. 
13. Hou R, Xu C, Zhang S, Wu M, Zhang W. Distribution of human papillomavirus genotype and cervical neoplasia among women with abnormal cytology in Beijing, China. Int J Gynaecol Obstet 2012;119:257261.

14. Vet JN, Boer MA, van den Akker BE et al. Prevalence of human papillomavirus in Indonesia: a population-based study in three regions. $\mathrm{Br}$ J Cancer 2008;99:214-218.

15. Seoud M. Burden of human papillomavirus-related disease in the extended middle East and north Africa - a comprehensive literature review. J Low Genit Tract Dis 2012;16:106-120.

16. Smith JS, Melendy A, Rana RK, Pimenta JM. Age-specific prevalence of infection with human papillomavirus in females: a global review. J Adolesc Health 2008;43:(4 Suppl):S5-25, S25.e21-41.

17. Trottier H, Ferreira $S$, Thomann $P$ et al. Human papillomavirus infection and reinfection in adult women: the role of sexual activity and natural immunity. Cancer Res 2010;70:8596-8577.

18. Rositch AF, Burke AE, Viscidi RP, Silver MI, Chang K, Gravitt PE. Contributions of recent and past sexual partnerships on incident human papillomavirus detection: acquisition and reactivation in older women. Cancer Res 2012;DOI: 10.1158/0008-5472.CAN-12-2635.

19. Rumbold AR, Tan SE, Condon JR et al. Investigating a cluster of vulvar cancer in young women: a cross-sectional study of genital human papillomavirus prevalence. BMC Infect Dis 2012;12:243.

20. Clifford GM, Rana RK, Franceschi S, Smith JS, Gough G, Pimenta JM. Human papillomavirus genotype distribution in low-grade cervical lesions: Comparison by geographic region and with cervical cancer. Cancer Epidemiol Biomarkers Prev2005;14:1157-1164.

21. De Boer MA, Peters LA, Aziz MF et al. Human papillomavirus type 18 variants: histopathology and E6/E7 polymorphisms in three countries. Int J Cancer 2005;114:422-425.

22. Cuzick J, Arbyn M, Sankaranayaran R et al. Overview of himan papillomavirus-based and other novel options for cervical cancer screening in developed and developing countries. Vaccine 2008;26 Suppl 10:K29-41.

23. Goldie SJ, Gaffikin L, Goldhaber-Fieber JD, Gordillo-Tobar A, Levin C, Mahe $\mathrm{C}$ et al. Cost-effectiveness of cervicl-cancer screening in five developing countries. N Engl J Med 2005;353:2158-2168. 
24. Scarinci IC, Garcia FA, Kobetz E et al. Cervical cancer prevention: new tools and old barriers. Cancer 2010;116:2531-2542.

25. Ogilvie GS, Patrick DM, Schulzer M, Sellors JW, Petric M, Chambers K et al. Diagnostic accuracy of self collected vaginal specimens for human papillomavirus compared to clinician collected human papillomavirus specimens: a meta-analysis. Sex Trans Infect 2005;81:207-212.

26. Sankaranarayanan R, Nene BM, Shastri SS, Jayant K, Muwonge R, Budukh AM et al. HPV screening for cervical cancer in rural India. N Engl J Med 2009;360:1385-1394.

27. Low JJ, Ko Y, Ilancheran A, Zhang XH, Singhal PK, Tay SK. Health and econiômic burden of HPV-related disease in Singapore. Asian Pac J Cancer Prev 2012;13:305-308.

28. Temrungruanglert W, Havanond P, Khemapech N et al. Model for predicting the burden and cost of treatment in cervical cancer and HPVrelated diseases in Thailand. Eur J Gynaecol Oncol 2012;33:391-394.

29. Hu D, Goldie S. The economic burden of noncervical human papillomavirus disease in the United States. Am J Obstet Gynecol 2008;198:500.e1-7.

30. Fleurence RL, Dixon JM, Milanova TF, Beusterien KM. Review of the economic and quality-of-life burden of cervical human papillomavirus disease. Am J Obstet Gynecol 2007;196:206-212.

31. Winer RL, Hughes JP, Feng Q, O'Reilly S, Kiviat NB, Holmes KK et al. Condom use and the risk of genital human papillomavirus infection in young women. N Engl J Med 2006 ;354:2645-2654.

32. Hogewoning CJ, Bleeker MC, van den Brule AJ, Voorhorst FJ, Snijders PJ, Berkhof $\mathrm{J}$ et al. Condom use promotes regression of cervical intraepithelial neoplasia and clearance of human papillomavirus: a randomized clinical trial. J Cancer 2003;107:811-816.

33. Backes DM, Bleeker MC, Meijer CJ, Hudgens MG, Agot K, Bailey RC et al. Male circumcision is associated with a lower prevalence of human papillomavirus-associated penile lesions among Kenyan men. Int J Cancer 2012;130:1888-1897.

34. Hernandez BY, Wilkens LR, Zhu X, McDuffie K, Thompson P, Shvetsov YB et al. Circumcision and human papillomavirus infection in men: a sitespecific comparison. J Infect Dis 2008;197:787-794.

35. Hernandez BY, Shvetsov YB, Goodman MT, Wilkens LR, Thompson P, Zhu X et al. Reduced clearance of penile human papillomavirus infection in uncircumcised men. J Infect Dis 2010;201:1340-1343. 
36. Jayasinghe Y, Garland SM. Genital warts in children: what do they mean? Arch Dis Child 2006;91:696-700.

37. Syrjänen S et al. Current concepts on human papillomavirus infections in children. APMIS 2010;118:494-509.

38. Lacour DE, Trimble C. Human papillomavirus in infants: transmission, prevalence, and persistence. J Pediatr Adolesc Gynecol 2012;25:93-97.

39. Syrjänen S, Puranen M. Human papillomavirus infections in children: the potential role of maternal transmission. Crit Rev Oral Bio Med 2000;11:259-274.

40. Boone JD, Erickson BK, Huh WK. New insights into cervical cancer screening. J Gynecol Oncol 2012;23:282-287.

41. Kyrgiou M, Koliopoulos G, Martin-Hirsch P, Arbyn M, Prendiville W, Paraskevaidis E. Obstetric outcomes after conservative treatment for intraepithelial or early invasive cervical lesions: systematic review and meta-analysis. Lancet 2006;367:489-498.

42. Ortoft G, Henriksen T, Hansen E, Petersen L. After conisation of the cervix, the perinatal mortality as a result of preterm delivery increases in subsequent pregnancy. BJOG 2010;117:258-267.

43. Grimm C, Polterauer S, Natter C et al. Treatment of cervical intraepithelial neplasia with topical imiquimod: a randomized controlled trial. Obstet Gynecol 2012;120:152-159.

44. Hernandez BY, McDuffie K, Wilkens LR, Kamemoto L, Goodman MT. Diet and premalignant lesions of the cervix: evidence of a protective for folate, riboflavin, thiamin, and vitamin B12. Cancer Causes Control 2003;14:859-870.

45. Bruinsma FJ, Quinn MA. The risk of preterm birth following treatment for precancerous changes in the cervix: a systematic review and metaanalysis. BJOG 2011;118:1031-1041.

46. Rao D, Gela N, Daley EM, Kattezham R, Rodriguez G, Cella D. Developing a measure of health-related quality of life for women with cervical dysplasia resulting from human papillomavirus infection. Int J STD AIDS 2010;21: 697-701.

47. Markowitz LE, Dunne EF, Saraiya M, Lawson HW, Chesson H, Unger ER. Quadrivalent human papillomavirus vaccine: recommendations of the advisory committee on immunization practices (ACIP). MMWR Recomm. Rep 2007:56:1-24. 
48. Centers for Disease Control and Prevention (CDC). FDA licensure of bivalent human papillomavirus vaccine (HPV2, Cervarix) for use in females and updated HPV vaccination recommendations from the advisory committee on immunization practices (ACIP). MMWR Morb Mortal Wkly Rep 2010;59:626-629.

49. Goldie SJ, Grima D, Kohli M, Wright TC, Weinstein M, Franco E. A comprehensive natural history model of HPV infection and cervical cancer to estimate the clinical impact of a prophylactic HPV-16/18 Vaccine. Int J Cancer 2003;106:896-904.

50. Brotherton JM, Fridman M, May CL, Chappell G, Saville AM, Gertig DM. Early effect of the HPV vaccination programme on cervical abnormalities in Victoria, Australia: an ecological study. Lancet 2011;377:2085-2092.

51. Downs LS Jr, Scarinci I, Einstein MH, Collins Y, Flowers L. Overcoming the barriers to HPV vaccination in high-risk populations oin the US. Gynecol Oncol 2010;117:486-490.

52. DiAngi YT, Panozzo CA, Ramogola-Masire D, Steenhoff AP, Brewer NT. A cross-sectional study of HPV vaccine acceptability in Gabarone, Botswana. PLoS One 2011;6:e25481.

53. Pandey D, Vanya V, Bhagat S, Vs B, Shetty J. Awareness and attitude towards human papillomavirus (HPV) vaccine among medical students in a premier medical school in India. PLoS One 2012;7:e40619.

54. Jaspers L, Budiningsih S, Wolterbeek R, Henderson FC, Peters AA. Parental acceptance of human papillomavirus (HPV) vaccination in Indonesia. Vaccine 2011;29:7785-7793.

55. Tsu V, Murray M, Franceschi S. Human papillomavirus vaccination in low-resource countries: lack of evidence to support vaccinating sexually active women. Br J Cancer 2012;107:1445-1450. 



\section{Chlamydia Trachomatis Infection in Women}

Rizalinda Sjahril, Department of Microbiology, Faculty of Medicine

Hasanuddin University, South Sulawesi, Indonesia

\section{Introduction}

Chlamydiae causes the most prevalent sexually transmitted bacterial disease in women and men, most of which are asymptomatic. Infections are localized on the columnar epithelial cells such as urethra and cervix that may eventually progress into chronic inflammation causing tissue damage, pelvic inflammatory disease (PID), ectopic pregnancy, tubal infertility, epididymitis, proctitis and Reiter's syndrome, and reactive arthritis $[1,2]$. Chlamydia infection is very important to diagnose and treat in women because even though it is mostly asymptomatic, but potential to cause serious damage to the reproductive organs. Concurrent antibiotic treatment to the sex partner is also important to stop Chlamydia re-infections [3].

Infection in some people may directly undergo natural clearance, in some it can continue being asymptomatic, and in the event of repeated infections it poses more harm due to scarring and other serious sequelae such as tubal infertility and ectopic pregnancy.

Bacterial virulence, environmental cofactors and host immunity determines the rate of inflammation, susceptibility and the development of disease. It is interesting 
that despite the virulence of Chlamydia, persistent and silent infection may occur because of its ability to evade the host's immune system [2]. The fact that the majority of infections are asymptomatic also hampers diagnosis and adequate treatment.

Table 1: The prevalence of Chlamydia infection in women in Indonesia reported in separate publications [References 7-16]

\begin{tabular}{|c|c|c|c|c|}
\hline $\begin{array}{c}\text { Location of } \\
\text { Survey }\end{array}$ & $\begin{array}{l}\text { Subject of } \\
\text { sampling }\end{array}$ & $\begin{array}{l}\text { Methods of } \\
\text { examination }\end{array}$ & $\begin{array}{c}\text { Prevalence } \\
(\%)\end{array}$ & Reference \\
\hline $\begin{array}{l}\text { Surabaya } \\
(1992-1993)\end{array}$ & $\mathrm{FSW} *(\mathrm{n}=1873)$ & & 18 & [7] \\
\hline \multirow[t]{2}{*}{ Bali (2001) } & $\begin{array}{l}\text { Low Risk women } \\
\qquad(\mathrm{n}=312)\end{array}$ & Gen Probe & 5.6 & [8] \\
\hline & $\mathrm{FSW}(\mathrm{n}=625)$ & $\begin{array}{l}\text { Abbott LCx } \\
\text { probe system }\end{array}$ & 39 & [9] \\
\hline Manado (2001) & $\begin{array}{l}\text { Low Risk women } \\
\qquad(\mathrm{n}=357)\end{array}$ & DNA Probe & 2.5 & [10] \\
\hline Kupang (1999) & FSW $(n=288)$ & PACE2 test & 23.9 & [11] \\
\hline $\begin{array}{l}\text { In nine provinces } \\
\text { in Indonesia } \\
(2005)\end{array}$ & FSW $(n=2500)$ & PCR & 43.5 & [12] \\
\hline Riau and Salatiga & $\mathrm{FSW}(\mathrm{n}=580)$ & PCR & 33.7 & [13] \\
\hline Manado (2010) & FSW $(n=221)$ & PCR & 26.7 & [14] \\
\hline Timika & $\operatorname{FSW}(\mathrm{n}=3086)$ & LCR & $33-41$ & [15] \\
\hline Makassar & $\operatorname{PROM} * *(\mathrm{n}=30)$ & PCR & 30 & [16] \\
\hline
\end{tabular}

Incidence and prevalence varies in different areas. The magnitude depends on the population, country, surveillance methods and reporting. The European Centre for Disease Prevention and Control described the prevalence among 7 European countries range between $1.4-3 \%$ in people at age $18-44$ years. The prevalence in a large group of sexually active women in Italy during 2000-2009 was 5.2\%. It showed an association with multiple life time sexual partners, younger age $(<40$ years), nulliparae, smoking, use of oral contraceptives, and coinfection with HPV 
and Trichomonas vaginalis [4]. A prevalence of $34 / 100$ person years was reported among the high-risk adolescents in the US, with reinfections up to $84 \%[5,6]$.

In Indonesia, separate studies have reported the prevalence of Chlamydia infections in different population targets (See Table 1). Although much focus of research was given to the female sex workers (FSW) considering the risk of transmission is higher in those groups, the other normal low risk populations as well as the adolescent groups and men who have sex with men (MSM) must be given similar attention considering that these different groups have the same possibility of getting infected by sexual activity.

\section{Bacteriology}

Chlamydiaceae have been classified separately in a family because of their special growth cycle. It comprises of the Chlamydia and the Chlamydophyla genera. Chlamydia is known to infect mammals only, while the Chlamydophila may infect mammals, birds, reptiles and amphibian $[17,18]$. The causative agent of urogenital infection and trachoma is Cblamydia trachomatis, meanwhile Cblamydophila pneumoniae is a pathogen increasingly known causing respiratory infections. The other species pathogenic for humans but actually a primary pathogen to animals is C. psittaci. Chlamydophila pecorum is associated with infectious polyarthritis, encephalitis, pneumonia and diarrhoea in ruminants [19].

The cell membrane of Chlamydiae has different lipopolysaccharides on its surface that separates it into 19 serovariants [19]. Chlamydia trachomatis type A, B, Ba and $C$ are found in conjunctivitis and trachoma, serovars $D, D a, E, F, G, G a, H, I$, $\mathrm{Ia}, \mathrm{J}$ and $\mathrm{K}$ are predominant among the genital tract infections (urethritis and cervicitis) and neonatal infections (inclusion conjunctivitis or neonatal pneumonitis, and serovars L1, L2, L2a, and L3 cause genital infection and are associated with lymphogranuloma venerum. Although certain serotypes may cause different diseases, it is unclear whether the serotypes have a correlation with any specific clinical manifestations. Based on the biological characteristic, the $C$. trachomatis serovars are distinguished into the lymphogranuloma venereum biovar (the LGV serovars) and the trachoma biovar [19,20].

The chromosome of Chlamydiae consists of approximately 1.000 .000 base pairs encoding for about 600 proteins. Some of the genes have been characterized and showed the ability of the bacteria to adjust and survive intracellularly. Molecular mimicry between the chlamydial proteins and the host cells may be related to specific disease syndromes [1].

Chlamydiae are present in two morphologically and functionally different forms. The infective form is the Elementary Body (EB) which is stable at the environment outside the cells and ready for attachment and invasion into new cells. The invasion starts by endocytosis of adhering EB and becomes internalized by the host cell in a vacuole where it is transformed into a replicative form, which is called 
Reticulate Body (RB). The life cycle then involves remodeling of the parasitophorous vacuole into an inclusion and replication, expansion of inclusion and transition of $\mathrm{RB}$ to $\mathrm{EB}$, and finally release of $\mathrm{EB}$ from the host cell [19, 21, 22].

The release of EB from the cell initiates subsequent rounds of infection. Egress can occur via two discrete mechanisms. Cell lysis involves the sequential disruption of inclusion and cellular membranes by cysteine proteases. Alternatively, the inclusion can remain membrane-bound and be pushed out, or "extruded", from the host cell. This process is dependent on actin-polymerization and myosin, allowing the host cell to remain intact [23].

\section{Pathomechanism of infection}

Adherence of an infectious EB to the epithelial cell surface of the urethra or cervix initiates infection through phagocytosis or receptor-mediated endocytosis or pinocytosis. Chlamydia remains inside the host cell within the parasitophorous vacuole. Early gene expression allows modification of the vacuole into an inclusion enable the EB to access the exocytic pathway. Then EB reconverts into RB which further replicates by binary fissions, becomes matured and changed into EB, before it is released from the host cell. Once again, the released EBs are able to infect other epithelial cells [1].

Infection of the cervical or urethral epithelial cells results in mucopurulent secretions, although most of chlamydial infections remain asymptomatic, especially in women. This perhaps is due to minimal secretions despite chlamydia infection and being camouflaged to the presence of normal fluor albus that is difficult to be distinguished from true infections.

Infection of the cervix leads to complications: 1) ascending infections causing PID, 2) ascending infections during pregnancy causing premature rupture of the membrane (PROM), chorioamnionitis and premature delivery, puerperal and neonatal infections, and 3) Chlamydia infection possess a co-factor role of the development of cervical neoplasia [1].

\section{Immunopathogenesis}

Chlamydial Elementary Bodies (EB) infect columnar (epithelial) cells in the genital tract. In some women clearance does not happen, resulting in persistence. Longer exposure to Chlamydia increases the risk to spread from the cervix and urethra to the upper genital tract such as the fallopian tube where it can cause tubal factor subfertility $[1,2,24]$.

After entering the host cell Chlamydial EB remain in the inclusion body and are not fused with the lysosome. As a response to endocytosis, epithelial cells release chemokines to attract leukocytes to the inflammation site, as well as proinflammatory mediators such as cytokines, interleukins, GM-CSF (granulocyte mac- 
rophage colony stimulating factor), TNF (tumor necrosis factor) and GROa (growth related oncogene) which induce a cellular inflammatory response and cause damage to the tissues $[2,25]$.

The humoral and cellular acquired immunity starts soon after the first recognition of the pathogen. B lymphocytes start to recognize the antigen presented by the antigen presenting cells (macrophages and dendritic cells) and then produce antibodies against it. After activation by antigen presenting cells, T-helper lymphocytes produce proinflammatory cytokines. A Th1-type response plays a role in resolution of infection, while a Th2-type response is involved in persistence and disease. However, the Th1-type response through the production of IFN-y apart from protecting infection can also inhibit intracellular chlamydial replication, thus causing persistence and unapparent infection [2,26].

During persistence, chlamydia stops most of its active replication but continue to produce a $60 \mathrm{kDa}$ heat shock protein which is responsible for delayed type hypersensitivity-induced disease. Hsp60 activates endothelial cells and macrophages to release proinflammatory cytokines and TNF- $\alpha$. While the infected cells release Hsp60 into the bloodstream, immune cells react and produce antibodies against Chlamydial hsp60, which unfortunately resembles the human Hsp60. Therefore, in chronic persistent infections inflammation continues to occur, although no clinical symptoms are present. A similar mechanism exists during reinfections by either a new strain or the same strain of Chlamydia. This leads to repeated inflammation and induced scarring of the infected tissues [26].

\section{Diagnosis of Chlamydia infection in women}

Chlamydia infections cause cervicitis and urethritis $(50 \%)$, cervicitis only $(33 \%)$, or urethritis only (15-25\%), and most episodes of cervicitis are asymptomatic or minimally symptomatic. Although there is no specific clinical manifestation, presumptive diagnosis can be made upon the presence of endocervical mucopus, erythema, oedema and induced cervical mucosal bleeding [1].

\subsection{Traditional methods of diagnosis}

Traditional methods of diagnosis involve clinical symptoms, microscopic examination and culture. These were once popular because of the cheap equipment and their simplicity. The enzyme immunoassay (EIA) and the molecular technique provide better accuracy and are quick and convenient [27]. However, the costs consideration is still the major problem for their application in countries with limited resources.

In regards to clinical diagnosis, colposcopy may show yellow pus, signs of inflammation at the cervix surface and friable when touched with the swab, and yellow discoloration of the swab inserted in the cervix. Histology can reveal meta- 
plastic atypical cells, infiltration of plasma cells to the cervical stroma, intraepithelial and intraluminal inflammation and intracervical follicles made of transformed lymphocytes $[1,28]$.

Clinical features include subclinical endometritis, obvious salpingitis, abscess of the ovarium and the fallopian tube, peritonitis, periappendicitis and perihepatitis. Perihepatitis might be seen as Fitz-Hugh-Curtis-syndrome during surgery procedures, for example during laparoscopy for infertility. Many silent infections due to Chlamydia occur in the fallopian tubes and can cause abscess formations even after years of infection.

Several algorithms which were developed by the WHO in 1994 are aimed to guide health care providers for syndromic management of patients suffering from sexually transmitted diseases. They provide the choice of management for patients based on the present symptoms, signs and risk assessment, and are very useful in places where speculum examination is not available and if the laboratory facility is lacking [29]. However, the algorithm for women with symptoms of vaginal discharge cannot be applied for case finding or used as screening among asymptomatic patients [30]. The algorithm, without the supporting laboratory diagnostic means for Neisseria gonorrhea and Chlamydia trachomatis may fail to identify infections in pregnant women [31]. In addition, the high rate of asymptomatic infections in developing countries have necessitated the availability of supporting rapid diagnostic tests [32]. Furthermore, the algorithm allows multiple antibiotic prescription despite lack of sufficient laboratory tests, thus enabling to increase antibiotic resistance. It was also indicated that an effective communication skill is essential in syndromic management to obtain true information regarding the symptoms [33].

Figure 1 shows the algorithm for the diagnosis and management of pelvic inflammatory disease (PID) with one of its common causative agents is Chlamydia trachomatis. Although it is possible to obtain false-positive diagnosis if using this algorithm alone without the support of Gram or Giemsa staining, antibiotic treatment is justified considering the severe consequences of PID if is left untreated or receiving late treatment [28].

\subsection{Direct diagnosis}

Laboratory diagnosis is essential in order to adequately treat the disease and prevent spread of the sexually transmissible infection. About 4 decades ago, cell culture was a reference method. However, even though the sensitivity is $70-85 \%$ being laborious and requiring a few days - nowadays many non-culture-based assays are preferable. These are less costly and faster [34]. 


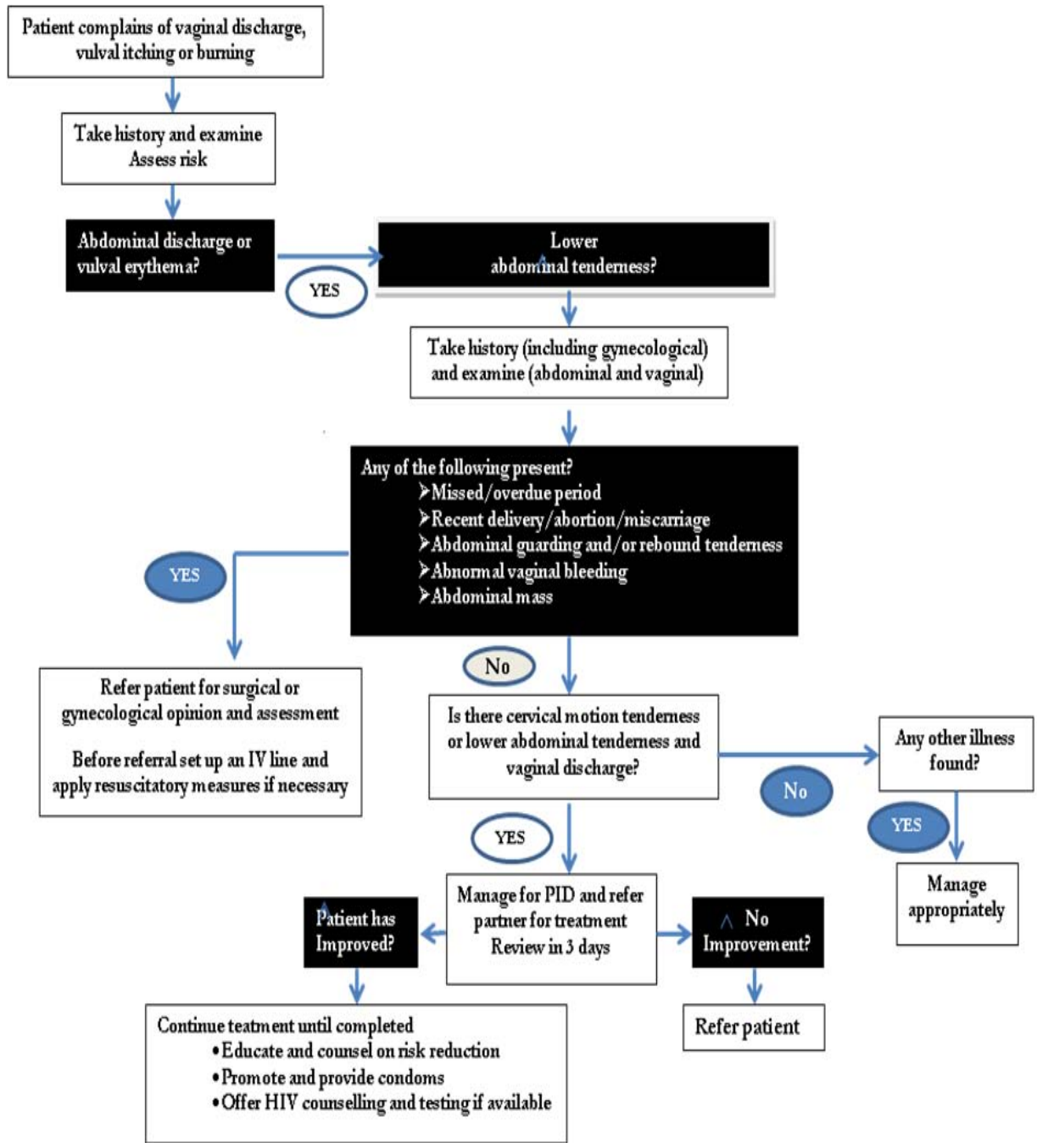

Figure 1. The algorithm for diagnosis of pelvic inflammatory disease commonly caused by Chlamydia, Neisseria gonorrhea or other anaerobe bacteria 
Table 2: Methods for direct detection of Chlamydia trachomatis

\begin{tabular}{|c|c|c|c|}
\hline Method & $\begin{array}{l}\text { Turn- } \\
\text { around } \\
\text { time }\end{array}$ & Advantages & Limitations \\
\hline Cell Culture & 72 hours & Specificity & Sensitivity $70-85 \%$, laborious \\
\hline \multicolumn{4}{|l|}{ Antigen detection } \\
\hline DFA & $45 \mathrm{~min}$ & Simple, unit test & Sensitivity $75-80 \%$ \\
\hline & & & Subjective reading \\
\hline EIA & $4 \mathrm{~h}$ & Automation & Sensitivity $75-80 \%$ \\
\hline Point of care & $30 \mathrm{~min}$ & $\begin{array}{l}\text { Low cost, unit } \\
\text { test }\end{array}$ & Low specificity (confirmatory test) \\
\hline \multicolumn{4}{|l|}{ Molecular methods } \\
\hline DNA probe & $2 \mathrm{~h}$ & Easy to perform & Sensitivity $75-80 \%$ \\
\hline Hybrid capture & $4 \mathrm{~h}$ & $\begin{array}{l}\text { Sensitivity } 95 \% \\
\text { Specificity } 99 \%\end{array}$ & Only for cervical specimen (FDA) \\
\hline $\begin{array}{l}\text { NAAT (PCR, SDA, } \\
\text { TMA, NASBA) }\end{array}$ & $2-4 \mathrm{~h}$ & $\begin{array}{l}\text { Sensitivity }>95 \% \\
\text { Specificity } 99 \%\end{array}$ & Contamination, costly \\
\hline
\end{tabular}

*'Table is adapted from reference: [35] and [36]

\subsection{Microbiological laboratory preparation}

Sampling methods (collecting and processing Chlamydia specimens)

To keep the viability of chlamydiae, specimens must be processed quickly within 24-48 hours and should be stored at $4^{\circ} \mathrm{C}$ until processed. Specimens which will be processed later than 48 hours should be stored at $-70^{\circ} \mathrm{C}$.

i. Urethral colonization in women is about $5-30 \%$ of any suspected patient. The first void urine (FVU) which is the first $10-30 \mathrm{~mL}$ of urine can therefore be used $[35,37]$. The best urine is obtained between 2-6 hours after the last micturition. In cases where the patient has just recently urinated, ask them to wait for 2 hours [38].

ii. Endocervical or urethral swab and specimens from the upper genital tract, liquid from Douglas's pouch, endometrium and tubal specimens. Dacron, cotton or calcium alginate swabs or cytobrush are used to collect the endocervical tissue. Swabs should have plastic or metal shafts and not wooden shafts because wooden components have toxic effects on cell culture $[34,38]$. The swab should be inserted into the cervical os past the squamocolumnar junction 1-2 cm deep, rotated for 15-30 seconds and removed without touching the vaginal mucosa [36]. In nonpregnant women, the use of a cytologic brush is superior because the cytobrush can scrape more cells allowing to obtain a better result for direct fluorescentantibody stain (DFA), however, the cytobrush may cause bleeding which potential- 
ly inhibit PCR diagnosis and is not allowed for use in pregnant women [36, 38, 39]. Cervical scraping is best obtained from the cervical transitional zone or within the opening of the cervix. The swab should be placed directly into a tube containing 2 SP medium $(0.2 \mathrm{M}$ sucrose phosphate containing $10 \mu \mathrm{g}$ of gentamicin $/ \mathrm{mL}, 25 \mu \mathrm{g}$ of vancomycin $/ \mathrm{mL}$ and $25 \mathrm{U}$ of nystatin $/ \mathrm{mL}$ ) or Stuart medium. Antibiotics in the transport medium inhibit other bacterial overgrowth in culture, but keeping in mind that broad-spectrum antibiotics (tetracyclines, macrolides and penicillins) should be avoided. To reduce the contamination rate, it is recommended to remove ectocervical mucopus before taking the endocervical sample [36, 38].

iii. Other sites of infection (conjunctiva, nasopharynx, or deeper respiratory tract) are for cases other than cervicitis and urethritis in women, or from anal swabs.

\subsection{Diagnostic methods}

The choice of the diagnostic method depends on the bacterial load or amount of bacteria that are possibly present in the specimens. The source of specimens from genitourinary infections are the cervical cells, vaginal mucosal cells and the firstvoid urine, with the latter having the least bacterial load. Because of its sensitivity, the nucleic acid amplification tests (NAAT) are applicable to use on scrapings from any suspected infected tissues including the first-void urine, vaginal, introital and vulvar swabs. Meanwhile the non-nucleic acid amplification (non-NAA) methods, being less sensitive, are best used on scrapings from conjunctiva, nasopharynx, cervix, urethra and rectum specimens [38].

1. Cytological examination

Cytological examination reveals the intracytoplasmic inclusion stained by Giemsa solution. The smear is dried followed by fixation with methanol prior to flooding it in diluted Giemsa solution. Ethanol is used to clear the Giemsa solution and dried off. The basophilic inclusion bodies are visualized pinkish-blue under the microscope.

2. Cell culture

Specimens are mixed with cycloheximide-treated MacCoy, HeLa, BHK-21 or Buffallo green monkey kidney cell lines then centrifuged to enhance bacterial absorption to the cells. Following 2 hours of incubation at $35^{\circ} \mathrm{C}+5 \% \mathrm{CO}_{2}$, the cell's medium is changed into cycloheximide-containing medium and incubation is continued to 48-72 hours. The inclusion body that is formed inside the infected cells is visualized after binding to specific immunofluorescent monoclonal antibodies specific for Chlamydia trachomatis and viewed under a fluorescence microscope. Inclusion bodies can also be visualized by Giemsa or Iodine stains [34, 36, 38, 39].

3. Antigen detection

Enzyme immuno assay (EIA) applies monoclonal or polyclonal antibodies to bind with soluble chlamydial lipopolisacharides (LPS). Because of the low sensitivity $(65-75 \%)$ of EIAs, a second confirmatory test is required. The confirmatory test 
applies monoclonal antibodies directed at binding a group-specific epitope on the LPS or using fluorescent-labeled monoclonal antibodies (direct fluorescent antibody $=$ DFA) directed against the outer membrane protein (MOMP) $[38,39]$.

4. Nucleic acid hybridization (NAH)

A nucleic acid hybridization test, for example PACE2 (Gen Probe, USA) applies DNA-RNA hybridization in the attempt to detect RNA of Chlamydia. The test has the same sensitivity as EIAs and the cell culture methods.

5. Nucleic acid amplification tests (NAAT)

Some molecular tests have been developed and used for clinical diagnosis, such as the polymerase chain reaction Amplicor (Roche Molecular Systems, USA), ligase chain reaction LCx assay (Abbott Laboratories, USA), transcription-mediated amplification AMP-CT and APTIMA Combo 2 (Gen Probe Inc, USA) and strand displacement amplification ProbeTec (BD Diagnostic Systems, USA), and real time PCR Cobas TaqMan CT (Roche, USA) [40]. Molecular detection systems allow faster, and more sensitive methods enabling to detect Chlamydiae in urine or self-administered vaginal swabs [34]. The result of NAAT when compared to the previous gold standard cell culture was significantly more sensitive, and is acceptable as the new gold standard for the diagnosis of Chlamydia trachomatis infections $[41,42]$.

\section{Antibody detection}

Generally, serological examination is not recommended to determine whether a person has genitourinary infection because the $\operatorname{IgG}$ antibodies detected could have been formed in a previous infection. IgM is usually not present in an active infection because it is likely that the patient has previously been infected with Chlamydia trachomatis or now is infected with Chlamydia pneumonia potentially causing an anemnestic response. The microimmunofuorescence assay (MIF) for detection of antichlamydial IgM is useful in the case of neonatal conjunctivitis, but not for detection of antichlamydial $\operatorname{IgG}$ in babies, because of the possession of maternal IgG. The Chlamydia trachomatis serological markers of persistent infections IgA, cHSP60 $\operatorname{IgG}$ and CRP were found significantly more often in women with tubal pathology as compared to those without pathology [24, 43, 44]. The other serological assays such as complement fixation test (CFT), enzyme immune assays (EIA), indirect hemagglutination, neutralization, precipitation, gel diffusion, enzyme linked fluorescence, immunoperoxidase and immunoelectrophoresis are available, some as inhouse methods and only a few methods have been commercialized [34, 36, 38].

\section{Diagnostic considerations of urogenital infections in adult women}

The clinical specimens for Chlamydia tests in adult women are the first void urine or endocervical or vaginal swabs. Culture techniques are the preferred methods for the detection of Chlamydia trachomatis infections, but they have been replaced by the nucleic acid amplification test because of the high sensitivity (85\%) and specificity $(94-99.5 \%)$ of NAAT when used with endocervical swabs and urine. Other tests 
such as direct immunofluorosence, EIA and nucleic acid hybridization can be used with endocervical swabs and urine. Some NAATs can be used with vaginal swabs as well as the liquid-based cytology specimens collected for Pap smears $[45,46]$.

\section{Diagnostic considerations of chlamydial infections in infants}

Ophthalmia neonatorum usually appears within 5-12 days or may appear late until 30 days after birth, and chlamydial pneumonia may appear at age 1-3 months. Conjunctivitis is characterized by oedema in one or both eyes, with mucopurulent discharge. Hemorrhagic eye discharge is a typical sign of chlamydial infection of the eyes [47]. Prophylaxis with silver nitrate or antimicrobial ointment used for the prevention of gonococcal infection does not protect against chlamydial ophthalmia neonatorum. It is important to evert the eye lid and scrape using a dacron-tipped swab from the mucous surface of the eyelid of babies suspected of suffering from ophthalmia neonatorum [46]. Although diagnosis can be done by a NAAT, none of the available molecular tests are approved by the FDA for use in conjunctival or nasopharyngeal specimens. PCR on conjunctival specimens demonstrated a good result, but the recommended technique for diagnosis of infection in infants is cell culture [48, 49]. Chlamydial pneumonia is characterized by staccato cough, no wheezing, and no fever. There is hyperinflation and bilateral diffuse infiltrates on a chest radiograph and peripheral eosinophilia may be present. The test performed should be directed at specimens from the nasopharynx, or tracheal aspirates or lung biopsies [46].

\section{Treatment}

Several factors which must be considered before choosing the antibiotic treatment of urogenital infections are:

1. Site of infection

2. Age of the patient

3. Complicated or uncomplicated infections

4. Pregnancy

\subsection{Treatment of uncomplicated infections in adults}

The efficacy of antibiotics refers to either the bacteriologic eradication or resolution of clinical signs and symptoms. Several studies have reported that the bacteriologic eradication rate of azithromycin was $95-100 \%$ within $2-4$ weeks of treatment and the clinical cure rate was $80-95 \%$. Similarly, the bacteriologic eradication rate of doxycycline was $99 \%$ at 2 weeks, and the clinical cure rate was $83 \%$, but in addition there was a small percentage of failures [50]. Although both antibiotics showed the same efficacy, the single dose administration of azithromycin allows 
direct observation by the physician and it appears to be more cost effective. It was reported that $25 \%$ of patients taking doxycyclin showed strict compliance, $51 \%$ took intermediate amounts of medication, and $24 \%$ were noncompliant, suggesting that azithromycin is potentially more beneficial [50-52].

Refraining from sexual intercourse is advised to the patients during the first week after the initiation of treatment. Retesting for chlamydial infection is not suggested until 3-4 months after the completion of the antibiotic course unless there are signs and symptoms of persistence or if the patient is pregnant. Although CDC recommends that women who are under 25 years old or at increased risk of STDs are screened annually to interrupt progression or reducing transmission [53], there is no single accepted screening strategy. Interestingly, despite the screening programs, the Chlamydia infection rate is increasing in countries such as the USA, Sweden and Canada. This may be related to changes in sexual behaviour, improved reporting systems, failure in treatment and notification to the sexual partner, and changes in natural immunity [54].

The recurrence of chlamydial infection and persistence of symptoms due to other bacterial causes are treated with $2 \mathrm{~g}$ metronidazole (Flagyl) orally in a single dose plus $500 \mathrm{mg}$ erythromycin base orally four times per day for seven days or $800 \mathrm{mg}$ erythromycin ethylsuccinate orally four times per day for seven days [46].

Table 3: CDC-recommended regimens for uncomplicated urogenital chlamydial infection in adults

\begin{tabular}{l|cc}
\hline & ROUTE & DOSAGE \\
\hline Recommended Regimens & & \\
Azithromycin (Zithromax) & Orally & $1 \mathrm{~g}$ single dose* \\
$\begin{array}{c}\text { Doxycycline (Vibramycin) } \\
\text { Alternative Regimens }\end{array}$ & Orally & $100 \mathrm{~g}$ twice per day, 7 days \\
Erythromycin base & Orally & $500 \mathrm{mg}$ four times per day, 7 days \\
Erythromycin ethylsuccinate & Orally & $800 \mathrm{mg}$ four times per day, 7 days \\
Ofloxacin (Floxin) & Orally & $300 \mathrm{mg}$ twice per day, 7 days \\
Levofloxacin (Levaquin) & Orally & $500 \mathrm{mg}$ once per day \\
\hline
\end{tabular}

Consider alternative regimen if vomiting occurs within 1-2 hours of taking azithromycin (Adapted from reference: [45].)

\subsection{Treatment of uncomplicated infections in pregnancy}

Doxycycline, ofloxacin and levofloxacin are contraindicated in pregnant women. Recent studies have provided data on the safety, efficacy and tolerability of $1 \mathrm{~g}$ single dose azithromycin in pregnant women. Erythromycin, the previously recommended regimen, is well known to cause high intolerance rates in pregnant women resulting in a low compliance, thus recommended only as an alternative therapy. Disagreement on the efficacy of amoxycillin was supported by cell culture 
experiments demonstrating that exposure to penicillin could induce the conversion to a persistent form of Chlamydia, which results negative in culture test but still viable and thus possibly causing recurrent infections at a later time. However, it was difficult to provide clinical evidence of chlamydial persistence in a patient unless a nonculture test is used, and because it is possible that reinfections were obtained from an untreated partner. Therefore, amoxycillin remains as the other recommended antibiotic regimen because its efficacy and tolerability are similar to azithromycin $[45,51]$. All pregnant women must have a follow-up test 3 weeks after the completion of therapy to ensure therapeutic cure $[46,50]$. All pregnant women with chlamydial infection during the first trimester who had been retested upon completion of the first course of treatment must be retested after 3 months after the completion of therapy. All those pregnant women at the age $\leq 25$ years, and those with the risk of chlamydial infections should be tested during their third trimester of pregnancy [45].

Table 4: CDC-recommended regimens for uncomplicated urogenital chlamydial infection in pregnant women *

\begin{tabular}{l|ll}
\hline & ROUTE & DOSAGE \\
\hline $\begin{array}{l}\text { Recommended Regimens } \\
\text { Azithromycin (Zithromax) }\end{array}$ & Orally & 1 gram single dose \\
Amoxycillin & Orally & $500 \mathrm{mg}$ three times per day, 7 days \\
Alternative Regimens & & \\
Erythromycin base & Orally & $500 \mathrm{mg}$ four times per day, 7 days \\
Erythromycin base & Orally & $250 \mathrm{mg}$ four times per day, 14 days \\
Erythromycin ethylsuccinate & Orally & $800 \mathrm{mg}$ four times per day, 7 days \\
Erythromycin ethylsuccinate & Orally & $400 \mathrm{mg}$ four times per day, 14 days \\
\hline
\end{tabular}

Adapted from reference: [45]

\subsection{Treatment of chlamydial infection in infants}

Ophthalmia neonatorum and chlamydial pneumonia are treated with erythromycin (base or ethylsuccinate) $50 \mathrm{mg} / \mathrm{kg}$ per day divided into 4 doses for 14 days [53]. The cure rate is $80 \%$, and usually another course of therapy is necessary. Topical antibiotic treatment or with silver nitrate for ophthalmia neonatorum does not prevent chlamydial infection of the conjunctiva because the nasopharynx and the genital tract of the infected newborn usually are also colonized by Chlamydia trachomatis $[46,55]$. 


\section{Complication and Sequelae}

The most important complication of sexually transmitted chlamydial infections is PID, which resulted from the ascending spread of the microorganisms from the vagina and the cervix to the upper genital tract. Damage to the fallopian tubes predisposes a person to ectopic pregnancy and infertility, recurrent PID and chronic pelvic pain [34]. The time span from genitourinary infection until the presence of PID is unclear because a great proportion of chlamydial infections is asymptomatic, and there are several risk factors influencing the rate of progression to PID. It varies from 2-5\% for the high risks who turn up with PID within 2 weeks after diagnosis to a very low incidence among the low risks which may occur not before a year after the initial infection. A mathematical model study suggested that clinical PID can occur already at the beginning, at the end or throughout the duration of chlamydial infection [56]. Other impacts of chlamydial infection in pregnancy are stillbirth, premature delivery, premature rupture of the membrane and low birth weight. These complications strongly support the importance of screening at the earliest stage.

\section{Concluding remarks}

Chlamydia trachomatis strains $\mathrm{D}$ to $\mathrm{K}$ cause genitourinary infection, most of which are asymptomatic. Undetected and untreated infections, most commonly occurring in women, may become a source of infection to her sexual partner, and cause severe long-term complications for herself, thus adding a burden to the national health program $[57,58]$. In many developing countries, screening has not become a routine examination. Considering the advantage of screening, great efforts are required involving the government, health authorities, the specialists and general practitioners. The choice of screening must be cost-effective and feasible using non-invasive procedures. The annual screening should target all women at high risks, e.g. $\leq 25$ years old sexually active women, and $>25$ years old women if they are at increased risk for infection, and repeating the test 3 months after completion of a course of antibiotic treatment because repeated infections are common. It is important to treat male sex partners of the infected females. Education of the public is also important to raise self awareness of the screening program [59]. Constraints are great especially in Asian countries like Indonesia because STD remains a stigma, but the advantage of screening definitely outweighs the negative responses. 


\section{References}

1. Paavonen, J. and W. Eggert-Kruse, Chlamydia trachomatis: impact on human reproduction. Human Reproduction Update. 1999;5:433-447.

2. Mascellino, M.T., P. Boccia, and A. Oliva, Immunopathogenesis in Chlamydia trachomatis infected women. International Scholarly Reseach Network Obstetrics and Gynecology. 2011: 436936. doi: 10.5402/2011/436936. Epub 2011 Nov 24.

3. Adetunde, I.A., et al., Epidemiology of Chlamydia bacteria infections - a review. Journal of American Science. 2009;5:55-64.

4. Marcone, V., et al., Epidemiology of Chlamydia trachomatis endocervical infection in a previously unscreened population in Rome, Italy, 2000-2009. Euro Surveillance. 2011;17:1-8.

5. Batteiger, B., et al., Repeated Chlamydia trachomatis genital infections in adolescent women. Journal of Infectious Diseases. 2010;201:45-51.

6. Walker, J., et al., Chlamydia trachomatis incidence and reinfection among young women - behavioural and microbiological characteristics. PLoOS ONE, 2012. 7(5): e37778. doi: 10.1371/journal.pone.0037778. Epub 2012 May 25.

7. Joesoef, M.R., et al., Patterns of sexually transmitted diseases in female sex workers in Surabaya, Indonesia. International Journal of STD AIDS. 1997:8:576-80.

8. Patten, J. and I. Susanti, Reproductive health and STDs among clients of a women's health mobile clinic in rural Bali, Indonesia. International Journal of STD AIDS. 2001;12:47-49.

9. Reed, B., K. Ford, and D. Wirawan, The Bali STD/AIDS study: association between vaginal hygiene practices and STDs among sex workers. Sexually Transmitted Infections. 2001;77:46-52.

10. Joesoef, M.R., et al., High rate of bacterial vaginosis among women with intrauter-ine devices in Manado. Contraception. 2001;64:169-72.

11. Davies, S.C., et al., Sexually transmitted infections among female Sex workers in Kupang, Indonesia. Sexually Transmitted Diseases. 2003;30:671-679.

12. Tanudyaya, F.K., et al., Prevalence of sexually transmitted infections and sexual risk behavior among female sex workers in nine provinces in Indonesia, 2005. South East Asean Journal of Tropical Medicine and Public Health. 2010;41:463-473. 
13. Bollen, L.J.M., et al., Addressing the high prevalence of gonorrhoea and chlamydia among female sex workers in Indonesia: results of an enhanced, comprehensive interven-tion. Sexual Transmitted Infections. 2010;86:61-65.

14. Mawu, F.O., et al., Sexually transmissible infections among female sex workers in Manado, Indonesia, using a multiplex polymerase chain reaction-based reverse line blot assay. Sexual Health. 2011;8:52-60.

15. Silitonga, N., et al., Prevalence over time and risk factors for sexually transmissible infections among newly-arrived female sex workers in Timika, Indonesia. Sexual Health. 2011;8:61-64.

16. Ritung, M., The correlation between vitamin $C$ and cervicovaginal bacterial growth with the premature rupture of the membrane, in obstery and gynecology. 2011; University of Hasanuddin: Makassar. p. 51.

17. Everett, K.D.E., R.M. Bush, and A.A. Andersen, Emended description of the order Chlamydiales, proposal of parachlamydiaceae fam. nov and Simkaniaceae fam. nov each containing one monotypic genus, revised taxonomy of the family Chlamydiaceae, including a new genus and five new species, and standard for the identification of organisms. International Journal of Systematic Bacteriology. 1999;49:415-40.

18. Corsaro, D. and G. Greub, Pathogenic potential of novel Chlamydiae and diagnostic approaches to infections due to these obligate intracellular bacteria. Clinical Microbiology Reviews. 2006;19:283-297.

19. Cevenini, R., M. Donati, and V. Sambri, Chlamydia trachomatis - the agent. Best Practice and Research: Clinical Obstetrics and Gynaecology. 2002;16:761773.

20. Dunlop, E.M.C., S. Darougar, and J.D. Treharne, Epidemiology of infection by serotypes $\mathrm{D}$ to $\mathrm{K}$ of Chlamydia trachomatis. The British Journal of Venereal Diseases. 1980;56:163-8.

21. Cocchiaro, J.L. and R.H. Valdivia, New insights into Chlamydia intracellular survival mechanisms. Cellular Microbiology. 2009;11:1571-1578.

22. Fredlund, H., et al., Molecular genetic methods for diagnosis and characterisation of Chlamydia trachomatis and Neisseria gonorrhoeae: impact on epidemiological surveil-lance and interventions. APMIS. 2004;112:771-84.

23. Hybiske, K. and R.S. Stephens, Mechanisms of host cell exit by the intracellular bacterium Chlamydia. PNAS. 2007;104:11430-11435.

24. denHartog, J., S. Morre, and J. Land, Chlamydia trachomatis-associated tubal factor subfertility: immunogenetic aspects and serological screening. Human Reproduction Update. 2006;12:719-730. 
25. Darville, T. and T.J. Hiltke, Pathogenesis of genital tract disease due to Chlamydia trachomatis. Journal of Infectious Diseases. 2010;201(Suppl 2):S114S125.

26. Linhares, I.M. and S.S. Witkin, Immunopathogenic consequences of Chlamydia trachomatis $60 \mathrm{kD}$ a heat shock protein expression in the female reproductive tract. Cell Stress and Chaperones. 2010;15:467-473.

27. Su, W.H., et al., Diagnosis of Chlamydia infection in women. Taiwanese Journal of Obstetrics and Gynecology. 2011;50:261-267.

28. WHO, Sexually transmitted and other reproductive tract infections - a guide to essential practice. 2005: Geneva. p. 1-193.

29. Ndowa, F.J., Designing and evaluating clinical algorithm for STI case management, training course in sexual and reproductive health research, Geneva. 2011. www.gfmer.ch

30. Passey, M., et al., Screening for sexually transmitted diseases in rural women in papua New Guinea: are WHO theraputic algorithms appropriate for case detection? Bulletin of the World Health Organization. 1998;76:401-411.

31. Romoren, M., et al., Chlamydia and gonorhoea in pregnant Batswana women:time to discard the syndromic approach? BMC Infectious Diseases. 2007;7:1-11.

32. Fesseha, B.K., Diagnosis of sexually transmitted infections in developing nations using syndromic management: Is it working?, in School of Public Health. 2008. University of Texas: Dallas. p. 42.

33. Mahmood, M.A. and A. Saniotis, Use of syndromic management algorithm for Sexually transmitted infections and reproductive tract infections management in community settings. Karachi Journal of Pakistan Medical Association. 2011;61:453-457.

34. Darville, T., Chlamydia trachomatis genital infection in adolescents and young adults, in: Hot topics in infection and immunity in children, A.J. Pollard and A. Finn, Editors. 2006, Springer: New York. p. 85-100.

35. Bebear, C. and B. deBarbeyrac, Genital Chlamydia trachomatis infections. Clinical Microbiology and Infectious Diseases. 2009;15:4-10.

36. Black, C.M., Current methods of laboratory diagnosis of Chlamydia trachomatis infections. Clinical Microbiology Reviews. 1997;10:160-184.

37. Monif, G.R.G., A new gold standard for the detection of Chlamydia trachomatis? Infectious Diseases in Obstetrics and Gynecology. 1998;6:44-45. 
38. Chernesky, M.A., The laboratory diagnosis of Chlamydia trachomatis infections. Canadian Journal of Infectious Diseases and Medical Microbiology. 2005;16:39-44.

39. Barnes, R.C., Laboratory diagnosis of human Chlamydial infections. Clinical Microbiology Reviews. 1989;2:119-136.

40. Skidmore, S., P. Horner, and H. Mallinson, Testing specimens for Chlamydia trachomatis. Sexually Transmitted Infections. 2006;82:272-275.

41. Jesperson, D.J., et al., Prospective comparison of cell culture and nucleic acid amplification tests for laboratory diagnosis of Chlamydia trachomatis infections. Journal of Clinical Microbiology. 2005;43:5324-5326.

42. Schachter, J., et al., Confirming positive results of nucleic acid amplification tests (NAATs) for Chlamydia trachomatis: All NAATs are not created equal. Journal of Clinical Microbiology. 2005;43:1372-1373.

43. Bax, C.J., et al., Chlamydia trachomatis heat shock protein 60 (cHSP60) antibodies in women without and with tubal pathology using a new commercially available assay. Sexually Transmitted Infections. 2004;80:415421.

44. Cappello, F., et al., Chlamydia trachomatis infection and anti-Hsp60 immunity: The two sides of the coin. PLoS Pathogens. 2009;5:1-9.

45. CDC, Sexually transmitted diseases treatment guidelines, 2010. Morbidity and Mortality Weekly Report. 2010;59 (RR-12):44-49.

46. Miller, K.E., Diagnosis and treatment of Chlamydia trachomatis infection. American Family Physician. 2006;73:1411-1416.

47. Chang, K., V.Y.W. Cheng, and N.S. Kwong, Neonatal haemorrhagic conjunctivitis: a specific sign of chlamydial infection. Hong Kong Medical Journal. 2006;12:27-32.

48. Hammerschlag, M.R., Chlamydial and gonococcal infections in infants and children. Clinical Infectious Diseases. 2011;53 (Suppl 3):S99-102.

49. Yip, T.P.P., et al., Incidence of neonatal chlamydial conjunctivitis and its association with nasopharyngeal colonization in a Hong Kong hospital, assessed by polymerase chain reaction. Hong Kong Medical Journal. 2007;13:22-6.

50. Adimora, A.A., Treatment of uncomplicated genital Chlamydia trachomatis infections in adults. Clinical Infectious Diseases. 2002;35 (Suppl 2):S183-6.

51. Geisler, W., Management of uncomplicated Chlamydia trachomatis infections in adolescents and adults: Evidence reviewed for the 2006 Centers for Disease 
Control and Prevention Sexually Transmitted Diseases Treatment Guidelines. Clinical Infectious Diseases. 2007;44 (Suppl 3):S77-83.

52. Workowski, K.A. and S.M. Berman, CDC Sexually transmitted diseases treatment guidelines. Clinical Infectious Diseases. 2002;35 (Suppl 2):S135-7.

53. CDC, CDC Grand Rounds: Chlamydia prevention: challenges and strategies for reducing disease burden and sequelae. Morbidity and Mortality Weekly Report. 2011;66:371-373.

54. Taylor, B.D. and C.L. Haggerty, Management of Chlamydia trachomatis genital tract infection:screening and treatment challenges. Infection and Drug Resistance. 2011;4:19-29.

55. Zar, H.J., Neonatal chlamydial infections prevention and treatment. Pediatric Drugs. 2005;7:103-110.

56. Herzog, S.A., et al., Timing of progression of Chlamydia trachomatis infection to pelvic inflammatory disease: a mathematical modelling study. BMC Infectious Diseases. 2012;12:187.

57. Haggerty, C.L., et al., Risk of sequelae after Chlamydia trachomatis genital infection in women. Journal of Infectious Diseases. 2010;201(S2):S134-S155.

58. Shaw, K., et al., Public health policies and management strategies for genital Chlamydia trachomatis infection. Risk Management and Healthcare Policy. 2011;2011:57-65.

59. Mylonas, I., Female genital Chlamydia trachomatis infection: where are we heading? Archives of Gynecology and Obstetry. 2012;285:1271-1285. 



\title{
23. The Burden of Streptococcal Diseases in Maternal-Child Health in West Africa
}

\author{
Nicholas T.K.D. Dayie, University of Ghana Medical School, Department of Microbi- \\ ology, Accra, Ghana \\ Gabriel Y.K. Ganyaglo, Department of Obstetrics and Gynaecology, Korle-Bu Teaching \\ Hospital, Accra, Ghana
}

\section{Abstract}

Infections and malnutrition continue to conspire against children in the West African sub-region celebrating their 5th birthday. Living beyond the first month of life is becoming a formidable hurdle in most communities within the sub-region. National and donor efforts appear to focus on nutrition. Despite improvements in vaccination programs across the region, infections continue to account for a high number of under 5 years-old deaths. This study was conducted to demonstrate the impact of infections on maternal and child health by streptococci which are important pathogens, known to affect the early days of life. 


\section{Background and aim}

Every year, 4.6 million children in sub-Saharan Africa loose their lives before attaining the age of five years (UNICEF, 2004). Due to a lack of appropriately equipped health facilities that enable to carry out basic microbiological investigations, the causes of most of these deaths could not properly be identified. Hence, the data on invasive streptococcal infections are very limited, although they are associated with maternal and child health. They play a leading role in the causes of maternal and neonatal deaths, particularly in the developing world. Among the pathogens associated with maternal and child health are group A streptococci (GAS), group B streptococci (GBS) and Streptococcus pneumoniae. Many of the streptococcal-associated diseases and deaths among neonates and their mothers are preventable by the administration of prophylactic intrapartum antibiotics and appropriate vaccines. This chapter gives an account of the burden of 3 species of streptococci on maternal and child health in West Africa.

\section{Methods}

We reviewed articles in the Medline database from 1992 to 2012 and contacted experts in Ghana to seek for unpublished data on GAS, GBS and Streptococcus pneumoniae in order to get a broader picture of the burden of the diseases associated with GAS, GBS and S. pneumoniae.

\section{Group A streptococcus (GAS)}

Group A streptococcus, also called Streptococcus pyogenes, is the most common bacterial cause of pharyngitis and can lead to acute rheumatic fever (Bisno et al., 1996). GAS is also associated with life-threatening illnesses such as streptococcal toxic shock syndrome, necrotizing fasciitis, and acute post-streptococcal glomerulonephritis. Approximately 9,700 cases of invasive disease and 1,300 deaths are associated with GAS each year (O’Brien et al, 2002; Hoge et al., 1993). Several studies have compared GAS strains that cause pharyngitis in children of school-going age with isolates from cases associated with invasive disease in the community (Haukness et al., 2002; Muotiala et al, 1997; Cockerill et al., 1997). The results of these studies suggest that children of school-going age serve as a reservoir of infection for the community. Hence, the introduction of an appropriate GAS vaccine will not only prevent pharyngitis and invasive disease in children of school- going age, but may also reduce carriage and transmission of the organism in the community (McNeil et al. 2005; Haber et al., 2007). Donkor et al. (2006) reported a 2\% nasal carriage rate of GAS among 100 Ghanaian children $<5$ years of age who visited the Korle-Bu Teaching Hospital for treatment of various diseases and who were not on antibiotics for the past two weeks. Since then, no data has been published 
on the prevalence of GAS in Ghana. Based on this information, it can be assumed that there is no burden of GAS in Ghana. Referring to other West African countries, there is also no data on the prevalence of GAS either. Infections due to GAS may be emerging but it seems that they are not in the scope of public health interest yet.

\section{Streptococcus pneumoniae}

Streptococcus pneumoniae is also called pneumococcus. Pneumococcal diseases remain a major cause of morbidity and mortality among children under 5 years and constitute a substantial burden on health care systems in resource-limited countries. They are the leading cause of bacterial pneumonia, meningitis, and sepsis in children. Although there are over 90 serotypes, only 20 serotypes are known to cause invasive pneumococcal disease (Donkor et al., 2010). The drug of choice is penicillin and its derivatives. O'Brien et al. (2009) gave a global estimate of pneumococcal disease burden. According to their report in the year 2000, about 14.5 million episodes of serious pneumococcal diseases were estimated to occur worldwide. More than 800,000 children aged 1-59 months lose their lives because of pneumococcal disease. Of this figure, 91,000 were HIV-positive and 735,000 were HIV-negative. Among the HIV-negative children, over 61\% died in ten African and Asian countries. Adetifa et al. (2012) reported that Nigeria has a rate of 52.5\% nasopharyngeal pneumococcal carriage among children who are less than five years of age. Donkor et al. (2010) reported a nasopharyngeal carriage rate of $15.3 \%$ in Ghana. Personal communication with Ghanaian researchers in the field of pneumococcal research as of today made us aware that the nasopharyngeal carriage rate is $34 \%$. Since carriage always precedes invasive infections with respect to pneumococci, it is imperative to look for appropriate vaccines to reduce carriage rates of serotypes that are globally known to be multiple antibiotic resistant with a view to reducing morbidity and mortality rates among the vulnerable age groups in Ghana. Holliman et al. (2007) investigated the epidemiology of invasive pneumococcal disease in Kumasi, Ghana. They observed that a very low resistance level to penicillin existed and that the theoretical vaccine coverage of the 7-, 9-, 11 - and 23-valent vaccines was $26 \%$, $63 \%, 64 \%$ and $76 \%$, respectively.

Between $26^{\text {th }}$ of April and $2^{\text {nd }}$ of May 2012, a series of activities and public sector consultations led to the launching and the introduction of the PCV-13 vaccine in Ghana by GAVI and the Ghanaian Ministry of Health. The usage of the PCV13 vaccine in Ghana became necessary because of a number of factors. First, current research findings suggest an increase in the nasopharyngeal pneumococcal carriage rate from 15.3\% in 2010 to $34 \%$ in 2012 among children who are less than six years of age. Second, there is a yearly outbreak of pneumococcal and meningococcal meningitis in Northern Ghana during the dry season. And third, Adetifa et al. (2012) reported that the neighboring country Nigeria has a 52.5\% nasopharyn- 
geal pneumococcal carriage rate among children less than five years of age. Since resources for child survival are increasing, estimates of pneumococcal disease burden based on comprehensive, clear, and rigorous methods are needed.

\section{Group B streptococcus (GBS)}

ftdffftf During the last few decades, group B streptococcus (GBS) has emerged as an important pathogen. The major reservoirs for GBS are the vagina and the perianal region or rectum. The colonization of these regions is a risk factor for subsequent infection in pregnant women and newborns. Early neonatal sepsis due to GBS is believed to be acquired from colonization of the perineum of the mother in the late third trimester.

In 2001 and by using vagino-rectal swabbing, Enweronu et al. showed in a pilot study at Korle-Bu Teaching Hospital in Ghana where 100 pregnant women at minimum stage of 28 weeks have been tested, that there was a GBS prevalence of $19 \%$. In 2012, Ganyaglo et al. obtained a GBS prevalence of 21\% after swabbing 1,200 pregnant women between 35-42 weeks in the same hospital (unpublished). In the Gambia, GBS prevalence was found to be $22.4 \%$ (Suara et al., 1990), whereas in Calabar Nigeria, GBS prevalence was found to be as low as 9\% (Nwachukwu et al., 2003). In rural Zimbabwe, GBS prevalence was as high as $60 \%$ whereas in urban Zimbabwe it was 46\% (Mavenyengwa et al., 2006). In Egypt, GBS prevalence was $25 \%$ (Shabayek et al., 2009). Global GBS prevalence is estimated to be around $20 \%$. Although nearly all the studies cited used pregnant women in their third trimester, the laboratory methods were not uniform.

In the 70's, early-onset neonatal sepsis due to GBS primarily acquired from a colonized mother was responsible for more than $50 \%$ of neonatal deaths in the United States of America. By the mid 80's by giving intrapartum antibiotics to risk mothers, the neonatal mortality rate due to early-onset disease had reduced by about $43 \%$. In recent times, the case fatality rate from GBS infection in the US is as low as $4 \%$. Thus, universal culture-based screening is the key to identify colonized mothers. Regularly updated guidelines prescribe appropriate intrapartum interventions which have helped to prevent early neonatal sepsis (CDC 2010, revised guidelines).

In resource-poor settings like the West African sub-region, although GBS prevalence is very high, routine screening for GBS colonization among pregnant women is not part of the general exercise. Although prevalence studies abound, there is a rareness of follow-up studies among colonized mothers. To this end, the incidence of GBS invasive disease has not been established and the annual rate of neonatal GBS disease is unknown. Although sepsis is an important cause of early childhood mortality, the causative agent in most cases is not known.

Global neonatal deaths accounted for $43 \%$ of deaths among children who are under five years of age in 2011. Over the same period in sub-Saharan Africa, neo- 
natal deaths accounted for 33\% of under five years old deaths (UNICEF progress report, 2012). In Ghana, neonatal deaths account for $27 \%$ of deaths in children under five (GDHS, 2008). It is increasingly more difficult to live beyond the first month in resource poor-settings.

To meet MDG 4, neonatal mortality should be tackled and neonatal sepsis cannot be ignored. There is an urgent need for research into the causes of sepsis in the neonatal period within the West African sub-region as a whole.

During the antenatal period, GBS colonization of the perineum can result in urinary tract infection. Severe maternal infection with GBS can lead to abortion and preterm delivery. Following delivery, GBS can cause endometritis and puerperal sepsis. Puerperal sepsis is an important cause of maternal morbidity and mortality accounting for $9.7 \%$ of maternal deaths in Africa (The Lancet, 2006).

It does appear the greatest burden of streptococcal diseases in maternal and child health within the West African sub-region is the lack of robust data to inform policy and provide clinical guidelines. Universal culture-based screening and intrapartum antibiotic prophylaxis is recommended for reducing the burden of GBS disease. However, in the sub-region, with rampant antibiotic abuse, there is already a high risk of developing antibiotic resistance which can worsen with the use of antibiotic prophylaxis. It may be useful to consider a risk-based approach to the screening. This can be followed by a stringent follow-up of babies of positive mothers. These babies with early signs of infection are then treated with appropriate antibiotics.

\section{Conclusion}

Over $13 \%$ of deaths in children under 5 years of age are associated with GAS each year. In the sub-region, such information is not known. Although some data is available on nasopharyngeal pneumococcal carriage rate, pneumococcal-specific morbidity and mortality rate is unknown. Also, though prevalence studies on maternal colonization with GBS abound, the rate of invasive early neonatal sepsis due to GBS is unknown. In conclusion, knowledge of the burden of streptococcal diseases in West Africa is not sufficient enough and requires concerted efforts by academia to fill the research gap.

\section{References}

Adetifa IM, Antonio M, Okoromah CA, Ebruke C, Inem V, Nsekpong D, Bojang A, Adegbola RA. Pre-vaccination nasopharyngeal pneumococcal carriage in a Nigerian population: epidemiology and population biology. PLoS One. 2012;7(1):e30548. Epub 2012 Jan 24.

Bisno AL. Acute pharyngitis: etiology and diagnosis. Pediatrics. 1996;97:949 _954. 
Cockerill FR III, MacDonald KL, Thompson RL, et al. An outbreak of invasive group A streptococcal disease associated with high carriage rates of the invasive clone among school-aged children. JAMA. 1997;277:38 - 43.

Causes of Maternal Death: a Systematic Review. The Lancet 2006; 367:1066-74.

Centers for Disease Control and Prevention. Prevention of perinatal Group B streptococcal disease. Revised guideline from CDC. MMWR 2010; 59 (No. RR-10): 1-23.

Donkor ES, Newman MJ, Oliver-Commey J, Bannerman E, Dayie NT, Badoe EV. Invasive disease and paediatric carriage of Streptococcus pneumoniae in Ghana. Scand J Infect Dis. 2010;42:254-9.

Donkor E.S, E. Nartey: Nasal colonisation of drug resistant bacteria in Ghanaian children less than five years. The Internet Journal of Microbiology. 2008 Volume 5 Number 2. DOI: 10.5580/16d5.

Enweronu-Laryea CC, Damale NRK, Newman MJ. Prevalence of group B streptococcus in pregnant women attending a tertiary hospital in Ghana in 2001. Archives of Clinical Microbiology 2011;vol.2 No. 2:5.

Ghana Statistical Service (GSS), Ghana Health Service (GHS), and ICF Macro. 2009. Ghana Demographic and Health Survey 2008. Calverton, Maryland, USA: GSS, GHS, and ICF Macro.

Haber M, Barskey A, Baughman W, et al. Herd immunity and pneumococcal conjugate vaccine: a quantitative model. Vaccine. 2007;25:5390 -5398

Haukness HA, Tanz RR, Thomson RB Jr, et al. The heterogeneity of endemic community pediatric group A streptococcal pharyngeal isolates and their relationship to invasive isolates. J Infect Dis. 2002;185:915-920.

Hoge CW, Schwartz B, Talkington DF, Breiman RF, MacNeill EM, Englender SJ. The changing epidemiology of invasive group A streptococcal infections and the emergence of streptococcal toxic shock-like syndrome: a retrospective population-based study. JAMA. 1993;269:384 -389.

Holliman RE, Liddy H, Johnson JD, Adjei O. Epidemiology of invasive pneumococcal disease in Kumasi, Ghana. Trans R Soc Trop Med Hyg. 2007;101:405-13.

McNeil SA, Halperin SA, Langley JM, et al. Safety and immunogenicity of 26valent group A streptococcus vaccine in healthy adult volunteers. Clin Infect Dis. $2005 ; 41: 1114-1122$

Muotiala A, Seppala H, Huovinen P, Vuopio-Varkila J. Molecular comparison of group A streptococci of T1M1 serotype from invasive and noninvasive infections in Finland. J Infect Dis. 1997;175:392-399 
Mavenyengwa RT, Masunga P, Meque E, Kudinha T, Moyo SR, Bevanger L, Bergh K, Nziramasanga P, Mapako T. Streptococcus agalactiae (group B streptococcus (GBS)) colonisation and persistence, in pregnancy; a comparison of two diverse communities (rural and urban). Central African Journal of Medicine. 2006;52:38-43

Nwachukwu NC, Utsalo SJ, Kanu I, Anyanwu EC. Genital colonization of group B streptococcus at term pregnancy in Calabar, Nigeria. The Internet Journal of Paediatrics and Neonatology. 2007 Volume 7 Number 2

O’Brien KL, Beall B, Barrett NL, et al. Epidemiology of invasive group A streptococcus disease in the United States, 1995-1999. Clin Infect Dis. 2002;35:268 - 276

O'Brien KL, Wolfson LJ, Watt JP, Henkle E, Deloria-Knoll M, McCall N, Lee E, Mulholland K, Levine OS, Cherian T; Hib and Pneumococcal Global Burden of Disease Study Team. Burden of disease caused by Streptococcus pneumoniae in children younger than 5 years: global estimates. Lancet. 2009;374(9693):893-902.

Suara RO, Adegbola RA, Mulholland EK, Greenwood BM, Baker CJ. Seroprevalence of antibodies to group B Streptococcal polysaccharides in Gambian mothers and their newborns. J Natl Med Assoc. 1998;90:109-1 14

Shabayek SA, Abdalla SM, Abouzeid AM.Vaginal carriage and antibiotic susceptibility profile of group B Streptococcus during late pregnancy in Ismailia, Egypt. Journal of Infection and Public Health. 2009;2:86-90.

UNICEF. The state of the world's children 2004. at http://www.unicef.org/publications/2004_OfficialSumm_ENG.pdf. [Accessed November 18, 2012]

UNICEF, Committing to Child Survival: A Promise Renewed - Progress Report 2012, New York, 2012. Available at

http://www.childinfo.org/mortality_neonatal.php [Accessed November 29, 2012] 



\section{Candidiasis in Pregnant Women}

Ichsan, Faculty of Medicine, Syiah Kuala University, Banda Aceh-Indonesia

Marut Tangwattanachuleeporn, Faculty of Allied Health Science, Burapha University, Chon Buri-Thailand

\section{Candidiasis and risk factors in pregnancy}

Candida albicans is the most prominent cause of human mycosis worldwide. This species can be found in most human individuals as a commensal in the gastrointestinal tract. Therefore, candidiasis or commonly called thrush or moniliasis, is an endogenous infection caused by C. albicans but also by other Candida species. In patients with local predisposing factors, the two most common infections are vulvovaginal and superficial oropharyngeal candidiasis. In immunocompromised patients, such as individuals suffering from human immunodeficiency virus (HIV) infection or some cancer patients, Candida could be a serious threat.

Pregnant women have a twofold higher prevalence rate of vulvovaginal candidiasis (VVC) compared with non-pregnant women (Sobel, 2007) because the vaginal condition is changed based on hormonal conversion that influences the respective microbial flora.

Other risk factors of VVC that have been identified are: 
1. Diabetic patients (Bohannon, 1998)

2. Previous history of VVC (Foxman, et al., 2000)

3. Higher education degree, intermediate age, oral contraceptive drugs (Foxman, et al., 2000, Reed, 1992, Gibb, et al., 1992, Foxman, 1990)

4. Sexual behavior ; age at first intercourse, frequency of oral-genital contact (Spinillo, et al., 1993)

5. Contraception devices such as; diaphragm, vaginal contraceptive sponge, intrauterine device acceptors(Reed, 1992, Foxman, et al., 2000, Sobel, 1993)

6. Use of antibiotics (Reed, 1992, Foxman, et al., 2000, Sobel, 1993), and

7. Use of corticosteroid

The leading cause of candidiasis is an excessive use of antibiotics which will harm the normal bacterial flora. Since yeasts are unaffected by those antibiotics, they will selectively outcompete the normal bacterial flora and subsequently convert from the commensal into the infective state. Newborns and young children also have a risk to develop oral candidiasis, especially when heavily immunosuppressed, e.g. by HIV infection (Fig.1).

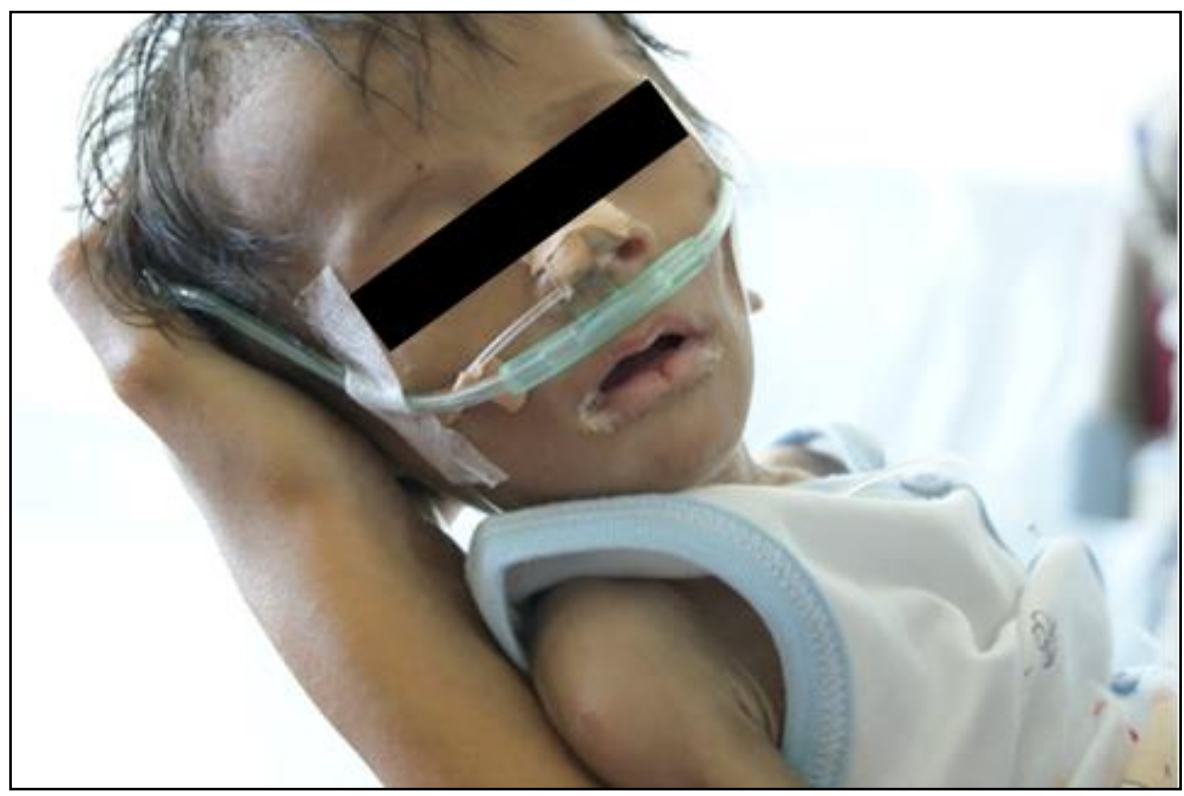

Figure 1: A child with oral candidiasis 


\section{Signs and symptoms in pregnant women with VVC}

The following symptoms might occur in pregnant women with VVC:

- Vaginal erythema

- Vulvar edema

- Excoriation

- Satellite lesions

- Vulvar pruritus

- Vaginal discharge

- Soreness and/or burning discomfort in the vagina and vulva

- Dysuria

- Dyspareunia

Table 1: Signs and Symptoms of Vaginal Candidiasis in Pregnant Women $(n=50)$

\begin{tabular}{|l|l|r|}
\hline No & Signs and symptoms & Number of Patients (\%) \\
\hline 1 & Vaginal discharge & $50(100 \%)$ \\
2 & Vulvar pruritus & $42(82 \%)$ \\
3 & Vulvar burning & $28(56 \%)$ \\
4 & Dyspareunia & $8(16 \%)$ \\
5 & Painful micturation(dysuria) & $6(12 \%)$ \\
\hline
\end{tabular}

$(\mathrm{n}=$ total number of cases); Source : Aslam, et al. 2008, own presentation

\section{Diagnosis and differential diagnosis}

Diagnosis of VVC is confirmed by the presence of Candida spp. in vaginal swabs by direct microscopic examination and/or culture. At present, culture is necessary because it can enhance the sensitivity of diagnosis and lead to the identification of the yeast species which could finally influence the choice for appropriate antifungal therapy (Aslam, Hafeez, et al., 2008). A positive Gram stain, the absence of a watery discharge, and patient self-diagnosis have been determined as the finest predictors of a positive culture for these patients (Abbott, 1995). In the past 25 years, some rapid diagnostic tests have been developed to speed up VVC diagnosis (Reed et. al., 1992).

However, the presence of nonspecific symptoms might complicate the correct differentiation between VVC and other infectious diseases, for instance bacterial vaginosis or Trichomonas vaginalis infection. Amongst others, the following differential diagnoses have to be taken into consideration when VVC is unlikely:

- Bacterial vaginosis

- Trichomonas vaginalis infection 
- Sexually transmitted diseases

- Urinary tract infection

- Atrophic vaginitis

- Helminthic infection (e.g. threadworm/pinworm in young woman)

- Contact dermatitis or eczema

- Mechanical irritation, e.g. long-distance cyclists, sexual abuse in girls

- Rectovesical fistula

- Underlying immunosuppression/undiagnosed diabetes mellitus

- Psoriasis

\section{The distribution and antifungal susceptibilities of Candida species}

Candida albicans is the most common cause of candidiasis including VVC. It is well known that $C$. glabrata is second most cause of candidiasis. The third frequently identified Candida species is either C. tropicalis or C. parasilosis depending on many factors such as the site of infection and country (Table 2). In a recent study, a mixed infection with two or more species of Candida in VVC was seen in $10.3 \%$ of patients and the most usual mixed causes were $C$. albicans and C. glabrata (Mahmoudi et al., 2011).

In most cases, $C$. albicans is sensitive to all antifungal drugs, except for some rare strains that are resistant against flucytosine. Candida glabrata and C.krusei show intrinsic resistance against the azoles. Candida parapsilosis is less susceptible to echinocandins. Amphotericin B and echinocandins are the most effective antifungals against Candida. However, amphotericin B has a lot of side effects and echinocandins are still very expensive. This information indicates that species identification in candidiasis is essential in order to decide on adequate antifungal therapy (Table 3).

There are various effective treatment options for VVC. Topical antifungal pessaries or vaginal tablets containing clotrimazole or miconazole are usually recommended. For mild cases, a single treatment of those topical drugs would be effective. Oral antifungal drugs containing fluconazole or itraconazole may be used if C.albicans infection is severe or recurrent. The application of topical drugs such as cream formulations is safer during pregnancy compared to a systemic application. 
Table 2. Selected epidemiological studies of Candida species isolates from various types of candidiasis and healthy women

VVC, vulvovaginal candidiasis; -, not mention

\begin{tabular}{|c|c|c|c|c|c|c|c|c|}
\hline \multirow[t]{2}{*}{ Country } & \multirow[t]{2}{*}{ Candidiasis } & \multirow[t]{2}{*}{$\begin{array}{l}\text { No. of } \\
\text { strains }\end{array}$} & \multirow[t]{2}{*}{$\begin{array}{l}\text { C. alb. } \\
(\%)\end{array}$} & \multicolumn{4}{|c|}{$\begin{array}{l}\text { non-albicans Candida } \\
\text { (NAC) }(\%)\end{array}$} & \\
\hline & & & & C.g. & C.t. & C.p. & other & \\
\hline Turkey & $\begin{array}{l}\text { VVC in pregnant } \\
\text { women }\end{array}$ & 189 & 61,9 & 19 & 13,2 & 0,5 & 5,4 & \multirow[t]{4}{*}{$\begin{array}{l}\text { Kalkanci et } \\
\text { al., } 2012\end{array}$} \\
\hline & acute VVC & 73 & 63 & 20,5 & 12,3 & 0 & 4,2 & \\
\hline & $\begin{array}{l}\text { symptomatic } \\
\text { recurrent VVC }\end{array}$ & 89 & 64 & 15,7 & 13,5 & 0 & 6,8 & \\
\hline & $\begin{array}{l}\text { asymptomatic } \\
\text { recurrent VVC }\end{array}$ & 27 & 51,9 & 25,9 & 14,8 & 3,7 & 3,7 & \\
\hline \multirow[t]{2}{*}{ Brazil } & $\begin{array}{l}\text { vaginal mucosa } \\
\text { in AIDS patients }\end{array}$ & 18 & 55,6 & 22,2 & 0 & 16,7 & 5,6 & \multirow[t]{2}{*}{$\begin{array}{l}\text { Oliveira et } \\
\text { al., } 2011\end{array}$} \\
\hline & $\begin{array}{l}\text { vaginal mucosa } \\
\text { in non-AIDS }\end{array}$ & 7 & 85,7 & 0 & 0 & 14,3 & 0 & \\
\hline Iran & VVC & 191 & 67 & 18,3 & 6,8 & 1,6 & 6,3 & $\begin{array}{l}\text { Mahmoudi et } \\
\text { al., } 2011\end{array}$ \\
\hline Italy & VVC & 518 & 62,7 & 28,3 & 1,7 & 2,4 & 4,9 & $\begin{array}{l}\text { Asticcioli et } \\
\text { al., } 2009\end{array}$ \\
\hline USA & $\begin{array}{l}\text { patients suspec- } \\
\text { ted of VVC }\end{array}$ & 93,775 & 89 & 7,9 & 1,4 & 1,7 & 0 & $\begin{array}{l}\text { Vermitsky et } \\
\text { al., } 2008\end{array}$ \\
\hline USA & VVC & 593 & 70,8 & 18,9 & 1,4 & 5 & 3,9 & $\begin{array}{l}\text { Richter et al., } \\
2005\end{array}$ \\
\hline USA & Candiduria & 530 & 51,8 & 15,6 & 7,9 & 4,1 & 20,6 & $\begin{array}{l}\text { Kauffman et } \\
\text { al., } 2000\end{array}$ \\
\hline Spain & Candiduria & 389 & 68,4 & 8,2 & 3,6 & - & 19,8 & $\begin{array}{l}\text { Alvarez- } \\
\text { Lerma et al., } \\
2003\end{array}$ \\
\hline Brazil & Candidemia & 282 & 38 & 9 & 17 & 23 & 13 & $\begin{array}{l}\text { Colombo et } \\
\text { al., } 2007\end{array}$ \\
\hline $\begin{array}{l}\text { World- } \\
\text { wide }\end{array}$ & Candidemia & 1,239 & 50 & 17,4 & 9,8 & 17,4 & 5,4 & $\begin{array}{l}\text { Pfaller et al., } \\
2010\end{array}$ \\
\hline China & $\begin{array}{l}\text { Vagina swab } \\
\text { from healthy } \\
\text { women }\end{array}$ & 128 & 78,9 & 7,8 & - & - & 13,3 & $\begin{array}{l}\text { Ji, Zhang et } \\
\text { al., } 2009\end{array}$ \\
\hline
\end{tabular}

Abbreviation: C.g.= C. glabrata; C.t.=C. tropicalis; C.p.=C.parapsilosis 
Table 3. In vitro susceptibilities of antifungal drugs

\begin{tabular}{|l|c|c|c|c|r|r|}
\hline & $\begin{array}{c}\text { C. } \\
\text { albicans }\end{array}$ & $\begin{array}{c}\text { C. } \\
\text { glabrata }\end{array}$ & $\begin{array}{c}\text { C. } \\
\text { tropicalis }\end{array}$ & $\begin{array}{c}\text { C. } \\
\text { parapsilosis }\end{array}$ & $\begin{array}{c}\text { C. } \\
\text { krusei }\end{array}$ & other \\
\hline Fluconazole & + & $+/-$ & + & + & - & + \\
\hline Itraconazole & + & $+/-$ & + & $+/-$ & $+/$ & + \\
\hline Posaconazole & + & $+/-$ & + & + & + & + \\
\hline Voriconazole & + & $+/-$ & + & + & + & + \\
\hline Ketoconazole & + & + & + & + & + & + \\
\hline Amphotericin B & + & + & + & + & + & + \\
\hline Flucytosine & $+/-$ & + & $+/-$ & + & + & + \\
\hline Nystatin & + & + & + & + & + & + \\
\hline Echinocandinsa & + & + & + & - & + & + \\
\hline
\end{tabular}

Source: data combined from Leventakos et al., 2010 and Richter et al., 2005.

$+=$ most strains are susceptible; $-=$ most or all strains are resistant

In some pregnant women, recurrent or persistent Candida infection could occur. This can be an indication that the patient suffers from other risk factors such as iron deficiency, diabetes mellitus, or even immune deficiency. Body hygiene is the best way to avoid VVC. There are some prevention steps that could be useful:

- Always wash your vaginal area with non-perfumed soap and water, or water alone. Avoid using highly-scented soaps, shower gels, vaginal deodorants or douches.

- Do not use latex condoms, spermicidal creams and lubricants if they cause irritation. Instead, try using non-allergenic condoms.

- Avoid wearing tight-fitting clothes made of artificial fibers such as nylon.

- Prefer to use cotton underwear and loose-fitting clothes.

- Clean your genital area gently with warm water. 


\section{References}

Abbott, J. (1995). Clinical and microscopic diagnosis of vaginal yeast infection: a prospective analysis. Ann. Emerg. Med. 25:587-591.

Alvarez-Lerma, F., J. Nolla-Salas, et al. (2003). Candiduria in critically ill patients admitted to intensive care medical units. Intensive Care Med 29: 1069-1076.

Aslam, M., R. Hafeez, et al. (2008). Vulvovaginal candidiasis in pregnancy. Biomedica 24 (Jan. - Jun.): 54-56.

Asticcioli, S., L. Sacco, et al. (2009). Trends in frequency and in vitro antifungal susceptibility patterns of Candida isolates from women attending the STD outpatients clinic of a tertiary care hospital in Northern Italy during the years 2002-2007. New Microbiol 32(2): 199-204.

Bohannon NJV.(1998). Treatment of vulvovaginal candidiasis in patients with diabetes. Diabetes Care 21:451-456.

Colombo, A. L., T. Guimaraes, et al. (2007). Prospective observational study of candidemia in Sao Paulo, Brazil: incidence rate, epidemiology, and predictors of mortality. Infect Control Hosp Epidemiol 28: 570-576.

Foxman B. (1990). The epidemiology of vulvovaginal candidiasis: risk factors. Am J Public Health 80:329-331.

Foxman B, Barlow R, d'Arcy H, Gillepsie B, Sobel JD. (2000). Candida vaginitis: self-reported incidence and associated costs. Sexually Transmitted Diseases 27:230-235.

Gibb D, Hockey S, Brown L, Lunt H. (1995). Vaginal symptoms and insulin dependent diabetes mellitus. N Z Med J 108:252-253

Geiger AM, Foxman B. (1998). Risk factors for vulvovaginal candidiasis: a case control study among university students. Epidemiol 7:182-187.

Kalkanci, A., A. B. Guzel, et al. (2012). Yeast vaginitis during pregnancy: susceptibility testing of 13 antifungal drugs and boric acid and the detection of four virulence factors. Med Mycol 50:585-593.

Kauffman, C. A., J. A. Vazquez, et al. (2000). Prospective multicenter surveillance study of funguria in hospitalized patients. The National Institute for Allergy and Infectious Diseases (NIAID) Mycoses Study Group. Clin Infect Dis 30: 14-18.

Leventakos, K., R. E. Lewis, et al. (2010). Fungal infections in leukemia patients: how do we prevent and treat them? Clin Infect Dis 50:405-415. 
Mahmoudi Rad, M., S. Zafarghandi, et al. (2011). The epidemiology of Candida species associated with vulvovaginal candidiasis in an Iranian patient population. Eur J Obstet Gynecol Reprod Biol 155:199-203.

Oliveira, P. M., R. E. Mascarenhas, et al. (2011). Candida species isolated from the vaginal mucosa of HIV-infected women in Salvador, Bahia, Brazil. Braz J Infect Dis 15:239-244.

Pfaller, M. A., M. Castanheira, et al. (2010). Variation in Candida spp. distribution and antifungal resistance rates among bloodstream infection isolates by patient age: report from the SENTRY Antimicrobial Surveillance Program (20082009). Diagnosis Microbiol Infect Diseases 68:278-283.

Reed B.D. (1992). Risk factors for Candida vulvovaginitis. Obstet Gynecol Surv 47:551-560.

Reed, B. D., and C. L. Pierson. (1992). Evaluation of a latex agglutination test for the identification of Candida species in vaginal discharge. J. Am. Board Fam. Pract. 5:375-380.

Richter, S. S., R. P. Galask, et al. (2005). Antifungal susceptibilities of Candida species causing vulvovaginitis and epidemiology of recurrent cases. J Clin Microbiol 43:2155-2162.

Sobel J.D. (1993). Candidal vulvovaginitis. Clin Obstet Gynecol 36:153-65.

Spinillo A, Pizzoli G, Colonna L, Nicola S, De Seta F, Guaschino S. (1993). Epidemiologic characteristics of women with idiopathic recurrent vulvovaginal candidiasis. Obstet Gynecol 81:721-727.

Vermitsky, J. P., M. J. Self, et al. (2008). Survey of vaginal-flora Candida species isolates from women of different age groups by use of species-specific PCR detection. J Clin Microbiol 46:1501-1503.

Zhang, et al. (2009). Investigation of the situation of vaginal microflora in healthy women population. Zhonghua Fu Chan Ke Za Zhi 44:9-12. 


\section{Salmonella Typhi Infection}

Yadi, Medical Faculty, Department Microbiology, Mulawarman University, Samarinda, East Kalimantan, Indonesia.

\section{Summary}

Salmonella enterica serotype Typhi is an important intracellular pathogen which causes typhoid or enteric fever in humans. The bacterium is serologically positive for lipopolysaccharide antigens $\mathrm{O} 9$ and $\mathrm{O} 12$, protein flagellar antigen $\mathrm{Hd}$, and polysaccharide capsular antigen Vi. Typhoid fever is a global problem, with more than 27 million cases worldwide each year resulting in an estimated 217,000 deaths. Typhoid fever is most common among children, especially in endemic areas. The clinical presentation of typhoid fever varies, ranging from fever with little morbidity to toxemia and other associated complications involving many systems. Diagnosis of the disease is still inadequate in developing countries where the disease is epidemic. The treatment with appropriate antimicrobial agents is important for reducing the mortality of enteric fever. Increasing antimicrobial use fosters resistance, an obstacle that became a major public health problem. 


\section{Introduction}

Salmonella enterica serotype Typhi that caused human typhoid is a worldwide problem. Salmonella enterica serotype Typhi infects only humans. It is transmitted by ingestion of food or water contaminated with feces from an infected person. Typhoid fever is most common among children, especially in areas of Asia and Africa (WHO, 2003). This article discusses literature related to organism, epidemiology, pathogenesis, symptoms, diagnosis and treatment of the disease.

\section{The organism}

Salmonella enterica serotype Typhi (S. Typhi) is a member of the family Enterobacteriaciae (Parry et al., 2002). S. Typhi grows best under aerobic conditions, but may also grow anaerobically. For the growth of S. Typhi the temperature ranges from 4 to $40^{\circ} \mathrm{C}$; the optimum is $37^{\circ} \mathrm{C}$ (Khan, 2004). These bacteria do not require sodium chloride for growth, but can grow in the presence of 0.4 to $4 \%$. They are sensitive to heat and often killed at temperatures of $70^{\circ} \mathrm{C}$ or above and grow in a $\mathrm{pH}$ range of 4 to 9 with the optimum between 6.5 and 7.5 (Pui et al., 2011).

Salmonellae are Gram-negative bacteria (WHO, 2003; Ohl \& Miller, 2001) and when isolated from clinical specimens, colonies of S. Typhi are non-lactose fermenting and produce a characteristic biochemical pattern in Kligler iron agar: acid without gas in butt, an alkaline and a moderate amount of $\mathrm{H}_{2} \mathrm{~S}$ production in slant (Parry, 2006). S. Typhi can survive about a week in sewage-contaminated water and remains viable for 1-2 weeks in fecal materials (Khan, 2004).

The genus Salmonella is highly polymorphic, occurring in several distinct forms (Bisi-Johnson \& Obi, 2012). A schematic S. Typhi cell wall can be seen in Figure 1. The bacterium is serologically positive for lipopolysaccharide antigens O9 and $\mathrm{O} 12$, protein flagellar antigen $\mathrm{Hd}$, and polysaccharide capsular antigen $\mathrm{Vi}$ (Parry et al., 2002). The $\mathrm{O}$ antigens are lipopolysaccharides (LPS) of the outer bacterial membrane. They are heat stable, resistant to alcohol and diluted acids (Pui et al., 2011). The Vi capsular antigen is largely restricted to S. Typhi, although it is shared by some strains of $S$. enterica serotypes Hirschfeldii (Paratyphi C) and Dublin, and Citrobacter freundii (Parry, 2006).

$\mathrm{Vi}$ antigen appears to be an important determinant of virulence (Moudgil \& Narang, 1985). A unique flagella type, $\mathrm{Hj}$ is present in some $\mathrm{S}$. Typhi isolates from Indonesia (Parry, 2006; Parry et al., 2002). Recently, the other flagellin gene of S. Typhi named Ind, showed a close homology with the flagellin gene of Serratia marcescens. The Ind gene was detected in $21.8 \%$ of S. Typhi isolates from the East Indonesian archipelago, all of which contained the Hd gene (Hatta et al., 2011).

$\mathrm{H}$ antigens are heat-labile proteins associated with the peritrichous flagella and can be expressed in one of two phases. The phase $1 \mathrm{H}$ antigens are specific and associated with the immunological identitification of the particular serovars whereas phase 2 antigens are non-specific antigens containing different antigenic subunit 
proteins which can be shared by many serovars (Pui et al., 2011). Salmonella are classified into more than 50 serogroups based on the $\mathrm{O}$ (somatic) antigen, and divided into 2,400 serovars based on the $\mathrm{H}$ antigen (de Jong et al., 2012).

Substantial sequence heterogeneity exists between alleles encoding different flagellar antigens while alleles encoding the same flagellar antigen are homologous, suggesting that flagellin genes may be useful targets for the molecular determination of flagellar antigen type (Mcquiston, 2004). Complete genome sequences from multiple Salmonella strains are available (de Jong et al., 2012). The genome of S. Typhi is approximately 5 million base pairs long and codes for some 4,000 genes of which more than 200 are functionally inactive (Zhang et al., 2008; Baker \& Dougan, 2007). Differences in gene content among Salmonella enterica Serovar Typhi indicate that even within a highly clonal bacterial population the genomic reservoir is unstable (Boyd, 2003).

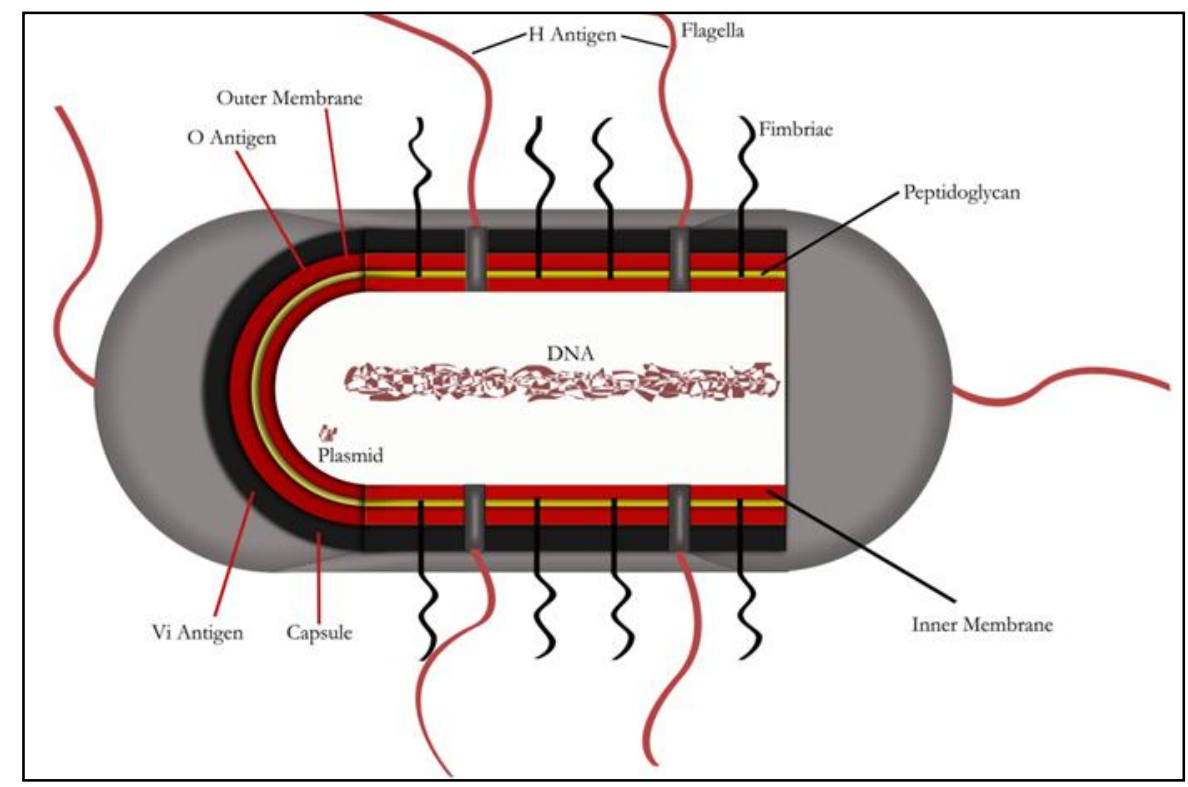

Figure 1: S.Typhi cell wall; own presentation

\section{$4 \quad$ Epidemiology}

Typhoid fever is a global health problem, with more than 27 million cases worldwide each year resulting in an estimated 217,000 deaths (de Jong et al., 2012; Crump \& Mintz, 2010). However, the true magnitude is difficult to quantify be- 
cause the clinical manifestation is confused with many other febrile illnesses and most typhoid endemic areas lack facilities to confirm the diagnosis (Parry, 2006; Crump \& Mintz, 2010; WHO, 2009).

Typhoid fever is most common among children, especially in areas of Asia and Africa (Ochiai et al., 2008) that lack clean water and adequate sanitation, and it is also an important travel-associated disease (de Jong et al., 2012). A study on fever of unknown origin in Ghana revealed that $\mathrm{S}$. Typhi remains the most prevalent bacterial species causing bacterial blood stream infections (BBSI), where 40.7\% (59/145) of BBSI were caused by S. Typhi (Groß et al., 2011). The same situation can be found in Kathmandu Valley where Salmonella infections are the leading cause of bloodstream infection among pediatric outpatients with fever (Pradhan et al., 2012). Clinical Studies in Minna (Nigeria) also revealed a high prevalence of typhoid fever (44.4\%) among children within the age group 1-10 years which is also similar with an earlier report of $43.9 \%$ prevalence rate in Cebu City, India. It is argued that children are the most vulnerable group, particularly in environments where inadequate water supply and poor environmental hygiene exists combined with a high level of ignorance (Adabara et al., 2012).

Typhoid fever is usually developed through the ingestion of contaminated food or water and thus, through carriers excreting S. Typhi by feces or urine. In addition, these bacteria can survive for prolonged periods in water, ice, dust and dried sewage and these may become sources of infection. In endemic areas, peaks of transmission occur during dry weather seasons or at the onset of rains. A multinational population-based cohort study observed that there is an increased risk for Salmonella bacteremia during the summer season, and due to increased travel abroad but also with food preparation related to outdoor cooking during that season. Several previous studies have documented increased colonization rates with Salmonella spp. in food animals during the summer months (Laupland et al., 2010).

Risk factors for disease include eating food prepared outside the home, such as ice creams or flavoured iced drinks from street vendors, drinking contaminated water and eating vegetables and salads that have been grown with human waste as fertilizer (Parry, 2006; WHO, 2009). After the tsunami in Aceh, Indonesia, clean water and drug availability were significant independent risk factors, while for the earthquake in Yogyakarta, Indonesia, contact with other typhoid patients and educational level was a significant risk. Although typhoid fever transmitted by food and water is a common problem in daily life, its characteristics and risk factors may differ in disaster-affected areas (Sutiono et al., 2010). A study on risk factors for clinical typhoid fever in South Sulawesi/Indonesia showed that limited education was highly associated with the occurence of typhoid fever. Risk of infection was significantly higher for those who had received no formal education or had attended primary school only. It has been stated that no cases occured among those who had attended secondary school (Hatta et al., 2009). This study indicates that knowledge obtained at secondary school helps to prevent communicable diseases such as typhoid fever. Education may help to improve general hygiene and avoid 
risky behaviour such as not washing hands before eating, not cooking of vegetables and not selecting good quality water for drinking. It also becomes clear that a person with a better knowledge about the transmission of disease most likely avoids contact with patients or takes precautions to prevent the infection.

With 274 cases per 100,000 persons Asia has the highest incidence of typhoid fever cases worldwide, especially in Southeast Asian countries and at the Indian subcontinent, followed by sub-Saharan Africa and Latin America with 50 cases per 100,000 persons. In an urban slum in Dhaka, the incidence of bacteremic typhoid fever was found to be 390/100,000 population, with a 9-fold higher risk for preschool children than for older persons (WHO, 2009). Recent prospective population-based disease surveillance studies at five sites in China, India, Indonesia, Pakistan and Vietnam revealed high rates of typhoid fever among children in urban slums, including children below 5 years of age. In three urban slums in Karachi, Kolkata and North Jakarta, incidence of blood-confirmed typhoid fever cases among children 5 to 15 years of age ranged from 180 cases to 494 cases per 100,000 (WHO, 2009; Ochiai et al., 2008). A study on an outbreak of typhoid fever among school children of a school in a military station in central India reported that the most susceptible group of the children were between 5 to 17 years of age (Dhadwal \& Shetty, 2008).

Typhoid fever is a common and significant cause of morbidity between 1 and 5 years of age (Sinha et al., 1999). In 2007, the Communicable Disease Centre (CDC) of Indonesia reported a prevalence of 358-810 per 100,000 population for typhoid fever with $64 \%$ occurring in 3 to 19 year olds. In South Sulawesi, the case detection rate increased from 257 per 100,000 population in 1991 to 386 per 100,000 population in 2007 (Moehario, 2009; Hatta \& Ratnawati, 2008).

Insofar as it is known only two countries, China and Vietnam, have incorporated typhoid vaccination into their routine immunization programs, and only in a limited fashion. The Dehli State in India also introduced vaccination in 2004 in 2-5 years old children through community-based campaigns. The reason why these efforts have not be more extended derives from the fact that most developing countries are uncertain of their true typhoid fever disease burden, due to the lack of rapid diagnostic tools, infrequency of laboratory testing and poor reporting systems (WHO, 2009).

Multidrug-resistant S. Typhi are still common in many areas, although in some regions fully sensitive strains have re-emerged (Parry, 2006). Prospective population-based disease-surveillance studies at five sites in China, India, Indonesia, Pakistan and Vietnam have shown a multidrug resistance (resistance to chloramphenicol, ampicillin and trimethoprim-sulfamethoxazole) in $83(65 \%)$ isolates from the site in Pakistan, $4(22 \%)$ from the site in Vietnam, and $9(7 \%)$ from the site in India. In contrast, all isolates from the sites in China and Indonesia were susceptible to all antimicrobial agents (Ochiai et al., 2008). 


\section{Pathogenesis}

S. Typhi causes typhoid fever, a fecal-oral infection leading to systemic disease (Baker et al., 2010). S. Typhi is adapted to humans and does not occur in animals (Bisi-Johnson \& Obi, 2012; Levine, 2009). This is mainly because S. Typhi are host-restricted to humans and there is no known zoonotic reservoir (Baker et al., 2010). The presence of virulence plasmids in host-adapted serovars suggests that virulence plasmid acquisition may have expanded the host range of Salmonella (Rafael \& Josep, 1999).

S. Typhi rapidly and efficiently pass through the intestinal mucosa of humans to eventually reach the reticuloendothelial system, where after a 8- to 14-days incubation period, S. Typhi precipitate a systemic illness (Levine, 2009). Susceptible human hosts ingest the causative organisms through contaminated food and water. The dissemination of salmonellosis to various organs depends on the serotype, the size of the inoculum, and the status of the host (Bisi-Johnson \& Obi, 2012). Once ingested, S. Typhi is able to survive exposure to gastric acid in the stomach before passage into the small intestine. S. Typhi have a two-stage inducible acid tolerance system that allows the bacterium to survive in conditions of severe low $\mathrm{pH}$ stress ( $\mathrm{pH} 3.3)$. The first stage involves the production of an inducible $\mathrm{pH}$ homeostasis system functional at external $\mathrm{pH}$ values below 4.0, and the second stage involves the synthesis of acid shock proteins that are essential for $\mathrm{pH} 3.3$ acid tolerance (Huang \& DuPont, 2005). After passing through the pylorus and reaching the small intestine, the bacilli rapidly penetrate the mucosa to arrive in the lamina propria. In the small intestine, S. Typhi penetrate the intestinal mucosa via $\mathrm{M}$ cells (Huang \& DuPont, 2005) and then enter the underlying lymphoid cells. Salmonella may sometimes take a paracellular route between enterocytes (Levine, 2009).

The initial early bacteremia apparently occurs within 24 to $72 \mathrm{~h}$ after ingestion of the organisms and is rarely detected in natural infections because the patients are usually asymptomatic at this early stage. This bacteremia is transient and is rapidly terminated as bacilli are phagocytosed by the macrophages and monocytes of the reticuloendothelial system. Multiplication at the intracellular site takes place and organisms re-enter the blood stream, producing continuous bacteremia for days or weeks. The reappearance of bacteremia corresponds with the onset of manifestations of the disease. This secondary bacteremia is the event which initiates the symptomatic phase of infection (Moudgil \& Narang, 1985). S. Typhi causes a systemic infection without stimulating a significant inflammatory response and transfer from the gastrointestinal lumen to the reticuloendothelial system in a relatively undetected fashion. Indeed, one may argue that the ability of S. Typhi to avoid immune detection constitutes the organism as a "stealth" pathogen and this has significant implications for diagnostics (Baker et al., 2010). S. Typhi causes intestinal necrosis which can ulcerate and result in perforation (Bisi-Johnson \& Obi, 2012). 
The pathogenesis of intestinal perforation in patients with typhoid fever is poorly understood with respect to the host and bacterial factors involved. It is generally believed that perforation occurs in the Peyer patches of the distal ileum (Chanh et al., 2004). Gross bleeding comes from eroded vessels in or near the Peyer's patches. This complication occurs late in the second week in about $1-3 \%$ of untreated patients and rarely in patients whose treatment with an appropriate antibiotic begun late in their clinical course (Levine, 2009). The endotoxin of S. Typhi was implicated in the pathogenesis of typhoid fever due to the similarities between the clinical features of typhoid fever and the clinical manifestations following an injection of bacterial endotoxin to human volunteers. Both typhoid fever and endotoxin injected into animals or human volunteers give rise to fever, headache, chills, malaise, myalgia, anorexia, abdominal discomfort, thrombocytopenia and leukopenia (Moudgil \& Narang, 1985).

The organisms that initiate relapse appear to come from the reticuloendothelial system. Typhoid bacilli can be isolated from liver biopsies during the relapse, and also from bone marrow many months after the patient has fully recovered from the symptoms. Relapses usually occur about 3 weeks after the last febrile day or about 2 weeks after cessation of antibiotics (Levine, 2009). Most relapses are milder than the initial illness, last for only a few days, and respond promptly to appropriate antibiotic therapy. Relapse after treatment with oral fluoroquinolones and azithromycin and after parenteral ceftriaxone therapy is distinctly uncommon. During the primary bacteremia that follows ingestion of typhoid bacilli and seeds the reticuloendothelial system, organisms also reach the gall bladder, an organ for which S. Typhi has a remarkable predilection (Levine, 2009). During the phase of persistent bacteremia, all organs are repeatedly exposed to S. Typhi. Bacteria occur in the gall bladder in almost all cases. Organisms multiply in the bile and are excreted with bile into the intestine. The intestinal tract may be infected with $\mathrm{S}$. Typhi either by direct bacteremic spread, as for example to Peyer's patches in the terminal ileum or through contaminated bile (Moudgil \& Narang, 1985).

S. Typhi can be readily cultured from bile or from bile-stained duodenal fluid in patients with acute typhoid fever. In approximately $2-5 \%$ of patients, the gall bladder infection becomes chronic. The propensity to become a chronic carrier is greater in females and increases with age at the time of acute S. Typhi infection, thereby resembling the epidemiology of gall bladder disease. The infection tends to become chronic in those individuals who have preexistent gall bladder pathology at the time of acute S. Typhi infection. Carriers shed as many as 109 organisms/g feces. These organisms are propelled the length of the gastrointestinal tract without penetrating and causing disease in the host (Levine, 2009). 


\section{Clinical features}

The clinical manifestation of typhoid fever is various, ranging from fever with little morbidity to marked toxemia and other associated complications involving many systems. In endemic regions, diagnosis can be missed because of non-specific features like diarrhoea and vomiting, or predominant respiratory symptoms (Bhanet al., 2005).

Many factors influence the severity and overall clinical outcome of the infection. They include the duration of illness before the initiation of appropriate therapy, the choice of antimicrobial treatment, age, the previous exposure or vaccination history, the virulence of the bacterial strain, the quantity of inoculum ingested, host factors (e.g. HLA type, AIDS or other immunosuppression) and whether the individual was taking other medications such as $\mathrm{H} 2$ blockers or antacids to diminish gastric acid (WHO, 2003).

An average case of acute non-complicated typhoid fever has an incubation period of 10-14 days and is usually associated with prolonged low-grade fever, dull frontal headache, malaise, myalgia, a dry bronchitic cough, anorexia and nausea (Bhanet al., 2005). Fever and malaise mark the onset of bacteraemia but patients are not usually present at the hospital until towards the end of the first week of symptoms (Parry C. M., 2006). High-grade fever can continue for up to 4 weeks if left untreated, followed by a return to normal temperature. Malaise and lethargy can continue for a couple of months later. Although not present consistently, relative bradycardia at the peak of high fever is an indicator of typhoid fever (Bhanet al., 2005).

A study in Vietnam reported that the median length of fever before the person was admitted to hospital and diagnostic samples were taken was 8 days (range 2 13). Headache was reported in $91.1 \%$ of the cases $(82 / 90)$, rose spots in $15.5 \%$ $(14 / 90)$ and $70 \%(63 / 90)$ had transient diarrhoea (median of three times per day [range 0-4])(Tran et al., 2005). Coated tongue, alteration of bowel habits varying from constipation in adults to diarrhoea in children, tender abdomen, hepatomegaly, and splenomegaly are often present (Bhanet al., 2005). Constipation is generally more common in adults, but in young children and adults with HIV infection diarrhoea predominates (Parry, 2006).

The complications include intestinal hemorrhage or perforation, pneumonia, myocarditis, hepatitis, acute cholecystitis, pancreatitis, pericarditis, endocarditis, orchitis, meningitis, parotitis, arthritis, osteomyelitis that are all associates with typhoid fever. Acute renal failure, early small bowel perforation and cochleovestibular impairment as rare complications of typhoid fever had been also reported (Khan, 2012). Short duration of symptoms prior to admission, leukopenia, inadequate treatment, and male gender are independent risk factors for perforation among hospitalized patients with typhoid fever (Hosoglu et al., 2004). 


\section{Diagnosis}

Typhoid (enteric) fever is still a common disease in many developing countries but current diagnostic tests are inadequate (Baker et al., 2010). In the regions where enteric fever is common, clinical diagnosis of typhoid fever is inadequate, as the symptoms it causes are nonspecific and overlap with those of other febrile illness, such as vector-borne malaria, dengue fever and rickettsioses as well as environmentally transmitted leptospirosis and melioidosis (Zhou \& Pollard, 2010; Parry., 2006). In developing countries most serotype Typhi infections are diagnosed purely on clinical grounds and treated presumptively. As a result, the diagnosis may be delayed or missed while other febrile illnesses are considered, and patients without typhoid fever may receive unnecessary and inappropriate antimicrobial therapy (Olsen et al., 2004).

A study in Jakarta, Indonesia has shown that over $80 \%$ of patients with typhoid fever or paratyphoid fever are treated in outpatient settings. They compared symptoms (with the aim to develop a clinical prediction rule that allows direct empirical antibiotic treatment to cases with suspected [para] typhoid fever) rather than all febrile patients or patients who have undergone additional diagnostic tests. Paratyphoid fever ( S. Paratyphi A) could not be distinguished clinically from typhoid fever (Vollaard et al., 2005). Therefore, clinical suspicion of typhoid fever must be confirmed by appropriate laboratory investigations.

\section{Isolation and identification of the organism}

Multiple blood cultures should be obtained from patients in whom the diagnosis is suspected clinically (Levine, 2009). The volume of blood cultured is one of the most important factors in the isolation of S. Typhi from typhoid patients: $10-15 \mathrm{ml}$ should be taken from school children and adults in order to achieve optimal isolation rates; $2-4 \mathrm{ml}$ is required from toddlers and preschool children. This is because children have higher levels of bacteraemia than adults (WHO, 2003).

Blood cultures are the standard diagnostic method and can be positive in 60$80 \%$ of cases (Parry, 2006). The current "gold standard" of bacteriological confirmation of typhoid fever is the bone marrow culture, which is positive in $85-95 \%$ of cases, even when the patient has received antibiotics (Levine, 2009). Bone marrow cultures may be attempted when blood cultures are negative for bacterial growth after three to four days of incubation (Farooqui et al., 1991).

Polymerase chain reaction (PCR) can be applied to clinical specimens to expedite a microbiological diagnosis of S. Typhi infection. Several studies have been reported since the first evaluation of PCR as a diagnostic tool for typhoid fever in 1993 when Song et al. successfully amplified the flagellin gene ( $f l i C-d)$ of S. Typhi in all cases of culture-proven typhoid fever but not in any of the healthy controls (Zhou \& Pollard, 2010). 
The multiplex PCRs proved to be $100 \%$ sensitive in detecting all of these true strains. The O serogrouping PCR was also 100\% sensitive in detecting other serovars within groups B and D (Levy et al., 2008). Nested PCR can be used as a gold standard to determine the cut-off titer of the Widal test for diagnosis of typhoid fever (Prakash et al., 2005). Another study in Makassar, Indonesia, using a nested PCR specific for S. Typhi to detect the pathogen in patients blood samples with clinical suspicion of typhoid fever showed a sensitivity of $84.5 \%$ (Hatta \& Smits, 2007). However, these methods have been amenable only to research and reference laboratories, since they are impractical for routine use in most laboratories in endemic areas that support clinical facilities.

At present, MALDI-TOF-based intact cell mass spectrometry (ICMS) advances to a widely used routine identification tool for bacteria and fungi. A recent study using blood culture isolates taken from a study population suffering from febrile illness in Ghana demonstrates that ICMS-based species identification is applicable to isolates and it is possible to discriminate clinically important subtypes rapidly, such as the serovars inside the $S$. enterica subspecies (Kuhns et al., 2012).

\section{Serological diagnostic methods}

The first typhoid diagnostic assay, the Widal test, was developed in 1896 (Baker et al., 2010). Widal and Sicard showed that the serum of patients with typhoid fever agglutinated typhoid bacilli (Levine, 2009). The Widal test, in which $\mathrm{O}$ and $\mathrm{H}$ agglutinins are demonstrated in serum, may be performed with appropriate antigens in tubes or on slides (Parry, 2006). The test has only moderate sensitivity and specificity. It can be negative in up to $30 \%$ of culture-proven cases of typhoid fever. This may be because of prior antibiotic therapy that has blunted the antibody response (WHO, 2003). Since S. Typhi is a member of the Enterobacteriaceae, many of the surface antigens are conserved and induce cross-reactive antibodies (Baker et al., 2010).

Detection of urinary Vi antigen with this ELISA shows promise for the diagnosis of typhoid fever, particularly when used within the first week after fever onset. However, positive reactions for Vi antigen in patients with brucellosis must be understood before urinary $\mathrm{Vi}$ antigen detection can be developed further as a useful rapid diagnostic test (Fadeel et al., 2004). The serological assays based on the detection of IgM antibodies against either serotype Typhi lipopolysaccharide (ELISA) or whole bacteria (dipstick) had a significantly higher sensitivity than the Widal test when used with a single acute-phase serum sample. These tests could be of use for the diagnosis of typhoid fever in patients who have clinical typhoid fever but are culture negative or in regions where bacterial culturing facilities are not available (House et al., 2001).

Recently, a variety of rapid antibody tests have shown promise and their value in routine practice is undergoing evaluation such as IDL Tubex ${ }^{\circledR}$ test, Typhidot ${ }^{\circledR}$ test, IgM dipstick test, latex agglutination test, immunochromatographic lateral 
flow assay, dot enzyme immunoassay (Dutta et al.,2006; Hatta et al., 2002; Abdoel et al., 2007; House et al., 2001; Rahman et al., 2007; Olsen et al., 2004).

The advantages of the dipstick assay are that the result can be obtained on the same day allowing a prompt treatment, that only a small volume of serum is needed, and that no special laboratory equipment is needed to perform the assay. The stability of the reagents of the dipstick and the simplicity of the assay allows its use in places that lack laboratory facilities (Hatta et al., 2002).

A latex agglutination assay for the serodiagnosis of typhoid fever was evaluated on the basis of samples collected from patients with clinical suspicion of typhoid fever in South Sulawesi, Indonesia, where the disease is endemic. The latex assay is very easy to use, gives a rapid result and may be used as a point-of-care diagnostic test (Abdoel et al., 2007).

\section{Treatment}

Early diagnosis of typhoid fever and prompt institution of appropriate antibiotic treatment are essential for optimal management, especially in children (Bhutta, 2006). More than $90 \%$ of patients can be managed at home with oral antibiotics, reliable care and close medical follow-up for complications or failure to respond to therapy (WHO, 2003; Bhan et al., 2005). Although most cases can be managed at home with oral antibiotics and regular follow-up, patients with severe illness, persistent vomiting, severe diarrhoea, and abdominal distension require hospitalization and parenteral antibiotic treatment (Bhutta, 2006; WHO, 2003; Bhan et al., 2005). Antimicrobial resistance is a major public health problem and timely treatment with appropriate antimicrobial agents is important for reducing the mortality of enteric fever (Parikh, 2012). Appropriate antibiotic treatment (the right drug, dose, and duration) is critical to curing typhoid fever with minimal complications (Bhutta, 2006).

Chloramphenicol was the drug of choice for several decades after its introduction in 1948. However, it was set aside due to the emergence of plasmid-mediated resistance (Parikh, 2012). Chloramphenicol, amoxicillin or co-trimoxazole are appropriate for the treatment of typhoid fever in areas of the world where bacteria are still susceptible to these drugs. These antibiotics are inexpensive, widely available and rarely associated with side effects. They produce relief of symptoms with defervescence usually within 5 to 7 days. These antibiotics produce cure rates of $95 \%$, relapse rates of $1-7 \%$ and convalescent carriage rates of $2-10 \%$ (Parry, 2006). Trimethoprim-sulfamethoxazole (TMP-SMZ) can be used orally or i.v. in adults at a dose of $160 \mathrm{mg}$ TMP plus $800 \mathrm{mg}$ SMZ twice daily or in children at 4 $\mathrm{mg}$ TMP per $\mathrm{kg}$ and $20 \mathrm{mg} \mathrm{SMZ}$ per $\mathrm{kg}$ for 14 days (WHO, 2003). In developing countries such as Ghana an extraordinarily high percentage of chloramphenicol resistance was obvious, but this drug was still a first choice treatment for typhoid fever for some years (Groß et al., 2011). Efficacy, availability and costs are im- 
portant criteria for the selection of first-line antibiotics to be used in developing countries (WHO, 2003).

Fluoroquinolones (ciprofloxacin, ofloxacin, and pefloxacin) are the most effective drugs for treatment of typhoid fever caused by isolates that are not quinolone resistant, with a clinical cure rate of about $98 \%$, fever clearance time of about 4 days, relapse and faecal carriage rates of less than $\%$ (Bhan et al., 2005). Fluoroquinolones are equally effective for treatment of typhoid fever in children and adults. However, these drugs are not registered for routine use in children because of evidence of articular damage in growing, weight-bearing joints in young beagles (Bhan et al., 2005).

Ampicillin and amoxicillin are used at 50 to $100 \mathrm{mg}$ per $\mathrm{kg}$ per day orally, i.m. or i.v., divided into three or four doses. No benefit has been reported to result from the addition of clavulanic acid to amoxicillin (WHO, 2003). In areas where multi-drug resistant (MDR) isolates are common many physicians start with a fluoroquinolone, usually ciprofloxacin or ofloxacin. Treatment can be completed in a week or less with minimal toxicity. Some authors also suggest fluoroquinolones should be used in areas where strains sensitive to chloramphenicol are still common, because of greater efficacy and likely compliance, but others disagree with this approach (Parry, 2006).

Of the third-generation cephalosporins, oral cefixime (15-20 mg per kg per day for adults, 100-200 mg twice daily) has been widely used in children in a variety of geographical settings and found to be satisfactory. However, a trial of cefixime in MDR S. Typhi in Vietnam indicated significantly higher treatment failure rates than with ofloxacin (WHO, 2003). Other agents, e.g. cefodoxime have been proved to be successful against typhoid fever. The cost and route of administration make ceftriaxone less suitable for patient treatment in some low- and middleincome countries, and the oral third-generation cephalosporin cefixime appears to be inferior to other oral agents both in terms of fever clearance time and treatment failure (Crump \& Mintz, 2010).

Since the rising rate of quinolone resistance there is a clear need to identify improved strategies for treating MDR typhoid in childhood. Recent data on the use of azithromycin in children indicate that it may be safe to give it as an alternative agent for the treatment of uncomplicated typhoid fever (WHO, 2003). Azithromycin in a dose of $500 \mathrm{mg}(10 \mathrm{mg} / \mathrm{kg})$ given once daily has been proved effective within the treatment of typhoid fever in adults and children (WHO, 2003). Azithromycin has given 95\% cure rates following 5 to 7 day treatment courses. Defervescence occurs in 4 to 6 days with relapse and convalescent fecal carriage rates of less than 3\% (Parry, 2006). 


\section{References}

Abdoel, T. H., Pastoo, R., Smits, H. L., \& Hatta, M. (2007). Laboratory evaluation of a simple and rapid latex agglutination assay for the serodiagnosis of typhoid fever. Transactions of the Royal Society of Tropical Medicine and Hygiene, 101, 1032-38

Adabara, N. U., Ezugwu, B. U., Momojimoh, A., Madzu, A., Hashiimu, Z., \& Damisa, D. (2012). Clinical study: The prevalence and antibiotic susceptibility pattern of Salmonella Typhi among patients attending a military hospital in Minna, Nigeria. Advances in preventive medicine, Volume 2012.

Baker, S., \& Dougan, G. (2007). The genome of Salmonella enterica serovar Typhi. Clinical Infectious Diseases, 45, S29-33.

Baker, S., Favorov, M., \& Dougan, G. (2010). Searching for the elusive typhoid diagnostic. BioMed Central Infectious Diseases, 10:45.

Bhan, M. K., Bahl, R., \& Bhatnagar, S. (2005). Typhoid and paratyphoid fever. Lancet, 366, 749-62.

Bhutta, Z. A. (2006). Current concepts in the diagnosis and treatment of typhoid fever. British Medical Journal, 333, 78-82.

Bisi-Johnson, M. A., \& Obi, C. L. (2012). Escherichia coli and Salmonella species: molecular landscape and therapeutic considerations: a review. Journal of Medical Science Advances, 1(1), 1-16.

Chanh, N. Q., Everest, P., Khoa, T. T., House, D., Murch, S., Parry, C., et al. (2004). A clinical, microbiological, and pathological study of intestinal perforation associated with typhoid fever. Clinical Infectious Diseases, 39:617.

Crump, J. A., \& Mintz, E. D. (2010). Global trends in typhoid and paratyphoid fever. Clinical Infectious Diseases, 50, 241-6.

de Jong, H. K., Parry, C. M., van der Poll, T., \& Wiersinga, W. J. (2012). Hostpathogen interaction in invasive salmonellosis. Public Library of Science Pathogen, 8(10), e1002933.

Dhadwal, B. S. \& Shetty, R. A. (2008). Epidemiological investigation of a typhoid outbreak. Medical Journal Armed Forces India, 64(3), 241-242.

Dutta, S., Sur, D., Manna, B., Sen, B., Deb, A. K., Deen, J. L., et al. (2006). Evaluation of new-generation serologic tests for the diagnosis of typhoid fever: data from a community-based surveillance in Calcutta, India. Diagnostic Microbiology and Infectious Disease, 56, 359-365. 
E. F. Boyd, S. P., F. Blackmer., \& M. McClelland (2003). Differences in gene content among Salmonella enterica serovar Typhi isolates. Journal Of Clinical Microbiology, 41(8), 3823-3828.

Fadeel, M. A., Crump, J. A., Mahoney, F. J., Nakhla, I. A., Mansour, A. M., Reyad, B., Et Al. (2004). Rapid diagnosis of typhoid fever by enzyme-linked immunosorbent assay detection of salmonella serotype typhi antigens in urine. American Journal Tropical Medicine and Hygiene, 70(3), 323-328.

Farooqui, B. J., Khurshid, M., Ashfaq, M. K., \& Khan, M. A. (1991). Comparative yield of Salmonella Typhi from blood and bone marrow cultures in patients with fever of unknown origin. Journal of Clinical Patholology, 44(3), 258-259.

Groß, U., Amuzu, S. K., Ciman, R. d., Kassimova, I., Groß, L., Rabsch, W., et al. (2011). Bacteremia and antimicrobial drug resistance over time, Ghana. Emerging Infectious Diseases, 17(10), 1879-82.

Hatta, M., Bakker, M., Van Beers, S., Abdoel, T. H., \& Smits, H. L. (2009). Risk factor for clinical typhoid fever in villages in rural south Sulawesi Indonesia. International Journal of Tropical Medicine , 4(3), 91-99.

Hatta, M., \& Ratnawati. (2008). Enteric fever in endemic areas of Indonesia: an increasing problem of resistance. The Journal of Infection in Developing Countries, 2(4), 279-282.

Hatta, M., \& Smits, H. L. (2007). Detection of Salmonella Typhi by nested polymerase chain reaction in blood, urine, and stool samples. American Journal of Tropical Medicine and Hygiene, 76(1), 139-143.

Hatta, M., Goris, M. G., Heerkens, E., Gooskens, J., \& Smits, H. L. (2002). Simple dipstick assay for the detection of Salmonella Typhi-specific IgM antibodies and the evolution of the immune response in patients with typhoid fever. American Journal of Tropical Medicine and Hygiene, 66(4), 416-421.

Hatta, M., Sultan, A. R., Pastoor, R., \& Smits, H. L. (2011). New flagellin gene for Salmonella enterica serovar Typhi from the East Indonesian archipelago. American Journal of Tropical Medicine and Hygiene, 84(3), 429-34..

Hosoglu, S., Aldemir, M., Akalin, S., Geyik, M. F., Tacyildiz, I. H., \& Loeb, M. (2004). Risk factors for enteric perforation in patients with typhoid fever. American Journal of Epidemiology, 160(1), 46-50.

House, D., Wain, J., Ho, V. A., Diep, T. S., Chinh, N. T., Bay, P. V., et al. (2001). Serology of typhoid fever in an area of endemicity and its relevance to diagnosis. Journal of Clinical Microbiology, 39(3), 1002-1007.

Huang, D. B., \& DuPont, H. L. (2005). Problem pathogens: extra-intestinal complications of Salmonella enterica serotype Typhi infection. Lancet Infectious Diseases, 5(6), 341-48. 
Khan, M. E. (2004). Typhoid fever in a south african in-patient population. Groningen.

Kuhns, M., Zautner, A. E., Rabsch, W., Zimmermann, O., Weig, M., Bader, O., et al. (2012). Rapid discrimination of Salmonella enterica serovar Typhi from other serovars by MALDI-TOF mass spectrometry. Public Library of Science One, 7(6), e40004.

Laupland, K. B., Schonheyder, H. C., Kennedy, K. J., Lyytikainen, O., Valiquette, L., Galbraith, J., et al. (2010). Salmonella enterica bacteraemia: A multi-national population-based cohort study. BMC Infectious Diseases, 10:95.

Levine, M. M. (2009). Typhoid fever. In P. S. Brachman, \& E. Abrutyn, Bacterial infections of humans epidemiology and control. Pennsylvania: Springer Science+Business Media, LLC. 913-937

Levy, H., Diallo, S., Tennant, S. M., Livio, S., Sow, S. O., Tapia, M., et al. (2008). PCR method to identify Salmonella enterica serovars Typhi, Paratyphi A, and Paratyphi B among salmonella isolates from the blood of patients with clinical enteric fever. Journal of Clinical Microbiology, 46(5), 1861-1866.

McQuiston, E.A. (2004). Sequencing and comparative analysis of flagellin genes fliC, fljB, and flpA from salmonella. Journal of Clinical Microbiology, 42(5), 1923-1932.

Moehario, L. H. (2009). The molecular epidemiology of Salmonella Typhi across Indonesia reveals bacterial migration. The Journal of Infectin in Developing Countries, 3(8), 579-584.

Moudgil, K., \& Narang, B. (1985). Pathogenesis of typhoid fever. Indian Journal of Pediatrics, 52, 371-378.

Ochiai, R. L., Acosta, C. J., Danovaro-Holliday, M. C., Baiqing, D., Bhattacharya, S. K., Agtini, M. D. et al. (2008). A study of typhoid fever in five Asian countries: disease burden and implications for controls. Bulletin of the World Health Organization, 86 , 260-268.

Ohl, M. E., \& Miller, S. I. (2001). Salmonella: A model for bacterial pathogenesis. Annual Review of Medicine, 52, 259-74.

Olsen, S. J., Pruckler, J., Bibb, W., Thanh, N. T., Trinh, T. M., Minh, N. T., et al. (2004). Evaluation of rapid diagnostic tests for typhoid fever. Journal of Clinical Microbiology, 42(5), 1885-1889.

Parikh, F. (2012). Management of enteric fever in 2012. Medicine Update 2012, Vol. 22.

Parry, C. M., Hien, T. T., Dougan, G., White, N. J., Farrarr J. J. (2002). Typhoid Fever. The New England Journal of Medicine, 347(22), 1770-82. 
Parry, C. M. (2006). Epidemiological and clinical aspects of human. In: P. Mastroeni, \& D. Maskell, salmonella infections clinical, immunological and molecular aspects (hal. 1-17). Cambridge: Cambridge University Press.

Pradhan, R., Shrestha, U., Gautam, S. C., Thorson, S., Shrestha, K., Yadav, B. K., et al. (2012). Bloodstream infection among children presenting to a general hospital outpatient clinic in urban Nepal. Public Library of Science One, 7(10), e47531.

Prakash, P., Mishra, O. P., Singh, A. K., Gulati, A. K., \& Nath, G. (2005). Evaluation of nested PCR in diagnosis of typhoid fever. Journal of Clinical Microbiology, 43(1), 431-432.

Pui, C. F., Wong, W. C., Chai, L. C., Tunung, R., Jeyaletchumi, P., Noor, H. M., et al. (2011). Salmonella: a foodborne pathogen. International Food Research Journal, 18, 465-473.

Rafael, R., \& Josep, C. (1999). The virulence plasmids of salmonella. International Microbioly, II, 177-184.

Rahman, M., Siddique, A. K., Tam, F. C.-H., Sharmin, S., Rashid, H., Iqbal, A., et al. (2007). Rapid detection of early typhoid fever in endemic community children by the TUBEX O9-antibody test. Diagnostic Microbiology and Infectious Disease, 58(3), 275-81.

Sinha, A., Sazawal, S., Kumar, R., Sood, S., Reddaiah, V. P., Singh, B., et al. (1999). Typhoid fever in children aged less than 5 years. Lancet, 354, 734-37.

Sutiono, A. B., Qiantori, A., Suwa, H., \& Ohta, T. (2010). Characteristics and risk factors for typhoid fever after the tsunami, earthquake and under normal conditions in Indonesia. BioMed Central Research Notes, Vol 3, 106.

Tran, H., Bjune, G., Nguyen, B., Rottingen, J., Grais, R., \& Guerin, P. (2005). Risk factors associated with typhoid fever in Son La province, northern Vietnam. Transactions of the Royal Society of Tropical Medicine and Hygiene, 99(11), 819-826.

Vollaard, A. M., Ali, S., Widjaja, S., van Asten, H. A., Visser, L. G., Surjadi, C., et al. (2005). Identification of typhoid fever and paratyphoid fever cases at presentation in outpatient clinics in Jakarta, Indonesia. Transactions of the Royal Society of Tropical Medicine and Hygiene, 99(6), 440—450.

WHO. (2003). Background document: The diagnosis, treatment and prevention of typhoid fever. Geneva: World Health Organization.

WHO. (2009). who. int. [Accessed: November 20, 2012].

http://www.who.int/vaccine_research/diseases/diarrhoeal/en/index $7 . h t m l$ 
Zhang, X.-L., Jeza, V. T., \& Pan, Q. (2008). Salmonella Typhi: from a human pathogen to a vaccine vector. Cellular \& Molecular Immunology, 5(2), 91-97.

Zhou, L., \& Pollard. (2010). A fast and highly sensitive blood culture PCR method for clinical detection of Salmonella enterica serovar Typhi. Annals of Clinical Microbiology and Antimicrobials, 9, 14. 



\title{
26. Epidemiology and Characterization of Rotavirus Strains Causing Diarrhoea in Indonesia
}

\author{
Hera Nirwati, Department of Microbiology, Faculty of Medicine, Universitas Gadjah \\ Mada, Yogyakarta, Indonesia \\ Yati Soenarto, Department of Paediatrics, Faculty of Medicine, Universitas Gadjah \\ Mada/Sardjito Hospital, Yogyakarta, Indonesia
}

\section{Summary}

Rotaviruses are the major cause of severe diarrhoea among children in developed and developing countries. Various efforts to improve sanitation and hygiene did not decrease the incidence of rotavirus diarrhoea. Therefore, the best strategy is prevention through using rotavirus vaccine. In order to provide maximum protection, the vaccine given should provide immunity against all strains circulating in the population. Surveillance to determine the strain circulations have been conducted in both developed and developing countries. Even though there are variations, the global circulations of rotavirus strains are dominated by G1P[8], G2P[4], G3P [8], G4P [8] and G9P[8]. This article presents some research findings on rotavirus strain circulations in Indonesia. Some of them are similar to the global strains, but some of them are uncommon. As a consequence, before administrating vaccina- 
tion by using available rotavirus vaccines, a study needs to be conducted to ensure that the available vaccines can prevent rotavirus infection in Indonesia.

\section{Introduction}

Rotaviruses cause severe diarrhoea in children under 5 years old in developed and developing countries. Studies published between 1986 and 1999 showed that rotaviruses cause $22 \%$ of childhood diarrhoea hospitalization. This proposition increased to 39\% from 2000 to 2004. Approximately 611,000 children under 5 years old die every year from rotaviruses, mostly in developing countries (Parashar et al., 2006).

Rotaviruses are members of the Rotavirus genus and Reoviridae family. The particle of a rotavirus is 1,000 A in size and has a capsid that surrounds the doublestranded RNA genome consisting of 11 segments. Rotavirus genome segments encode six structural proteins (VP1-4, VP6, VP7) which form the virus' particle and six non-structural proteins (NSP1-6) which have function in the replication process and influence the pathogenesis or the immune response to infection (Greenberg \& Estes, 2009).

A rotavirus has a capsid that is composed of three concentric layers of proteins. The innermost layer is composed of VP2 protein, the middle layer is composed of VP6 protein and the outer layer is composed of VP7 glycoprotein and VP4 forms spikes that protrude from the surface of outer layer. VP4 and VP7 are essential proteins that elicit immune responses and they are used as the basis for a binary classification system. Typing based on VP7 protein (a glycoprotein = G type antigen) is known as the G-typing while based on VP4 protein (proteasesensitive protein $=$ P-type antigen) is known as the P-typing. Until now, $19 \mathrm{G}$ types and $28[\mathrm{P}]$ types are known (Greenberg \& Estes, 2009).

Rotaviruses are generally transmitted through the faecal-oral route. Feces of an infected person may contain up to $10^{10}$ rotavirus particles per gram. The huge amount of rotaviruses contained in the stool is probably the reason why the improvement of hygiene and sanitation is not sufficient enough to decrease the incidence of diarrhoea caused by these viruses. Moreover, rotaviruses can be transmitted through the respiratory tract and through the contact with various objects (toys, hands, surfaces). Rotaviruses are also known as one of the causes of nosocomial infections in hospitals. A study in the United States reported that $20 \%$ of patients were hospitalized because of rotavirus nosocomial infection. Meanwhile, another study estimated that out of every 4 patients treated at the hospital, one of them suffers from this infection (Kapikian \& Chanock, 1996; Greenberg \& Estes, 2009).

Rotavirus infections mostly affect children between 6 months and 2 years old. The risk of rotavirus infection increases among groups of low socioeconomic conditions, malnutrition-effected people, infants with low birth weight and preterm 
birth, persons suffering from compromised immunity and co-infection with other enteric pathogens (Grimwood \& Lambert, 2009).

\section{Global situation of rotavirus diarrhoea}

Although the incidence of diarrhoea in developed and developing countries are almost the same, developing countries have a $50 \%$ higher mortality rate. This is mainly due to the longer time period infected people have to wait for medical support and hydration therapy. The top ten countries which have the highest mortality rate for rotavirus infections are (1) India (23.2\%), (2) Nigeria (9.5\%), (3) Congo (5.8\%), (4) Ethiopia (5.2\%), (5) China (5.2\%), (6) Pakistan (3.8\%), (7) Afghanistan (3.4\%), (8) Bangladesh (2.9\%), (9) Indonesia (2.5\%) and Angola (2.1\%) (Parashar et al, 2009).

Epidemiological studies that were generally hospital-based, showed that for the last 20 years rotavirus strains which causing severe diarrhoea in children were dominated by (G1P[8], G3P[8], G4P[8], G2P[4], and G9P[8]).

G1P[8] strains consistently appeared in most places and reached $65 \%$ in developed regions such as the United States, the United Kingdom, and Australia. However, in less developed regions such as South America and Africa they reached only $25 \%-50 \%$. In addition to strains that are common, several uncommon strains have been found in specific areas such as G5P[8] in Brazil, G8P[6] in Africa, G10P[11] and G12P[6] in India (Grimwood \& Lambert, 2009).

\section{Epidemiology of rotaviruses causing diarrhoea in children under 5 years old in Indonesia}

Diarrhoea which is caused by rotaviruses does not have a specific clinical manifestation and thus, the clinical manifestations alone cannot be used for diagnosis. Diagnosis requires detection of the existence of the virus particles, the antigen, or antibodies specific to rotaviruses. At first, the diagnosis of rotaviruses is established by using an electron microscope. This technique is not widely used because it requires expensive equipment and expert examiner. The techniques currently used are based on the detection of rotavirus antigen in faeces by using enzymelinked immunosorbent assay (ELISA) (Wall et al., 1982).

Rotavirus infection in Indonesia was first reported by Soenarto et al. (1981) who detected rotaviruses by using electron microscope. Of 334 samples of infants and children with diarrhoea in Yogyakarta, 126 of them (38\%) were rotavirus positive.

In Indonesia, several researchers who used enzyme immunoassay technique for rotavirus detection reported various levels of incidence. Subekti et al. (2002) who conducted a study in Jakarta from 1997 to 1999 found that 170 out of 236 
children with diarrhoea $(72 \%)$ were infected by rotaviruses. Putnam et al. (2007) who conducted a study in Medan, Makassar, Jakarta, Yogyakarta, Surabaya, Bali and Nusa Tenggara found that 748 out of 1,660 children under five years old $(45.1 \%)$ suffered from diarrhoea due to rotaviruses. Moreover, Soenarto et al. (2009) who conducted a study with 2,240 children under 5 years old in Yogyakarta, Jakarta, Bandung, Mataram, Denpasar and Palembang found that $60 \%$ of them suffered from diarrhoea due to rotaviruses. In addition, Nirwati et al. (2009) who conducted a study in Yogyakarta found that $54.81 \%$ of children under 5 years old had diarrhoea caused by rotaviruses. Lastly, Widowati et al. (2012) reported that $32.68 \%$ of children under 5 years old in Yogyakarta and 64\% in Palembang got diarrhoea because of rotaviruses.

Rotaviruses usually produce sporadic episodes of diarrhoea. The incubation period ranged between 1 to 3 days and the duration of illness is generally 5 to 7 days. In general, children with rotavirus gastroenteritis present fever and vomiting early in the illness. Low-grade fever is seen in approximately $30 \%$ to $50 \%$ of children. Vomiting is a prominent clinical feature. Vomiting is almost 4 times more common and is prolonged among children with rotavirus gastroenteritis compared with gastroenteritis due to other infectious agents (Leung et al., 2005).

Soenarto et al. (2009) compared the clinical symptoms of children under 5 years old who suffered from diarrhoea and were either rotavirus positive and or negative in Yogyakarta, Jakarta, Bandung, Mataram, Denpasar and Palembang in 2006 and found that rotavirus-positive patients had a 2.3 times higher risk of dehydration than rotavirus-negative patients. In addition, those infected with rotaviruses were proven to vomit 3 times more than persons without rotavirus infection (Table 1).

With $72 \%$, rotaviruses mostly affect 6-23 month old children (Table 1, Soenarto et al., 2009). On the other hand, rotaviruses rarely infect children younger than 6 months of age. This is likely due to protective maternal antibodies which are mediated through diaplacental transfer and breast feeding. Research conducted by Chan et al. (2011) in Yogyakarta proved that most pregnant women already have antibodies against rotaviruses and pass antibodies through the placenta, colostrum and breast milk.

Meanwhile, several studies conducted in Asian countries showed varying results in rotavirus-positive diarrhoea. In Pakistan, Qazi et al. (2009) found that 17\% of 575 children with diarrhoea were rotavirus positive. In Taiwan, Wu et al. (2009) reported that $25 \%$ of the 3,435 patients with diarrhoea were rotavirus positive. Zaman et al. (2009) reported that rotaviruses were detected in 33\% of the 4,519 children with diarrhoea in Bangladesh from the year 2000 to 2006. Li et al. (2009) reported that in China $52 \%$ of 2,328 children investigated in 2007 proved to be rotavirus positive. Tamura et al. (2010) reported that $47.5 \%$ of 183 children with diarrhoea were infected by rotaviruses in Vietnam. Nyambat et al. (2009) reported that $56 \%$ of the 2,281 examined children suffered from rotavirus-positive diar- 
rhoea in Cambodia. Meanwhile, Moe et al. (2009) reported that in Miyanmar 57\% of 1,004 children have been tested positive for rotavirus.

Countries with tropical climates usually show seasonal variation in the incidence of rotavirus infections compared to countries with a temperate climate. In Indonesia, such infections occur throughout the year with an increase in the rainy season, as reported by Ofoyo et al. (2002), Subekti et al. (2002), and Soenarto et al. (2009). Similar patterns have been reported in many countries in Asia such as in Pakistan, Bangladesh, China, Taiwan, Vietnam,Cambodia, and Myanmar (Qazi et al., 2009; Zaman et al., 2009, Li et al., 2009; Wu et al., 2009; Tamura et al., 2010; Nyambat et al., 2009; Moe et al., 2009).

Table 1: Characteristics and clinical symptoms of hospitalized children aged $<5$ years with rotavirus diarrhoea in Indonesia, from 1 January to 31 December 2006 (Source: Soenarto et al., 2009).

\begin{tabular}{|c|c|c|c|c|}
\hline Characteristic & $\begin{array}{c}\text { No. }(\%) \text { of patients } \\
\text { enrolled } \\
(n=2240)\end{array}$ & $\begin{array}{l}\text { No. }(\%) \text { of patients } \\
\text { with rotavirus-positive } \\
\text { diarrhea } \\
(n=1345)\end{array}$ & $\begin{array}{l}\text { No. }(\%) \text { of patients } \\
\text { with rotavirus-negative } \\
\text { diarrhea } \\
(n=895)\end{array}$ & Odds ratio $(95 \% \mathrm{Cl})$ \\
\hline \multicolumn{5}{|l|}{ Sex } \\
\hline Male & $1348(60)$ & $827(61)$ & $521(58)$ & $1.1(1.0-1.4)$ \\
\hline Female & $892(40)$ & $518(39)$ & $374(42)$ & \\
\hline \multicolumn{5}{|l|}{ Age, months } \\
\hline $0-2$ & $139(6)$ & $44(3)$ & $95(11)$ & $0.7(0.3-1.5)$ \\
\hline $3-5$ & $313(14)$ & $168(12)$ & $145(16)$ & $1.8(0.9-3.6)$ \\
\hline $6-11$ & 765 (34) & $490(36)$ & $275(31)$ & $2.7(1.4-5.4)$ \\
\hline $12-23$ & $718(32)$ & $483(36)$ & $235(26)$ & $3.1(1.6-6.2)$ \\
\hline $24-35$ & $193(9)$ & $115(9)$ & $78(9)$ & $2.3(1.0-4.7)$ \\
\hline $36-47$ & $69(3)$ & $28(2)$ & $41(7)$ & $1.0(0.5-2.5)$ \\
\hline $48-59$ & $43(2)$ & $17(1)$ & $26(3)$ & 1.0 (reference) \\
\hline \multicolumn{5}{|l|}{ Clinical symptom } \\
\hline Dehydration & $1953(87)$ & $1223(91)$ & $730(82)$ & $2.3(1.8-2.9)^{\mathrm{a}}$ \\
\hline Vomiting & $1750(78)$ & $1152(86)$ & $598(67)$ & $3.0(2.4-3.7)^{a}$ \\
\hline Stool mucous & $658(29)$ & $377(28)$ & $281(31)$ & $0.9(0.7-1.0)$ \\
\hline Bloody stool & $78(3)$ & $24(2)$ & $54(6)$ & $0.3(0.2-0.5)^{\mathrm{a}}$ \\
\hline Fever & 986 (44) & $591(44)$ & $395(44)$ & $1.0(0.8-1.2)$ \\
\hline
\end{tabular}

NOTE. $\mathrm{Cl}$, confidence interval.

a Statistically significant $(P<.05)$.

\section{Characterization of rotavirus strains causing diarrhoea in children under 5 years old in Indonesia}

Various efforts to improve hygiene and sanitation have been done but did not significantly reduce the morbidity rate of rotavirus infection (Greenberg \& Estes, 
2009). Thus, the prevention with a vaccine is the most effective long-term strategy. The plan to develop rotavirus vaccine is currently based on oral administration of live-attenuated viruses where the protection is expected to be similar to the natural rotavirus infection. This encourages the surveillance in several countries to recognize rotavirus strains which are circulating and it helps to monitor their changes. In the future implementation of rotavirus vaccine programmes, strain surveillance also plays a crucial role in detecting antibody-escape mutants as well as changes in the local distribution of rotavirus strains that co-circulate in humans. In addition, it could also cover the monitoring of the circulation of vaccine strains and potential reassortment between the vaccine and co-circulating wild-type rotaviruses (Kirkwood, 2010).

In Indonesia, rotavirus strains which cause diarrhoea were firstly reported by Bishop et al. (1989) who performed the characterization by using enzyme immunoassay (EIA). After that, other researchers used reverse transcriptase polymerase chain reaction (RT-PCR) to detect the circulating strains. Several circulating strains are listed in table 2 .

Table 2 shows that various rotavirus strains circule in Indonesia. In addition to common strains found globally, such as G1P[8], G2P[4], G3P[8], G4P[8] and G9P[8], uncommon rotavirus strains such as G1P[4], G1P[6], G1P[10], G2P[6], G2P[8], G2P[10], G3P[4], G4P[8] G9P[4] and G9P[6] were also found. These findings are in line with data from Gentsch et al (2005) who showed that the rotavirus strains circulating in developing countries are more diverse than in developed countries. As a consequence, there are greater possibilities that children in developing countries are infected with more than one type of strain.

Some studies in Asian countries reported a variation in rotavirus strains' circulation. Qazi et al. (2009) reported that the most common rotavirus strains in Pakistan were G9P[8] (15\%), G1P[8] (13\%) and G1P[4] (8.4\%). Zaman et al. (2009) who conducted a surveillance study in Bangladesh found that the predominant type were G2 (41\%), G1 (22\%) and G9 (22\%). Li et al. (2009) reported that in China G3P $[8](42 \%)$ was the most common genotype detected. Wu et al. (2009) who conducted a study in Taiwan found that the most prevalent rotavirus genotypes were G1P[8] (40\%), G3P[8] (27\%) and G9P[8] (17\%). In Vietnam, Tamura et al. (2010) found that G3P [8] was the most frequent genotype (38\%), followed by G1P[8] (20.7\%). Nyambat et al. (2009) found that in Cambodia G1P[8] strain was the most common genotype (53\%), followed by G2P [4] (10\%). Moe et al. (2009) reported that in Myanmar, the most common combination were G3P [8] (27\%) and G1P [8] (19\%) strains with some unusual combination of G1P[4] (19\%), G1P[6] and G3P[4].

Several studies have attempted to associate the type of infecting rotavirus strain with the clinical severity of the disease. Widowati et al. (2012) found that G1 and G9 strains cause more severe dehydration than other types of strains do. In contrast, Casio et al. (2001) found that G2 were associated with greater clinical severity whereas Uhno \& Svensson (1986) found that G2 as well as G1 and G4 
strains were associated with greater clinical severity. Meanwhile, Barnes et al. (1992) and Polanco et al. (2003) found that there was no correlation between the degree of severity of disease and the rotavirus strain.

Table 2: The identification of rotavirus strains that cause diarrhoea in Indonesia

\begin{tabular}{|c|c|c|c|c|c|c|}
\hline $\begin{array}{l}\mathbf{N} \\
\mathbf{O}\end{array}$ & Location & $\begin{array}{l}\text { Tech- } \\
\text { nique }\end{array}$ & $\begin{array}{l}\text { G type } \\
(\%)\end{array}$ & $\begin{array}{l}\text { P type } \\
(\%)\end{array}$ & $\begin{array}{l}\text { G\& P type } \\
(\%)\end{array}$ & References \\
\hline 1 & Yogyakarta & EIA & $\begin{array}{l}\text { G1 (1.6) } \\
\text { G2 (8.47) } \\
\text { G3 } \\
(52.54) \\
\text { G4 } \\
(35.59) \\
\text { mix in- } \\
\text { fextion } \\
(1.69)\end{array}$ & & & $\begin{array}{l}\text { Bishop et al } \\
(1989)\end{array}$ \\
\hline 2 & $\begin{array}{l}\text { Medan } \\
\text { Makassar } \\
\text { Jakarta } \\
\text { Yogyakarta } \\
\text { Surabaya } \\
\text { Bali } \\
\text { Nusa- } \\
\text { Tenggara }\end{array}$ & RT-PCR & $\begin{array}{l}\text { G1 (11.2) } \\
\text { G2 (17.4) } \\
\text { G3 (1.6) } \\
\text { G4 (2.3) } \\
\text { G8 (0.1) } \\
\text { G9 (9.2) } \\
\text { Mix } \\
(47.6) \\
\text { Untype- } \\
\text { able (7.1) }\end{array}$ & $\begin{array}{l}\mathrm{P}[4](16.2) \\
\mathrm{P}[6](11.3) \\
\mathrm{P}[8](24.7) \\
\mathrm{P}[9](0.7) \\
\mathrm{P}[10](1.4) \\
\mathrm{P}[11](0.5) \\
\text { Mix (3.7) } \\
\text { Untypeable } \\
(20.7)\end{array}$ & $\begin{array}{l}\text { G1P[10] } \\
(0.22) \\
\text { G1P[4] (0.98) } \\
\text { G1P[6] (5.03) } \\
\text { G1P[8] (0.55) } \\
\text { G2P[10] } \\
(7.22) \\
\text { G2P[6] (1.42) } \\
\text { G2P[8] (0.98) } \\
\text { G3P[4] (0.88) } \\
\text { G4P[8] (0.55) }\end{array}$ & $\begin{array}{l}\text { Putnam et al } \\
(2007)\end{array}$ \\
\hline 3 & $\begin{array}{l}\text { Yogyakarta } \\
\text { Jakarta } \\
\text { Bandung } \\
\text { Mataram } \\
\text { Denpasar } \\
\text { Palembang }\end{array}$ & RT-PCR & $\begin{array}{l}\text { G1 (30) } \\
\text { G2 (14) } \\
\text { G3 (0.4) } \\
\text { G4 (0.4) } \\
\text { G9 (29) } \\
\text { Mix (9) }\end{array}$ & $\begin{array}{l}\mathrm{P}[4](16) \\
\mathrm{P}[6](56) \\
\mathrm{P}[8](17) \\
\text { Mix (9) } \\
\text { Untypeable } \\
(21)\end{array}$ & $\begin{array}{l}\text { G1P[4] (2) } \\
\text { G1P[6] (34) } \\
\text { G1P[8] (11) } \\
\text { G2P[4] (17) } \\
\text { G2P[6] (3) } \\
\text { G3P[4] (1) } \\
\text { G9P[4] (2) } \\
\text { G9P[6] (21) } \\
\text { G9P[8] (9) }\end{array}$ & $\begin{array}{l}\text { Soenarto et } \\
\text { al (2009) }\end{array}$ \\
\hline 4 & Yogyakarta & RT-PCR & $\begin{array}{l}\text { G1 } \\
(80.36) \\
\text { G2 } \\
(16.07) \\
\text { G3 (3.57) }\end{array}$ & $\begin{array}{l}\mathrm{P}[4](17.7) \\
\mathrm{P}[6](7.1) \\
\mathrm{P}[8](69.6) \\
\text { Untypeable } \\
(5.3)\end{array}$ & $\begin{array}{l}\text { G1P[4] (1.78) } \\
\text { G1P[6] (7.14) } \\
\text { G1P[8] } \\
(66.07) \\
\text { G2P[4] } \\
(16.07) \\
\text { G3P[8] (3.57) }\end{array}$ & $\begin{array}{l}\text { Nirwati et al } \\
(2009)\end{array}$ \\
\hline
\end{tabular}


There are two rotavirus vaccines, Rotarix ${ }^{\circledR}$ and RotaTeq ${ }^{\circledR}$ which have been developed by GlaxoSmithKline and Merck, respectively. RotaTeq ${ }^{\circledR}$ is a live, reassortant, pentavalent human-bovine rotavirus vaccine containing 5 rotavirus reassortants: G1, G2, G3, G4 and P[8] derived from human and bovine strains. Rotarix ${ }^{\circledR}$ is a live, monovalent, human attenuated rotavirus vaccine derived from the most common human rotavirus strain G1P[8]. These vaccines are currently used in the routine childhood immunization schedules in many middle and high-income countries and several low-income countries have expressed their interest in introducing rotavirus vaccine (Tate \& Parashar, 2011).

Wilopo et al. (2009) conducted a study to assess rotavirus vaccination effectiveness and costs in Indonesia. Economic and disease burden data were collected from Purworejo and Yogyakarta. They calculated the total direct costs (medical and non-medical direct costs i.e. transportation) and indirect costs (lost earnings) resulting from rotavirus infection. They concluded that vaccination can effectively reduce the burden of disease and healthcare costs. This study provides information for decision-making processes to introduce the rotavirus vaccine into Indonesia's National Immunization Programe.

\section{Conclusion}

Rotaviruses remain the most common cause of sevesre childhood diarrhoea in the world as well as in Indonesia. Most of the children under 5 years old suffer from rotavirus diarrhoea and they need medical treatment, particularly because of high dehydration. Rotavirus infections occur throughout the year and mainly infect children with the age of 6 to 23 months. Therefore, prevention with vaccines is an effective long-term strategy in the management of rotavirus infection. The research findings show that both common and uncommon rotavirus strain circulations are found in Indonesia. As a consequence, before administrating vaccination by using available rotavirus vaccines, a study needs to be conducted to demonstrate and ensure that the available vaccines are able to prevent and control rotavirus infection in Indonesia.

\section{References}

Barnes,G.L., Unicomb,L. and Bishop,R.F. (1992). Severity of rotavirus infection in relation to serotype, monotype and electropherotype. Journal of Paediatrics and Child Health, 28, 54-57.

Bishop,R.F., Unicomb,L.E., Soenarto,Y., Suwardji,H., Ristanto, and Barnes,G.L. (1989). Rotavirus serotypes causing acute diarrhoea in hospitalized children in Yogyakarta, Indonesia during 1978-1979. Archives Virology, 107, 207-213. 
Casio,A., Vizzi,E., Alaimo,C. and Arista,S. (2001). Rotavirus gastroenteritis in Italian children: can severity of symptoms be related to the infecting virus?. Clinical Infectious Disease, 32, 1126-1132.

Chan, J., Nirwati, H., Triasih, R., Bogdanovic-Sakran, N., Soenarto, Y., Hakimi, M., Duke, T., Buttery, J.P., Bines, J.E., Bishop, R.F., Kirkwood C.D. \& Danchin, M.D. (2011). Maternal antibodies to rotavirus: Could they interfere with live rotavirus vaccines in developing countries? Vaccine, 29, 1242-1247.

Gentsch,J.R., Laird,A.R., Bielfelt,B., Griffin,D.D., Banyai,K., Ramachandran,M., Jain,V., Cunliffe,N.A., Nakagomi,O., Kirkwood,C.D., Fischer,T.K., Parashar,U.D., Bresee,J.S., Jiang,B. and Glass,R.I. (2005). Serotype diversity and reassortment between human and animal rotavirus strains: implications for rotavirus vaccine programs. Journal of Infectious Diseases 192(S1), S146S159.Greenberg, H. B. \& Estes, M. K. (2009). Rotaviruses: from Pathogenesis to vaccination. Gastroenterology, 136, 1939-1951.

Greenberg, H. B. \& Estes, M. K. (2009). Rotaviruses: from pathogenesis to vaccination. Gastroenterology, 136, 1939-1951.

Grimwood,K. and lambert,S.B. (2009). Rotavirus vaccines. Human Vaccines 5 (2), 57-69.

Kirkwood, C.D. (2010). Genetic and antigenic diversity of human rotaviruses: potential impact on vaccination programs. Journal of Infectious Diseases 202(S1), S43-S48.

Leung,A.K.C., Kellner,J.D., \&Davies,H.D. (2005). Rotavirus gastroenteritis. Advances in theraphy, 22 (5), 476-487.

Li,D.D., Liu,N., Yu,J.M., Zhang,Q., Cui,S.X., Zhang,D.L., Yang,S.H., Cao,D.J., Xu,Z.Q. \& Duan,Z.J. (2009). Molecular epidemiology of G9 rotavirus strains in children with doarrhoea hospitalized in Mainland China from January 2006 to December 2007. Vaccine, 27 Suppl 5, F40-F45.

Moe,K., Thu,H.M., Oo,W.M., Aye,K.M., Shwe,T.T., Mar,W. \& Kirkwood,C.D. (2009). Genotyping of rotavirus isolates collected from children less than 5 years of age admitted for diarrhoea at the Yangon Children's Hospital, Myanmar. Vaccine, 27 Suppl 5, F89-F92.

Nirwati, H., Aman, A.T., Aminah, S. \& Soenarto, Y. (2009). Isolation and identification of rotavirus causing diarhea in children under 5 years old in Yogyakarta. Presentation on Annual Scientific Meeting, Indonesian Society for Clinical Microbiology, Yogyakarta.

Nyambat,B., Meng,C.Y., Vansith,K., Vuthy,U., Rin,E., Kirkwood,C., BogdanovicSakran,N. \& Kilgore,P.E. (2009). Hospital-based surveillance for rotavirus 
diarrhoea in Phnom Penh, Cambodia, March 2005 trough February 2007. Vaccine, 27 Suppl 5, F81-F84.

Ofoyo, B.A., Subekti,D,. Tjaniadi,P., Machpud,N., Komalarini,S., Setiawan,C., Simanjuntak,C., Punjabi,N., Corwin,A.L., Wasfy,M., Campbell,J.R. \& Lesmana,M. (2002). Enteropathogens associated with acute diarrhoea in community and hospital patients in Jakarta, Indonesia. FEMS Immunology and Medical Microbiology 34, 139-146.

Parashar,U.D., Gibson,C.J., Bresee,J.S., and Glass,R.I. (2006), Rotavirus and severe childhood diarrhoea. Emerging Infectious Diseases, 12 (2), 304-306.

Parashar,U.D., Burton,A., Lanata,C., Boschi-Pinto,C., Shibuya,K., Steele,D., Birmingham,M. ang Glass,R.I. (2009). Global mortality associated with rotavirus disease among children in 2004. The Journal of Infectious Diseases, 200, S9S15.

Polanco-Marin,G., Gonzalez-Losa Mdel R, Rodriguez-Angulo E, ManzanoCabrera I, Camara-Mejia J, Puerto-Solis M. (2003). Clinical manifestations of the rotavirus infection and his relation with the electropherotypes and serotypes detected during 1998 and 1999 in Merida, Yucatan, Mexico. Journal of Clinical Virology, 27, 242-246.

Putnam,S.D., Sedyaningsih, E.R., Listiyaningsih,E., Pulungsih,S.P., Komalarini, Soenarto, Y., Salim, O.C., Subekti, D., Riddle, M.S., Burgess,T.H. \& Blair, P.J. (2007). Group A rotavirus-associated diarrhoea in children seeking treatment in Indonesia. Journal of Clinical Virology, 40, 289-294.

Qazi,R., Sultana,S., Sundar,S., Warraich,H., un-Nisa,T., Rais,A \& Zaidi,A.K. (2009). Population-based surveillance for severe rotavirus gastroenteritis in children in Karachi, Pakistan. Vaccine, 27 Suppl 5, F25-F30.

Soenarto,Y., Sebodo,T., Ridho,R., Alrasjid,H., Rohde,J.E., Bugg,H.C., Barnes,G.L. \& Bishop,R. (1981). Acute diarrhoea and rotavirus infection in newborn babies and children in Yogyakarta, Indonesia, From June 1978 to June 1979. Journal of Clinical Microbiology, 14, 123-129.

Soenarto,Y., Aman,A.T., Bakri,A., Waluya,H., Firmansyah,A., Kadim,M., Martiza,I., Prasetyo,D., Mulyani,N.S., Widowati,T., Soetjiningsih, Karyana,I.P., Sukardi,W., Bresee,J. \& Widdowson,M.A. (2009). Burden of severe rotavirus diarrhoea in Indonesia. The Journal of Infectious Diseases, 200 Suppl 1, S188194.

Subekti,D., Lesmana,M., Tjaniadi,P., Safari,N., Frazier,E., Simanjuntak,C., Komalarini,S., Taslim,J., Campbell,J.R. \& Oyofo,B.A. (2002). Incidence of norwalk-like viruses, rotavirus and adenovirus infection in patients with acute 
gastroenteritis in Jakarta, Indonesia. FEMS Immunology and Medical Microbiology, 33, 27-33.

Tamura,T., Nishikawa,M., Anh,D.D., \& Suzuki,H. (2010). Molecular epidemiological study of rotavirus and norovirus infections among patients with acute gastroenteritis in Nha Trang, Vietnam, December 2005-June 2006. Japan Journal of Infectious Diseases, 63, 405-411.

Tate,J.E. and Parashar,U.D. (2011). Monitoring impact and effectiveness of rotavirus vaccination. Expert Reviews Vaccines 10(8), 1123-1125.

Uhno,I. and Svensson L. (1986). Clinical and epidemiological features of acute infatile gastroenteritis associated with human rotavirus subgroups 1 and 2 . Journal of Clinical Virology, 23,551-555.

Wall,R.A., Mellars,B.J., Luton,P., and Boulding,S. (1982). Comparison of ELISA, SPACE, and electron microscopy for the routine diagnosis of rotavirus infection. Journal of Clinical Pathology, 35, 104-106.

Widowati,T., Bakrie,A., Nirwati,H. \& Soenarto,Y. (2012). Surveillance of rotavirus diarrhoea. Paediatrica Indonesiana, 52 (1), 22-27.

Widowati,T, Mulyani,N.S., Nirwati,H., \& Soenarto,Y. (2012). Diare Rotavirus pada anak usia balita. Sari Pediatri 13 (5), 340-345.

Wilopo,S.A., Kilgore,P., Kosen,S., Soenarto,Y., Aminah,S., Cahyono,A., Ulfa,M., Tholib,A. (2009). Economic evaluation of a routine rotavirus vaccination programme in Indonesia. Vaccine, 27S, F67-F74.

Wu,F.T., Liang,S.Y., Tsao,K.C., Huang,C.G., Lin,C.Y., Lin,J.S., Su,C.Y., Eng,H.L., Yang,J.Y., Chen,P.J. \& Yang,C.F. (2009). Hospital-based surveillance and molecular epidemiology of rotavirus infection in Taiwan, 2005-2007. Vaccine, 27 Suppl 5, F50-F54.

Zaman,K., Yunus,M., Faruque,A.S., El Arifeen,S., Hossain,I., Azim,T., Rahman,M., Podder,G., Roy,E., Luby,S. \& Sack,D.A. (2009). Surveillance of rotavirus in a rural diarrhoea treatment centre in Bangladesh, 2000-2006. Vaccine, 27 Suppl 5, F31-F4. 



\section{Tuberculosis in Children}

Micha Loebermann, University of Rostock, Department of Tropical Medicine, Infectious Diseases and Nephrology, Germany

Hilte Geerdes-Fenge, University of Rostock, Department of Tropical Medicine, Infectious Diseases and Nephrology, Germany

\section{Introduction}

Children in contact to subjects with active pulmonary tuberculosis are at a high risk to acquire the infection and to develop active disease themselves. In comparison to adults with active tuberculosis, the diagnosis of tuberculosis in children has numerous drawbacks. Médecins Sans Frontières, an internationally acting nongovernmental organization (NGO), have estimated that $93 \%$ of the applied tests fail to produce true results in children (2012).

Children may fail to develop classical signs and symptoms of active disease, making the decisions for treatment difficult (Rahman et al., 2012). Additionally, young children have a higher risk to develop active disease after contact to tuberculosis (Brent, 2012) and one third of all tuberculosis-associated deaths occur in children (Burgner et al., 2006). Most cases of active disease in children are detected by case contact investigations (del Rosal et al., 2010). The total progression rate 
from latent tuberculosis to active disease in children during the first 15 years of age is estimated to be $23 \%$ in a Chinese study, with the highest progression rate during the first year of age (5.5\%) (Cao et al., 2012). An older review found that after recent tuberculin skin test (TST) conversion the risk of progression to active tuberculosis to be $17 \%$ in children aged $0-5$ years, and $8 \%$ in children aged $6-15$ years (Horsburgh, 2004).

The WHO estimates that 10-15\% of all annual Mycobacterium tuberculosis infections occur in children, leading to $900000-1450000$ new cases of tuberculosis in children each year (2011). Children generally contract the infection from close family members (Batra et al., 2012), however, in low incidence countries caretakers originating from countries with high incidence rates may play an important role in transmitting tuberculosis to children. We present data and a review of the literature to evaluate the risks and consequences associated with tuberculosis in caretakers.

\section{The source of infection in children}

An Indian study showed that in $66.1 \%$ of tuberculosis cases the source of infection in children was associated with a woman living in the household - mostly the mother - emphasizing the significance of close contact for $\mathrm{Tb}$ transmission (Batra et al., 2012). The risk for acquiring active tuberculosis was estimated to be 30 times higher in children with a household contact to tuberculosis compared with children in the general population in Peru (Becerra et al., 2012). In high-income countries extra child care is provided by nursery schools or nannies. One form of caretaking is the employment of au-pairs - young women or men from abroad choose to take care of the children in guest families where they are provided with lodging, food and a small salary. Within this context, au-pairs get to know the culture and the language of their destinations country. In Germany around 75\% of au-pairs come from countries with a high prevalence and incidence of tuberculosis and may be infected without currently displaying symptoms of disease (Geerdes-Fenge and Loytved, 2011). A Spanish study showed, that active tuberculosis in foreigners is mostly diagnosed within 2 years after immigration (Arce Arnaez et al., 2005). A thirty year observation showed that the number of children who developed tuberculosis after extra-familial contacts increased and one source of infection have been immigrant caretakers (del Rosal et al., 2010).

\section{Survey of tuberculosis in caretakers}

In a survey from Germany and Austria, reports from public health departments revealed 22 au-pairs with active tuberculosis between 2002 and 2010 (GeerdesFenge and Loytved 2011). In all these cases, the screening of contacts was performed by using the tuberculin skin test and/or interferon-gamma release assays (IGRA). In accordance with national guidelines all subjects with positive screening 
results had a chest x-ray and possible further diagnostic procedures. Subjects newly infected with $M$. tuberculosis were offered prophylactic treatment.

All au-pairs detected with active tuberculosis were women aged between 19 and 27 years. Sputum-smear positive tuberculosis was found in 17/22 women (77\%), $3(13.6 \%)$ had extra-pulmonic manifestations and $2(9 \%)$ were asymptomatic. The time between arrival from abroad and the onset of tuberculosis ranged between 0 and 19 months (mean 7.5 months). Screening of contacts revealed 47 infections (21 in children and 26 in adults) and the number of infected contacts increased with the time between onset of symptoms and diagnosis (Fig. 1).

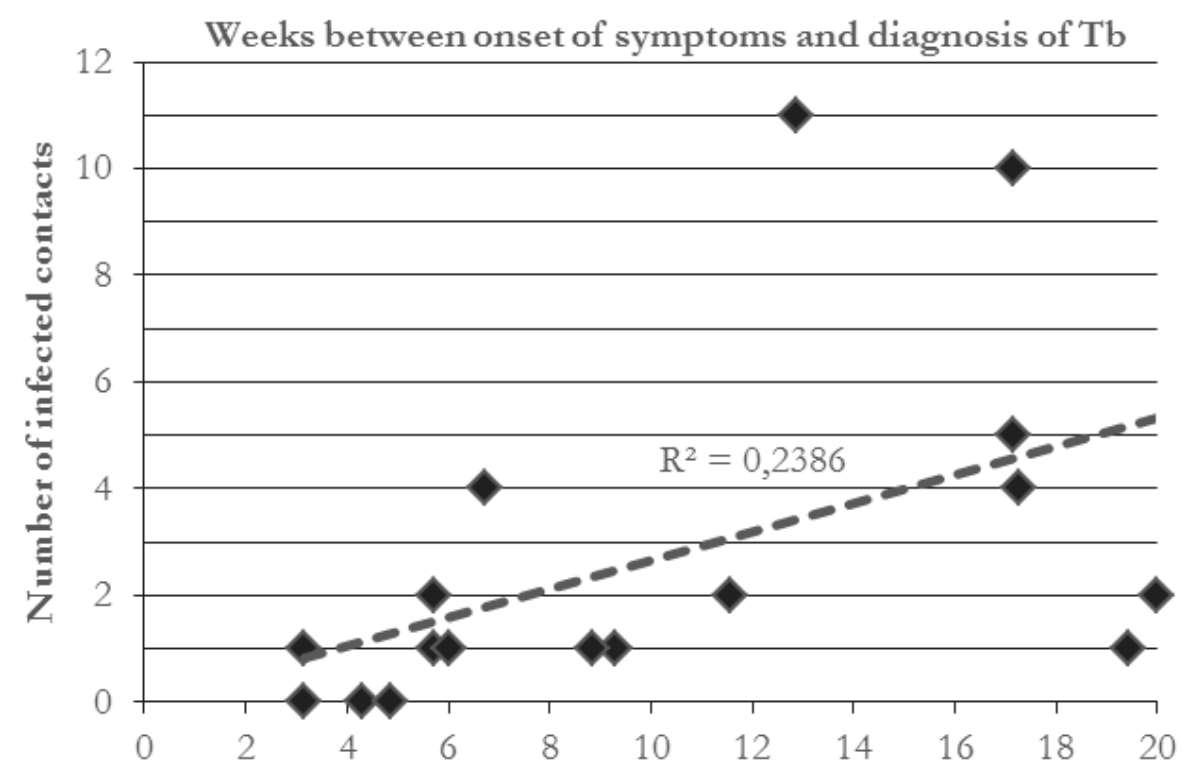

Figure 1: Infections caused by 17 au-pairs with sputum-smear positive tuberculosis.

In patients with pulmonary tuberculosis, the time between onset of disease and diagnosis of disease ranged between 3 and 20 weeks (mean $10 \pm 6.1$ weeks). The risk for transmission increased greatly with the time elapsing between onset of disease and diagnosis. Those subjects who were diagnosed within 5 weeks after the onset of symptoms only 1 person contracted the infection, whereas infected contacts were found in all subjects who were diagnosed more than 5 weeks after the onset of symptoms. Au-pairs with pulmonary tuberculosis without positive sputum smears did not cause infections in contacts, neither adults, nor children.

Following active tuberculosis, initially 47 contacts were found with positive skin test or IGRA. Initially. 32 adults and 38 children were identified as contacts to 17 au-pairs with sputum-smear positive tuberculosis. Among the 25 adult contacts with $M$. tuberculosis infection only one accepted isoniazide (INH) prevention treat- 
ment, whereas 17 of 21 children accepted INH prevention treatment. One additional contact was primarily not diagnosed. This school teacher developed pulmonary tuberculosis and infected another 2 adults and 11 children. The outcome of screening and prevention treatment are summarized in Figure 2 (adults) and Figure 3 (children).

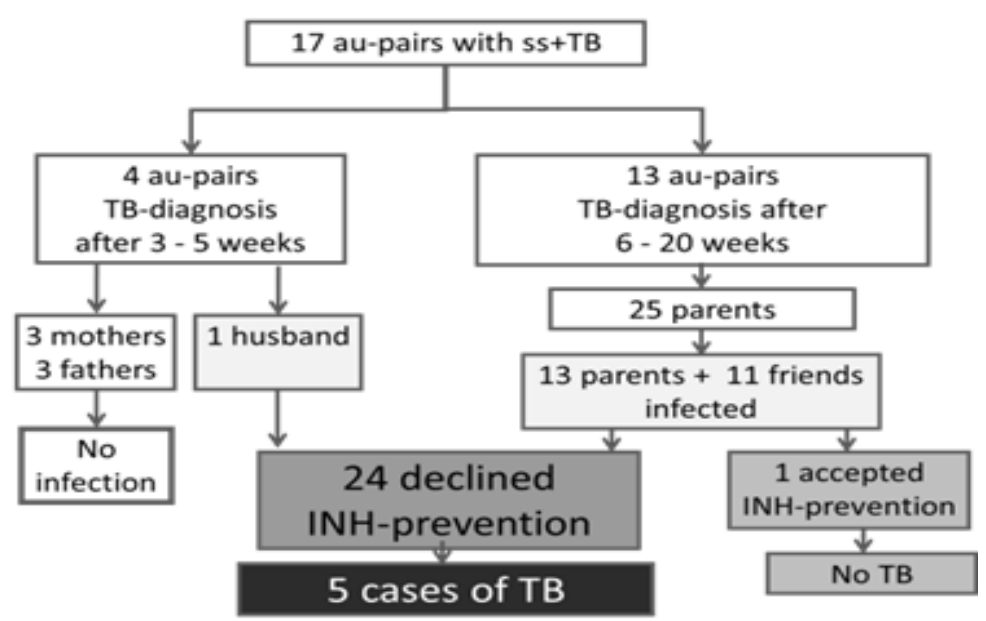

Figure 2: Outcome of adult contacts to sputum smear positive (ss + ) tuberculosis.

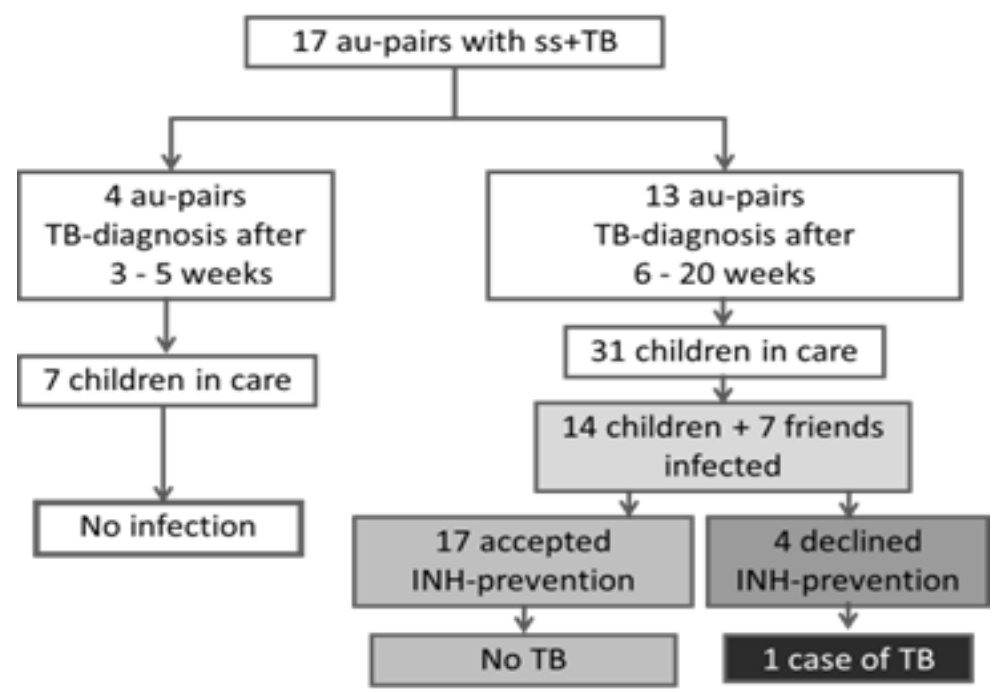

Figure 3: Outcome of child contacts to sputum smear positive (ss + ) tuberculosis. 
Table 1 Characteristics of studies investigating contact screening (TST: tuberculin skin testing, IGRA: interferon gamma release assay).

\begin{tabular}{|c|c|c|c|c|c|c|}
\hline Country, author & $\begin{array}{l}\text { Screening } \\
\text { method }\end{array}$ & $\begin{array}{l}\text { Number } \\
\text { of index } \\
\text { cases }\end{array}$ & $\begin{array}{l}\text { Number of } \\
\text { contacts } \\
\text { screened }\end{array}$ & $\begin{array}{l}\text { Infected or active } \\
\text { tuberculosis in } \\
\text { contacts }\end{array}$ & $\begin{array}{l}\text { Tb developed } \\
\text { with chemo- } \\
\text { prophylaxis }\end{array}$ & $\begin{array}{l}\text { Tb developed } \\
\text { without } \\
\text { chemoprophy- } \\
\text { laxis }\end{array}$ \\
\hline $\begin{array}{l}\text { Spain (Vidal et } \\
\text { al., 1997) }\end{array}$ & $\begin{array}{l}\text { TST, chest } \\
\text { radiography }\end{array}$ & 635 & 3,071 & $1,440(47 \%)$ & 1 in $296(0.3 \%)$ & $\begin{array}{l}13 \text { in } 60 \\
(21.7 \%)\end{array}$ \\
\hline $\begin{array}{l}\text { The Gambia } \\
\text { (Lienhardt et } \\
\text { al., 2003) }\end{array}$ & TST & 206 & $\begin{array}{l}384 \\
(<5 \text { years })\end{array}$ & $135(35 \%)$ & ND & ND \\
\hline $\begin{array}{l}\text { Germany } \\
\text { (Geerdes-Fenge } \\
\text { and Loytved, } \\
2011 \text { ) }\end{array}$ & IGRA & 17 & 32 (adults) & $25(78 \%)$ & $0 / 1$ & $5 / 25(20.8 \%)$ \\
\hline $\begin{array}{l}\text { Germany } \\
\text { (Geerdes-Fenge } \\
\text { and Loytved, } \\
2011 \text { ) }\end{array}$ & IGRA & 17 & $\begin{array}{l}38 \text { (chil- } \\
\text { dren) }\end{array}$ & $21(55 \%)$ & $0 / 17$ & $1 / 4(25 \%)$ \\
\hline $\begin{array}{l}\text { Zimbabwe } \\
\text { (Mtombeni et } \\
\text { al., 2002) }\end{array}$ & $\begin{array}{l}\text { TST, chest } \\
\text { radiography }\end{array}$ & 102 & 174 & $\begin{array}{l}\text { TST: 109/174 } \\
(62.6 \%) \\
\text { Chest X-ray: } \\
73 / 174(42 \%) \\
\end{array}$ & & \\
\hline $\begin{array}{l}\text { Philippines } \\
\text { (Salazar-Vergara } \\
\text { et al., 2003) }\end{array}$ & $\begin{array}{l}\text { TST, chest } \\
\text { radiography }\end{array}$ & 62 & $\begin{array}{l}153 \text { (chil- } \\
\text { dren } 0-15 \\
\text { years) }\end{array}$ & $112(73 \%)$ & ND & ND \\
\hline $\begin{array}{l}\text { Indonesia } \\
\text { (Rutherford et } \\
\text { al., 2012) }\end{array}$ & $\begin{array}{l}\text { TST, IGRA } \\
\text { (Quanti- } \\
\text { feron) }\end{array}$ & & 304 & $\begin{array}{l}\text { TST: } \\
\text { 145/302 (48\%) } \\
\text { IGRA: } \\
\text { 152/299 (51\%) } \\
\text { both tests: } \\
\text { 180/304 }(59 \%)\end{array}$ & & \\
\hline $\begin{array}{l}\text { Pakistan (Batra } \\
\text { et al., 2012) }\end{array}$ & $\begin{array}{l}\text { Children } \\
<15 \text { years }\end{array}$ & $\begin{array}{l}\mathrm{Tb} \\
\text { screen- } \\
\text { ing }\end{array}$ & 6613 & $\begin{array}{l}\text { Suspected Tb: } \\
317(4.8 \%) \\
\text { diagnosed Tb: } \\
121(1.8 \%) \\
\end{array}$ & & \\
\hline $\begin{array}{l}\text { Egypt, } \\
\text { (Mohamed, } \\
\text { 2012) }\end{array}$ & TST & 197 & $\begin{array}{l}297 \\
\text { (children } \\
<5 \text { years) }\end{array}$ & $136 / 252(54.0 \%)$ & \multicolumn{2}{|c|}{$\begin{array}{l}8 / 217(3.7 \%) \text {, most subjects with } \\
\text { short preventive INH chemothera- } \\
\text { py }\end{array}$} \\
\hline $\begin{array}{l}\text { Southeast Asia } \\
\text { (Triasih et al., } \\
\text { 2012) }\end{array}$ & $\begin{array}{l}\text { Systematic } \\
\text { review } \\
\text { (11 studies), } \\
\text { TST } \\
\end{array}$ & & $\begin{array}{l}\text { children } \\
\text { (0-15 years) }\end{array}$ & $24.4 \%-69.2 \%$ & & \\
\hline
\end{tabular}

\section{Transmission}

A source for tuberculosis with symptomatic infection can be identified in 21 to $48 \%$ of affected children (Toppet et al., 1991; Driver et al., 1995). For the majority 
of children, the source are adults living in the same house. Mothers are generally in closer contact with young children and play a major role in transmitting tuberculosis to children (Titone et al., 2003; Triasih et al., 2012). Table 1 summarises different studies with children screened for tuberculosis after contact to individuals with disease. A study from Zimbabwe showed that $77 \%$ of the children under 5 years whose parent had smear-positive tuberculosis had suspected or probable tuberculosis themselves at the time of evaluation (Mtombeni et al., 2002). In The Gambia, a study found 135 of $384(35 \%)$ children below 5 years with contact to tuberculosis cases to have positive TST responses (Lienhardt et al., 2003). In this study, children were 2.8 times more likely to acquire tuberculosis if they had a first degree relative with tuberculosis compared to other household contacts, $93 \%$ of the children with a parent with tuberculosis were TST positive, whereas only $33 \%$ of children in contact to other household members with tuberculosis has a positive TST reaction (Lienhardt et al., 2003).

Table 2 summarises risk factors associated with acquiring tuberculosis in children. Screening of pregnant women for the presence of current cough, fever, weight loss, and night sweats is a low-cost, but effective intervention to identify and treat maternal tuberculosis (Getahun et al., 2012), thus significantly reducing the risk of exposure to M. tuberculosis in young children.

Table 2: Factor associated with the risk of transmitting tuberculosis to children [modified from (Lienhardt et al., 2003; WHO, 2006)]

\begin{tabular}{|l|l|l|}
\hline & Risk factor & Odds ratio \\
\hline Nutrition status & $\begin{array}{l}\text { Severe malnutrition } \\
\text { Vitamin D deficiency }\end{array}$ & ND \\
\hline Immune status & HIV infection & ND \\
\hline Genetic proximity to Tb case & $1^{\text {st } \text { degree family member }}$ & OR 4.7 \\
\hline Geographic proximity to Tb case & Sharing beds & OR 4.9 \\
\hline Number of people in household & $<5$ & OR 6.9 \\
\hline Duration of cough of index & $>10$ weeks & OR 2.1 \\
\hline
\end{tabular}

\section{Tuberculosis diagnosis in children}

In the beginning of the last century, physicians found that tuberculosis in children was highly underdiagnosed despite symptoms and signs that may hint to active infection with $M$. tuberculosis, like night sweats, loss of appetite and fatigue (Williams, 1909). The diagnosis of tuberculosis has substantially improved since then. However, establishing a definite diagnosis in children may even today be associated with some difficulties. 
For the diagnosis of tuberculosis it is important to distinguish between latent infection (LTBI) and active disease. The infection leads to a primary complex 3-8 weeks after exposure, at this time point TSTs and IGRA turns positive in the majority of infected subjects. In most subjects, the infection is contained for years without signs of illness. Only in a few subjects, infection primarily leads to active disease, but may also develop years after infection. The risk of developing active illness once infected with $M$. tuberculosis is dependent on age and immune status. Children and patients with immunosuppression have a higher risk of developing active tuberculosis. In contrast to LTBI, active disease is diagnosed by detection of $M$. tuberculosis microscopically, in culture, or using a rapid molecular test (Gene Xpert MTB/RIF) endorsed by WHO in 2010 that can detect the bacteria and rifampicin resistance within 100 minutes (WHO, 2012). To establish the diagnosis of active tuberculosis in children, these laboratory methods are supplemented by clinical findings and chest radiography (Shingadia, 2012), in contrast to adults where gastric washing may additionally be needed to collect adequate specimens for mycobacterial culture (Rahman et al., 2012).

Following infection, children under the age of 10 years are at an increased risk of progression of the disease. This is true especially for children under 5 years and the most common age of children to present with tuberculosis is between 1 and 4 years (WHO, 2006). The WHO suggest that three of the following conditions are strong indicators of active disease: chronic symptoms or physical signs suggestive of tuberculosis, positive TST, and chest radiograph suggestive of tuberculosis (WHO, 2006). Children with proven disease should be offered treatment according to national guidelines that are generally in accordance with WHO guidelines (WHO, 2006 and 2010).

A major measure to prevent disease in children is to provide isoniazid preventive therapy (IPT) to those children exposed to $M$. tuberculosis. The only way to diagnose infection before progression of the disease is by using TST or IGRA (Amanatidou et al., 2012). Both tests may provide false-negative or false-positive results in some cases, but IGRAs have a higher specificity excluding those patients with positive TST due to previous bacille Calmette-Guerin (BCG) vaccination (Shingadia, 2012; Yassin et al., 2012). Many national programs therefore recommend IPT to all children under the age of five, independent of screening results when they do not have active disease (WHO, 2006). ITP delivery has been proven highly effective in reducing the risks of developing active tuberculosis in different settings (Mandalakas et al,. 2012; Mohamed, 2012; Shingadia, 2012; Uluk et al., 2012). In HIV-infected children, ITP seems to be particularly efficient and isoniazid may be used as primary prophylaxis in settings with a high burden of both HIV and tuberculosis (WHO, 2007; Gray et al., 2009; Zar et al., 2012). 


\section{Conclusions}

Children are at a special risk for $M$. tuberculosis infection and disease after contact to adults with tuberculosis. Transmission of $M$. tuberculosis mainly occurs by household contacts. Screening for latent infection and sequential isoniazid preventive treatment are essential measures to avoid active tuberculosis in children.

\section{References}

Amanatidou, V., G. Syridou, et al. (2012). "Latent tuberculosis infection in children: diagnostic approaches.” Eur J Clin Microbiol Infect Dis 31(7): 12851294.

Arce Arnaez, A., J. Inigo Martinez, et al. (2005). "[Tuberculosis and immigration in a health sanitary area in Madrid, Spain. Trends in 1994-2003]." Med Clin (Barc) 125(6): 210-212.

Batra, S., A. Ayaz, et al. (2012). "Childhood tuberculosis in household contacts of newly diagnosed TB patients.” PLoS One 7(7): e40880.

Becerra, M. C., M. F. Franke, et al. (2012) "Tuberculosis in children exposed at home to multidrug-resistant tuberculosis.” Pediatr Infect Dis J DOI: 10.1097/INF.0b013e31826f6063.

Brent, A. J. (2012). "Childhood Tb surveillance: Bridging the knowledge gap to inform policy." J Trop Med 2012: 865436.

Burgner, D., S. Cherian, et al. (2006). "Pulmonary tuberculosis: management in children has special considerations." BMJ 332(7554): 1392-1393.

Cao, H., Y. Zhou, et al. (2012). "Estimates of tuberculosis progression rate of children in China." J Biol Dyn 6(2): 663-673.

del Rosal, T., F. Baquero-Artigao, et al. (2010). "Impact of immigration on pulmonary tuberculosis in Spanish children: a three-decade review." Pediatr Infect Dis J 29(7): 648-651.

Driver, C. R., J. J. Luallen, et al. (1995). "Tuberculosis in children younger than five years old: New York City.” Pediatr Infect Dis J 14(2): 112-117.

Geerdes-Fenge, H. and G. Loytved (2011). "[Tuberculosis in 22 Au-pairs in Germany and Austria - rapid diagnosis reduces risk of infection for host families].” Dtsch Med Wochenschr 136(37): 1837-1841.

Getahun, H., D. Sculier, et al. (2012). "Prevention, diagnosis, and treatment of tuberculosis in children and mothers: evidence for action for maternal, neonatal, and child health services." J Infect Dis 205 Suppl 2: S216-227. 
Gray, D. M., H. Zar, et al. (2009). "Impact of tuberculosis preventive therapy on tuberculosis and mortality in HIV-infected children." Cochrane Database Syst $\operatorname{Rev}(1):$ CD006418.

Horsburgh, C. R., Jr. (2004). "Priorities for the treatment of latent tuberculosis infection in the United States." N Engl J Med 350(20): 2060-2067.

Jackson-Sillah, D., P. C. Hill, et al. (2007). “Screening for tuberculosis among 2381 household contacts of sputum-smear-positive cases in The Gambia." Trans R Soc Trop Med Hyg 101(6): 594-601.

Lienhardt, C., J. Sillah, et al. (2003). "Risk factors for tuberculosis infection in children in contact with infectious tuberculosis cases in the Gambia, West Africa." Pediatrics 111(5 Pt 1): e608-614.

Mandalakas, A. M., A. C. Hesseling, et al. (2012). "Modelling the cost-effectiveness of strategies to prevent tuberculosis in child contacts in a high-burden setting." Thorax.

Médecins Sans Frontières. (2012). "Uncounted and untreated: Children with Tuberculosis”. Geneva, Switzerland, Médecins Sans Frontières.

Mohamed, A. M. (2012). "Adherence to and outcome of isoniazid chemoprophylaxis among household contact children of adults having pulmonary tuberculosis in Alexandria, Egypt.” J Egypt Public Health Assoc 87(3-4): 71-78.

Mtombeni, S., A. Mahomva, et al. (2002). "A clinical evaluation of children under the age of five years who are household contacts of adults with sputum positive tuberculosis in Harare, Zimbabwe." Cent Afr J Med 48(3-4): 28-32.

Rahman, N., K. K. Pedersen, et al. (2012). "Challenges in diagnosing tuberculosis in children." Dan Med J 59(7): A4463.

Rutherford, M. E., P. C. Hill, et al. (2012). "Risk factors for Mycobacterium tuberculosis infection in Indonesian children living with a sputum smearpositive case." Int J Tuberc Lung Dis 16(12): 1594-1599.

Salazar-Vergara, R. M., I. G. Sia, et al. (2003). “Tuberculosis infection and disease in children living in households of Filipino patients with tuberculosis: a preliminary report." Int J Tuberc Lung Dis 7(12 Suppl 3): S494-500.

Shingadia, D. (2012). “Tuberculosis in childhood." Ther Adv Respir Dis 6(3): 161 171.

Sinfield, R., M. Nyirenda, et al. (2006). "Risk factors for TB infection and disease in young childhood contacts in Malawi." Ann Trop Paediatr 26(3): 205-213.

Titone, L., A. Romano, et al. (2003). “[Epidemiology of paediatric tuberculosis today].” Infez Med 11(3): 127-132. 
Toppet, M., A. Malfroot, et al. (1991). "Tuberculosis in children: a 13-year follow up of 1714 patients in a Belgian home care centre." Eur J Pediatr 150(5): 331335.

Triasih, R., M. Rutherford, et al. (2012). "Contact investigation of children exposed to tuberculosis in South East Asia: a systematic review." J Trop Med 2012: 301808.

Uluk, T., W. E. Allison, et al. (2012). "Evaluation of an interferon gamma release assay in children with suspected tuberculosis in Papua New Guinea." Pediatr Infect Dis J.

Underwood, B. R., V. L. White, et al. (2003). "Contact tracing and population screening for tuberculosis--who should be assessed?” J Public Health Med 25(1): 59-61.

Verver, S., J. H. van Loenhout-Rooyackers, et al. (2005). "Tuberculosis infection in children who are contacts of immigrant tuberculosis patients." Eur Respir J 26(1): 126-132.

Vidal, R., M. Miravitlles, et al. (1997). "Increased risk of tuberculosis transmission in families with microepidemics." Eur Respir J 10(6): 1327-1331.

WHO (2006). Guidance for national tuberculosis programmes on the management of tuberculosis in children. Geneva, Switzerland, World Health Organisation.

WHO (2007). "Chapter 4: childhood contact screening and management.” Int J Tuberc Lung Dis 11(1): 12-15.

WHO (2010). "Treatment of tuberculosis: guidelines for national programmes". WHO. Geneva, Switzerland, World Health Organisation.

WHO (2011). "Better tests urgently needed." Bull World Health Organ 83(3): 164165.

WHO (2012). “Global tuberculosis report 2012.” Geneva, Switzerland, WHO.

Williams, M. H. (1909). "Pulmonary tuberculosis in children.” Br Med J 1(2511): 387-391.

Yassin, M., R. Petrucci, et al. (2012). "Added value of TST, IGRAS and IP-10 to identify children with TB infection.” Eur Respir J.

Zar, H. J., B. Eley, et al. (2012). "Advances in childhood tuberculosis contributions from the University of Cape Town." S Afr Med J 102(6): 518521. 


\title{
28. Diagnosis of Children Lung Tuberculosis based on Rapid Molecular Methods (MAS-PCR and Xpert MTB/RIF System)
}

\author{
Leli Saptawati, Faculty of Medicine, Sebelas Maret University Surakarta,Indonesia \\ Mubammad Nasrum Massi, University of Hasanuddin, Medical Microbiology, Makas- \\ sar, Indonesia
}

\section{Introduction}

Tuberculosis is one of many infectious diseases that affect children around the world. Morbidity and mortality caused by this infection is high, especially when it affects infants and young children. Many factors including population density, poverty and HIV prevalence contribute to increasing incidence rates of tuberculosis worldwide. The number of tuberculosis cases is the highest in poor countries and throughout the last decade, it increased among children, particularly in SubSaharan Africa (Walls et al., 2004). Based on a number of 2000 reports, the incidence in children under 15 years of age is approximately 884,019 cases $(11 \%$ of total cases). Approximately $75 \%$ of emergency cases occurred in the 22 countries with the highest number of tuberculosis cases. Children under 5 years are especially 
at risk because at this age tuberculosis often proceeds rapidly from latent to active infection (Chaulet, 1992). Source of transmission in children derives mainly from adult patients with Mycobacterium-tuberculosis-positive sputum (World Health Organization, 2006).

Among children, tuberculosis is rarely followed by a condition of post-primary pulmonary disease. Children under 2 years are experiencing hematogenous tuberculosis, which is an extrapulmonary disease. Data presented by the World Health Organization (WHO) showed that $70-80 \%$ of children develop pulmonary tuberculosis, while the rest experience extrapulmonary disease. If a child has pulmonary tuberculosis, it rarely has cavitas and therefore often does not secrete infectious sputum. As a consequence, only 3-13\% of children showed positive acid-fast bacilli (AFB) staining, while $29 \%$ present with a positive culture. In children, the primary infection is usually characterized by mediastinal lymphadenopathy (Adams et al., 2012).

\section{Pathogenesis of pulmonary tuberculosis}

Pulmonary $M$. tuberculosis infection can be divided into different pathomechanisms including primary, secondary or reactivated tuberculosis, and the spread of $M$. tuberculosis. In primary tuberculosis, there are 5 stages of disease progression:

1. M. tuberculosis infect humans through inhalation of infectious droplets and reaches the alveoli (Engleberg et al., 2007). The droplet size is $5 \mu \mathrm{m}$ which contains 1-3 AFB. The minimum dose for infection is about 10 bacteria (Bauman, 2009).

2. These bacteria will be phagocyted by macrophages through a variety of receptors including the receptors for complement and surfactants, as well as some Toll-like receptors (TLR); TLR 2 and TLR4 (Krutzik et al., 2004). These processes will be enhanced by surfactant, which is a glycoprotein found at the surface of the alveoli. The surfactant A will enhance the activity of the mannose receptor (Gaynor et al., 1995). The surfactant $\mathrm{D}$ is also found in the alveoli which would inhibit phagocytosis by blocking mannosyl-oligosaccharide residues on the surface of bacterial cells. This will lead to the inhibition of the interaction of $M$. tuberculosis with mannose receptors on the surface of macrophages (Ferguson et al., 1999). In the macrophage, the bacteria will be destroyed or inhibited from growth through the activation of macrophages and the production of reactive oxygen (Ehrt et al., 2001). However, this process can not destroy all mycobacteria, because they are capable to secrete a so-called repetitive protein which can inhibit the fusion between the phagosome and lysosomes (Bauman, 2009).

3. In the nhe next stage, mycobacteria replicate inside macrophages, and gradually destroy their host cells. After that, the bacteria will be released from the dead macrophages and these will be phagocyted by others. Then M. tuberculosis will start a new replication cycle (Bauman, 2009). In the lymphatic system, infected macrophages will be transported to the regional lymph nodes. In some patients, 
this process may lead to the formation of a so-called Ghon complex, which represents an enlarged hilar lymph node in the inflammed lung. In case of individuals with a decreased immune system, the bacteria may spread hematogenously. In the early onset of lympho-hematogenous spread, bacteria are only present in a variety of locations such as the lymph nodes, the lining of the brain, the vertebral column and most often in the apex of the lung (Engleberg et al., 2007). This early stage of infection may last for several weeks without any symptoms or only mild fever (Bauman, 2009).

4. Chemokines produced by infected macrophages will attract neutrophils, lymphocytes and monocytes to the site of infection, but none of these is able to kill the bacteria efficiently (van Crevel et al., 2002; Fenton and Vermeulen, 1996). Subsequently formed granulomas limit the spread of M. tuberculosis (Smith, 2003). Research conducted by Flynn et al. concluded that granulomas have a role in maintaining a latent phase of infection, while the Tumor Necrosis Factor- $\alpha$ (TNF- $\alpha$ ) is required for the granuloma to function properly (Flynn et al., 1995). A host cellular immune response towards $M$. tuberculosis appears after growing for 2-12 weeks or when the number of bacteria reaches a number of $10^{3}$ to $10^{4}$. The emerging cellular immune response is characterized by a positive tuberculin skin test result (American Thoracic Society, 2000). Infected macrophages present mycobacterial antigen to $T$ lymphocytes. Subsequently activated lymphocytes attract and activate macrophages in greater numbers and will form the inflammatory response. Macrophages surrounding the site of infection will form tubercles (Bauman, 2009). Tubercles are surrounded by granuloma tissue fibrosis and may end up in fibrosis and calcification (Levinson, 2008).

5. The cellular immune response destroys infected macrophages, then generates cheese formation with granulomas in the middle and surrounded by fibroblasts, lymphocytes and monocytes. Mycobacteria can not replicate in this cheese formation because the $\mathrm{pH}$ is acidic, oxygen levels are low, and toxic substances are present. However, some bacteria may still survive and persist in a dormant stage for years. This stage is therefore called latent or persistent infection without causing clinical symptoms. The host immune response will determine whether the infection remains at this stage or progress to the next stage. For individuals who have a good cellular immune response, further granulomas will disappear by the process of calcification and formation of fibrotic tissue. However, if the host cellular immune cells are decreasing, the central part of the granuloma will melt by an unknown process and will be an excellent medium for the replication of M. tuberculosis. In this case, bacteria will be released from the granuloma and spread throughout the lung fields and even through the pulmonary veins and lymph nodes. In this circumstances, the individual will experience secondary tuberculosis or reactivation of a former latent infection, respectively (Smith, 2003). Systemic spread of bacteria may occur when infected macrophages carry $M$. tuberculosis through blood and lymph vessels to various organs such as the bone marrow, spleen, kidneys, brain 
and spinal cord. Symptoms and signs vary depending on the location of the infection (Bauman, 2009).

\section{Representative specimens for diagnosis}

Children with tuberculosis usually have difficulties to collect their sputum. Therefore, additional procedures are needed to obtain samples from the lower respiratory tract. Some approaches are based on using three consecutive early morning gastric lavage (GL) or gastric aspirate (GA) samples for attempting microbiological diagnosis. However, GL is unpleasent, relatively invasive, requires trained staff and hospitalization for an overnight, although it may be performed in on outpatient setting. This procedure is not feasible in many high-burden countries like Indonesia, and the yield of M. tuberculosis has been dissappointingly low. More recently, a number of less invasive alternative methods have been proposed, including induced sputum (IS), nasopharyngeal aspiration (NPA) and the string test (Berggren et al., 2004 and Brisson-Noel et al., 1989).

\section{Diagnosis of tuberculosis}

Currently, the diagnosis of pulmonary tuberculosis in children is mainly based on the clinical condition, radiological findings, and results of the Tuberculin Skin Test (TST). However, the clinical picture has low specificity, while the radiological interpretations vary and TST is subjective and can not differentiate between an active infection or previous exposure to $M$. tuberculosis. Various laboratory methods can be done to support the diagnosis of tuberculosis in children. However, there are also some disadvantages that make laboratory methods less effective. Acid-fast stain often gives negative results in children with positive culture results. Cultures from the sputum are good enough to make the diagnosis of pulmonary tuberculosis in children, but it will take quite a long time (Nicol et al., 2011). However the liquid culture system has a significant advantage over solid culture media. In studies on adult patients with tuberculosis, the mean time to detection is subtantially shorter for automatic liquid culture systems (13.2 days by MGIT; Becton Dickinson, compared with typical Lowenstein Jensen (LJ) media with 25,8 days). It is likely that these findings can be extrapolated to children with tuberculosis, although there is paucity of data in children (Brisson-Noel et al., 1989).

Rapid diagnostic tests mycobacteria based on nucleid acid amplification techniques have been developed for direct detection from clinical specimens (sputum induction, SI; nasopharyngeal aspiration, NPA; gastric aspiration, GA; gastric lavage, GL; stool; urine and blood) (Soini and Musser, 2001; Woods, 1999; Gardiner and Beavis, 2000;).

The best-known nucliec amplification test for detection of M. tuberculosis is PCR which first has used the $65-\mathrm{kDa}$ gene as target (Brisson-Noel et al., 1989). 
Eisenach et al. used IS6110 PCR to detect mycobacteria in clinical specimens because this target is a repetitive DNA sequence of the M. tuberculosis complex genome (Eisenach et al., 1991). Many researchers have used this target (IS6110) for PCR to detect M. tuberculosis DNA from clinical samples obtained from adults. More recently, several alternative nucleic acid amplification tests have been published and are commercially available, including PCR-based Amplicor (Roche), ligase chain reaction (Lcx; Abbott), transcription-mediated amplification (TMA; Gen-probe), and strand displacement amplification (BDProbe; Tec-SDA) (18-23). Their high costs and - in some cases - low sensitivities have so far precluded their use in the diagnosis of tuberculosis in children (Montenegro et al., 2003).

The efficacy of PCR-based test varies considerably according to the method used, sampling processing for DNA extraction, the gene target, and the people who run this molecular technology. Other considerations that influence the sensitivity and specificity of the assay are the strict precautions required to prevent contamination, the use of suitable controls, and re-testing of clincal samples with doubtful results. All factors related to the test itself can be optimized, and accurate reproducible results can be obtained under established controlled conditions (Montenegro et al., 2003).

We have optimized the rapid method for detection of M. tuberculosis DNA from GL specimens of children with the diagnosis of suspected tuberculosis. This method is a multiplex allele-specific PCR (MAS-PCR) that could detect mutations in the rpoB, katG, oxyR-inhA and mabA-inhA genes/promoter of M. tuberculosis.

This method is based on a mismatch between a nucleotide on the 3' end of a primer with a mutated nucleotide in the DNA template. Under stringent annealing conditions, the "wild-type" primer will not anneal to the mutated template because the temperature of annealing exceeds the melting temperature of the primer. This will then result in the absence of an amplification product (Mokrousov et al., 2003). The possibility of an unsuccessful amplification reaction, e.g. because of the presence of inhibitors or the absence of a DNA template, can be excluded by the use of internal controls included in the reaction.

The concentration of all reagents, especially the primer and magnesium concentration, and the PCR conditions will be optimized to obtain the successfully generated amplification products. Th primer concentration will be titrated and PCR condition will be assessed using various annealing temperatures until the condition is suitable for amplification of all amplicons. As a guide, we have used formula and conditions described by Yang et al. (2005).

In our study, (unpublished data) we found that from $50 \mathrm{GA}$ samples which were applied to MAS-PCR, we got a positive result for M. tuberculosis in $13(26 \%)$ samples, from which $12(24 \%)$ were tested susceptible, and $1(2 \%)$ resistant to INH. The remaining 37 (74\%) samples were tested negative for M. tuberculosis (NTM). Therefore, gastric aspirates are representative specimens that could be used for the detection of M. tuberculosis in children suffering from tuberculosis. 
Xpert MTB / RIF (Cepheid, Sunnyvale, CA, USA) is a new molecular method with good sensitivity and specificity and can provide the results in less than 2 hours. This method allows the detection of $M$. tuberculosis and multi-drug resistant (MDR) M. tuberculosis.

\section{Xpert MTB/RIF system}

Xpert MTB/RIF system is a molecular-based method using real-time PCR. This method has been recommended by the WHO since December 2010 (Cepheid, 2012 and Theron et al., 2011). This system is useful for the detection of both $M$. tuberculosis and MDR-M-tuberculosis, because it can detect resistance against rifampin (RIF). Almost all processes run automatically, so that manual works needed are minimal. For sure this is very beneficial for laboratories that lack technicians experienced in molecular methods (Cepheid, 2012). This method was designed in such a way that it can be applied directly to a clinical specimen, including purification process, concentrate detection and identification of nucleic acid targets as well as the report result (Cepheid, 2012). Specimens that can be examined are not only respiratory samples (sputum) but also non-respiratory samples (pleural fluid, urine, stool etc.) (Lawn et al., 2011).

\section{Xpert MTB/RIF mechanism}

Principally, the Xpert MTB/RIF system works by detecting M. tuberculosis and resistance against RIF. The detection is done simultaneously by using real-time PCR, with five probes and complementary with the target resistance gene rpoB. A mutation in the amplified rpoB fragment of 81 bp length shows that there is a resistance against RIF. The PCR amplification process is hemi-nested, and the amplified target is detected in real time by six color fluorescent molecular beacons. In short, the specimen examination process is done as follows: the clinical specimen is added to the reagent consisting of $\mathrm{NaOH}$ and isopropanole. After homogenization, the reaction mixture is incubated for 15 minutes at room temperature. This incubation process will decrease the risk of aerosol production and will decrease the viability of M. tuberculosis, so that it will increase the safety of the examiner. After that, the specimen is transferred to the cartridge manually. The cartridge is then inserted to the Xpert MTB/RIF machine. Afterward, the identification will run automatically, starting from purification process, DNA extraction and heminested real-time PCR. All processes run automatically and are integrated within a cartridge without the need of amplicon manipulation, making cross contamination risks unlikely.

The algorithm identifies a sample as M.tuberculosis positive if at least two of the five $\mathrm{rpoB}$ probes are positive within two cycles of each other. A positive result does not necessarily mean that viable M. tuberculosis are present. The machine de- 
tects RIF resistance if there is at least one rpoB probe that does not result in a signal (drop out) or if the difference between the first $r p o B$ signal and the last $r p o B$ signal is 3.5 cycle or more in a cycle threshold. The result of the examination will be displayed on a computer screen: M. tuberculosis negative or positive, semiquantitative M. tuberculosis concentrate estimation (low, medium or high), and RIF sensitive or resistant (Rie et al., 2010).

\section{The time to get result and safety}

Since the examination using the Xpert MTB/RIF method is easy to perform and does not need a Biosafety Cabinet (BSC), it is suitable for almost all laboratories in developing countries, which usually lack a BSC and skillful technicians. The time to get results is less than 2 hours and much shorter than the time of 7-42 days that is required for a culture.

Benada et al did a study aiming to compare the number of aerosols produced in the Xpert MTB/RIF process to those produced while preparating the acid fast staining directly from sputum: if the 15-minutes incubation procedure was done correctly, no bacteria ( $0 \mathrm{CFU} / \mathrm{m}^{3}$ air) were found in the Xpert MTB/RIF procedure, but in the acid fast staining process, $16-325 \mathrm{CFU} / \mathrm{m}^{3}$ air were identified (the measurement was done by using Andersen Impactor/Thermo Scientific, MA, USA) and BioSamplerTN (SKC Inc, PA, USA). These results show that if the Xpert MTB/RIF procedure is done correctly, it can be done safely without using a BSC (Rie et al., 2010).

\section{Xpert MTB/RIF sensitivity and specificity}

The limit for detection of mycobacteria using the Xpert MTB/RIF is 131 bacteria (95\% CI : 106-176)/ml sputum, whereas the limit for detection of M. tuberculosis using culture with liquid media is $10-100 \mathrm{CFU} / \mathrm{ml}$ and using microscopic assessment it is $10,000 \mathrm{CFU} / \mathrm{ml}$. Two studies were done to measure the specificity by using either 20 non-tuberculous mycobacteria (NTM) isolates or 89 different microorganisms (including bacteria, fungi and viruses) with different strains commonly found in the respiratory tract, respectively. In those two studies, Xpert MTB/RIF results were negative in all samples (100\% specificity). Furthermore, to measure the accuracy of RIF resistance detection, a study using DNA genomes of M. tuberculosis isolates with 23 different kinds of mutations within the rpoB gene was done. All these samples could correctly be diagnosed as RIF resistant.

Laboratory-based studies have been done in 4 different laboratories in the USA, France, Spain and the Netherlands. Those studies aimed to test the sensitivity and specificity of Xpert MTB/RIF in sputum samples. The sensitivity of positive smear samples was $98-100 \%$ while negative smear samples had a sensitivity of $57-83 \%$. An assessment of specificity in laboratory-based studies is limited, since 
obtaining further samples and clinical follow-up is often impossible. At the same time, laboratory-based studies were also done in 4 laboratories in Germany, France and Spain aiming to measure the performance of Xpert MTB/RIF to nonrespiratory samples such as pleural fluid, gastric fluid, tissue, cerebrospinal fluid, urine and stool. Based on those studies, the sensitivity was $77.3-99 \%$, while the specificity was $98.2-100 \%$. A similar study done in India resulted in a sensitivity of $81 \%$ and a specificity of $99.6 \%$ (Lawn et al., 2011).

In prior studies, most samples were taken from adult patients. There are not many reports about the Xpert MTB/RIF performance in children with tuberculosis. One of the reports about the accuracy of Xpert MTB/RIF in TB diagnosis in children was done by Nicol and Zar (2011). The result of one of their studies is presented in Table 1.

Tab. 1: Xpert MTB/RIF performance compared to that of microscopic assessment

\begin{tabular}{|c|c|c|c|c|c|c|}
\hline & $\begin{array}{l}\text { Sensi- } \\
\text { tivity }\end{array}$ & $\begin{array}{l}\text { Speci- } \\
\text { ficity }\end{array}$ & PPV & NPV & $\begin{array}{l}\text { Sensitivity } \\
\text { in smear } \\
(+)\end{array}$ & $\begin{array}{l}\text { Spesificity } \\
\text { in smear } \\
(-)\end{array}$ \\
\hline \multicolumn{7}{|c|}{ All children with a complete result from at least one sputum (535) } \\
\hline $\begin{array}{l}\text { Xpert } \\
\text { All }\end{array}$ & $\begin{array}{l}64 / 87 \\
73.6 \% \\
(64.1-83.0)\end{array}$ & $\begin{array}{l}443 / 448 \\
98.9 \% \\
(97.9-99.9)\end{array}$ & 92.8 & 95.1 & $\begin{array}{l}29 / 30 \\
96.7 \% \\
(89.9-100)\end{array}$ & $\begin{array}{l}35 / 57 \\
61.4 \% \\
(48.4-74.4)\end{array}$ \\
\hline $\begin{array}{l}\text { HIV } \\
(+)\end{array}$ & $\begin{array}{l}14 / 15 \\
93.3 \% \\
(79.0-100)\end{array}$ & $\begin{array}{l}102 / 102 \\
100 \% \\
(96.4-100)\end{array}$ & 100 & 99.0 & $\begin{array}{l}9 / 9 \\
100 \% \\
(66.4-100)\end{array}$ & $\begin{array}{l}5 / 6 \\
83.3 \%\end{array}$ \\
\hline $\begin{array}{l}\text { HIV } \\
(-)\end{array}$ & $\begin{array}{l}50 / 72 \\
69.4 \% \\
(58.5-80.3)\end{array}$ & $\begin{array}{l}340 / 345 \\
98.6 \% \\
(97.3-99.8)\end{array}$ & 90.9 & 93.9 & $\begin{array}{l}20 / 21 \\
95.2 \% \\
(85.3-100)\end{array}$ & $\begin{array}{l}30 / 51 \\
58.8 \% \\
(44.8-72.8)\end{array}$ \\
\hline
\end{tabular}

$\mathrm{PPV}=$ positive-predictive balue, $\mathrm{NPV}=$ negative-predictive value

In Indonesia, we do also a study about the performance of Xpert MTB/RIF to detect $M$. tuberculosis in children from induced sputum samples (unpublished data). From that study we found that among 41 smear-negative, culture negative (S-C-) samples, all of them were detected correctly as negative by Xpert MTB/RIF.

\section{References}

Adams, LV., Starkey, JR. (2012). Tuberculosis disease in children. www.uptodate.com/contents/tuberculosis-disease-in-children.

American Thoracic Society. (2000). Diagnostic standards and classification of tuberculosis in adults and children. Am. J. Respir. Crit. Care Med, 161(4 pt 1): 1376-95. 
Bauman, RW. (2009). Microbiology with diseases by body system. 2nd ed. San Francisco, Pearson Education, Inc, 673-4.

Berggren, PI., Gudetta, B., Bruchfeld, J., Eriksson, M., Giesecke, J. (2004).

Detection of Mycobacterium tuberculosis in gastric aspirate and sputum collected from Ethiopian HIV-positive and HIV-negative children in a mixed in - and outpatient setting. Acta Paediatr, 93:311-315.

Brisson-Noel, A., Gicquel, B., Lecossier, D., Levy-Frebault, V., Nassif, X., Hance, AJ. (1989). Rapid diagnosis of tuberculosis by amplification of mycobacterial DNA in clinical samples. Lancet, 4:1069-71.

Cepheid. (2012). Cepheid system. http://www.cepheid.com/systems-andsoftware/systems-overview.

_ (2012). GeneXpert FAQ. http://www.cepheid.com/resources-andsupport/support/technical-faq/genexpert-faq.

Chaulet, P. (1992). Epidemiology of tuberculosis in children. Child Trop, (196197):7-19.

Crevel van, R., Ottenhoff Tom, HM., Van der Meer Jos, WM. (2002). Innate immunity to Mycobacterium tuberculosis. Clin. Microbiol, 15(2): 294-309.

Ehrt, S., Schnappinger, D., Bekiranov, S., Drenkow, J., Shi, S., Gingeras, TR et al. (2001). Reprogramming of the macrophage transcriptome in response to interferon-gamma and Mycobacterium tuberculosis: signaling roles of nitric oxide synthase-2 and phagocyte oxidase. J Exp. Med, 194(8): 1123-40.

Eisenach, KD., Sifford, MD., Cave, MD., Bates, JH., Crawford, JT. (1991). Detection of Mycobacterium tuberculosis in sputum samples using a polymerase chain reaction. Am. Rev. Respir. Dis;144:1160-63.

Engleberg, NC., DiRita, V., Dermody, TS. (2007). Schaechter's Mechanism of Microbial Disease. 4th ed. Philadelphia: Lippincott Williams \& Wilkins, 253-4.

Fenton, MJ., Vermeulen, MW. (1996). Immunopathology of tuberculosis: roles of macrophages and monocytes. Infect. Immun, 64(3): 683-90.

Ferguson, JS., Voelker, DR., McCormack, FX., Schlesinger, LS. (1999). Surfactant protein D binds to Mycobacterium tuberculosis bacilli and lipoarabi-nomannan via carbohydrate-lectin interactions resulting in reduced phagocytosis of the bacteria by macrophages. J. Immunol, 163(1): 312-21. Abstract.

Flynn, JL., Goldstein, MM., Chan, J., Triebold, KJ., Pfeffer, K., Lowenstein, CJ., et al. (1995). Tumor necrosis factor- $\alpha$ is required in the protective immune response against Mycobacterium tuberculosis in mice. Immunity, 2(6): 561-72.

Gardiner, DE., Beavis, KG. (2005). Laboratory diagnosis of mycobacterial infection. Semin. Respir. Infec; 15:132-43. 
Gaynor, CD., McCormack, FX., Voelker, DR., McGowan, SE., Schlesinger, LS. (1995). Pulmonary surfactant protein A mediates enhanced phagocytosis of Mycobacterium tuberculosis by a direct interaction with human macrophages. J. Immunol, 155(11): 5343-51. Abstract.

Krutzik, SR, Modlin, RL. (2004). The role of Toll-like receptors in combating mycobacteria. Semin. Immunol, 16(1): 35-41. Abstract.

Lawn, SD., Nicol, MP. (2011). Xpert巴 MTB/RIF assay: development, evaluation and implementation of a new rapid molecular diagnostic for tuberculosis and rifampicin resistance. Future Microbiol, 6(9): 1067-82.

Levinson, W. (2008). Review of Medical Microbiology and Immunology. United States,The McGraw-Hill Companies, Inc, 164.

Mokrousov, I., et al. (2003). PCR-based methodology for detecting multidrugresistant strains of Mycobacterium tuberculosis Beijing family circulating in Russia. Eur. J. Clin. Microbiol. Infect. Dis. 22(6): p. 342-8.

Montenegro, SH., Gilman, RH., Sheen, P., Cama, R., Caviedes, L., Hopper, T., Chamber, R., Oberhelman, R. (2003). Improved detection of Mycobacterium tuberculosis in Peruvian children by use of a heminested IS6110 polymerase chain reaction assay. Clin. Inf. Dis; 36:16-23.

Nicol, MP., Workman, L., Isaac, W., Munro, J., Black, F., Eley, B., et al. (2011). Accuracy of the Xpert MTB/RIF test for the diagnosis of pulmonary tuberculosis in children admitted to hospital in Cape Town, South Africa: a descriptive study. www.thelancet.com/infection.

Nicol, MP., Zar, HJ. (2011). New specimens and laboratory diagnosis for childhood pulmonary TB: progress and prospects. Paediatr. Respr. Rev.;12(1): 16-21.

Rie, AV., Shipp, LP., Scott, L., Sanne, I., Stevens, W. (2010). Xpert® MTB/RIF for point-of care diagnosis of TB in high-HIV burden, resource-limited countries: hype or hope? Expert Rev. Mol. Diagn, 10(7), 937-46.

Smith I. (2003). Mycobacterium tuberculosis Pathogenesis and Molecular Determinants of Virulence. Clin. Microbiol. Rev, 16(3):463-96.

Soini, H., Musser, JM. (2001). Molecular diagnosis of mycobacteria. Clin. Chem, 47:809-14.

Theron, G., Peter, J., Zyl-Smit, RV., Hridesh Mishra, H., Streicher, E., Murray, S. (2011). Evaluation of the XpertMTB/RIF Assay for theDiagnosis of Pulmonary Tuberculosis in a High HIV Prevalence Setting. Am. J. Respir. Crit. Care. Med, 184:132-40. 
Walls, T., Shingadia, D. (2004). Global epidemiology of paediatric tuberculosis. J. Infect, 48(1):13-22.

World Health Organization. (2006). Chapter 1: Introduction and diagnosis of tuberculosis in children. Int. J. Tuberc. Lung Dis, 10(10):1091-7.

Woods, GL. (1999). Molecular methods in the detection and identification of mycobacterial infection. Arch. Pathol. Lab. Med, 123:1002-6.

Yang, Z., et al. (2005). Simultaneous detection of isoniazid, rifampin, and ethambutol resistance of Mycobacterium tuberculosis by a single multiplex allelespecific polymerase chain reaction (PCR) assay. Diagn. Microbiol. Infect. Dis, 53(3):201-8. 



\section{The Role of the Tuberculosis Laboratory in Supporting the Clinical Examination of Various Specimens of Tuberculosis Patients in North Sumatera, Indonesia}

Kusumawati, R.L, Department of Microbiology, Faculty of Medicine, University of Sumatera Utara, Indonesia

\section{Introduction}

Tuberculosis (TB) is a highly contagious disease which is transmitted through the air. If left untreated, a person with active tuberculosis will infect about 10 to 15 people a year. Globally more than 2 billion people are infected with the causative agent Mycobacterium tuberculosis. Ten percent of those infected will develop tuberculosis disease during their lifetime; the highest risk groups are those living in poverty, malnourished, or with a weakened immune system (i.e. HIV and other diseases). Indonesia is ranked fourth in the global tuberculosis burden. The five countries with the largest number of incident cases in 2010 were India, China, South Africa, Indonesia and Pakistan (Figure 1).

Indonesia has an estimated total prevalence of 690,000 or 289 per 100,000 population and an estimated annual incidence of 450,000 new cases per year (189 
per 100,000 population). The annual mortality of tuberculosis is approximately 64,000 deaths (WHO Global Tuberculosis Control 2011).

Directly Observed Treatment Short course (DOTS) is a key component of the Stop TB Strategy. Ensuring early case detection and diagnosis through qualityassured bacteriology is the second key element of tuberculosis control emphasized in the DOTS strategy. Thus, laboratory services should be fully integrated within the tuberculosis control program, which in turn should form an essential part of the overall primary health programs in the countries.

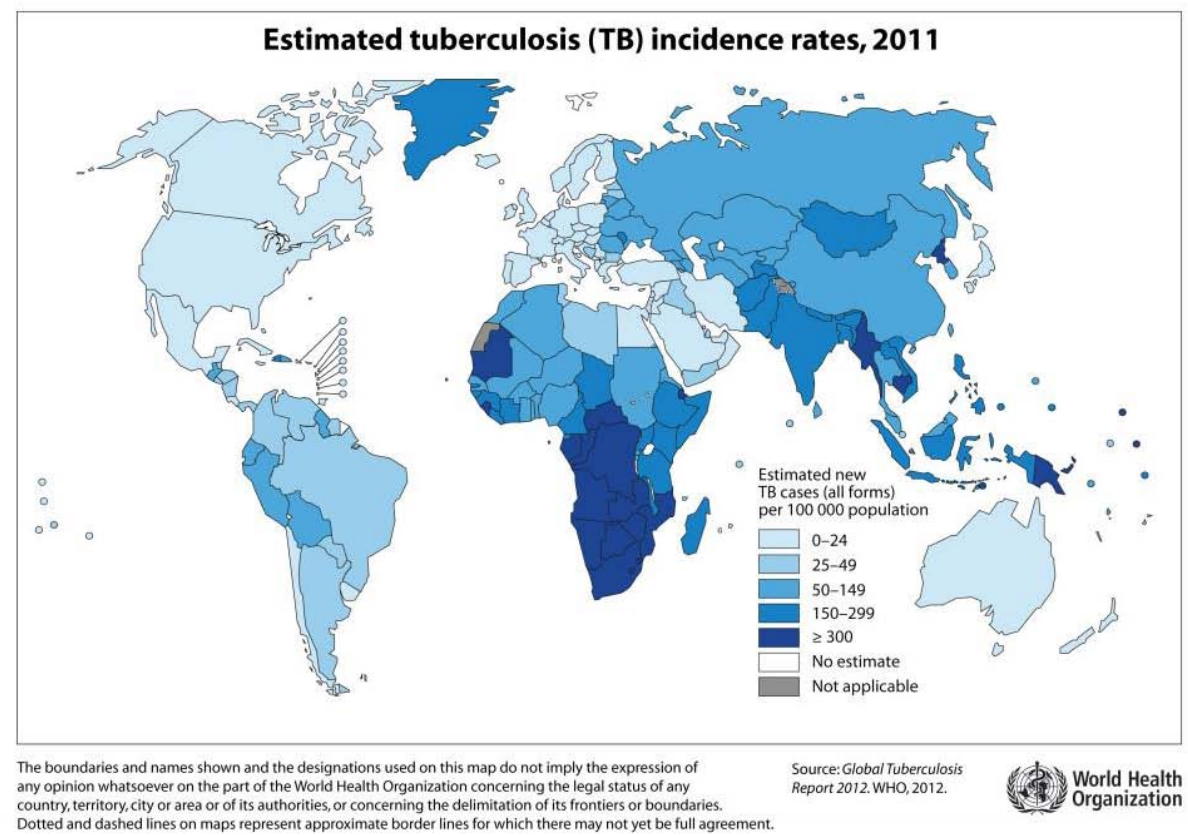

Figure 1. High tuberculosis-burden countries

Source: (WHO Global Tuberculosis Report, 2012)

\section{MDR-TB Laboratory}

Multidrug-resistant $M$. tuberculosis (MDR-TB) was defined resistant to two first-line drugs: isoniazid and rifampicin. Drug resistance in M. tuberculosis is attributed primarily to the accumulation of mutations in the drug target genes, these mutations lead either to an altered target or to a change in the titration of the drug (Rattan et 
al., 1998). Indonesia ranks number 9 on the list of the 27 high MDR-TB burden countries (WHO Global TB Control, 2011).

One of the greatest threats to the global control of tuberculosis is the growing prevalence of drug-resistant bacilli (Pablos-Mendez et al., 1998). For MDR-TB patients, WHO recommends a treatment of at least 20 months with a regimen that includes second-line antimycobacterial drugs.

In Indonesia, the total numbers of estimated MDR-TB cases in 2010 were 6,100 cases per year. The spread of MDR-TB is a challenging problem. The Indonesian National Tuberculosis Program is currently implementing the Programmatic Management of Drug-Resistant Tuberculosis (PMDT) in several sites to increase the number of diagnosed MDR-TB patients as well as the treatment with plans to scale up additional sites over the coming few years.

However, there are still many other remaining challenges in the program. Thirty percent of the estimated tuberculosis cases are still not being detected and many patients are diagnosed too late. Only a small portion of the estimated number of MDR-TB cases is under treatment. There are limitations in the health system including laboratory quality, human resources, logistics and data quality.

The basic package of DOTS for control of tuberculosis has been expanded to include components that address additional challenges such as the handling of $M$. tuberculosis/HIV co-infection and multidrug-resistant tuberculosis; contributions to the health-system strengthening, engagement of all care providers, empowerment of patients and communities, as well as enabling and promoting research. The emphasis on expanding laboratory capacity (smear microscopy first, followed by culture/drug sensitivity testing) and the use of quality-assured drugs are important parts of this more comprehensive approach to tuberculosis control (Central TB Division, New Delhi, 2010) .

Diagnosis and treatment of tuberculosis is provided in specialized facilities but until now the management of MDR-TB represents a serious problem.

The central MDR-TB laboratory at H. Adam Malik General Hospital in Medan District, North Sumatera Province is part of the PMDT and the 2010-2014 Indonesian National Tuberculosis Program Strategic Plan. The laboratory uses Bio Safety Level $2+$ standard with anteroom, weather vestibule, cooling and ventilation facilities (heating ventilating air-conditioning-HVAC) and air filters (HEPA filter), completed with control equipment for the negative pressure system. This laboratory's primary function is the management of MDR-TB cases.

Tuberculosis typically affects the lungs (pulmonary tuberculosis) but it can also affect other organs (extrapulmonary tuberculosis) such as pleura, lymph nodes, genitourinary tract, meninges, skin, abdomen, joint and bones. Acid-fast bacilli (AFB) smear microscopy has been assessed by the Ziehl-Neelsen staining method, followed by culture/drug sensitivity testing.

GenXpert instruments and Xpert MTB/RIF cartridges have been used for deciding on appropriate treatment and care of tuberculosis, MDR-TB and TB/HIV patients. Xpert MTB/RIF test can detect $M$. tuberculosis as well as mutations, pre- 
senting resistance to rifampicin directly from the sputum in the assay that provides results within 2 hours.

\section{Anti-tuberculosis drug resistance patterns in H. Adam Malik General Hospital, 2011.}

This study examined the susceptibility patterns of drug-resistant tuberculosis cases in H. Adam Malik General Hospital, a tertiary care hospital including a DOTS TB center and Voluntary Counseling and Testing (VCT) facility for HIV prevention. Mycobacterium tuberculosis was retrospectively identified through drug susceptibility test (DST) and clinical data were analyzed for a period of one year from January to December 2011. Samples were obtained from sputum, bronchoalveolar lavage, pleural fluid, pus, ear swab, biopsy, gastric lavage and cerebrospinal fluid samples of the patients with suspected tuberculosis. All samples were cultured in Lowenstein-Jensen medium and followed by DST according to the Indirect Proportion Method. Of 2,221 samples, 225 (10.1\%) were AFB smear-positive and 311 (14\%) were culture positive. Of these culture-positive samples, 290 (93.2\%) were identified as M. tuberculosis and 21 (6.8\%) were Mycobacterium Other Than Tuberculosis (MOTT) (Table 1). SD TB Ag MPT64 and Niacin rapid test were used to differentiate $M$. tuberculosis complex bacteria from MOTT among culture-positive samples.

For this report, the laboratory results from 266 M. tuberculosis isolates were further analyzed.

Table 1: AFB smear and culture examination results from all specimens in H. Adam Malik General Hospital, 2011.

\begin{tabular}{|l|c|}
\hline Examination & No. $(\%)$ samples; $\mathrm{n}=2,221$ \\
\hline AFB smear positive & $225(10.1 \%)$ \\
AFB culture positive & $311(14.0 \%)$ \\
\hline Species identification & $290(93.2 \%)$ \\
- M. tuberculosis & $21(6.8 \%)$ \\
- Mycobacterium Other Than & \\
Tuberculosis (MOTT) & \\
\hline
\end{tabular}

Among the 266 M. tuberculosis isolates, $146(55 \%)$ were sensitive to all first-line antimycobacterial agents (isoniazid, rifampicin, streptomycin and ethambutol), 59 $(22 \%)$ were mono-resistant, $44(17 \%)$ were poly-resistant and $17(6 \%)$ were MDRTB (Table 2). Eight (47\%) of the MDR-TB cases were new patients. 
Table 2: Antimycobacterial drug resistance patterns in H. Adam Malik General Hospital, 2011.

\begin{tabular}{|l|c|}
\hline $\begin{array}{l}\text { Drug susceptibility test pattern against } 1^{\text {st }} \text { line } \\
\text { antimycobacterial agents }\end{array}$ & No. (\%) of isolates, $\mathrm{n}=266$ \\
\hline Sensitive & $146(55 \%)$ \\
\hline Monoresistant & $59(22 \%)$ \\
\hline Polyresistant & $44(17 \%)$ \\
\hline Multidrug-resistant TB (MDR-TB) & $17(6 \%)$ \\
\hline
\end{tabular}

Seventy percent of tuberculosis patients were male; $97 \%$ were $>18$ years of age, and $26 \%$ were receiving retreatment for tuberculosis (Table 3 ).

Table 3: Demographic data and history of antimycobacterial treatment of patients suffering from tuberculosis, H. Adam Malik General Hospital, North Sumatera, January-December 2011.

\begin{tabular}{|l|c|}
\hline Patient data & $\begin{array}{l}\text { No. }(\%) \text { of patients with culture-positive tuberculosis } \\
(\mathrm{n}=311)\end{array}$ \\
\hline Sex & $218(70 \%)$ \\
Male & $93(30 \%)$ \\
Female & $9(3 \%)$ \\
\hline Age, $y$ & $302(97 \%)$ \\
$<18$ & \\
$\geq 18$ & $230(74 \%)$ \\
\hline History of TB treatment & $81(26 \%)$ \\
New case & \\
Retreatment case & \\
\hline
\end{tabular}

Generally, lower case notifications among women suggest that they may be at lower risk of acquiring tuberculosis than males. Given the known barriers that many women face when accessing healthcare, it is important to verify if woman are truly at lower risk of acquiring tuberculosis, or if they are less likely to be diagnosed. Moreover, the increasing feminization of the HIV epidemic potentially increases the number of women and children with HIV/TB co-infection (WHO Tuberculosis: Women and TB, 2009).

HIV co-infection is the most powerful known risk factor for susceptibility to M. tuberculosis infection and progression to active disease. Of 116 sputum samples of HIV patients in VTC during six months from January to June 2011 showed that $20(17 \%)$ were smear-positive. HIV-infected tuberculosis cases are less likely to be smear-positive than those cases uninfected with HIV, and that the illness typically progresses more quickly, so that the infectious period before diagnosis is shorter. 
Reporting DST result is an important part for the correct management of patients' therapy. A rapid communication of resistance patterns, for example, will allow the prompt switch from ineffective therapy to an effective one.

Without early diagnosis and effective treatment, these new cases will continue to generate further infections in the community. North Sumatera Province must therefore urgently scale up prevention measures for MDR-TB through better infection control, enhancement of directly-observed treatments, universal use of appropriate diagnosis and treatment. Because MDR-TB is a laboratory diagnosis, the central laboratory as the highest level of the service should be capable of performing microscopy, mycobacterial culture, and drug susceptibility testing as well as species identification. Early detection, effective treatment and infection control measures for MDR-TB are needed to reduce transmission.

Continuous surveillance of drug-resistant tuberculosis and better case management are necessary to reduce the high prevalence of drug-resistant tuberculosis and drug-resistant TB/HIV in the country.

\section{References}

Central TB Division, Directorate General of Health Services, Ministry of Health \& Family Welfare, Nirman Bhavan, New Delhi (2010). Revised National Tuberculosis Control Programme DOTS-Plus Guidelines.

Pablos-Mendez A et al (1998). Global surveillance for antituberculosis-drug resistance, 1994-1997. N Engl J Med,338,1641

Rattan A, Kalia A, Ahmad N (1998). Multidrug-resistant Mycobacterium tuberculosis: molecular perspectives. Emerg Infect Dis, 4,195

World Health Organization (2009) Tuberculosis: Women and TB

World Health Organization (2011) Global TB Control.

World Health Organization Report (2012) Global tuberculosis report. 


\section{Buruli Ulcer: A Disease which Leads to Severe Disabilities and Mostly Affects Children Living in Wet Agro-Ecosystems of West and Central Africa}

Solange Kakou-Ngaroa; Tchigossou Genevieve; Kamgang Guy Richard; Sownnmi Akindayo; Yessoufou Akadiri; Adeoti Razack; Fening Opoku Joseph; Goergen Georg; Tamò Manuele; Djouaka Roussean

The Agro-Eco-Health Platform for Buruli Ulcer Control in West and Central Africa. International Institute of Tropical Agriculture, Benin, West Africa (Corresponding author:Djonaka Rousseau)

\section{Introduction- What is Buruli Ulcer?}

Buruli Ulcer (BU) is a devastating skin disease with serious necrotizing cutaneous infection caused by Mycobacterium ulcerans. BU is the most common mycobacterium infection of humans after leprosy and tuberculosis. The disease affects rural poor people living or working close to rivers and stagnant water. About $70 \%$ of those affected are children under the age of 15 years. The start of the illness is painless 
while the bacteria invade further into the skin and soft tissue without any warning signs and then even attacks bones. When the ulcer becomes more prominent and if it is not adequately treated through effective antibiotics, surgically removal of the damaged tissue followed by skin transplants and eventually amputations are necessary. The healing process is considered a continuation of the suffering which can be associated with serious handicaps. What remains are most of the time severely disabled children who require long treatment and continuously bear the stigma of disability for the rest of their lives.

$\mathrm{BU}$ is a disease less documented and most neglected with high prevalence in wet agro-ecosystems of West and Central Africa. Reported cases range from 900 in Cameroon to 11,000 in Ghana and 28,000 in Côte d'Ivoire.

\section{The prevalence of Buruli Ulcer in West and Central Africa}

Well identified and described in 30 countries in the world, the prevalence and the incidence of $\mathrm{BU}$ varies significantly where it occurs but remains relatively high in West and Central Africa making these two regions the most affected in the world. The National Program for Buruli Ulcer Control in Côte d'Ivoire reports 28,000 cases with an average of 2,000 new cases every year, making Côte d'Ivoire the most endemic country in the world.

In Ghana, results of a national Buruli Ulcer survey, published in 2002, identified 5,619 patients with 6,332 clinical lesions at various stages. The overall crude national prevalence rate of active lesions was 20.7 per 100,000 but the rate was 150.8 per 100,000 in the most disease-endemic district. The case search in endemic areas demonstrated widespread disease and gross underreporting compared to the routine reporting system.

In the endemic locality of Lalo in southwestern Benin, a total of $752 \mathrm{BU}$ patients were detected during a study in 2005. Of these patients, 160 were active cases making an overall extreme prevalence of 86.6 per 10,000 inhabitants for this locality.

In Cameroon, national data for 2005 revealed a total number of notified cases of 932 patients. In total, 914 new cases were reported with 209 cases being recorded in the most endemic locality of Ayos in South Cameroon.

The world report on BU incidence indicates a total of 5,000 new cases per year with a cumulative number of 60,000 cases found for the African continent in 2008. The report also highlighted underreporting of numerous cases in most countries due to low diagnostic facilities in most endemic localities. 


\section{BU-induced disabilities}

According to the World Health Organization, a disability is defined as any longterm limitation in activity resulting from a condition or health problem. A disability could be associated either with a partial or a full limitation of movements.

$\mathrm{BU}$ causes extensive destruction of the skin and soft tissues and patients who are not treated early enough are often left with severe scarring and with long-term disabilities. About a quarter of patients are left with permanent disabilities. In Africa, $25 \%$ of BU lesions are recorded on upper limbs, $63 \%$ on lower limbs and on other parts of the body such as head, neck regions, chest and breast.

A study conducted in Ghana analyzed the appearance of BU ulcers on head and neck regions. This study revealed that most patients $(55.3 \%, N=21)$ with $\mathrm{BU}$ lesions on the head or neck regions present deformities such as stiffness of the neck $(N=4)$, loss of part of the ear $(N=2)$, loss of part the nose $(N=1)$, loss of part of the lower lips $(N=2)$, loss of the eyeball $(\mathrm{N}=5)$, loss of the eyelid $(\mathrm{N}=4)$, and baldness $(N=3)$ at the frontal part of the head.

$\mathrm{BU}$ disabilities are generally accompanied with severe scars due to surgeries. These scars cause psychological effects (e.g., low self-esteem, shyness, and inferiority complex) which can affect the victims in their education (especially in children), marriage, and work. Particularly on the face, plastic surgery for BU is more challenging than applied to other body parts. Establishing surveillance teams in remote endemic localities is necessary for rapid diagnosis and a prompt management of $\mathrm{BU}$ for the prevention of disabilities.

\section{Agricultural intensification and resulting human diseases such as BU}

Wetlands in West Africa have recently been targeted for increased food security as they appear to become new pathways to poverty reduction through increased agricultural productivity and higher food production. However, the intensification of agriculture in wetlands is accompanied by inappropriate practices such as poor water management, inappropriate land use and inadequate use of agricultural inputs which have resulted in a significant disruption of the local agro-ecosystems and a poor supply of ecosystem services. (i) The aggressive soil use (excessive tillage) in farming systems such as maize, roots and tubers has increased water beds in agricultural settings and has disturbed the natural fauna and flora capable of biocontrolling some vectors and reservoirs of human diseases. (ii) Poor irrigation systems and excess watering in vegetable and rice farming have increased the level of standing water bodies and favored the development of vectors and reservoirs of several human diseases (e.g. malaria, schistosomiasis, filariasis). (iii) and finally, misuse and overuse of agricultural inputs such as fertilizers and pesticides have 
increased the selection of population of insects capable of withstanding lethal doses of insecticides used for vector control in public health.

Several published papers have revealed associations between the intensification of agriculture in lowlands and the increased incidence of human diseases such as malaria, schistosomiasis, filariasis, and BU. To date we do know that BU transmission is associated with changes in land use, and exacerbated by poor agricultural practices. But what remains unknown is the mechanism through which humans get infected with $M$. ulcerans, the bacteria responsible for $\mathrm{BU}$. To solve this cross cutting problem, studies need to be conducted by multidisciplinary teams of medical, environmental, agricultural and social scientists for clearly analyzing the contributions of poor agricultural practice to the increased incidence of $\mathrm{BU}$ as well as developing innovative, cost effective and environmentally friendly technologies for mitigating BU risks exposures during farming activities.

\section{BU social rejection and economic cost}

$\mathrm{BU}$ is associated with disabilities, disfiguration and large lesions which in most cases stigmatize the patient and prevent him from undergoing treatments. As a consequence, most patients seek treatment too late, and both the direct and indirect costs for managing $\mathrm{BU}$ are considerable. The long hospital stay, often more than three months per patient, represents a huge loss in productivity for adult patients and family caregivers, and the loss of educational opportunities for children. The long-term care of those disabled, most of whom are children aged 15 years, place an additional costly load on affected families.

BU imposes a serious economic burden on affected households and on health systems that are involved in diagnosing the disease and treating patients. For example, in Ghana in 2001-2003, the mean annual total costs of BU for a household by stage of disease ranged from US\$ 76.20 (16\% of a work-year) per patient with a nodule to US\$ 428 ( $89 \%$ of a work-year) per patient who had undergone amputation. The average cost of treating a BU case was estimated to be US\$ 780 per patient in 1994-1996, an amount which far exceeded per capita government spending on health.

In Benin, a survey conducted in rural communities revealed that healing of BU by traditional practitioners range from US $\$ 40$ to US $\$ 120$ excluding surgeries.

In Cameroon, studies on the economic cost of BU have revealed that $25 \%$ of annual earning of household is spent on each patient suffering from BU. This cost burden clearly surpasses the cost threshold of $10 \%$, which has shown to be catastrophic for a household economy and likely leads to further impoverishment.

In Côte d'Ivoire, the typical cost of treatment can exceed $\$ 400$ a month and may last for 2 to 3 month. This cost is economically devastating in a region where many people live on less than $\$ 2$ a day. 
Understanding the transmission pattern of $\mathrm{BU}$ will facilitate the prevention of the disease and will reduce related expenses and the burden on the household income.

\section{Understanding the transmission of BU - a puzzle with several uncovered pieces}

BU transmission is poorly understood and reportedly coupled with rapid environmental changes to the landscape and exacerbated by poor agricultural practices. In rural agricultural areas, some hypothesis highlight that (i) inappropriate intensification of agricultural practices such as excess tillage allows $M$. ulcerans to move from the internal soil layers to the surface soil then after; (ii) the bacteria brought at the surface soil is extensively spread in the environment through poor watering systems in irrigated agriculture.

Although the disease can be successfully managed at clinical level if detected early enough, one of the missing pieces of the puzzle is to understand how $M$. ulcerans is transmitted from the environment to humans. This missing element is crucial to identify the main exposure factors, and analyze the potential vectors/hosts implicated in the transmission of the disease. The clear understanding and description of the BU transmission mechanism will be a strong scientific advancement which will help developing programs for preventing the disease. It is also certain that the combination of a BU preventive package to existing BU management strategies will foster the control of this disease also known as "the mysterious disease" in most West African communities.

\section{BU - an orphan disease}

BU is considered as a neglected or orphan disease because of its confined geographical occurrence which results in low interest to funding bodies and a relatively low attraction to policy makers. Malaria has been long considered the most important target disease in Africa, and is still attracting a lot of attention because of its morbidity. However, most people ignore that BU significantly reduces farmer's productivity in wet agro-ecosystems and affects the schooling rates of children, endangers household's financial stability, and jeopardizes family's livelihoods in several West and Central African rural communities. We advocate that funds have to be raised for a better understanding of the diseases transmission and for the implementation of a sustainable BU preventive program in areas of endemicity. Considering the fact that $\mathrm{BU}$ is exacerbated by the intensification of agriculture for food security, it will increasingly become necessary to mobilize funds for joint research activities conducted by a multidisciplinary team of health, environment, agriculture and social scientists to better understand and mitigate risks associated 
with poor agricultural practices and increased $\mathrm{BU}$ incidence in wet agroecosystems.

\section{Funding research on BU transmission and prevention}

An encouraging initiative focussing on the study of $\mathrm{BU}$ transmission has recently been launched in West and Central Africa namely "The AgroEcoHealth systems thinking initiative for fighting BU". The initiative brings together several scientists with different and complementary background knowledge to research on the identification of risk factors associated with BU transmission in wet-agro-ecosystems and analyse agricultural practices likely to favour the spread of $M$. ulcerans and the incidence of $\mathrm{BU}$ in wet agro-ecosystems. Target activities are gradually being implemented in selected pilot countries with relatively high prevalence of BU such as Benin, Côte d'Ivoire, Ghana and Cameroon. However, the implementation rate of planned activities remains unacceptably slow due to limitations in financial resources and the poor support of funding bodies.

We conclude that such an initiative which is focused on the control and prevention of the disease for containing new cases of $\mathrm{BU}$ calls for adequate resource mobilisation to meet the needs of a 5 years strategic program which has been budgeted with an estimated 1.7 million USD.

\section{References for further readings}

1. Ajoulat I, Johnson C, Zinsou C, Guédénon A, Portaels F (2003) Psychosocial aspects of health seeking behaviors of patients with Buruli ulcer in southern Benin. Trop Med and Int Health 8(8): 750-759.

2. Yadouleton AWM, Asidi A, Djouaka RF, Braïma J, Agossou CD, Akogbeto MC (2009). Development of vegetable farming: a cause of the emergence of insecticide resistance in populations of Anopheles gambiae in urban areas of Benin. Malaria J 8: 103 doi:10.1186/1475-2875-8-103.

3. Boelee, E, Madsen H (2006) Irrigation and schistosomiasis in Africa: Ecological aspects. Colombo, Sri Lanka: International Water Management Institute. 39p. (IWMI Research Report 99).

4. Walsh DS, Portaels F, Meyers WM (2010) Recent advances in leprosy and Buruli ulcer (Mycobacterium ulcerans infection). Curr Opin Infect Dis 23: $445-455$.

5. Duker AA, Portaels F, Hale M (2006) Pathways of Mycobacterium ulcerans infection: A review. Environ Int 32: 567-573. 
6. Hayman J, Asiedu K (2000) Epidemiology. In: Asiedu K, Scherpbier R, Raviglione M, editors. Mycobacterium ulcerans infection. Geneva, Switzerland: World Health Organization. pp. 9-14.

7. Marsollier L, Robert R, Aubry J, Saint André JP, Kouakou H, Legras P, Manceau AL, Mahaza C, Carbonnelle BSO (2002). Aquatic insects as a vector for Mycobacterium ulcerans. Appl Environ Microbiol 68(9): 4623.

8. Matthys B, Tschannen AB, Tian-Bi NT, Comoé H, Diabaté S, Traoré M, Vounatsou P, Raso G, Gosoniu L, Tanner M, Cissé G, N'Goran EK, Utzinger J (2007). Risk factors for Schistosoma mansoni and hookworm in urban farming communities in western Côte d'Ivoire. Trop Med Int Health 12(6): 709-723.

9. Merritt RW, Walker ED, Small PLC, Wallace JR, Johnson PDR, et al. (2010) Ecology and transmission of Buruli Ulcer disease: A systematic review. PLoS Negl Trop Dis 4(12): e911.

doi:10.1371/journal.pntd.0000911.

10. Noeske J, Kuaban C, Rondini S, Sorlin P, Ciaffi L, Mbuagbaw J, Portaels F, Pluschke G (2004) Buruli Ulcer disease in Cameroon rediscovered. Am J Trop Med Hyg 70(5): 520-526.

11. Peeters Grietens K, Um Boock A, Peeters H, Hausmann-Muela S, Toomer E, et al. (2008) "It is me who endures but my family that suffers": Social isolation as a consequence of the household cost burden of Buruli Ulcer free of charge hospital treatment. PLoS Negl Trop Dis 2(10): e321. doi:10.1371/journal.pntd.0000321.

12. Porten K, Sailor K, Comte E, Njikap A, Sobry A, Sihom F, Meva'a A, Eyangoh S, Myatt M, Nackers F, Grais RF (2009). Prevalence of Buruli ulcer in Akonolinga health district, Cameroon: results of a cross sectional survey. PLoS Negl Trop Dis 3(6): e466.

13. Prescott N (1999) Coping with catastrophic health shocks. Washington: Inter American Development Bank. Conference on Social Protection and Poverty.

14. Ranson K (2002) Reduction of catastrophic health care expenditures by a community-based health insurance scheme in Gujurat India: current experiences and challenges. Bull WHO 80: 613-621.

15. Johson RC, Makoutode M, Hougnihin R, Guedenon A, Ifebe D, Boko M, Portaels F (2004). Le traitement traditionnel de l'ulcere de buruli au Benin. Med Trop 64 : 145-150.

16. Djouaka RF, Bakare AA, Coulibaly ON, Akogbeto MC, Ranson H, Hemingway J, Strode C (2008). Expression of the cytochrome P450s, 
CYP6P3 and CYP6M2 are significantly elevated in multiple pyrethroid resistant populations of Anopheles gambiae s.s. from Southern Benin and Nigeria. BMC Genomics 2008, 9:538 doi:10.1186/1471-2164-9-538.

17. Russell S (2004) The economic burden of illness for households in developing countries: A review of studies focusing on malaria, tuberculosis, and immunodeficieny virus/acquired immunodeficiency symdrom. Am J Trop Med Hyg 71: 147-155.

18. Stienstra Y, van der Graaf WT, Asamoa K, van der Werf TS (2002) Beliefs and attitudes toward Buruli Ulcer in Ghana. Am J Trop Med Hyg 67: 20713.

19. Wagner T, Benbow ME, Burns M, Johnson RC, Merritt R, et al. (2008) A landscape-based model for predicting Mycobacterium ulcerans infection (Buruli Ulcer disease) presence in Benin, West Africa. EcoHealth 5: 69-79.

20. WHO, BU Resolution WHA57.1 Surveillance and control of Mycobacterium ulcerans disease (Buruli ulcer). In: Fifty-seventh World Health Assembly, Geneva, 17-22 May 2004. Resolutions and decisions. Geneva, World Health Organization, 2004 (WHA57/2004/REC/1):1-2.

21. WHO, Book of abstract, annual meeting on Buruli Ulcer 14-17March 2005 - WHO headquarter Geneva, Switzerland.

22. WHO, Book of abstract, annual meeting on Buruli Ulcer March 2011 WHO headquarter Geneva, Switzerland.

23. World Health Organization. Buruli Ulcer disease. 2007. Available at: http://www.who.int/mediacentre/factsheets/fs199/en/print.html (Accessed on April 30, 2010). 


\section{Arboviral Infection during Pregnancy and Childhood: Implications for the Mother and Child}

Eric Bertrand Fokam, Department of Zoology and Animal Physiology, University of Buea, Cameroon

Yolande Puepi Djike, Department of Paediatrics, Faculty of Medicine and Biomedical Sciences, University of Yaoundé I, Cameroon

\section{Introduction}

A virus is an obligate intracellular infective agent that typically consists of a nucleic acid in a protein coat. The term virus originates from Latin language meaning "slimy liquid or poison" (Hughes, 1977). These agents are extremely diverse and can infect virtually any kind of cellular organisms, and several species are known to affect human health. Walter Reed and his team were the first to recognise a viral infection of humans in Cuba, the yellow fever virus (Reed et al., 1901 a,b). Since then, viruses have been incriminated as a cause of several diseases in humans, often occurring as massive outbreaks or being endemic in certain regions. They have sometimes caused high mortality and severe morbid conditions, due to their ability 
to induce cellular proliferation or provoke cellular destruction (Levine, 2001). In 1941, the rubella virus was the first virus blamed for inducing the occurrence of a congenital disease characterized by cataract in a child whose mother got infected during pregnancy (Gregg, 1941). When a viral infection occurs during pregnancy, concerns about the effects on the foetus certainly equates with attention to the health of the mother (Hurley, 1974; Hanshaw and Dudgeon, 1978). Some arboviruses such as the dengue viruses (DENVs), American equine encephalitis viruses, the Crimean-Congo haemorrhagic fever virus (CCHFV) and the Colorado tick fever virus (CTFV) cause foetal maternal infections (Chastel, 1999). Waterson (1979) believes that the foetus is seldom infected. However, when vertical transmission eventually occurs, the results may be catastrophic. Infection may also occur at delivery if the mother is viraemic.

Arboviruses (arthropod-borne viruses) are "viruses that are maintained in nature principally, or to an important extent, through biological transmission between susceptible vertebrate hosts by haematophagous arthropods; they multiply and produce viraemia in the vertebrates, multiply in the tissues of arthropods, and are passed on to new vertebrates by the bites of arthropods after a period of extrinsic incubation" (Calisher et al., 1986). Mosquitoes are the main vectors for most arboviruses, although other biting flies, midges, and ticks may also transmit the disease (Calisher, 1994). Humans are considered to be incidental host (dead-end) because they often do not produce significant viraemia to contribute to the transmission cycle. Figure 1 recapitulates the major events involved in a typical arbovirus transmission cycle.

Of the almost 600 species of arboviruses described, only about 100 have been found to infect humans worldwide (Beaty et al., 1995). Agents of major diseases of public health significance belong to the families of Togaviridae (genus Alphavirus), Flaviviridae (genus Flavivirus), and Bunyaviridae (genera Phlebovirus and Nairovirus). Although the public health significance of this group of viruses is globally recognised, little is known of their potential for vertical transmission to the foetus or as teratogens (Tsai, 2006). The recognition of congenitally acquired infections may have been obscured in developing countries in general and in Africa in particular by high infant mortality rates, but perhaps as importantly, where these infections are transmitted in an endemic pattern, acquired immunity may limit infections in females by the time they reach childbearing age.

In addition, different arboviruses infect different patients in relation to the age: for instance Western equine encephalitis (WEE) and Easter equine encephalitis (EEE) are illnesses of infants and the elderly; Saint Louis encephalitis is rare in children, and La Crosse (LAC) causes paediatric disease (Aghar, 1995). While infection with some of these agents is generally self-resolving, early childhood infection may have dramatic consequences for the patients, with uncertain prognosis. As with other tropical diseases, the disabling sequelae of arboviruses disproportionately affect resource-poor communities through chronic impairment of individual performance in activities of daily living (Labeaud, 2008). Long-term longitu- 
dinal studies of the impact of childhood arbovirus infections are few in number, particularly for post-encephalitic complications, meaning that the lifetime burden of these infections may remain poorly understood for long (Labeaud et al., 2011).

\section{Arbovirus Transmission Cycle}

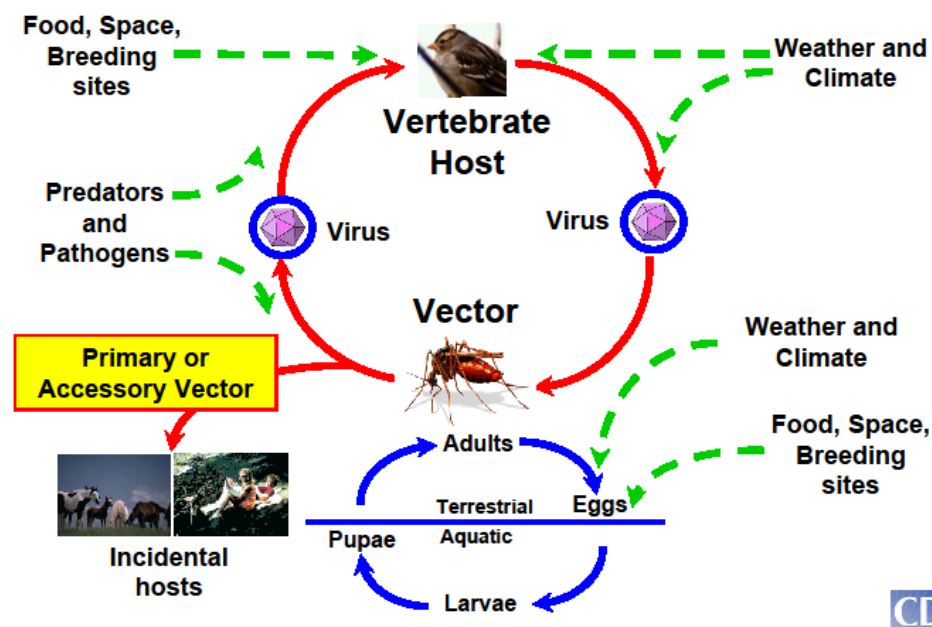

Figure 1: Typical arbovirus transmission cycle (www.cdc.gov/ncidod/dvbid/arbor/schemat.pdf)

\section{Alphaviral infections}

\subsection{Chikungunya virus infections}

Prior to the sudden 2005-2007 explosive emergence of the chikungunya virus (CHIKV) in the Indian Ocean and subcontinent, especially on the islands of La Réunion and Mayotte (Paquet et al., 2006), there had been no reports of mother to child transmission of this virus. In actual fact, this alphavirus was considered a minor pathogen, often overlooked and mistaken as a variant of the dengue virus (DENV) (Freeman, 1985). Its ability to cause fatal encephalitis in children was however recognised (Carey et al., 1969). The outbreak of La Réunion was immense, affecting the whole territory, infecting a third of its population (about 800,000 inhabitants), revealing previously unknown symptoms of the disease and displaying unusual severity and mortality (Brücker et al., 2007; Enserick, 2007; Fritel et al., 2010).

CHIKV infection in pregnant women does not seem to present specific gravity for the health of the mother (Tran and Chastel, 2008; Fritel et al., 2010), however 
may have a serious impact on the outcome of the pregnancy, and maternal-foetal transmission of the virus has been demonstrated, sometimes with dramatic effect. Retrospective studies of cohorts of pregnant women infected with the CHIKV on La Réunion have revealed among other manifestations of the disease increased miscarriage rates, abnormally high rates of caesarean section for foetal distress, and mother to child transmission of the virus confirmed by placental or cerebral detection of the virus in the brain of the neonates (Tran and Chastel, 2008). Malformation of the foetuses however did not occur (Lenglet et al., 2006). For women whose pregnancies ended before 22 weeks of gestation virally-induced miscarriage was diagnosed, and confirmed by reverse-transcriptase polymerase chain reaction (RT-PCR) in the amniotic fluid, placenta and brain of some foetuses. In pregnancies terminated after the 22nd week, caesarean section was more often performed to rescue the foetus (Table 1). The caesarean section however did not protect the newborns from infection with CHIKV from their mothers (Gerardin et al., 2007). Congenital CHIKV infection in newborns manifests with observable clinical symptoms between the 3rd and 7th day post-partum with inconstant fever, prostration, pain, inability to breastfeed, rash and swelling on the limbs (Touret et al., 2006a). Complications in the babies include seizures, severe thrombocytopaenia and disseminated intravascular coagulation (DIC) (Touret et al., 2006a, 2006b).

Chikungunya appears to be mostly a disease of the elderly, with age above 60 years and underlying medical conditions as independent risk factors for severe complications and age above 85 years as the only risk factor for mortality (Tandale et al., 2006; Chia et al., 2010). However, the La Réunion epidemic was particular instructive for understanding manifestations of CHIK. Major symptoms displayed by children were essentially neurological (Table 2). Onset of these neurological symptoms in children is usually early, within 24 hours after onset of fever (Arpino et al., 2009). In a 2006 retrospective hospital-based study on 30 children, the more common neurological complications with infection included encephalitis $(40 \%)$, febrile fits (33\%), meningitis (13\%) and acute encephalopathy (13\%) (Robin et al., 2008). Other neurological complications were encephalomyelitis, Guillain-Barré syndrome, acute flaccid paralysis, optic neuritis and encephalomyeloradiculitis. Mortality was around $10 \%$ in a review focusing on the LaRéunion outbreak (Tyler, 2009).

\subsection{Other alphaviral infection}

Transplacental infection with alphaviruses has previously been demonstrated with the Ross River virus (RRV). During an outbreak of RRV infection in Fiji, vertical transmission of the virus was serologically demonstrated (specific $\operatorname{IgM}$ ) in the cord blood of neonates, indicative of the transplacental infection of the foetuses (Aaskov et al., 1981). The viruses for American equine encephalitis are essentially neurotropic. As such, WEE has long been shown to cause encephalitis in newborns, infected transplacentally by the pregnant women shortly before delivery (Chastel, 
1999). While maternal infection may be benign, foetal infection may be catastrophic for the newborn. Foetal infections with the Venezuelan encephalitis virus (VEE) carry an even sombre prognosis. Severe encephalitis was described in babies born of mothers infected with VEE, and they did not survive their first week of life, owing to massive necrotic brain lesions (Wenger, 1967).

\section{Flaviviral infections}

Flaviviruses are probably the most medically significant arboviruses. Dengue (DEN) is recognised as the most rapidly expanding disease in the world, and presently poses a threat to the health and life of almost 2.5 billion people in the world. Although a very effective vaccine has been available for yellow fever (YF) for almost a century, the disease still re-emerges periodically, especially in Africa causing massive outbreaks, sometimes with heavy mortality. Japanese encephalitis (JE) is mostly prevalent in Asia, and maternal-foetal transmissions of the virus are rare (Bran and Chastel, 2008). The disease, however, causes severe neurological manifestations in $0.1-2.5 \%$ of infected people. West Nile virus (WNV) had been prevalent in Africa and Europe for decades, but gained much fame when it established in the USA in 1999 (Deubel et al., 2001).

\subsection{Dengue}

The origin of the word dengue is uncertain, although often attributed to the Swahili language of East Africa to mean breakbone fever because of the painful symptoms (Christie, 1872). DEN is a mosquito-borne infection found in tropical and sub-tropical regions around the world. Dengue has affected humans for a very long time (Howe, 1977). However, in recent years, transmission has increased predominantly in urban and semi-urban areas and DEN has become a major international public health concern. DENVs occur as four distinct serotypes (DENV 1, DENV 2, DENV 3 and DENV 4). The incidence of DEN has grown dramatically around the world in recent decades. Over 2.5 billion people (over $40 \%$ of the world's population) are now at risk from DEN. The World Health Organization (WHO) currently estimates that there may be 50-100 million infections worldwide every year (WHO, 2012). DENV infection in humans causes a spectrum of illness, ranging from unapparent or mild febrile illness to severe and fatal haemorrhagic disease. Classical dengue fever (DF) is a severe, flu-like illness that affects infants, young children and adults, but seldom causes death. It is characterised by sudden onset of fever and a variety of non-specific signs and symptoms, including frontal headache, retro-orbital pain, body aches, nausea and vomiting, joint pains, weakness, and rash (Gubler, 1997). Patients may be anorectic, have altered taste sensation and mild sore throat, injected conjunctivae and inflamed pharynx. Haemorrhagic manifestations may occur in DF patients and range from mild to severe. 
Skin haemorrhages are the most common, including petechiae and purpura, as well as gum bleeding, epistaxis, menorrhagia, and gastrointestinal haemorrhage. Haematuria occurs infrequently, and jaundice is rare. Dengue fever is generally self-limited and rarely fatal. A more serious form of DEN is DEN haemorrhagic fever (DHF), a disease of children under the age of 15 years (WHO, 1997), which may be complicated with DEN shock syndrome (DSS) (Gubler, 2006). Shock is caused by systemic capillary leakage, thrombocytopaenia and hypovolaemic shock plasma leakage (WHO, 1997). An estimated 500,000 people with severe dengue require hospitalisation each year, a large proportion of them are children (WHO, 2012). About $2.5 \%$ of those affected die.

Jain and Chatuverdi (2010) reviewed clinical manifestations of DEN in infants. The burden of severe illness lies predominantly in infants 4-9 months of age (Halstead et al., 2002; Hongsiriwon, 2002; Capeding et al., 2010). Severe clinical manifestations of DHF/DSS and DSS alone are more prevalent in infants, followed by children and then adults (Hammond et al., 2005). Manifestations such as convulsions and hepatic dysfunction more commonly affect infants than older children, and fatality rate is 4 times higher in infants (Kalayanarooj and Nimmannitya, 2003). The risk of presenting with signs of DHF/DSS by year of age was estimated and it was seen that infants and children aged 5-7 years are at significant risk for severe dengue, whereas in adults and adolescents 14 years and older, the risk is significantly less (Hammond et al., 2005). Coryza, nausea/vomiting, rash, petechiae and other haemorrhagic manifestations (table 2) are significantly higher in infants and younger children than in older children (Witayathawornwong, 2005, 2006). Very high fever, hepatosplenomegaly, rashes, oedema of the lower extremities, retro-orbital puffiness, vomiting and convulsions are commoner in infants than in older children (Kabilan et al., 2003; Soundravally et al., 2010). Infants with DHF/DSS present with some bleeding (87.5\% of cases), cyanosis and ascites $(37.5 \%)$ and shock $(25 \%)$.

The ability of all four serotypes of DENVs to interfere with the normal course and outcome of pregnancy is now widely recognised (Bunyaveschevin et al., 1997; Chye et al., 1997; Carles et al., 1999, 2000; Boussemart et al., 2001; Ahmed, 2003; Chotigeat et al., 2003; Berberian et al., 2011; Chitra and Panicker, 2011). Near-term pregnant women who are infected with DENV can transmit the disease to the foetus through the placenta and this can result in congenital infection (Wiwanitkit, 2010). The dengue virus is an RNA virus with small molecular size $(40-85 \mathrm{~nm})$ that can permit vertical transmission of disease, which is concordant with the basic rule of vertical transmission in nanomedicine (Wiwanitkit, 2006). Maternal-foetal transmission of DENV was convincingly demonstrated for the first time during the 1971 epidemic in French Polynesia. That outbreak attributed to DENV 2 saw the manifestation of several forms of haemorrhagic uterine bleeding followed by abortion (Moreau et al., 1973). Maternal-foetal transmission was confirmed for the first time during the 1989-1990 epidemic in the same area. During the outbreak caused by DENV 3, simultaneous infection in mothers and newborns was demon- 
strated (Poli et al., 1991). Some of the infected babies were premature while others were born at term. The infants were viraemic, while their mothers' serum had antibodies indicative of primary and secondary infection with DENV 3. All babies were febrile, thrombocytopaenic, cyanotic, and they had mottling skin and cold extremities. The neonates had hepatomegaly and some of them presented haemorrhagic symptoms seen in DEN (purpura, bleeding in the stomach). All infected mothers and babies during the Polynesian outbreak survived the infection. Maternal-foetal transmission since then has been observed in Malaysia, Thailand, Sudan, Guadeloupe, India, French Guiana (Adam et al., 2010; Kariyawasam and Senanayake, 2010; Chitra and Panicker, 2011). Severe infection in some of the infected mothers required administration of plasma and platelets. Children who died developed after birth severe renal impairment, respiratory distress, DIC, and eventually died of cerebral haemorrhage. Death often occurred between 1-6 days. Infection during pregnancy has been reported from several areas, each complementing the array of manifestations previously observed (Table 1). Hence for mothers, the frequency of preterm delivery, caesarean section and major risk of bleeding (especially when caesarean section was required) was significantly increased (Carles et al., 1999, 2000). For the foetuses, the frequency of prematurity, in utero death and foetal distress during labour increased and sometimes low birth weight (LBW) has been observed. The risk of maternal-foetal transmission is also increasing and most likely when the delivery occurs during or shortly after the febrile episode in the mother. When infection in the mother occurs early or very late in pregnancy, the risk of a negative prognosis is increasing. Infections of pregnant women during the first trimester have resulted in a significant increase in the number of neural tube defects and death of a child of athrogryposis during the 1989 and 2005 epidemic respectively. Dead foetuses are often macerated, but scarcely display malformations; more or less complex congenital malformations have been recorded during the 1998 outbreak in Colombia (Restrepo et al., 2003).

\subsection{Yellow fever}

Approximately 200,000 cases of YF are reported annually, 90\% of which occur in Africa (Barnett, 2007) with 30,000 deaths. The presentation of YF disease ranges from subclinical infection to systemic disease including fever, jaundice, haemorrhage, and renal failure. Differences in virus strains as well as incompletely understood host immune factors are likely responsible for the range of clinical symptoms. Three phases of YF are known. The first phase during the virus is presented in blood and it is characterised by fever, malaise, generalised myalgia, nausea, vomiting, irritability, dizziness, and a generally toxic appearance. 
Table 1: Summary of the effect of infection with major arboviruses during pregnancy on pregnant women and foetuses

\begin{tabular}{|c|c|c|}
\hline $\begin{array}{l}\text { Viral infec- } \\
\text { tion }\end{array}$ & $\begin{array}{l}\text { Consequence/symptoms in } \\
\text { pregnant mothers }\end{array}$ & $\begin{array}{l}\text { Consequences/ symptoms in } \\
\text { foetuses/newborns }\end{array}$ \\
\hline Chikungunya & $\begin{array}{l}\text { - Fever, arthralgia, headache, } \\
\text { oedema, diarrhoea, aphthae, epi- } \\
\text { staxis or gingivorrhagia, rash. } \\
\text { - More frequent and longer hospi- } \\
\text { tal admission. } \\
\text { - Higher frequency of miscarriages } \\
\text { and premature births. } \\
\text { - More frequent caesarean deliv- } \\
\text { ery. }\end{array}$ & $\begin{array}{l}\text { - Increased risk of stillbirth and } \\
\text { foetal distress. } \\
\text { - Congenital infection with post- } \\
\text { partum symptoms (inconstant fever, } \\
\text { prostration, pain, inability to breast- } \\
\text { feed, rash and swelling on the } \\
\text { limbs). } \\
\text { - Seizures, severe thrombocytopae- } \\
\text { nia and DIC. } \\
\text { - Increased risk of congenital mal- } \\
\text { formation }\end{array}$ \\
\hline Dengue & $\begin{array}{l}\text { - Fever, chills, headache, arthral- } \\
\text { gia, backache, anorexia, retro- } \\
\text { orbital pains, vomiting. } \\
\text { - Low platelet count to severe } \\
\text { thrombocytopaenia. } \\
\text { - More frequent premature births. } \\
\text { - More frequent caesarean deliv- } \\
\text { ery. }\end{array}$ & $\begin{array}{l}\text { - Higher frequency of low birth } \\
\text { weight } \\
\text { - Thrombocytopaenia, leukopaenia, } \\
\text { fever, diarrhoea. } \\
\text { - Fever, irritability, purpurea, exan- } \\
\text { thema. } \\
\text { - Gastrointestinal and intracerebral } \\
\text { haemorrhages, DSS }\end{array}$ \\
\hline Yellow fever & $\begin{array}{l}\text { - Fever, jaundice, and conjunctival } \\
\text { suffusion. } \\
\text { - Liver enzymes elevated. } \\
\text { - Mild anaemia. }\end{array}$ & $\begin{array}{l}\text { - Fever, cyanosis, hypotension, } \\
\text { tachycardia, cutaneous paleness, } \\
\text { jaundice, hepatomegaly. } \\
\text { - Haematemesis, melena, bleeding at } \\
\text { sites of venipuncture, hypoglycemia, } \\
\text { and oliguria. } \\
\text { - Liver and renal failure, DIC, sei- } \\
\text { zures, coma, and death. } \\
\text { - Liver necrosis, pulmonary haem- } \\
\text { orrhage, acute tubular necrosis. }\end{array}$ \\
\hline West Nile & $\begin{array}{l}\text { - Meningoencephalitis. } \\
\text { - More frequent premature births. }\end{array}$ & - Severe brain lesions. \\
\hline $\begin{array}{l}\text { Japanese } \\
\text { encephalitis }\end{array}$ & $\begin{array}{l}\text { - Neurological manifestations, } \\
\text { especially hemiplegia. } \\
\text { - More frequent abortion of foe- } \\
\text { tuses (especially during first tri- } \\
\text { mester). }\end{array}$ & $\begin{array}{l}\text { - Congenital infection with post- } \\
\text { partum symptoms } \\
\text { - Increased mortality of the foetuses } \\
\text { especially when infection occurs in } \\
\text { first semester of pregnancy). }\end{array}$ \\
\hline
\end{tabular}

Laboratory abnormalities include leukopenia, present at the onset of illness, and elevation of serum transaminase levels before the onset of jaundice. The second phase is characterised by the improvement of symptoms including a reduction of 
fever; this may last up to $48 \mathrm{~h}$ but is not noted in all cases. Some infected individuals recover at this phase without developing jaundice. The third phase occurs in about $15 \%$ of cases and it is characterised by the return of fever, nausea, vomiting, jaundice, and bleeding diathesis. Antibodies appear in the blood as the virus disappears. Multiorgan system involvement is typical. Serum transaminase levels are proportional to the severity of the disease; they peak early during the second week of illness in patients who recover. Case-fatality rates vary widely but were in the range of 20\% for West African patients with jaundice in several studies (Marfin et al.,, 2006). A dramatic resurgence of YF has occurred since the 1980s and in subSaharan Africa several outbreaks have been recorded (Robertson et al., 1996; Wiysonge et al., 2008). Despite the recurrence of epidemics of YF in tropical Africa and South America, vertical transmission of the virus in humans has not been conclusively documented.

Reports of YF during pregnancy are scarce (Sicé and Rodallec, 1940) and there is only one report that describes vertical transmission to newborns (Bentline et al., 2011) from natural infection in Brazil. In this case, the mother, a 30-year-old woman (exposed to YF in late pregnancy) had never received YF vaccination. She had fever, headache, and jaundice; 3 days after the onset of jaundice, she delivered an apparently normal female child through vaginal partum. She was mildly anaemic, but leukocyte and platelet counts were normal. She was discharged after 7 days with complete recovery. The girl child was born asymptomatic with a birth weight of 3,800 g and Apgar scores of 9-10. She was discharged from the hospital after 2 days. On the 3rd day of life, she had fever and cyanosis and was readmitted to the hospital with suspected pneumonia. On the 8th day of life, she had haematemesis, melena, bleeding at venipuncture sites, hypoglycemia, and oliguria. The child developed hypotension, tachycardia, cutaneous paleness, jaundice, melena, and hepatomegaly. The mother's diagnosis prompted doctors to investigate possible YF. The infant was intubated for ventilatory support and received volume expansion, vasoactive amines, antimicrobial drugs, blood components (erythrocytes, platelets, fresh frozen plasma, cryoprecipitate) and drugs to control bleeding. Despite therapy, the newborn experienced liver and renal failure, DIC, seizures, and finally coma. She died on the 12th day of life and autopsy specimens showed massive liver necrosis, pulmonary haemorrhage, and acute tubular necrosis. Diagnosis of YFV infection was confirmed in the mother and infant's sera by IgM capture ELISA; and in the infant by RT-PCR. YF vaccine virus has equally been isolated from asymptomatic newborns from pregnant women who were inadvertently administered 17D vaccine strain (Tsai et al., 1993). Transmission of the YFV through breast-feeding has also been recently reported MMWR, 2009; Kuhn et al., 2011).

Infancy and older age are associated with increased severity and lethality of infection with YFV. Most infections in endemic countries occur in persons younger than 15 years because this group contains the largest population of non-immune individuals (Gardner and Ryman, 2010). Occupational or recreational exposure may as well be increased among children. 


\subsection{Japanese encephalitis}

Infection with the Japanese encephalitis virus (JEV) is widely distributed in Asia, and often manifests with severe neurological symptoms. JE virus infection can begin as a mild febrile illness or aseptic meningitis. However, the most common presentation to hospital in humans is due to acute meningomyeloencephalitis (Watts et al., 2006a). In endemic areas JEV infection occurs primarily among children, particularly within the age group of 5-10 years old children (Solomon et al., 2000).

Maternal-foetal transmission of the JEV is rare. Cases of mother-child transmission were observed for the first time in India during the epidemics of 1978 and 1980 (Chatuverdi et al., 1980; Mathur et al., 1985). In the first of these epidemics, responsible for approximately 100 deaths, five pregnant women were hospitalised with diagnosis of JE, confirmed virologically and/or serologically. In three cases where the pregnancy was at 28 weeks, 30 weeks and term, the mothers gave birth to apparently healthy children, although one of the mothers was hemiplegic. In two cases where the pregnancies were at earlier stage (20 and 22 weeks) the foetuses were aborted. JEV was isolated from the placenta of one of the women, while it was equally recovered from the brain and liver of the other aborted foetus. Both mothers recovered without sequelae. During the 1980 outbreak, four pregnant women in the first trimester of pregnancy were infected with JEV, of which two (8 and 10 weeks of gestation) aborted while the other two (28 and 30 weeks of gestation) gave birth to apparently normal children. JEV was isolated from the placenta of one of the cases with abortion, however all mothers completely recovered. It is then quite obvious that the JEV can infect the placenta and foetus, causing abortion and death of the foetus, especially when infection occurs during the early phase of the pregnancy (i.e. first 12 weeks). Placental and foetal infections by the JEV have severally been induced in laboratory animals and observed in nature, mostly in sows in Japan during outbreaks of JE and during inter-epidemic periods.

\subsection{West Nile}

The West Nile virus (WNV) is widely distributed in Africa, Europe and Asia and gained much fame when it crossed the Atlantic and firmly established in the United States (New York) in 1999 (Deubel et al., 2001). Since then, it has attracted much more attention than it had previously and eventually, the first cases of maternal-foetal transmission have been starting in 2002 (CDC, 2002). 
Table 2: Summary of the effect of infection with major arboviruses during childhood

\begin{tabular}{l|l}
\hline Viral infection & \multicolumn{1}{|c}{ Consequence/symptoms in children } \\
\hline Chikungunya & $\begin{array}{l}\text { - Neurological symptoms including: encephalitis, febrile fits, meningi- } \\
\text { tis, and acute encephalopathy, encephalomyelitis, Guillain-Barre, acute } \\
\text { flaccid paralysis, optic neuritis and encephalomyeloradiculitis. } \\
\text { - Higher mortality. }\end{array}$ \\
\hline Dengue & $\begin{array}{l}\text { - Higher death rate. } \\
\text { - Potentially deadly plasma leakage, fluid accumulation, respiratory } \\
\text { distress, severe bleeding, or organ impairment. }\end{array}$ \\
& $\begin{array}{l}\text { - Severe clinical manifestations including severe abdominal pain, nau- } \\
\text { sea, persistent vomiting, rapid breathing, bleeding gums, fatigue, rest- } \\
\text { lessness, blood in vomit. }\end{array}$ \\
& - DHF/DSS \\
- Thrombocytopaenia and lymphocytosis. \\
- Cynosis and Convulsion. \\
- Hepatosplenomegaly and Hepatic dysfunction. \\
- Rash and petechiae, oedema of lower limbs. \\
- High fever, retrobulbar puffiness and coryza \\
- Death.
\end{tabular}

A young pregnant woman was hospitalised with a meningoencephalitis induced by the WNV. She eventually gave birth to an apparently normal baby at 38 weeks of gestation. However bilateral chorio-retinal lesions as well as severe brain lesions of the newborn were detected. The presence of WNV-specific IgM antibodies in the baby's blood and CSF was proof of existence of a congenital WNV infection (Alpert et al., 2003). Two other cases of meningoencephalitis due to WNV were later on observed in Louisiana and Illinois, in women who were at the 11 th and 16th week of pregnancy respectively (Chapa et al., 2003; Bruno et al., 2004). They gave birth to living children, including a premature infant, which showed no detectable neurological impairment. While WNV expanded its geographical range in 
the USA, the Centers for Disease Control (CDC) set up a surveillance programme to specifically monitor pregnant women infected with the virus. Transmission from the mother to the newborn via breast milk has also been suspected (CDC, 2002). WNV appears less dangerous than some other viruses for the pregnant woman and the foetus (O'Leary et al., 2006; Paisley et al., 2006). It has nevertheless been shown to cause abortion, stillbirths and lethal deformities in newborn animals.

Most children who become infected with WNV are likely to be either asymptomatic or have a mild febrile illness (Hayes and O'Leary, 2004). Detailed clinical descriptions of WNV encephalitis in children are scarce, but the clinical course was well-described for a 7-year-old girl with WN encephalitis in India who was admitted to a hospital with a history of 4 days of fever and convulsions that progressed to coma (Carey et al., 1968). She gradually regained consciousness but developed a facial palsy by the time she left the hospital 3 weeks after admission. In the USA, the 11,822 cases of WN neuroinvasive disease reported to CDC between 1999 and 2007, only 4\% occurred in children, of which 3 died (Lindsey et al., 2009, 2010). WNV infection in children may lead to West Nile Fever (with unspecific symptoms) or West Nile neuroivasive disease. The later is more serious and characterised by meningitis, meningoencephalitis, acute flaccid paralysis (Lindsey et al., 2009).

\section{Bunyaviral infections}

The Bunyaviridae family comprises close to 300 viruses with at least 167 in the genus Bunyavirus (Calisher, 1994; Alatoom and Payne, 2009). The family is divided into 5 genera of which 3 are arthropod-borne: Phlebovirus (sandfly fever, Rift Valley fever, etc.), Nairovirus (Crimean-Congo haemorrhagic fever) and Bunyavirus. Most viruses that belong to these groups cause fever, rash, arthritis, encephalitis and hepatitis and they are common in Africa and South America (MMWR, 1998). The Rift Valley fever virus (RVFV) causes abortion in almost $100 \%$ of pregnant ewes (Watts et al., 2006b). Although several outbreaks of RVF have occurred in humans throughout African and the Arabic peninsula, similar risk has not yet been conclusively demonstrated in infected pregnant women.

For infection with the CCHFV between the 8th-38th week of pregnancy, abortion has ensued either during the haemorrhagic phase of the disease or during convalescence, or even more sadly the pregnant women succumbed the disease (Chastel, 1999; Nabeth et al., 2004; Ergonul et al., 2009). Advances in patient care have recently improved the survival of the pregnant mother, but not yet for the foetus or newborn. Perinatal infection has been demonstrated (Ergonul et al., 2009)

In the USA, LaCrosse virus (LAC) is a paediatric pathogen. Infections occur in young people and may be asymptomatic or cause meningitis or encephalitis (Calisher, 1994). 


\section{Other arboviral infections}

Infection of pregnant women with Colorado tick fever virus (CTFV), a Reoviridae, has also been reported. In some cases, women lost their pregnancy during the convalescence phase of the disease (Poland, 1985; Chastel, 1999). In one case, a baby, born to a woman who has been infected with the virus during the first month of pregnancy, showed multiple malformations, although the CTFV was not detected in the baby. In another case, a baby who has been delivered 6 days after infection of his mother, developed a self-limited febrile illness with leucopaenia, probably caused by the CTFV (Eklund et al., 1959; Chastel, 1999). While the role of the CTFV has not been conclusively proven in the aforementioned conditions, it is important to remain vigilant in the follow-up of pregnant women infected with this agent.

\section{Treatment and prevention}

\subsection{Treatment}

In the absence of appropriate medication, treatment for arbovirus infection is essentially symptomatic. Enough rest, water and the use of analgesics and antipyretics may relieve symptoms of fever and aching. It should be taken care to avoid certain medications such as aspirin or nonsteroidal anti-inflammatory drugs which may increase the risk for bleeding. Infected people should be protected from further arthropod exposure (staying indoors or under a mosquito net) during the first few days of illness, so they do not contribute to the transmission cycle.

\subsection{Prevention}

In the absence of treatment for any arboviral disease, our arsenal of vaccines against these agents is rather limited. Yellow fever and JE are preventable diseases. The 17D live-attenuated vaccine against YFV and inactivated JE-MB/JE-VC vaccine (depending on whether it was grown in mouse brain or Vero cell lines) are relatively safe and effective. Yellow fever vaccine is recommended for people aged 9 months and above (WHO, 2011) and in several countries in Africa it is now included in routine vaccination programmes for infants who are 5 years old or younger. The implementation of this recommendation for instance in Cameroon has enabled the country raise its YF immunisation coverage from 59\% in 2004 to over $72 \%$ in 2006 (Wiysonge et al., 2008) and probably significantly above this figure owing to mass immunisation campaigns following recent outbreaks of YF in several areas of the country. However, for pregnant women, the 17D vaccine and its 17DD heat resistant variant are to be avoided during the first trimester, since it is a live vaccine that sometimes causes abortions. It should also be avoided during 
the third quarter of pregnancy due to a risk of placental abruption. The use of 17D vaccination during pregnancy should be carefully considered if the risk of acquiring YF is greater than the theoretical risk of teratogenicity (as would be the case during an epidemic). A recent study of women who were vaccinated with yellow fever vaccine early in their pregnancies found no major malformations in their infants (Cavalcanti et al., 2007). A slight increased risk was noted for minor, mostly skin, malformations in infants. There is no information about the possible risks of the inactivated vaccine against JE in pregnant women (WHO, 1994). This vaccine is especially recommended for children older than one year in endemic area (Vietnam, China, Japan). For pregnant women - because the risk of infection with JE virus, for herself and the foetus is very high (epidemics, repeated exposure to mosquito bites in rural or suburban) - it is preferable to vaccinate. For the other arboviruses posing a major threat to human health, various vaccine candidates are at different stages of development. For DEN, there is the need to develop a tetravalent vaccine in order to circumvent the higher risk of developing DHF, posed by antibody-dependent enhancement and observed when a person gets a secondary infection through a serotype of DENV other than through the one that caused the primary infection.

Communities and public health authorities should endeavour to prevent mosquitoes from accessing egg-laying habitats by means such as environmental management and modification, proper disposing of solid waste and the removal of artificial man-made habitats, as well as the frequent covering, emptying and cleaning of domestic water storage containers and appropriate application of insecticides towards water storage outdoor containers. Other actions include appropriate household and personal protection measures such as window screens, long-sleeved clothes, insecticide-treated materials, coils and vaporisers. Additionally, community participation and mobilisation for sustained vector control should be improved, applying insecticides as space spraying during outbreaks as one of the emergency vector control measures, as well as carrying out measure dealing with surveillance and monitoring of vectors to determine effectiveness of control interventions (WHO, 2012).

Pregnant women and younger children should also avoid haematophagous arthropod exposure. Additionally to these community-level vector control measures, individual protection against arthropod bites (especially mosquitoes) are essential (WHO, 2011; Zielinski-Gutierrez et al., 2012). Refering to the use of impregnated bed nets (pyrethroids) although there are essential, it has been stated that they are not totally effective against Aedes mosquitoes and vectors of YFV, DENV and CHIKV because these mosquitoes easily bite outdoors and during the day. Pregnant women, more than other people, must therefore wear light and long clothing and avoid staying outdoors during periods of peak mosquito activity at dawn and dusk. Mosquito repellents should be applied to the skin of uncovered parts of the body (hands, ankles, neck) (Zielinski-Gutierrez et al., 2012). A repellent- compatible with pregnancy- must be selected. N, N-diethyl-M-toluamide or DEET re- 
mains the reference product but it should be noted that it is forbidden to use it by pregnant women in some countries. The transfer of DEET to the foetus is well documented in experimental animals (Schaeffer and Peters, 1992). It is worth mentioning that the use of repellent is not an absolute guarantee against arthropod bites, especially as it was demonstrated during the La Réunion outbreak where $20 \%$ of all mosquito bites were sustained through clothing (Girard, 2006).

\section{Conclusion}

While vertical transmission of some arboviruses transmitted by mosquitoes is a rare event in pregnancy, the consequences can be severe enough for us not to neglect a phenomenon whose importance is likely to increase in the future. Luckily, several of the tragic events summarised by Parsonson et al. (1981) including abortion, foetal death, premature birth and teratogenic changes happen less often in humans than they have been observed in animals in nature and in the laboratory.

As the human population increases and human activities take us even closer to sylvatic foci, human population are more likely to get exposed to new pathogens (including arboviruses) with the potential for some of them to urbanise. The current climatic changes observed will likely continue to alter the ecology and distribution of populations of several haematophagous arthropods, their associated pathogens and reservoir host, with additional risk of infection for humans. We are unfortunately poorly therapeutically and prophylactically equipped to deal with these accidents; our ignorance of the pathogenesis of several arboviruses incites us to conduct new epidemiological surveys and to develop better experimental models. While undesired, further epidemics of disease (such as the Indian Ocean outbreak of $\mathrm{CHIK}$ ) are likely to provide us with further insight into the pathology and epidemiology of several arboviruses.

\section{References}

Aaskov, J.G., Nair, K., Lawrence, G.W., Dalglish, D.A. \& Tucker, M. (1981). Evidence of transplacental transmission of Ross River virus in humans. Medical Journal of Australia, 2: 20-21.

Adam, I., Jumaa, A.M., Elbashir, H.M. \& Karsany, M.S. (2010). Maternal and perinatal outcomes of dengue in Port Sudan, Eastern Sudan. Virology Journal, 7: 153 .

Agha, R. (1995). Arbovirus infections. Pediatrics in Review,16: 353. 
Ahmed, S. (2003). Vertical transmission of dengue: first case report from

Bangladesh. Southeast Asian Journal of Tropical Medicine and Public Health, 34: 800-803.

Alatoom, A \& Payne D. (2009). An overview of Arboviruses and Bunyaviruses. Labmedicine, 40(4): DOI: 10.1309.

Alpert, S.G., Fergerson, J. \& Noël L.P. (2003). Intrauterine West Nile virus: ocular and systemic findings. American Journal of Ophthalmology, 136: 733-735.

Arpino, C., Curatolo, P. \& Rezza, G. (2009). Chikungunya and the nervous system: what we do and do not know. Review of Medical Virology, 19: 121-29.

Barnett, E.D. (2007).Yellow fever: epidemiology and prevention. Clinical Infectious Diseases, 44: 850-6.

Beaty, B.J., Calisher, C.H. \& Shope, R.E., (1995). Arboviruses . In Lennette, E.H., Lennette, D.A. \& Lennette, E.T. (Eds). Diagnostic procedures for viral, rickettsial, and chlamydial infections, 7th edition, Washington, DC: American Public Health Association, 189-212.

Bentlin, M.R., de Barros Almeida, R.C.M., Coelho, K.I.R., Ribeiro, A.F., Siciliano, M.M., Suzuki, A. \& Fortaleza, C.M.C.B. (2011). Perinatal transmission of yellow fever, Brazil, 2009. Emerging Infectious Diseases, 17(9): 1779-1780.

Berberiana, G., Fariñaa, D., Rosanovaa, M.T., Hidalgob, S., Enríac, D., Mitchenkod, A., Morenoe, J. \& Sotoe, I.S. (2011). Dengue perinatal. Archivos Argentinos de Pediatria, 109(3): 232-36.

Boussemart, T., Babe, P., Sibille, G., Neyret, C. \& Berchel, C. (2001). Case report, perinatal transmission of dengue: two new cases. Journal of Perinatalogy, 21: 255-257.

Brücker, G., Dellagi, K. \& Gaüzère, B.A. (2007). Chikungunya et autres arboviroses en milieu tropical, Saint- Gilles, la Réunion, 3 et 4 décembre 2007. Bulletin de la Société de Pathologie Exotique, 100: 315-316.

Bruno, J., Rabito, F.J. \& Dildy, G.A. (2004). West Nile virus meningoencephalitis. Journal of the Louisiana State Medical Society, 156: 204-205.

Bunyaveschevin, S., Tanawattanacharoen, S., Taechakraichana, N., Thisyakorn, U., Tannirandorn, Y. \& Limpaphayom, K. (1997). Dengue hemorrhagic fever during pregnancy: antepartum, intrapartum management. Journal of Obstetrics and Gynecologic Research, 23: 445-448.

Calisher, C.H. (1994). Medically important arboviruses of the United States and Canada. Clinical Microbiology Reviews, 7: 89-116.

Capeding, R.Z., Brion, J.D., Caponpon, M.M., Gibbons, R.V., Jarman, R.G., Yoon, I.K. \& Libraty, D.H .(2010). The incidence, characteristics, and presentation of 
dengue virus infections during infancy. American Journal of Tropical Medicine and Hygiene, 82: 330-336.

Carey, D.E., Myers, R.M., de Ranitz C.M. \& Jadhav M. (1969). The 1964

Chikungunya epidemic at Vellore, South India, including observations on concurrent dengue. Transactions of the Royal Society of Tropical Medicine and Hygiene, 63: 424-445.

Carey, D.E., Rodrigues, F.M., Myers, R.M. \& Webb, J.K.(1968). Arthropod-borne viral infections in children in Vellore, South India, with particular reference to dengue and West Nile viruses. Indian Pediatrics, 5: 285-296.

Carles, G., Peiffer, H. \& Talarmin, A. (1999). Effects of dengue fever during pregnancy in French Guiana. Clinical Infectious Diseases, 28: 637-640.

Carles, G., Talarmin, A., Peneau, C. \& Bertsch, M. (2000). Dengue et grossesse, Étude de 38 cas en Guyane française. Journal de Gynecologie Obstetrique et Biologie de Reproduction, 29: 758-762.

Cavalcanti, D.P., Salomao, M.A., Lopez-Camelo, J. \& Pessoto, M.A. (2007). Early exposure to yellow fever vaccine during pregnancy. Tropical Medicine and International Health, 12(7): 833-7.

CDC. (1998). Arboviral infections of the central nervous system-United States, 1996-1997. Morbidity and Mortality Weekly Report, 47: 517-22.

CDC. (2002). Intrauterine West Nile virus infection, New York, 2002. Morbidity and Mortality Weekly Report, 51: 1135-1136.

Chapa, J.B., Ahn, J.T., DiGiovani, L.M. \& Imail, M.A. (2003). West Nile virus encephalitis during pregnancy. Obstetrics and Gynecology, 102: 229-231.

Chastel, C. (1999). Arbovirus, arénavirus et virus voisins. In Denis, F. (Ed.) Les virus transmissibles de la mère à l'enfant, John Libbey Eurotext, Paris, pp. 365394.

Chaturvedi, U.C., Mathur, A., Chandra, A., Das, S.K., Tandon, H.O. \& Singh, U.K. (1980). Transplacental infection with Japanese encephalitis virus. Journal of Infectious Diseases, 141: 712-715.

Chia, P.Y., Ng, M.M.-L. \& Chu, J.J.H. (2010). Chikungunya fever: A review of a reemerging mosquito-borne infectious disease and the current status. Current research, technology and education topics. Applied Microbiology and Microbial Biotechnology, 597-606.

Chitra, T.V. \& Panicker, S. (2011). Maternal and fetal outcome of dengue fever in pregnancy. Journal of Vector Borne Diseases, 48: 210-13. 
Chotigeat, U., Khaoluang, S., Kanjanapatanakul, V. \& Nisalak, A. (2000). Vertical transmission of dengue virus. Journal of Infectious Diseases and Antimicrobial, 17: 33-34.

Christie, J. (1872). Remarks on "Kidinga Pepo": A peculiar form of exanthematous disease. British Medical Journal, 577.

Chye, J.K., Lim, C.T., Kwee, B., Ng, K.B., Lim, J.M.H., George, R. \& Lam, S.K. (1997). Vertical transmission of dengue. Clinical Infectious Diseases, 25: 13741377.

Deubel, V., Gubler, D.J., Layton, M. \& Malkinson, M. (2001). West Nile virus: a newly emergent epidemic disease. Emerging Infectious Diseases, 7: 536.

Eklund, C.M., Kohls, G.M., Jellison, W.L., Burgdorfer, W., Kennedy, R.C. (1959). The clinical and ecological aspects of Colorado tick fever. Proceedings of the 6th International Congress on Tropical Medicine and Malaria, Lisbon, 5:197203. Lisbon.

Enserink, M. (2007). Chikungunya: no longer a third world disease. Science, 318: 1860-1861.

Ergonul, O., Celikbas, A., Yildirim, U., Zenciroglu, A., Erdogan, D., Ziraman, I., Saracoglu, F., Demirel, N., Cakmak, O. \& Dokuzoguz, B. (2010). Pregnancy and Crimean-Congo haemorrhagic fever. Clinical Microbiology and Infection, 16(6): 647-50.

Freeman, B.A. (1985). Burrows textbook of Microbiology. 20th Edition, WB Saunders Company, Philadelphia. 1038 p.

Fritel, X., Rollot, O., Gerardin, P., Gauzere, B.A., Bideault, J., Lagarde, L., Dhuime, B., Orvain, E., Cuillier, F., Ramful, D., Samperiz, S., Jaffar-Bandjee, M.C., Michault, A., Cotte, L., Kaminski, M. \& Fourmaintraux, A. (2010). Chikungunya virus infection during pregnancy, Reunion, France, 2006. Emerging Infectious Diseases, 16: 418-425.

Gardner, C.L. \& Ryman K.D. (2010). Yellow fever: A reemerging threat. Clinical Laboratory Medicine, 30: 237-260.

Girard, P. (2006). Chikungunya et répulsifs. La lettre d'Infectiologie, 21: 142-143.

Gregg, N.M. (1941). Congenital cataract following German measles in the mother. Transactions of the Ophthalmological Society of Australia, 3: 35-46.

Gubler, D.J. (1997). Dengue and dengue haemorrhagic fever: its history and resurgence a global public health problem. In Gubler, D.J. \& Kuno, G. (Eds.): Dengue and dengue haemorrhagic fever, CAB International, London. 
Gubler, D.J. (2006). Dengue and Dengue Hemorrhagic Fever. In Guerrant, R.L., Walker, D.H. and Weller, P.F. (Eds.): Tropical Infectious Diseases, 2nd edition, Elsevier Churchill-Livingstone, Philadelphia, pp 813-822.

Halstead, S.B., Lan, N.T., Myint, T.T., Shwe, T.N., Nisalak, A., Kalyanarooj, S., Nimmannitya, S., Soegijanto, S., Vaughn, D.W. \& Endy, T.P. (2002). Dengue hemorrhagic fever: research opportunities ignored. Emerging Infectious Diseases, 8(12): 1474-9.

Hammond, S.N., Balmaseda, A., Perez, L., Tellez, Y., Saborro, S.I., et al. (2005) Differences in dengue severity in infants, children, and adults in a 3-year hospital-based study in Nicaragua. American Journal of Tropical Medicine and Hygiene, 73: 1063-70.

Hanshaw, J.B. \& Dudgeon, J.A. (1978). Viral diseases of the fetus and newborn. W B Saunders, Philadelphia.

Hayes, E.B. \& O’Leary, D.R. (2004). West Nile virus infection: a pediatric perspective. Pediatrics, 113(5): 1375-1381.

Hongsiriwon, S. 2002. Dengue hemorrhagic fever in infants. Southeast Asian Journal of Tropical Medicine and Public Health, 33: 49-55.

Howe, G.M. (1977). A world geography of human diseases. New York, Academic Press, 302 p.

Hughes, S.S. (1977). The virus: A history of the concept. London: Heinemann Education Books.

Hurley, R. (1974). Viral diseases in pregnancy. British Journal of Hospital Medicine, 12: 86-92.

Jain, A. \& Chaturvedi, U.C. (2010). Dengue in infants: an overview. FEMS Immunology Medicine and Microbiology, 59: 119-130.

Kabilan. L., Balasubramanian, S., Keshava, S.M., Thenmozhi, V., Sekar, G., Tewari, S.C., Arunachalam, N., Rajendran, R. \& Satyanarayana, K. (2003). Dengue disease spectrum among infants in the 2001 dengue epidemic in Chennai, Tamil Nadu, India. Journal of Clinical Microbiology, 41: 3919-3921.

Kalayanarooj, S. \& Nimmannitya, S. (2003) Clinical presentations of dengue hemorrhagic fever in infants compared to children. Journal of the Medical Association of Thailand, 86: S673-S680.

Kariyawasam, S. \& Senanayake, H. (2010). Dengue infections during pregnancy: case series from a tertiary care hospital in Sri Lanka. Journal of Infection in Developing Countries, 4(11): 767-775.

Kuhn, S., Twele-Montecinos, L., MacDonald, J., Webster, P. \& Law, B. (2011). Case report: probable transmission of vaccine strain of yellow fever virus to an 
infant via breast milk. Canadian Medical Association Journal, 183(4), DOI:10.1503/cmaj.100619.

LaBeaud, A.D. (2008). Why arboviruses can be neglected tropical diseases. PLoS Neglected Tropical Diseases, 2: e247.

LaBeaud, A.D., Bashir, F. \& King, C.H. (2011). Measuring the burden of arboviral diseases: the spectrum of morbidity and mortality from four prevalent infections. Population Health Metrics, 9: 1.

Lenglet, Y., Barau, G., Robillard, P.Y., Randrianaivo, H., Michault, A., Bouveret, A., Gérardin, P., Boumahni, B., Touret, Y., Kauffmann, E., Schuffenecker, I., Gabriele, M. and Fourmaintraux, A. (2006). Infection à Chikungunya chez la femme enceinte et risque de transmission materno-foetale. Journal de Gynecologie Obstetrique et de Biologie de la Reproduction (Paris), 35(6): 57883.

Levine, A.J. (2001). The origins of virology. In Knipe, D.M. and Howley, P.M. (Eds.): Fields Virology, 4th edition, LWW, Philadelphia, PA, USA, pp. 3-18.

Lindsey, M.P., Hayes, E.B., Staples, E.J., and Fischer, M. (2009). West Nile Virus disease in children, United States, 1999-2007. Pediatrics, 123:e1084.

Lindsey, M.P., Staples, E.J., Lehman, J.A. and Fischer, M. (2010). Surveillance for human West Nile Virus disease-United States, 1999-2008. Morbidity and Mortality Weekly Report, 59(SS-2): Surveillance Summaries 1

Marfin, A.A., Eidex, R.B. \& Monath, T.P. (2006). Yellow fever. In Guerrant, R.L., Walker, D.H. \& Weller, P.F. (Eds.): Tropical Infectious Diseases, 2nd edition, Elsevier Churchill-Livingstone, Philadelphia, pp. 797-812.

Mathur, A., Tandon, H.O., Mathur, K.R., Sarkari, N.B.S., Singh, U.K. \& Chaturvedi, U.C. (1985). Japanese encephalitis virus infection during pregnancy. Indian Journal of Medical Research, 81: 9-12.

Moreau, J.P., Rosen, L., Saugrain, J. \& Lagraulet, J. (1973). An epidemic of dengue on Tahiti associated with hemorrhagic manifestations. American Journal Tropical Medicine and Hygiene, 22: 237-241.

Nabeth, P., Ould Cheikh, D., Lo, B., Faye, O., Vall, I.O.M., Niang, M., Wague, B., Diop, D., Diallo, M., Diallo, B., Diop, O.M. \& Simon, F. (2004). CrimeanCongo Hemorrhagic fever, Mauritania. Emerging Infectious diseases, 10(12): 2143-9.

O’Leary, D.R., Kuhn, S., Kniss, K.L., Hinckley, A.F., Rasmussen S.A., Pape, W.J., Kightlinger, L.K., Beecham, B.D., Miller, T.K., Neitzel, D.F., Michaels, S.R., Campbell, G.L. Lanciotti, R.S. \& Hayes, E.B. (2006). Birth outcomes following West Nile Virus infection of pregnant women in the United States: 2003-2004. Pediatrics, 117(3): e537-45. 
Paisley, J.E., Hinckley, A.F., O’Leary, D.R., Kramer, W.C., Lanciotti, R.S., Campbel, G.L. \& Hayes, E.B. (2006). West Nile virus infection among pregnant women in a northern Colorado community, 2003 to 2004. Pediatrics, 117(3): 814-820.

Paquet, C., Quatresous, I., Solet, J.L., Sissoko, D., Renault, P., Pierre, V., Cordel, H., Lassale, C., Thiria, J., Zeller, H. \& Schuffenecker, I. (2006). Épidémiologie de l'infection par le virus Chikungunya à l'Île de la Réunion : point de la situation au 8 janvier 2006. Euro Surveillance, 11(2): 2-3.

Parsonson, M., Della-Porta, A.J.\& Snowdon A.A. (1981). Developmental disorders of the fetus in some Arthropod-borne virus infections. American Journal of Tropical Medicine and Hygiene, 31: 3.

Poland, J.D. (1985). Colorado tick fever. In Conn, R.B. (Ed.): Current diagnosis, Saunders, Philadelphia: pp. 195-97.

Poli, L., Chungue, E., Soulignac, O., Gestas, P., Kuo, P. \& Papouin-Rauzy, M. (1991). Dengue materno-foetale ; à propos de 5 cas observés pendant l'épidémie de Tahiti (1989). Bulletin de la Société de Pathologie Exotique, 84: 513-521.

Reed, W., Carroll, J. \& Agramonte, A. (1901b). The etiology of yellow fever: An additional note. Journal of the American Medical Association, 36: 431-440.

Reed. W., Carroll, J., Agramonte, A. \& Lazear, J. (1901a). Senate Documents, 66(822): 156.

Restrepo, B.N., Isaza, D.M., Salazar, C.L., Ramirez, J.L., Upegui, GE, Ospina, M. \& Ramirez, R. (2003). Dengue en el embarazo: efectos en el feto y el recién nacido. Biomedica, 23: 416-423.

Robertson, S.E., Hull, B.P. \& Tomori, O. (1996). Yellow fever: a decade of reemergence. Journal of the American Medical Association, 276(14): 1157-62.

Robin, S., Ramful, D., Le Seach', F., Jaffar-Bandjee, M.C., Rigou, G. \& Alessandri, J.L. (2008). Neurologic manifestations of pediatric chikungunya infection. Journal of Child Neurology, 23: 1028-35.

Schaeffer, C. \& Peters, P.W. (1992). Intrauterine diethyltoluamide exposure and fetal outcome. Reproductive Toxicology, 6: 175-176.

Sicé, A. \& Rodallec, C. (1940). Manifestations hémorragiques de: la fièvre jaune (typhus amaril). Répercussions de l'infection maternelle sur l'organisme foetal. Bulletin de la Societe de Pathologie exotique, 33: 66-9.

Solomon, T., Dung, N.M., Kneen, R., Gainsborough, M., Vaughn, D.W. and Khanh, V.T. (2000). Japanese encephalitis. Journal of Neurology Neurosurgical Psychiatry, 68: 405-415. 
Soundravally, R., Narayanan, P., Bhat, B.V., Soundraragavan, J. \& Setia, S. (2010). Fulminant hepatic failure in an infant with severe dengue infection. Indian Journal of Pediatrics, 77(4): 435-7.

Tandale, B.V., Sathe, P.S., Arankalle, V.A., Wadia, R.S., Kulkarni, R., Shah, S.V., Shah, S.K., Sheth, J.K., Sudeep, A.B., Tripathy, A.S. \& Mishra, A.C. (2009). Systemic involvements and fatalities during Chikungunya epidemic in India, 2006. Journal of Clinical Virology, 46: 145-49.

Touret, Y., Randrianaivo, H., Michault, A., Schuffenecker, I., Kauffmann, E. \& Lenglet, Y. (2006a). Infection maternelle par le virus Chikungunya et mort foetale in utero. Bulletin de la Société de Pathologie Exotique, 99: 140-141.

Touret, Y., Randrianaivo, H., Michault, A., Schuffenecker, I., Kauffmann, E., Lenglet, Y., Barau, G. \& Fourmaintraux, A. (2006b). Transmission maternofoetale précoce du virus Chikungunya. Presse Medicale, 35: 1656-1658.

Tran, A. \& Chastel, C. (2008). Mosquito-borne arboviruses and pregnancy: Pathological consequences for the mother and infant, A general review. Bulletin de la Société de Pathologie Exotique, 101(5): 418-24.

Tsai, T.F. (2006). Congenital arboviral infections: Something new, something old. Pediatrics, 117: 936.

Tsai, T.F., Paul, R., Lynberg, M.C. \& Letson, G.W. (1993). Congenital yellow fever virus infection after immunization in pregnancy. Journal of Infectious Diseases, 168: 1520-1523.

Tyler, K.L. (2009). Emerging viral infections of the central nervous system: part 2. Archives of Neurology, 66: 1065-74.

Tyler, K.L. (2009). Emerging viral infections of the central nervous system: part 2. Archives of Neurology, 66: 1065-74.

Waterson, A.P. (1979). Virus infections (other than rubella) during pregnancy. British Medical Journal, 13: 564-566.

Watts, D.M., Flick, R., Peters, C.J. and Shope, R.E. (2006). Bunyaviral fevers: Rift valley fever and Crimean-Congo hemorrhagic fever. In Guerrant, R.L., Walker, D.H. \& Weller, P.F. (Eds.): Tropical Infectious Diseases, 2nd edition, Elsevier Churchill-Livingstone, Philadelphia, pp. 756-761.

Watts, D.M., Granwher, B.P., Shope, R.E., Solomon, T. \& Tesh R.B. 2006. Japanese encephalitis and West Nile, and other flavivirus infections. In Guerrant, R.L., Walker, D.H. \& Weller, P.F. (Eds.): Tropical Infectious Diseases, 2nd edition, Elsevier Churchill-Livingstone, Philadelphia, pp. 823830. 
Wenger, F., (1967). Necrosis cerebral masiva del feto en casos de encefalitis equina Venezolana. Investigaccion Clinico (Maracaibo), 21: 13-31.

WHO. (2011). Yellow fever. Fact sheet, $\mathrm{N}^{\circ} 100$.

WHO. (2012). Dengue and severe dengue. Fact sheet, $N^{\circ} 117$.

Witayathawornwong, P. (2003). Parturient and perinatal dengue hemorrhagic fever. Southeast Asian Journal of Tropical Medicine and Public Health, 34: 797-799.

- (2006). Dengue haemorrhagic fever among infants in Petchabun province, Thailand: 2003-2005. Dengue Bulletin, 30: 35-41.

Wiwanitkit, V. (2010). Non vector-borne transmission modes of dengue. Journal of Infection in Developing Countries, 4(1): 051-4.

Wiysonge, C.S., Nomo, E., Mawo, J., Ofal, J., Mimbouga, J., Ticha, J. \& Ndumbe, P.M. (2008). Yellow fever control in Cameroon: where are we now and where are we going? BMC Medicine, $6: 3$.

Zielinski-Gutierrez, E., Wirtz, R.A., Nasci, R.S. \& Brogdon, W.G. (2012).

Protection against mosquitoes, ticks, and other insects and arthropods. In Brunette, G.W. (Ed.) CDC health information for international travel 2012, Oxford University Press, New York, pp. 86-89. 



\section{Social-Related and General Aspects of Ebola Virus in African Communities}

Matthias Grade, Christliches Krankenhaus Quakenbrück, Teaching Hospital of the Medical School of Hannover, Germany

\section{Summary}

The goal of this article is to determine to what extent recurrent outbreaks of potentially life-threatening diseases such as Ebola virus infection can change the life of a society and families. A special focus is given to the social life in general as well as to family relations. Additionally to relevant own data and sources (mainly data from a stay in Uganda during Ebola outbreak in 2000/2001) a Medline query under the inclusion of the following keywords has been performed: Ebola-Virus, fear, social stigma, society, outbreak, tribal life.

Key words:

Ebola Virus/Fever, Outbreak, Fear, Social Affection, Social Stigma, Traditional Tribal Life 


\section{Introduction}

The Ebola virus belongs to the most virulent pathogens known today. Together with the Marburg virus it belongs to the family of the Filoviridae and so far only these two representatives are known. The first Filovirus infection (Marburg virus) is documented in 1967 (1). At that time, laboratory assistants with flu-like symptoms, coupled with diffuse bleeding were admitted to hospitals in Marburg/Germany. The town in Germany where the disease first occurred gave it its name, the Marburg virus. After the inclusion of other similar cases, the connection of the patient to a specific monkey-species, the so-called Green monkeys (Cercopithecus aethiops) became clear and the chain of infection could be proved through evidence of close contact between them. These monkeys came from Africa and were also delivered to Frankfurt and Belgrade. After all monkeys have been killed, the infection could be stopped. A total of 31 persons have been infected by the new infection. $23 \%$ of the infected died. Until today, there were only 5 documented outbreaks of Marburg epidemic, all without exception and without major proportions in rural Africa e.g in Watsa with 97 cases (DRC, 2001/2 \# 002) and in Angola with 65 cases in 2004 (2, 3).

The first infection with the Ebola virus was documented in 1976 in the former Republic of Zaire now DRC and in the Sudan (4) precisely in the regions Yambu$\mathrm{ku}$ (Zaire) and Nzara (Sudan). It was the first time it came to numerically larger outbreaks with a higher mortality rate than in the previous Marburg virus outbreaks. Especially in nurses, medical assistants and doctors, the mortality rate exceeded 90\%. Since 1976 numerous outbreaks in rural areas in sub-saharan Africa have been documented (Figures 1 and 2, Table 1).

In recent times there has been an increased research on the transmission and contagiosity of the disease. The methodological approach to identify infection chains, especially in hospitals with the aim to uncover infections in health workers and reduce their infection rates, firstly found expression in the newly defined "barrier- nursing" methods (Figure 3). Data which has been available on the major African Ebola outbreak in 1976 were predominantly retrospective information. Therefore, the outbreak in Zaire (now DRC) from 1995 permitted for the first time the opportunity to examine more closely the development of the disease. During that time novels were also published such as Richard Preston's "the hot zone" (10) in which the public was wrapped into a pseudoscientific reality. This lurid representation of the Ebola virus itself came across with "half truths" about Ebola infection rather based on rational thinking.

Due to these and similar publications "the mystification" and rumors about Ebola virus have been increased. However, international organizations such as the World Health Organization (WHO) or the American Centers for Disease Control and Prevention (CDC) pushed the public interest towards the disease and therefore, realizing greater funds by the public sector and industry. In addition, recent events within the context of terrorism and the role of biologically effective weap- 
ons pushed the subject forward. The possibility of using Ebola as a threat, more precisely as a weapon of mass destruction brought the discussion into a new dimension (21).

\section{Social aspects of Ebola in Eastern/Central Africa}

Besides the purely medical findings on the disease there are also socio-cultural and socio-political relationships which have to be taken into account when discussing Ebola outbreaks and its influence on the life of society. In fact, there are not many studies performed, so far. Only in the last few years the number of publications increased mainly from the Anglo-American region $(9,18)$ but also from African countries themselves $(11,13)$. Most of these publications were covering the immediate threats which were more of a technical rational nature such as the lack of protective clothing. Only a minority of authors described behaviour pattern and general (mostly diffuse) fear of the disease and its manifestation in the society and community. Social discrimination and stigma are mentioned (8) for example a "social cripple" within traditional structures. In addition, there is a special focus on the still very rooted African tribal life, in which the majority of the Ebola outbreaks took and take place.

Authors such as Loscin et al. were dealing primarily with the question of how the people handle the anticipation of the adopted safe death. The authors are describing how they were interacting with their helplessness and with the knowledge of how to deal with the loss of close relatives.

Hewlett et al. (20) comes to a similar conclusion in which he investigated three large Ebola outbreaks - Kikwitt/ DRC in 1995; Gulu/Uganda in 2000/2001 and in Republic of Congo/ROC in 2003. He performed open and semi-structured interviews and questionaires. Hewlett put the focus primarily on the group of helpers in particular doctors and nurses, which are both observers and helpers as well as affected people and therefore victims (Figure 3).

Kinsmann et al. (11) describes a retrospective analysis on the basis of a newspaper survey which has been carried out after the numerically largest outbreak in Uganda in 2000/2001 in Gulu, Mbarara and Masindi (Figures 1 and 2). For the purpose of this report the two English-speaking magazines of the country, the Daily Monitor and the New Vision have been used for the research. Relevant articles were cut and computerized ( $n=693$ ) according to key words such as confusion, anger, serious stigma in affected communities. In the analysis it became clear that the reactions during Ebola outbreaks in large parts have been disproportionally compared to the realistic danger the disease could cause.

Preventive education of the population as a precaution must therefore be a solution of the problem in the long term. In addition, already existing medical early warning systems (e.g. virological-based early warning) and the WHO case-finding definition should be used, and also taking into account the importance of educa- 
tional aspects and "rational enlightenment" on irrational aspects of the contagiosity of the disease. Rooted in the African tribal life, the set of believes about the causes and the effect must be raised against authentic experiences expressed by survivors of the affected community.

\section{Socially-related aspects concerning the tribal family living in the affected regions}

African families contain quite often 2-3 generations in one household (14). This system of the "bulk-family" is frequently found in Africa's rural parts. Village communities with families of approximately 15-20 members appear quite often and the male family-members represent a social safeguarding for the parents and grandparents.

A retrospective report on the analysis of executed antibodies has been launched by the author (14) in the region Masindi/Uganda (Figures 1 and 2) in 2002. One goal was to find contact persons and survivors from the large Ebola outbreak in 2000/2001. A family originally immigrating from Kenya and then moved and settled to the rural area of Masindi in the late 1990s has been surveyed in the study. This " $\mathrm{K}$ " family was a bulk-family with 26 members including three generations. They were working as smallholders and drove cattle-raising in the region (Figure 4).

During the Ebola outbreak in 2000, the father and the mother were infected by the Ebola virus and died in the domestic environment. The father has been the index case as he lived up to his death nearby his wife and the other family members. Unfortunately, and also after diagnosis and the organization and implementation of standard procedures such as decisions on who is carrying out nursing measures, a further 14 family members died, including small children as well as the grandparents.

This example impressively illustrates the social collapse within an African bulkfamily when the actual breadwinner (in this case the adult male) died and children, women and only two adolescent remained. In addition to the devastating results for the family itself the remaining family members were exposed to social stigma deriving from society in the sense of being the " $\mathrm{K}$ " family which came from Kenya to Uganda as foreign strangers and carried the virus and therefore "shifting all evil" into these African communities.

\section{Lessons learned and outlook}

It becomes obvious that an outbreak situation always poses a special challenge to the society. In particular, in situations where many factors such as contagiosity and the mode of infection - due to the lack of knowledge - are not clearly defined yet, 
the entire scenario becomes unpredictable. Due to the special situation during Ebola outbreak, the main vector of transmission (fruit bats) has not been detected up to the year $2005(4,5,9,10)$. Mystification and the uncertainty about the mode of infection has been used as a strong engine for a whole entertaining industry. Pseudoscientific representations from this uncertainty of the people created movies like "Outbreak" (US-movie of 2005) and reinforced the difficult situation.

In an increasingly globalised world, the outermost corners of the world can be reached via digital media. Therefore, fear and grief in almost apocalyptical extent will be created in the affected and vulnerable minds of the people and repeatedly evoked in the memory of a society. Many authors $(11,18,19,20)$ do agree that a society can be paralyzed by this enormous irrational fear coupled with a morbid interest over a long time.

However, it is true that the specific attitude of the disease causes the protection of the population before curative medical assistance to the individual. According to epidemiological aspects, priorities have been clarified by authorities of the WHO and other organizations. However, and with regards to the understanding of active curative medical practice, conflicts arise within different treatment measures, especially when looking at the differences between various regions of the world. The question remains on how this problem can be solved? How does a society deal with a rational fear to a comprehensible life-threatening infection of a potentially deadly virus? There can be only a polypragmatic solution! Public educational measures must be the main focus in endemic countries with high illiteracy rate in the society. Therefore, better policies at the community and national level must be implemented.

Special programs, like those of the Ugandan Red Cross (URC) or MSF (doctors without borders) have already been implemented and they are considered a real and valuable approach (22).

In addition, so-called post-trauma relief programs exist in different ways e.g. in Uganda (23). These programs can help to overcome the loss of family members. Another interesting aspect, suggested by Loscin et al. (2003) is to tackle the fear by artistically expressive workout (8).

It is hoped for the future that in the process of globalization also remote rural areas in Africa will be in the position to fight Ebola disease. Additionally, and with reference to a well-educated and globalized world, the Ebola burden should not longer be considered as a huge threat to everyone or something that only plagues a minority of people in local Africa. 


\section{Figures and Tables}

A. Geographical distribution of the outbreaks in Central and Eastern Africa since 1974 (Selection)

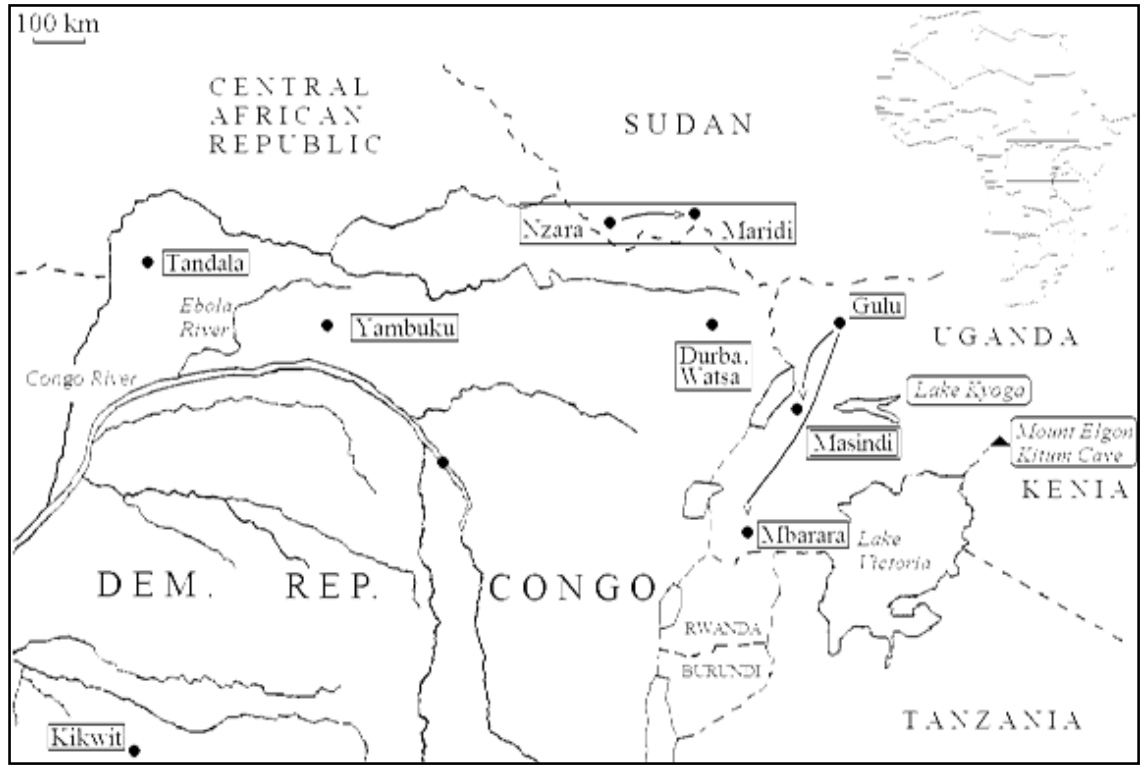

Figure 1: Map of affected areas

Source: Grade M. Ebola in Afrika, Suedwestdeutscher Verlag für Hochschulschriften 


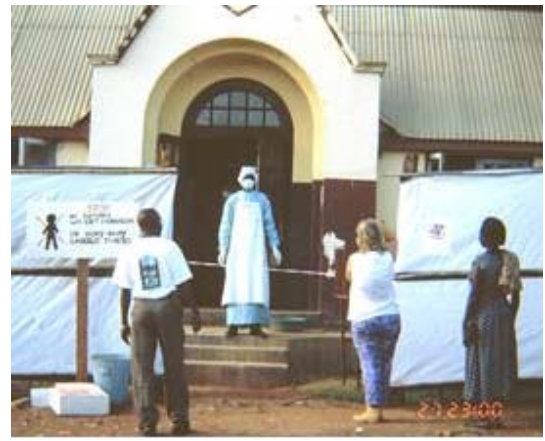

a. Main entrance of Gulu General Hospital.

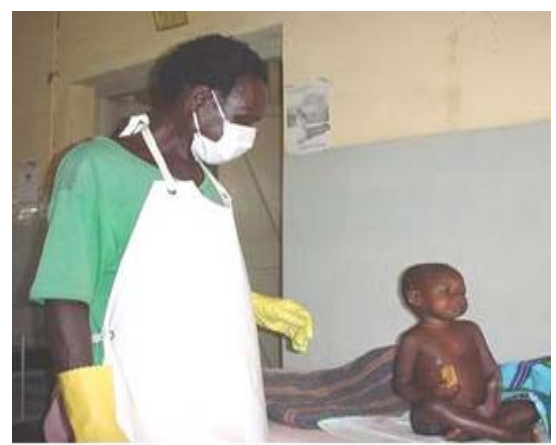

c. Breaches of barrier nursing: uncovered skin and lack of protection glasses

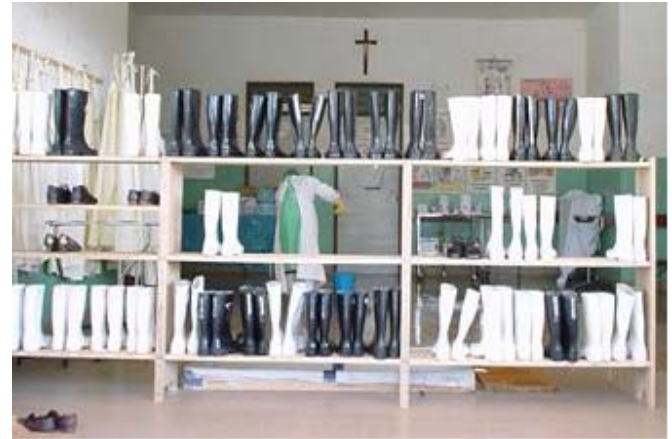

b. Necessary equipment like rubber boots

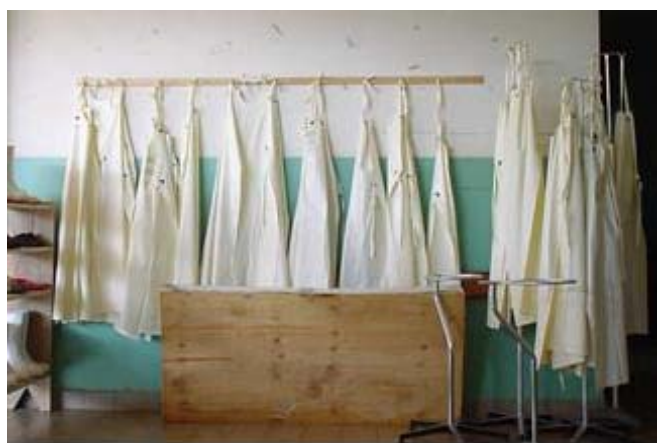

d. Necessary equipment like aprons

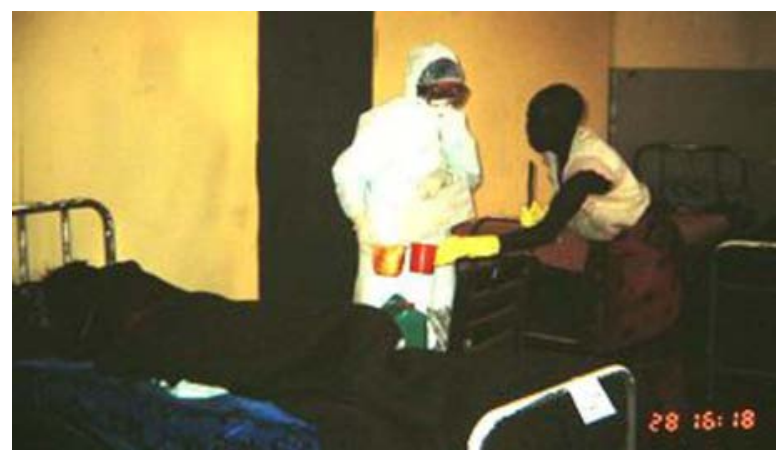

e. Non-protected caregiver

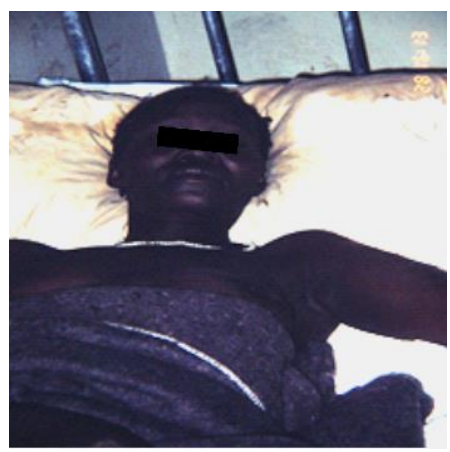

f. Ebola patient

Figure 2: Impressions by the author from the Ugandan Ebola outbreak 2000/2001 in Gulu 
B. Chronology of major Ebola haemorrhagic fever (EHF) outbreaks

Table 1: Ebola Outbreaks in Sub-Sharan Africa since 1976 (Major outbreaks)

\begin{tabular}{|c|c|c|c|c|c|}
\hline Year & Country & Virus subtype & Cases & Deaths & $\begin{array}{l}\text { Case } \\
\text { fatality }\end{array}$ \\
\hline 2011 & Uganda & Ebola Sudan & 1 & 1 & $100 \%$ \\
\hline 2008 & DRC & Ebola Zaire & 32 & 14 & $44 \%$ \\
\hline 2007 & Uganda & Ebola Bundibugyo & 149 & 37 & $25 \%$ \\
\hline 2007 & DRC & Ebola Zaire & 264 & 187 & $71 \%$ \\
\hline 2005 & Congo & Ebola Zaire & 12 & 10 & $83 \%$ \\
\hline 2004 & Sudan & Ebola Sudan & 17 & 7 & $41 \%$ \\
\hline $2003,11 / 12$ & Congo (ROC) & Ebola Zaire & 35 & 29 & $83 \%$ \\
\hline $2003,1-4$ & Congo (ROC) & Ebola Zaire & 143 & 128 & $90 \%$ \\
\hline $2001-2002$ & Congo & Ebola Zaire & 59 & 44 & $75 \%$ \\
\hline $2001-2002$ & Gabon & Ebola Zaire & 65 & 53 & $82 \%$ \\
\hline $2000-2001$ & Uganda & Ebola Sudan & 425 & 224 & $53 \%$ \\
\hline 1996 & $\begin{array}{l}\text { South Africa (ex- } \\
\text { Gabon) }\end{array}$ & Ebola Zaire & 1 & 1 & $100 \%$ \\
\hline $1996(7-12)$ & Gabon & Ebola Zaire & 60 & 45 & $75 \%$ \\
\hline $1996(1-4)$ & Gabon & Ebola Zaire & 31 & 21 & $68 \%$ \\
\hline 1995 & DRC & Ebola Zaire & 315 & 254 & $81 \%$ \\
\hline 1994 & Cote d'Ivoire & Ebola Ivory Coast & 1 & 0 & $0 \%$ \\
\hline 1994 & Gabon & Ebola Zaire & 52 & 31 & $60 \%$ \\
\hline 1979 & Sudan & Ebola Sudan & 34 & 22 & $65 \%$ \\
\hline 1977 & Zaire (Today DRC) & Ebola Zaire & 1 & 1 & $100 \%$ \\
\hline 1976 & Sudan & Ebola Sudan & 284 & 151 & $53 \%$ \\
\hline 1976 & Zaire (Today DRC) & Ebola Zaire & 318 & 280 & $88 \%$ \\
\hline
\end{tabular}

Source: Adapted to the WHO 2012 Fact sheet;

DRC $=$ Democratic Republic of Kongo (former Zaire) 
C. Hospital staff: Doctors, nurses and volunteers - helpers and victims at the same time

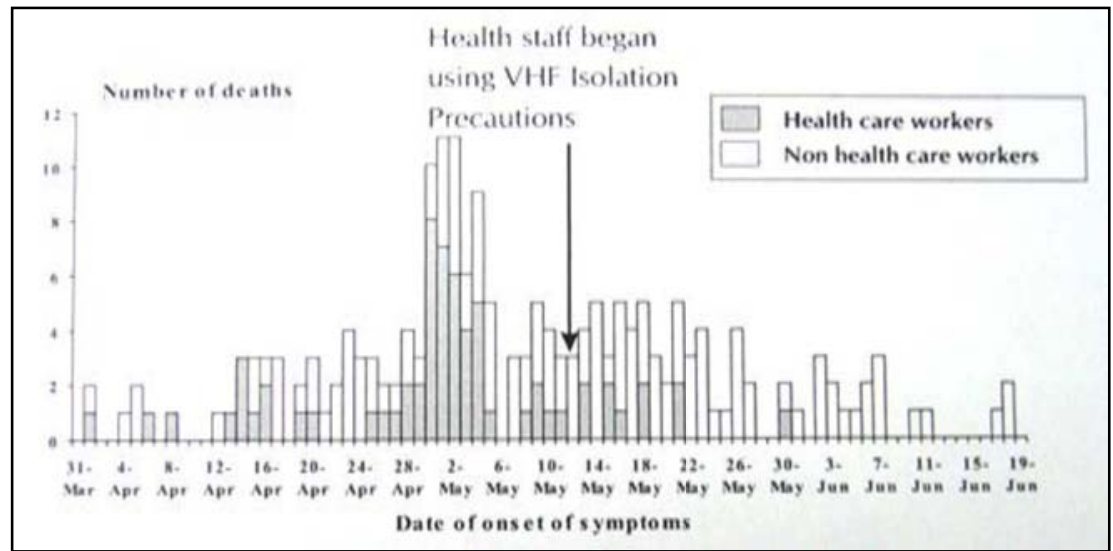

Figure 3:

The number of infected health care workers declined after barrier nursing practices were begun during the Ebola haemorrhagic fever outbreak at Kikwit, 1995. One case resulted from an inappropriate use of barrier nursing practices. Infection Control for Viral Haemorrhagic Fevers in the African Health Care Setting (JID 1999; 179, Suppl 1)

Source: World Health Organisation / U.S. Department of Health \& Human ServicesPublic Health Services, CDC 1998; 1-198 (92)

\section{The „K“ Family 2001/North Masindi / Uganda}

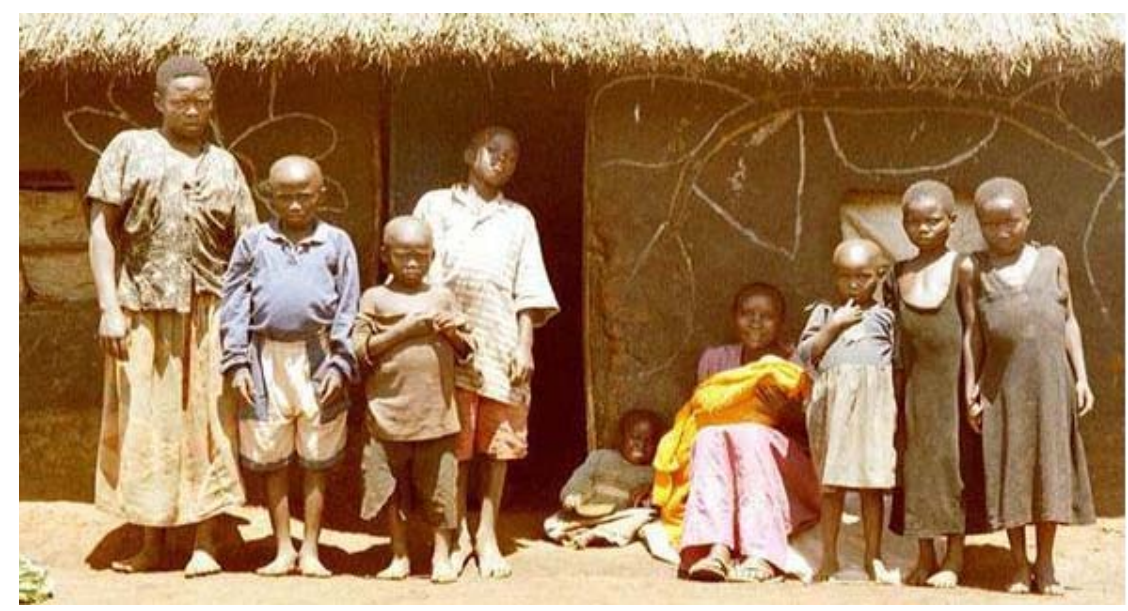

Figure 4: The 10 survivors of the originally 26 family members 


\section{References}

1. Martini GA, Siegert R. Marburg virus disease. Berlin: Springer Verlag, 1971.

2. K Leroy EM, Gonzalez JP, Baize S. Ebola and Marburg haemorrhagic fever viruses: major scientific advances, but a relatively minor public health threat for Africa. Clin Microbiol Infect 2011; 17:964-76.

3. Conrad JL, Isaacson M, Smith EB, et al. Epidemiologic investigation of Marburg virus disease, southern Africa, 1975. Am J Trop Med Hyg 1978; 27:1210-5.

4 World Health Organisation. Ebola haemorrhagic fever in Zaire, 1976. Report of an international commission. Bull WHO 1978;56:271-93.

5 Leroy EM, Epelboin A, Mondonge V, et al. Human Ebola outbreak resulting from direct exposure to fruit bats in Luebo, Democratic Republic of Congo, 2007. Vector Borne Zoonotic Dis 2009;9:723-8.

6 Heymann DL. Social, behavioural and environmental factors and their impact on infectious disease outbreaks. J Public Health Policy 2005; 26:133-9.

7 Groseth A, Feldmann H, Strong JE. The ecology of Ebola virus. Trends Microbiol 2007;15:408-16.

8 Locsin RC, Barnard A, Matua AG et al. Surviving Ebola: understanding experience through artistic expression. Int Nurs Rev 2003;50:156-66.

9 Loscin RC, Matua AG et al. The lived experience of waiting- to- know: Ebola at Mbarara, Uganda- hoping for life, anticipating death. J Adv Nurs 2002; 37:173-8.1

10 Preston R. The hot zone. New York: Random house, 1994.

11 Kinsman J. A time of fear: local, national, and international responses to a large Ebola outbreak in Uganda [epub ahead of print] [Record Supplied By Publisher] Global Health, 2012 Jun 13;8:15. doi: 10.1186/1744-8603-8-15.

12 Walsh PD, Biek R, Real LA.Wave-like spread of Ebola Zaire. PLoS Biol, Nov 2005, 3(11) pe371.

13 Kebede S, Duales S, Yokouide A, et al. Trends of major disease outbreaks in the African region, 2003-2007. East Afr J Public Health 2010; 7:20-9.

14 Grade M. Ebola in Afrika, Suedwestdeutscher Verlag für Hochschulschriften (11. Mai 2010) ISBN 10: 3838116631.

15 Dowell SF, Mukunu R, Ksiazek TG, et al. Transmission of Ebola hemorrhagic fever - a study of risk factors in family members, Kikwit, Democratic Republic of the Congo, 1995. J Infect Dis 1999;179 (suppl 1):S87-91. 
16 Borchert M, Mutyaba I, Van Kerkhove MD, et al. Ebola haemorrhagic fever outbreak in Masindi District, Uganda: outbreak description and lessons learned. BMC Infect Dis 2011;11:357.

17 MacNeil A, Farnon EC, Wamala J, et al. Proportion of deaths and clinical features in Bundibugyo Ebola virus infection, Uganda. Emerg Infect Dis 2010;16:1969-72.

18 Jeppsson A. Defend the human rights of the Ebola victims! Trop Doct 2002;32:181-2.

19 Bruce J; Brysiewicz P Ebola fever: The African emergency. Int J Trauma Nurs 2002;8:36-41.

20 Hewlett BL, Hewlett BS. Providing care and facing deaths: nursing during Ebola outbreaks in central Africa. J Transcult Nurs 2005;16:289-297.

21 Leffel EK, Reed DS. Marburg and Ebola viruses as aerosol threats. Biosecur Bioterror 2004;2:186-91.

22 http://www.aerzte-ohne-grenzen.de/informieren/einsatzlaender/afrika/d-rkongo/2012/2012-09-17-drkongo-uganda-ebola/index.html [Accessed January 19, 2013].

23 http://www.redcrossug.org/component/content/article/363-red-crossdelivers-relief-for-ebola-victims.html [Accessed January 19, 2013]. 



\title{
33. Neglected Tropical Diseases (NTDs) in Women and Children
}

\author{
Josephine Meister, Goettingen International Health Network, University Medical Center \\ Goettingen, Georg-August-University Goettingen, Germany.
}

\section{Introduction NTDs}

Neglected Tropical Diseases (NTDs)are a group of 17 infectious diseases which approximately affect one in six people worldwide, trapping the poorest people in poverty because of their impact on socio-economic development and health, particularly in women and children. NTDs are causing pregnancy complications, threaten newborns and reduce childhood development and growth. 1,2,3,4 NTDs are responsible for long-term disability, stigmatization and prevent individuals from being able to care for themselves or their families. The number of people infected by NTDs exceeds 1 billion in 149 endemic countries and causing billions of dollars in lost wages. NTDs hit predominantly people in impoverished regions, and they propagate poverty among those infected. 5 The disease severity worsen in cases of inadequate housing, poor water sanitation and vector control, rapid urbanization and lack of access to needed healthcare and case management. ${ }^{1}$ Additionally, global migration or tourist travels further allow the spread of NTDs. 
Table: 1: Overview Neglected Tropical Diseases

\begin{tabular}{|ll|}
\hline \multicolumn{2}{|c|}{ 17 Neglected Tropical Diseases } \\
Buruli Ulcer (Mycobacterium ulcerans infection) & Leprosy \\
Chagas disease & Lymphatic filariasis \\
Dengue/Severe dengue & Onchocerciasis (River blindness) \\
Dracunculiasis (guinea-worm disease) & Rabies \\
Echinococcosis & Schistosomiasis \\
Foodborne trematodiases & Soil-transmitted helminthiases \\
Human African trypanosomiasis (Sleeping sick- & Taeniasis/Cysticercosis \\
ness) & \\
Leishmaniosis & Trachoma \\
Yaws (Endemic treponematoses) & \\
\hline
\end{tabular}

These diseases are neglected because of lacking health care services to patients affected by NTDs. NTDs are also neglected in terms of research and development for more effective diagnostics and treatment and there is no sufficient funding available concerning the support of initiatives that address the whole of diseases.

Among the 17 diseases are trachoma and onchocerciasis, the most common causes of infectious blindness worldwide. One of the most severe NTDs are soiltransmitted helminthes (roundworm, whipworm and hookworms) which are the major source of malnutrition, iron deficiency and anemia worldwide, causing immense risks for pregnant women and preventing children from physical growth and development.

The transmission cycle and persistence of pathogens accountable for NTDs depend on vectors and intermediate hosts. The vector-borne NTDs such as Chagas disease, lymphatic filariasis, dengue, leishmaniosis and onchocerciasis account for an estimated $16 \%$ of the total burden of infectious diseases. Dengue fever is spreading and the global incidence increased 30 -fold. ${ }^{6}$ It mainly affects people in urban areas and in the case of dengue the access to medicine alone is not enough but integrated vector control is necessary. Chagas disease is the main source for changing conditions of the cardiovascular system, often underdiagnosed and continuously spreading not only in South America but among migrants all over the world. Among the most deadly NTDs are Human African trypanosomiasis (HAT) and visceral leishmaniosis (VL) that continue to be extremely difficult to handle and they are very costly. HAT starts with neurological disorders such as severe headaches and sleep disturbances and eventually results in coma and death. VL causing debilitating skin conditions and it has been stated that in 2012, 35 diseaseaffected countries reported VL (kala-azar) coinfection with HIV. Similarly, there is an infestation of skin and deformation of extremities evoked by leprosy, Buruli 
Ulcer and elephantiasis (lymphatic filariasis). The three diseases are life-threating, causing high stigmatization, social exclusion and loss of productivity. ${ }^{8,9}$ The impact of NTDs in conflict situations is even more severe. Refugees and displaced person are reservoirs of pathogens and conflict delays the delivery of health care services, puts the life of health workers at risk and case management and disease surveillance is not possible.

Many of NTDs are coendemic and one should emphasize the need to deliver preventive chemotherapy (defined as the widespread distribution of safe, singledose, quality-assured medicines) and if necessary in combination as NTDs can increase susceptibility to diseases such as tuberculosis and HIV/AIDS, and hinder their treatment.

NTDs are increasingly associated with other obstacles and risks such as resistance to medicines and pesticides, especially within the context of the scaling up of therapeutic dose (preventive chemotherapy) or controlling application in a population.

NTDs lack sufficient Research and Development (R\&D) as the current scientific information does not adequately support all aspects of disease control and management, particularly by considering existing (non-adequate) capacities in (i) the given number of treatments available, (ii) delivery of effective chemotherapy, (iii) hygiene services, (iv) trained health care personnel (v) early diagnosis, and (vi) sustainable preventive measures. ${ }^{10}$ In addition, approaches to pediatric medicines development and sex-specific study design remain underrepresented in NTDs intervention strategies. There is also almost no evaluation of the measurement on the impact of NTDs intervention. Similarly, analysis of the burden of NTDs and research on under-diagnosis is insufficient.

For controlling NTDs and intensified disease-management it can be stated that financial capacities improved throughout the past decade, however these financial contributions are far behind the needs to enhance integrated approaches to human, animal and environmental health, all part in reducing transmission and disease impact of NTDs.

\section{Neglected Tropical Diseases in women and children}

Women and children are disproportionally affected by NTDs. However, when addressing the Millennium Development Goal 3 and 5, controlling NTDs have not been adequately addressed. NTDs cause severe pregnancy complication and threaten neonatal and child health. NTDs increase the susceptibility to other diseases and affect the physical and cognitive growth of children. NTDs disfigure and disable and hinder women to take care of themselves and their family members. 


\subsection{NTDs in women}

Effective NTDs control and elimination requires the collection of epidemiological data that shows the differential impact according to sex and age. Particularly in resource-poor settings women have a higher than average risk to become infected by NTDs. This is mainly due their specific role in the family concerning reproductive health and caretaking associated with greater exposure to all family members (as potential carrier of the disease), as well as with household and agricultural activities. Today, two thirds of the food in most developing countries is produced by women and referring to the latest FAO report, women account for an average of 43 per cent of the agricultural labour force. ${ }^{11}$ NTDs thrive in areas with warm and moist climates, spread by insects and contaminated water and soil (e.g. infested eggs or larvae of worms). Women and girls are particularly on risk as they carry the main responsibility for collecting water and wood and they are spending long hours in fields and markets, all places where the transmission of disease occurs more frequently. Common NTDs among women that lead to severe disability and thus, affects the socio-economic situation of a family are trachoma, onchocerchiasis (river blindness) and lymphatic filariosis [LF] (elephantiasis). Taking the example of LF-related disability, it affects 40 million people globally, many of them are women. The disease can cause an enlargement of the entire genitals, vulva and breasts or the entire arm and leg. The most common LF-related disability issues include impact on work, stigma surrounding LF within local communities, depression, feelings of shame/humiliation, fear and reduced social status. ${ }^{12,13}$ Person et al. showed that women described a broad spectrum of stigma including the denied access to education and meaningful work roles. Without the burden of NTDs, mothers would have more likely healthy children as they are being better able to care for them. ${ }^{14}$

Most important, NTDs critically impair reproductive health in developing countries. In Africa, an estimated 10 million pregnant women are affected by schistosomiasis. Transmitted by freshwater snails, the disease is a chronic disease causing abdominal pain, fever, diarrhoea and blood in stool or urine. Half of these women with schistosomiasis develop anaemia and complications during pregnancy. 15 Salawu et al. estimates that 9-13 million women are afflicted with urogenital schistosomiasis. The typical genital changes, such as sandy patches and pathological blood vessels increase women's susceptibilty to super-infection, cause contact bleeding, decreased fertility, abortions, discharge and bleeding. ${ }^{16}$

In a study with 313 pregnant women in Nigera, 20,8\% were tested positive for Schistosoma haematobium. Women aged 24 years $(31,5 \%)$ in the first trimester of pregnancy had the highest intensity of infection $(33,1 \%)$. Women with urogenital schistosomiasis are also 3 to 4 times more likely to become infected with HIV than others. ${ }^{6}$ Therefore, Hegertun et al. argues that anti-schistosomal treatment should be already made available for girls at young age as a study with 1,057 schoolgirls between 10 and 12 years of age showed that they had similar genital symptoms to 
those reported for adult urogenital infection. Early treatment can therefore prevent chronic genital damage and secondary infections such as HIV. ${ }^{17}$

Referring to NTDs, high-risk groups for hookworm infection are preschool and school-age children and women of childbearing age (including pregnant women in the 2 nd and 3 rd trimesters and lactating women). ${ }^{18}$ Today, an estimated 44 million pregnant women are infected with hookworms. Hookworm infection in pregnant women is leading to malnutrition, iron deficiency and anemia. In Africa, hookworm infection is responsible for about $7 \%$ of the $20 \%$ of maternal deaths caused by anaemia. ${ }^{6}$ This supports the thesis that deworming during pregnancy has a significant influence on reducing maternal and perinatal morbidity and mortality.

Regarding the prevalence of intestinal worms, it mainly affects maternal nutrition and thus, influences the foetal growth and development. More than a quarter of all neonatal deaths occur because of foetal growth restriction, causing 800,000 deaths in the first month of life every year. A mean to reduce child mortality with largest predicted effects is therefore the treatment of severe acute malnutrition in the mother caused by hookworm infection. ${ }^{19}$

Another serious health concern among pregnant women is leishmaniosis. There are several reports on congenital transmission of $\mathrm{VL}$ from the mother to the child. ${ }^{20}$ Taking the example of a study on American VL, classical manifestations of the disease are persistent fever, weight loss, progressive anemia or pancytopenia, hypergammaglobulinemia, hepatomegaly and splenomegaly. Regarding the wellbeing of the foetus, great concern about the effect of drugs used for the treatment has been expressed by Mescouto-Borges et al. ${ }^{21}$ Due to the fact that pregnant women are usually excluded in clinical trials, recommendations for treatment which implies reasonable outcome such as liposomal amphotericin B are difficult as epidemiological data are lacking. ${ }^{22,23}$ In cases of congenital infection, the majority of children present the illness within the first year of life.

\subsection{NTDs in children}

Children are more vulnerable to NTDs due to their immature immune system and exposure to daily activities. Taking the example of trachoma, it is estimated that 325 million children live in areas where trachoma is confirmed or suspected. It is a bacterial infection of the eye and young children are the main reservoirs of infection (through direct contact with infected eye, nose, or throat and certain flies) and they have a 3 to 4 times higher chance of developing blindness in later life than others. ${ }^{6,24}$

Another NTD is dengue as the leading cause of childhood hospitalization and mortality. In a study of Teixera et al., 26,6\% of 625 children between $0-3$ years of age in Salvador, Brazil have been tested seropositive for dengue, showing a significant association between the seroprevalence of dengue and age. Consequently, 
children in early childhood run a higher risk of developing haemorrhagic fever, the most severe form of dengue. ${ }^{25}$

The total number of new cases of leprosy in 2011 was 219,075 mainly in India and Brazil. However, only in Indonesia more than 20,000 cases have been reported in 2011 with an incidence rate of $12.25 \%$ children among new cases and $39.79 \%$ females. ${ }^{6}$

Among all NTDs, soil-transmitted helminth infections remain to have one of the greatest impacts on children's growth, development, and physical fitness. Parasitic worm infections during pregnancy reduce child survival by causing severe anaemia which leads to low birthweight and increased infant mortality. Further, in a study with 1,004 children aged 7 months to 3 years in Ecuador, Mehta et al. hypothesize that a child of a mother infected with soil-transmitted helminthes (STH) has a greater risk of infection when compared to a child of an uninfected mother because individuals have an increased susceptibility to re-infection after treatment. 26 According to the World Health Organization, about 890 million children require treatment with preventive chemotherapy every year. ${ }^{6,27}$ In a recent survey by Wang et al., 1,707 children in 141 impoverished rural areas (95 villages and 46 schools) of Guizhou and Sichuan provinces in Southwest China have been screened with $21,2 \%$ of pre-school children and $22,9 \%$ of school-aged children infected with at least one of the three types of soil-transmitted helminths. ${ }^{28}$

Another public health problem with severe outcome for children is congenital transmission of Chagas disease. Traditionally endemic in the American continent, the estimated prevalence of Chagas disease has been estimated to 8 to 10 million in 2005 and according to a study of Holguin et al., the incidence of congenital cases was 130 per 100,000 births with a risk for transmission from the mother to the child of about 1 and 10\% with approx. 12,500 deaths per year. ${ }^{29}$ Detecting the disease in young women at child-bearing age may reduce vertical transmission. Most of the Trypanosoma cruzi infections are asymptomatic in the mother and infants who survive acute infection, carry a 20-30 risk of cardiac or gastrointestinal diseases. ${ }^{30}$ In a study from Argentina by Blanco et al. comprised 16,842 pregnant women who were seroreactive to $T$. cruqi. The incidence of congenital infection among those infants born to seroreactive mothers was $7.1 \%$. ${ }^{31}$ In Europe, the majority of infected individuals are immigrants and a recent study in Switzerland showed a prevalence of Chagas disease of $9.7 \%$ among 72 Latin Americans tested during pregnancy. ${ }^{32}$ Estimations about the number of infections in Europe reach between 68,000 and 123,000 cases with congenital transmission ranges from 0 to 3 cases per 1,000 pregnancies. In all studies, the authors acknowledged the problem of underdiagnosis. According to Holguin et al. in 2009, only 4,300 cases have been diagnosed in Europe, most of them in Spain. Thus, the average rate of underdiagnosis has been $94 \%$ and $96 \% .{ }^{29}$ Similar results have been outlined by Otero et al. in a recent study in a hospital in Barcelona. Global coverage of diagnosis in this hospital has only been $43 \%$ with 1,473 pregnant women involved and $3.5 \%$ seroprevalence rate for T. cruzi. ${ }^{30}$ 


\section{NTDs solutions and policy recommendation}

An enabling environment for action against NTDs in maternal and child health requires timely data, evidences coordination and coherence between the actors at clinical level as well as on international, national and subnational level. This also requires sustainable public and private funding.

The impetetus for improving the fight against NTDs is stronger today than 5 years ago. Countries have managed to improve NTDs status. Today, there are Mass Donation Programs (MDA) make available by government institutions such as the US Agency for International Development (USAID) and the UK Department for International Development (DFID) or by major pharmaceutical companies such as GlaxoSmithKline, Johnson and Johnson, Sanofi, Merck \& Co.Inc. and Gilead Sciences. ${ }^{5,33}$ Advocacy, resource mobilization and R\&D support is provided through private philanthropy organizations and product-development partnerships such as FIND (Foundation for Innovative New Diagnostics), DNDi (Drugs for Neglected Disease initiative), BMGF (Bill \& Melinda Gates Foundation) and the GNNTD (Global Network for Neglected Tropical Disease Initiative). 34,35,36,37. To support national programs for the control of NTDs and to highlight their public health importance, several key documents have been launched throughout the past years. It is worth mentioning the first WHO Report on NTDs in 2010, WHO's roadmap to accelerate work on NTDs in 2011, as well as the London Declaration on NTDs (2012). These were followed by the second WHO Report on NTDs in January 2013. The report sets the milestones for reaching the elimination and control targets of NTDs and also lists all relevant NTDs resolutions by the World Health Assembly from 1948 to 2012 6,8,38,39 On 27 May 2013, the World Health Assembly finally adopted the resolution WHA66.12 which addresses all 17 NTDs. 40

Despite these achievements and successes, challenges remain. Concerning maternal and child health, an approach that targets a whole of diseases and a strategy for integrated work in several disciplines is needed. First, this approach requires a good understanding about the impact of the different Neglected Tropical Diseases on mothers and children and how to control them. It requires that stakeholders continue to build alliances, create innovative mobilization of resources and utilize ONE Health principles for enhancing knowledge on serious challenges to the health of people, animals and ecosystems and to promote inter-agency and crosssectoral collaboration and partnerships. Networks such as GIHN (Goettingen International Health Network) focus on the threat disease poses to people, their economies, their water and food supplies, and the biodiversity essential to maintaining healthy environments and to prevent the exacerbation of disease severity. ${ }^{41}$

Globally, the more timely data, evidence coordination and coherence between the actors exist, the more progress can be achieved to sustain, expand and extend programs and decrease the burden of NTDs for maternal and child health. 
The following interventions are essential to maintaining this progress:

(a) To provide technical support, tools and resources for NTDs prevention, control, elimination and eradication to national programs, health organizations, medical and non-medical personnel. This approach requires the overall commitment to solve access issues towards health care services including diagnostics, essential medicines and therapy, particularly for children and pregnant women. It also includes the implementation of increased vector control programs, appropriate veterinary public health measures, save water, sanitation and hygiene provision and training of health personnel. This includes improving diagnostics, necessary supply of drugs, expansion of preventive chemotherapy and sustainable capacity building into national health plans. The provision of health care for patients and interrupting transmission of disease should be of greatest interest. It is also about national and international entities to reduce stigma and discrimination.

(b) To advance Research and Development. Current scientific information is not enough to substantiate all effects of NTDs control. This approach requires commitment by governments, health organizations, donors, pharmaceutical industry and academic leaders to support drug development, vaccines, diagnostics and focus on epidemiological data, NTDs impact as well as evaluation of NTDs intervention. The approach also includes an identification of actors, strategies and programs at national level and the analysis of each country's potential (also nonendemic) to contribute to the management and control of NTDs at a global scale by existing national competences and capacity. ${ }^{10}$

(c) To increase cooperation and partnership. This approach should focus on intersectoral collaboration and increased advocacy efforts for global NTDs initiatives and progressive scaling up of intervention. It also includes working with endemic countries, to engage with the post-2015 agenda and to ensure sustainable funding and assured quality of affordable prices.

For maternal and child health and with regards to the clinical level, a strategy for integrated work among several disciplines of physicians is necessary including obstetricians, pediatricians, microbiologists, epidemiologists, midwifes and infectiologists. ${ }^{30}$ Furthermore, an increased antenatal care and routine implementation of screening programs as well as the assurance of neonatal/infant care is needed. In cases of infections with soil-transmitted helmints, schistosomiasis, onchocericasis, lymphatic filariosis or trachoma, mass drug distribution programs should continue. This will help to decrease the number of re-infections and co-infection prevalence. The approach also includes an enhancement of diagnostic screening programs for NTDs such as dengue, visceral leishmaniosis and Chagas disease to prevent vertical transmission and ensure high cure rates for children. However, most of the interventions are not suitable for children and thus, an increased focus on paediatric care and appropriate medicines and treatment for children with NTDs is a must. 


\section{References}

1. Mackey TK Liang, BA: Threats from emerging and re-emerging neglected tropical diseases (NTDs), Infect Ecol Epidemiol. 2012; 2: 10.

2. Global Network for Neglected Tropical Diseases, Sabin Vaccine Institute: NTDs - The Facts.

http://www.globalnetwork.org/sites/default/files/AboutNTDs2013.pdf [Accessed June 15, 2013].

3. Hotez, PJ: Fighting neglected tropical diseases in the southern United States, BMJ. 2012 Sep 13;345:e6112. doi: 10.1136/bmj.e6112.

4. Hotez PJ, Dumonteil E, Heffernan MJ, Bottazzi ME: Innovation for the 'bottom 100 million': eliminating neglected tropical diseases in the Americas, Adv Exp Med Biol. 2013;764:1-12.

5. Barry MA, Simon GG, Mistry N, Hotez PJ: Global trends in neglected tropical disease control and elimination: impact on child health, Arch Dis Child Published Online First: 22 June 2013, doi:10.1136/archdischild2012-302338 [Accessed June 24, 2013].

6. WHOa: Sustaining the drive to overcome the global impact of neglected tropical diseases. Second WHO report on neglected tropical diseases, Geneva, World Health Organization, 2013 (document WHO/HTM/NTD/2013.1.).

7. Geiger A Simo G, Grébaut P, Peltier JB, Cuny G, Holzmuller P: Transcriptomics and proteomics in human African trypanosomiasis: Current status and perspectives, J Proteomics, 2011;74:1625-43

8. WHOb: Working to overcome the global impact of neglected tropical diseases. First WHO report on neglected tropical diseases, Geneva, World Health Organization, 2010, Department of Control of Neglected Tropical Diseases.

9. WHOc: World Health Assembly, resolution on all 17 Neglected Tropical Diseases, Geneva, World Health Organization (document WHA66.12) http://www.who.int/neglected_diseases/mediacentre/WHA_66.12_Eng. pdf [Accessed June 13, 2013].

10. Meister J: Neglected Tropical Diseases Action in Germany and Brazil, submitted for the degree Master of Arts by Josephine Meister to Ottovon-Guericke University Magdeburg/Germany, Faculty of Humanities, Social Sciences and Education, Institute for Political Science, Department Human Rights Education, (September 27, 2011), unpublished. 
11. Nije NI, Ndiaye T: Women and agricultural water resource management, United Nations Chronicle, 04.2013

http://www.un.org/wcm/content/site/chronicle/home/archive/issues20

13/water/womenandagriculturalwaterresourcemanagement [Accessed June 14, 2013].

12. Zeldenryk L, Gordon S, Gray M, Speare R, Melrose W: Disability measurement for lymphatic filariasis: A review of generic tools used within morbidity management programs. PLoS Negl Trop Dis. 2012; 6(9): e1768. doi:10.1371/journal.pntd.0001768.

13. Person B, Bartholomew LK, Gyapong M, Addiss DG, van den Borne B: Health-related stigma among women with lymphatic filariasis from the Dominican Republic and Ghana, Social Science \& Medicine, Volume 68, Issue 1, January 2009, Pages 30-38.

14. Global Network Neglected Tropcial Diseases: Links to maternal and child health

http://www.globalnetwork.org/sites/default/files/MaternalandChildHeal th.pdf [Accessed June 14, 2013].

15. Danso-Appiah A, Stolk WA, Bosompem KM, Otchere J, Looman CWN, et al.: Health seeking behaviour and utilization of health facilities for Schistosomiasis-related symptoms in Ghana. PLoS Negl Trop Dis. 2010;4(11): e867.

16. Kjetland EF, Leutscher PD, Ndhlovu PD.: A review of female genital schistosomiasis. Trends Parasitol. 2012 Feb;28(2):58-65.

17. Hegertun IEA, Sulheim Gundersen KM, Kleppa E, Zulu SG, Gundersen SG, et al:: S. haematobium as a common cause of genital morbidity in girls: A Cross-sectional Study of Children in South Africa. PLoS Negl Trop Dis. 2013;7(3): e2104.

18. Centers for Disease Control and Prevention: What is hookworm? http://www.cdc.gov/parasites/hookworm/gen_info/faqs.html [Accessed June 14, 2013]

19. Black RE, Alderman H, Bhutta ZA, Gillespie S, Haddad L, Horton S, Lartey A, Mannar V, Ruel M, Victora CG, Walker SP, Webb P (Maternal and Child Nutrition Study Group, Group members): Maternal and child nutrition: building momentum for impact, The Lancet, 2008 http://www.thelancet.com/series/maternal-and-child-nutrition [ Accessed June 14, 2013]. 
20. Meinecke CK, Schottelius J, Oskam L, Fleischer B: Congenital transmission of visceral leishmaniasis (kala-azar) from an asymptomatic mother to her child. Pediatrics. 1999;104:e65.

21. Mescouto-Borges, MR, Maués É, Costa DL, Pranchevicius MC, Romero GA: Congenitally transmitted visceral leishmaniasis: report of two Brazilian human cases. Braz J Infect Dis. 2013;17:263-6

22. Osorio Y, Rodriguez LD, Bonilla DL, Peniche AG, Henao H, et al: Congenital transmission of experimental leishmaniasis in a hamster model. Am J Trop Med Hyg. 2012;86: 812-820.

23. Boehme C, Hain U, Novosel A, Eichenlaub S, Fleischmann E, Löscher T: Congenital visceral leishmaniasis [letter]. Emerg Infect Dis. 2006; 12: 359_ 360 .

24. International Trachoma Initiative: Children as agents of change in trachoma communities. http://trachoma.org/sites/default/files/guidesandmanuals/children-asagents-of-change-in-trachoma-control_1.pdf [Accessed June 15, 2013].

25. Teixeira MG, Morato V, Barreto FR, Mendes CM, Barreto ML, Costa MD: Risk factors for the incidence of dengue virus infection in preschool children. Trop Med Int Health. 2012 Sep 14. doi: 10.1111/j.13653156.2012.03086.x. [Epub ahead of print]

26. Mehta RS, Rodriguez A, Chico M, Guadalupe I, Broncano N, et al.: Maternal geohelminth infections are associated with an increased susceptibility to geohelminth infection in children: a case-control study. PLoS Negl Trop Dis. 2012; 6: e1753.

27. WHOd: Soil-transmitted helminthiases: number of children treated in 2010. Weekly Epidemiol Record. 2012; 87:225-232.

28. Wang X, Zhang L, Luo R, Wang G, Chen Y, et al.: Soil-transmitted helminth infections and correlated risk factors in preschool and schoolaged children in rural Southwest China. PLoS ONE. 2012; 7: e45939.

29. Holguín A, Norman F, Martín L, Mateos ML, Chacón J, López-Vélez R, Pérez-Molina JA: Dried blood as an alternative to plasma/serum for Trypanosoma cruzi -IgG detection in screening programs. Vlin Vaccine Immunol. 2013; 20: 1197-202.

30. Otero S, Sulleiro E, Molina I, Espiau M, Suy A, Martín-Nalda A: Congenital transmission of trypanosoma cruzi in non-endemic areas: evaluation of a screening program in a tertiary care hospital in Barcelona, Spain Concepción Figueras Am J Trop Med Hyg. 2012; 87: 832-836. 
31. Blanco SB, Segura EL, Cura EN, Chuit R, Tulián L, Flores I, Garbarino G, Villalonga JF, Gürtler RE: Congenital transmission of Trypanosoma cruzi: an operational outline for detecting and treating infected infants in north-western Argentina. Trop Med Int Health. 2000; 5: 293-301.

32. Jackson Y, Gétaz L, Wolff H, Holst M, Mauris A, et al.: Prevalence, clinical staging and risk for blood-borne transmission of Chagas disease among Latin American migrants in Geneva, Switzerland. PLoS Negl Trop Dis. 2010; 4(2): e592.

33. Access to Medicine Index: Overall Ranking Index 2012. http://www.accesstomedicineindex.org/ranking [Accessed June 15, 2013].

34. Foundation for Innovative New Diagnostics (FIND): Diseases and projects- Human African Trypanosomiasis (HAT) \& other neglected diseases (OND). http://www.finddiagnostics.org/programs/hat-ond/ [Accessed June 15, 2013].

35. Drugs for Neglected Diseases initiative (DNDi): R\&D Projects - 2013 Outlook. http://www.dndi.org/diseases-projects/portfolio.html [Accessed June 15, 2013].

36. The Bill \& Melinda Gates Foundation (BMGF): Neglected infectious diseases -strategy overview. http://www.gatesfoundation.org/What-WeDo/Global-Health/Neglected-Infectious-Diseases [Accessed June 24, 2013].

37. Global Network Neglected Tropical Diseases: Factsheets and publications.http://www.globalnetwork.org/factsheets-and-publications [Accessed June 15, 2013].

38. WHOe: Accelerating work to overcome the global impact of neglected tropical diseases: a roadmap for implementation. Geneva, World Health Organization, 2012 (document WHO/HTM/NTD/2012.1).

39. London Declaration on Neglected Tropical Diseases: London, 30 January 2012

http:/ / search.who.int/ search?q=london + declaration\&ie $=u t f 8 \&$ site $=$ defa ult_collection\&client $=$ _en\&proxystylesheet $=$ _en\&output $=$ xml_no_dtd\& oe $=$ utf8 [Accessed June 15, 2013].

40. WHOf: World Health Assembly, resolution on all 17 Neglected Tropical Diseases, Geneva, World Health Organization (document WHA66.12)http://www.who.int/neglected_diseases/mediacentre/WHA_ 66.12_Eng.pdf [Accessed June 15, 2013]. 
41. Goettingen International Health Network (GIHN): 1 Annual Report 2011/2012, Georg-August University Goettingen and University Medical Center Goettingen, Germany. www. gihn.net [Accessed June 15, 2013]. 

IV. Non-communicable Diseases 



\title{
34. Significance of Iron Deficiency for Pregnancy and Childbirth in Low-Resource Countries
}

\author{
Patricia Christoph and Daniel Surbek, Department of Obstetrics and Gynecolo- \\ gy, University Hospital Insel, University of Bern, Switzerland
}

\section{Maternal Anemia and iron deficiency - A global public health burden}

\subsection{Anemia and iron deficiency: Magnitude of the problem}

Anemia is a major public health problem affecting 1.62 billion people globally. This number represents a quarter of the population worldwide. In developing countries the prevalence is $43 \%$ opposed to $9 \%$ in developed countries. Children and women of reproductive age are most at risk. Anemia in pregnancy is thought to be one of the commonest problems affecting pregnant women in developing countries, and Africa and Asia are the continents accounting for more than $85 \%$ of the absolute anemia burden in high risks groups (McLean et al., 2009).

The high prevalence of anemia in developing countries could even be an underestimate; data from rural areas is still lacking, the actual prevalence rates for many 
individual countries are not known as there are very few community-based surveys (McLean et al., 2009).

Table 1: Global anemia prevalence

\begin{tabular}{|l|c|}
\hline Children $<5$ years & $47 \%$ \\
\hline Pregnant women & $42 \%$ \\
\hline Non-pregnant women (15-49 years) & $30 \%$ \\
\hline
\end{tabular}

Iron deficiency is the most common cause of anemia (Ezzati et al., 2006). In many cases, iron deficiency is combined or exaggerated by malnutrition, infectious diseases and genetic alterations (Tolentino et al. 2007).

Iron deficiency is number 15 in the WHO ranking of selected risk factors for preventable death and disability in the most recent report from the Global Burden of Disease (GBD) (Ezzati et al., 2006).

Iron is critically important in muscle, brain and red blood cells. Iron deficiency may occur at any age. Typically, it occurs if nutrition is based on staple foods with little meat, or when people are exposed to infections that cause blood loss. Young children and women of childbearing age are most commonly and most severely affected. An estimated $41 \%$ of pregnant women and $27 \%$ of preschool children worldwide have anemia caused by iron deficiency (Table 1, de Benoist et al., 2008).

Iron deficiency anemia in early childhood reduces intelligence in midchildhood; it can also lead to developmental delay and even disability. About 18\% of maternal mortality in low- and middle-income countries - almost 120,000 deaths - is attributable to iron deficiency (Ezzati et al., 2006). Adding this disease burden to that for iron deficiency anemia in children and adults results in 19.7 million disability-adjusted life years (DALYs), or 1.3\% of global total DALYs. Forty per cent of the total attributable global burden of iron deficiency occurs in the South-East Asia Region and almost another quarter in the African Region. (Rodgers et al., 2004)

\subsection{Definition of anemia}

In most published studies, the minimum hemoglobin in healthy pregnant women living at sea level is $11-12 \mathrm{~g} / \mathrm{dL}$. The acceptable minimum hemoglobin level during pregnancy by WHO criteria is taken to be $11 \mathrm{~g} / \mathrm{dL}$ in the first trimester of pregnancy, $10.5 \mathrm{~g} / \mathrm{dL}$ in the second and $11 \mathrm{~g} / \mathrm{dL}$ in the third trimester. The WHO further divides anemia in pregnancy into: mild anemia (hemoglobin 10-10.9 $\mathrm{g} / \mathrm{dL}$ ), moderate anemia (Hb 7.0-9.9 g/dL) and severe anemia (hemoglobin $<7 \mathrm{~g} / \mathrm{dL}$ ). This is of course an arbitrary classification. The definition of severe anemia in gen- 
eral and in pregnancy in the published literature typically is not uniform, and many authors defined it by $\mathrm{Hb}<8.0 \mathrm{~g} / \mathrm{dL}$.

The clinical symptoms and signs of anemia vary and depend on the cause of anemia, the severity and the speed of onset. Typically, patients are pale, tired, have dizziness and headache, and are intolerant to physical stress. Further symptoms may arise, such as lack of concentration ability, shortness of breath, tachycardia or even cardiac insufficiency. Medical history of signs and symptoms, clinical examination, blood tests, and additional investigations should ideally be done to confirm the diagnosis of anemia, along with further investigations to find the underlying cause. Due to lack of resources and lack of staff motivation, screening of anemia is often done solely by clinical examination of the conjunctivae or is not carried out at all. In developing countries, anemia is therefore often underdiagnosed. Regarding isolated iron deficiency without anemia, it is now generally accepted that symptoms such as fatigue typically occur, and that replenishment of iron stores alleviates or abrogates these symptoms (results of the PREFER study, see www.clintrials.gov study no. NCT01110356).

\section{Types and causes of anemia}

\subsection{Nutritional anemia}

\section{Iron deficiency}

Anemia during pregnancy is most often believed to result from nutritional deficiencies, especially iron deficiency. The definition and identification of iron deficiency may be difficult especially in situations in which chronic inflammation is present. It is estimated that iron deficiency anemia (IDA) affects as many as 200 million people in the world probably making this the commonest nutritional deficiency in the world (Peña-Rosas et al., 2012). Among pregnant women the majority of all anemia cases have been attributed to iron deficiency (van den Broek et al., 1998). The prevalence of iron deficiency may be 2-3 times that of anemia, ranging from about $50 \%$ in some countries to nearly $100 \%$ in parts of others.

The serum ferritin level correlates well with total body iron stores and is therefore the best and most appropriate parameter to estimate iron stores. Iron deficiency in pregnancy has been defined as low ferritin levels and it is considered the gold standard for the diagnosis of IDA in pregnancy, together with hemoglobin values (Seid et al., 2008).

A ferritin cut-off point of $30 \mu \mathrm{g} / \mathrm{L}$ should be used, which accurately reflects bone marrow iron stores with high sensitivity and specificity. However, serum ferritin is also an acute phase protein that is raised in both acute and chronic infec- 
tion. Therefore, C-reactive protein should be measured simultaneously to exclude "false-high" ferritin values (Baynes et al., 1986).

Table 2: Ferritin values reflecting iron storage (normal C-reactive protein values)

\begin{tabular}{|l|l|}
\hline Ferritin $<15 \mu \mathrm{g} / 1$ & Lack of iron, completly empty iron storages \\
\hline Ferritin $15-30 \mu \mathrm{g} / 1$ & Lack of or short of iron \\
\hline Ferritin $30-50 \mu \mathrm{g} / 1$ & Grey area, functional IDA is possible \\
\hline Ferritin $>50 \mu \mathrm{g} / 1$ & Sufficient iron \\
\hline
\end{tabular}

There is often evidence of iron deficiency before a drop in hemoglobin concentration is noted. As pregnancy proceeds, most women show haematological changes suggestive of iron deficiency especially if not receiving iron supplements. Decreasing maternal iron stores and therefore the demand for additional iron is due to the growing foetus, placenta and the increased maternal red cell mass. Iron requirement during first trimester is limited, $0.8 \mathrm{mg}$ per day, but this rises considerably during the second and third trimester to $6.3 \mathrm{mg} /$ day due to the expansion of the maternal red cell mass and development and maintenance of the maternal-foetalunit. The total iron requirement over the whole pregnancy is about $1,000 \mathrm{mg}$.

Iron deficiency is often nutritional in origin. One of the major contributory factors in less industrialised countries is consumption of plant-based food containing insufficient iron, especially insufficient available haem iron from meat. Iron is obtained in the form of non-heam iron from vegetables and as haem iron from meat. Heam iron is absorbed about two to three times better than non-haem iron. A small amount of heam iron in the diet will improve absorption of non-haem iron and thus the diet composition is an important determinant of the amount of iron actually absorbed. Iron is stored in the reticulo-endothelial system as ferritin and haemosiderin (van den Broek et al., 2003).

Infection can also contribute to anemia and iron deficiency in developing countries.

Anemia in chronic infections is a result of an abnormal distribution of the body`s iron. Infections increase the liver`s production of 'acute phase proteins' form (hepcidin), which inhibit mobilization of the iron stored in the liver. The iron is retained in the liver and the iron stores increase while there is an iron deficient erythropoiesis.

Infections causing chronic blood loss such as parasitic infestation with hookworm and to a lesser extent schistosoma, increase iron requirement. Viral and bacterial infections may also interfere with food intake, absorption, storage and use of many nutrients including iron. Repeated episodes of infection may thus contribute to the development of iron deficiency and anemia (McLean et al., 2009). 
Even in developed countries anemia during pregnancy is common (Milman et al., 2008). However, in developing countries this occurrence is compounded by early onset of childbearing, high numbers of births, short intervals between births, and poor access to antenatal care and supplementation (Kalaivani et al., 2009)

\section{Folic acid deficiency}

Folates are a heat-labile, light-sensitive family of water-soluble vitamins essential to red blood cell maturation. Folates are present in all foods but more plentiful in dark green vegetable leaves and in liver. A diet that is rich in other B vitamins and in vitamin $\mathrm{C}$ is usually rich in folate too. Some important staples in the developing world such as rice, cassava, millet, sorghum and maize are poor sources of folate.

Low serum and erythrocyte concentrations of folate can lead to changes in cell morphology and intramedullary death of erythrocytes and reduced erythrocyte lifespan. Folic acid is required for the synthesis and maturation of erythrocytes. Folic acid deficiency contributes to megaloblastic anemia, a condition characterised by cells with large and malformed nuclei resulting from impaired DNA synthesis. During pregnancy, folate demands increase, and women entering pregnancy with poor folate status often develop megaloblastic anemia; folic acid deficiency during pregnancy can lead to neural tube defects (NTD) in the foetus.

Furthermore, lactation places additional demands with preferential uptake of folate by mammary glands over maternal requirements.

Folic acid supplementation with 400-800 $\mu \mathrm{g}$ folic acid daly must be given to all women from onset of pregnancy, ideally even 1-3 months before conception, because this supplementation reduces the incidence of foetal malformations including NTD (spina bifida), cleft lip/palate, urinary tract and cardiac malformation.

Folate requirements approximately double during pregnancy, especially in the last trimester and in the puerperium. Since body stores of folate are limited and dietary folate is likely to be insufficient in developing countries, anemia may develop as a consequence (McLean et al., 2009). Many studies have demonstrated a steady fall in serum folate levels throughout pregnancy, especially in women from poor socio-economic groups, in multigravidae, in smokers, and in women with twin pregnancies. The recommended intake of folate in pregnancy is at least 300 $\mu \mathrm{g} /$ day.

The contribution of folate deficiency to anemia at the population level is unknown because few global data exist, although it is thought to be low in developing countries. Review of epidemiological studies and nutrition surveys from South America did not reveal population-level folate deficiency in Costa Rica, Guatemala, or Mexico, but identified high prevalence of serum folate deficiency in specific subpopulations of Cuban men (64-89\%) and Chilean women (25\%) before flour fortification. Consumption and preparation of folate-rich food varies greatly by region (Allen et al., 2004). 
The amount of folic acid supplementation that might cause harm is still unknown (Mason et al., 2007). There is some evidence that high levels of folic acid due to high intake can accelerate the growth of preneoplasmic lesions (Chustecka et al., 2009).

There is growing concern worldwide that prenatal high folic acid in the presence of low vitamin B12 causes epigenetic changes in the unborn predisposing them to metabolic syndromes, central adiposity and adult diseases such as Type 2 diabetes (Yajnik et al., 2008). Another active area of research and concern is that excess folic acid in utero causes epigenetic changes to the brain leading to autism spectrum disorders (Beard et al., 2011).

\section{Vitamin B12 deficiency}

Muscle, red cell and serum vitamin B12 concentrations fall during pregnancy. The recommended intake of vitamin B12 is $2.0 \mu \mathrm{g}$ per day in the non-pregnant and 3.0 $\mu \mathrm{g}$ per day during pregnancy. Vitamin B12 is synthesised only by microorganisms, and its primary source is from ingestion of animal products. Any diet containing animal products will meet this requirement. Absorption of vitamin B12 involves a complex process by which gastric enzymes and acid facilitate its release from food sources, before being bound by an intrinsic factor secreted by gastric parietal cells, followed by uptake in the distal ileum.

Vitamin B12 deficiency can result in a megaloblastic macrocytic anemia, which is more common in severe vitamin B12 deficiency (Metz et al., 2008). The global prevalence of vitamin B12 deficiency is unknown, but evidence from several developing countries suggests that deficiency is widespread and is present throughout life. In South America, at least 40\% of children and adults were vitamin B12 deficient, with prevalences greater than 70\% in Africa and Asia (Allen et al., 2004 and 2009). However, how much this deficiency contributes to anemia is unclear, with few data available for the haematological effect of increasing B12 intake at the population level (Metz et al., 2008).

The main causes of vitamin B12 deficiency are inadequate dietary intake, especially from vegetarian diets (Antony et al., 2003), pernicious anemia, an autoimmune disorder resulting from autoantibody against intrinsic factor, tropical sprue, and co-infection with Diphyllobothrium latum, Giardia lamblia, and $H$ pylori. Vitamin B12 deficiency is associated with lactovegetarianism in India, and the scarcity of meat products in many south Asian diets (Misra et al., 2002; Antony et al., 2001; Bondevik et al., 2001). The prevalence of pernicious anemia is unknown, but reports from several African countries suggest that it could be more prevalent than was previously thought (Savage et al., 1994; Stabler et al., 2004). In addition, even without full-blown pernicious anemia, more simple chronic gastritis syndromes may contribute to vitamine B12 deficiency in pregnancy. 


\section{Vitamin A deficiency}

Vitamin A deficiency results from low dietary intake of preformed vitamin A from animal products and carotenoids from fruits and vegetables. Vitamin A plays an important part in erythropoiesis and has been shown to improve hemoglobin concentration and increase the efficacy of iron supplementation. The mechanisms are not fully understood, but are suggested to operate through effects on transferrin receptors affecting the mobilisation of iron stores, increasing iron absorption, stimulating erythroid precursors in the bone marrow, and reducing susceptibility to infections (Fishman et al., 2000).

During pregnancy, serum retinol concentrations have been shown to drop below non-pregnancy concentrations. Serum retinol concentrations of 1.05, 0.7, and $0.35 \mu \mathrm{mol} / \mathrm{L}$ indicate inadequate, moderately inadequate, and very inadequate liver stores, respectively.

Although serum retinol is commonly used as an indicator of vitamin A status, it is under strict homeostatic control and more accurate ways of diagnosing vitamin A deficiency in pregnancy include dose response tests. Vitamin A deficiency is the leading cause of preventable blindness in children and increases the risk of disease and death from severe infections. In pregnant women, vitamin A deficiency causes night blindness and may increase the risk of maternal mortality.

Vitamin A deficiency is common in Africa and southeast Asia, affecting an estimated $21 \%$ of children of preschool age and $6 \%$ of pregnant women, and accounting for around 800,000 deaths of women and children worldwide (Ezzati et al., 2004).

\subsection{Infectious causes of anemia}

\section{Soil-transmitted helminths}

Infectious diseases can contribute to anemia through impaired absorption and metabolism of iron and other micronutrients or increased nutrient losses. Hookworm (Necator americanus and Ancylostoma duodenale) infection is described to be one of the principal causes of IDA in developing countries especially in children. It is prevalent throughout the tropics and subtropics, in sub-Saharan Africa and southeast Asia with an estimated 576-740 million infections. The hookworm is acquired mainly by skin contact with faecal-contaminated soil or vegetation. In the tropics and subtropics, where ecological conditions allow larval development, hookworm infections are overdispersed or highly aggregated in areas of poverty, where poor water, sanitation, and infrastructure result in endemicity, often concentrated in small populations within these areas (Hotez et al., 2004).

Adult hookworms live in the duodenum and jejunum of humans attached to the intestinal mucosa, causing mechanical and chemical damage to capillaries and arterioles and sucking blood. By secreting anticlotting agents, the parasite ingests 
the flow of extravasated blood, with some recycling of lysed erythrocytes and blood. Once they leave the attached site, this causes chronic blood loss from the mucosa, with the severity dependent on the intensity of infection and the species of hookworm (A. duodenale is more invasive than $N$. americanus). Co-infection with several species is common. Hookworm disease results when chronic blood loss exceeds iron reserves, inducing IDA (Albonjco et al., 1998).

In people whose dietary intake of iron is low and whose blood iron stores are already depleted, hookworm infection can presumably give rise to IDA in just a few weeks, especially during pregnancy, when iron requirements are increased.

A systematic review of 12 studies of deworming during pregnancy (Brooker et al., 2008) showed that women with light hookworm infection (1-1,999 eggs per $g$ of faeces) had a standardised mean difference of hemoglobin that was 0.24 lower ( $95 \%$ CI -0.36 to -0.13$)$ than in those with no hookworm. A meta-analysis of 14 randomised controlled trials (Gulani et al., 2007) of deworming in sub-Saharan Africa and Asia showed a significant increase in mean hemoglobin $(1.71 \mathrm{~g} / \mathrm{L}, 95 \%$ CI 0.70-2.73), with an increased response in those provided with iron supplementation. A more recent meta-analysis of deworming in non-pregnant populations (Smith et al., 2010) showed the beneficial effect of anthelmintic treatment, and the differential effects of co-administration with praziquantel to target other parasites, or iron supplementation to replenish iron stores, or both; for example, albendazole together with praziquantel increased mean hemoglobin by $2.37 \mathrm{~g} / \mathrm{L}$ (95\% CI 1.33 $3.50)$.

\section{Malaria}

Malaria causes 1-3 million deaths every year, a burden falling overwhelmingly on Africa. Malaria due to Plasmodium falciparum, the most pathogenic species, may cause severe anemia in pregnancy. It is estimated that in sub-Saharan Africa 23 million pregnant women are exposed to Plasmodium infection annually. Women in their first and second pregnancies living in an endemic area are at a higher risk of acquiring malaria than non-pregnant women or multi gravidae, due to reduction of an appropriate immune response to the malaria parasite.

Anemia associated with malaria is caused by haemolysis of the red blood cells. Hypersplenism, a condition characterised by exaggeration of the inhibitory or destructive functions of the spleen, contributes to the anemia in up to $25 \%$ of women who suffer from malaria in pregnancy. Several studies have shown that protection against malaria contributes to the prevention of anemia in pregnancy (Rogerson et al., 2000) thus highlighting the importance of chemoprophylaxis and other methods of malaria control. The adverse effects of malaria on maternal and foetal wellbeing are thought to be for the most part due to the associated severe anemia, and subsequent hypoxia and congestive heart failure (Menendez et al., 2000; McLean et al., 2009). 
There is evidence that malaria can induce iron deficiency by several mechanisms: possibly through immobilising iron in haemazoin complexes and loss of urinary iron, as well as reducing intestinal iron absorption during the acute illness period. However these effects exerted by malaria on body iron status are still poorly understood, in part because biochemical and haematological indices of iron status are confounded by the malaria infection.

The interaction between malaria and iron and folate supplementation has been the subject of intense research and controversy in recent years (Prentice et al., 2008), intensified by the results of two cluster-randomised double-blind intervention trials (Sazawal et al., 2006; Tielsch et al., 2006) of iron and folate in preschool children in Zanzibar and Nepal.

There are some controversies in the literature regarding the increased risk of severe morbidity and mortality in children receiving iron and folate supplementation (Sazawal et al., 2006). The subsequent WHO-led Expert Consultation (Allen et al., 2008) emphasised the need to exercise caution against universal iron supplementation for children younger than 2 years in malaria-endemic regions where appropriate screening and clinical care are scarce, together with other recommendations. Thus, the treatment of children with iron supplements should be accompanied by adequate screening for and treatment of malaria.

Other malaria control strategies have had a beneficial effect on anemia. A systematic review of the effect of insecticide-treated bednets for prevention of malaria (Lengeler et al., 2004) showed a beneficial effect on hemoglobin in children. Use of insecticide-treated bednets compared with no bednets increased absolute packedcell volume by $1.7 \%$, whereas use of treated bednets compared with untreated bednets increased absolute packed-cell volume by $0.4 \%$.

\section{HIV/AIDS}

Anemia is the most common haematological complication associated with HIV infection, and is a marker of disease progression and survival (Volberding et al., 2004). Where anemia is associated with leucopaenia and thrombocytopaenia, the antenatal health worker should be alerted to the possibility of AIDS. The mechanism of HIV/AIDS-related anemia is multifactorial, resulting from HIV infection and the induced anemia of chronic disease, AIDS-related illnesses, and antiretroviral treatment. The burden of HIV/AIDS-related anemia falls predominantly on sub-Saharan Africa, where women and children are most at risk (Calis et al., 2008).

Transmission of HIV by blood transfusion is possible in developing countries, where there is a high prevalence of HIV positivity among donors and where the ability to screen for HIV is suboptimal. This further highlights the importance of the antenatal clinic in the prevention of anemia early in pregnancy, which may avoid the need for a blood transfusion later in the pregnancy. 


\subsection{Genetic causes}

Genetic hemoglobin disorders, which result from structural variation or reduced production of globin chains of hemoglobin, can result in anemia. Estimates of the burden of hemoglobin disorders suggest that at least $5.2 \%$ of the global population, and more than $7 \%$ of pregnant women, are carriers of significant hemoglobin variant (Modell et al., 2008). There is substantial regional variation, with the burden falling overwhelmingly on the African and southeast Asian regions, where 18.2\% and $6.6 \%$ of the population, respectively, carry a significant hemoglobin variant. Although extensive research has investigated the distribution and functional consequences of these genetic hemoglobin disorders, their contribution to the global anemia burden remains unclear (Weatherall et al., 2008). Micromapping studies are needed to estimate their prevalence in different populations (Weatherall et al., 2010), which could also have implications for prevalence estimation of anemia in populations in which these variants are present.

Each year, more than 330,000 infants are born with these disorders $(83 \%$ sickle-cell disorders and 17\% thalassaemias). Sickle-cell disorders are associated with chronic haemolytic anemia, and an estimated 2.28 per 1,000 conceptions worldwide are affected by sickle-cell disorders (Modell et al., 2008). Thalassaemias are highly prevalent in many Mediterranean, middle eastern, and south and southeast Asian countries; an estimated 0.46 per 1,000 conceptions worldwide are affected by homozygous $\beta$ thalassaemia, hemoglobin $\mathrm{E} / \beta$ thalassaemia, homozygous $\alpha 0$ thalassaemia, and $\alpha 0 / \alpha+$ thalassaemia (hemoglobinH disease). Only $12 \%$ of transfusion-dependent patients with $\beta$ thalassaemia receive transfusions, and only $39 \%$ of those receive adequate iron chelation (Modell et al., 2008).

As child survival improves, inherited hemoglobin disorders could become an increasingly important disease burden and cause of anemia in the future (Weatherall et al., 2001). However, many of the recent advances involving genetic and pharmacological treatments are unlikely to be available in low-resource settings, in which health system requirements for diagnosis and management of these conditions remain poor (Weatherall et al., 2008).

\subsection{Socio-cultural and enviromental determinants}

Social risk factors may include the perceptions of policy makers and of the society of the problem of anemia during pregnancy, cultural food taboos, socio-economic status and literacy level.

One study in Nigeria showed that many foods that are avoided during pregnancy provide all the key nutrients (calcium, iron, vitamin A, folic acid, vitamin C) needed by pregnant women (Brabin et al., 2001). Nigerian women complaining of dizziness attributed this to insufficient blood and used traditional medicine for this condition. A study from Sierra Leone reported that certain illnesses, including worm infestations are believed to drain the blood or 'dirty' it (Bledsoe et al., 1985). 
Many pregnant women in India and Thailand believed that taking iron and vitamin tablets will cause them to have big babies, resulting in difficult deliveries. Behavioural risk factors mainly involve dietary pattern, and health seeking behaviour. Pregnancy is recognised as a special event in a woman's life by most societies and often dietary restrictions are imposed on women. These food taboos are culturally defined and can adversely affect the health of women during pregnancy.

In Malawi, most people seem to believe that the following food items should not be eaten by a pregnant woman for reasons as given: Pepper is prohibited because babies are born with sores all over the body and red eyes. Eggs should not be eaten because the head of the child is born without hair. Sweet potato leaves cause delay during birth of the child. When eating pig meat the baby is born with skin rashes. The mudfish, that has a slippery body as it has no scales, causes abortions. Sugar cane causes the baby to be born with grey skin.

A woman's own perceptions of pregnancy and her health status are major factors that will influence her health decision making. These health perceptions, not only reflect the cultural back ground but also a woman's role and status within the family and community. In many traditional societies one of the most important roles of women is to produce children. Pregnant women are expected to carry on with their daily activities the same way as they would in a non pregnant state and often the side effects of pregnancy are de-emphasized. In addition, the community may look unfavourably on women who complain about symptoms associated with pregnancy. Thus health problems associated with nutritional anemia may not be recognised or may be ignored. Cultural idioms may play an important role in a pregnant woman's decision to seek health care and to comply with a prescribed treatment regime (Elegbe et al., 1984).

\section{Consequences of anemia}

\subsection{Maternal and perinatal cosequences}

The consequences of morbidity associated with chronic anemia extend to loss of productivity from impaired work capacity, cognitive impairment, and increased susceptibility to infection (Haas et al., 2001), which also exerts a substantial economic burden. Anemia is estimated to contribute to more than 115,000 maternal deaths and 591,000 perinatal deaths globally per year (Ezzati et al., 2006).

\section{Pbysiological changes in pregnancy}

The hemoglobin concentration, haematocrit and red cell count fall during pregnancy because the expansion of the plasma volume is greater than that of the red cell mass. However, there is a rise in total circulating hemoglobin directly related to 
the increase in red cell mass. This in turn depends partly on the iron status of the individual (Baker et al., 1979).

Plasma volume rises progressively throughout pregnancy with a tendency to plateau in the last 8 weeks. Women with multiple pregnancies have proportionately higher increment of plasma volume and in contrast women with poorly growing foetuses have a correspondingly poor plasma volume. Red cell mass increases steadily between the end of the first trimester and term. As with plasma volume the extent of the increase is related to the size of the foetus (Baker et al., 1979). Thus women with multiple pregnancies are also at increased risk of anemia.

Few studies have comprehensively assessed the factors associated with anemia in pregnancy in developing countries. This lack of research may be due to 3 main factors (van den Broek et al., 1998).

1. Adequate diagnostic facilities are lacking in many health institutions in developing countries.

2. The aetiologic pattern is often complex such that, for example, infection and nutritional deficiencies co exist.

3. The contribution of each aetiologic factor is difficult to assess in pregnancy because maternal physiologic changes alter the indexes used to diagnose anemia and nutritional deficiencies.

The general clinical manifestations of anemia include skin or mucosal pallor, lack of energy and shortness of breath, fatigue, lack of concentration and decreased mental, physical and cognitive performance all of which can present in different grades depending on the severity of the anemia (Al-Momen et al., 1996).

Consequences of moderate to severe anemia in pregnancy are susceptibility to infection and premature delivery, intrauterine growth restriction and the consequences of prematurity; increased perinatal morbidity and mortality (van Wyck et al., 2007).

This also means that anemia at the time of the delivery results more often in the need for blood transfusion, increased cardiovascular risks, longer hospital stay and problems in the postpartum period like reduced lactation or postpartum mood disorder (Breymann et al., 2008).

As anemia is usually multi-factorial in developing countries it becomes difficult to establish atributable risk for specific causes. The definitions of anemia across different studies vary, as do the methods for estimation of hemoglobin $(\mathrm{Hb})$ and the type of study sample, which may be either hospital or community. This variation between studies complicates comparative analysis (Harrison et al., 1989).

Anemia is probably a chronic rather than acute condition in many cases. There is a resulting compensatory shift of the oxygen dissociation curve to the right. Thus, women may be seen with very low hemoglobin in their early antenatal period without any overt symptoms of cardiac failure. They will, however, easily become tired and may decompensate, e.g. as a result of labour. Should any adverse 
event such as bleeding occur, their risk of death is high. The available data relating maternal and neonatal mortality to pregnancy anemia is limited and in most of the cases causality is difficult to establish. Some times the pregnancy outcome is related to the underlying cause of anemia, e.g. HIV, ante-partum haemorrhage, etc. rather than to the anemia itself.

It must also be noted that maternal mortality and morbidity data are most often based on hospital data. Hospital-based studies do not represent the actual situation in developing countries and more community-based studies are needed. These must include appropriate methodology to establish a clear causal relationship. Finally it must be noted that there are currently no agreed international standards or sets of criteria for attributing death to anemia.

A United Nations expert panel considered severe chronic anemia $(<7 \mathrm{~g} / \mathrm{dL})$ an associated cause in up to half of the maternal deaths worldwide.

In combination with obstetric haemorrhage, chronic anemia is estimated to be responsible for $17-46 \%$ of cases of maternal death. The proportion of maternal deaths due to acute and chronic anemia has been estimated for some countries: India (16\%), Kenya (11\%), Nigeria (9\%) and Malawi (8\%) (Schulmann et al., 1996).

Maternal morbidity resulting from anemia is similarly difficult to establish. Diminished work capacity and physical performance have been reported as a result of anemia (Rush et al., 2000; Johnson et al., 1997). IDA leads to abnormalities in host defence and neurological dysfunction (Harrison et al., 1975 and 1985).

\section{Consequences for the foetus/neonate/child}

In low-income countries, easy-to-remedy nutritional deficiencies - like iron deficiency - prevent 1 in 38 newborns from reaching age 5 (Rodgers et al., 2004).

The transgenerational transfer of poor iron status from mother to child has been shown in several studies, with maternal iron deficiency increasing the vulnerability of infants to iron deficiency and anemia (Kalaivani et al., 2009; de Pee et al., 2002; Meinzen-Dett et al., 2006; Colomer et al., 1990).

For the neonate there is a risk of reduced iron stores with serious consequences for their development (Klebanoff et al., 1991; Libermann et al., 1988; van Wyck et al., 2007).

\subsection{Infant mortality and morbidity}

Data from industrialised countries suggest that maternal anemia and iron deficiency increases low birth weight and preterm birth risk. Low birth weight (either IUGR or prematurity) is the most important risk factor for infant mortality and significant detriment of childhood morbidity.

Information collected from 1,423 live-born singletons and from their mothers prior to delivery attending in two hospitals in Chickwawa district, Malawi suggest- 
ed that IUGR of the baby is significantly associated with moderately severe anemia at booking $(\mathrm{Hb}<8.9 \mathrm{~g} / \mathrm{dl})$ at first antenatal clinic visit (RR 1.6; 95\% CI 1.2-2.2). The study concludes that prevention of IUGR requires reduction of anemia during pregnancy. Furthermore, the prevention of IUGR requires efforts to reduce anemia prevalence prior to pregnancy considering the high prevalence of anemia at first antenatal visit and late attendance in pregnancy to antenatal clinics of many women. The findings depend on the definitions used in the study. Asymmetric growth retardation was defined as a ponderal index \{calculated as 100 times the birth weight (in grams) divided by the cube of birth length (cubic centimeters)\} $<10 \%$ of reference value. Serial scanning of the foetus, which is very useful in accurately diagnosing IUGR, was not used in this study.

Because of the poor reduction observed in child mortality rates in the past decade in sub Saharan Africa and the noted increase in post-neonatal infant mortality (PNIM defined as mortality occurring after 28 days and before 1 year of age) in Malawi, a cohort study of mothers and their infants was carried out to analyse the risk factors for PNIM. Information was collected on women attending the antenatal services of two hospitals in a rural area of Malawi and 561 of their babies were enrolled in a follow up study. There were 128 with low birth weight (LBW, $<2500 \mathrm{~g}$ ), 138 with foetal anemia (FA, hemoglobin $<12.5 \mathrm{~g} / \mathrm{dl}$ ), 42 with both and 228 with a normal birth weight and no foetal anemia. The study showed there was no significant association between post neonatal infant mortality and foetal anemia (RR 1.60, 95\% CI 0.78-3.27) (Reddaiah et al., 1988).

\section{Prevention and treatment}

\subsection{Dietary modification}

Dietary modification could be an important way of decreasing the incidence of nutritional deficiency, though this may be difficult due to the limited purchasing power of a household and problems in persuading people to change long established dietary habits. Increased consumption of normal foods so that energy needs are met can however increase iron consumption. Such a strategy was used in rural India and increased total iron consumption by $25-30 \%$. Enhancing the bioavailability of ingested iron rather than increasing the total amount in the diet is another possibility. This can best be achieved by promoting iron absorption enhancers such as haem iron (e.g. meat) and vitamin C. Any campaign to promote the intake of haem iron could run into the obstacles of unavailability and high cost, as well as religious cultural and philosophical objections to the consumption of meat. In contrast, efforts to increase the content of non-heam iron, which is found in food such as pulses and in green vegetables in conjunction with an increased vitamin $C$ intake, have perhaps a greater chance of success. In many communities vegetables and fruits are eaten only infrequently and in small amounts. Persuading 
families to add these foods to their diets can have a considerable impact on iron absorption. Pregnant women should also be encouraged to increase their intake of pulses and green vegetables. Prolonged cooking of vegetables should also be discouraged as this destroys much of the vitamin $\mathrm{C}$ and folic acid content: nutrition education should stress this and teach ways of avoiding it. Health workers should also stress the importance of reducing tea and coffee consumption, particularly during meals. This alone can have a considerable impact on bioavailability of iron

\subsection{Iron supplementation (with folic acid)}

The available data from controlled trials provide clear evidence of a reduction of iron deficiency, IDA, low birth weight and preterm birth in pregnant women receiving iron supplementation (Peña-Rosas et al., 2012). Therefore, at present there is evidence to advice a policy of routine iron supplementation in pregnancy. Routine iron supplementation during pregnancy is warranted at least in populations in which iron deficiency is common, eventually even in all pregnant women (Huddle et al., 1999).

Studies from developing countries suggest that $120 \mathrm{mg}$ elemental iron and 1 $\mathrm{mg}$ folic acid are the optimum daily dosages needed to prevent anemia in pregnant women. But WHO recommends, for all pregnant women, if there are no high risk factors a combination tablet containing $60 \mathrm{mg}$ of elemental iron and $250 \mu \mathrm{g}$ of folate to be taken twice a day. Where this is not available, tablets such as ferrous sulphate, containing $60 \mathrm{mg}$ of elemental iron, should be given twice a day together with one folic acid tablet (1 mg) (van den Broek et al., 2003).

The side effects of oral iron administration (gastrointestinal side effects such as nausea, cramps and constipation) or women's dislike of the tablets owing to smell or taste have often being blamed for treatment failure, although a literature review (Suharno et al., 1993) found out that side effects accounted for only 10\% of non compliance. Rather, in most cases, women did not take their tablets because they never received them or received inadequate quantities. The impact of iron supplementation could be improved by counselling on why, how and when to take iron tablets and by supplying the tablets

There are various possible forms of treatment for IDA. Oral iron is the preferred route of administration for mild anemia. Treatment with iron preparations is used routinely in pregnancy, if iron deficiency with or without anemia develops. However, oral iron supplementation often leads to adverse effects such as constipation, abdominal pain or sickness. If these unwarranted gastrointestinal effects arise, adherence to iron treatment decreases.

Intravenous iron preparations are promising, especially in cases of severe anemia. They provide a greater and more rapid iron supply than oral iron therapy without the gastrointestinal side effects of oral substitution and make it possible to avoid bloodtransfusion with associated risks. However, because large-scale prospective randomized trials including maternal and neonatal outcomes after oral vs. 
intravenous iron treatment are lacking, oral iron treatment shoud remain the first line treatment for iron deficiency and IDA. Intravenous iron treatment in pregnancy should be limited to women with gastrointestinal side effects or lack of appropriate hemoglobin increase after oral treatment, or to those with severe anemia or those in need of rapid anemia correction for obstetric reasons (e.g. placenta praevia with antepartum bleeding), aiming to avoid the need for peripartum blood transfusion. Intravenous iron treatment should not be used in the first trimester of pregnancy to avoid any possibly yet unknown negative effect on the foetus' development.

In the majority of cases, anemia can be treated effectively with oral iron preparations. Many patients tolerate oral intake of iron supplements well; however, up to $40 \%$ have side effects related to oral iron treatment. The incidence of adverse reactions is dose dependent. The main adverse effects are of gastrointestinal nature ( $\mathrm{Al}$ et al., 2005; Bayoumeu et al., 2002; Breymann et al., 2008), the most common being constipation, diarrhoea, epigastric discomfort, nausea, severe abdominal pain or vomiting. These secondary effects can be lessened by the intake of tablets after meals, although this leads to a concomitant reduction of iron absorption. Typically, these adverse effects lead to poor treatment adherence especially in pregnancy when similar gastrointestinal complaints are often a problem prior to iron treatment due to the physiological changes in pregnancy. Intolerance to oral iron intake leads to a greater percentage of failure in the treatment. In addition, even with strict oral iron treatment adherence, there are still quite a number of patients who do not respond with an appropriate hemoglobin increase (i.e., hemoglobin increase below $10 \mathrm{~g} / \mathrm{L}$ within 14 days) (Puolakka et al., 1980).

Others possible indications for i.v. iron treatment include the necessity of rapid increase of hemoglobin, for example pregnancies in the third trimester with high risk of peripartum hemorrhage (e.g. placenta previa or placenta increta), or Jehovah's witnesses (van Wyck et al., 2007).

In these clinical situations described above, intravenous (i. v.) iron administration is indicated. A faster increase in hemoglobin, ferritin and iron stores by intravenous iron therapy has been reported by different authors (van Wyck et al., 2007; Bayoumeu et al., 2002; Bencaiova et al., 2009; Breymann et al., 2010; Christoph et al., 2012; Khalafallah et al., 2010; Singh et al., 1989; van Wyck et al., 2007 ).

Severe anemia mainly occurs in developing countries where it has been attributed to poor nutrition and concurrent conditions, especially infectious diseases such as Malaria. These women particularly benefit from high-dose intravenous iron substitution, because availability of blood transfusions is very limited and blood transfusions still bare certain risks. Intravenous iron substitution in this low resource setting seems to be a very good and safe alternative (Christoph et al., 2012). 


\subsection{Vitamin A supplementation}

The influence of vitamin A and iron supplementation was studied in anaemic pregnant women in West Java, in a randomized double masked placebo controlled field trial. 251 women aged 17-35 years, parity 0-4, gestation 16-24 weeks and hemoglobin between 80 and $109 \mathrm{~g} / \mathrm{L}$ were randomly allocated to four groups: vitamin A (2.4 mg retinol) and placebo iron tablets; iron (60 mg elemental iron) and placebo vitamin A ; vitamin A and iron; or both placebos, all daily for 8 weeks (Suprapto et al., 2002). Maximum hemoglobin was achieved with both vitamin A and iron supplementation (12.78 g/L, 95\% CI 10.86-14.70), with one third of the response attributable to vitamin A (3.68 g/L, 95\% CI 2.03-5.33) and two thirds to iron $(7.71 \mathrm{~g} / \mathrm{L}, 95 \%$ CI 5.97-9.45). After supplementation, the proportion of women who became non-anaemic was 35\% in the vitamin A-supplemented group, $68 \%$ in the iron-supplemented group, $97 \%$ in the group supplemented with both, and $16 \%$ in the placebo group. Therefore this study concluded that improvement in vitamin A status may contribute to the control of anemia in pregnant women (Suprapto et al., 2002).

\subsection{Control of parasitic infections}

Because anthelmintic treatment has been proven to slow the decrease of haemoglobin levels during pregnancy, WHO recommends that anthelminthic treatment should be included in strategies to control maternal anemia in most of the developing countries

\subsection{Blood Transfusion}

Although it is difficult to give absolute recommended hemoglobin level at which to transfuse, it should be considered in women at or above 34 weeks gestation who have extremely low hemoglobin levels (less than $6-7 \mathrm{~g} / \mathrm{dl}$ ), as it is important that severe anemia is corrected prior to delivery, although these women are also candidates for antepartum high-dose intravenous iron treatment to avoid blood transfusions. There is no role for treatment of severe IDA at community level. The community health workers must immediately refer pregnant women with severe anemia to appropriate health care level (McDermott et al., 1996).

\subsection{Potential barriers to effective anemia prevention and control}

The challanges to implemet efficient anemia prevention and control strategies are found at different levels. Often there is insufficient political priority due to poor awareness of the magnitude and consequences of anemia burden followed by inflexibility of financial disbursements for related health issues (e.g. malaria, reproductive health) to be used towards anemia control. 
There is limited leadership and capacity of existing human resources to champion anemia prevention and control and to meet the challenge of coordination of necessary multisectoral policy response (food and agriculture, health systems, water and sanitation, etc). There is a lack of institutional and operational capacity for scaling up the coverage of high-quality maternal and child health services and adressing the needs of vulnerable groups. Poor knowledge about how to coordinate the policy and implementation response to the context-specific causes of anemia may have an influence as well.

Often little individual and community awareness of anemia status due to latent symptoms and signs are found and therefore at the society level there is only limited mobilisation to demand policy attention to address anemia resulting in poor coordination of resources to support multisectoral response. The lack of education and information about anemia prevention, and awareness of benefits of appropriate intervention aggravate these conditions. Restricted financial access makes it difficult to have access to iron-rich food sources, iron supplements, health services, etc.

\section{Conclusion}

Screening for IDA in pregnancy has been based on the association with increased risk of preterm delivery and low birth weight, and maternal signs and symptoms of anemia. While the consequences of mild IDA for the foetus may be overestimated (Al-Momen et al., 1996), moderate to severe IDA leads to increase in morbidity of pregnant women and their children in developing as well as in developed countries. Understanding causes of anemia and potential mechanisms are crucial to our ability to intervene to reduce this burden. In the past decade, our understanding of the etiology and mechanisms of anemia in less developed countries has advanced significantly (Tolentino and Friedman, 2007)

Interventions to combat anemia in pregnancy have most recently focussed on antenatal supplementation with iron and vitamin A and on prevention of malaria during pregnancy. Meta-analysis of available studies suggest that oral iron supplementation should be given to all pregnant women, at least in countries with low dietary iron intake or high prevalence of iron deficiency. Malaria is not a contraindication against iron supplementation in pregnancy, as there is no evidence of increase of placental malaria by iron treatment. Intravenous iron treatment in pregnancy should be limited to women with gastrointestinal side effects or lack of appropriate hemoglobin increase after oral treatment, or to those with severe anemia or those in need of rapid anemia correction for obstetric reasons (e.g. placenta praevia with antepartum bleeding), aiming to avoid the need for peripartum blood transfusion. Intravenous iron treatment should not be used in the first trimester of pregnancy. 


\section{References}

Al RA, Unlubilgin E, Kandemir O, Yalvac S, Cakir L, Haberal A. Intravenous versus oral iron for treatment of anemia in pregnancy: a randomized trial. Obstet Gynecol. 2005 Dec;106(6):1335-40.

al-Momen AK, al-Meshari A, al-Nuaim L, Saddique A, Abotalib Z, Khashogii T, Abbas M. Intravenous iron sucrose complex in the treatment of iron deficiency anemia during pregnancy. Eur J Obstet Gynecol Reprod Biol. 1996 Nov;69(2):121-4.

Albonico M, Stoltzfus RJ, Savioli L, Tielsch JM, Chwaya HM, Ercole E, Cancrini G. Epidemiological evidence for a differential effect of hookworm species, Ancylostoma duodenale or Necator americanus, on iron status of children. Int J Epidemiol. 1998 Jun;27(3):530-7.

Allen L, Black RE, Brandes N, Brittenham G, Chazot G, Chunming C, Crawley J, de Benoist B, Dalmiya N, Darnton-Hill I, Dewey K, El-Arifeen S, Fontaine O, Geissler C, Haberle H, Harvey P, Hasler J, Hershko C, Hurrell R, Juma MA, Lönnerdal B, Lozoff B, Lynch S, Martines Salgado H, McLean E, Metz J, Oppenheimer S, Premji Z, Prentice A, Ramsan M, Ratledge C, Stoltzfus R, Tielsch J, Winachagoon P; Secrétariat de l'Organisation mondiale de la Santé, au nom des participants à la consultation. [Conclusions and recommendations of a WHO expert consultation meeting on iron supplementation for infants and young children in malaria endemic areas]. Med Trop (March). 2008 Apr;68(2):182-8. French.

Allen LH. How common is vitamin B-12 deficiency? Am J Clin Nutr. 2009 Feb;89(2):693S-6S. doi: 10.3945/ajcn.2008.26947A.

Allen LH. Folate and vitamin B12 status in the Americas. Nutr Rev. 2004, Jun;62(6 Pt 2):S29-33; discussion S34.

Antony AC. Vegetarianism and vitamin B-12 (cobalamin) deficiency. Am J Clin Nutr. 2003 Jul;78(1):3-6.

Antony AC. Prevalence of cobalamin (vitamin B-12) and folate deficiency in India-audi alteram partem. Am J Clin Nutr. 2001 Aug;74(2):157-9.

Baker SJ, DeMaeyer EM. Nutritional anemia: its understanding and control with special reference to the work of the World Health Organization. Am J Clin Nutr. 1979 Feb;32(2):368-417.

Baynes RD, Meriwether WD, Bothwell TH, Fernandes Costa FJ, Bezwoda WR, MacPhail AP. Iron and folate status of pregnant black women in Gazankulu. S Afr Med J. 1986 Aug 2;70(3):148-51. 
Bayoumeu F, Subiran-Buisset C, Baka NE, Legagneur H, Monnier-Barbarino P, Laxenaire MC. Iron therapy in iron deficiency anemia in pregnancy: intravenous route versus oral route. Am J Obstet Gynecol. 2002 Mar;186(3):518-22.

Beard CM, Panser LA, Katusic SK. Is excess folic acid supplementation a risk factor for autism? Med Hypotheses. 2011 Jul;77(1):15-7. doi: 10.1016/j.mehy.2011.03.013.

Bencaiova G, von Mandach U, Zimmermann R. Iron prophylaxis in pregnancy: intravenous route versus oral route. Eur J Obstet Gynecol Reprod Biol. 2009 Jun;144(2):135-9. doi: 10.1016/j.ejogrb.2009.03.006.

Bledsoe CH, Goubaud MF. The reinterpretation of Western pharmaceuticals among the Mende of Sierra Leone. Soc Sci Med. 1985;21(3):275-82.

Bondevik GT, Schneede J, Refsum H, Lie RT, Ulstein M, Kvåle G. Homocysteine and methylmalonic acid levels in pregnant Nepali women. Should cobalamin supplementation be considered? Eur J Clin Nutr. 2001 Oct;55(10):856-64.

Brabin BJ, Hakimi M, Pelletier D. An analysis of anemia and pregnancy-related maternal mortality. J Nutr. 2001 Feb;131(2S-2):604S-614S; discussion 614S615S.

Breymann C, Honegger C, Holzgreve W, Surbek D. Diagnosis and treatment of iron-deficiency anemia during pregnancy and postpartum. Arch Gynecol Obstet. 2010 Nov; 282(5):577-80. doi: 10.1007/s00404-010-1532-z.

Breymann C, Gliga F, Bejenariu C, Strizhova N. Comparative efficacy and safety of intravenous ferric carboxymaltose in the treatment of postpartum iron deficiency anemia. Int J Gynaecol Obstet. 2008 Apr; 101(1):67-73. doi: 10.1016/j.ijgo.2007.10.009.

Brooker S, Hotez PJ, Bundy DA. Hookworm-related anemia among pregnant women: a systematic review. PLoS Negl Trop Dis. 2008 Sep 17;2(9):e291. doi: 10.1371/journal.pntd.0000291.

Calis JC, van Hensbroek MB, de Haan RJ, Moons P, Brabin BJ, Bates I. HIVassociated anemia in children: a systematic review from a global perspective. AIDS. 2008 Jun 19;22(10):1099-112. Doi 10.1097/QAD.0b013e3282fa759f.

Christoph P, Schuller C, Studer H, Irion O, De Tejada BM, Surbek D. Intravenous iron treatment in pregnancy: comparison of high-dose ferric carboxymaltose vs. iron sucrose. J Perinat Med. 2012 May 13;40(5):469-74. doi: 10.1515/jpm2011-0231.

Colomer J, Colomer C, Gutierrez D, Jubert A, Nolasco A, Donat J, Fernandez Delgado R, Donat F, Alvarez-Dardet C. Anemia during pregnancy as a risk 
factor for infant iron deficiency: report from the Valencia Infant Anemia Cohort (VIAC) study. Paediatr Perinat Epidemiol. 1990 Apr;4(2):196-204.

De Pee S, Bloem MW, Sari M, Kiess L, Yip R, Kosen S. The high prevalence of low hemoglobin concentration among Indonesian infants aged 3-5 months is related to maternal anemia. J Nutr. 2002 Aug;132(8):2215-21.

Elegbe I, Ojofeitimi EO, Olegbe IA. Traditional treatment of pregnancy anemia in Nigeria. An indication for modern therapeutics. Trop Doct. 1984 Oct;14(4):175-7.

Ezzati M, Hoorn SV, Lopez AD, Danaei G, Rodgers A, Mathers CD, Murray CJL. Comparative quantification of mortality and burden of disease attribuable to selected risk factors. In: Lopez AD, Mathers CD, Ezzati M, Jamison DT, Murray CJL, editors. Global Burden of Disease and Risk Factors. Washington (DC): World Bank; 2006. Chapter 4.

Ezzati M, Lopez AD, Rodgers A, Vander Hoorn S, Murray CJ; Comparative risk assessment collaborating group. Selected major risk factors and global and regional burden of disease. Lancet. 2002 Nov 2;360(9343):1347-60.

Fishman SM, Christian P, West KP. The role of vitamins in the prevention and control of anemia. Public Health Nutr. 2000 Jun;3(2):125-50.

Gulani A, Nagpal J, Osmond C, Sachdev HP. Effect of administration of intestinal anthelmintic drugs on hemoglobin: systematic review of randomized controlled trials. BMJ. 2007 May 26;334(7603):1095. Epub 2007 Apr 13.

Haas JD, Brownlie T 4th. Iron deficiency and reduced work capacity: a critical review of the research to determine a causal relationship. J Nutr. 2001 Feb;131(2S-2):676S-688S; discussion 688S-690S.

Harrison KA. The struggle to reduce high maternal mortality in Nigeria. Afr J Reprod Health. 2009 Sep;13(3):9-20.

Harrison KA. Tropical obstetrics and gynaecology. 2. Maternal mortality. Trans R Soc Trop Med Hyg. 1989 Jul-Aug;83(4):449-53.

Harrison KA, Rossiter CE, Tan H. Family planning and maternal mortality in the Third World. Lancet. 1986 Jun 21;1(8495):1441.

Hirsch S, Sanchez H, Albala C, de la Maza MP, Barrera G, Leiva L, Bunout D. Colon cancer in Chile before and after the start of the flour fortification program with folic acid. Eur J Gastroenterol Hepatol. 2009 Apr;21(4):436-9. doi: 10.1097/MEG.0b013e328306ccdb.

Hotez PJ, Brooker S, Bethony JM, Bottazzi ME, Loukas A, Xiao S. Hookworm infection. N Engl J Med. 2004 Aug 19;351(8):799-807. 
Huddle JM, Gibson RS, Cullinan TR. The impact of malarial infection and diet on the anemia status of rural pregnant Malawian women. Eur J Clin Nutr. 1999 Oct;53(10):792-801.

Johnson JW, Ojo OA. Amniotic fluid oxygen tensions in severe maternal anemia.Am J Obstet Gynecol. 1967 Feb 15;97(4):499-506.

Kalaivani K. Prevalence and consequences of anemia in pregnancy. Indian J Med Res. 2009 Nov;130(5):627-33.

Khalafallah A, Dennis A, Bates J, Bates G, Robertson IK, Smith L, Ball MJ, Seaton $\mathrm{D}$, Brain T, Rasko JE. A prospective randomized, controlled trial of intravenous versus oral iron for moderate iron deficiency anemia of pregnancy. J Intern Med. 2010 Sep;268(3):286-95. doi: 10.1111/j.1365-2796.2010.02251.x. Epub 2010 May 19.

Lengeler C. Insecticide-treated bed nets and curtains for preventing malaria. Cochrane Database Syst Rev. 2004;(2):CD000363.

Liljestrand J, Bergström S, Birgegård G. Anemia of pregnancy in Mozambique. Trans R Soc Trop Med Hyg. 1986;80(2):249-55.

Mason JB, Dickstein A, Jacques PF, Haggarty P, Selhub J, Dallal G, Rosenberg IH. A temporal association between folic acid fortification and an increase in colorectal cancer rates may be illuminating important biological principles: a hypothesis. Cancer Epidemiol Biomarkers Prev. 2007 Jul;16(7):1325-9.

McDermott JM, Slutsker L, Steketee RW, Wirima JJ, Breman JG, Heymann DL. Prospective assessment of mortality among a cohort of pregnant women in rural Malawi. Am J Trop Med Hyg. 1996;55(1 Suppl):66-70.

McLean E, Cogswell M, Egli I, Wojdyla D, de Benoist B. Worldwide prevalence of anemia, WHO vitamin and mineral nutrition information system, 1993-2005. Public health nutr. 2009 Apr;12(4):444-54. doi: 10.1017/S1368980008002401. Epub 2008 May 23.

Meinzen-Derr JK, Guerrero ML, Altaye M, Ortega-Gallegos H, Ruiz-Palacios GM, Morrow AL. Risk of infant anemia is associated with exclusive breast-feeding and maternal anemia in a Mexican cohort. J Nutr. 2006 Feb;136(2):452-8.

Menendez C, Fleming AF, Alonso PL. Malaria-related anemia. Parasitol Today. 2000 Nov;16(11):469-76.

Metz J. A high prevalence of biochemical evidence of vitamin B12 or folate deficiency does not translate into a comparable prevalence of anemia. Food Nutr Bull. 2008 Jun;29(2 Suppl):S74-85.

Milman N. Prepartum anemia: prevention and treatment. Ann Hematol. 2008 Dec;87(12):949-59. doi: 10.1007/s00277-008-0518-4. Epub 2008 Jul 19. 
Misra A, Vikram NK, Pandey RM, Dwivedi M, Ahmad FU, Luthra K, Jain K, Khanna N, Devi JR, Sharma R, Guleria R. Hyperhomocysteinemia, and low intakes of folic acid and vitamin B12 in urban North India. Eur J Nutr. 2002 Apr;41(2):68-77.

Modell B, Darlison M. Global epidemiology of hemoglobin disorders and derived service indicators. Bull World Health Organ. 2008 Jun;86(6):480-7.

Peña-Rosas JP, De-Regil LM, Dowswell T, Viteri FE. Daily oral iron supplementation during pregnancy. Cochrane Database Syst Rev. 2012 Dec 12;12:CD004736. doi: 10.1002/14651858.CD004736.pub4.

Prentice AM. Iron metabolism, malaria, and other infections: what is all the fuss about? J Nutr. 2008 Dec;138(12):2537-41. doi: 10.3945/jn.108.098806.

Puolakka J, Jänne O, Pakarinen A, Vihko R. Serum ferritin in the diagnosis of anemia during pregnancy. Acta Obstet Gynecol Scand Suppl. 1980;95:57-63.

Reddaiah VP, Raj PP, Ramachandran K, Nath LM, Sood SK, Madan N, Rusia U. Supplementary iron dose in pregnancy anemia prophylaxis. Indian J Pediatr. 1989 Jan-Feb;56(1):109-14.

Rodgers A, Ezzati M, Vander Hoorn S, Lopez AD, Lin RB, Murray CJ; Comparative risk assessment collaborating group. Distribution of major health risks: findings from the global burden of disease study. PLoS Med. 2004 Oct;1(1):e27. Epub 2004. Oct 19.

Rush D. Nutrition and maternal mortality in the developing world. Am J Clin Nutr. 2000 Jul;72(1 Suppl):212S-240S. Review. Erratum in: Am J Clin Nutr $2001 \mathrm{Jan} ; 73(1): 134$.

Savage D, Gangaidzo I, Lindenbaum J, Kiire C, Mukiibi JM, Moyo A, Gwanzura C, Mudenge B, Bennie A, Sitima J, et al. Vitamin B12 deficiency is the primary cause of megaloblastic anemia in Zimbabwe. Br J Haematol. 1994 Apr;86(4):844-50.

Sazawal S, Black RE, Ramsan M, Chwaya HM, Stoltzfus RJ, Dutta A, Dhingra U, Kabole I, Deb S, Othman MK, Kabole FM. Effects of routine prophylactic supplementation with iron and folic acid on admission to hospital and mortality in preschool children in a high malaria transmission setting: community-based,randomised, placebo-controlled trial. Lancet. 2006 Jan 14;367(9505):133-43. Erratum in: Lancet. 2006 Jan 28;367(9507):302.

Seid MH, Derman RJ, Baker JB, Banach W, Goldberg C, Rogers R. Ferric carboxymaltose injection in the treatment of postpartum iron deficiency anemia: a randomized controlled clinical trial. Am J Obstet Gynecol. 2008 Oct;199(4):435.e1-7. doi: 10.1016/j.ajog.2008.07.046. 
Singh K, Fong YF, Kuperan P. A comparison between intravenous iron polymaltose complex (Ferrum Hausmann) and oral ferrous fumarate in the treatment of iron deficiency anemia in pregnancy. Eur J Haematol. 1998 Feb;60(2):119-24.

Smith JL, Brooker S. Impact of hookworm infection and deworming on anemia in non-pregnant populations: a systematic review. Trop Med Int Health. 2010 Jul;15(7):776-95. doi: 10.1111/j.1365-3156.2010.02542.x. Epub 2010 May 25.

Stabler SP, Allen RH. Vitamin B12 deficiency as a worldwide problem. Annu Rev Nutr. 2004;24:299-326.

Suharno D, West CE, Muhilal, Karyadi D, Hautvast JG. Supplementation with vitamin A and iron for nutritional anemia in pregnant women in West Java, Indonesia. Lancet. 1993 Nov 27;342(8883):1325-8.

Suprapto B, Widardo, Suhanantyo. Effect of low-dosage vitamin A and riboflavin on iron-folate supplementation in anaemic pregnant women. Asia Pac J Clin Nutr. 2002;11(4):263-7.

Tielsch JM, Khatry SK, Stoltzfus RJ, Katz J, LeClerq SC, Adhikari R, Mullany LC, Shresta S, Black RE. Effect of routine prophylactic supplementation with iron and folic acid on preschool child mortality in southern Nepal: communitybased, cluster-randomised, placebo-controlled trial. Lancet. 2006 Jan 14;367(9505):144-52.

Tolentino K, Friedman JF. An update on anemia in less developed countries. Am J Trop Med Hyg. 2007 Jul; 77(1):44-51.

van den Broek N. Anemia and micronutrient deficiencies. Br Med Bull. 2003;67:149-60.

van den Broek NR, Graham WJ. Quality of care for maternal and newborn health:the neglected agenda. BJOG. 2009 Oct;116 Suppl 1:18-21. doi: 10.1111/j.1471-0528.2009.02333.x.

van den Broek NR, Rogerson SJ, Mhango CG, Kambala B, White SA, Molyneux $\mathrm{ME}$. Anemia in pregnancy in southern Malawi: prevalence and risk factors. BJOG. 2000Apr;107(4):445-51.

van den Broek NR, Letsky EA, White SA, Shenkin A. Iron status in pregnant women: which measurements are valid? Br J Haematol. 1998 Dec;103(3):81724.

Van Wyck DB, Martens MG, Seid MH, Baker JB, Mangione A. Intravenous ferric carboxymaltose compared with oral iron in the treatment of postpartum anemia: a randomized controlled trial. Obstet Gynecol. 2007 Aug;110(2 Pt 1):267-78. Erratum in: Obstet Gynecol. 2008 Apr;111(4):996. 
Volberding PA, Levine AM, Dieterich D, Mildvan D, Mitsuyasu R, Saag M; Anemia in HIV Working Group. Anemia in HIV infection: clinical impact and evidence-based management strategies. Clin Infect Dis. 2004 May 15;38(10):1454-63. Epub 2004 Apr 27.

Weatherall DJ. The inherited diseases of hemoglobin are an emerging global health burden. Blood. 2010 Jun 3;115(22):4331-6. doi: 10.1182/blood-2010-01251348. Epub 2010 Mar 16.

Weatherall DJ. Hemoglobinopathies worldwide: present and future. Curr Mol Med. 2008 Nov;8(7):592-9.

Weatherall DJ, Clegg JB. Inherited hemoglobin disorders: an increasing global health problem. Bull World Health Organ. 2001;79(8):704-12. Epub 2001 Oct 24.

Yajnik CS, Deshmukh US. Maternal nutrition, intrauterine programming and consequential risks in the offspring. Rev Endocr Metab Disord. 2008 Sep;9(3):203-11. doi: 10.1007/s11154-008-9087-z. Epub 2008 Jul 26. 



\section{The Impact of Contraception on Maternal Mortality in Indonesia}

Shofwal Widad, Department of Obstetrics and Gynaecology, Faculty of Medicine, Gadjah Mada University, Yogyakarta, Indonesia

\section{Maternal mortality in Indonesia}

Indonesia is an archipelagic country in Southeast Asia, comprising approximately 17,508 islands, with a land area of 1,910,931 square kilometers. The distance from Sabang, the west endpoint, to Merauke, the east endpoint, is approximately 5,233 $\mathrm{km}$. It has 33 provinces, 399 regencies (districts) and 98 municipalities: It has a population growth rate of 1.49 percent and a total of $237,641,326$ people (the world's fourth most populous country) with a female population of 118,010,410. In 2010, the country's national human development index was 72.27 with a GDP (per capita gross domestic product) of US $\$ 3.542^{2}$

Currently, maternal mortality remains high in Indonesia compared to other Southeast Asian countries in general. The United Nations (UN) estimates that every year about 350,000 women die as a result of pregnancy or childbirth worldwide. Indonesia is one of 11 countries that account for 65 percent of all maternal deaths worldwide. ${ }^{3,4}$ The maternal mortality decline over the 16 years period from 1991 to 2007 was notably slow, both in terms of the maternal mortality ratio (MMR, defined as the number of maternal deaths per 100,000 live births per year) 
and in terms of absolute number of deaths. According to the Indonesian Demographic and Health Survey (IDHS), the MMR declined just 162 points, from 390 in 1991 to 228 in 2007 (Figure 1)..$^{5}$ The absolute number of death was about 19,000 in 2005, seventh-ranked largest number of global maternal death after India, Nigeria, the Democratic Republic of Congo, Afghanistan, Ethiopia, and Bangladesh. ${ }^{4}$ The National official report of the Ministry of Health stated that the absolute number of death was 11,534 in 2010. About 50 percent of maternal deaths occurred in five provinces, 19.8 percent in West Java, 15.3 percent in Central Java, 5.6 percent in East Nusa Tenggara, 4.7 percent in Banten and 4.3 percent in East Java. ${ }^{6}$ Referring to the continuous slow decrease of MMR, the Millennium Development Goal (MDG) target 5A achievement of 102/100,000 live births looks unlikely to be achieved, if there are not extraordinary efforts. Even the WHO, UNICEF, UNFPA and the World Bank estimated that the MMR in Indonesia was 420/100,000 live births in 2005 with a range of uncertainty between 240-600/100,000 live births, information based on adjusted estimation for countries which use the direct sisterhood method as a source of maternal mortality due to a lack of complete registration of death within the country, leading to underreporting of maternal mortality. ${ }^{2}$

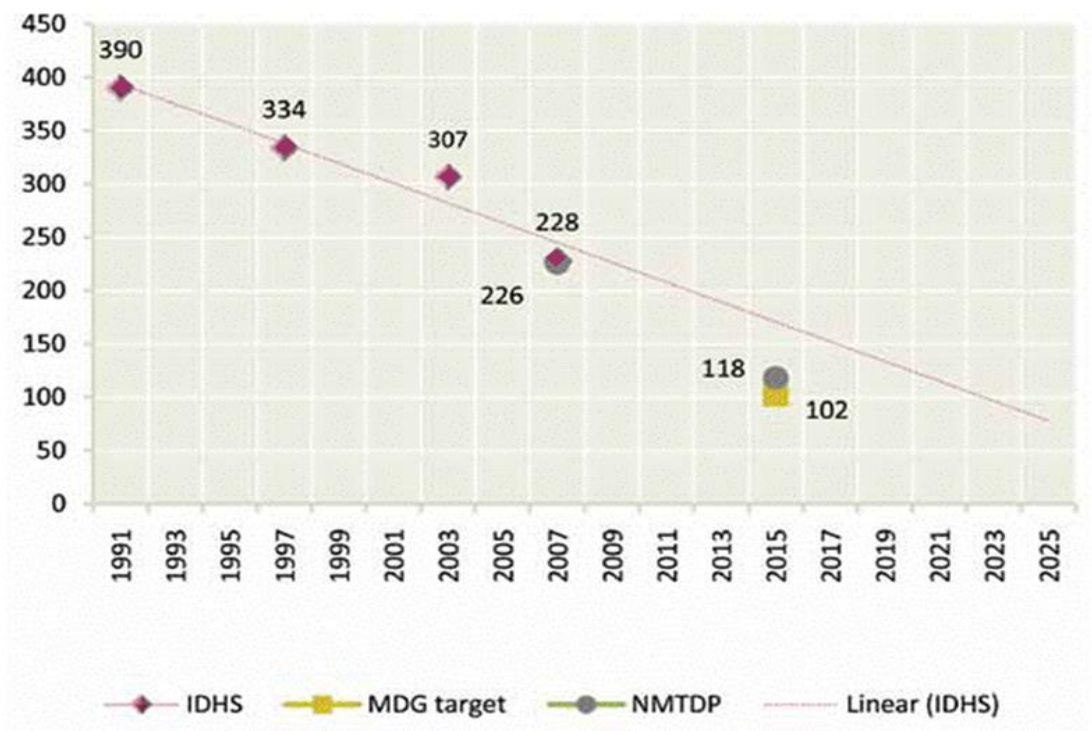

Figure 1. National trends and projections for the maternal mortality ratio 1991-2025. IDHS: Indonesia Demographic Health Survey; MDG: Millennium Development Goal, NMTDP: National Medium-Term Development Plan.

Source: Indonesian Central Bureau of Statistics (BPS/Biro Pusat Statistik), IDHS several years $^{5}$ 


\section{Causes of maternal mortality}

The direct medical causes of maternal mortality in Indonesia were hemorrhage (27 percent), hypertensive disorders (23 percent), infection (11 percent), puerperial complication ( 8 percent), obstetric trauma ( 5 percent), dystocia or prolonged labour ( 5 percent), abortion complication ( 5 percent), obstetric emboli ( 5 percent), and others (11 percent; Figure 2). ${ }^{6}$ Haemorrhage is a major cause of death, probably related to delays in getting emergency obstetric care. Hypertensive disorders have different etiopathogenesis and are more similar to the leading causes of maternal death in developed countries. Both causes, which contribute to 50 percent of total maternal death, usually occur around the time of childbirth and the key to handle this issue is to attain timely comprehensive emergency obstetric care to avoid maternal death. ${ }^{7}$

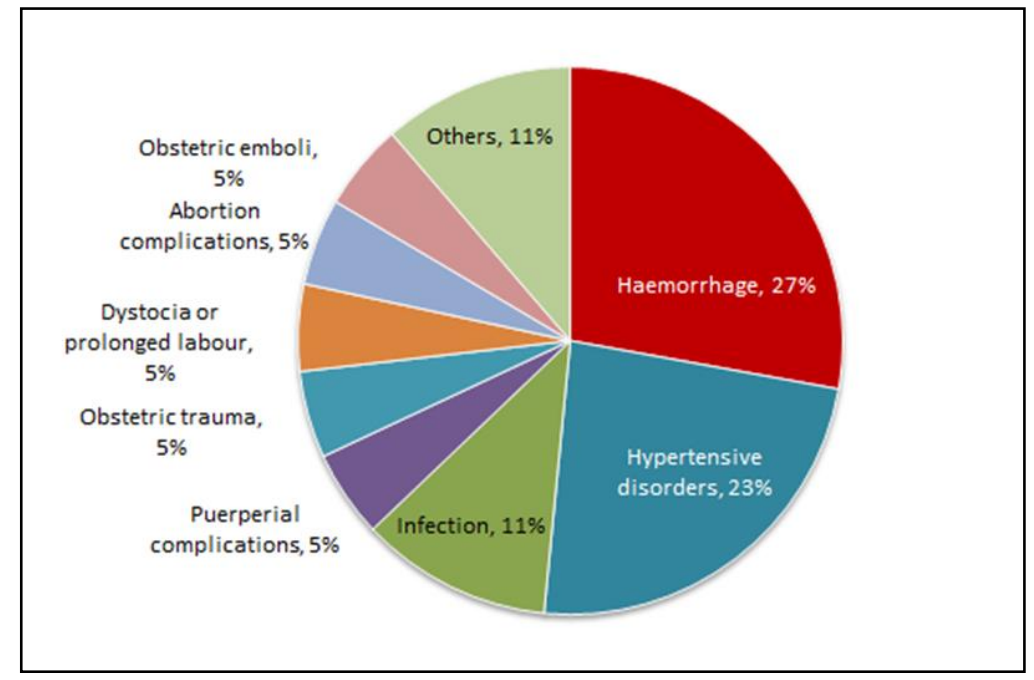

Figure 2. Causes of maternal mortality in Indonesia.

Source: Hernawati ${ }^{6}$

\section{Current situation and some efforts to reduce maternal mortality}

There are large disparities within the Indonesian population, especially with regards to woman's education, socio-economic level, region, province and geography (e.g. urban versus rural) which cause inequities towards the proper access to skilled birth attendance, health facility deliveries and other maternal health service indicators. Currently, 82.2 percent of births are assisted by a skilled health attendant. ${ }^{8}$ The 2009 National Socio-Economic Survey data showed that the highest propor- 
tion of births assisted by health personnel is DKI Jakarta, the capital city of Indonesia, and the lowest one is Maluku, a province in eastern Indonesia. Nowadays, 92.7 percent of pregnant women get antenatal care from a health professional during pregnancy, but only 61.4 percent of them have complied with the recommended minimum of four antenatal care visits. 5,8

In the last 30 years, there have been many policies and strategies affecting maternal health in Indonesia, including the Safe Motherhood Initiative in the 1980s, Gerakan Sayang Ibu (Mother Friendly Movement) in the 1990s, Making Pregnancy Safer and the district decentralization in the 2000s, as well as the campaign Healthy Indonesia in 2010.9 To increase skilled birth attendance, the Indonesian government launched the village-based midwife programme in 1989. The goal of this programme was to place a skilled birth attendant in every village to provide antenatal and perinatal care, family planning, other reproductive health services, and nutrition counseling (Figure 3). The attendants were also there to facilitate basic primary health care services, including immunization and nutrition interventions. At its inception in 1989, the village-based midwife programme faced the staggering target of training and placing 54,000 midwives throughout a vast archipelago within 7 years. By 1997, over 96 percent of the population of Indonesia had access to 54,000 village-based midwives, many of whom were equipped with small birthing units. Midwife density increased from 0.2 to 2.6 per 10,000 people between 1986 and 1996. In rural areas, births which have been attended by skilled midwives increased from 22 percent to 55 percent between 1990 and 2003, and socioeconomic inequalities were reduced for professional attendance at births. ${ }^{10}$

Even though the rates for births attendance by skilled health personnel and antenatal care coverage are relatively high, several factors still need attention, especially since maternal mortality ratio remains high, such as quality of antenatal care services in ensuring early diagnosis and prompt treatment for high risk pregnancies and complications during pregnancy and around deliveries. ${ }^{5}$ In Indonesia, the provision of skilled birth attendants was not followed by increased facility and referral level capacities. Not all primary health centers can provide basic obstetric care, and about 34 percent of district public hospitals do not have an obstetrician, indicating limited provision of 24 hours continuum of care necessary for dealing with emergency situations and comprehensive obstetric care. 3,11

Regarding the pre-pregnancy period, contraceptive and reproductive health will be the crucial issues to be improved. Nationally, the contraceptive prevalence rate (CPR) increased from 49.7 percent in 1991 to 61.4 percent in 2007. The use of modern methods increased from 47.1 percent in 1991 to 57.4 percent in 2007. The lowest CPR for modern methods is in Papua (24.5 percent), while the highest is in Bengkulu (70.4 percent, Figure 4). Disparity of CPR among provinces indicates the unequal coverage of family planning programs. ${ }^{5}$ Among modern methods, injectables are the most commonly used contraceptive method (57.7 percent), followed by the pill ( 24.7 percent), intrauterine device ( 6.8 percent), implant (4.6 percent), and tubectomy ( 2.93 percent). ${ }^{12}$ 


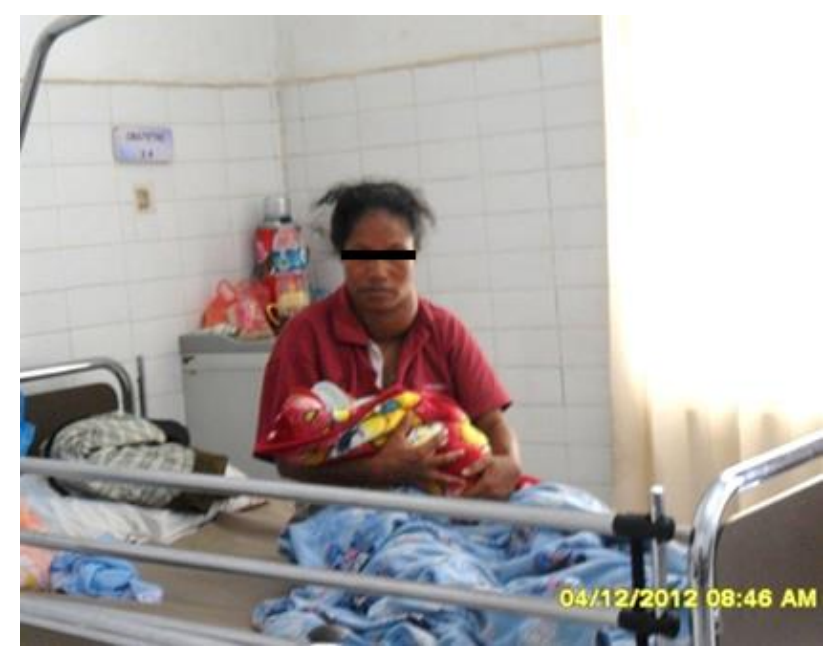

Figure 3. High parity mother and her baby: beneficiaries of skilled attendance in Bajawa District Hospital, East Nusa Tenggara, Indonesia

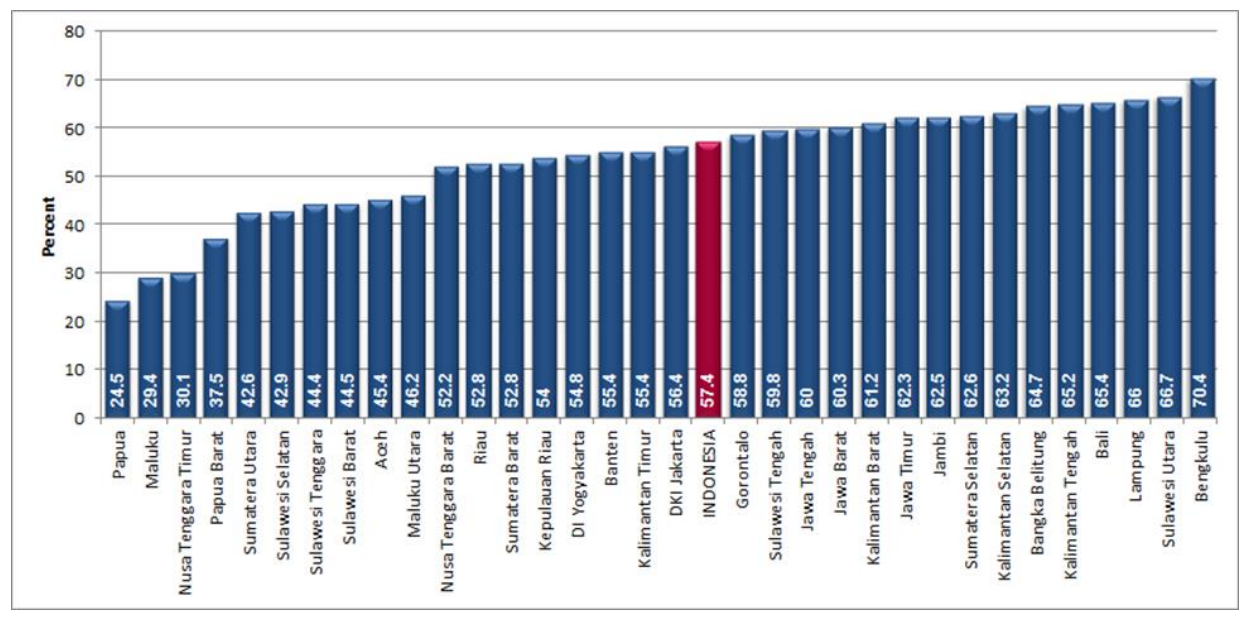

Figure 4. Contraceptive Prevalence Rate (CPR) by province, 2007 Source: BPS, IDHS $2007^{5}$

The number of couples of reproductive age who want to avoid, space pregnancy or limit childbearing, but do not use any method of contraception (unmet need for contraception) is 9.1 percent (4.3 percentage points are for spacing and 4.7 percentage points for limiting). IDHS 2007 shows that 60 percent of married women with 2 children, 75 percent of married women with 3-4 children, and 80 percent of married women with 5 or more children do not want any more children; however they are not applying any contraceptives methods. ${ }^{5}$ 
The probable causes of unmet need for contraception are access problems to services, social and cultural obstacles, negative perception about contraception, side effects and the inconvenience of using contraceptives. The unmet need for contraception varies greatly among provinces, regions, and socio-economic status. The more educated and wealthy group has better access to information and services of family planning and reproductive health. The lowest unmet need is found in Bangka Belitung (3.2 percent) and the highest in Maluku (22.4 percent, Figure 5). Higher unmet need was found in rural areas (9.2 percent) compared with urban districts (8.7 percent). In addition, higher unmet need was found in the lowest socioeconomic level (13 percent) compared to the highest socio-economic level (8 percent). Moreover, 12.3 percent of unmet need in women is because of side effects, 10.1 percent because of health problems, and 3.1 percent because of husband's prohibition. ${ }^{5}$ The unmet need for contraception is caused by side effects and the inconvenience of using contraceptives, reflected by the low quality of family planning services.

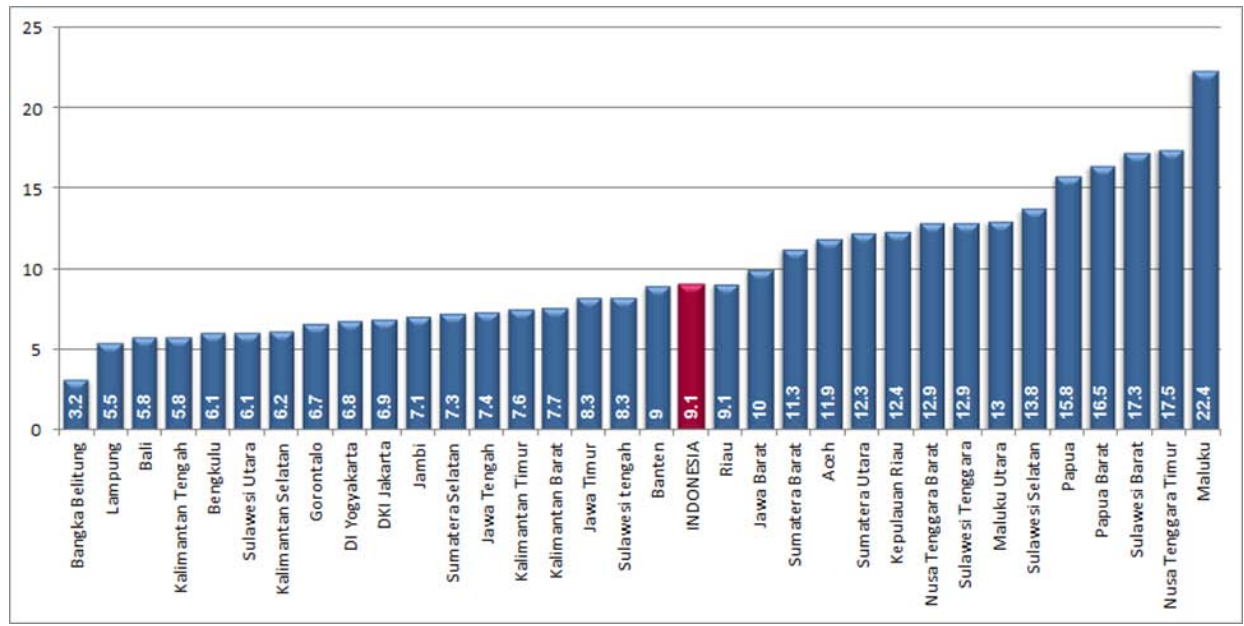

Figure 5. Unmet need for contraception by province, Indonesia 2007. Source: BPS, IDHS 20075

Although substantial progress and some efforts has been made for the fifths Millennium Development Goal (MDG5), focusing on the reduction of maternal mortality ratio by three-quarters between 1990 and 2015, it it unlikely that this goal can be achieved. An agenda to reduce maternal mortality beyond 2015 is needed including continuous efforts for regarding family planning as something important and thus, improve the unequal coverage of family planning among provinces within the country. 


\section{How contraceptive use affects maternal mortality}

In 1987, The Safe Motherhood Initiative, a global campaign and movement to reduce maternal mortality was launched. It identified family planning as one of four strategies to reduce maternal mortality in developing countries, where 99 percent of all maternal deaths took place. The other strategies were antenatal care, safe delivery, and postnatal care. In 1994, the International Conference on Population and Development repeated the importance of family planning for the improvement of women's health. Family planning programs that support the use of modern contraceptives have direct and indirect effects to reduce maternal mortality. ${ }^{13}$

Firstly, family planning directly reduces the number of maternal deaths because it reduces the chance of getting pregnant and pregnancy-related complications, as well as lowering the risk of unwanted pregnancies and unsafe abortions. Contraception coverage is 61 percent in the world, while unmet need for contraception ranges from 6 percent in European countries to 23 percent in the countries of subSaharan Africa. The unmet need for contraception leads to unwanted pregnancies, which in turn lead to termination of pregnancies. The WHO data showed, from the 190 million pregnancies in the world, almost 41 percent of them are unwanted pregnancies, with 22 percent resulting in induced abortion each year. In turn, abortion complications contributed to 13 percent of total maternal deaths worldwide. These data show that about 20-25 percent of maternal deaths could be eliminated if unplanned and unwanted pregnancies were prevented. So, fewer births lead to lower risk of maternal death and finally results in less total number of deaths. As abortion is illegal in Indonesia, many pregnant women seek unsafe abortion services. In places where induced abortion is prohibited or illegal, actual events can only be estimated indirectly. Statistics about abortion often do not reflect actual number of events, as many events are hidden. Official data from the Ministry of Health (Figure 2) showed that 5 percent of maternal deaths were caused by abortion complications, but actual events were estimated higher, about 6-16 percent. $5,13,14,15,16$

Secondly, family planning indirectly reduces the number of maternal death by avoiding high-risk births that are related to "too young, too old, too many or too close", and finally lead to a lower risk of mortality associated with each birth. Maternal mortality risk is higher than average in young women (less than 18 years; "too young") because pelvic development is incomplete. Adolescent mothers are at greater risk of pregnancy-induced hypertension and its complications, anemia, miscarriage, and obstetric complications. Maternal mortality risk is higher also in older women (older than 35 years; "too old"), in those of higher parity (more than four births; "too many") and in those of closer spacing period between pregnancies (less than two years apart; "too close") because their health could be more compromised. Studies have shown that advanced maternal age is associated with increased incidence of medical complications such as hypertension and diabetes, as well as obstetric complications. Pregnancies occurring after four births are associ- 
ated with increased risks to serious complications of pregnancy and delivery, including hemorrhage, infection, anemia, and hypertensive disorder. Frequent pregnancies potentially lead to low mother's nutritional status. ${ }^{13,15,17}$

Empirical studies from Bangladesh showed that if women younger than 20 years and those older than 39 years avoided having children, maternal mortality would decrease by 34 percent, and elimination of births in mothers with five or more children could reduce the number of maternal deaths by 58 percent. In 1971, the Population Council Annual Report stated that the elimination or restriction of births to women aged 18-35 years in developing countries could reduce maternal mortality by 20 percent. Prolonged birth spacing as a result of contraceptive use is also likely to contribute to maternal survival because it enables a longer recovery period between pregnancies, providing more time for mothers to improve their nutritional status. ${ }^{13}$

Stover et al. showed evidence that family planning lowers the risk per birth, the maternal mortality ratio (MMR), by preventing high-risk, high-parity births. They use estimates from UN organizations of MMRs and data from 146 Demographic and Health Surveys on contraceptive use and the distribution of births by risk factor over the period from 1990-2005. They found, that the drop in the total fertility rate (TFR) from 1990 to 2005, primarily due to increasing contraceptive use, resulted in 1.2 million fewer maternal deaths, more precicely 15 percent fewer than would have occurred with no fertility decline. These 1.2 million additional deaths averted between 1990 and 2005 can be added to those that were already being averted by the level of contraceptive use in 1990. There are strong evidences that contraceptive use affects the maternal mortality rate. Furthermore, by reducing demographically high-risk births in particular, especially high-parity births, family planning reduced the MMR and thus, averted additional maternal deaths indirectly.

Time-series survey data show a clear pattern of smaller percentages of highrisk births as contraceptive use increases. The greatest effect is the reduction of high-parity births, which are actually eliminated at high levels of contraceptive use. Cross-national data show that the MMR highly correlated with the percentage of high-risk births. This evidence suggests that the reduction in high-risk births, resulted by contraceptive use, lowers the MMR. ${ }^{15}$

More interestingly, Ahmed et al. developed statistical analysis models, using data extracted from the Maternal Mortality Estimation Inter-Agency Group (MMEIG) database, the UN World Contraceptive Use 2010 database, and the UN World Population Prospect 2010 database, to estimate maternal deaths averted by contraceptive use (statistical analysis model I) and to estimate the effect of satisfying unmet need for contraception (statistical analysis model II) in 172 countries. They estimated, using the analysis model I, that 342,203 women died of maternal causes in the world in 2008, but that contraceptive use averted 272,040 maternal deaths (44 percent reduction). So, without contraceptive use, the number of maternal death in the world would have been 1.8 times higher than the 2008 total. They also calculated, by using model II, that satisfying unmet need for contracep- 
tion could prevent another 104,000 maternal death per year (29 percent reduction), which is equivalent to an increase in global contraceptive use from 64 percent to 75 percent. Using Ahmed's statistical analysis model I, there were 11,491 maternal deaths in Indonesia in 2008, but that contraceptive use averted 15,917 maternal deaths (58.1 percent reduction). So, without contraceptive use, the number of maternal death in Indonesia would have been 2.4 times higher than the 2008 total. ${ }^{13}$

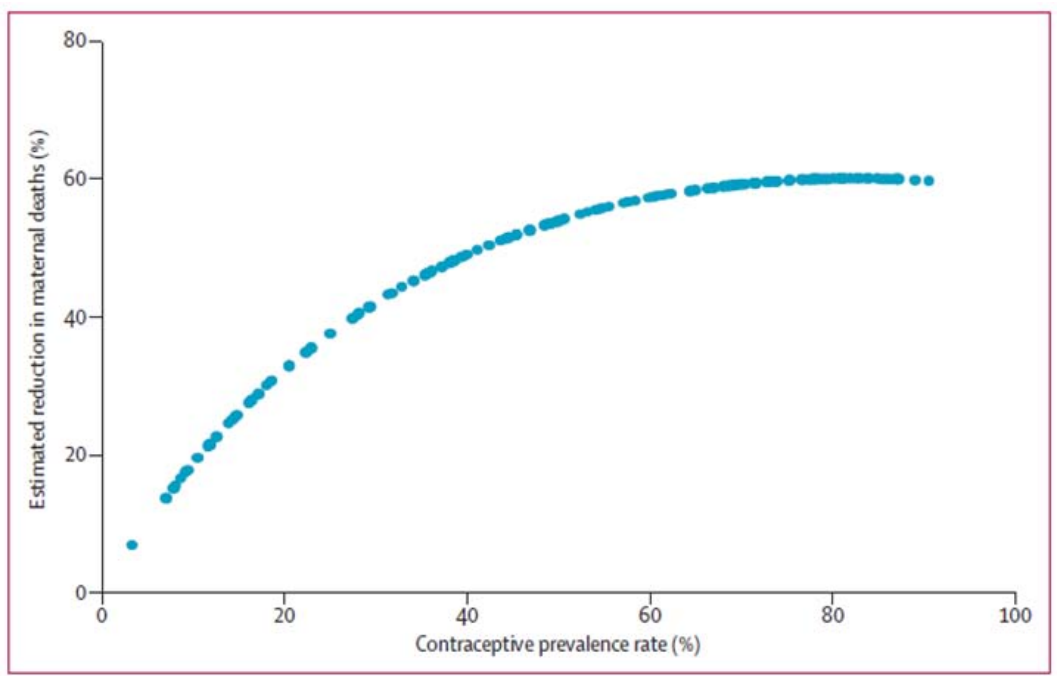

Figure 6. CPR and estimated mortality reduction. ${ }^{13}$

\section{What should we take as lesson?}

In fact, contraception is not only related to demographic solution but also significantly reduces the risk of maternal mortality. Family planning is one of four essential strategies to reduce maternal mortality in developing countries, beside antenatal care, safe delivery, and postnatal care.

There are two roles of contraception in reducing maternal mortality, with direct and indirect effects. Direct effect is by lowering the pregnancy chance and hence, it generally may reduce the childbirth risks. Indirect effect is by reducing the high-risk pregnancy. Some evidences suggest that increasing CPR and satisfying unmet need for contraception can reduce maternal mortality significantly. 


\section{References}

1. BPS. Trends of selected socio-economic indicators of Indonesia. August 2012.http://www.bps.go.id/aboutus.php?booklet=1 [Accessed January 7, 2013]

2. BPS. Pertumbuhan Ekonomi Indonesia (Indonesia Economic Growth). Berita Resmi Statistik, 2012. http:/ /www.bps.go.id [Accessed January 7, 2013]

3. Acuin CS, Khor GL, Liabsuetrakul T, et al. Maternal, neonatal, and child health in southeast Asia: towards greater regional collaboration. Lancet 2011:377;516-25.

4. World Health Organization. Maternal mortality in 2005: estimates developed by WHO, UNICEF, UNFPA, and the World Bank. 2007.

5. Ministry of National Development Planning (BAPPENAS). Report on the achievement of the Millennium Development Goals Indonesia 2010.

6. Hernawati I, Analysis on maternal mortality in Indonesia 2010. Seminar paper, 2011.

7. Filippi V, Ronsmans C, Campbell OMR, et al. Maternal health in poor countries: the broader context and a call for action. Lancet 2006;368:153541.

8. Ministry of Health. Riset Kesehatan Dasar (Research on Basic Health), 2010.

9. Thomsen S, Hoa DT, Malqvist M, et al. Promoting equity to achieve maternal and child health. Reproductive Health Matters 2011;19(38):176182.

10. Shankar A, Sebayang S, Guarenti L, et al. The village-based midwife programme in Indonesia. Lancet 2008:371;1226-1229.

11. Data and Information Center, Ministry of Health. Data and Information, 2011.

12. BPS. National Socio-Economic Survey, 2009. http://www.bps.go.id [Accessed January 8, 2013]

13. Ahmed S, Li Q, Liu L, et al. Maternal death averted by contraceptive use: an analysis of 172 countries. Lancet 2012;380:111-25. 
14. WHO. Unsafe abortion: global and regional estimates of the incidence of unsafe abortion and associated mortality in 2008. Geneva: World Health Organization, 2011.

15. Stover J and Ross J. How increased contraceptive use has reduced maternal mortality. Matern Child Health J 2010;14:687-695.

16. Campbell OMR and Graham WJ. Strategies for reducing maternal mortality: getting on with what works. Lancet 2006;368:1284-99.

17. Senanayake P and Potts M. Atlas of contraception. Second edition. Informa, 2008. 



\section{Basic Sonography in Maternal Care in Resource-Poor Settings}

Werner Stein, Gynaecological Hospital, Klinikum Fulda, Germany

\section{Introduction}

Maternal and neonatal mortality and morbidity in resource-poor countries have been improved over the past decade but nevertheless they are still very high ${ }^{1}$. Taking the example of the African region, it accounts for 480 maternal deaths per 100,000 live births in comparison to 20 maternal deaths per 100,000 live births in Europe. Neonatal mortality is 34 per 1,000 live births, stillbirth rate 104 per 1,000 live births in the Africa Region compared to 7 and 27 per 1,000 live births respectively in Europe.

There are various reasons for high mortality and morbidity rates and they are all interconnected. There are physical barriers, lack of equipment or medication, lack of qualified staff, corruption and mismanagement which results in lower effectiveness of health facilities. Conventional maternal care in resource-poor countries is unable to properly identify certain potential life-threatening conditions.

\footnotetext{
1 World health statistics 2012. http://www.who.int/gho/publications/world_health_statistics/EN_
} WHS2012_Full.pdf; [Accessed December 5, 2012]. 
Sonography is an inexpensive and safe method. It provides valuable information in the management of pregnant women and would therefore be an ideal diagnostic tool in resource-poor settings.

Sonography facilitates the early identification of high-risk pregnancies and the implementation of adjusted management as in placenta praevia or multiple pregnancies and thus, reduces considerable risks of maternal and foetal complications. Nevertheless it is difficult to apply sonography in the developing world due to a lack of human capacities and resources.

In order to enhance further maternal care in resource-poor settings the implementation of basic sonography, as an essential part of maternal care, should be carefully considered. Basic sonography in resource-poor settings enables midwives to detect additional potential life-threatening maternal and foetal health problems in time and helps them to make important decisions for further management.

Ideally, sonography should be implemented and used in settings which work already well with existing resources and means. The proper use of existing technologies should probably be a precondition before focusing on the implementation of sonography. Common and important health issues that are easily treatable through basic technologies are for example neonatal hypothermia and subpartual hypoxia. After birth, newborns face the danger of low temperature due to an unfavourable relationship of volume and surface. Hence hypothermia is a very serious problem, especially for a slightly wet newborn. Nevertheless, hypothermia can easily be prevented by applying simple measures such as immediately drying after delivery. The newborn should be wrapped or be laid down on the belly of the mother using her as a heating source. In addition, cleaning of the newborn in the labour room should be avoided because of thermal loss. Another mean which is easy to perform and helps to monitor the foetal well-being includes the intermittent auscultation of the foetal heart rate during delivery. It helps to detect subpartual foetal hypoxia. As a consequence - and as these steps are easy to perform - clinical practices need to change. If existing technologies are not used sufficiently, a new and more sophisticated technology such as sonography should probably not be implemented. In cases where maternal care is performed with inadequate effectiveness sonography will most likely show no benefit.

This article describes the requirements for the application of sonography in maternal health care in less developed countries. The author is convinced that sonography would be most powerful in the hands of clinical decision makers such as midwives and nurses and not predominantly by medical doctors. The author does not favour the implementation of a special sonographer who is not directly involved in clinical decisions and has no appropriate knowledge of the clinical condition of the patient.

Sonography should focus on easy learnable and reproducible outcomes that have the potential to significantly improve the treatment of a patient. It is of high importance to keep sonography as simple as possible to perform and to embed 
sonography entirely in the clinical decision-making processes. Using sonography as a fancy technology would simply miss the point.

The author discusses the application of sonography in maternal care in resource-poor settings such as in rural Sub-Saharan Africa. It is proposed that sonography should be performed primarily by midwives on a very basic level. Indications should be simple and clearly addressed.

\section{Basic Sonography}

Basic sonography enables to address the following issues:

viability of the foetus, multiple pregnancy, exclusion of placenta praevia, severe polyhydramnios or severe oligohydramnios, intrauterine growth retardation.

\section{Indications for basic sonography are:}

non-detectable foetal heartbeats, vaginal bleeding, suspected polyhydramnios, suspected multiple pregnancy, discrepancy of fundal height and gestational age, preeclampsia, unclear foetal position.

The main obstacles against the effectiveness of sonography are the following:

the impact of sonography is inadequately understood sonography is poorly implemented in substantial health care management decisions regular non-use of sonography ("the device is caged") sonography generates many but not always usable information sonographic examination does not focus on obvious and easy findings poor training and inadequate follow-up education of health workers the perception of sonography as a status symbol of doctors is a main obstacle for midwives to use sonography in their daily routine the perception of sonography as a primary money-generating tool would deter poor people from its usage.

Basic sonography in maternal health care addresses several health issues such as 1) severe amniotic fluid abnormalities, such as polyhydramnios and oligohydramnios, 2) placenta praevia and 3) placental dysfunction. 


\section{Amniotic fluid abnormalities}

\subsection{Polyhydramnios}

By applying basic sonography severe polyhydramnios can be identified. Polyhydramnios occur in about $1 \%$ of pregnancies.

Semiquantitative assessment of amniotic fluid volume is part of routine ultrasound examination. Either the single deepest uninterrupted pocket (Maximum Vertical Pocket (MVP) of amniotic fluid is measured or the Amniotic Fluid Index (AFI) is calculated adding the depth of amniotic fluid in all four abdominal quadrants. The best technique to measure amniotic fluid remains controversial. Polyhydramnios means an excess of amniotic fluid and is defined as a MVP $>8 \mathrm{~cm}$ or an AFI $>24 \mathrm{~cm}$. MVP exceeds $16 \mathrm{~cm}$ in severe polyhydramnios. Severe polyhydramnios can be easily recognized through a uterine cavity, just like if there would be enough space for another foetus (Fig. 1).

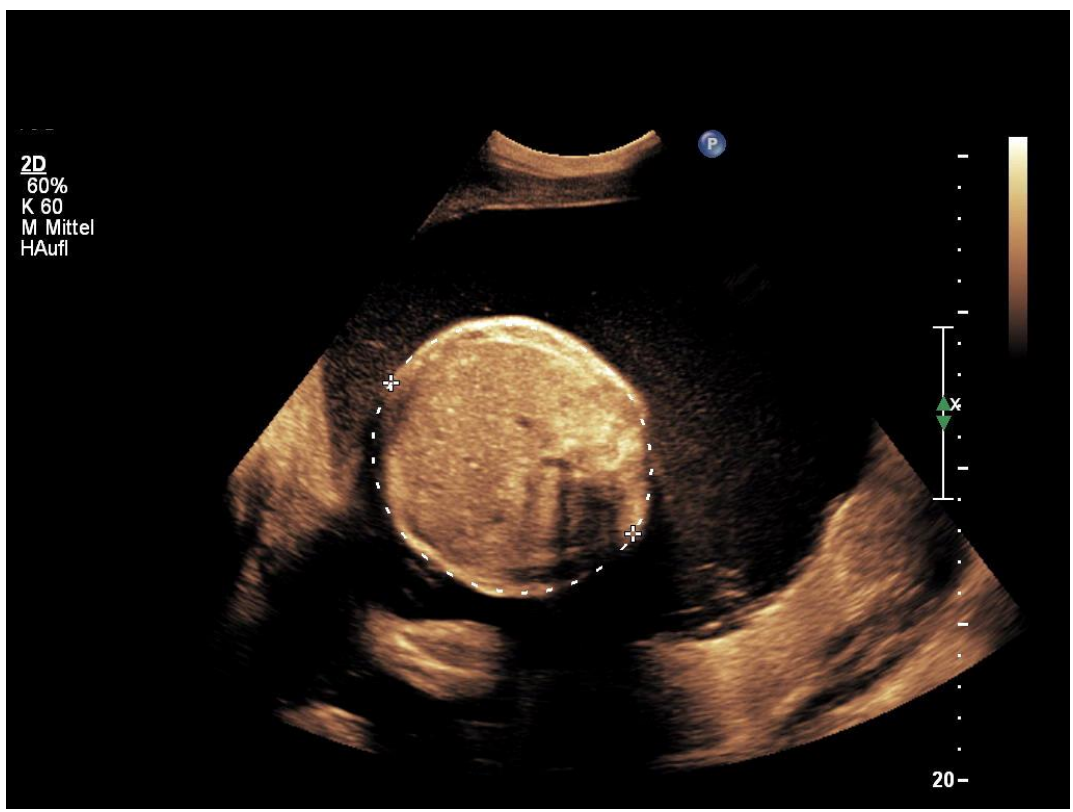

Figure 1: Polyhydramnios

In the case of polyhydramnios, the uterine size outpaces the gestational age. Severe cases may lead to shiny skin, premature rupture of membranes and premature labour. Foetal heart sounds might be scarcely audible.

Pregnancies complicated by severe polyhydramnios increase maternal and neonatal morbidity. Polyhydramnios is associated with preterm delivery and may provoke abnormal foetal lie and abruptio placentae during labour. 
In most cases of polyhydramnios an exact cause cannot be identified. Some cases are due to maternal diabetes mellitus, which causes foetal hyperglycemia resulting in foetal polyuria. Nevertheless a pronounced polyhydramnios can also be associated with an identifiable cause. It is therefore prudent to perform a targeted foetal ultrasonography. Severe cases may lead to the detection of foetal malformations that impair the ability of the foetus to swallow such as foetal gastrointestinal obstruction (oesophageal atresia, annular pancreas or thoracic masses compressing the oesophagus) or neurological abnormalities such as anencephaly with an impaired swallowing reflex. Foetal movements should be observed sonographically to exclude akinesia syndromes. The possibility of a twin-twin transfusion syndrome in twin pregnancies should be considered. The stuck twin is usually located next to the uterine wall and might be missed. Chorioangioma of the placenta can also cause polyhydramnios. When foetal hydrops is associated with polyhydramnios a maternal antibody screening should be done. If the screening is negative, the cause of the foetal hydrops is most likely nonimmune.

In cases of symptomatic polyhydramnios with a firm and tense maternal abdomen or when there are preterm contractions or a shortened cervix is present, transabdominal decompression should be performed before the onset of labour or in early labour. The transabdominal decompression prevents cord prolapse or placental abruption, secondary to sudden uterine decompression after rupture of membranes.

The uterine cavity is tapped with a 20-gauge spinal needle under sonographic guidance. The site chosen should be near the midline of the abdomen and below the umbilicus. Not more than 1.51 should be removed in one session because of the risk of placental abruption. If sonographic findings after completion of the procedure show persistence of the polyhydramnios, the procedure should be repeated the next day.

The major indication for serial amniocenteses is severe polyhydramnios secondary to twin-twin transfusion syndrome or foetal anomaly.

Maternal indomethacin therapy ( $25 \mathrm{mg}$ every $6 \mathrm{hrs})$ might be an alternative to symptomatic polyhydramnios. Indomethacin is a potent inhibitor of prostaglandin synthesis and acts mainly via reduced foetal urine production. Nevertheless, accurate surveillance, especially for fetal side effects is required. The risk of ductal arteriosus constriction increases to 50 percent by the $32 \mathrm{nd}$ week of gestation ${ }^{2}$ and should therefore not be given beyond this gestational age.

\subsection{Oligohydramnios}

Oligohydramnios is defined as an AFI of $<5 \mathrm{~cm}$ or deepest pocket $<2 \mathrm{~cm}$, in severe cases $<0.5 \mathrm{~cm}$. The visual criterion for oligohydramnios is an obvious lack of fluid

\footnotetext{
2 Van den Veyver I, Moise KJ, Ou C-N, et al. The effect of gestational age and fetal indomethacin levels on the incidence of constriction of the ductus arteriosus. Obstet Gynecol. 1993; 82:500-503.
} 
(Fig. 2). The sonographic demonstration of the foetal stomach usually indicates the ability of the foetus to swallow sufficient amniotic fluid.

Oligohydramnios is caused either by reduced amniotic fluid production or by increased loss. Decreased production is usually the result of urinary tract malformations, uteroplacental insufficiency, and twin to twin transfusion as well as maternal dehydration. Fetal urinary tract anomalies resulting in oligohydramnios include bilateral renal agenesis, multicystic dysplastic kidneys, infantile polycystic kidney disease, and lower urinary tract obstruction. Increased loss may result in early rupture of the membranes. The earlier the gestational age the lower is the incidence of premature rupture of membranes as an explanation of oligohydramnios.

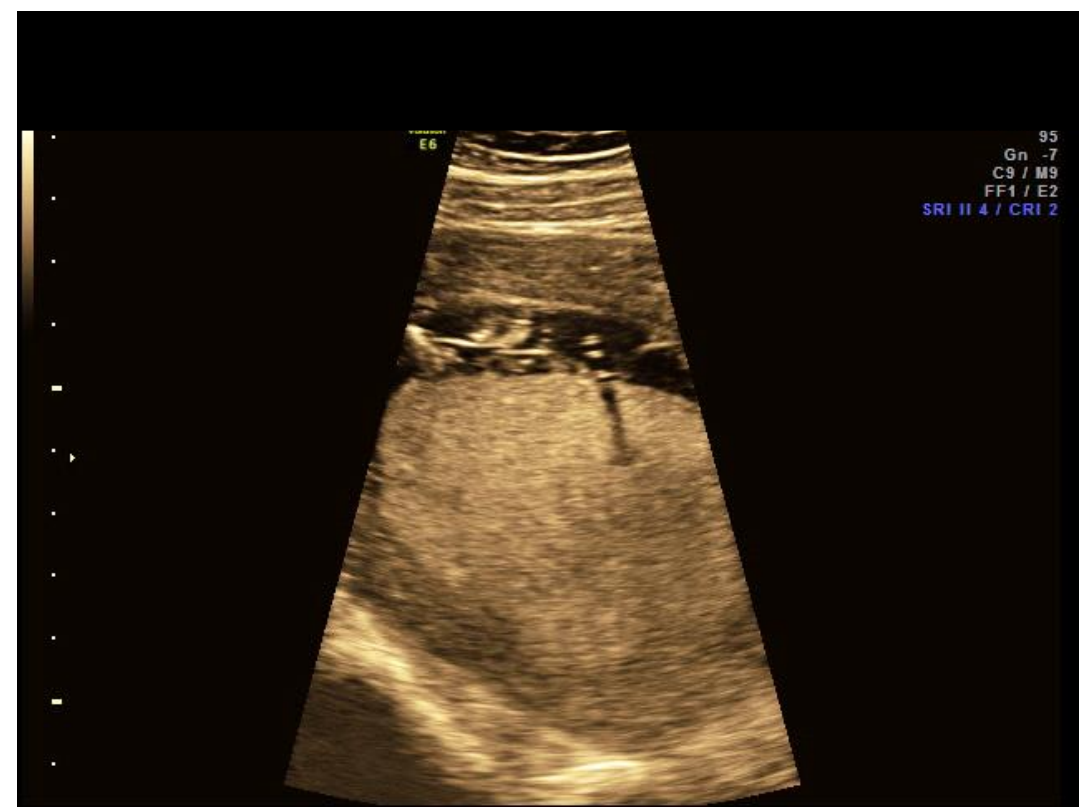

Figure 2: Oligohydramnios

Prolonged oligohydramnios in early second trimester can cause pulmonary hypoplasia and skeletal deformity. The prevalence of pulmonary hypoplasia after oligohydramnios depends on the gestational age at onset, the severity, and the duration of oligohydramnios. Isolated oligohydramnios during the third trimester is not necessarily associated with poor perinatal outcome.

Fetal kidneys and bladder should be carefully examined. The pregnant women should be asked for a history suggestive of rupture of membranes and examination to exclude early rupture of membranes. If these findings are normal, fetal growth and placental function should be assessed. 
Amniotic fluid volume is easily estimated by sonographic means. In resource-poor settings, focus should be primarily laid on gross changes of amniotic fluid.

\section{Placenta praevia}

Placenta praevia is defined as a placenta that lies in the lower uterine segment either directly over or in close proximity to the cervix (Fig. 3). Women with placenta praevia often present painless, bright red vaginal bleeding during the second half of pregnancy. Massive haemorrhage associated with placenta praevia occurs not only during pregnancy, but also at and shortly after Cesarean section. Placenta praevia complicates up to about $1 \%$ of all births. Uterine scaring due to a prior caesarean section or uterine curettage are important risk factors for placenta praevia.

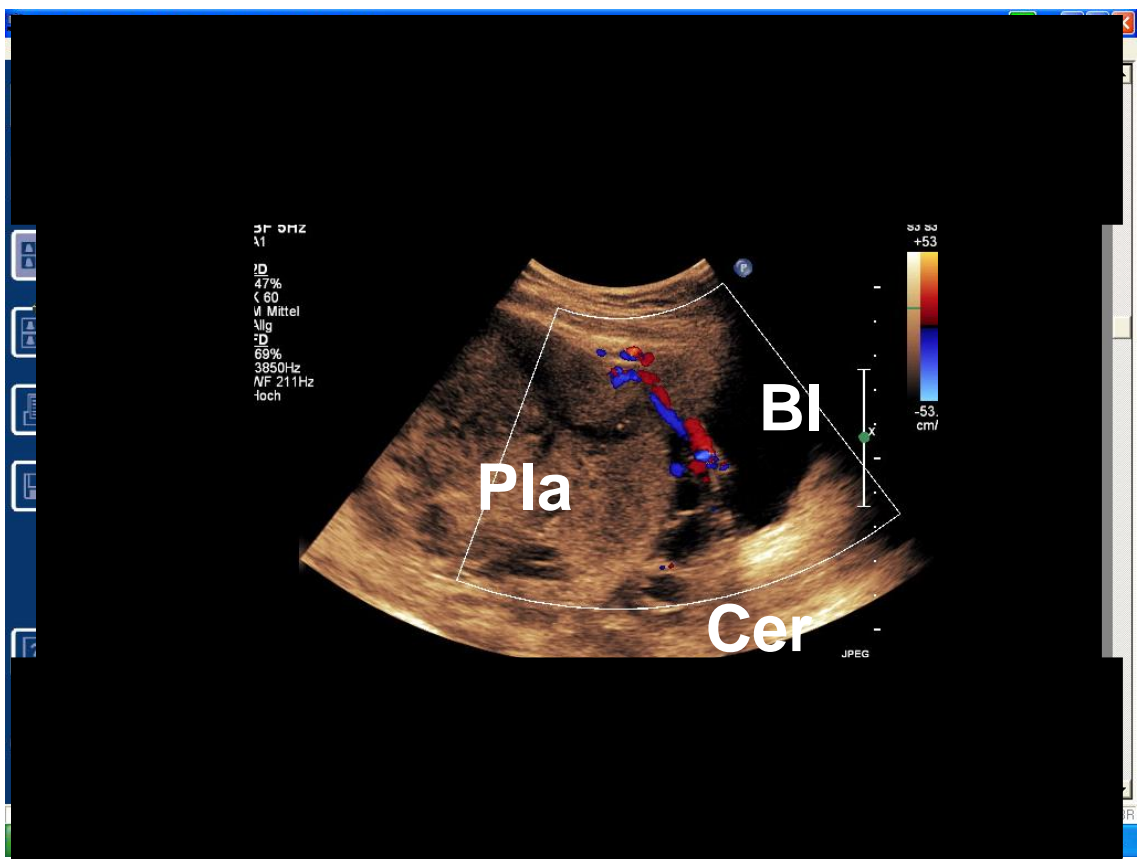

Figure 3: Placenta praevia (Pla) covers internal os of cervix (Cer) and lies next to the bladder (B1)

Sonography is the method of choice for diagnosing placenta praevia. The placental edge overlaps or is within $20 \mathrm{~mm}$ of the internal cervical os. Transvaginal sonography is superior to transabdominal sonography. A short uterine cervical length in the third trimester and sinus venosus at the margin of the placenta may predict an increased risk of antenatal bleeding in placenta praevia ${ }^{3}$.

\footnotetext{
${ }^{3}$ Hasegawa J, Nakamura M, Hamada S, et al. Prediction of hemorrhage in placenta previa. Taiwan J Obstet Gynecol. 2012;51:3-6.
} 
If the placenta is overlapping or reaching the internal cervical os during the second trimenon, sonography should be repeated in late pregnancy to rule out placenta praevia 4 .

In case the placental edge is located within $10 \mathrm{~mm}$ of the internal cervical os at term, an elective caesarean section should be performed around 36 to 37 weeks of gestation. In case caesarean sections are used very reluctant it would be prudent for trial of labour in women with a distance of less than $10 \mathrm{~mm}$ distance of placental edge and internal os.

\section{Placental dysfunction}

Placental dysfunction is one of the leading causes of maternal and foetal morbidity and mortality. Placental dysfunction may affect the foetus via intrauterine growth restriction (IUGR), oligohydramnios, hypoxic-ischemic injury, and eventually foetal death. It is the inability to meet an increasing demand for oxygen and substrate of the developing foetus due to insufficient blood flow to the placenta. According to the time of onset of an adverse intrauterine nutrition, an IUGR foetus may be classified as early-onset or late-onset IUGR. Early-onset IUGR foetuses tend to be symmetrically small and late-onset IUGR foetuses show a distinct smaller abdomen and/or shorter femur than head measurements. Placental dysfunction may also affect the pregnant women causing preeclampsia, a pregnancy-induced hypertensive disorder. The mother and the foetus may be independently or together being affected. Therefore, an evaluation of both foetus and mother would be in each case mandatory.

The identification of placental dysfunction and the timing of delivery are the most critical aspects of antenatal management.

Comprehensive foetal evaluation in IUGR includes foetal biometry, amniotic fluid volume, heart rate patterns, arterial and venous Doppler and biophysical variables. In the setting of resource-poor countries the extent of these investigations has to be reduced and should preferably focus on the Doppler measurement of umbilical artery, the measurement of the abdominal circumference compared to the head circumference and on the evaluation of amniotic fluid volume.

\subsection{Doppler measurement of umbilical artery}

Even for a sonographic beginner it is easy to identify the umbilical cord and to display blood flow (Fig. 4). The measured curve of the blood flow and Doppler indices are automatically presented by the scanner. Commonly used indices are Resistance Index and Pulsatility Index. Pulsatility Index (PI) is preferable, because

\footnotetext{
${ }^{4}$ Bhide A, Thilaganathan B. Recent advances in the management of placenta previa. Curr Opin Obstet Gynecol. 2004;16:447-51.
} 
it gives a broader range of values in describing a range of waveform shapes when there is a reduced or no end-diastolic flow (Fig. 5).

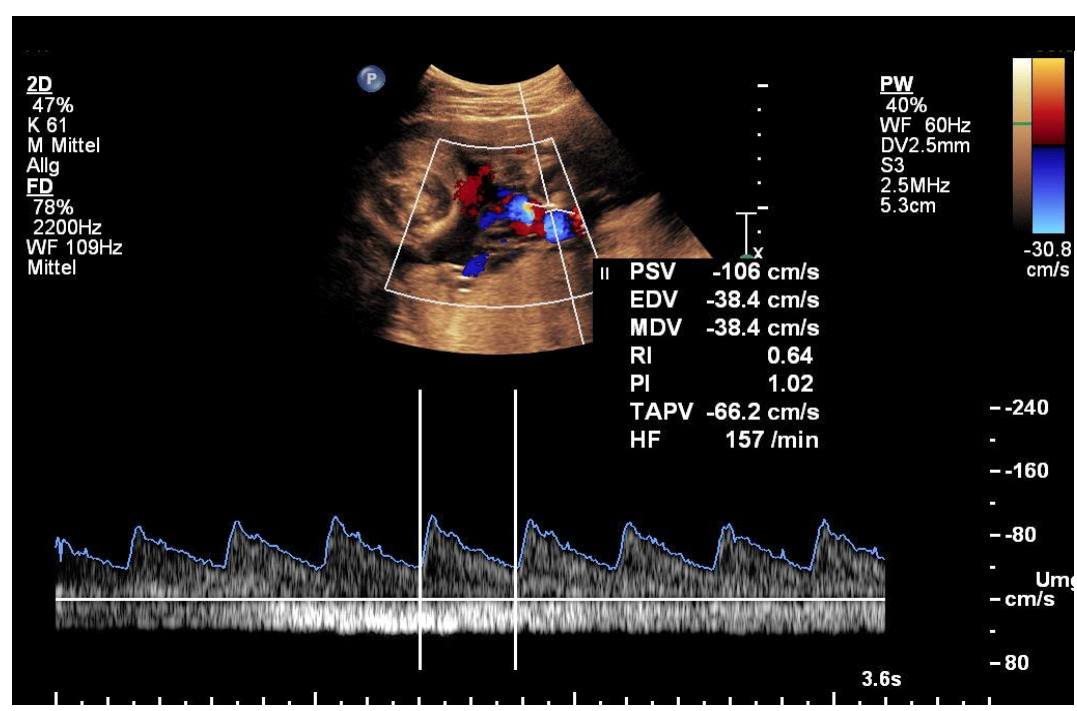

Figure 4: Doppler Measurement of umbilical artery, Pulsatility Index normal range with positive enddiastolic flow

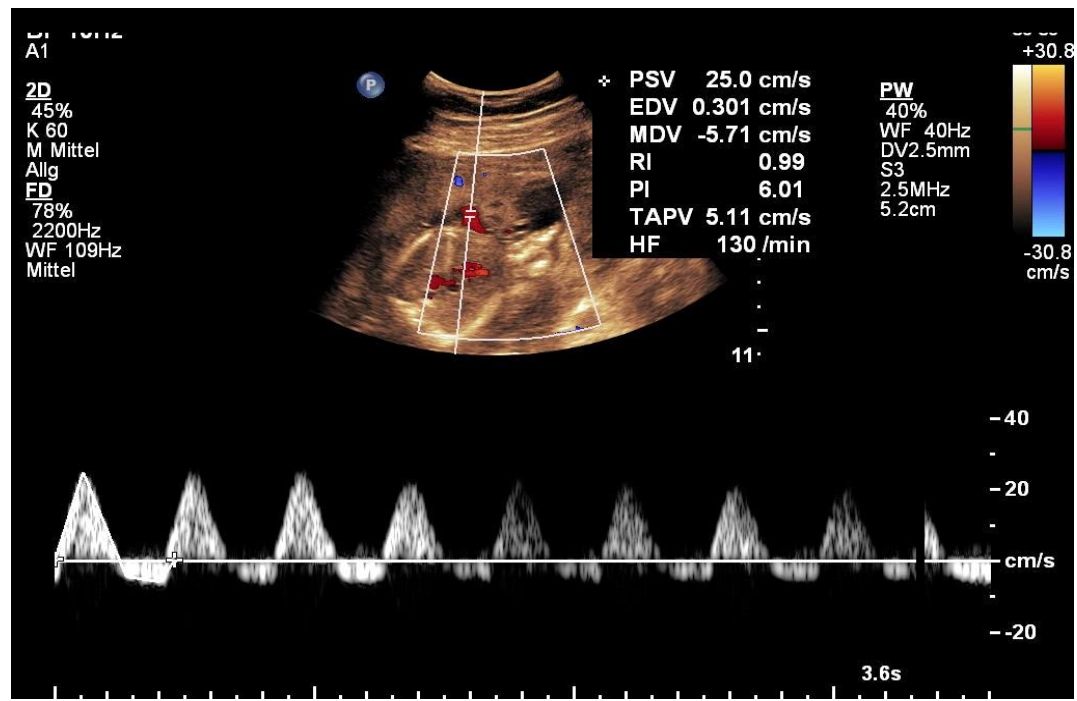

Figure 5: Pathologic blood flow of umbilical artery with reverse flow 
The PI of the umbilical artery increases in relation to the amount of damaged villous vascular tree of the placenta. Absence or even reversal of end-diastolic velocities can occur when $60-70 \%$ of the villous vascular tree is damaged ${ }^{5}$. A high PI indicates therefore an increased vascular resistance and a low nutritional supply for the fetus.

\subsection{Measurement of Abdominal Circumference}

The correct display of the foetal abdominal cross section and hence, the correct measurement of the abdominal circumference (AC) are challenging. The use of an oblique foetal cross section results in an overestimation of the $\mathrm{AC}$ and would therefore miss the true low AC. Nevertheless the aberration is only one-sided and the underestimation of an $\mathrm{AC}$ would be unusual. The display of a round cross section with symmetric vertebra and the confluence of portal vein and Ductus venosus ensure the correct measurement plane (Fig. 6).

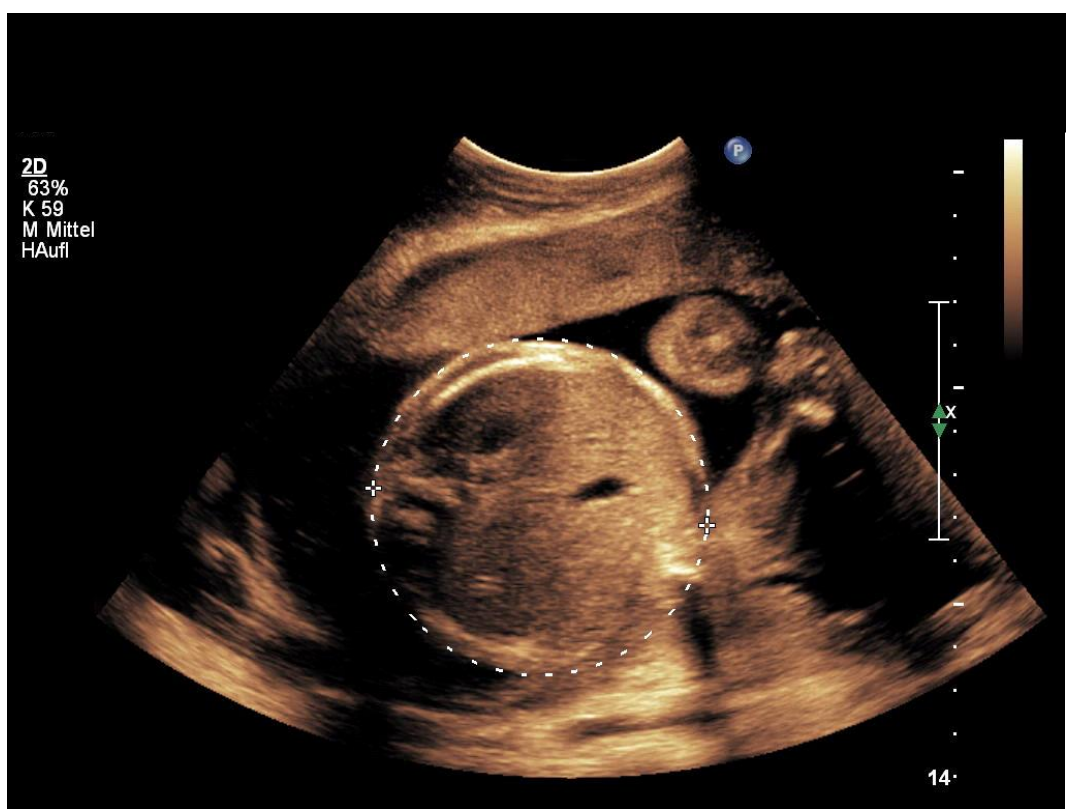

Figure 6: Measurement of abdominal circumference

A small foetus is characterized by an abdominal circumference below the 2.5th percentile and may be classified as an IUGR foetus or as a healthy constitutional small for gestational age (SGA) foetus. The diagnosis of a SGA foetus is made by

\footnotetext{
${ }^{5}$ Wilcox G, Trudinger B, Cook CM, Wilcox W, Conelly A. Reduced fetal platelet counts in pregnancies with abnormal Doppler umbilical flow waveforms. Obstet Gynecol 1989;73:639- 643.
} 
exclusion of pathological findings. When an anomaly scan and umbilical artery Doppler are normal, the small foetus is likely to be a normal small foetus.

The sonographical estimation of foetal weight is strongly discouraged because of a discrepancy of clinical importance and potential measurement errors. Foetal biometry consists of measurement of three or sometimes four foetal dimensions - abdominal circumference, femur length, biparietal diameter or head circumference and consists in calculation of an estimated foetal weight by using a weight formula. There is no "best" formula. Sonographic foetal weight estimates are relatively inaccurate $(95 \%$ limits of agreement are about $20 \%$ ) irrespective of the used formula6. The main disadvantage by using several biometric measurements is the potentiation of the error due to measurements of several potentially inaccurate foetal planes.

The diagnosis of an IUGR foetus is made on the basis of sonographic findings such as low abdominal circumference, an increased Pulsatility Index of the umbilical artery and often additionally an oligohydramnios.

When the diagnosis of a small foetus is made, based on the measurement of the abdominal circumference, the umbilical artery Doppler should be used as the primary surveillance tool and vice versa. This is the main diagnostic tool to differentiate a growth-restricted foetus from a constitutionally small healthy foetus.

Once an IUGR foetus is identified, serial evaluation every two weeks or on a weekly schedule should be done (or on a more frequent interval if necessary) with Doppler studies. In a resource-poor setting the compliance would probably be very low and would be feasible only in selected cases.

When the resistance in cerebral blood vessels declines, foetal cerebral blood flow improves. Increased right ventricular afterload results in increased venous Doppler indices. The measurement of Doppler indices of foetal vessels, i.e. middle cerebral artery and Ductus venosus should be subject of a more experienced sonographer. One should be aware that the diagnosis of an IUGR foetus is probably beyond the reach of a resource-poor setting.

\section{Two level sonography}

Basic sonography in maternal health care performed by midwives covers foetal viability, foetal position, multiple pregnancies, severe amniotic fluid abnormalities, placenta praevia and placental dysfunction (Doppler measurement of umbilical artery and measurement of Abdominal Circumference). Ideally, pathologic findings of the basic sonography should lead to a more detailed sonography by an experienced sonographer. This proposed two level sonography would have two advantages: On the one hand, the quality of basic sonography would be controlled

\footnotetext{
${ }^{6}$ Anderson NG, Jolley IJ, Wells JE. Sonographic estimation of fetal weight: comparison of bias, precision and consistency using 12 different formulae. Ultrasound Obstet Gynecol. 2007;30:173-9.
} 
and potentially be improved ${ }^{7}$. On the other hand, foetal structural anomalies could be detected. Nevertheless, it should be kept in mind that more detailed sonographic foetal studies are very time consuming. Foetal sonography that focuses on structural anomalies should probably only be offered in cases when therapeutic options are available. Newborn babies with a gastroschisis or spina bifida for instance usually die soon after birth due to an almost inevitable bacterial infection and before a lifesaving operation can be started. In cases with foetal gastroschisis or spina bifida (Fig. 7) an intrauterine transfer to a facility capable of an immediate operative treatment after birth should be considered.

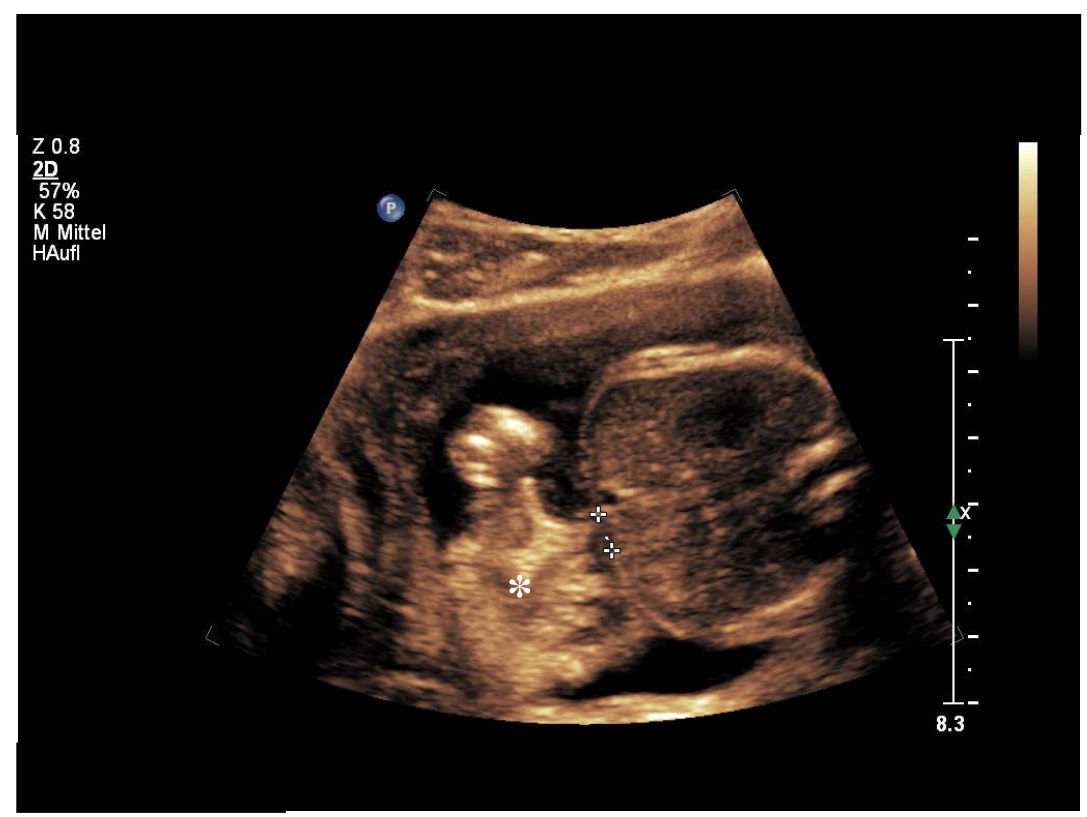

Figure 7: Gastroschisis, a congenital fetal structural anomaly with a defect in the anterior abdominal wall $(++$ through which viscera $(*)$ protrude).

\section{Summary}

Sonography should be implemented and used in settings which work already well with existing resources and means. Midwives should primarily perform sonography. Indications have to be clear and should influence further management. The objective of sonography depends very much on local preconditions. Primarily basic sonography should focus on conditions that are potential life threatening for the

\footnotetext{
7 Stein W, Katunda I, Butoto C. A two-level ultrasonographic service in a maternity care unit of a rural district hospital in Tanzania. Trop Doct. 2008;38:125-6.
} 
pregnant woman such as placenta previa. Clinical doubtful cases of foetal viability, foetal position or multiple pregnancies are easily clarified by sonography. Identification and management of placental insuffiency are challenging and demand high compliance of the pregnant woman. Basic sonography in maternal health care covers foetal viability, foetal position, multiple pregnancies, severe amniotic fluid abnormalities, placenta praevia and placental dysfunction (Doppler measurement of umbilical artery and Measurement of Abdominal Circumference). Pathologic findings should lead to a more detailed sonography by an experienced sonographer. 



\section{Neonatal Health: The Key to Achieving Millennium Development Goal 4?}

Carsten Krüger, St. Franæiskeus Hospital Ablen, Department of Paediatrics, Germany

\section{Introduction}

Protecting children and their families and enabling them to live healthy lifes are core responsibilities of all societies in the world. Nevertheless, maternal and child health is under constant threat, especially in many of the low-and-middle-income countries (LMICs). Therefore, in the year 2000 the United Nations General Assembly endorsed the Millennium Development Goals (MDGs). Two of the MDGs specifically focus on maternal and child health: MDG 5 promotes the aim to reduce maternal deaths by 75\% between 1990 and 2015; MDG 4 calls for the reduction of under-five deaths by two-thirds during this period (table 1).1,2 
Table 1: Millennium Development Goal 4 - expanded targets and indicators (adapted from references 1 and 2)

\begin{tabular}{lll}
\hline Goal & Targets & Indicators \\
\hline Reduce child & Reduce by two-thirds, & - Under-five mortality rate \\
mortality & between 1990 and 2015, the & - Infant mortality rate \\
& under-five mortality rate, & - Proportion of 1-year-old children \\
& and ensuring faster progress & immunised against measles \\
& among the poor and other & - Neonatal mortality rate \\
& marginalised groups & - Prevalence of underweight children \\
& & under five years \\
\hline
\end{tabular}

Table 2: Perinatal, neonatal and childhood vital events in different regions and countries of the world (most recent available data, compiled from references 3-11)

\begin{tabular}{|c|c|c|c|c|c|c|c|}
\hline Indicator & World & $\begin{array}{l}\text { Indus- } \\
\text { trialised } \\
\text { coun- } \\
\text { tries }\end{array}$ & $\begin{array}{l}\text { Low- } \\
\text { income } \\
\text { coun- } \\
\text { tries }\end{array}$ & $\begin{array}{l}\text { Gha- } \\
\text { na* }\end{array}$ & India* & $\begin{array}{l}\text { Indo- } \\
\text { nesia* }\end{array}$ & $\begin{array}{l}\text { Tan- } \\
\text { zania* }\end{array}$ \\
\hline $\begin{array}{l}\text { Live births } \\
(\mathrm{x} 1,000)\end{array}$ & 134,754 & 11,425 & 120,617 & 770 & 27,165 & 4,372 & 1,862 \\
\hline $\begin{array}{l}\text { Skilled attendance } \\
\text { at birth }(\%)\end{array}$ & 67 & $\sim 99$ & 66 & 57 & 53 & 79 & 49 \\
\hline $\begin{array}{l}\text { MMR (/100,000 } \\
\text { live births) }\end{array}$ & 260 & 14 & 290 & 350 & 230 & 240 & 790 \\
\hline LBW rate $(\%)$ & 15 & $\sim 10$ & 15 & 13 & 28 & 9 & $>10$ \\
\hline $\begin{array}{l}\text { Prematurity rate } \\
(\%)\end{array}$ & 11.1 & 9.3 & 11.8 & 14.5 & 13 & 15 & 11.4 \\
\hline $\begin{array}{l}\text { Stillbirths } \\
(\mathrm{x} 1,000)\end{array}$ & 2,640 & 36.4 & $\sim 2,560$ & 17 & 613 & 64 & 47 \\
\hline $\begin{array}{l}\text { Neonatal deaths (x } \\
1,000)\end{array}$ & 3,072 & 53 & 3,019 & 22 & 875 & 73 & 48 \\
\hline $\begin{array}{l}\text { PMR } \\
\text { (/1,000 live births) }\end{array}$ & 47 & 10 & 50 & 45 & 70 & 30 & 69 \\
\hline $\begin{array}{l}\text { NMR } \\
\text { (/1,000 live births) }\end{array}$ & 23 & 3 & 25 & 28 & 42 & 17 & 26 \\
\hline $\begin{array}{l}\text { IMR } \\
\text { (/1,000 live births) }\end{array}$ & 40 & 5 & 44 & 50 & 48 & 27 & 50 \\
\hline $\begin{array}{l}\text { U5MR } \\
\text { (/1,000 live births) }\end{array}$ & 57 & 6 & 63 & 74 & 63 & 35 & 76 \\
\hline
\end{tabular}

Abbreviations: IMR - infant mortality rate; LBW - low birth weight; MMR - maternal mortality ratio; NMR - neonatal mortality rate; PMR - perinatal mortality rate; U5MR- under-five mortality rate *: Current Göttingen International Health Network partner countries 
Despite considerable progress in the reduction of maternal deaths during the last 30 years, still around 343,000 women died during pregnancy, delivery or shortly afterwards in 2008 (down from 526,000 in 1980 and 432,000 in 1990), more than $98 \%$ of them in $\mathrm{LMICs}^{3}$. At the current rate of reduction, MDG 5 will not be met globally, but importantly not be met in many LMICs in Sub-Saharan Africa and South Asia which carry the largest burden. ${ }^{3}$

Progress in the reduction of under-five deaths has been equally slow. Nevertheless, compared to 1990 when 12.5 million children under five died, ${ }^{1}$ this number has decreased to an estimated 7.6 million deaths in 2010.4 Despite the impressive reduction by almost 5 million under-five deaths, this translates to only $40 \%$ fewer deaths after 20 years. As just five years are left for meeting MDG 4, it is obvious that this rate of reduction is too small to achieve the goal in most parts of the world. The largest burden of childhood deaths (>98\%) remains with the LMICs (Table 2), again affecting Sub-Saharan Africa and South Asia the most. ${ }^{4}$

Similarly to the unequal progress within the different world regions, the changes in the causes of under-five deaths have not been distributed evenly. Whereas in 1990 infectious causes contributed more than $65 \%$ to the total numbers of deaths, in 2010 this rate has fallen to less than $40 \%$ in children older than one month.,12 On the contrary, perinatal and neonatal causes of under-five deaths have increased from around 30\% in 1990 to $40 \%$ in 2010, exceeding even 50\% in many Asian countries. ${ }^{4,12}$ An analysis by Black and colleagues provided evidence that the proportion of neonatal deaths (all deaths occurring during the first 28 days of life), and thus the importance of preventing them, increases more and more the lower the under-five mortality rate is in a given country. ${ }^{13}$

As with child mortality, the burden of neonatal diseases and deaths (>98\%) rests on the LMICs. ${ }^{4}$ More than $90 \%$ of all births take place in these countries, but often less than $60 \%$ are supervised by skilled birth attendants (Table 2). Low rates of skilled attendants at birth are an important factor for higher stillbirth rates, neonatal and maternal morbidity and mortality. ${ }^{14-18}$ For numerous reasons, low birth weight (LBW: $<2.5 \mathrm{~kg}$ ) and prematurity rates are considerably higher in LMICs, which explains their importance as another major cause of neonatal mortality (tables $2+3) \cdot 4,7,9,19$ Infections are a special threat to all neonates, thus taking also a considerable toll during this early period of life (table 3).4,20 Birth-related trauma, such as birth asphyxia is an important cause of neonatal mortality as well, especially during the first few days of life (Table 3). $4,15,16,21$ While up to $75 \%$ neonatal deaths occur during the first week, more than $50 \%$ of neonatal deaths occur during the first 24 hours after birth. ${ }^{22}$ Not surprisingly, birth asphyxia, besides prematurity, rapidly progressing infections and severe congenital malformations, is the main cause of death during these first critical hours of life, causing up to $80 \%$ in some studies. ${ }^{15,16,22,23}$ 
Table 3: Global causes of 3.2 million neonatal deaths in 2010 (adapted from reference 4)

\begin{tabular}{|c|c|}
\hline Cause & Percentage \\
\hline Prematurity/LBW & 35 \\
\hline Birth-related trauma/Asphyxia & 23 \\
\hline Infections & 27 \\
\hline Sepsis/Meningitis & 12 \\
\hline Pneumonia & 10 \\
\hline Diarrhoea & 2.5 \\
\hline Tetanus & 2.5 \\
\hline Congenital disorders & 10 \\
\hline Others & 5 \\
\hline
\end{tabular}

Abbreviations: LBW - low birth weight

\section{Birth aspyxia and neonatal resuscitation}

Given that birth asphyxia is one of the leading causes of early neonatal death, interventions are urgently needed to reduce this preventable complication. One approach is to improve obstetric care (preferably in health facilities and provided by skilled birth attendants), including the use of the partograph and foetal heart rate monitoring as abnormalities detected early during the course of delivery predict adverse outcomes for the child (and the mother). ${ }^{15-18,21,24-27}$ Most important, referral to emergency Caesarean section must be available and accessible for all women in labour who experience unexpected and serious complications in order to save the lives of both the mother and the neonate as the majority of these complications are not foreseeable even with functioning antenatal care programs. ${ }^{28}$ Equally important for the survival of the neonates is the presence of skilled birth attendants who are trained in neonatal resuscitation and thus, assist the neonate during the critical adaptation phase and initiate resuscitative measures if necessary (Figure 1). ${ }^{15-18,21,24-27}$.

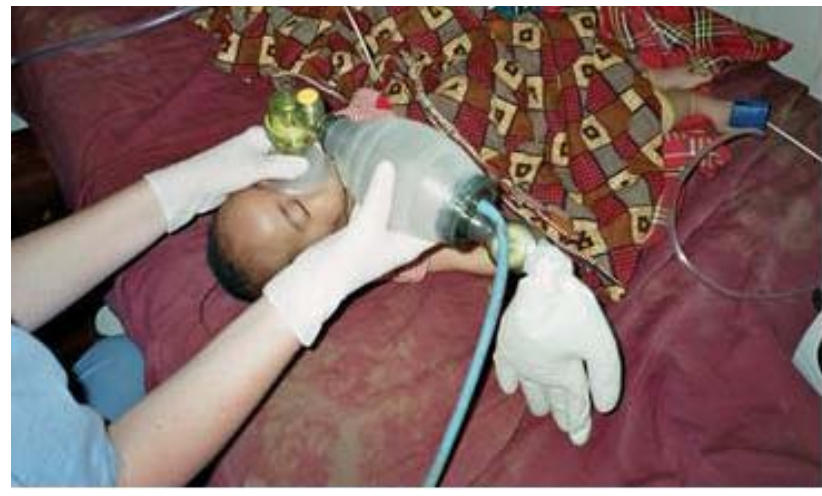

Figure 1: Neonatal resuscitation in a Tanzanian rural hospital 
Only basic equipment is required for neonatal resuscitative interventions: a table or another firm, even surface with sufficient light, clean, dry and warm blankets, head cover for LBW and premature infants, sterile cord clamps/ties with scissors/scalpel, a suction device (manual type sufficient), and a ventilation bag ("ambu bag") with a neonatal-size face mask. ${ }^{29}$ Despite a long history of usage, oxygen is not necessary for the successful resuscitation in neonates and may be even harmful instead. ${ }^{30}$

The most critical question with regard to neonatal resuscitation in birth asphyxia is who should and is able to provide this care in LMIC settings. Qualified physicians are rare and midwives are usually busy with assisting the mothers who just gave birth. ${ }^{31,32}$ Nonetheless, in health facilities usually midwives and nurses are the first who could assist the neonate if experiencing any difficulties (for the discussion on those neonates who are born outside health facilities see the comments under heading 4). ${ }^{15,16,33}$ But many of these are not well trained in neonatal resuscitation. Therefore, the American Academy of Pediatrics, together with other partners from around the world, developed a training program on neonatal resuscitation directed towards health staff in LMICs working with limited resources (www.helpingbabiesbreathe.org). ${ }^{34}$ This practical training course teaches the essential basics of neonatal resuscitation and uses booklets, manikins and the essential equipment mentioned in the previous paragraph, always keeping in mind the difficult circumstances under which health care is delivered in most parts of LMICs (Figure 2).

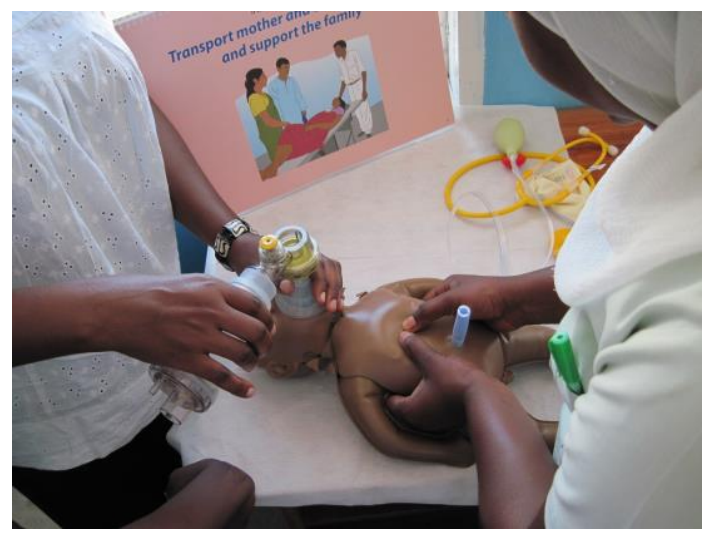

Figure 2: Training nurses and midwives in neonatal resuscitation in Tanzania

First studies from Tanzania and Nepal indicate that if this program is applicable, it reduces the number of neonatal deaths due to birth asphyxia and seems viable over quite a long period, e.g. more than two years. 35,36 These studies confirmed that in most neonates, vigorous stimulation and face mask ventilation is sufficient for successful resuscitation. Recently, a study from Rwanda revealed that health workers trained in this program may loose their practical skills already after three months if not being re-trained and practising their skills constantly. ${ }^{37}$ This parallels 
the results of other studies which showed unfavourable outcomes of neonatal resuscitation programs if no attention was paid to continuing supervision and retraining which needs to be incorporated into daily routine practice and given high priority by the leadership. ${ }^{38-40}$

\section{Neonatal care in health facilities}

After the initial adaptation phase including resuscitation measures for some neonates, continuous basic care is important to ensure the health and survival of the neonates within the facilities. The pillars of neonatal care in LMICs consist of a safe, clean environment (clothes and good hand hygiene), warmth (not bathing the neonate shortly after birth, changing wet clothes frequently; see below for kangaroo mother care), breast-feeding (preferably exclusive and initiated within the first hour of life) and support for the new mother, the baby and the extended family. ${ }^{29}$ But the question is whether these facilities are prepared to provide good neonatal care. Typically, they suffer from a lack of qualified staff, basic equipment like suction machines, ventilation bags and masks or oxygen supply, and drugs, let alone other more sophisticated equipment like mechanical ventilators or infusion pumps. ${ }^{41}$ In addition, many of the facilities are overwhelmed by huge numbers of patients, including high-risk referrals. Not surprisingly, the numbers of stillbirths, neonatal and maternal deaths are unacceptably high even at university hospitals, exceeding often national mortality rates..$^{42}$ Under such circumstances, the introduction of appropriate technology may play an important role in supporting the staff and achieving better results. Examples could be a locally produced cot (Figure 3) which provides sufficient warmth for premature, LBW and/or sick neonates, and oxygen concentrators. ${ }^{43}$ Incubators which are based on western technology are usually not appropriate due to a higher risk of nosocomial infection and other difficulties. ${ }^{29}$ Another approach could be the introduction of standardised care and treatment protocols which reduced neonatal death rates in Tanzania and Papua New Guinea significantly even if it is not possible to improve the equipment very much. ${ }^{44,45}$

LBW and premature infants (and sometimes sick term neonates) need special attention as they are prone to hypothermia, feeding difficulties, poor weight gain, infections and respiratory problems (Figure 4). ${ }^{29}$ Birth weight is fairly easy to measure, at least in health facilities, and defines whether the neonate is LBW or not. More important for the prognosis is the neonate's gestational age, though, as preterm infants tend to have more problems than term LBW infants. ${ }^{719,29}$ As many mothers do not plan the pregnancy, do not know gestational age and do not have a first-trimester ultrasonography performed for determining gestational age, the use of maturity scores (Table 4) is essential to estimate the potential risk of the neonate. ${ }^{19,46}$ 


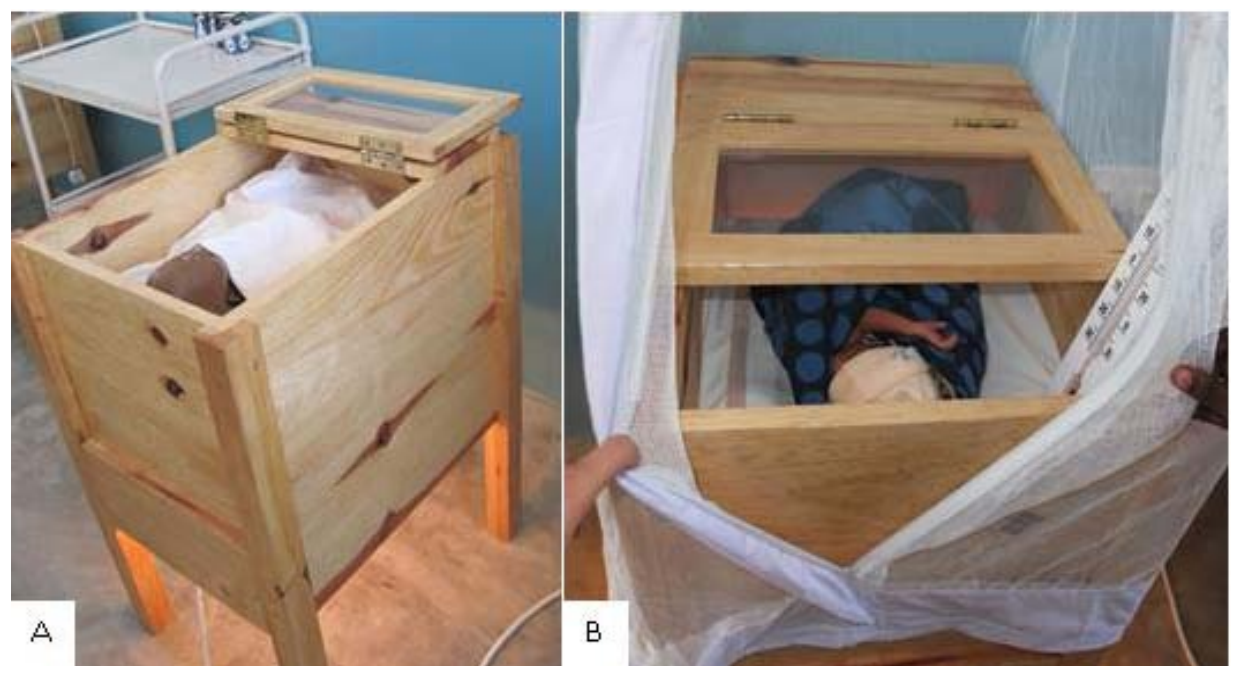

Figure 3A+B: Appropriate technology - the "Moshi Baby Cot" in Bagmoyo/ Tanzania

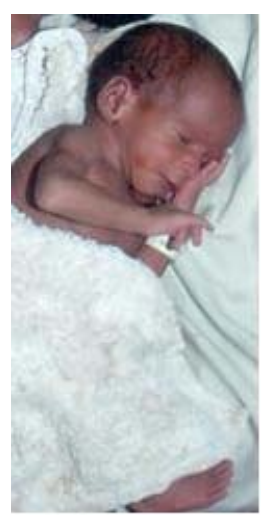

Figure 4: A LBW neonate in rural Tanzania

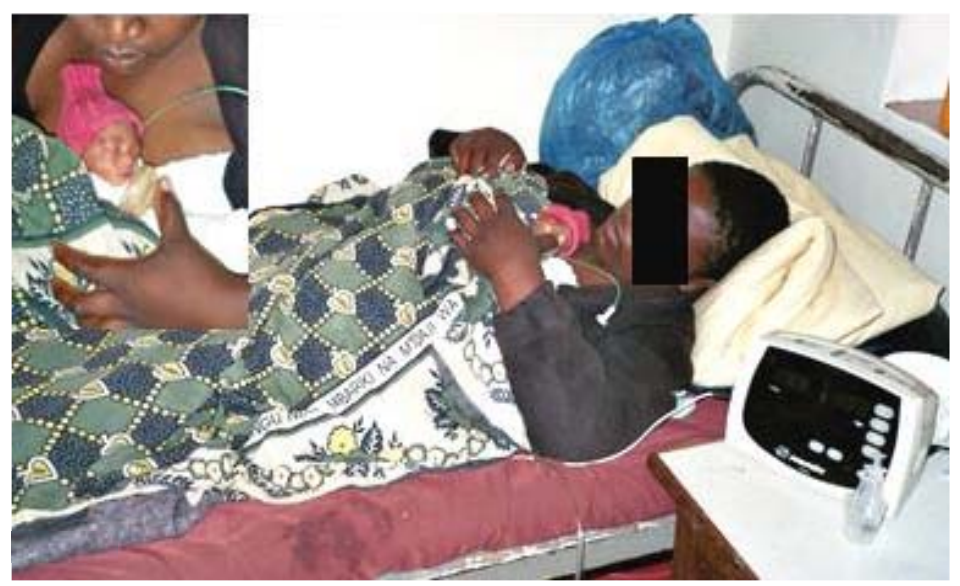

Figure 5: Kangaroo mother care in Tanzania

During recent years, several studies could prove that the technique of kangaroo mother care (Figure 5) is very effective in the care of LBW and premature infants in LMICs: hypothermia, bradycardia, apnoea, respiratory disorders, and infections are reduced in frequency, breast feeding and weight gain is facilitated, and the final outcome of these vulnerable neonates is considerably better. ${ }^{47-51}$ Nevertheless, mothers (and fathers) who adopt this technique need continuous support as this type of care needs to be provided for several weeks 24 hours a day. 
Table 4: Finnström maturity score in preterm and term neonates (adapted from reference 46)

\begin{tabular}{|c|c|c|c|c|}
\hline Score & 1 & 2 & 3 & 4 \\
\hline Breast size & $<5 \mathrm{~mm}$ & $5-10 \mathrm{~mm}$ & $>10 \mathrm{~mm}$ & \\
\hline $\begin{array}{l}\text { Nipple for- } \\
\text { mation }\end{array}$ & $\begin{array}{l}\text { No areola nipple } \\
\text { visible }\end{array}$ & $\begin{array}{l}\text { Areola present, } \\
\text { nipple well formed }\end{array}$ & $\begin{array}{l}\text { Areola raised, } \\
\text { nipple well formed }\end{array}$ & \\
\hline $\begin{array}{l}\text { Skin } \\
\text { opacity }\end{array}$ & $\begin{array}{l}\text { Numerous veins } \\
\text { and venules } \\
\text { present }\end{array}$ & $\begin{array}{l}\text { Veins and tributar- } \\
\text { ies seen }\end{array}$ & $\begin{array}{l}\text { Large blood ves- } \\
\text { sels seen }\end{array}$ & $\begin{array}{l}\text { Few blood } \\
\text { vessels seen } \\
\text { or none at all }\end{array}$ \\
\hline Scalp hair & Fine hair & $\begin{array}{l}\text { Coarse and silky } \\
\text { individual strands }\end{array}$ & & \\
\hline Ear cartilage & $\begin{array}{l}\text { No cartilage in } \\
\text { antitragus }\end{array}$ & $\begin{array}{l}\text { Cartilage in antit- } \\
\text { ragus }\end{array}$ & $\begin{array}{l}\text { Cartilage present } \\
\text { in antihelix }\end{array}$ & $\begin{array}{l}\text { Cartilage in } \\
\text { helix }\end{array}$ \\
\hline Fingernails & $\begin{array}{l}\text { Do not reach } \\
\text { finger tips }\end{array}$ & Reach finger tips & $\begin{array}{l}\text { Nails pass finger } \\
\text { tips }\end{array}$ & \\
\hline $\begin{array}{l}\text { Plantar } \\
\text { skin creases }\end{array}$ & No skin creases & $\begin{array}{l}\text { Anterior trans- } \\
\text { verse crease only }\end{array}$ & $\begin{array}{l}\text { Two-thirds anteri- } \\
\text { or sole creases }\end{array}$ & $\begin{array}{l}\text { Whole sole } \\
\text { covered }\end{array}$ \\
\hline
\end{tabular}

Total points scored:

7891011121314151617181920212223

Days of gestation:

191198204211217224230237243250256263269276282289295

Weeks of gestation:

$27+28+29303132333435-36-36 \frac{1}{2}$ 371/2 381/2 391/2 40+ 41+ 42+

Notes: Test fingernails by scratching them along your hand. Skin creases are the deep creases, not the fine lines.

Besides the prevention and treatment of birth asphyxia, and the special care for LBW and preterm infants, prevention and timely treatment of neonatal infections is of utmost importance. Hygienic conditions around the time of birth are as important as meticulous hand hygiene, good skin care and clean, dry clothing during the later phase. As the clinical signs and symptoms of neonatal infections are often subtle and inconclusive (Table 5), a high index of suspicion, along with good history taking and physical exam, is needed to detect neonatal infections early and to initiate antibiotic treatment in time. ${ }^{29,52,53}$

Typical treatment regimens consist of a combination of parenterally administered ampicillin and gentamicin or penicillin $G$ and gentamicin. ${ }^{29,54}$ In case of suspected staphylococcal disease (Figure 6), cloxacillin should be added. ${ }^{29,54}$ Cefotaxime is a good alternative in severe cases, if available, whereas ceftriaxone, when being given over several days, should be avoided in neonates due to its side effects. ${ }^{54}$ 
Table 5: Clinical signs and symptoms of neonatal infections (sepsis, pneumonia, meningitis, diarrhoea, skin infections and others)

\begin{tabular}{llll}
\hline $\begin{array}{l}\text { Causative } \\
\text { bacteria }\end{array}$ & Ports of entry & Signs and symptoms & Diagnosis \\
\hline E.coli & Umbilicus & Skin infections (umbilicus) & History \\
$\begin{array}{l}\text { B-Streptococci } \\
\text { Staph. aureus }\end{array}$ & Skin & Fever, hypothermia & Clinical \\
Non-typhoidal & Airway & Apathy & Laboratory \\
Salmonella & GIT & Difficult feeding, vomiting, & Lumbar punct. \\
(esp. in Africa) & (be aware of & distended abdomen & Cultures \\
& traditional "treat- & Grey skin color, poor periph- & X-ray \\
Others & ment" practices) & Tachy-/dyspnoea & Ultrasound \\
& & Tachy-/bradykardia & Others \\
& & Bulging fontanel, stiff neck & \\
& & Seizures & \\
& & Impaired consciousness, & \\
& & coma & \\
\hline
\end{tabular}

Abbreviations: ENT - ears, nose, throat; GIT - gastrointestinal tract

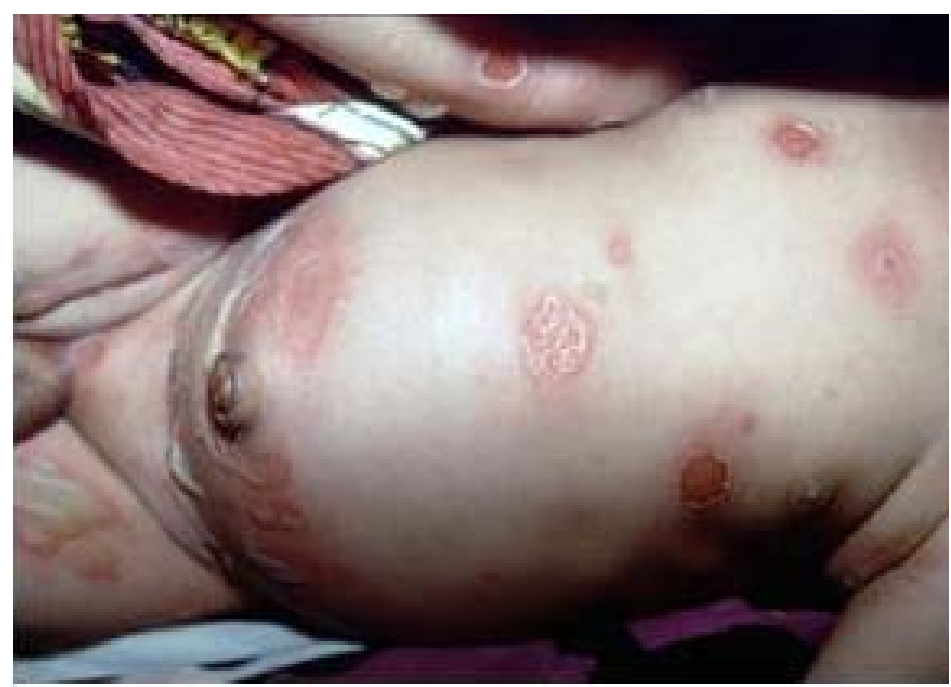

Figure 6: Staphylococcal skin infection in a neonate

\section{Neonatal care in the community}

For decades, neonatal care in LMICs was a neglected issue on the global health agenda as it was assumed that based on the experience from the industrialised world, only high-tech, costly interventions would bring about any significant 
changes which would not be affordable in a LMIC setting. This view changed when it became clear how important neonatal health is for reducing child deaths in LIMCs. ${ }^{22}$ And given the fact that world-wide around $40 \%$ of births take place outside health facilities without skilled attendance and mostly at home, ${ }^{50}$ the critical question is who can and should take care of these neonates (and of their mothers) during and after delivery (Figure 7).

This situation has attracted even more attention as half of the neonatal deaths occur on the first day of life and another quarter during the following week, ${ }^{22}$ and even in case of serious complications, only a minority of the neonates are brought to the attention of qualified health workers. ${ }^{56-58}$ Routine home visits by health staff are even rarer, be it in Sub-Saharan Africa or South Asia where most neonatal deaths occur. ${ }^{22,59}$ In several African countries, these figures vary from 2 to $36 \%$.

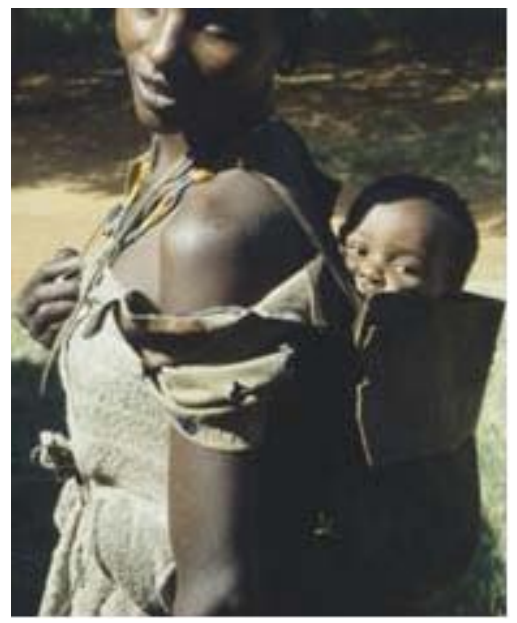

Figure 7: Infant life in a rural Tanzanian pastoralist community

Family members, traditional birth attendants, community health workers or formally trained health staff like midwives deployed to the communities do play a role in different LMIC settings.14- 18,21,26,60-62 They could assist in resuscitation, prevent and treat infections and support families to care for $\mathrm{LBW}$ and premature infants.

Other types of interventions like mobilising the communities themselves and/or women's groups may also be of interest. 14-18,21,26,60-64 These new approaches which are covered by the term "community-based neonatology/newborn care" nowadays, can be divided into medical interventions, strategies directed towards changes in community behaviour and a mixture of both..$^{21,26,62-64}$ In 1999, a large pioneering study by Bang and colleagues from Gadchiroli/India was the first to demonstrate that treatment of neonatal infections by village health workers in the 
community can significantly reduce neonatal mortality. ${ }^{65}$ These results were sustained over several years, and could be replicated in other parts of Asia. $63,64,66$ Some studies conducted in Pakistan, Nepal and Bangladesh suggest that chlorhexidine cleansing of the umbilicus after home delivery decreases the number of deaths and infection rates in neonates significantly, although the optimal timing and frequency has not been determined yet.67-70 The data base for neonatal resuscitation in the community conducted by community health workers is weaker, but some positive results have been reported from Pakistan and India. ${ }^{64,66}$

In 2004, a large study from Nepal was the first to report significant reductions in neonatal mortality $(>30 \%$ ) after community interventions facilitated by women's group..$^{71}$ Other studies from Asia which either focussed on community interventions alone or used a mixed approach produced similar results. ${ }^{63,64}$

During the last years, two meta-analyses examined the effect of communitybased neonatal care. ${ }^{63,64}$ For several medical interventions, in particular treatment of neonatal infections, a significant reduction of neonatal mortality rates by $30 \%$ could be found, also for interventions directed towards community behaviour changes and for a mixed approach. The data base for routine postnatal visits, although endorsed by WHO and UNICEF, is still weak. ${ }^{72}$ At present it is not yet clear which approach (medical vs. community vs. combined interventions) is the best for a given setting. Most likely, there will be no single approach for all settings.

It must be stated, however, that significant reductions of neonatal mortality rates in the community were only observed in LMIC settings where these mortality rates were initially higher than 30/1000 live births, and that rates below 20/1000 live births have not been achieved yet. ${ }^{63,64}$ Thus this approach is a necessary and effective intermediate step in improving neonatal health in the community, but it must be accompanied by continuous efforts to link home deliveries with emergency and routine facility-based intra-partum and postnatal care, ${ }^{73-76}$ to increase the number of births in health institutions and to provide high-quality care at these facilities. $2,15-18,21,24-26,28,41,44,45,61,62,74,76$

\section{Conclusion}

Improving neonatal health is a fundamental prerequisite in order to accelerate progress towards the achievement of MDG 4 world-wide, especially in SubSaharan Africa and South Asia. As birth asphyxia, neonatal infections and high rates of LBW and prematurity are the major causes of stillbirth and neonatal deaths, appropriate routine and emergency skilled obstetric care, neonatal resuscitation, prevention and timely treatment of infections and special support for LBW and premature infants are essential. Health facilities must be prepared to care for these vulnerable patients, with regard to qualified staff, essential equipment and appropriate technology. Community-based care needs further research and refinement, and close links between the community and health facilities are needed. 


\section{References}

1. UN. The Millennium Development Goals Report 2010. New York: United Nations, 2010. [Available online at http://www.un.org/millenniumgoals/pdf/MDG\%20Report $\% 202010 \% 2$ 0En\%20r15\%20-low\%20res\%2020100615\%20-.pdf ]

2. UN Millennium Project 2005. Who's got the power? Transforming health systems for women and children. Task force on child health and maternal health. [http://www.unmillenniumproject.org/documents/maternalchildcomplete.pdf ]

3. Lozano R, Wang H, Foreman KJ, Rajaratnam JK, Naghavi M, Marcus JR, et al. Progress towards Millennium Development Goals 4 and 5 on maternal and child mortality: an updated systematic analysis. Lancet 2011 378(9797):1139-65.

4. Liu L, Johnson HL, Cousens S, Perin J, Scott S, Lawn JE, et al. Global, regional, and national causes of child mortality: an updated systematic analysis for 2010 with time trends since 2000 . Lancet 2012;379(9832):2151-61. Erratum in: Lancet 2012;380(9850):1308.

5. UNICEF. State of the world's children 2012. New York: UNICEF, 2011. [http://www.unicef.org/sowc2012/pdfs/SOWC\%202012Main\%20Report_EN_13Mar2012.pdf]

6. UN Interagency group for child mortality estimation. Levels and trends in child mortality. Report 2011. New York: UNICEF, 2011. [http://www.childinfo.org/files/Child_Mortality_Report_2011.pdf ]

7. Blencowe H, Cousens S, Oestergaard MZ, Chou D, Moller AB, Narwal R, et al. National, regional, and worldwide estimates of preterm birth rates in the year 2010 with time trends since 1990 for selected countries: a systematic analysis and implications. Lancet 2012;379(9832):2162-72.

8. Cousens S, Blencowe H, Stanton C, Chou D, Ahmed S, Steinhardt L, et al. National, regional, and worldwide estimates of stillbirth rates in 2009 with trends since 1995: a systematic analysis. Lancet 16;377(9774):1319-30.

9. Lawn JE, Gravett MG, Nunes TM, Rubens CE, Stanton C; GAPPS Review Group. Global report on preterm birth and stillbirth (1 of 7): definitions, description of the burden and opportunities to improve data. BMC Pregnancy Childbirth 2010;10 Suppl 1:S1.

10. Oestergaard MZ, Inoue M, Yoshida S, Mahanani WR, Gore FM, Cousens S, et al. Neonatal mortality levels for 193 countries in 2009 with trends since 1990: a systematic analysis of progress, projections, and priorities. PLoS Med 2011;8(8):e1001080. 
11. WHO. Perinatal and neonatal mortality. Country, regional and global estimates. Geneva: WHO, 2006.

[http://whqlibdoc.who.int/publications/2006/9241563206_eng.pdf ]

12. WHO. The World Health Report 1995: Bridging the gaps. Geneva: WHO, 1995. [http://www.who.int/whr/1995/en/whr95_en.pdf]

13. Black RE, Morris SS, Bryce J. Where and why are 10 million children dying every year? Lancet 2003;361(9376):2226-34.

14. Wilson A, Gallos ID, Plana N, Lissauer D, Khan KS, Zamora J, et al. Effectiveness of strategies incorporating training and support of traditional birth attendants on perinatal and maternal mortality: metaanalysis. BMJ 2011;343:d7102.

15. Wall SN, Lee AC, Carlo W, Goldenberg R, Niermeyer S, Darmstadt GL, et al. Reducing intrapartum-related neonatal deaths in low- and middleincome countries-what works? Semin Perinatol 2010;34:395-407.

16. Lawn JE, Lee AC, Kinney M, Sibley L, Carlo WA, Paul VK, et al. Two million intrapartum-related stillbirths and neonatal deaths: where, why, and what can be done? Int J Gynaecol Obstet 2009;107 Suppl 1:S5-18, S19.

17. Kerber KJ, de Graft-Johnson JE, Bhutta ZA, Okong P, Starrs A, Lawn JE. Continuum of care for maternal, newborn, and child health: from slogan to service delivery. Lancet 2007;370(9595):1358-69.

18. Koblinsky M, Matthews Z, Hussein J, Mavalankar D, Mridha MK, Anwar I, et al. Going to scale with professional skilled care. Lancet 2006;368(9544):1377-86.

19. Born too doon: global action report for preterm birth. New York: MoD, PMNCH, Save the Children, WHO, 2012.

[http://www.who.int/pmnch/media/news/2012/201204_borntoosoonreport.pdf ]

20. Thaver D, Zaidi AK. Burden of neonatal infections in developing countries: a review of evidence from community-based studies. Pediatr Infect Dis J 2009;28(1 Suppl):S3-9.

21. Osrin D, Prost A. Perinatal interventions and survival in resource-poor settings: which work, which don't, which have the jury out? Arch Dis Child 2010;95:1039-46.

22. Lawn JE, Cousens S, Zupan J; Lancet neonatal survival steering team. 4 million neonatal deaths: when? where? why? Lancet 2005;365(9462):891900. 
23. Ersdal HL, Mduma E, Svensen E, Perlman J. Birth asphyxia: a major cause of early neonatal mortality in a Tanzanian rural hospital. Pediatrics 2012;129(5):e1238-43.

24. Srofenyoh E, Ivester T, Engmann C, Olufolabi A, Bookman L, Owen M. Advancing obstetric and neonatal care in a regional hospital in Ghana via continuous quality improvement. Int J Gynaecol Obstet 2012;116:17-21.

25. Evjen-Olsen B, Olsen OE, Kvåle G. Achieving progress in maternal and neonatal health through integrated and comprehensive healthcare services - experiences from a programme in northern Tanzania. Int J Equity Health 2009;8:27.

26. McCoy D, Storeng K, Filippi V, Ronsmans C, Osrin D, Matthias B, et al. Maternal, neonatal and child health interventions and services: moving from knowledge of what works to systems that deliver. Int Health 2010;2:87-98.

27. Langli Ersdal H, Mduma E, Svensen E, Sundby J, Perlman J. Intermittent detection of fetal heart rate abnormalities identify infants at greatest risk for fresh stillbirths, birth asphyxia, neonatal resuscitation, and early neonatal deaths in a limited-resource setting: a prospective descriptive observational study at Haydom Lutheran Hospital. Neonatology 2012;102:235-42.

28. Olsen ØE, Ndeki S, Norheim OF. Availability, distribution and use of emergency obstetric care in northern Tanzania. Health Policy Plan 2005;20:167-75.

29. WHO. Pocket book of hospital care in children. Geneva: WHO, 2005. [http://whqlibdoc.who.int/publications/2005/9241546700.pdf ]

30. Davis PG, Dawson JA. New concepts in neonatal resuscitation. Curr Opin Pediatr 2012;24:147-53.

31. Olsen ØE, Ndeki S, Norheim OF. Human resources for emergency obstetric care in northern Tanzania: distribution of quantity or quality? Hum Resour Health 2005;3:5.

32. WHO. World health report 2006. Working together. Geneva: WHO, 2006. [ Available online at http://www.who.int/whr/2006/whr06_en.pdf ]

33. Wall SN, Lee AC, Niermeyer S, English M, Keenan WJ, Carlo W, et al. Neonatal resuscitation in low-resource settings: what, who, and how to overcome challenges to scale up? Int J Gynaecol Obstet 2009;107 Suppl 1:S47-62, S63-4. 
34. Singhal N, Lockyer J, Fidler H, Keenan W, Little G, Bucher S, et al. Helping babies breathe: global neonatal resuscitation program development and formative educational evaluation. Resuscitation 2012;83:90-6.

35. Ersdal HL, Mduma E, Svensen E, Perlman JM. Early initiation of basic resuscitation interventions including face mask ventilation may reduce birth asphyxia related mortality in low-income countries: a prospective descriptive observational study. Resuscitation 2012;83:869-73.

36. Kc A, Målqvist M, Wrammert J, Verma S, Aryal DR, Clark R, et al. Implementing a simplified neonatal resuscitation protocol-helping babies breathe at birth (HBB) at a tertiary level hospital in Nepal for an increased perinatal survival. BMC Pediatr 2012;12:159.

37. Musafili A, Essén B, Baribwira C, Rukundo A, Persson LÅ. Evaluating helping babies breathe: training for healthcare workers at hospitals in Rwanda. Acta Paediatr 2013;102:e34-8.

38. Nzinga J, Ntoburi S, Wagai J, Mbindyo P, Mbaabu L, Migiro S, et al. Implementation experience during an eighteen month intervention to improve paediatric and newborn care in Kenyan district hospitals. Implement Sci 2009;4:45.

39. Opiyo N, Were F, Govedi F, Fegan G, Wasunna A, English M. Effect of newborn resuscitation training on health worker practices in Pumwani Hospital, Kenya. PLoS One 2008;3(2):e1599.

40. Carlo WA, Goudar SS, Jehan I, Chomba E, Tshefu A, Garces A, et al. Newborn-care training and perinatal mortality in developing countries. $\mathrm{N}$ Engl J Med 2010;362:614-23.

41. Opondo C, Ntoburi S, Wagai J, Wafula J, Wasunna A, Were F, et al. Are hospitals prepared to support newborn survival? - An evaluation of eight first-referral level hospitals in Kenya. Trop Med Int Health 2009;14:116572.

42. Kidanto HL, Massawe SN, Nystrom L, Lindmark G. Analysis of perinatal mortality at a teaching hospital in Dar es Salaam, Tanzania, 1999-2003. Afr J Reprod Health 2006;10:72-80.

43. Duke T, Peel D, Graham S, Howie S, Enarson PM, Jacobson R. Oxygen concentrators: a practical guide for clinicians and technicians in developing countries. Ann Trop Paediatr 2010;30:87-101.

44. Krüger C, Niemi M, Espeland H, Naman N, Malleyeck I. The effects of standardised protocols of obstetric and neonatal care on perinatal and 
early neonatal mortality at a rural hospital in Tanzania. Int Health 2012;4:55-62.

45. Duke T, Willie L, Mgone JM. The effect of introduction of minimal standards of neonatal care on in-hospital mortality. P N G Med J 2000;43:127-36.

46. Finnström O. Studies on maturity in newborn infants. IX. Further observations on the use of external characteristics in estimating gestational age. Acta Paediatr Scand 1977;66:601-4.

47. Conde-Agudelo A, Belizán JM, Diaz-Rossello J. Kangaroo mother care to reduce morbidity and mortality in low birthweight infants. Cochrane Database Syst Rev 2011 Mar 16;:CD002771.

48. Blackwell K, Adriano Cattaneo A. What is the evidence for kangaroo mother care of the very low birth weight baby? Melbourne: International child health review collaboration, 2007.

[http://www.ichrc.org/sites/www.ichrc.org/files/kangaroo.pdf ]

49. Bergman NJ, Linley LL, Fawcus SR. Randomized controlled trial of skinto-skin contact from birth versus conventional incubator for physiological stabilization in 1200- to 2199-gram newborns. Acta Paediatr 2004;93:779-85.

50. Ibe OE, Austin T, Sullivan K, Fabanwo O, Disu E, Costello AM. A comparison of kangaroo mother care and conventional incubator care for thermal regulation of infants $<2000 \mathrm{~g}$ in Nigeria using continuous ambulatory temperature monitoring. Ann Trop Paediatr 2004;24:245-51.

51. Arif MA, Arif K. Low birthweight babies in the Third World: maternal nursing versus professional nursing care. J Trop Pediatr 1999;45:278-80.

52. Bhutta ZA, Zaidi AK, Thaver D, Humayun Q, Ali S, Darmstadt GL. Management of newborn infections in primary care settings: a review of the evidence and implications for policy? Pediatr Infect Dis J 2009;28(1 Suppl):S22-30.

53. Young Infants Clinical Signs Study Group. Clinical signs that predict severe illness in children under age 2 months: a multicentre study. Lancet 2008;371(9607):135-42.

54. Darmstadt GL, Batra M, Zaidi AK. Parenteral antibiotics for the treatment of serious neonatal bacterial infections in developing country settings. Pediatr Infect Dis J 2009;28(1 Suppl):S37-42.

55. Countdown to 2015 Coordinating Committee. Countdown to 2015 decade report (2000-2010): taking stock of maternal, newborn and child survival. New York: UNICEF, 2010. 
[http://www.countdown2015mnch.org/documents/2010report/Countd ownReportAndProfiles.pdf ]

56. Bari S, Mannan I, Rahman MA, Darmstadt GL, Serajil MH, Baqui AH, et al. Trends in use of referral hospital services for care of sick newborns in a community-based intervention in Tangail District, Bangladesh. J Health Popul Nutr 2006;24):519-29.

57. Sharkey AB, Chopra M, Jackson D, Winch PJ, Minkovitz CS. Pathways of care-seeking during fatal infant illnesses in under-resourced South African settings. Trans R Soc Trop Med Hyg 2012;106:110-6.

58. Bazzano AN, Kirkwood BR, Tawiah-Agyemang C, Owusu-Agyei S, Adongo PB. Beyond symptom recognition: care-seeking for ill newborns in rural Ghana. Trop Med Int Health 2008;13:123-8.

59. UNICEF. Progress for children: A world fit for children statistical review (No. 6). Nw York: UNICEF, 2007. [http://www.unicef.org/publications/files/Progress_for_Children_No_6 _revised.pdf ]

60. Darmstadt GL, Lee AC, Cousens S, Sibley L, Bhutta ZA, Donnay F, et al. 60 million non-facility births: who can deliver in community settings to reduce intrapartum-related deaths? Int J Gynaecol Obstet 2009;107 Suppl 1:S89-112.

61. Byrne A, Morgan A. How the integration of traditional birth attendants with formal health systems can increase skilled birth attendance. Int J Gynaecol Obstet 2011;115:127-34.

62. Ekman B, Pathmanathan I, Liljestrand J. Integrating health interventions for women, newborn babies, and children: a framework for action. Lancet 2008;372(9642):990-1000. Erratum in: Lancet 2008;372(9649):1544.

63. Bhutta ZA, Ali S, Cousens S, Ali TM, Haider BA, Rizvi A, et al. AlmaAta: Rebirth and Revision 6. Interventions to address maternal, newborn, and child survival: what difference can integrated primary health care strategies make? Lancet 2008;372(9642):972-89.

64. Gogia S, Ramji S, Gupta P, Gera T, Shah D, Mathew JL, et al. Community based newborn care: a systematic review and metaanalysis of evidence: UNICEF-PHFI series on newborn and child health, India. Indian Pediatr 2011;48:537-46.

65. Bang AT, Bang RA, Baitule SB, Reddy MH, Deshmukh MD. Effect of home-based neonatal care and management of sepsis on neonatal mortality: field trial in rural India. Lancet 1999;354(9194):1955-61. 
66. Bang AT, Reddy HM, Deshmukh MD, Baitule SB, Bang RA. Neonatal and infant mortality in the ten years (1993 to 2003) of the Gadchiroli field trial: effect of home-based neonatal care. J Perinatol 2005;25 Suppl 1:S92107.

67. Arifeen SE, Mullany LC, Shah R, Mannan I, Rahman SM, Talukder MR, et al. The effect of cord cleansing with chlorhexidine on neonatal mortality in rural Bangladesh: a community-based, cluster-randomised trial. Lancet 2012;379(9820):1022-8.

68. Tielsch JM, Darmstadt GL, Mullany LC, Khatry SK, Katz J, LeClerq SC, et al. Impact of newborn skin-cleansing with chlorhexidine on neonatal mortality in southern Nepal: a community-based, cluster-randomized trial. Pediatrics 2007;119:e330-40.

69. Mullany LC, Darmstadt GL, Khatry SK, Katz J, LeClerq SC, Shrestha S, et al. Topical applications of chlorhexidine to the umbilical cord for prevention of omphalitis and neonatal mortality in southern Nepal: a community-based, cluster-randomised trial. Lancet 2006;367(9514):910-8.

70. Soofi S, Cousens S, Imdad A, Bhutto N, Ali N, Bhutta ZA. Topical application of chlorhexidine to neonatal umbilical cords for prevention of omphalitis and neonatal mortality in a rural district of Pakistan: a community-based, cluster-randomised trial. Lancet 2012;379(9820):102936.

71. Manandhar DS, Osrin D, Shrestha BP, Mesko N, Morrison J, Tumbahangphe KM, et al. Effect of a participatory intervention with women's groups on birth outcomes in Nepal: cluster-randomised controlled trial. Lancet 2004;364(9438):970-9.

72. Costello A, Tripathy P. Community based newborn care. Indian Pediatr 2012;49:73; author reply 74.

73. Schmid T, Kanenda O, Ahluwalia I, Kouletio M. Transportation for maternal emergencies in Tanzania: empowering communities through participatory problem solving. Am J Public Health 2001;91:1589-90.

74. Lervik O. Too Far to Walk: A study of the ambulance service in the Haydom Area [student thesis]. Bergen: Faculty of Medicine, University of Bergen, 2001.

75. Krüger C, Olsen OHE. Inequities among the very poor: effect of young age on care-seeking. Lancet 2003;361(9370):1744-5.

76. Hounton S, Chapman G, Menten J, De Brouwere V, Ensor T, Sombie I, et al. Accessibility and utilisation of delivery care within a skilled care 
initiative in rural Burkina Faso. Trop Med Int Health 2008;13 Suppl 1:4452.

\section{Appendix: Glossary of Terms in Neonatology}

Gestational age (GA)

Term: $37+0$ weeks $-41+6$ weeks (259-293 days)

Preterm/premature (moderate/late) $:<37+0$ weeks $(<259$ days)

very preterm/premature: $<32+0$ weeks $(<224$ days $)$

immature, extremely preterm/premature: $<28+0$ weeks $(<196$ days)

extremely low gestational age newborn (ELGAN): $<26+0$ weeks $(<182$ days)

Postterm, overdue: $>41+6$ weeks (> 293 days)

\section{Weight definitions}

Appropriate for GA (AGA): 10th-90th percentile for GA

Small for GA (SGA): < 10th percentile for GA

Large for GA (LGA): > 90th percentile for GA

Macrosomia: $>4500(4000) \mathrm{g}$

Low birth weight (LBW): $<2500 \mathrm{~g}$

Very low birth weight (VLBW): $<1500 \mathrm{~g}$

Extremely/very very low birth weight (ELBW/VVLBW): $<1000 \mathrm{~g}$

Intrauterine growth retardation (IUGR): failure of normal foetal growth caused by multiple adverse effects on the foetus.

\section{Definitions of vital events related to pregnancy, birth and infancy}

- Abortion: death of an embryo (early) or a foetus up to $28\left(22^{*}\right)$ completed weeks of gestation (late)

- Stillbirth (intrauterine/foetal death; fresh/macerated): after completed 28 $\left(22^{*}\right)$ weeks of gestational age (or with $>1000 \mathrm{~g}\left(500^{*}\right)$ birth weight or with $>35\left(25^{*}\right)$ cm length)

- Perinatal period/death: after completed $28\left(22^{*}\right)$ weeks of gestation until 7 days after delivery; deaths defined as occurring during this period

- Neonatal period/death: days of life 1-28; deaths defined as occurring during this period (early: days 1-7; late: days 8-28)

- Perinatal mortality rate (PMR): all stillbirths after completed $28\left(22^{*}\right)$ weeks of gestational age (or with $>1000\left(500^{*}\right) \mathrm{g}$ birth weight or with $>35\left(25^{*}\right)$ $\mathrm{cm}$ length) and all deaths of live-born neonates during the first 7 days of life per 1000 live births

- Neonatal mortality rate (NMR): deaths of live-born neonates during the first 28 days of life (early NMR: day 1-7; late NMR: day 8-28) per 1000 live births 
- Infant mortality rate (IMR): deaths below 1 year of age per 1000 live births

- Postneonatal mortality rate (PNMR): IMR - NMR

- $\quad[*$ : lower threshold applies to industrialised countries] 


\section{Ovarian Cancer in Pregnancy: What Should We Do?}

Hasanuddin, Department of Obstetrics and Gynecology, Medical Faculty of Syiah Kuala University, Banda Aceh, Indonesia

\section{Introduction}

Cancer remains a major global health problem, especially in developing countries. It is one of the leading causes of non-accidental death in women of child-bearing age. In the USA, this disease accounts for about $19 \%$ of mortality in women between 15 and 34 years. Nevertheless, cancer in pregnancy is uncommon. Although the exact incidence and prevalence are unknown, estimates suggest one to two cases of cancer in every 1000 pregnancies. ${ }^{1}$

The most common cancers in pregnancy are cervical, breast, and ovarian cancer, followed by malignant melanoma, lymphoma, and leukemia. However, small series of colorectal, renal, and thyroid cancer have been reported and there are case reports of almost all known types of cancer in pregnant women. ${ }^{1}$

Ovarian cancer is the second most frequent gynecological cancer complicating pregnancy. The occurrence of ovarian cancer in pregnancy is uncommon, but exceedingly rare. Overall incidence of ovarian cancer during pregnancy is estimated between $1 / 10,000$ to $1 / 150,000$. But this number increased since childbearing is more and more frequently postponed to a later maternal age. Around $40 \%$ of these 
tumors are germ cell tumors. When an ovarian germ cell cancer is diagnosed during pregnancy, several conflicts arise concerning the health and life of the mother and the foetus by the diagnosed tumor and its therapy. The best reference material currently available on this issue is the Guidelines of an "International Consensus Meeting on Gynecologic Cancers in Pregnancy" hold in Leuven, Belgium. 2, 3

In a review of English literature from 1882 onwards, 24 primary ovarian cancers were found and 10 new cases were added from the records of Toronto Hospitals. One study reported 23 cases of ovarian cancer from Israel between 1960 and 1984 among 1,083,652 deliveries that took place 4,5

Another study found 6 cases among 160,889 deliveries over a period of 20 years. A large series of 60 malignant ovarian tumors in pregnancy was reported by Novak et al., but did not give any detailed information. Several case reports of malignant ovarian neoplasms of various types in pregnancy have been reported in the last decade. ${ }^{6}$

Gynecological cancer during pregnancy is a demanding problem, and multidisciplinary expertise should be available. Counseling both parents on the maternal prognosis and foetal risk is needed. When there is a firm desire to continue the pregnancy, gynecological cancer can be treated in selected cases. The staging and treatment should follow the standard approach as much as possible. Guidelines for safe pelvic surgery during pregnancy are presented. Mainly in cervical and ovarian cancer, chemotherapy and an alternative surgical approach need to be considered. Administration of chemotherapy during the second or third trimester maybe not increase the incidence of congenital malformation. Until now, the long-term outcome of children in utero exposed to oncological treatment modalities is poorly documented, but preterm birth on its own is associated with congenital anomalies. Delivery should be postponed preferably until after a gestational age of 34 weeks. ${ }^{7}$

\section{Diagnostic evaluation}

The majority of ovarian cancer during pregnancy are diagnosed at an early stage, when disease is still confined to the ovary. It seems that the distribution of different histologic types of ovarian cancers during pregnancy is the same as in nonpregnant women in the corresponding reproductive-age group. ${ }^{8,9}$

Mostly, the diagnosis of ovarian cancer in pregnancy is made during routine prenatal examinations in asymptomatic pregnant women during the first trimester, or based on lateral pelvic pain unexplained by the pregnancy. Rarely the diagnosis has been established after an adnexal mass was found obstructing labor. Physical examination is helpful, however, especially in the first and early second trimesters with limited value. ${ }^{9}$ Confirmation of the presence of a pelvis mass and the morphologic characteristics of the mass depend on the radiological appearance. The most common diagnostic instrument is ultrasonography. Various scale or scoring systems are used for ultrasonographic diagnosis, but the usefulness of these criteria 
in pregnancy has not been confirmed. In practice, a suspected malignancy in the case of adnexal lesion is based on these criteria: tumor size (with a cut-off at $5 \mathrm{~cm}$ or $6 \mathrm{~cm}$ to define a subgroup of lesion for which surgical exploration is needed), tumor morphological characteristics, and the presence of extraovarian disease. The use of color Doppler imaging is helpful in order to examine a vascular map of the ovarian mass that can improve the accuracy of ultrasound. ${ }^{11,12}$

Magnetic Resonance Imaging (MRI) is a valuable imaging modality safe to use during pregnancy. In case where the suspicious process is confined to the adnexa and the dignity is unclear, a laparoscopic procedure can be performed for diagnostic clarification. Although not definitively proven by large studies, MRI is widely used in pregnancy and is considered safe. MRI should be used as the method of choice for abdominal and especially pelvic imaging for diagnostic and staging purpose in ovarian cancer during pregnancy. It is noteworthy that gadoliniumenhanced magnetic resonance procedures can be safely applied during pregnancy. ${ }^{3}$, 13

Pelvic CT is contraindicated because of the high level of ionizing radiation and the risks of exposure to the foetus, therefore it should be avoided in ovarian cancer diagnosis and staging during gestation. The approximate foetal dose absorbed during abdominal or pelvic CT is up to $3 \mathrm{cGy}$ and up to $8.9 \mathrm{cGy}$, respectively. The latter is near, but does not reach the lower threshold for foetal damage, which is estimated to be between $10 \mathrm{cGy}$ and $20 \mathrm{cGy}$. Thus, major concerns do not focus on deterministic effects, but especially on threshold dose-unrelated, so called stochastic effects which are associated with increased lifetime risk for cancer and high risk for childhood cancer after abdomino-pelvic CT scan is performed, particularly during the third trimester. In principle, the use of positron emission tomography combined with CT is possible during pregnancy and procedures generate approximate foetal exposure of 1.9 cGy. However, in ovarian cancer this option is rarely necessary to make a proper diagnosis and should therefore be considered only in selected cases. ${ }^{3}$

Clinicians often use tumor markers to monitor ovarian cancer during pregnancy, but these tumor markers can also be physiologically raised during pregnancy. In one small study, concentrations of ovarian cancer antigen 125 (CA-125) were higher than $35 \mathrm{IU} / \mathrm{ml}$ in 11 of $46(24 \%)$ pregnant women. Concentrations greater than $65 \mathrm{IU} / \mathrm{ml}$ are recorded in $16 \%$ of patients during the first trimester, with wide variations between week 5 and 8 . CA-125 normalizes during the second and third trimesters but rises immediately after delivery, also with wide fluctuations. A return to baseline is recorded after 2-10 weeks post partum. Thus, CA-125 concentrations are not helpful during the first trimester, but can be used during the second and third trimester. ${ }^{14}$ 


\section{Expectant management}

The treatment of cancer diagnosed during pregnancy is complex and it depends upon multiple factors including the physical state of the patient, the tumor type and the stage, the gestational week, as well as maternal and foetal prognosis. But evidence-based clinical guidelines on how best to manage ovarian cancer in such patients have yet to be developed due to a lack of prospective randomized trials and cohort studies. ${ }^{7}$

When considering management of the ovarian cancer in this setting there are two key issues: how the pregnancy affects the behavior of the cancer, and second, how the cancer and its treatment affect the pregnancy. According to the literature, hypotheses have been made that pregnancy might affect the biological behaviour of tumours, although evidence for changes in prognosis has not been convincing. ${ }^{1}$

The most important consideration when deciding whether to continue the pregnancy or not is the need for adjuvant chemotherapy. Depending on the gestational age and diagnosis, a short delay (4 to 6 weeks) may be appropriate to allow the pregnancy to progress beyond the first trimester or maturnity. 1,2,3

Although a theoretical case can be made for terminating pregnancy, evidence of subsequent beneficial changes in the biological behaviour of the tumor is lacking. A pregnancy can be terminated as a therapeutic abortion until 24 weeks of gestation and after 28 to 30 weeks by a planned delivery of a viable preterm infant. However, before 33 weeks neonatal mortality is high with a substantial risk of major handicap in foetuses that survive. ${ }^{1}$

Surgery and anesthesia are safe during pregnancy if physiologic adaptations are considered. Adequate maternal monitoring is crucial in preventing hypoxia, hypotension, and hypoglycemia. Pregnant patients should be positioned in left lateral tilt to prevent caval compression. Foetal monitoring during surgery is always difficult to interpret and only useful if clinically relevant. Foetal monitoring during surgery for gynecological cancers is mostly not feasible. A cardiotocography, dopton, or ultrasound just before and after the surgery may be useful to exclude direct foetal damage timely associated with surgery. With regards to foetal resuscitation, the local policy needs to be followed. ${ }^{15}$

Surgery may increase preterm delivery but numbers are difficult to interpret because no comparison was made with a normal pregnant population. But previous data suggest that surgery does not increase the risk for miscarriage and congenital anomalies. Laparoscopic surgery during pregnancy is safe and effective when performed in experienced hands. However, most of the reported surgeries did not include the reproductive tract or were indicated for cancer treatment. Therefore, conclusion should be interpreted cautiously. ${ }^{7}$

There is no literature supporting the prophylactic use of tocolysis in case of surgery during pregnancy. But nevertheless it is useful to prevent preterm labour with prophylactic tocolytic agents after 24 weeks of gestation. When preterm labor is diagnosed perioperatively, tocolytic agents like nifedipine, atosiban, hexoprenalin 
(Gynipral) or indomethacin should be considered when the gestational age is before 32 weeks. ${ }^{15}$

After surgery, all ovarian cancer in pregnancy need adjuvant chemotherapy with the exception of grade 1-2 stage $1 \mathrm{~A}$ or $1 \mathrm{~B}$ epithelial malignancies, stage $1 \mathrm{~A}$ grade 1 immature teratoma and stage $1 \mathrm{~A}$ grade 1 dysgerminoma. Given the poor outcome of pregnancies associated with ovarian malignancies, treatment cannot be delayed in affected patients. ${ }^{2}$

\section{Case report}

A 27 -years old housewife, gravida 2, para 1 pregnant woman was transfered to our hospital with a chief complaint of vague abdominal pain for three month prior admitted to our hospital. Clinical examination showed an enlarged abdomen and accumulation of ascites. Ultrasound evaluation revealed a normal foetus at 26 weeks of gestation. In sonographic evaluation, a tumor mass of the right adnexa measured $15 \times 11 \times 10 \mathrm{~cm}$ was found and suggested malignancy with accumulation of ascites. We performed paracentesis due to increasing dyspnea and cytologic examination revealed malignant cells in the ascites. She was $155 \mathrm{~cm}$ tall and her weight was $49 \mathrm{~kg}$. Her blood pressure was $110 / 70 \mathrm{mmHg}$ and her pulse rate was $88 /$ min. In laboratory tests, the blood type was $\mathrm{B}, \mathrm{Rh}$-positive, $\beta$-human chorionic gonadotropin ( $\beta$ hCG) was $6,580 \mathrm{mIU} / \mathrm{ml}, \mathrm{LDH}$ was $402 \mathrm{U} / \mathrm{L}$, AFP was 12,650 $\mathrm{ng} / \mathrm{ml}$ and CA-125 was $46 \mathrm{U} / \mathrm{ml}$.

We performed right salpingo-oophorectomy and omentectomy; diagnosis by peritoneal cytology was stage $1 \mathrm{C}$ grade 2 dysgerminoma. After surgery, we discussed with the patient and her family about the risk and benefits of adjuvant chemotherapy during pregnancy. Then she was scheduled to receive three cycles of $15 \mathrm{mg}$ of bleomycin, $100 \mathrm{mg} / \mathrm{m} 2$ per day, etoposide and $20 \mathrm{mg} / \mathrm{m} 2$ per day and cisplatin for 5 days every 3 weeks, starting 3 weeks after surgery. After she received three courses of chemotherapy, we performed caesarian section and surgical staging two months after her first visit in our hospital. And than we gave 3 courses of chemotherapy afterwards. After finishing chemotherapy, the patient and her baby are still in a good condition.

\section{Discussion}

Ovarian dysgerminoma originates from the germ cell of the ovary. The name dysgerminoma was proposed by Meyer in 1931. Dysgerminoma is investigated histologically. Diagnosis is made by determination of serum lactate dehydrogenase $(\mathrm{LDH})$, as well as alpha-fetoprotein (AFP) as a tumor marker. Serum LDH is considered to be an indicator of therapeutics. Dysgerminoma is considered to be one of the most common ovarian tumors in reproductive age and tends to occur in young women, though the incidence of pregnancy with dysgerminoma is extremely 
low, but unfortunately the management of dysgerminoma during pregnancy is not well established. ${ }^{16}$

There is no evidence that pregnancy increases the incidence of cancer and no evidence of an adverse effect of pregnancy upon the prognosis or biology of cancer. It has been discussed how pregnancy influences dysgerminoma or how dysgerminoma influences pregnancy. Bakri et al. reported that the association of pure dysgerminoma and pregnancy did not adversely affect the tumor prognosis or foetal outcome. Ovarian cancer during pregnancy tends to progress asymptomatically and there are no differences with benign ovarian tumors in early stages. Therefore, we are facing some difficulties in making a correct diagnosis of ovarian cancer in pregnancy because of a huge gravid uterus that make pelvic examinations insufficient, and also because it is not recommended to conduct computed tomography (CT) scan in pregnancy. Occasional ultrasonography should be performed in order to confirm whether an ovarian tumor is present or not. It is very useful to evaluate whether an existing tumor is cystic or solid. In general, non-enhanced MRI is relatively accurate and also recommended. The $\mathrm{LDH}$ value is hard to use as an indicator of ovarian cancer when the patient is pregnant. ${ }^{16,17}$

The lines of treatment of cancer in pregnancy are essentially the same as in the non-pregnant state, namely surgery and chemotherapy. Pregnancy is no contraindication to surgery as surgical intervention is least likely to affect pregnancy, especially if done during the second and third trimesters. ${ }^{16}$

The treatment of ovarian cancer in pregnancy is as difficult as it is the diagnosis. Because of very few cases there is lack of statistically significant experience world-wide. The surgical treatment needs to be individualized and its exact extend would depend upon the stage of the disease and gestational age of the baby at diagnosis. After surgical treatment, all patients with disease beyond stage $1 \mathrm{~A}$ would require adjuvant chemotherapy. The standard adjuvant chemotherapy for dygerminoma consisting of bleomycin, etoposide and cisplatin has proven to be highly effective and have an acceptable toxicity for the patient and foetus. Even though all the chemotherapeutic drugs are potentially teratogenic, the use of bleomycin, etoposide and cisplatin in the second and third trimester of pregnancy is safe for the mother and the foetus as several studies concluded. 2,7,18

\section{Conclusions}

Dysgerminoma is the most common germ-cell malignancy associated with pregnancy, accounting for approximately $30 \%$ of ovarian cancer in pregnancy. Cancer during pregnancy is uncommon and need a rapid approach by a multidisciplinary team involving anaesthetists, obstetricians, neonatologist, and clinical and medical oncologists. Counseling about any harmful risk to the foetus should be performed in a supportive and informative manner. A detailed targeted ultrasound examina- 
tion is often used to accurately date the pregnancy and to terminate the pregnancy. 7,19

The risk of applying chemotherapy during pregnancy must be weighed against the impact of delayed treatment on maternal survival. Multiple studies have shown that chemotherapy administered during the first trimester increase the risk of spontaneous, foetal death, abortion and major malformations, but if chemotherapy is administered during the second and third trimester it is supposed to be safe. However, a definitive conclusion can not be derived yet because of the limited number of cases currently available in the literature and the long-term outcome of children in utero exposed to oncological treatment is poorly documented. 2,19

\section{References}

1. Moran BJ, Yano H, Zahir NA, Farqurharsan M. (2007). Conflicting priorities in surgical intervention for cancer in pregnancy. Lancet Oncol, 8, 536-544

2. Zarchi MK, Behtash N, Gilani MM. (2008). Good pregnancy outcome after prenatal exposure to bleomycin, etoposide and cisplatin for ovarian immature teratoma: a case report and literature review. Arch Gynecol Obstet, 277, 75-78

3. Zeimet AG, Marth C. (2012). Ovarian malignancies in pregnancy. In: Ayhan A, Reed N, Gultekin M, Dursun P (eds). Textbook of gynaecological oncology. Gunes Publishing, Istanbul, 82, 492-493

4. Lee YK, Yoo CY, Lee G, Park DC. (2011). Ovarian dysgerminoma associated with pregnancy. A case report. Korean J Obstet Gynecol, 54, 218-222

5. Novak ER, Lambrou CD, Woodruff JD. (1975). Ovarian tumors in pregnancy. An ovarian tumor registry review. Obstet Gynecol, 46, 401406

6. Rahman MS, Sibai MH, Rahman J, Sulaiman SA, Yahya AR et al. (2002). Ovarian carcinoma associated with pregnancy. A review of cases. Acta Obstet Gynecol Scan, 81, 260-264

7. Crispens MA, Jones HW. (2003). Management of cancer during pregnancy. CME J of Gynecol Oncol, 8, 143-149

8. Zanotti KS, Belinson JL, Kennedy AW. (2006). Treatment of gynecologic cancers in pregnancy. Semin Oncol, 27, 686-698 
9. Partridge EE, Phillips JL, Menck HR. (1996). The National Cancer Data Base report on ovarian cancer treatment in United States hospitals. Cancer, 78, 2236-2246

10. Tangir J, Schwartz P. (2002). Embryonal carcinoma: Diagnosis and management during pregnancy and fertility after the diagnosis. CME J of Gynecol Oncol, 7, 225-227

11. Sayar H, Lhomme C, Verschraegen CF. (2005). Malignant adnexal masses in pregnancy. Obstet Gynecol Clin North Am, 32, 569-593

12. Marret H, Lhomme C, Lecuru F. (2010). Guidelines for the management of ovarian cancer during pregnancy. Eur J Obstet Gynecol Reprod Biol, $149,18-21$

13. Kier R, McCarthy SM, Scoutt LM, Viscarello RR, Schwartz PE. (1990). Pelvic masses in pregnancy: MR imaging. Radiol, 1763, 709

14. Morice P, Uzan C, Gouy S, Verschraegen C, Meder CH. (2012). Gynaecological cancers in pregnancy. Lancet, 379, 558-569

15. Amant et al. Write all authors up to five, if more then write et al (2009) Gynecological cancers in pregnancy: guidelines of an international consensus meeting. Int J Gynecol Cancer, 19, S1-S12

16. Hady ESA, Hemida RAH, Gamal A, El-Zafarani M, Toson E, et al. (2012). Cancer during pregnancy: perinatal outcome after in utero exposure to chemotherapy. Arch Gynecol Obstet, 286, 283-286

17. Lee YK, Yoo CY, Lee GL, Park DC. (2011). Ovarian dysgerminoma associated with pregnancy. Korean J Obstet Gynecol, 4, 218-222

18. Raghunath R, Shashi R, Vijaykumar M. (2006). A case of pregnancy with epithelial ovarian cancer. J Obstet Gynecol India, 56, 446-448

19. Hubalek M, Schindelwig CS, Zeimet AG, Sergi C, Brezinka C, et al. (2007). Chemotherapeutic treatment of a pregnant patient with ovarian dysgerminoma. Arch Gynecol Obstet, 276, 179-183 


\title{
39. Breast Cancer in Countries with Limited Resources
}

\author{
Kantelhardt EJ', Bogale $S^{2}$, Mathewos $A^{2}$, Tariku $W^{3}$, Hanson $C^{4}$, Adamu $A^{5}$ \\ ${ }^{1}$ Department of Gynaecology, Martin-Luther-University Halle (Saale), Germany \\ ${ }^{2}$ Radiotherapy Center, Addis Ababa University, Ethiopia \\ ${ }^{3}$ Department of Surgery, Aira Hospital, Ethiopia \\ ${ }^{4}$ Global Health, Karolinska Institute, Stockholm, Sweden \\ ${ }^{5}$ School of Public Health, Addis Ababa University, Etbiopia
}

\section{Summary}

International women's health has for a long term focused on maternal mortality (MDG V). There is a growing need to approach non-communicable diseases in the world: cardio-vascular and chronic lung disease, diabetes and cancer are now the leading causes of death $(60 \%)$. Out of 35 Mio deaths half of them occur among people who are under the age of 70 years and the other half are females. In total, $80 \%$ of chronic diseases occur in low- and middle-income countries which also carry the burden of infectious diseases. In 2010, the annual incidence of breast cancer was estimated to be 1.2 Mio new cases per year. The increase is mainly seen in low- and middle-income countries. Resource limitations with regard to funding, 
personnel, infrastructure and political instability are tremendous. There is little attention given to breast care in low-resource settings due to other health priorities. However, with increasing life-expectancy and the reduction of mortality because of infectious diseases, more people are confronted with non-communicable diseases and thus, the topic of cancer in developing countries emerges more and more. Specific guidelines for breast cancer diagnosis and treatment were published by the Global Breast Health Initiative differentiating them according to the availability of resources in different settings. Taking into account public awareness and resources of health care facilities, obtaining correct diagnosis, and deciding on how to put strategies of treatment into practice - it becomes clear that these conditions substantially differ between developed and developing countries. For the upcoming ten years, further research is essential to meet the global challenges of diagnosis and treatment of breast cancer.

\section{Epidemiologic facts}

Worldwide, breast cancer is the leading entity among different cancers in women likewise in developed and developing countries. In 2010, besides 1.2 million new cases per year the prevalence is likely to be 5.2 million in the same year [Bray et al., 2012]. It is the primary cause of cancer death in women and it is responsible for 425,000 annual deaths worldwide. The incidence is estimated to vary up to ten times worldwide. There are many speculations on the reasons why these differences occur. Some suspected factors may be life-expectancy, genetic, reproductive, hormonal and nutritional factors as well as health system differences (e.g. examination skills, mammography screening) and women's status in society.

The observation of migrants showed that environmental influence is high. The low-risk population from Asia migrating to the high-risk country USA will soon have descendants with high incidence in breast cancer. Although data is scarce, there is consensus about increasing incidence in developing countries when changes in life style occur. Parkin and colleagues have studied cancer registries from Latin America, Asia and Africa comparing the 1960ies with the 1990ies incidences. Latin America has intermediate rates, however in some areas like Colombia the number of inicidences almost doubled. Asia's incidence rate is diverse having low incidence areas like India or China (less than 50 per 100,000) but they are increasing as well. The main reason for this trend is a higher incidence in younger birth cohorts. Africa shows an increase from a formerly low incidence in the Northern part, the Southern parts do increase slowly, but rates are altered by the AIDS epidemic, promoting different cancers such as cervical cancer or Kaposi Sarcoma [Parkin, 1994].

The known risk factors such as early age at menarche, low parity and higher age at first birth, late menopause or less breast feeding relate mostly to the level of the oestrogen milieu of a woman. Higher socio-economic background plays a ma- 
jor role for greater incidence as well - but also leading to a reduced mortality because of earlier presentation and treatment options [Adams et al., 2004]. These patterns are changing in the developing world too. There are also more aggressive types of estrogen-receptor negative and early onset breast cancer seen in developing countries and thus, may also explain higher fatality rates [Huo et al., 2009].

\section{Case report from 2007}

During her third pregnancy a 35 years old patient from rural Ethiopia presented with a palpable mass in the right breast. Being a nurse, she consulted three doctors who all told her this was a physiological situation due to hormonal changes. Again after delivery, she consulted a surgeon in Addis Abeba because the mass was increasing in size. Mammography was not available. A fine needle aspiration was done, the result was indeterminate. After a second consultation, again a specimen was taken, and the patient herself brought it to a renowned pathologist in town. The result was "malignancy suspected on cytology". Now being four weeks after normal delivery, she went to the university hospital for surgery. Mastectomy was offered and done within 24 hours. The result was a fast growing stage II breast cancer (pT2pN2a(5/6)MxG3), ductal carcinoma of the breast. The waiting period for radiotherapy at the only institution available was long since there was only one machine. The carcinoma was later identified abroad as hormone receptor negative, HER2 negative. According to the current data (www.adjuvant-online.de), the absolute benefit of chemotherapy is estimated being nearly $40 \%$ progression-free survival after ten years. The patient completed three cycles of FEC100 chemotherapy and three cycles of docetaxel. Standard radiotherapy was given. Most patients would probably have only noticed the mass and watched and waited. Some might have consulted doctors who would not recognise the problem. If the diagnosis was confirmed histologically, some might have received surgery. Some patients would have received tamoxifen if receptor positive and maybe oral cyclophosphamide. Fortunately by now in the year 2012, facilities have improved and more oncologists are available in Addis Abeba (four colleagues). For now, pathology service is possible for hormone-receptor status and much effort is put on decentralizing oncologic care.

\section{What about breast cancer in countries with limited resources?}

In 2011, the United Nations Summit talked about non-communicable diseases. World leaders agreed to set goals to reduce cardio-vascular and chronic respiratory diseases, diabetes and cancer. A 48-page booklet came out after the meeting showing the need of a comprehensive approach which is not always straight- 
forward like communicable diseases (see the 8 Millennium Development Goals). Important factors for achieving the set of goals and targets include the commitment of national governments to provide sufficient financial resources. What is to be said about breast cancer, taking into accout various obstacles? As gynaecologists and obstetricians, we are aware about the tragedy having a life-time risk for maternal death of 1 in 27 women in Ethiopia [WHO, 2005]. Can we discuss about breast cancer cases there? Having in mind these tremendous basic problems throughout the world - we are certainly obliged to discuss with carefulness not to miss the target improving women's health worldwide.

Cancer is not the major problem in very low-income countries today, but eventually numbers will increase. Many middle-income countries in South-East Asia, Latin-America and Northern Africa are more and more experiencing a "health transition" as they have successfully decreased the burden of communicable diseases. The ratio of communicable to non-communicable diseases is 3.8/1 in highmortality countries in Africa but $0.5 / 1$ in low-mortality countries in South-East Asia [WHO, 2003; Ferlay et al., 2000]. Moreover, the middle and upper class in the poorest countries are facing similar health needs than people in developed countries due to changing socio-demographic characteristics, life-styles and nutrition.

And there are also the patients in such countries who increasingly start to address this issue in public. The globalization prozess will accelerate interaction between the different parts of the world. Our patient from Ethiopia had a cousin in the United States with breast cancer - of course she knows, this cousin had received chemotherapy. So of course, she will start to search for it in Ethiopia. There is an enormous amount of information in the internet. To avoid promotion of procedures and treatment with merely commercialized intentions, the scientific community has to discuss before other interests are driving the development. The more attention is given to the enormous progress which is seen in developed countries right now, the better is the battle against the disease. Key points within this battle include appropriate allocation of scarce resources, the adaptation of programs to the need of people and culture-specific community applications as well as realistic long-term considerations of manpower and political issues.

\section{Guidelines for breast cancer in countries with limited resources}

To improve breast health care and cancer treatment worldwide, the Breast Health Global Initiative BHGI [http://www.fhcrc.org/science/phs/bhgi/] was founded in 2002. There was a growing need for strategies that can be implemented in countries with limited resources according to their individual options. Since 2003 and in regular meetings, guidelines based on methods of evidence-based medicine are agreed on and published. These recommendations are structured for four levels of resources to address the different circumstances (Tables 1-4). For each aspect, the 
individual site can decide on which level service should be implemented [Anderson et al., 2006]. The following sub-chapters provide an overview of these guidelines.

\subsection{Women getting access to care}

The main goal is to detect breast cancer at the earliest stage possible according to the existing resources. When mammography is available, prognosis is better for non-palpable lesions compared to palpable ones. Likewise, the smaller the palpable lesion the better is the prognosis [Nyström et al., 2002; Elkin et al., 2005]. A clinical trial focusing on down-staging of breast cancer by implementing clinical breast examination, clearly shows benefit in identifying cancer at an earlier stage [Sankaranarayanan et al., 2011]. Although training in breast self examination (BSE) has not shown a reduction of mortality in breast cancer [Thomas et al., 2002] and BSE cannot be recommended on the basis of current evidence, it has a value in raising self-awareness for breast symptoms and might change behaviors. The outcome of any program implemented (clinical breast examination or BSE) definitely has to be adequately evaluated, measuring facts about the findings and the impact on mortality and morbidity in the long term [Smith et al., 2006; Albert et al., 2003].

There are some more premises that need to be taken into account: Women have to be empowered to seek health care as such. There has to be an infrastructure for the diagnosis and the treatment of breast cancer. Education and awareness has to be promoted by local authorities. Higher sensitivity will be achieved when awareness and response to symptoms is carried out by as many as possible members of the population and thus, throughout increasing opportunity that is based on attentive health professionals at all levels of care [Smith et al., 2006].

Low-income countries have a low incidence of breast cancer especially in the young population with dense breast tissue not accessible by mammography. Therefore, the efficacy of mammography screening programs has to be questioned by methodological reasons. It has been shown that mammography screening reduces mortality - when accompanied by quality assurance and audits of all participating health professionals [Smith et al., 2004]. Therefore, mammography screening is more adequate for countries with better resources and older patients [Duffy et al., 2006]. In addition, ultrasound adds useful information on palpable tumors (Fig. 1). 


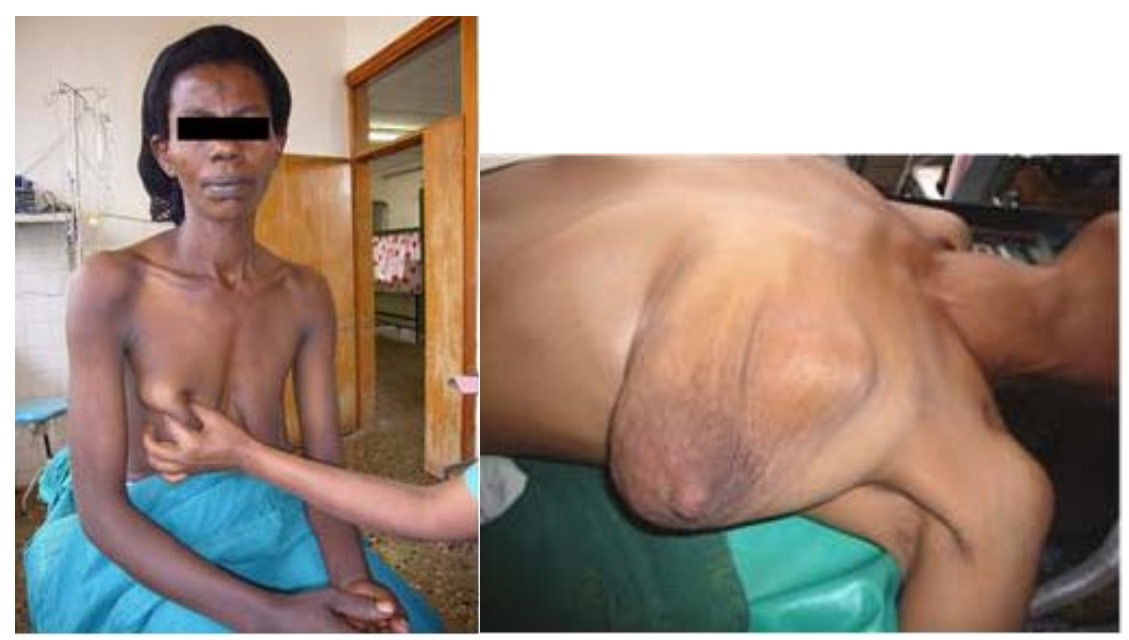

Fig 1: Patients presenting with palpable masses on the breast (pictures provided by Eva J Kantelhardt).

Altogether, it can be stated that programs for clinical breast examinations should be always considered to be implemented - ideally integrated in other running programmes such as HIV, maternal and child health or others (see Table 1).

\begin{tabular}{|c|c|c|c|}
\hline Level & Public education & Detection Method & Evaluation Goal \\
\hline Basic & $\begin{array}{l}\text { Teach early detection, risk } \\
\text { factors and breast health } \\
\text { awareness }\end{array}$ & Clinical history and CBE & $\begin{array}{l}\text { Breast health aware- } \\
\text { ness }\end{array}$ \\
\hline Limited & $\begin{array}{l}\text { Encourage CBE for age } \\
\text { groups at higher risk } \\
\text { through field workers }\end{array}$ & $\begin{array}{l}\text { Ultrasound in women with } \\
\text { positive }\end{array}$ & $\begin{array}{l}\text { Down-staging of } \\
\text { symptomatic women }\end{array}$ \\
\hline Enhanced & $\begin{array}{l}\text { Link regional awareness to } \\
\text { generally women's health } \\
\text { programmes }\end{array}$ & $\begin{array}{l}\text { Mammography screening } \\
\text { every } 2 \text { years/yearly depend- } \\
\text { ing on the population }\end{array}$ & $\begin{array}{l}\text { Down-staging of } \\
\text { asymptomatic wom- } \\
\text { en especially in target } \\
\text { groups }\end{array}$ \\
\hline Maximal & $\begin{array}{l}\text { National awareness cam- } \\
\text { paigns using media }\end{array}$ & $\begin{array}{l}\text { Mammography screening } \\
\text { yearly depending on popula- } \\
\text { tion, other technologies for } \\
\text { high risk groups }\end{array}$ & $\begin{array}{l}\text { Down-staging of } \\
\text { asymptomatic wom- } \\
\text { en especially in all } \\
\text { risk groups }\end{array}$ \\
\hline
\end{tabular}

Table 1: Resource allocation for early detection of breast cancer (adapted from the Breast Health Global Initiative, BHGI)

$\mathrm{CBE}=$ clinical breast examination; target group selection for mammographic screening should consider breast cancer demographics and resource constraints within the population.[...] Breast magnetic 
resonance imaging is more sensitive than mammography in detecting tumors in asymptomatic women who have an inherited susceptibility to breast cancer. Note that the table stratification scheme implies incrementally increasing resource allocation at the basic, limited, and enhanced levels. Maximal level resources should not be targeted for implementation in LMCs, even though they may be used in some higher income settings. (Adapted from Anderson et al. 2008. Guideline implementation for breast healthcare in low-income and middle-income countries: overview of the Breast Health Global Initiative Global Summit 2007. Cancer 113(8 Suppl): 2221-2243. The American Cancer Society. Reproduced with permission of Wiley-Less, Inc., a subsidiary of John Wiley \& Sons, Inc.)

\subsection{Cultural perspectives}

Many factors are known to influence people's behaviour towards health care. Major categories are culture, socioeconomic status and health experience. Especially when confronted with the diagnosis of cancer, patients experience fear, underestimation, fatalism and pessimism [Lannin et al., 2002]. Some cultures for example do not have a word for cancer. There is the fear of personal stigma or stigma towards the family, fear of contagion or exclusion from the society. In many societies in Africa, patients prefer to first visit a traditional healer and believe in traditional explanations like witchcraft, or ideas related to bad air or misbehaviour of the patient. Some patients think that knowledge of an incurable disease could lead to depression which might force them to commit suicide.

Also gender issues need to be taken into account such as the dependency from the husband and the fear of breakage of marriage if the diagnosis has been positive. Altogether it is of major importance to consider all these factors when trying to implement a strategy at any level of care. It is not a solution, to blame the patients for their beliefs. Careful and appropriate design and implementation of breast cancer care is needed [Dein 2004].

\subsection{The challenge of diagnostics}

The Breast Health Global Initiative has given some standards for diagnostic procedures [Shyyan et al., 2006]. As in all other diseases, history and physical examination is standard. Special attention is given to the clinical examination of the breast. Staging for metastatic disease should be performed in patients with stage III disease whenever possible since treatment may differ. In patients with small tumors stage I and II, it is unlikely to find metastasis by staging. Laboratory tests are not recommended in general.

Introduction of diagnostic mammography needs to consider the following aspects:

- Is there enough equipment and personnel available?

- Are the costs for the film covered?

- What is the size of the lesion patients present?

- Which age group is addressed (young women have dense breasts!)?

- Are there alternatives to diagnostic mammography? 
- Can breast-conserving therapy be offered?

- How to ensure quality of care?

Ultrasound is generally more often available so that it can serve as a diagnostic tool in distinguishing cysts, evaluating whether a mass is suspicious and may guide tissue sampling (Fig. 2). Also the liver should be evaluated for metastases by ultrasound. A problem that may arise is that through clinical examination and ultrasound alone, possibly too many lesions will be detected. Needle biopsy or core biopsy should to be one of the first methods to be implemented.

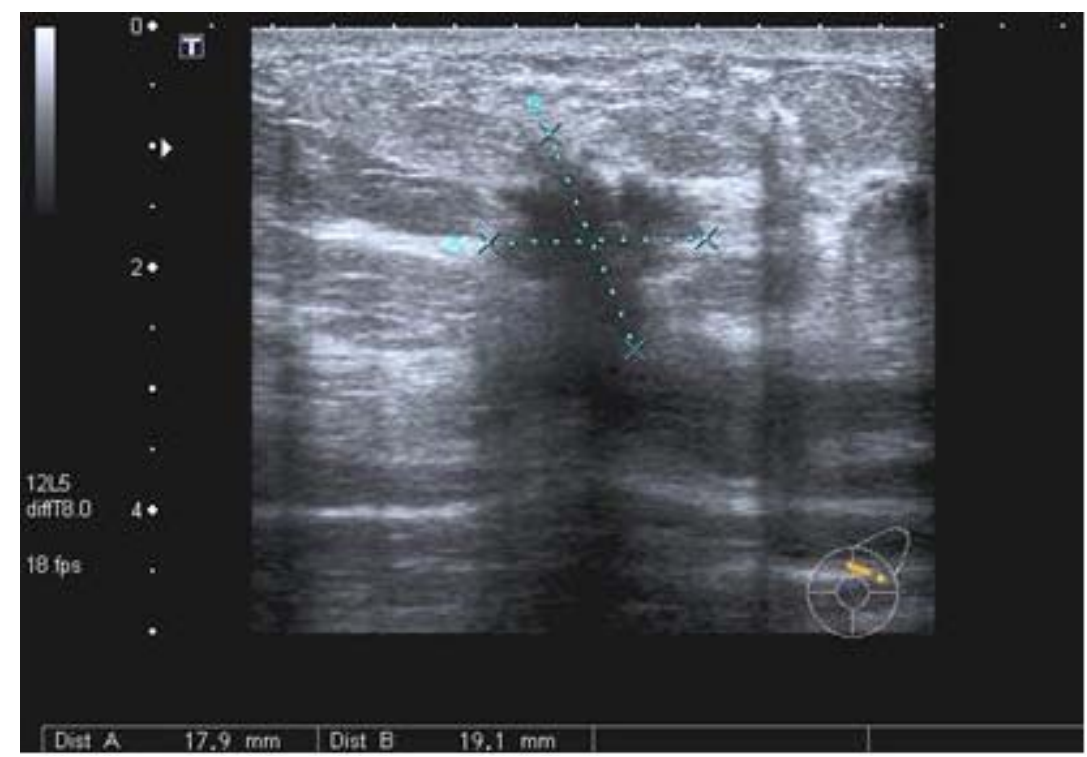

Figure 2: Breast cancer on ultrasound (picture courtesy of Regina Grosse).

The correct microscopic evaluation of tissue specimen is the main essential step ahead. It should be guaranteed for all women to distinguish a malignant lesion from a benign one. To provide such service, there are several factors of organization that need to be addressed like transportation, labeling, archiving, documentation and communication.

The minimal-invasive biopsy is an optimal, less invasive and rather inexpensive tool for diagnosis without having the need for operation (compared to surgical biopsy). The least invasive and least expensive tool represents fine-needle aspiration, however the specimens are sometimes small and difficult to interpret. In this case, the specimen could be sent to a central lab. Core-needle biopsy gives more material for the pathologist but is more costly. In low-resource settings, surgical biopsy might be the only option to give histological information. However, a pathologist must be available then. As a minimal standard, assessment of the tumor size, histological type and grading should be performed. Estrogen- and pro- 
gesterone-receptor status do need more financial options. The pathologist has to keep records and assess the outcome to verify the accuracy of diagnosis. When only few personnel is available, staff could be trained depending on the tasks that are needed. This also entails options for improving their status and income [Shyyan et al., 2004].

\subsection{Treatment depending on resources}

For the individual patient, a TNM classification should be done. Depending on the resources, therapy should follow international guidelines. Effective local treatment could be a modified radical mastectomy. Breast-conserving therapy needs careful assessment, and the access to ultrasound and mammography should be provided. Surgical margins have to be evaluated and postoperative radiotherapy should be offered (Fig. 3). Certain patients may also need radiotherapy after modified radical mastectomy.
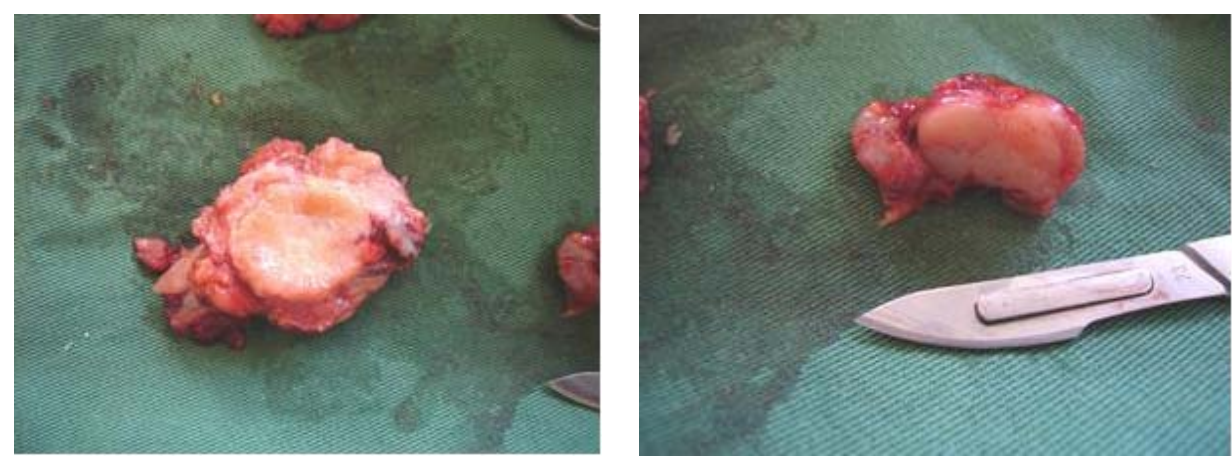

Figure 3: Invasive carcinoma (left) and lymph node (right) after surgical resection (picture courtesy of Regina Grosse)

Appropriate treatment to prevent a relapse depends on individual diagnostic and predictive factors of the patient. Endocrine treatment with few side effects is recommended for hormone receptor-positive patients in countries with basic resources. Tumors should be considered hormone receptor positive if the status is unknown. Ovarian ablation is an option in pre-menopausal women. Other options like aromatase inhibitors immediately raise costs and side effects must be considered.

If feasible, chemotherapy could be added. This does need a skilled physician for administration. Depending also on resources, CMF, anthracyclines, taxanes and others should be given according to the standards (Table 2). 
Table 2: Therapy for stage I breast cancer for different levels (adapted from BHGI: Treatment resource allocation table for stage $I$ breast cancer).

\begin{tabular}{|c|c|c|c|c|c|}
\hline Level & Surgery & Radiation & Chemotherapy & Endocrine & Biological \\
\hline 1 Basic & $\begin{array}{l}\text { Modified radical } \\
\text { mastectomy }\end{array}$ & & & $\begin{array}{l}\text { Oophorec- } \\
\text { tomy in pre- } \\
\text { menopausal } \\
\text { women, } \\
\text { Tamoxifen* } \\
\end{array}$ & \\
\hline 2 Limited & $\begin{array}{l}\text { Breast-conserving } \\
\text { surgery (if referral } \\
\text { to center with } \\
\text { radiotherapy pos- } \\
\text { sible) }{ }^{+}, \text {SLN biopsy } \\
\text { with blue dye }{ }^{++}\end{array}$ & & $\begin{array}{l}\text { Classical CMFo, } \\
\text { AC, EC, FAC }\end{array}$ & & oo \\
\hline 3 Enhanced & $\begin{array}{l}\text { Sentinel biopsy }{ }^{++} \text {, } \\
\text { Breast reconstruc- } \\
\text { tion }\end{array}$ & $\begin{array}{l}\text { Breast- } \\
\text { conserving } \\
\text { whole-breast } \\
\text { irradiation }^{+}\end{array}$ & Taxanes & $\begin{array}{l}\text { Aromatase } \\
\text { inhibitors } \\
\text { LH-RH ago- } \\
\text { nists }\end{array}$ & $\begin{array}{l}\text { Trastuzumb } \\
\text { for HER2- } \\
\text { pos. disease }\end{array}$ \\
\hline 4 Maximal & & & $\begin{array}{l}\text { Growth factors } \\
\text { dose-dense } \\
\text { chemotherapy }\end{array}$ & & \\
\hline
\end{tabular}

SLN $=$ sentinel lymph node; $\mathrm{CMF}=$ cyclophosphamide, methotrexate, and 5-fluorouracil; AC = doxorubicin and cyclophosphamide; EC = epirubicin and cyclophosphamide; FAC $=5$-fluorouracil, doxorubicin, and cyclophosphamide; $\mathrm{LH}-\mathrm{RH}=$ luteinizing hormone-releasing hormone; HER-2/neu $=$ human epidermal growth factor receptor 2. ${ }^{*} \mathrm{ER}$ testing by IHC is preferred for establishing hormone receptor status and is cost-effective when tamoxifen is available. When tamoxifen is available at the basic level, then IHC testing of ER status should also be provided. ${ }^{+}$Breast-conserving surgery can be provided as a limited-level resource but requires breast-conserving radiation therapy. If breastconserving radiation is unavailable, then patients should be transferred to a higher level facility for postlumpectomy radiation. ${ }^{++}$The use of SLN biopsy requires clinical and laboratory validation of the SLN technique. ${ }^{\circ}$ Systemic chemotherapy requires blood chemistry profile and complete blood count testing for safety. When chemotherapy is available at the basic level, these tests also should be provided. ooIf the costs associated with trastuzumab were substantially lower, trastuzumab would be used as a limited-level. In this case, measurement of HER-2/neu overexpression and/or gene amplification would also need to be available at the limited level in order to properly select patients for this highly effective but expenxive HER-2/neu-targeted therapy. Note that the table stratification scheme implies incrementally increasing resource allocation at the basic, limited, and enhanced levels. An empty matrix box indicates that additional resource allocation is not mandated beyond those resources required at lower levels. Maximal level resources should not be targeted for implementation in LMCs, even though they may be used in some higher income settings. (Adapted from Anderson et al. 2008. For more informations see Table 1; reproduced with permission of Wiley-Less, Inc., a subsidiary of John Wiley \& Sons, Inc.)

Only minor modifications of the therapeutic strategies are recommended for stage II breast cancer (Table 3). 
Table 3: Therapy for stage II breast cancer for different levels (adapted from BHGI: Treatment resource allocation table for stage II breast cancer).

\begin{tabular}{|c|c|c|c|c|c|}
\hline Level & Surgery & Radiation & $\begin{array}{l}\text { Chemo- } \\
\text { therapy }\end{array}$ & Endocrine & Biological \\
\hline 1 Basic & $\begin{array}{l}\text { Modified radical } \\
\text { mastectomy }\end{array}$ & $* *$ & $\begin{array}{l}\text { Classical } \\
\text { CMFo, } \\
\text { AC, EC, } \\
\text { FAC }\end{array}$ & $\begin{array}{l}\text { Oophorec- } \\
\text { tomy in pre- } \\
\text { menopausal } \\
\text { women, } \\
\text { Tamoxifen* }\end{array}$ & \\
\hline 2 Limited & $\begin{array}{l}\text { Breast-conserving } \\
\text { surgery (if referral } \\
\text { to ctr. with radio- } \\
\text { therapy possible) } \\
\text { SLN biopsy with } \\
\text { blue dye }{ }^{++}\end{array}$ & $\begin{array}{l}\text { Postmastectomy } \\
\text { irradiation of } \\
\text { the chest wall } \\
\text { and regional } \\
\text { nodes for high- } \\
\text { risk cases** }\end{array}$ & & & oo \\
\hline 3 Enhanced & $\begin{array}{l}\text { Sentinel biopsy }{ }^{++} \text {, } \\
\text { Breast reconstruc- } \\
\text { tion }\end{array}$ & \begin{tabular}{|l|} 
Breast- \\
conserving \\
whole-breast \\
irradiation \\
\end{tabular} & Taxanes & $\begin{array}{l}\text { Aromatase } \\
\text { inhibitors } \\
\text { LH-RH } \\
\text { agonists } \\
\end{array}$ & $\begin{array}{l}\text { Trastuzumb } \\
\text { for HER2- } \\
\text { pos. disease }\end{array}$ \\
\hline 4 Maximal & & & \begin{tabular}{|l|} 
Growth \\
factors \\
dose-dense \\
chemother.
\end{tabular} & & \\
\hline
\end{tabular}

Abbreviations and general comments see also Table 2. ${ }^{* *}$ Chest wall and regional lymph node irradiation substantially decreases the risk of post-mastectomy local recurrence. If available, it should be used as a basic-level resource. (Adapted from Anderson et al. 2008. Reproduced with permission of Wiley-Less, Inc., a subsidiary of John Wiley \& Sons, Inc.)

The same applies to locally advanced (Table 4) or metastatic breast cancer: surgery will be widely available, probably tamoxifen and/or ovarian ablation (Fig. 4 and 5). Chemotherapy should carefully be considered taking the palliative situation into account. The balance of effect, side effects, safety and costs must be maintained [Eniu et al., 2006]. A national consensus has to be found which should be treated with priority! 


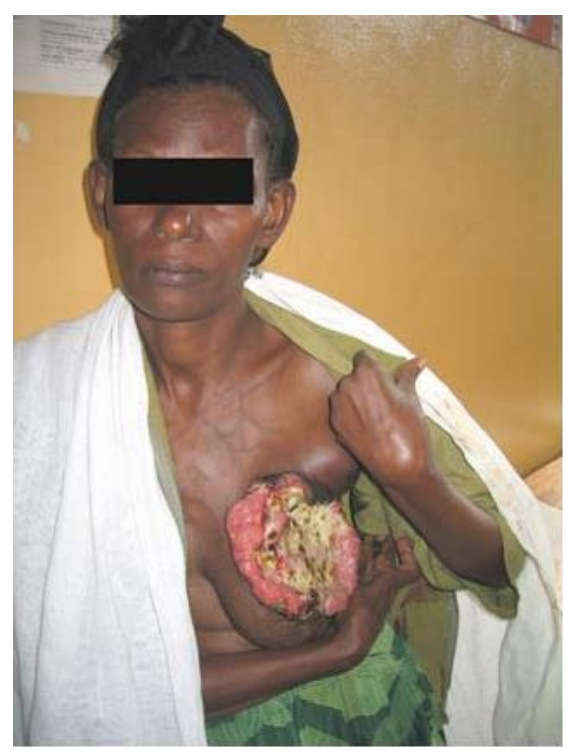

Figure 4: Patient with locally advanced breast cancer (picture courtesy of Erik Erichsen).

Table 4: Therapy for locally advanced breast cancer for different levels (adapted from BHGI: Treatment resource allocation table for locally advanced breast cancer).

\begin{tabular}{|c|c|c|c|c|c|}
\hline Level & Surgery & Radiation & Chemotherapy & Endocrine & Biological \\
\hline 1 Basic & $\begin{array}{l}\text { Modified } \\
\text { radical } \\
\text { mastectomy }\end{array}$ & ** & $\begin{array}{l}\text { Pre-operative } \\
\text { chemotherapy } \\
\text { with EC, FAC } \\
\text { or CMFo }\end{array}$ & $\begin{array}{l}\text { Oophorecto- } \\
\text { myin premeno- } \\
\text { pausal women }\end{array}$ & \\
\hline 2 Limited & & $\begin{array}{l}\text { Postmastectomy } \\
\text { irradiation of the } \\
\text { chest wall and } \\
\text { regional nodes** }\end{array}$ & & & oo \\
\hline 3 Enhanced & $\begin{array}{l}\text { Breast- } \\
\text { conserving } \\
\text { surgery, } \\
\text { Breast re- } \\
\text { construction }\end{array}$ & $\begin{array}{l}\text { Breast- } \\
\text { conserving } \\
\text { whole-breast } \\
\text { irradiation }\end{array}$ & Taxanes & $\begin{array}{l}\text { Aromatase } \\
\text { inhibitors } \\
\text { LHRH } \\
\text { agonists }\end{array}$ & \\
\hline 4 Maximal & & & $\begin{array}{l}\text { Growth factors } \\
\text { dose-dense } \\
\text { chemotherapy }\end{array}$ & & $\begin{array}{l}\text { Trastuzumab } \\
\text { for HER2- } \\
\text { pos. disease }\end{array}$ \\
\hline
\end{tabular}

Abbreviations and general comments see also Table 2 and 3. (Adapted from Anderson et al. 2008. Reproduced with permission of Wiley-Less, Inc., a subsidiary of John Wiley \& Sons, Inc.) 

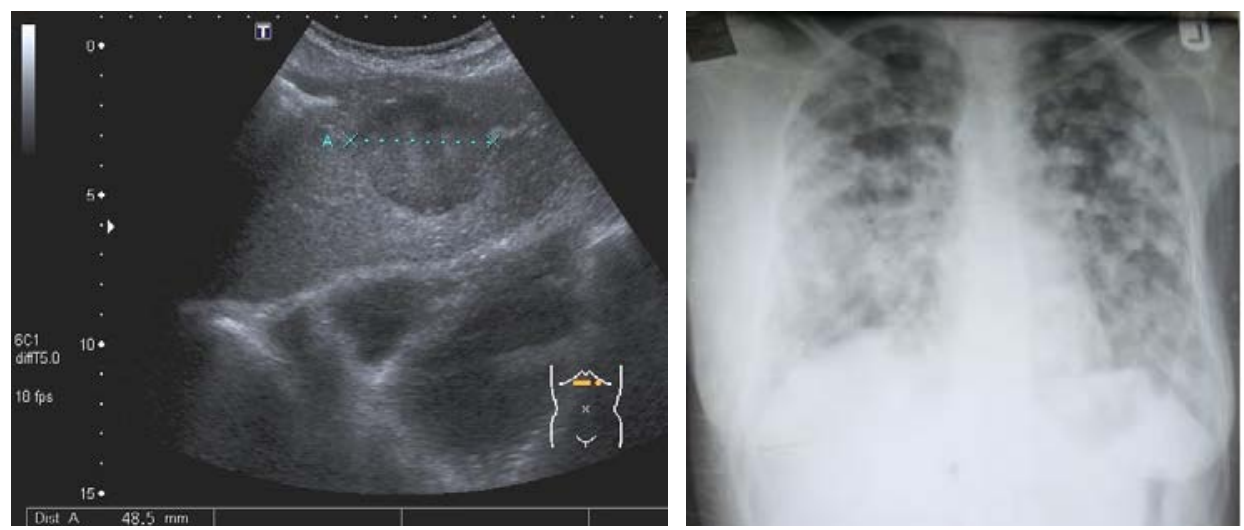

Figure 5: Hepatic (left) and pulmonary (right) metastasis from breast cancer (pictures courtesy of Hans Strauß).

\section{Politics and health care system}

Prior to any intervention concerning breast cancer, the availability of a populationbased cancer registry including data on the cause of death should be guaranteed [European Commission, 2000]. In most countries it is still absent, and thus, there is a need to create tools to measure the effects of health outcome. Policy guidelines for treatment of cancer are given by WHO [WHO, 2002]. The question is whether the treatment of cancer will be available in the private sector for selected people or whether there are enough reasons to make it a national health care priority [WHO, 2005].

Health care professionals accumulate in places with higher income - like bigger cities, financially strong neighboring countries or in developed countries. In countries with limited resources, insufficient salaries, a lack of career opportunities, lack of infrastructure or awkward living conditions make it difficult to attract and keep professionals, especially in remote areas where health care is needed. The number of nurses per inhabitant varies 200-fold worldwide [WHO, 2004]. Migration is a major problem - from rural to urban, from public to private, from poor to rich countries. The government could try to support centers of excellence according to the specific burden of breast cancer and the national resources for health - having a gynaecologic department where doctors have increasing possibilities to provide health care to the affected patients. Necessary skills, instruments, a pathologist as well as possible radiology and chemotherapy facilities should be available.

Implementing a new health care program needs political will and a strategy. New structures can be developed or breast care can be integrated in existing programs [Victoria et al., 2004]. Cost-effectiveness is a major factor to be considered. So far, not much data on the possibilities and calculations in these countries with 
limited resources is available. Therefore, it is most important that resource allocation is done in a reasonable manner consistent with the setting and not for prestige or other reasons [Anderson et al., 2006].

Governmental as well as non-governmental organizations should be involved. Private-public partnership is an emerging approach in recent years. An example of a non-governmental organisation is the famous Cancer Centre in Egypt, $140 \mathrm{~km}$ east from Cairo [Omar et al., 2003]. Noticing an increase in the prevalence of breast and urinary bladder cancer, volunteers started a charity clinic in 1988. Today $\mathrm{x}$-ray, ultrasound, mammography, image-guided biopsies, pathologic examination, endoscopies, surgery, radiotherapy and chemotherapy are possible. Over 15 years nearly 140,000 patients have been seen, and 20,000 have been admitted. Support is given by WHO, occasional government grants and USAID for research. Programs for public awareness are conducted including door to door visits.

\section{Perspectives for transnational collaboration}

Breast cancer will continuously increase in numbers all over the world. The highest burden will be in countries with limited resources. Up to now, the WHO has proposed general guidelines for the treatment of cancer. The BHGI has issued an updated guideline using current literature to give recommendations depending on the amount of resources.

More evidence-based research is needed from basic epidemiology up to efficient treatment in environments other than Europe or USA. This task must be put into the hands of the scientific community to be fulfilled and partnerships between developed and developing countries are necessary. Pharmaceutical companies will not take the challenge to implement clinical trials with huge costs in countries with a small market for the product later on. Organizations like the WHO, the European Commission and others must fill this gap.

A health care system must adapt to local resources and find out the most effective interventions for women affected by breast cancer. A good possibility is a partnership between national societies of gynaecologists to prepare strategies. A partnership has been established between the German Society of Obstetrics and Gynaecology (DGGG) and the Ethiopian Society of Obstetrics and Gynaecology (ESOG) through the DGGG working group International Women's Health (AG FIDE: www.ag-fide.de). In that way, transnational projects can be carried out for research and treatment as steps to global improvement.

The University of Addis Ababa and the University of Halle have as well implemented research collaboration on breast and gynaecologic cancer. Physicians and medical students have experienced great benefit in working together on questions of mutual interest. 


\section{References}

Adams J, White M, Forman D: Are there socioeconomic gradients in stage and grade of breast cancer at diagnosis? Cross sectional analysis of UK cancer registry data. 2004.

Albert AS 2003: Breast cancer in limited-resource countries: Early detection and access to care. The Breast Journal; 9, Suppl 2: S90-S93.

Anderson BO, Shyyan R, Eniu A, Smith RA, Yip CH, Bese NS, Chow LWC, Masood S, Ramsay S, Carlson RW: Breast cancer in limited-resource countries: An overview of the Breast Health Global Initiative 2005 Guidelines. The Breast Journal 2006; 12 Suppl. 1: 3-15.

Anderson BO, Yip CH, Ramsey SD, Bengoa R, Braun S, Fitch M, Groot M, Sancho-Garnier H, Tsu VD: Breast cancer in limited-resource countries: Health care systems and public policy. The Breast Journal 2006; 12 Suppl.1: 54-69.

Bray F, Ren JS, Masuyer E, Ferlay J. Global estimates of cancer prevalence for 27 sites in the adult population in 2008. Int J Cancer. 2013; 132: 1133-45.

Dein S: Explanatory models of an attitudes towards cancer in different cultures. Lancet Oncol 2004; 5: 119-24.

Duffy SW, Tabar L, Vitak B, Warwick J: Tumor size and breast cancer detection: What might be the effect of a less sensitive screening tool than mammography? The Breast Journal 2006; 12 Suppl.1: 91-95.

Elkin EB, Hudis C, Begg CB, Schrag D: The effect of changes in tumor size on breast carcinoma survival in the U.S.: 1975-1999. Cancer 2005; 104: 472-475.

Eniu A, Carlson RW, Aziz Z, Bines J, Hortobágyi GN, Bese NS, Love RR, Vikram B, Kurkure A, Anderson BO: Breast cancer in limited-resource countries: Treatment and allocation of resources. The Fred Hutchinson Cancer Research Center. The Breast Journal 2006; Volume 12 Suppl. 1: 38-53.

European Commission: Evaluation and monitoring of screening programmes. Luxembourg: European Commission, Europe Against Cancer Programme, 2000 .

Ferlay J, Bray F, Pisani P, Parkin DM: GLOBOCAN 2000: Cancer incidence, mortality and prevalence worldwide. IARC cancer base No. 5. Lyon, France: IARC 2001.

Huo D, Ikpatt F, Khramtsov A, Dangou JM, Nanda R, Dignam J, Zhang B, Grushko T, Zhang C, Oluwasola O, Malaka D, Malami S, Odetunde A, Adeoye AO, Iyare F, Falusi A, Perou CM, Olopade OI. Population differences 
in breast cancer: survey in indigenous African women reveals over-

representation of triple-negative breast cancer. J Clin Oncol. 2009; 27: 4515-21.

Lannin DR, Mathews HF, Mitchell J, Swanson MS: Impacting cultural attitudes in African-American women to decrease breast cancer mortality. Am J Surg 2002; 184: 418-423.

Nyström L, Andersson I, Bjurstam N, Frisell J, Nordenskjold B, Rutqvist LE: Long-term effects of mammography screening: updated overview of the Swedish randomised trials. Lancet 2002; 359: 909-19.

Omar S, Khaled H, Gaafar R, Zekry AR, Eissa S, el-Khatib O: Breast cancer in Egypt: a review of disease presentation and detaction strategies. East Mediterr Health J 2003; 9; 448-463.

Parkin DM: Cancer in developing countries. Cancer Surveys 1994; 19/20: 519-561.

Sankaranarayanan, R.; Sauvaget, C.; Ramadas, K.; Ngoma, T.; Teguete, I.;

Muwonge, R. et al. : Clinical trials of cancer screening in the developing world and their impact on cancer healthcare. In: Ann Oncol 2011; 22 Suppl 7: 20-28.

Sankaranarayanan R, Swaminathan R, Brenner H, Chen K, Chia KS, Chen JG, Law SC, Ahn YO, Xiang YB, Yeole BB, Shin HR, Shanta V, Woo ZH, Martin N, Sumitsawan Y, Sriplung H, Barboza AO, Eser S, Nene BM, Suwanrungruang K, Jayalekshmi P, Dikshit R, Wabinga H, Esteban DB, Laudico A, Bhurgri Y, Bah E, Al-Hamdan N. Cancer survival in Africa, Asia, and Central America: a population-based study. Lancet Oncol. 2010; 11: 165-73.

Shyyan R, Masood S, Badwe RA, Errica KM, Liberman L, Ozmen V, Stalsberg $\mathrm{OH}$, Vargas H, Vass L: Breast cancer in limited-resource countries: Diagnosis and pathology. The Fred Hutchinson Cancer Research Center. The Breast Journal 2006; 12 Suppl. 1: 24-37.

Smith AR, Caleffi M, Albert US, Chen THH, Duffy SW, Franceschi D, Nyström L: Breast cancer in limited-resource countries: early detection and access to care. The Breast Journal 2006; 12 Suppl. 1: 16-26.

Smith RA, Duffy SW, Gabe R, Tabar L; Yen AM; Chen TH: The randomized trials of breast cancer screening: what have we learned? Radiol Clin North Am 2004; 42: 793-806.

Thomas DB, Gao DL, Ray RM, Wang WW, Allison CJ, Chen FL, Porter P, Hu YW, Zhao GL, Pan LD, Li W, Wu C, Coriaty Z, Evans I, Lin MG, Stalsberg H, Self SG. Randomized trial of breast self-examination in Shanghai: final results. J Natl Cancer Inst. 2002; 94: 1445-57.

Victoria CG, Hanson K, Bryce J, Vaughan JP: Achieving unicersal coverage with health interventions. Lancet 2004; 364: 1541-1548. 
World Health Organisation. Global Health Atlas, 2004. Available at http://www.who.international/globalatlas/DataQuery/default.asp;accessed October 3,2005.

World Health Organisation: Maternal Mortality in 2005. Estimates developed by WHO, UNICF, UNFPA, and The World Bank. WHO, Geneva 2007

World Health Organisation: The World Health Report 2003. Shaping the future. WHO, Geneva 2003

World Health Organization: Cancer prevention and control. Provisional agenda item 13.12. 58th World Health Assembly, Geneva, Switzerland May 16-25, 2005.

World Health Organization: Executive summery. National cancer control programmes: Policies and managerial guidelines. Geneva, Switzerland: World Health Organization 2002:i-xxiv. 



\section{List of Authors}

The Authors of this book are coming from the following 14 countries: Cameroon, Cote d'Ivoire, Ethiopia, Germany, Ghana, Indonesia, Kenya, Nigeria, Rwanda, Sweden, Switzerland, Tanzania, Thailand, and the Republic of Benin.

Adamu, Addissie. Ass. Professor, MD, PPH, MA. (Ethiopia)

Addis Ababa University, School of Public Health, Department of Preventive Medicine, Epidemiology Unit. Specialization: Epidemiology. Interests: NCDs, Ethics, HI, E-Mail: adamuaddis@yahoo.com

Adeoti, Razack. PhD. (Republic of Benin)

International Institute for Tropical Agriculture (IITA), Benin. Specialization: Agricultural Economist. Interests: Impact assessment of technological changes on food security and poverty reduction. E-Mail: r.adeoti@cgiar.org

\section{Adeyemo,Temitayo. Mrs. (Nigeria)}

University of Ibadan, Department of Agricultural Economics, Ibadan.Specialization: Rural Development. Interests: Food Security, Gender, Health, Natual Resources. E-Mail: adeyemotemitayo@gmail.com, temmytee11@yahoo.com 
Afful-Mensah, Gloria. Mrs. (Ghana)

University of Professional Studies, Department of Banking and Finance, Accra. Specialization: Health Economics, Public Sector Economics and Agricultural Economics. Special Interest: Economics of Child and Maternal Health Care Utilization, Economic Growth and Developmental Issues, Food Security, Social Protection and Safety Nets. E-Mail: gaffulmensah@yahoo.com: gloriaaffulmensah@gmail.com

\section{Al Audhah, Nelly. Dr. M.Sc (Indonesia)}

Lambung Mangkurat University, Medical Faculty, Banjarmasin. Specialization: Parasitogy. Interests: Malaria, Scabies, Tropical Medicine. E-mail: Audhah_fkunlam@yahoo.co.id

\section{Anika, Silva. Prof. Dr. (Nigeria)}

University of Nigeria, Faculty of Veterinary Medicine, Department of Physiology and Pharmacology, Nsukka. Specialisation: Environmental Toxicology. Interests: Trypanosomiasis, Phytopharmacology, Polution. E-Mail: silvanik@yahoo.com

\section{Asenso Barnieh, Beatrice. Mrs. (Ghana)}

Ghana Education Service, Nkawie-Kumasi. Specialisation: Public Health. Interests: Nutrition and Health. E-Mail: Kod222@yahoo.com

\section{Bogale, Solomon. MD. (Ethiopica)}

Radiotherapy Center, Addis Abeba University. Specialization: Radio-Oncology. Interests: Breast Cancer. E-Mail: bsolomond@ethionet.et

\section{Chalid, Maisuri T. Dr.MD. (Indonesia)}

University of Hasanuddin Makassar. Specialization: Obtetrics and Gynecology, Maternal Fetal Medicine. Interests: Preeclampsia, Tropical diseases in pregnancy. E-Mail: maisurichalid@yahoo.com, or maisurichalid@gmail.com.

\section{Chigbu, Chibuike O. Dr. (Nigeria)}

University of Nigeria Enugu Campus, Obstetrics and Gynaecology, Enugu. Specialization: Obstetrics and Gynaecology. Interests: Global Health, Gynaecologic Oncology, Reproductive Health. E-Mail: chchigbu@yahoo.com

\section{Christoph, Patrizia. Dr. med. (Switzerland)}

Universitätsfrauenklinik Bern Inselspital, Bern. Specialization: Obstetrics and Gynaecology. Interests: Fetomaternal Medicine, Global Health. E-Mail: Patricia.christoph@web.de 
Dayie, Nicholas T.K.D. Mr. (Ghana)

University of Ghana Medical School, Department of Microbiology, Accra. Specialization: Medical Microbiology. Interests: Monitoring antibiotic resistance issues in developing countries and developing alternative antimicrobial agents from natural products. Organisms of interest are Streptococcus pneumoniae, Group B Streptococci and Enterococcus faecalis and faecium. E-Mail: nicholasdayie@yahoo.com

Djouaka, Rousseau. PhD. (Republic of Benin)

International Institute for Tropical Agriculture (IITA), Benin. Specialization: Cell Biologist. Interests: Tropical Diseases Research. E-Mail: r.djouaka@cgiar.org

Fening, Ken Okwae. Dr. (Ghana)

University of Ghana, Soil and Irrigation Research Centre, Instititute of Agricultural Research, College of Agriculture and Consumer Sciences. Specialization: Agricultural Entomology. Interests: Integrated Pest Management, Food Safety and Public Health, Organic farming and Stored Products Entomology. E-Mail: fokwae@daadalumni.de

Fening, Opoku Joseph. PhD. (Republic of Ghana)

ICSIR - Soil Research Institute, Kumasi Ghana Specialization: Soil and Environmental Sciences. Interests: Soil Sciences. E-Mail: kofifening@yahoo.com

\section{Flora, Rostika, S.Kep, M.Kes, DR. (Indonesia)}

Universitas Sriwijaya, RSUP Moh. Hoesin, Medical Physiology, Palembang. Specialization: Medical Physiology. Interests: Malaria, Plasmodium vivax, PregnancyRelated Malaria. Email: rostikaflora@gmail.com

Fokam, Eric B. Mr. (Cameroon)

University of Buea, Department of Zoology and Animal Physiology, Faculty of Science, Buea, Specialization: Medical and Veterinary Entomology. Interests: Ecology and Mechanisms of Emergence of Arboviral Infections. E-mail: efokam@daad-alumni.de

\section{Ganyaglo, Gabriel Yao-Kumah. Dr. (Ghana)}

Korle Bu Teaching Hospital, Department of Obstetrics and Gynaecology, Accra. Specialization: Obstetrics \& Gynaecology. Interests: Global Health, Urogynaecology. E-Mail: gganyaglo@hotmail.com

\section{Geerdes-Fenge, Hilte. Dr. (Germany)}

University Medical Center Rostock, Dept. of Tropical Medicine and Infectious Diseases, Rostock. Specialization: Medicine, Infectious Diseases. Interests: Tuberculosis, Pneumonia, Sepsis, Public Health. E-Mail: hilte.geerdes-fenge@unirostock.de 
Ghiffari, Ahmad. Dr. M.Sc. (Indonesia)

Universitas Sriwijaya, RSUP Moh.Hoesin, Medical Parasitology, Palembang. Specialization: Medical Parasitology. Interests: Malaria, Entomology, Ecology. E-Mail: dokter.ghi@gmail.com, ahmad.ghiffari@senckenberg.de

\section{Goergen, Georg PhD. (Republic of Benin)}

International Institute of Tropical Agriculture (IITA), Benin. Specialization: Insect Systematics. Interests: Insects morphological identification. E-Mail: G.Goergen@cgiar.org

Grade, Matthias. Dr. med. DTM\&H FEBG. (Germany).

Christliches Krankenhaus Quakenbrück, Specialization: Gastroenterology/Hepatology, Infectious Diseases and Tropical Medicine. Interests: Clinical Tropical Medicine, Clinical Tropical Virology, Global Health, E-Mail: m.grade@ckq-gmbh.de, matthiasgrade@gmx.de

Groß, Uwe. Prof. Dr. (Germany)

University Medical Center Goettingen, Medical Microbiology, Goettingen. Specialization: Medical Microbiology. Interests: Toxoplasmosis, Mycoses, Campylobacteriosis, Global Health. E-Mail: ugross@gwdg.de

Günthert, Andreas. Prof. Dr. (Switzerland)

Cantonale Hospital Lucerne LUKS, Department of Obstetrics and Gynecology, Lucern. Specialization: Gynecology. Interests: Gynecology, Gyneco-Oncology, Senology and Breast Cancer. E-Mail: Andreas.guenthert@luks.ch

\section{Hanson, Claudia. MD, MSc IH\&MSc Epidemiology (Sweden)}

Karolinska Institute, Global Health, Stockholm. Specialization: Gynecologist. Interests: Quality Assurance. E-mail: Claudia.hanson@ki.se

\section{Hasanuddin. M.D (Indonesia)}

Medical Faculty of Syiah Kuala University Banda Aceh, Department of Obstetrics and Gynecology, Division of Gynecologic Oncology, Banda Aceh. Specialization: Gynecologic Oncology. Interests: Cancer in Pregnancy. E-Mail: hasan.spog@yahoo.co.id

Ichsan. M.D. M.Sc. (Indonesia)

Syiah Kuala University Banda Aceh, Medical Faculty. Banda Aceh. Specialization: Clinical Microbiology. Interests: Candidiasis, Tuberculosis. E-Mail: ichsanmd_aceh@yahoo.com 
Jahn, Gerhard. Prof. Dr. (Germany)

University Hospital Tuebingen, Institute of Medical Virology and Epidemiology of Viral Disease, Tuebingen. Specialization: Medical Virology. Interests: Cytomegalovirus, Antiviral Resistance, Viral Infection in Pregnancy, Immunotherapy. E-Mail: Gerhard.jahn@med.uni-tuebingen.de

\section{Kamau-Mbuthia, Elizabeth. Dr. (Kenya)}

Egerton University, Department of Human Nutrition, Egerton. Specialization: Human Nutrition. Interests: Maternal/Child Nutrition, Infant Feeding, Food Security.E-Mail: ekambu@yahoo.com

\section{Kamgang, Guy Richard. MSc. (Republic of Cameroon)}

University of Abomey - Calavi (UAC), Benin. Specialization: Hydrologist. Interests: Water management, Environmental Impact Assessment. E-Mail: guykamgang@yahoo.fr

\section{Kantelhardt, Eva Johanna. Dr. Med. (Germany)}

Martin Luther University Halle, Department of Gynecology, Halle (Saale). Specialization: Gynecology. Interests: Gynecologic Oncology and Breast Cancer. E-Mail: Eva.kantelhardt@medizin.uni-halle.de

\section{Krüger, Carsten. Dr. med., MIH, FRCPCH (Germany)}

St. Franziskus Hospital, Department of Paediatrics, Ahlen. Specialization: Paediatrics, Neonatology. Interests: Global Child Health, Neonatology in Low-Income Countries, Immunisation, Infectious Diseases, Quality of Care. E-Mail: thea.carsten.krueger@web.de

\section{Kusumawati, R.L. SpMK(K), M. Biomed, Dr. (Indonesia)}

University of Sumatera Utara, Faculty of Medicine, Department of Microbiology, Medan. Specialization: Medical Microbiology. Interests: Tuberculosis, Dengue, Mycosis. E-Mail: liaiswara@yahoo.com

\section{Loebermann, Micha. Dr. med. (Germany)}

University of Rostock, Department of Tropical Medicine, Infectious Diseases and Nephrology. Specialisation: Infectious Diseases, Tropical Medicine, Antibiotic Stewardship. Interests: Viral Hepatitis, HIV, Schistosomiasis, Vaccines. Email: micha.loebermann@uni-rostock.de

\section{Makoi, Andrew. Dr. (Tanzania)}

SIKIKA Company LTD (a health advocacy NGO), Dar es Salaam. Specialization: Public Health, Global Health, Quality Control, Quality testing of medicines circulating in the country including Oxytocin used in reduction of post-partum haemorrhage (PPH), Human Resources for Health (HRH), Hospital Management. E-Mail: amakoi2001@yahoo.com 
Massi, Muhammad Nasrum. Prof. Dr. (Indonesia)

University of Hasanuddin, Medical Microbiology, Makassar. Specialization: Medical Microbiology. Interests: Tuberculosis. E-mail: Nasrumm2000@yahoo.com

Mathewos, Assefa. MD. (Ethiopia)

Radiotherapy Center, Addis Abeba University, Addis Abeba. Specialization: RadioOncology. Interests: Radio-Oncology. E-Mail: mathewosassefa80@hotmail.com

Meister, Josephine. M.A. (Germany)

Göttingen International Health Nework, University Medical Center Göttingen, University of Göttingen. Specialization: Political Science. Interests: Global Health, Neglected Tropical Diseases. E-Mail: josephine.meister@hotmail.de

\section{Moturi, Wilkister Nyaora. Dr. (Kenya)}

Egerton University, Department of Environmental Science, Egerton. Specialization: Environmental Health. Interests: Child Environmental Health, Water, Sanitation and Hygiene, Geochemistry and Health. E-Mail: moturi33@yahoo.com

\section{Msuya, John. Prof. (Tanzania)}

Sokoine University of Agriculture (SUA), Department of Food Science \& Technology, Morogoro. Specialization: Nutrition in Development Economics. Interests: Food Security: E-Mail: j_msuya@yahoo.com

Muljono, David H. Prof. Dr. MD, PhD. (Indonesia)

Eijkman Institute for Molecular Biology, Jakarta. University of Hasanuddin Makassar. Sydney Medical School, University of Sydney, Sydney. Specialization: Internal Medicine, Hepatology, Molecular Medicine. Interests: Hepatology, Viral Hepatitis, Emerging Diseases. E-Mail: dhmuljono@gmail.com, davidhm@eijkman.go.id

\section{Muthoka, Stellamaris. Dr. (Kenya)}

Egerton University, Department of Human Nutrition, Egerton. Specialization: Human Nutrition. Interests: Applied Human Nutrition, Rights to Food, Maternal and Child Health and Nutrition. E-Mail: skaveni@yahoo.com

Ndu, Pat Obiageli. Dr. (Nigeria)

University of Nigeria, Enugu Campis, Center for Environmental Management and Control (CEMAC). Specialization: Environmental Health, Public Health with Food Hygiene Bias. Interests: Water and Sanitation, Maternal and Child Health, Food Hygiene. Email: adaikenga@yahoo.co.uk

Ngotho, Douglas. Dr. (Kenya)

Egerton University, Dept of Reproductive Health, Egerton. Specialization: Reproductive Health. Interests: Maternal and Child Health, HIV/AIDS, Family Planning. E-Mail: douglasngotho@yahoo.com 
Nirwati, Hera. Dr, M.Kes. (Indonesia)

Universitas Gadjah Mada, Faculty of Medicine, Department of Microbiology, Yogyakarta. Specialization: Medical Microbiology. Interests: Rotavirus. E-mail: hnirwati@yahoo.com

Nketiah-Amponsah, Edward. Dr. (Ghana)

University of Ghana, Department of Economics, Legon, Accra. Specialization: Healthcare Economics, Development Economics and Public Sector Economics. E-Mail: enamponsah@ug.edu.gh, enamponsah@uni-bonn.de

\section{Nkurikiyimana, Edmond. Mr. (Rwanda)}

Rwanda Ministry of Health/Kabutare District Hospital. Specialization: Hospital and Healthcare Management. Interests: Health Economics and Health Financing. E-Mail: nkuredi@yahoo.fr

\section{Noubactep, Chicgoua. PD Dr. (Germany)}

University of Göttingen, Applied Geology. Specialization: Hydrogeochemistry. Interests: Water Supply, Environmental Remediation. E-Mail: cnoubac@gwdg.de

\section{Noviyanti, Rintis, S.Si, Ph.D (Indonesia)}

Eijkman Institute for Molecular Biology, RS Cipto Mangunkusumo Jakarta. Specialization: Molecular Biology. Interests: Molecular Biology, Plasmodium falciparum, Placental Malaria. Email: rintis@eijkman.go.id

\section{Olajide, O. Adeola. Dr. (Nigeria)}

University of Ibadan, Department of Agriculture and Economics, Faculty of Agriculture and Forestry, Ibadan Oyo State. Specialization: Farming and Rural Systems Development. Interests: Gender and Development, Intra-Household Studies, Poverty and Living Standard Analysis, Food Security, Agricultural Policy Analysis, Impact or Evaluation Studies. E-Mail: preciousfunso@yahoo.com

\section{Onwasigwe, Chika. Prof. Dr. (Nigeria)}

University of Nigeria Enugu Campus/University of Nigeria Teaching Hospital, College of Medicine, Department of Community Medicine, Enugu. Specialization: Public Health, Community Medicine. Interests: Epidemiology, Biostatistics. EMail: chikaonwasigwe@yahoo.com

\section{Paulick, Jan. (Germany)}

University of Goettingen, Faculty of Economic Sciences, Göttingen. Specialization: Economics. Interests: Development Economics, Global Health, Finance EMail: jan.paulick@gmail.com 
Priosoeryanto, Bambang Pontjo. Prof. DVM, MSc, Ph.D, APVet. (Indonesia)

Bogor Agricultural University, Division of Veterinary Pathology, Department of Veterinary Clinic, Reproduction and Pathology (CRP), Faculty of Veterinary Medicine; Jalan Agatis Kampu IPB Darmaga, Bogor. Specialization: Veterinary Pathology. Interests: Cancer, Infectious Animal Diseases, Herbal Medicine, Neurodegeneration. E-mail: bpontjo4@gmail.com

Raihan. Dr. SpA. (Indonesia)

Syiah Kuala University, Faculty of Medicine, Dr. Zainoel Abidin Hospital, Department of Child Health, Banda Aceh. Specialization: Paediatrics. Interests: Dengue, Malaria, Typhoid, Paediatric Infectious and Tropical Diseases. E-Mail: raihan_rais@yahoo.com

Saptawati, Leli. Dr. Sp.MK. (Indonesia)

Faculty of Medicine, Sebelas Maret University Surakarta. Specialization: Clinical Microbiology. Interests: Bacteriology. E-Mail: llsapt_md@yahoo.co.id

Schwab, Fabienne Dominique. Dr. (Switzerland)

University of Basel, Department of Obstetrics and Gynaecology, Basel. Specialization: Obstetrics and Gynaecology. Interests: Obstetrics, Gynaecology, Global Maternal and Child Health. E-Mail: fabsch@rocketmail.com

\section{Sjahril, Rizalinda. Dr. M.Sc. PhD. (Indonesia)}

Hasanuddin University, Faculty of Medicine, Makassar. Specialization: Medical Microbiology. Interests: Japanese Encephalitis, Cytomegalovirus, Hepatitis B Chlamydiae, Helicobacter pylori. E-Mail: Rizalinda_sjahril@yahoo.com

\section{Solange Kakou Ngazoa. Ph.D (Cote d'Ivoire)}

Pasteur Institute Abidjan, Molecular Biology Unit Specialization: Molecular Biology. Interests: Tropical Diseases Research. E-Mail:ngazoa_solange@yahoo.fr

\section{Sowunmi, Akindayo Ph.D (Nigeria)}

University of Iberdan, Department of Zoology, Hydrobiology and Fisheries Unit, Ibadan. Specialization: Hydrobiology. Interests: Aquatic Biomonitoring, Aquatic Ethnozoology. E-mail: aa.sowunmi@ui.edu.ng

\section{Stein, Werner. Dr. (Germany)}

Klinikum Fulda, Frauenkklinik, Fulda. Specialization: Obstetrics and Gynecology. Interests: Maternal and Neonatal Mortality, Ultrasonography in Resource-Poor Countries, Global Health, Laproscopy. E-Mail: steinwka@t-online.de, werner.stein@klinikum-fulda.de 


\section{Surbek, Daniel. Prof. Dr. M.D. (Switzerland)}

Department of Obstetrics and Gynecology. University Hospital Insel, Bern. Specialization: Obstetrics and Gynecology, Feto-Maternal Medicine. Interests: Perinatal Stem Cells, Anemia, Preeclampsia. E-Mail: daniel.surbek@insel.ch

Tamcke, Martin. Prof. Dr. Dr. h.c. (Germany)

University of Goettingen, Department of Ecumenical Theology and Oriental Church and Mission History, Göttingen. Specialization: Intercultural Theology. Interests: Interculturality, Transculturality, Ecumenical Theology. E-Mail: mtamcke@gwdg.de

\section{Tamò, Manuele, PhD. (Republic of Benin)}

International Institute of Tropical Agriculture (IITA), Benin. Specialization: Insect Ecologist. Interests: Biological Control, Bio-pesticides. E-Mail: m.tamo@cgiar.org

\section{Tangwattanachuleeporn, Marut, Dr. (Thailand)}

Burapha University, Faculty of Allied Health Sciences, Chonburi, Specialization: Medical Microbiology. Interests: Mycoses, Immunogenetics. E-Mail: tp.marut@gmail.com

\section{Tariku, Wakuma. MD (Ethiopia)}

Aira Hospital, Wollega. Specialization: Surgery. Interests: Breast Cancer. E-Mail: tarwakuma@yahoo.com

Tchigossou Genevieve. MSc. (Republic of Benin).

International Institute for Tropical Agriculture (IITA), Benin. Specialization: Cell Biologist. Interests: Tropical Diseases Research. E-Mail: tchigossougenevieve@yahoo.fr

\section{Tiuria, Risa. Ph.D. MSc. DVM. (Indonesia)}

Bogor Agricultural University, Division of Veterinary Parasitology; Department of Animal Infectious Animal Diseases and Veterinary Public Health (IPHK), Faculty of Veterinary Medicine, Bogor. Specialization: Veterinary Parasitology. Interests: Helminths, Imunoparasitology, Mucosal Immunity, Zoonotic Parasites. E-Mail: risatiprio@gmail.com

\section{Vollmer, Sebastian. J-Prof. Dr. (Germany)}

University of Göttingen, Department of Economics, Göttingen. Specialization: Economics. Interests: Development Economics, Population Economics, Global Health, Poverty and Inequality. E-Mail: svollmer@uni-goettingen.de 
Widad, Shofwal. Dr. SpOG.KFER. (Indonesia)

Gadjah Mada University, Dr. Sardjito Hospital, Department of Obstetrics and Gynaecology, Yogyakarta. Specialization: Obstetrics and Gynaecology. Interests: Reproductive Health, Reproductive Endocrinology and Infertility, Endometriosis, Minimally Invasive Surgery. E-Mail: sh_widad@yahoo.com

\section{Wydra, Kerstin. Prof. Dr. (Germany)}

Erfurt University of Applied Sciences, President, Erfurt. Specialization: Plant Pathology. Interests: Sustainable Development and Resource Management, Interdisciplinary Approach to “One Health”, E-Mail: kerstin.wydra@fh-erfurt.de

Yadi. MD. M.Si. (Indonesia)

Mulawarman University, Department of Microbiology, Samarinda. East Kalimantan. Specialization: Medical Microbiology. Interests: Salmonellosis, Tuberculosis, Leprosy. E-Mail: dryadi02@yahoo.com

\section{Yessoufou Akadiri, PhD. (Republic of Benin)}

Université de Abomey-calavi. Faculté des Sciences et Techniques, Département de Biochimie et de Biologie Cellulaire. Specialization: Immunology. Interests: Tropical Diseases Research. E-Mail: yeskad2001@yahoo.fr 
$M$ aternal-Child Health is one of the greatest challenges the world has to cope with today. Every year, thousands of women, newborns and children die unnecessarily, particularly in resource-poor settings. There is a great disparity caused by food insecurity and hunger, environmental health risks, sanitation challenges, cultural barriers and non-accessibility to diagnosis and treatment. "Maternal-Child Health: Interdisciplinary Aspects within the Perspective of Global Health" addresses these issues. The contributions of this book are based on the ONE HEALTH concept by focusing on infectious and non-communicable diseases and to present interdisciplinary views from more than 60 authors who come from 14 countries. The aim is to shape our understanding on Maternal-Child Health Solutions by looking at $\bullet$ agricultural and environmental - economic, social and theological • biomedical and nutritional - clinical human and veterinary as well as - epidemiology and - public health expertise. The Göttingen International Health Network is corresponding to a variety of different geographic regions and programs to improve global health perspective and health of the most vulnerable: mothers and their children. 